\title{
Symmetric Homotopy Theory for Operads and
}

\author{
Weak Lie 3-Algebras
}

\begin{abstract}
Dissertation
zur Erlangung des mathematisch-naturwissenschaftlichen Doktorgrades

Doctor rerum naturalium der Georg-August-Universität Göttingen

im Promotionsstudiengang Mathematical Sciences

der Georg-August University School of Science (GAUSS)
\end{abstract}

vorgelegt von

Malte Dehling

aus Boston, Massachusetts

Göttingen, 2020 


\section{Betreuungsausschuss}

Erstbetreuerin: $\quad$ Prof. Dr. Chenchang Zhu

Mathematisches Institut

Georg-August-Universität Göttingen

Zweitbetreuer:

Prof. Dr. Thomas Schick

Mathematisches Institut

Georg-August-Universität Göttingen

\section{Mitglieder der Prüfungskommission}

Referentin:

Prof. Dr. Chenchang Zhu

Mathematisches Institut

Georg-August-Universität Göttingen

Korreferent:

Prof. Dr. Bruno Vallette

Institut Galilée

Université Sorbonne Paris Nord

Weitere Mitglieder:

Prof. Dr. Madeleine Jotz Lean

Mathematisches Institut

Georg-August-Universität Göttingen

Prof. Dr. Russell Luke

Institut für Numerische und Angewandte Mathematik

Georg-August-Universität Göttingen

Prof. Dr. Victor Pidstrygach

Mathematisches Institut

Georg-August-Universität Göttingen

Prof. Dr. Thomas Schick

Mathematisches Institut

Georg-August-Universität Göttingen

Tag der mündlichen Prüfung: 16. November 2020 




\section{Contents}

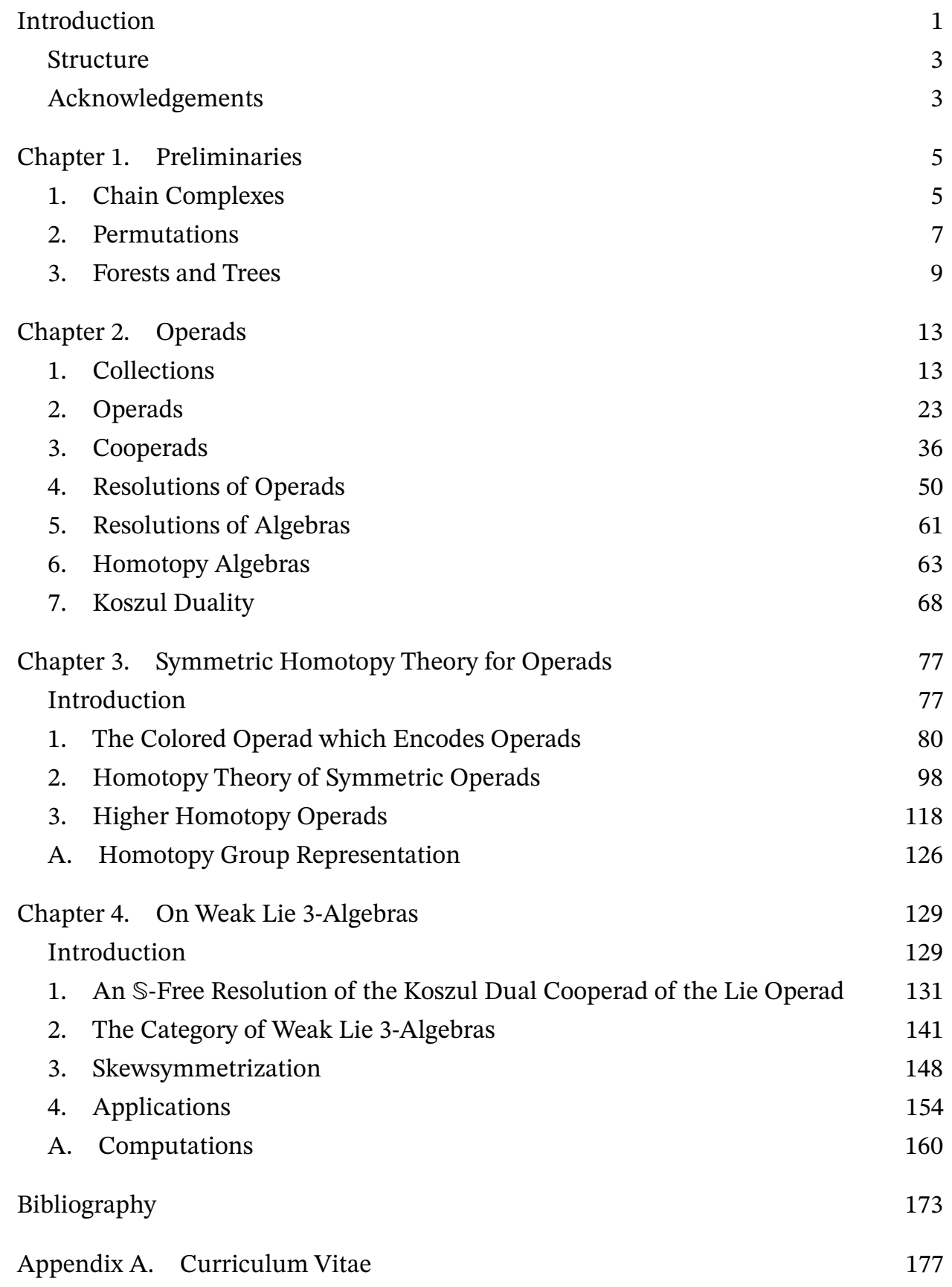





\section{Introduction}

The study of algebraic structures up to homotopy combines the fields of algebra and homotopy theory. The objects of study are types of algebras and their invariance properties with respect to certain homotopy operations on their underlying spaces. In our setting, the underlying base category of spaces is a symmetric monoidal model category $\mathrm{C}$, and the algebraic structures considered are algebras over an operad $\mathcal{P}$ in $\mathrm{C}$. Operads model many input-single output operations and their composition and are therefore suitable to describe many of the classical types of algebras, e.g., associative, commutative, and Lie algebras (see [46, 51]).

In general, algebras over an operad are rigid structures, meaning they do not play nice with homotopy operations on their underlying spaces. However, algebras over some operads $Q$ do have good homotopy properties; this is the case in particular over those that are cofibrant in the model structure on operads in C (see [5, 28, 29]). This model structure exists under some assumptions on the underlying model category $C$ and some restrictions on the operads (see op. cit. for details). For such a cofibrant operad $Q$, we can also equip its category $Q$-Alg of $Q$-algebras with a model structure, and in this category a version of the Boardman-Vogt homotopy invariance property holds: given a homotopy equivalence of cofibrant-fibrant spaces $X, Y$ in C, a structure of $Q$-algebra on either induces a homotopy equivalent $Q$-algebra structure on the other [5. Theorem 3.5].

We will often be interested in the homotopy category of $Q$-algebras, i.e., the localization Ho $Q$-Alg $=\mathcal{Q}-\operatorname{Alg}\left[W^{-1}\right]$ with respect to the class $W$ of weak equivalences. An isomorphism $A \rightarrow A^{\prime}$ in the homotopy category is a zigzag of weak equivalences in the category $Q$-Alg,

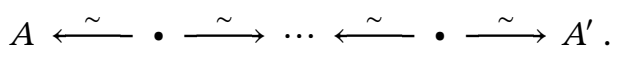

Given cofibrant (resp. fibrant) replacement functors $Q$ (resp. $R$ ) on the category $Q$-Alg, it is a consequence of a general result on model categories (see, e.g., [32. Theorem 1.2.10]) that

$$
\operatorname{Hom}_{\text {Ho Q-Alg }}\left(A, A^{\prime}\right) \cong \operatorname{Hom}_{\text {Q-Alg }}\left(Q A, R A^{\prime}\right) / \sim_{h},
$$

where the relation $\sim_{h}$ is homotopy of morphisms. We call a morphism $Q A \rightarrow R A^{\prime}$ of $Q$-algebras a homotopy morphism from $A$ to $A^{\prime}$ and denote it by $A \rightsquigarrow A^{\prime}$.

In this thesis, we work in the differential graded framework. The category $\mathrm{C}$ will be the category of chain complexes equipped with the standard projective model structure, i.e., weak equivalences are quasi-isomorphisms, fibrations are degreewise epimorphisms, and cofibrations are determined by the left lifting property with respect to acyclic fibrations. This model structure can be transferred to give model structures 
on dg $\$$-modules, dg operads, and dg algebras over a dg operad by defining the weak equivalences (resp. fibrations) to be those maps that are weak equivalences (resp. fibrations) on all underlying chain complexes. The cofibrations are then again determined by their lifting property. Note that the lifting property defining cofibrations depends on the structures, not just the underlying chain complexes. In particular, while it is clear from the above definition that all operads in C are fibrant, cofibrancy is an entirely different question.

On the category of augmented dg operads, we have functorial cofibrant resolutions given by the counit $\Omega \mathrm{B} \mathcal{P} \sim \mathcal{\rightarrow} \mathcal{P}$ of the cobar-bar adjunction

$$
\Omega: \text { conil dg Coopd } \rightleftharpoons \text { aug dg Opd : B , }
$$

provided the operad $\mathcal{P}$ in question is already $\mathbb{S}$-cofibrant, i.e., cofibrant in the underlying category of $\mathbb{S}$-modules. When working with chain complexes over a field $\mathbf{k}$ of characteristic 0 , this $\mathbb{S}$-cofibrancy condition is always satisfied. A downside of such cobar-bar resolutions is that they are very large, and therefore algebras over them have many generating operations. However, one of the main breakthroughs in the theory of operads is the development of Koszul duality for operads [24, 25], which provides us with an explicit construction of a small cooperad $\mathcal{P}^{i}$ as a candidate to replace the bar construction $\mathrm{B} \mathcal{P}$ in the cobar-bar resolution. The cooperad $\mathcal{P}$ is given by a presentation dual to a choice of presentation for $\mathcal{P}$. The presentation is called Koszul if in fact $\mathcal{P} i \hookrightarrow B \mathcal{P}$ is a weak equivalence in a Hinich-type model structure on dg cooperads (see [40]). In this case, the cobar construction on the Koszul dual cooperad $\mathcal{P}^{i}$ provides us with a small resolution

$$
\Omega \mathcal{P} i \stackrel{\sim}{\longrightarrow} \Omega \mathrm{BP} \stackrel{\sim}{\longrightarrow} \mathcal{P},
$$

called a Koszul resolution, and algebras over this type of resolution are known as $\mathcal{P}_{\infty}$ algebras.

For the classical types of associative, commutative, or Lie algebras, Koszul duality provides us with the following $\infty$-analogues: For associative algebras, we recover the $\mathrm{A}_{\infty}$-algebras introduced by Stasheff [65] and the bar construction of Eilenberg-MacLane [14]. For commutative algebras, we obtain the definition of $\mathrm{C}_{\infty}$-algebras introduced by Kadeishvili [33] and present in rational homotopy theory [60 66], along with the Harrison complex [27]. For Lie algebras, Koszul duality leads to the notion of $\mathrm{L}_{\infty}$-algebras as introduced by Hinich-Schechtman [30] (see also [13]) and crucial in deformation theory [23. 38], and to the Chevalley-Eilenberg complex [10]. However, of these classical operads Ass, Com, and Lie, only Ass is $\mathbb{S}$-cofibrant over any commutative ring $\mathbf{k}$, since its structural operations satisfy no symmetries.

For commutative algebras, there is also the notion of $\mathrm{E}_{\infty}$-algebras going back to May [52] and Boardman-Vogt [8]. An $\mathrm{E}_{\infty}$-operad is any $\mathbb{S}$-cofibrant resolution of the operad Com, such as the Barratt-Eccles operad $\mathcal{E}$ in dg operads (see, e.g., [4]). We may use the Barratt-Eccles operad for our purposes of constructing cofibrant resolutions for operads that are not $\mathbb{S}$-cofibrant already: the tensor product of any $\mathrm{dg}$ operad $\mathcal{P}$ with $\mathcal{E}$ 
is $\mathbb{S}$-cofibrant, and thus one obtains a cofibrant resolution

$$
\Omega \mathrm{B}(\mathcal{P} \otimes \mathcal{E}) \stackrel{\sim}{\longrightarrow} \mathcal{P} \otimes \mathcal{E} \stackrel{\sim}{\longrightarrow}
$$

over an arbitrary commutative ring $\mathbf{k}$.

The reason for the $\mathbb{S}$-cofibrancy condition is essentially that the cobar-bar resolution of an operad $\mathcal{P}$ resolves only the operadic composition, not the symmetries of the operations. This is a consequence of the classical treatment of operads as $\mathbb{S}$-modules equipped with composition structure. In Chapter 3 , we take a different perspective, viewing both the symmetry and the composition as part of the structure of an operad. Among other findings, we obtain a new cobar-bar adjunction whose counit resolves both structures simultaneously.

One of our original goals in this thesis was to give an explicit definition of homotopy Lie algebras over any commutative ring $\mathbf{k}$, also known as $\mathrm{EL}_{\infty}$-algebras. Unfortunately, in the context of our new cobar-bar adjunction, a Koszul duality approach is not yet available. In Chapter 4 we use yet another technique to attempt to construct a resolution of the Lie operad: we consider the classical Koszul dual cooperad Liei of the Lie operad and construct a small $\mathbb{S}$-cofibrant resolution $\mathrm{Lie}^{\diamond} \rightarrow \mathrm{Lie}^{\mathrm{i}}$ on the cooperad side-at least in low degrees. This allows us to explicitly define weak Lie 3-algebras, i.e., $\mathrm{EL}_{\infty}{ }^{-}$ algebras on underlying 3-term complexes, and their homotopy morphisms. As 2-term truncations, we recover Roytenberg's weak Lie 2-algebras [63], thereby providing a more conceptual construction for them. Among other results, we prove the desired homotopy invariance property for weak Lie 3-algebras. We end the chapter with some initial applications of weak Lie 3-algebras in higher differential geometry.

\section{Structure}

We begin this thesis by presenting some preliminary material in Chapter 1 fixing notation, and introducing conventions followed throughout the rest of the work. In Chapter 2 we present the classical theory of operads and Koszul duality. We take great care to introduce concepts and state results in such a way that they hold when working with chain complexes over commutative rings. Chapter 3 comprises our joint work with Bruno Vallette [12] on Symmetric Homotopy Theory for Operads. Finally, in Chapter 4. we reproduce our work On Weak Lie 3-Algebras [11].

\section{Acknowledgements}

The author wishes to express his appreciation to the Laboratoire J.A. Dieudonné of the University of Nice Sophia Antipolis; the Laboratoire Analyse, Géométrie et Applications of the Université Paris 13; and the Isaac Newton Institute for Mathematical Sciences for the invitations and the excellent working conditions. The author would also like to thank Chris Rogers and Dmitry Roytenberg for their insightful remarks and Yunhe Sheng for his inspiration. The author is most grateful to Chenchang Zhu for many helpful discussions and suggestions over the years, and to Bruno Vallette for his collaboration, valuable insights, and hospitality on numerous occasions. 



\section{CHAPTER 1}

\section{Preliminaries}

The aim of this chapter is to introduce notation and conventions used throughout this thesis.

We denote by $\mathbf{k}$ an arbitrary commutative unital ring and by $\mathbf{k}$-Mod its category of modules. In practice, for any computations we will work over the integers $\mathbf{k}=\mathbb{Z}$. Since $\mathbb{Z}$ is the initial object in the category of unital commutative rings, this ensures that our results hold over any such ring $\mathbf{k}$.

\section{Chain Complexes}

A $\mathbb{Z}$-graded chain complex $(V, \mathrm{~d})$ in $\mathbf{k}$-modules is a collection of $\mathbf{k}$-modules $\left\{V_{i}\right\}_{i \in \mathbb{Z}}$ with k-linear maps $\mathrm{d}_{i}: V_{i} \rightarrow V_{i-1}$ satisfying $\mathrm{d}_{i-1} \circ \mathrm{d}_{i}=0$ for all $i \in \mathbb{Z}$. The index $i$ is referred to as (homological) degree, and we use the notation $|v|=i$ for elements $v \in V_{i}$. A morphism of chain complexes in $\mathbf{k}$-modules $f:\left(V, \mathrm{~d}^{V}\right) \rightarrow\left(W, \mathrm{~d}^{W}\right)$ is a collection of k-linear maps $\left\{f_{i}: V_{i} \rightarrow W_{i}\right\}_{i \in \mathbb{Z}}$, such that $\mathrm{d}_{i}^{W} \circ f_{i}=f_{i-1} \circ \mathrm{d}_{i}^{V}$ for all $i \in \mathbb{Z}$. The category of chain complexes in $\mathbf{k}-$ Mod is denoted by $\mathbf{k}-\mathrm{Ch}$.

1.1. Abelian structure. It is a standard result that $\mathbf{k}-\mathrm{Ch}$ is an abelian category with biproduct the degreewise direct sum of k-modules $(V \oplus W)_{i}:=V_{i} \oplus W_{i}$ equipped with the componentwise differential $\mathrm{d}^{V \oplus W}(v+w)=\mathrm{d}^{V}(v)+\mathrm{d}^{W}(w)$. Kernels and cokernels are computed degreewise, i.e., $\operatorname{ker}(f)_{i}=\operatorname{ker}\left(f_{i}\right)$ and $\operatorname{coker}(f)_{i}=\operatorname{coker}\left(f_{i}\right)$.

1.2. Symmetric monoidal structure. The category $\mathbf{k}-\mathrm{Ch}$ can be equipped with a monoidal product

(1) $(V \otimes W)_{i}:=\bigoplus_{j \in \mathbb{Z}} V_{j} \otimes W_{i-j}, \quad \mathrm{~d}^{V \otimes W}(v \otimes w)=\mathrm{d}^{V}(v) \otimes w+(-1)^{|v|} \cdot v \otimes \mathrm{d}^{W}(w)$.

Slightly abusing notation, the chain complex given by $\mathbf{k}$ in degree 0 and 0 in all other degrees is denoted again by $\mathbf{k}$. It acts as the unit object with respect to the monoidal product. The monoidal product satisfies a certain symmetry: we denote by $\tau$ the natural isomorphism with components given on homogeneous elementary tensors by

$$
\tau^{V, W}: V \otimes W \longrightarrow W \otimes V, \quad v \otimes w \longmapsto(-1)^{|v||w|} \cdot w \otimes v .
$$

Clearly $\tau^{V, W} \circ \tau^{W, V}=\mathrm{id}$, and together with the above monoidal structure this turns $(\mathbf{k}-\mathrm{Ch}, \otimes, \mathbf{k})$ into a symmetric monoidal category.

REMARK 1.2.1. The sign in the differential of the tensor product in Equation (1) is necessary to ensure that $\mathrm{d}^{V \otimes W}$ squares to zero, and, as a consequence, the sign in the symmetry isomorphism in Equation (2) is required such that the components of $\tau$ are 
in fact morphisms of chain complexes. This is the basis for what is known as the Koszul sign rule, often phrased somewhat vaguely as: "whenever symbols $x, y$ of degree $|x|$ resp. $|y|$ change their relative order, a factor $(-1)^{|x||y|}$ is introduced."

1.3. Closed monoidal structure. By definition, the morphisms in $\mathbf{k}$-Ch preserve degrees and commute with the differentials. In addition, there exists another notion of so called internal homomorphisms, defined as follows. Let hom $(V, W)$ be the complex defined by

(3) $\operatorname{hom}(V, W)_{i}:=\prod_{j \in \mathbb{Z}} \operatorname{Hom}\left(V_{j}, W_{i+j}\right), \quad \mathrm{d}_{i}^{\operatorname{hom}(V, W)}(f)_{j}:=\mathrm{d}_{i+j}^{W} \circ f_{j}-(-1)^{i} \cdot f_{j-1} \circ \mathrm{d}_{j}^{V}$.

The index $i$ is referred to as the (homological) degree of the internal morphisms $f \in$ $\operatorname{hom}(V, W)_{i}$ and we use the notation $|f|=i$. The differential is sometimes written as $\partial$ or as the graded commutator $\left[\mathrm{d}_{-}\right]$. It is easy to verify that the functor $\_\otimes V$ is left adjoint to $\operatorname{hom}\left(V,{ }_{-}\right)$for any $V$ and, hence, $(\mathbf{k}-\mathrm{Ch}, \otimes, \mathbf{k})$ is a closed symmetric monoidal category. Explicitly, the adjunction is given by the bijections

$$
\begin{aligned}
& \operatorname{Hom}(U \otimes V, W) \longleftrightarrow \stackrel{\operatorname{Hom}}{\cong} U, \operatorname{hom}(V, W)) \\
& f \longmapsto\left(u \mapsto\left(f\left(u \otimes_{-}\right): v \mapsto f(u \otimes v)\right)\right) \\
& (u \otimes v \mapsto g(u)(v)) \longleftarrow g \text {. }
\end{aligned}
$$

REMARK 1.3.1. The homomorphisms of chain complexes are precisely the 0-cycles of the internal homomorphism complex,

$$
\operatorname{Hom}(V, W)=\left\{f \in \operatorname{hom}(V, W)_{0} \mid \partial(f)=0\right\}=\operatorname{Hom}(\mathbf{k}, \operatorname{hom}(V, W)) .
$$

1.4. Enriched structure. Like any closed symmetric monoidal category, $\mathbf{k}-\mathrm{Ch}$ is actually enriched over itself [35]. The counit of the internal hom adjunction provides us with evaluation maps for internal homomorphisms: the identity morphisms

$$
\text { id: } \operatorname{hom}(V, W) \rightarrow \operatorname{hom}(V, W)
$$

correspond to morphisms

$$
\mathrm{ev}: \operatorname{hom}(V, W) \otimes V \rightarrow W, \quad f \otimes v \mapsto \operatorname{ev}(f, v),
$$

which, for $v \in V_{i}$, evaluate to $\operatorname{ev}(f, v)=f_{i}(v)$. In particular, these allow us to define composition maps

$$
\left(\varsigma^{\circ}{ }\right): \operatorname{hom}(V, W) \otimes \operatorname{hom}(U, V) \longrightarrow \operatorname{hom}(U, W),
$$

for internal homomorphisms, corresponding to the successive evaluations

$$
\operatorname{hom}(V, W) \otimes \operatorname{hom}(U, V) \otimes U \stackrel{\mathrm{id} \otimes \mathrm{ev}}{\longrightarrow} \operatorname{hom}(V, W) \otimes V \stackrel{\text { ev }}{\longrightarrow} W .
$$

Let $f \in \operatorname{hom}(V, W), g \in \operatorname{hom}\left(V^{\prime}, W^{\prime}\right)$ be internal homomorphisms. Naturally, $f \otimes g$ is an element in $\operatorname{hom}(V, W) \otimes \operatorname{hom}\left(V^{\prime}, W^{\prime}\right)$. We will however denote by $f \otimes g \in$ 
$\operatorname{hom}\left(V \otimes V^{\prime}, W \otimes W^{\prime}\right)$ the image corresponding to the evaluation map $\operatorname{hom}\left(V, V^{\prime}\right) \otimes \operatorname{hom}\left(W, W^{\prime}\right) \otimes V \otimes W \stackrel{1 \otimes \tau \otimes 1}{\longrightarrow} \operatorname{hom}\left(V, V^{\prime}\right) \otimes V \otimes \operatorname{hom}\left(W, W^{\prime}\right) \otimes W$ $\stackrel{\mathrm{ev} \otimes \mathrm{ev}}{\longrightarrow} V^{\prime} \otimes W^{\prime}$.

Explicitly, this means $(f \otimes g)(v \otimes w)=(-1)^{|g||v|} \cdot f(v) \otimes g(w)$ on homogeneous simple tensors in accordance with the Koszul sign rule.

REMARK 1.4.1. Using this notation we can write the differential for the tensor product of chain complexes as $\mathrm{d}^{V \otimes W}=\mathrm{d}^{V} \otimes 1+1 \otimes \mathrm{d}^{W}$.

1.5. Suspension and desuspension functors. We denote by $s \mathbf{k}$ the chain complex given by $(s \mathbf{k})_{1}=\mathbf{k}$ and $(s \mathbf{k})_{i}=0$ for $i \neq 1$. Using this notation, we define the suspension functor $s:=\left(s \mathbf{k} \otimes_{\_}\right): \mathbf{k}-\mathrm{Ch} \rightarrow \mathbf{k}$-Ch. Analogously, we denote by $s^{-1} \mathbf{k}$ the chain complex given by $\left(s^{-1} \mathbf{k}\right)_{-1}=\mathbf{k}$ and $\left(s^{-1} \mathbf{k}\right)_{i}=0$ for $i \neq-1$. We define the desuspension functor $\mathrm{s}^{-1}:=\left(s^{-1} \mathbf{k} \otimes_{-}\right): \mathbf{k}-\mathrm{Ch} \rightarrow \mathbf{k}-\mathrm{Ch}$.

In addition, we define the (internal) suspension isomorphism

$$
\uparrow^{V}: V \rightarrow s V, \quad \uparrow^{V}(v):=s \otimes v,
$$

and the (internal) desuspension isomorphism

$$
\downarrow^{V}: V \rightarrow s^{-1} V, \quad \downarrow^{V}(v):=s^{-1} \otimes v .
$$

Note that suspension and desuspension are 1-cycles and (-1)-cycles, respectively. By abuse of notation, we will often denote the suspension and desuspension isomorphisms by $s$ resp. $s^{-1}$.

\section{Permutations}

We denote by $\mathbb{S}_{n}$ the symmetric group on $n$ elements, i.e., the group of bijections of the set $\underline{n}=\{1, \ldots, n\}$. An element $\sigma \in \mathbb{S}_{n}$ is usually denoted by its values $[\sigma(1), \ldots, \sigma(n)]$ or as a product of cycles $\left(i, \sigma(i), \ldots, \sigma^{r}(i)\right)$. Another way we depict symmetric group elements and their multiplication is using a graphical notation as indicated in Figure 1 A permutation $\sigma$ is denoted by a diagram of strands connecting their $i$-th input to their

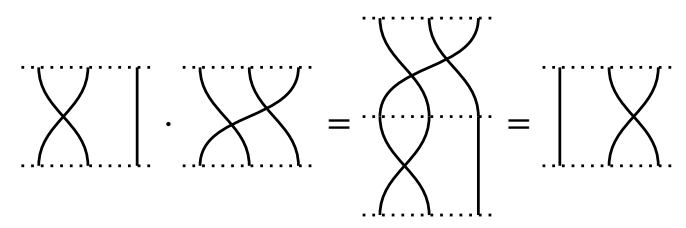

FIGURE 1. Example of multiplication: $[213] \cdot[231]=[132]$

$\sigma(i)$-th output. Diagrams are read top to bottom, i.e., inputs are at the top, outputs are at the bottom. Multiplication $\sigma \cdot \tau$ can be computed graphically by placing the diagram for $\tau$ on top of the diagram for $\sigma$. 
2.1. Composition. It will sometimes be convenient to view the groups $\mathbb{S}_{n}$ as oneobject categories and denote by $\mathbb{S}$ the symmetric groupoid $\mathbb{S}:=\coprod \mathbb{S}_{n}$. In addition to the groupoid multiplication, we may define a composition map on $\mathbb{S}$ as follows. Given a permutation $\sigma \in \mathbb{S}_{n}$ and an $n$-tuple $\left(\tau_{1}, \ldots, \tau_{n}\right)$ of permutations $\tau_{j} \in \mathbb{S}_{k_{j}}$, we define their composition $\sigma \circ\left(\tau_{1}, \ldots, \tau_{n}\right) \in \mathbb{S}_{k_{1}+\cdots+k_{n}}$ by

(8) $\left(\sigma \circ\left(\tau_{1}, \ldots, \tau_{n}\right)\right)(i)=\tau_{j}\left(i-\left(k_{1}+\cdots+k_{j-1}\right)\right)+\left(k_{\sigma^{-1}(1)}+\cdots+k_{\sigma^{-1}(\sigma(j)-1)}\right)$,

$$
\text { for } k_{1}+\cdots+k_{j-1}<i \leq k_{1}+\cdots+k_{j} \text {. }
$$

In terms of graphical notation, this composition can be constructed as indicated in Figure 2 and described in the following. As the first step, for each $j=1, \ldots, n$, we thicken the $j$-th strand of $\sigma$ to $k_{j}$ parallel strands. In this way, we obtain a permutation of $\mathbb{S}_{k_{1}+\cdots+k_{n}}$ sometimes denoted $\sigma\left(k_{1}, \ldots, k_{n}\right)$. The second step is to apply $\tau_{j}$ locally to the corresponding $k_{j}$ strands. This amounts to multiplication of $\sigma\left(k_{1}, \ldots, k_{n}\right)$ by $\left(\tau_{1}, \ldots, \tau_{n}\right) \in \mathbb{S}_{k_{1}} \times \cdots \times \mathbb{S}_{k_{n}} \subset \mathbb{S}_{k_{1}+\cdots+k_{n}}$.

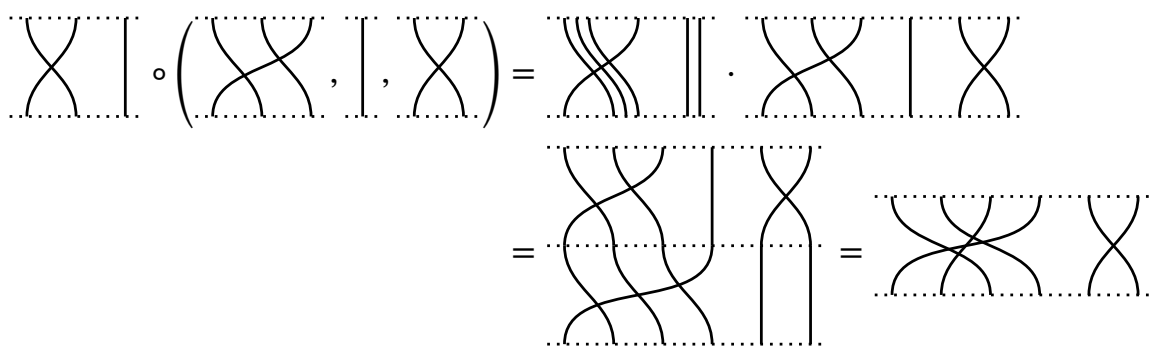

FIGURE 2. Example of composition: [213]॰ $([231],[1],[21])=[342165]$.

A special case we will often encounter is the composition where all but one permutation $\tau_{j}=\tau \in \mathbb{S}_{k}$ are the identity on a single strand $\mathrm{id}_{1} \in \mathbb{S}_{1}$. This composition is called the $j$-th partial composition and denoted

$$
\sigma \circ_{j} \tau:=\sigma \circ\left(\mathrm{id}_{1}, \ldots, \mathrm{id}_{1}, \tau, \mathrm{id}_{1}, \ldots, \mathrm{id}_{1}\right) \in \mathbb{S}_{n+k-1} .
$$

In terms of graphical notation, it amounts to thickening the $j$-th strand of $\sigma$ to $k$ parallel strands and applying $\tau$ there, extended by the identity on all other strands.

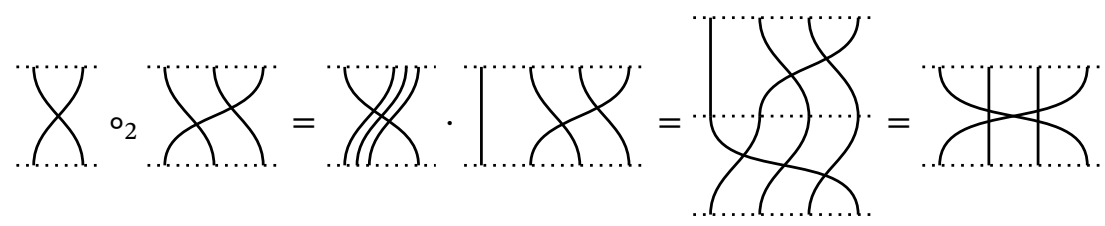

FIGURE 3. Example of partial composition: $[21] \circ_{2}[231]=[4231]$.

2.2. Shuffle permutations. Let $n_{1}, \ldots, n_{m}$ be natural numbers such that $n=n_{1}+$ $\cdots+n_{m}$. We call $\sigma \in \mathbb{S}_{n}$ an $\left(n_{1}, \ldots, n_{m}\right)$-shuffle, if $\sigma(i)<\sigma(i+1)$ for all $1 \leq i<n$ 
except when $i=N_{j}:=n_{1}+\cdots+n_{j}$ for some $1 \leq j<m$. We denote by $\operatorname{Sh}\left(n_{1}, \ldots, n_{m}\right) \subset$ $\mathbb{S}_{n}$ the subset of these $\left(n_{1}, \ldots, n_{m}\right)$-shuffles. The shuffles $\operatorname{Sh}\left(n_{1}, \ldots, n_{m}\right)$ form a set of representatives for the cosets $\mathbb{S}_{n} /\left(\mathbb{S}_{n_{1}} \times \cdots \times \mathbb{S}_{n_{m}}\right)$.

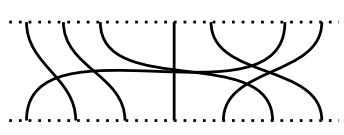

(A) Unreduced shuffle.

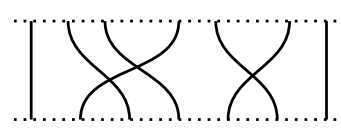

(в) Reduced shuffle.

FIGURE 4. Examples of $(3,2,2)$-shuffle permutations.

We call an $\left(n_{1}, \ldots, n_{m}\right)$-shuffle $\sigma$ reduced, if $\sigma\left(N_{j}\right)<\sigma\left(N_{j+1}\right)$ for all $1 \leq j<m$. The set of these reduced shuffles is denoted as $\overline{\operatorname{Sh}}\left(n_{1}, \ldots, n_{m}\right)$. The inverse of a (reduced) shuffle is called a (reduced) unshuffle and the set of these is denoted by $\mathrm{Sh}^{-1}\left(n_{1}, \ldots, n_{m}\right)$ resp. $\overline{\mathrm{Sh}}^{-1}\left(n_{1}, \ldots, n_{m}\right)$.

2.3. Representations. We use the notation $\mathbf{k}\left[\mathbb{S}_{n}\right]$ for the group algebra and the (right) regular representation of $\mathbb{S}_{n}$. We denote by $\mathbf{k} \cdot \mathrm{sgn}_{n}$ the one-dimensional signature represenation of $\mathbb{S}_{n}$, i.e., its underlying module is $\mathbf{k}$ and the adjacent transpositions $\sigma_{i}=$ $(i i+1)$ act by multiplication with -1 . We implicitely extend the group representations to representations of the group algebra by $\mathbf{k}$-linearity and write e.g., $x^{-\sigma+\tau}=-x^{\sigma}+x^{\tau}$.

\section{Forests and Trees}

A forest $F=(X, p)$ consists of a finite set edge $(F):=X$ of edges with a parent map $p: X \rightarrow X$, such that for some $n \geq 0$ the property $p\left(p^{n}(x)\right)=p^{n}(x)$ holds for all edges $x \in X$. By definition, the set of edges comes with a partition $X=\coprod_{i \geq 0} X^{i}$, where

$$
X^{0}:=\{x \in X \mid p(x)=x\} \text { and } X^{i}:=\left\{x \in X \mid p\left(p^{i}(x)\right)=p^{i}(x)\right\} \backslash X^{i-1},
$$

and the parent map $p$ decomposes as $\coprod_{i \geq 0} p_{i}$ with $p_{i}: X^{i} \rightarrow X^{i-1}$ and $p_{0}=$ id. As a result, a forest is equivalent to a diagram $X^{0} \leftarrow \cdots \leftarrow X^{n}$ of finite sets where $X^{n} \neq \varnothing$. When the components $p_{i}$ of the parent map are all surjective, we call $F$ an $n$-forest.

We call elements of $\operatorname{root}(F):=X^{0}$ roots for $F$ and edges in edge $(F)_{i}:=X^{i}$ its $i$-th generation edges. Elements of vert $(F):=p\left(X \backslash X^{0}\right)$ are referred to as vertices and those of leaf $(F):=X \backslash p\left(X \backslash X^{0}\right)$ as leaves. For a vertex $f$, we call elements of in $(f):=p^{-1}(f) \backslash X^{0}$ its input edges and $\operatorname{out}(f):=f$ its output edge. A tree $T=(X, p)$ is a particularly simple forest with a unique root resp. a diagram $1 \leftarrow X^{1} \leftarrow \cdots \leftarrow X^{n}$ of finite sets. When the $p_{i}$ are all surjective, we call $T$ an $n$-tree.

Note that in our terminology vertices appear only implicitly, and by definition they have at least one input and exactly one output edge. As a result, the roots and leaves-together called external edges or the boundary-of a forest are incident on fewer than two vertices. The remaining edges are incident on two vertices and called internal edges. A more general definition of rooted trees with boundary allowing for 0 input vertices can be found in [37]. For our purposes, this simpler but more restrictive version will suffice. 


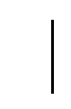

(A) Trivial tree.

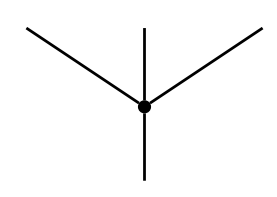

(B) Corolla.

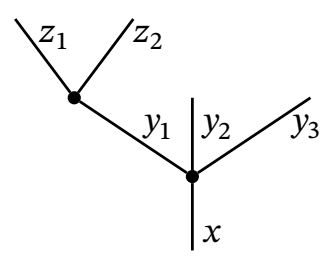

(c) Tree with inner edge.

FIGURE 5. Examples of trees

EXAMPLES. (i) The empty forest $X=\varnothing$ has no edges and thus no vertices. Since it has no root, it is not a tree. (ii) The trivial tree $X=1$ consists of a single edge and no vertices (Figure 5A). Its only edge is both its root, as well as a leaf. (iii) The corolla $X=1 \amalg X^{1}$ with $p(x)=1$ for all $x \in X$ has a single vertex whose set of input edges is $X^{1}$ and whose output edge is the root (Figure 5B). Note that $X^{1} \neq \varnothing$ is assumed. (iv) See Figure 5C The set of edges for this tree is edge $(T)=\left\{x, y_{1}, y_{2}, y_{3}, z_{1}, z_{2}\right\}$ and the parent map is given by $p(x)=x, p\left(y_{i}\right)=x$, and $p\left(z_{i}\right)=y_{1}$. This makes $x$ the root, leaf $(T)=\left\{z_{1}, z_{2}, y_{2}, y_{3}\right\}$ the leaves, and $y_{1}$ the only internal edge for $T$.

REMARK 3.0.1. By drawing a tree $T$ in the plane such as we have done in Figure 5 , we place a (total) order on the incoming edges in $(f)$ at each vertex $f \in \operatorname{vert}(T)$. Such a choice is called a planar structure (see Section 3.4 for details) and a tree with fixed choice of planar structure is known as a planar tree. When working with nonplanar trees, we disregard this additional structure.

3.1. Morphisms and subtrees. Let $F=(X, p)$ and $G=(Y, q)$ be forests. An isomorphism of forests $\varphi: F \rightarrow G$ is a bijection of edges $\varphi: X \rightarrow Y$ compatible with the parent maps: $\varphi \circ p=q \circ \varphi$. We denote by Forest (resp. Tree) the category of forests (resp. trees) with isomorphisms as morphisms and by $n$-Forest (resp. $n$-Tree) their full subcategories of $n$-forests (resp. $n$-trees).

In case $S=F=(X, p)$ is actually a tree, we define another type of morphism. An inclusion of a tree $\iota: S \hookrightarrow G$ is an injective map $\iota: X \hookrightarrow Y$ of edges, such that

(i) for $x \in$ edge $(S) \backslash 1$ a non-root edge: $\iota(p(x))=q(\iota(x))$; and

(ii) for $f \in \operatorname{vert}(S)$ any vertex: $\operatorname{in}(\iota(f)) \subset \iota(X)$.

Two inclusions $\iota: S \hookrightarrow G$ and $\iota^{\prime}: S^{\prime} \hookrightarrow G$ are considered equivalent if there exists an isomorphism $\varphi: S \rightarrow S^{\prime}$ such that $\iota^{\prime} \circ \varphi=\iota$. A subtree $S \subset G$ is an equivalence class of inclusions $S \hookrightarrow G$.

Let $F=(X, p)$ be a forest and $a \in X$ a marked edge. The subset

$$
\left.X\right|_{a}=\left\{x \in X \mid \exists n \geq 0: p^{n}(x)=a\right\}
$$

with induced parent map

$$
\left.p\right|_{a}:\left.\left.X\right|_{a} \longrightarrow X\right|_{a}, \quad x \longmapsto \begin{cases}a, & \text { if } x=a, \\ p(x), & \text { otherwise },\end{cases}
$$


forms a subtree $\left.F\right|_{a} \subset F$. Given any collection $\left\{F_{a}=\left(X_{a}, p_{a}\right)\right\}_{a \in A}$ of trees, their disjoint union is a forest $X=\coprod X_{a}$ with $X^{0}=A$. In particular, for any forest $F=(X, p)$, we have a canonical isomorphism

$$
\left.F \cong \coprod_{a \in X^{0}} F\right|_{a} .
$$

3.2. Grafting of trees. Given a tree $S$ with a marked leaf $l \in \operatorname{leaf}(S)$ and a second tree $T$, the grafting of $S$ with $T$ at $l$ is denoted by $S{ }_{l} T$ and obtained by identifying the marked leaf $l$ of $S$ with the root of $T$ as shown in Figure 6 Formally, given $S=\left(X_{S}, p_{S}\right)$, $T=\left(X_{T}, p_{T}\right)$, and a choice of leaf $l \in \operatorname{leaf}(S)$, we define the grafting $S \circ_{l} T$ of $S$ with $T$ at leaf $l$ as follows:

$$
X_{S^{\circ} T}:=\left(X_{S} \amalg X_{T}\right) /\left(l=1_{T}\right), \quad p_{S_{\circ} T}([x]):= \begin{cases}p_{S}(x), & \text { if } x \in X_{S}, \\ p_{S}(l), & \text { if } x=1_{T}, \\ p_{T}(x), & \text { if } x \in X_{T} \backslash 1_{T},\end{cases}
$$

where $1_{T}$ denotes the root of $T$.

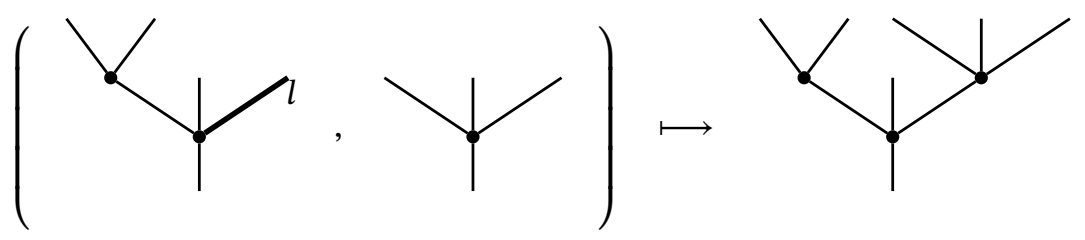

FIGURE 6. Grafting of trees.

3.3. Contraction of subtrees. Let $T$ be a tree and let $\iota: S \hookrightarrow T$ represent a subtree of $S \subset T$. A subtree is uniquely determined by its set of vertices $\operatorname{vert}(S) \subset \operatorname{vert}(T)$. The contraction of $S$ in $T$ is denoted $T / S$ and formed by identifying the vertices of $S$ as indicated in Figure 7 In terms of edges, this amounts to removing the inner edges of $S$ and appropriately redefining the parent map on leaves of $S$ as follows:

$$
X_{T / S}:=X_{T} \backslash \iota(\text { inner edges of } S), \quad p_{T / S}(x):= \begin{cases}\iota\left(1_{S}\right), & \text { if } x \in \iota(\text { leaf }(S)), \\ p_{T}(x), & \text { otherwise } .\end{cases}
$$

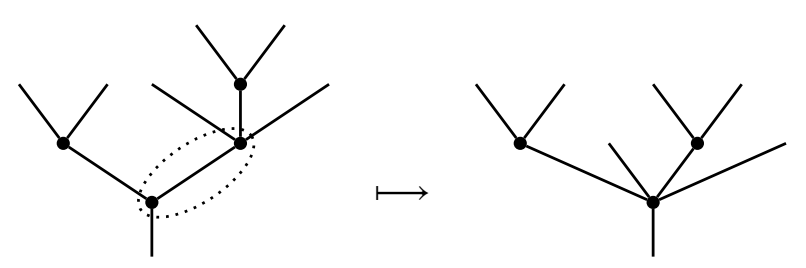

FIGURE 7. Contraction of the marked subtree.

Since all of our morphisms are injective, we cannot expect to obtain a quotient map $S \hookrightarrow T \rightarrow T / S$. This ad-hoc construction, however, will be enough for our purposes. 
3.4. Planar structure. Let $T=(X, p)$ be a tree. A planar structure on $T$ is a collection of ordering bijections $\chi(f): \operatorname{in}(f) \rightarrow \underline{n}$ for all $f \in \operatorname{vert}(T)$. A tree $T$ together with a choice of planar structure is called a planar tree. Morphisms $\varphi: S \rightarrow T$ of such trees are assumed to respect the planar structure in the sense that $\chi_{T}(\varphi(f)) \circ \varphi=\chi_{S}(f)$.

Equivalently, a planar tree is a diagram $1 \leftarrow X^{1} \leftarrow \cdots \leftarrow X^{n}$ in the category FOrd of finite ordered sets and order-preserving maps. Similarly, we define a planar forest as a diagram $X^{0} \leftarrow \cdots \leftarrow X^{n}$ in FOrd.

3.5. Coloring. Let $F$ be a forest and $C$ a fixed set whose elements we refer to as colors. A $C$-coloring for $F$ is a map $c$ : $\operatorname{edge}(F) \rightarrow C$. A forest equipped with a $C$-coloring is called a $C$-colored forest. Morphisms $\varphi: F \rightarrow G$ of such colored forests are assumed to be compatible with the colorings in the sense that $c_{G} \circ \varphi=c_{F}$.

In the colored context, there exist trivial trees $I_{c}$ for each color $c \in C$ and there are no morphisms between trivial trees of different colors. The constructions on trees we have seen earlier carry over to the colored case with the following caveat: we can only graft trees when the colors of their relevant leaf and root match. 


\section{Operads}

In this chapter, we recall basic definitions and results of the theory of algebraic operads. For a textbook introduction to algebraic operads we refer the reader to [46], upon which large parts of this chapter are heavily based. The constructions here are given in enough generality to obtain results for operads in chain complexes over a commutative ring $\mathbf{k}$.

This chapter is organized as follows. In Section 1, we introduce various categories of collections underlying different types of operads. In Section 2 we give the basic definitions of operads as well as their modules and algebras. We consider some constructions, including that of free operads. In Section 3. we introduce the dual concepts for cooperads. In Section 4 we recall the classical cobar-bar adjunction between cooperads and operads and the resolutions it provides. In Section 5 we define the cobar-bar adjunction for algebras over an operad. Its counit provides us with resolutions for operadic algebras. In Section 6 we give a treatment of the theory of homotopy algebras from an operadic perspective. Finally, in Section 7, we give a short overview of the classical Koszul duality theory for quadratic operads and its curved Koszul duality extension for quadratic-linear-constant operads.

\section{Collections}

We define categories of collections as modules over groupoids of 1-trees. Recall that for a groupoid $\mathcal{G}$, its category of left (resp. right) modules in $\mathcal{C}$ is the functor category $[\mathcal{G}, \mathcal{C}]$ (resp. $\left.\left[\mathcal{G}^{\mathrm{op}}, \mathcal{C}\right]\right)$. We work in the differential graded framework, i.e. we take the category $\mathcal{C}$ to be the category $\mathbf{k}$ - $C h$ of chain complexes.

The basic case we consider is the category of $d g$ symmetric collections or simply $d g$ collections

$$
\text { dg Coll }=\left[(1-\text { Tree })^{\text {op }}, \mathbf{k}-\mathrm{Ch}\right] .
$$

In addition, the categories

$$
\text { dg ns Coll }=\left[(\text { planar } 1-\text { Tree })^{\mathrm{op}}, \mathbf{k}-\mathrm{Ch}\right]
$$

of $d g$ nonsymmetric or $d g$ ns collections and

$$
\operatorname{dg} C \text {-col Coll }=\left[(C \text {-colored } 1 \text {-Tree })^{\text {op }}, \mathbf{k}-\mathrm{Ch}\right]
$$

of $d g$-colored collections play an important role. For now, we will give definitions and introduce constructions only for basic dg collections in order to reduce repetition. In 
Section 1.4 (resp. Section 1.5), we go over the necessary changes for dg ns collections (resp. dg $C$-colored collections).

Since $\mathbf{k}-\mathrm{Ch}$ is a monoidal category, we can equip the category of dg symmetric collections with the pointwise abelian and symmetric monoidal structures, i.e. for $\mathrm{dg}$ collections $M, N$ we define

$$
\begin{aligned}
& (M \oplus N)[1 \leftarrow X]:=M[1 \leftarrow X] \oplus N[1 \leftarrow X], \\
& (M \otimes N)[1 \leftarrow X]:=M[1 \leftarrow X] \otimes N[1 \leftarrow X] .
\end{aligned}
$$

Given another dg collection $K$ with a morphism (natural transformation) $g: M \otimes N \rightarrow K$, its components satisfy

$$
\begin{aligned}
\operatorname{Hom}((M \otimes N)[1 \leftarrow X], K[1 \leftarrow X]) & \triangleq \operatorname{Hom}(M[1 \leftarrow X] \otimes N[1 \leftarrow X], K[1 \leftarrow X]) \\
& \cong \operatorname{Hom}(M[1 \leftarrow X], \operatorname{hom}(N[1 \leftarrow X], K[1 \leftarrow X])) .
\end{aligned}
$$

We define a dg collection $\operatorname{hom}(N, K)$ pointwise by,

$$
\operatorname{hom}(N, K)[1 \leftarrow X]:=\operatorname{hom}(N[1 \leftarrow X], K[1 \leftarrow X])
$$

and on morphisms $\varphi^{\mathrm{op}}:(1 \leftarrow X) \longrightarrow\left(1 \leftarrow X^{\prime}\right)$ in 1-Tree ${ }^{\mathrm{op}}$,

$$
\operatorname{hom}(N, K)\left[\varphi^{\mathrm{op}}\right]: \operatorname{hom}(N, K)[1 \leftarrow X] \longrightarrow \operatorname{hom}(N, K)\left[1 \leftarrow X^{\prime}\right]
$$

is given by conjugation:

$$
\operatorname{hom}(N, K)\left[\varphi^{\mathrm{op}}\right](f)=K\left[\varphi^{\mathrm{op}}\right] \circ f \circ N\left[\varphi^{-1, \mathrm{op}}\right] .
$$

With this definition, the components $g[1 \leftarrow X]$ correspond to

$$
h[1 \leftarrow X]: M[1 \leftarrow X] \longrightarrow \operatorname{hom}(N, K)[1 \leftarrow X]
$$

and these form a natural transformation $h: M \rightarrow \operatorname{hom}(N, K)$. In fact, this isomorphism

$$
\operatorname{Hom}(M \otimes N, K) \cong \operatorname{Hom}(M, \operatorname{hom}(N, K))
$$

is natural in $M, N$, and $K$, and thus hom $\left({ }_{\lrcorner},{ }_{-}\right)$provides an internal hom functor with respect to the pointwise tensor product $\_\otimes_{\longleftarrow}$ on dg collections.

1.1. Composite product. We equip the category of dg collections with another monoidal product called the composite product. To do so, we first extend the domain category of a collection $N$ from $1-$ Tree $^{\text {op }}$ to 1 -Forest ${ }^{\text {op }}$ via

$$
N[B \leftarrow X]:=\operatorname{colim}_{\chi: B \rightarrow \underline{m}} N\left[1 \leftarrow X_{1}\right] \otimes \cdots \otimes N\left[1 \leftarrow X_{m}\right],
$$

where the colimit is taken over bijections $\chi: B \rightarrow \underline{m}$. We denote here by $b_{i}$ the unique element of $B$ for which $\chi\left(b_{i}\right)=i$, and write $X_{i}$ as shorthand for $X_{b_{i}}:=p^{-1}\left(b_{i}\right) \subset X$ with parent map $p$. The colimit can be computed as

$$
N[B \leftarrow X]=\left(\bigoplus_{\chi: B \rightarrow \underline{m}} N\left[1 \leftarrow X_{1}\right] \otimes \cdots \otimes N\left[1 \leftarrow X_{m}\right]\right)_{S_{m}},
$$


where the $\mathbb{S}_{m}$-action is given by

$$
\sigma \cdot\left(\chi, \nu_{1} \otimes \cdots \otimes \nu_{m}\right)= \pm\left(\sigma \chi, \nu_{\sigma^{-1}(1)} \otimes \cdots \otimes \nu_{\sigma^{-1}(m)}\right)
$$

with the appropriate Koszul sign. For a morphism $\varphi^{\mathrm{op}}:(B \leftarrow X) \longrightarrow\left(B^{\prime} \leftarrow X^{\prime}\right)$ of 1 -Forest ${ }^{\mathrm{op}}$, we define $N\left[\varphi^{\mathrm{op}}\right]$ to be the universal morphism mapping a component $\chi: B \rightarrow \underline{m}$ to $\chi^{\prime}:=\varphi^{*} \chi: B^{\prime} \rightarrow \underline{m}$ by application of

$$
N\left[\varphi_{1}^{\mathrm{op}}\right] \otimes \cdots \otimes N\left[\varphi_{m}^{\mathrm{op}}\right]
$$

where $\varphi_{i}$ are the appropriate restrictions of $\varphi$.

With this new notation in place, we define the composite product of dg collections $M, N$ by

$$
(M \circ N)[1 \leftarrow X]:=\operatorname{colim}_{\substack{1 \leftarrow B \leftarrow X \\ \in 2-\operatorname{Tree}(X)^{\mathrm{op}}}} M[1 \leftarrow B] \otimes N[B \leftarrow X],
$$

where the category 2-Tree $(X)$ consists of 2-trees with a given set $X$ of leaves and morphisms acting identically on the leaves. Note that this can be interpreted as a colimit over the slice category $2-$ Tree $^{\text {op }} /(1 \leftarrow X)$.

We now provide a simple and explicit way of computing the colimit above. Consider a partition $P$ of $X$, i.e. a set of disjoint nonempty subsets covering $X$. Such a partition defines a 2-tree $1 \leftarrow P \leftarrow X$ where the map from $X$ to $P$ sends each element to its containing subset. On the other hand, any 2 -tree $1 \leftarrow B \leftarrow X$ gives rise to a partition $P:=\left\{X_{b} \mid b \in B\right\}$ of $X$, and any two isomorphic objects of 2-Tree $(X)$ define the same partition $P$. In other words, the discrete category $\operatorname{Part}(X)$ provides a skeleton for the category 2-Tree $(X)$. As a result, the composite product $M \circ N$ of $\operatorname{dg}$ collections $M, N$ can be computed as

$$
\begin{aligned}
(M \circ N)[1 \leftarrow X] & =\bigoplus_{P \in \operatorname{Part}(X)} M[1 \leftarrow P] \otimes N[P \leftarrow X] \\
& =\bigoplus_{P \in \operatorname{Part}(X)} M[1 \leftarrow P] \otimes\left(\bigoplus_{\chi: P \rightarrow \underline{m}} N\left[1 \leftarrow P_{1}\right] \otimes \cdots \otimes N\left[1 \leftarrow P_{m}\right]\right)_{\mathbb{S}_{m}},
\end{aligned}
$$

where we used the notation $P_{i}:=\chi^{-1}(i)$. A typical element of $(M \circ N)[1 \leftarrow X]$ is denoted as $\mu \circ_{\chi}\left(\nu_{1}, \ldots, \nu_{m}\right)$.

We define a dg collection $I$ by

$$
I[1 \leftarrow X]:= \begin{cases}\mathbf{k}, & \text { if } X \cong 1 \\ 0, & \text { otherwise }\end{cases}
$$

This provides a unit for the composite product and turns (dg Coll, o, I) into a monoidal category. The monoidal structure is nonsymmetric, and the associativity isomorphisms $(M \circ N) \circ K \cong M \circ(N \circ K)$ involve reordering elements (see Figure 1) and hence the Koszul sign rule. 


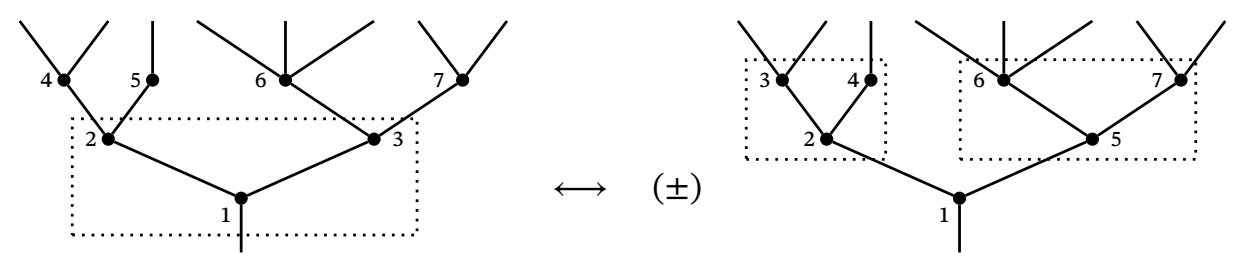

FIGURE 1. Associativity of the composite product.

1.2. Infinitesimal composite product. Note that the composite product is not linear in its right argument, i.e. in general

$$
M \circ\left(N_{1} \oplus N_{2}\right) \neq M \circ N_{1} \oplus M \circ N_{2}
$$

for dg collections $M, N_{1}, N_{2}$. We introduce here the notation $M \circ\left(N_{1} ; N_{2}\right)$ for the sub $\mathrm{dg}$ collection that is linear in $N_{2}$, i.e. that is spanned by elements $\mu{ }^{\circ} \chi\left(\nu_{1}, \ldots, \nu_{m}\right)$ where $v_{i} \in N_{2}$ for exactly one of $\nu_{1}, \ldots, \nu_{m}$ and $\nu_{j} \in N_{1}$ for $j \neq i$. More precisely, one might define a functor $\left(N_{1} ; N_{2}\right)$ on 1 -forests with a marked tree:

$$
\left(N_{1} ; N_{2}\right)[b \in B \leftarrow X]:=N_{1}\left[B \backslash b \leftarrow X \backslash X_{b}\right] \otimes N_{2}\left[1 \leftarrow X_{b}\right]
$$

and extend it to give

$$
\begin{aligned}
\left(N_{1} ; N_{2}\right)[B \leftarrow X] & =\bigoplus_{b \in B} N_{1}\left[B \backslash b \leftarrow X \backslash X_{b}\right] \otimes N_{2}\left[1 \leftarrow X_{b}\right] \\
& \cong\left(\bigoplus_{\chi: B \rightarrow \underline{n}} \bigoplus_{i=1}^{n} N_{1}\left[1 \leftarrow X_{1}\right] \otimes \cdots \otimes N_{2}\left[1 \leftarrow X_{i}\right] \otimes \cdots \otimes N_{1}\left[1 \leftarrow X_{n}\right]\right)_{\mathbb{S}_{n}} .
\end{aligned}
$$

The infinitesimal composite product can then be defined as

$$
M \circ\left(N_{1} ; N_{2}\right)[1 \leftarrow X]:=\operatorname{colim}_{\substack{1 \leftarrow B \leftarrow X \\ \in \operatorname{Tree}(X)^{\text {op }}}} M[1 \leftarrow B] \otimes\left(N_{1} ; N_{2}\right)[B \leftarrow X] .
$$

As shorthand notation, we introduce $M \circ{ }_{(1)} N:=M \circ(I ; N)$ since it appears so frequently, and we write $\mu \otimes_{\chi, i} \nu$ for $\mu \circ_{\chi}(1, \ldots, 1, \nu, 1, \ldots, 1)$ with $\nu$ in $i$-th place.

On morphisms, we introduce two types of infinitesimal composite product. Given $f: M \rightarrow M^{\prime}, g_{1}: N_{1} \rightarrow N_{1}^{\prime}$, and $g_{2}: N_{2} \rightarrow N_{2}^{\prime}$, we define $f \circ\left(g_{1} ; g_{2}\right)$ as the composition

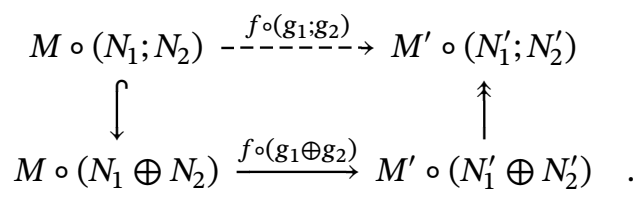

Consider now $f: M \rightarrow M^{\prime}$ and $g: N \rightarrow N^{\prime}$. We denote by $f{ }^{\circ}(1) g$ the morphism $f \circ(1 ; g)$, i.e. $\left(f \circ{ }_{(1)} g\right)\left(\mu \otimes_{\chi, i} \nu\right)=(-1)^{|g||\mu|} \cdot f(\mu) \otimes_{\chi, i} g(\nu)$. Given the same data, we also define a morphism

$$
f \circ \circ^{\prime} g: M \circ N \longrightarrow M \circ(N ; N) \stackrel{f \circ(1) g}{\longrightarrow} M^{\prime} \circ\left(N ; N^{\prime}\right),
$$

and when $N^{\prime}=N$ we implicitly postcompose with

$$
M^{\prime} \circ(N ; N) \longrightarrow M^{\prime} \circ(N \oplus N) \longrightarrow M \circ N,
$$


where the second map is just addition. In this way we obtain $f \circ^{\prime} g: M \circ N \rightarrow M^{\prime} \circ N$.

REMARK 1.2.1. With the above notation, we can write the differential of the full composite product as $\mathrm{d}^{M \circ N}=\mathrm{d}^{M} \circ 1+1 \circ^{\prime} \mathrm{d}^{N}$.

1.3. $\mathbb{S}$-Modules. Let $\mathbb{S}:=\coprod_{n \geq 1} \mathbb{S}_{n}$ be the symmetric groupoid and denote by

$$
\operatorname{dg} \mathbb{S}-\operatorname{Mod}=\left[\mathbb{S}^{\text {op }}, \mathbf{k}-\mathrm{Ch}\right]
$$

the category of right $\mathbb{S}$-modules in $\mathbf{k}$-Ch or $d g \mathbb{S}$-modules for short. Explicitly, a $d g \mathbb{S}$ module $M$ is a collection of chain complexes $M(n)$ equipped with right $\mathbb{S}_{n}$-actions for each arity $n \geq 1$. A morphism of $d g \mathbb{S}$-modules $f: M \rightarrow N$ is a collection of equivariant morphisms $f(n): M(n) \rightarrow N(n)$ of chain complexes. We will often write a dg $\mathbb{S}$-module as a sequence $(M(1), M(2), \ldots)$.

The symmetric groupoid $\mathbb{S}$ provides a skeleton for the category 1 -Tree via the obvious embedding functor mapping $n \in \mathbb{S}$ to the 1-tree $1 \leftarrow \underline{n}$. In the following, we use this to equip dg $\mathbb{S}$-Mod with extra structure in such a way that it becomes equivalent to $\mathrm{dg}$ Coll in the sense of Lemmas 1.3.1 and 1.3.2

As for dg collections, the category of $\mathrm{dg} \mathbb{S}$-modules inherits the abelian and symmetric monoidal structure of $\mathbf{k}$-Ch, i.e. for $\operatorname{dg} \mathbb{S}$-modules $M, N$ we have

$$
\begin{aligned}
& (M \oplus N)(n):=M(n) \oplus N(n), \\
& (M \otimes N)(n):=M(n) \otimes N(n),
\end{aligned}
$$

with the diagonal $\mathbb{S}_{n}$-action. Given another $\operatorname{dg} \mathbb{S}$-module $K$, we define

$$
\operatorname{hom}(N, K)(n):=\operatorname{hom}(N(n), K(n))
$$

with $\mathbb{S}_{n}$-action given by

$$
\operatorname{hom}(N, K)\left(\sigma^{\mathrm{op}}\right): f \longmapsto f^{\sigma}=K\left(\sigma^{\mathrm{op}}\right) \circ f \circ N\left(\sigma^{-1, \mathrm{op}}\right)
$$

resp. $f^{\sigma}(\nu)=f\left(\nu \cdot \sigma^{-1}\right) \cdot \sigma$. This again gives an internal homomorphism adjunction

$$
\operatorname{Hom}(M \otimes N, K) \cong \operatorname{Hom}(M, \operatorname{hom}(N, K)) \text {. }
$$

Lemma 1.3.1. The categories $\mathrm{dg} \mathbb{S}$-Mod of $d g \mathbb{S}$-modules and $\mathrm{dg}$ Coll of $d g$ collections are equivalent.

ProOF. Precomposition of dg collections with the embedding $\mathbb{S}^{\text {op }} \hookrightarrow 1$-Tree ${ }^{\text {op }}$ defines a restriction functor and this functor admits a left adjoint

$$
\widetilde{(\lrcorner)}: \operatorname{dg} \mathbb{S}-\operatorname{Mod}=\left[\mathbb{S}^{\mathrm{op}}, \mathbf{k}-\mathrm{Ch}\right] \rightleftharpoons\left[1-\text { Tree }^{\mathrm{op}}, \mathbf{k}-\mathrm{Ch}\right]=\mathrm{dg} \text { Coll }:\left.\left({ }_{(}\right)\right|_{\mathbb{S}_{\mathrm{op}}}
$$

defined as follows. For an $\mathbb{S}$-module $M$, consider its left Kan extension $\widetilde{M}$ as in the diagram

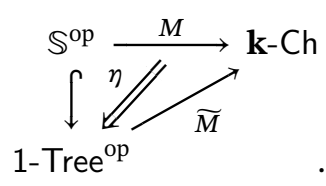


Since $\mathbb{S}^{o p}$ is small and $\mathbf{k}$-Ch is cocomplete, these Kan extensions exist for all $\mathbb{S}$-modules $M$, and they can be computed pointwise as

$$
\tilde{M}[1 \leftarrow X]=\operatorname{colim}_{\chi: X \rightarrow \underline{n}} M(n)=\left(\bigoplus_{\chi: X \rightarrow \underline{n}} M(n)\right)_{\mathbb{S}_{n}},
$$

where the colimit is taken over the comma category ( $\mathbb{S}^{\text {op }} \downarrow 1$-Tree ${ }^{\text {op }}$ ). The universal property of the Kan extension gives a bijection

$$
\operatorname{Nat}(\tilde{M}, N) \cong \operatorname{Nat}\left(M,\left.N\right|_{\mathbb{S}^{o p}}\right),
$$

thereby proving the adjunction. Since $\mathbb{S}^{\mathrm{op}} \hookrightarrow 1$-Tree ${ }^{\text {op }}$ is fully faithful, the components $\eta_{n}$ are isomorphisms. This proves that the adjunction is in fact an equivalence of categories.

We now equip the category of $\mathrm{dg} \mathbb{S}$-modules with a composite product as we did for $\mathrm{dg}$ collections. For dg $\mathbb{S}$-modules $M, N$, we define

$$
(M \circ N)(n):=\bigoplus_{m \geq 1} M(m) \otimes_{\mathbb{S}_{m}} \bigoplus \operatorname{Ind}_{\mathbb{S}_{\mathbb{n}_{1}} \times \cdots \times \mathbb{S}_{n_{m}}+\cdots+n_{m}}^{\mathbb{S}_{n}}\left(N\left(n_{1}\right) \otimes \cdots \otimes N\left(n_{m}\right)\right),
$$

where the (left) $\mathbb{S}_{m}$-action on the right is given by transposing factors while honoring the Koszul sign rule. The $\mathbb{S}$-module $I=(\mathbf{k}, 0, \ldots)$ acts as a (two-sided) unit with respect to the composite product. This turns the category of $\mathrm{dg} \mathbb{S}$-modules into a monoidal category (dg $\mathbb{S}$-Mod, o, I).

Note that, since the unshuffles $\operatorname{Sh}^{-1}\left(n_{1}, \ldots, n_{m}\right)$ form a set of representatives for the right cosets $\mathbb{S}_{n} /\left(\mathbb{S}_{n_{1}} \times \cdots \times \mathbb{S}_{n_{m}}\right)$, the composite product admits an expansion

(21) $(M \circ N)(n)=\bigoplus_{m \geq 1} M(m) \otimes_{\mathbb{S}_{m}} \bigoplus_{n=n_{1}+\cdots+n_{m}} N\left(n_{1}\right) \otimes \cdots \otimes N\left(n_{m}\right) \otimes \mathbf{k}\left[\operatorname{Sh}^{-1}\left(n_{1}, \ldots, n_{m}\right)\right]$, and, since the $\mathbb{S}_{m}$-action on the right is free,

$$
=\bigoplus_{\substack{m \geq 1 \\ n=n_{1}+\cdots+n_{m}}} M(m) \otimes N\left(n_{1}\right) \otimes \cdots \otimes N\left(n_{m}\right) \otimes \mathbf{k}\left[\overline{\operatorname{Sh}}^{-1}\left(n_{1}, \ldots, n_{m}\right)\right] .
$$

We denote an element $\mu \otimes_{\mathbb{S}_{m}} \nu_{1} \otimes \cdots \otimes \nu_{m} \otimes \sigma$ of $(M \circ N)(n)$ by $\mu \circ\left(\nu_{1}, \ldots, \nu_{m}\right)^{\sigma}$.

Lemma 1.3.2. The adjoint equivalence

$$
\widetilde{(\longleftarrow)}: \mathrm{dg} \mathbb{S}-\text { Mod } \rightleftharpoons \mathrm{dg} \text { Coll }:\left.\left({ }_{(}\right)\right|_{\mathbb{S}^{o p}}
$$

of Lemma 1.3.1 is an equivalence of monoidal categories.

Proof. It is enough to show that $\left.(\triangleleft)\right|_{\mathbb{S}^{\circ}}$ is strong monoidal, i.e. admits a natural isomorphism

$$
\phi:\left.\left.\left.\left(\_\right)\right|_{\mathbb{S o p}} \circ\left({ }_{\smile}\right)\right|_{\mathbb{S o p}} \Longrightarrow\left({ }_{\longleftarrow} \circ_{-}\right)\right|_{\mathbb{S o p}} .
$$

For dg symmetric collections $M, N$, we have

$$
\begin{aligned}
& \left(\left.\left.M\right|_{\mathbb{S}_{\text {op }}} \circ N\right|_{\mathbb{S}^{\text {op }}}\right)(n) \\
& \quad=\bigoplus_{m \geq 1} M[1 \leftarrow \underline{m}] \otimes_{\mathbb{S}_{m}} \bigoplus_{n=n_{1}+\cdots+n_{m}} \operatorname{Ind}_{\mathbb{S}_{n_{1}} \times \cdots \times \mathbb{S}_{n_{m}}}^{\mathbb{S}_{n}}\left(N\left[1 \leftarrow \underline{n_{1}}\right] \otimes \cdots \otimes N\left[1 \leftarrow \underline{n_{m}}\right]\right),
\end{aligned}
$$




$$
\begin{aligned}
& \left.(M \circ N)\right|_{\mathbb{S}^{\mathrm{op}}}(n) \\
& \quad=\bigoplus_{P \in \operatorname{Part}(\underline{n})} M[1 \leftarrow P] \otimes\left(\bigoplus_{\chi: P \rightarrow \underline{m}} N\left[1 \leftarrow P_{1}\right] \otimes \cdots \otimes N\left[1 \leftarrow P_{m}\right]\right)_{\mathbb{S}_{m}} .
\end{aligned}
$$

The main ingredients for the definition of the components $\phi_{M, N}$ are, for each element $\mu \circ\left(v_{1}, \ldots, v_{m}\right)^{\sigma} \in\left(\left.\left.M\right|_{\text {Sop }} \circ N\right|_{\text {Sop }}\right)(n)$, a partition $P$ of $\underline{n}$ and bijections $\chi: P \rightarrow \underline{m}$ and $\chi_{i}: P_{i} \rightarrow \underline{n_{i}}$. From such data we then obtain morphisms

$$
\begin{aligned}
& M\left[\chi^{\mathrm{op}}\right]: M[1 \leftarrow \underline{m}] \longrightarrow M[1 \leftarrow P], \\
& N\left[\chi_{i}^{\mathrm{op}}\right]: N\left[1 \leftarrow \underline{n_{i}}\right] \longrightarrow M\left[1 \leftarrow P_{i}\right] .
\end{aligned}
$$

Consider the subsets $P_{i} \subset \underline{n}$ defined by

$$
\begin{aligned}
& P_{1}=\sigma^{-1}\left(\underline{n_{1}}\right)=\sigma^{-1}\left(\left\{1, \ldots, n_{1}\right\}\right), \\
& P_{2}=\sigma^{-1}\left(n_{1}+\underline{n_{2}}\right)=\sigma^{-1}\left(\left\{n_{1}+1, \ldots, n_{1}+n_{2}\right\}\right), \quad \text { etc. }
\end{aligned}
$$

These $P_{i}$ form a partition $P=\left\{P_{1}, P_{2}, \ldots, P_{m}\right\}$, i.e. $\underline{n}=\coprod_{i} P_{i}$. Now denote by $\chi$ the map

$$
\chi: P \rightarrow \underline{m}, \quad P_{i} \mapsto i,
$$

and by $\chi_{i}: P_{i} \rightarrow \underline{n_{i}}$ the bijections preserving the induced order on $P_{i} \subset \underline{n}$.

It remains to verify that the above construction leads to welldefined morphisms $\phi_{M, N}(n)$, these morphisms are, in fact, isomorphisms, and the construction is natural in $n$ as well as $M, N$. We leave the rest of the proof to the reader.

The notation for the infinitesimal composite product of dg collections still makes sense for dg $\mathbb{S}$-modules and we adopt it in this context.

1.4. Nonsymmetric collections and $\mathbb{N}$-modules. Consider now the category $d g$ ns Coll introduced earlier. It carries the same pointwise abelian and closed symmetric monoidal structures as given in Equations (9) (11) The composite product is defined as before via Equations (12) and (14) with the colimits understood over corresponding planar trees. We denote by $\operatorname{OrdPart}(X)$ the discrete category of ordered partitions, i.e. of ordered sets $P$ of disjoint nonempty subsets covering $X$ such that the map $P \leftarrow X$ mapping elements to their containing subset is order preserving. This category forms a skeleton for the category of planar 2-trees with leaves $X$, and we obtain

$$
(M \circ N)[1 \leftarrow X]=\bigoplus_{P \in \operatorname{OrdPart}(X)} M[1 \leftarrow P] \otimes\left(N\left[1 \leftarrow P_{1}\right] \otimes \cdots \otimes N\left[1 \leftarrow P_{m}\right]\right),
$$

where $P=\left\{P_{1}<\cdots<P_{m}\right\}$.

Let $\mathbb{N}$ be the discrete groupoid of natural numbers $n \geq 1$ and denote by

$$
\operatorname{dg} \mathbb{N}-\operatorname{Mod}:=\left[\mathbb{N}^{o p}, \mathbf{k}-\mathrm{Ch}\right]
$$

the category of right $\mathbb{N}$-modules resp. $d g \mathbb{N}$-modules. Explicitly, a $\operatorname{dg} \mathbb{N}$-module $M$ is a collection of chain complexes $M(n)$ for each arity $n \geq 1$ and a morphism of dg $\mathbb{N}$ modules $f: M \rightarrow N$ is a collection of morphisms $f(n): M(n) \rightarrow N(n)$ of chain complexes. The abelian and closed symmetric monoidal structure on $\mathrm{dg} \mathbb{N}$-modules is again given 
pointwise as in Equations (15) (17) and the composite product of $\mathbb{N}$-modules $M$ and $N$ is defined by

$$
(M \circ N)(n):=\bigoplus_{m \geq 1} M(m) \otimes \bigoplus_{n=n_{1}+\cdots+n_{m}}\left(N\left(n_{1}\right) \otimes \cdots \otimes N\left(n_{m}\right)\right) .
$$

The $\mathbb{N}$-module $I=(\mathbf{k}, 0, \ldots)$ acts as a unit with respect to the composite product, and this turns (dg $\mathbb{N}$-Mod, o, I) into a monoidal category. As is the case for dg collections and $\mathrm{dg} \mathbb{S}$-modules, there is an adjoint equivalence

$$
\widetilde{(\lrcorner)}: \mathrm{dg} \mathbb{N}-\operatorname{Mod} \rightleftharpoons \mathrm{dg} \text { ns Coll }:\left.\left({ }_{(}\right)\right|_{\mathbb{N o p}}
$$

between the categories of $\mathrm{dg}$ ns collections and $\mathrm{dg} \mathbb{N}$-modules, and it is an equivalence of monoidal categories.

1.5. Colored collections and colored $\mathbb{S}$-modules. We now turn our attention to the category of $\operatorname{dg} C$-colored collections for some fixed set $C$ of colors. While planar structures on trees simplify their category of modules, $C$-colorings add to the bookkeeping involved. We define the same pointwise abelian and closed symmetric monoidal structures as for dg collections using Equations (9) (11) The composite product is defined as before via Equations (12) and (14), with the colimit taken over appropriate $C$-colored trees. We denote by $C$-col $\operatorname{Part}(X)$ the discrete category of $C$-colored partitions of $X$, i.e. of partitions $P$ of $X$ with a coloring map $P \rightarrow C$. This category is a skeleton for $C$-col 2-Tree $(X)$ and this gives us the following expansions for the composite product:

$$
\begin{aligned}
(M \circ N)[1 \leftarrow X] & =\bigoplus_{P \in C \text {-col Part }(X)} M[1 \leftarrow P] \otimes N[P \leftarrow X] \\
= & \bigoplus_{P \in C \text {-col Part }(X)} M[1 \leftarrow P] \otimes\left(\bigoplus_{\chi: P \rightarrow \underline{m}} N\left[1 \leftarrow P_{1}\right] \otimes \cdots \otimes N\left[1 \leftarrow P_{m}\right]\right)_{S_{m}} .
\end{aligned}
$$

Let $\mathbb{S}^{+} / C$ denote the slice category for

$$
\mathbb{S}^{+}: \mathbb{S} \rightarrow \text { Set }, \quad n \mapsto \underline{n}^{+}=\{0, \ldots, n\}
$$

over the constant functor $C$. An object of $\mathbb{S}^{+} / C$ is a tuple $(n, c)$ consisting of an object $n \in \mathbb{S}$ with a coloring $c: \underline{n}^{+} \rightarrow C$. The category $\mathbb{S}^{+} / C$ is a skeleton for the category of $C$-colored 1-trees via the embedding

$$
\mathbb{S}^{+} / C \longrightarrow C \text {-col 1-Tree }, \quad(n, c) \longmapsto\left(1 \leftarrow \underline{n}, 1 \amalg \underline{n} \cong \underline{n}^{+} \rightarrow C\right) .
$$

We denote by

$$
\operatorname{dg} C \text {-col } \mathbb{S}-\operatorname{Mod}:=\left[\left(\mathbb{S}^{+} / C\right)^{\mathrm{op}}, \mathbf{k}-\mathrm{Ch}\right]
$$

the category of right $\mathbb{S}^{+} / C$-modules in $\mathbf{k}$-complexes or $d g C$-colored $\mathbb{S}$-modules. Explicitly, a $d g C$-colored $\mathbb{S}$-module $M$ consists of, for each arity $n \geq 1$, a collection of chain complexes $M\left(c_{0} ; c_{1}, \ldots, c_{n}\right)$ indexed by $(n+1)$-tuples of colors $c_{i} \in C$ equipped with right $\mathbb{S}_{n}$-actions

$$
\left({ }_{(}\right)^{\sigma}: M\left(c_{0} ; c_{1}, \ldots, c_{n}\right) \longrightarrow M\left(c_{0} ; c_{\sigma(1)}, \ldots, c_{\sigma(n)}\right) .
$$


A morphism $f: M \rightarrow N$ is a collection of equivariant morphisms

$$
f\left(c_{0} ; c_{1}, \ldots, c_{n}\right): M\left(c_{0} ; c_{1}, \ldots, c_{n}\right) \longrightarrow N\left(c_{0} ; c_{1}, \ldots, c_{n}\right)
$$

of chain complexes. The abelian and closed symmetric monoidal structures on dg $C$-colored $\mathbb{S}$-modules are defined pointwise, i.e.

$$
\begin{aligned}
& (M \oplus N)\left(c_{0} ; c_{1}, \ldots, c_{n}\right):=M\left(c_{0} ; c_{1}, \ldots, c_{n}\right) \oplus N\left(c_{0} ; c_{1}, \ldots, c_{n}\right), \\
& (M \otimes N)\left(c_{0} ; c_{1}, \ldots, c_{n}\right):=M\left(c_{0} ; c_{1}, \ldots, c_{n}\right) \otimes N\left(c_{0} ; c_{1}, \ldots, c_{n}\right),
\end{aligned}
$$

with the diagonal $\mathbb{S}_{n}$-actions, and

$$
\operatorname{hom}(M, N)\left(c_{0} ; c_{1}, \ldots, c_{n}\right):=\operatorname{hom}\left(M\left(c_{0} ; c_{1}, \ldots, c_{n}\right), N\left(c_{0} ; c_{1}, \ldots, c_{n}\right)\right) \text {. }
$$

The composite product for $\mathrm{dg} C$-colored $\mathbb{S}$-modules is given by

$$
\begin{aligned}
& (M \circ N)\left(c_{0} ; c_{1}, \ldots, c_{n}\right) \\
& \quad:=\bigoplus_{m \geq 1}\left(\bigoplus_{d_{i} \in C}\left(M\left(c_{0} ; d_{1}, \ldots, d_{m}\right) \otimes \bigoplus_{n=n_{1}+\cdots+n_{m}} \operatorname{Ind}_{\mathbb{S}_{n_{1}} \times \cdots \times \mathbb{S}_{n_{m}}}^{\mathbb{S}_{n}}\left(N\left(d_{1} ; c_{1}, \ldots, c_{n_{1}}\right) \otimes \cdots\right)\right)\right)_{\mathbb{S}_{m}},
\end{aligned}
$$

and the $\operatorname{dg} C$-colored $\mathbb{S}$-module $I$ defined by $I(c ; c)=\mathbf{k}$ for $c \in C$ and zero otherwise acts as a unit with respect to it, turning ( $\operatorname{dg} C$-col $\mathbb{S}$-Mod, o, $I$ ) into a monoidal category. As before, there is an adjoint equivalence

$$
\widetilde{(\smile)}: \mathrm{dg} C \text {-col } \mathbb{S} \text {-Mod } \rightleftharpoons \mathrm{dg} C \text {-col Coll : (๘) }\left.\right|_{\left(\mathbb{S}^{+} / C\right)^{\text {op }}}
$$

and it is an equivalence of monoidal categories.

1.6. Weightgraded collections. A weightgraded chain complex is a chain complex $V$ with an additional decomposition $V=\bigoplus_{w \geq 0} V^{(w)}$ into its weight $w$ components $V^{(w)}$. The direct sum and tensor product of weightgraded chain complexes $U$ and $V$ are again weightgraded with components

$$
(U \oplus V)^{(w)}=U^{(w)} \oplus V^{(w)} \quad \text { resp. } \quad(U \otimes V)^{(w)}=\bigoplus_{w=u+v} U^{(u)} \otimes V^{(v)} .
$$

The category of weightgraded dg collections or wdg collections is the category of $\mathrm{dg}$ collections taking values in weightgraded chain complexes. Given a wdg collection $M$, we introduce its sub dg collection $M^{(w)}[1 \leftarrow X]:=M[1 \leftarrow X]^{(w)}$ of weight $w$ elements and denote by $\operatorname{wdeg}(\mu)=w$ the weight of a weighthomogeneous element $\mu \in M^{(w)}$. Slightly abusing notation, we introduce the extension

$$
N^{(v)}[B \leftarrow X]:=\left(\bigoplus_{\substack{\chi: B \rightarrow \underline{m} \\ v=v_{1}+\cdots+v_{m}}} N^{\left(v_{1}\right)}\left[1 \leftarrow X_{1}\right] \otimes \cdots \otimes N^{\left(v_{m}\right)}\left[1 \leftarrow X_{m}\right]\right)_{\mathbb{S}_{m}},
$$

and obtain for the composite product the components

$$
(M \circ N)^{(w)}[1 \leftarrow X]:=\operatorname{colim}_{\substack{1 \leftarrow B \leftarrow X \\ \in 2-\operatorname{Tree}(X)^{\mathrm{op}}}}\left(\bigoplus_{w=u+v} M^{(u)}[1 \leftarrow B] \otimes N^{(v)}[B \leftarrow X]\right)
$$




$$
=\left(\bigoplus_{w=u+v} M^{(u)} \circ N^{(v)}\right)[1 \leftarrow X] .
$$

Explicitly, this means that the weight of an element of the composite product $M \circ N$ is given by

$$
\operatorname{wdeg}\left(\mu \circ\left(\nu_{1}, \ldots, \nu_{m}\right)\right)=\operatorname{wdeg}(\mu)+\operatorname{wdeg}\left(\nu_{1}\right)+\cdots+\operatorname{wdeg}\left(\nu_{m}\right) .
$$

Analogous definitions can be made for decorated collections.

1.7. Schur functors. We consider the category

$$
\left[0-\text { Tree }^{\text {op }}, \mathbf{k}-\mathrm{Ch}\right]
$$

whose objects we think of as spaces. Given such a space $V$, we extend its domain to 0 -Forest ${ }^{\text {op }}$ via

$$
\begin{aligned}
V[X] & :=\operatorname{colim}_{\chi: X \rightarrow \underline{n}} V\left[X_{1}\right] \otimes \cdots \otimes V\left[X_{n}\right] \\
& =\left(\bigoplus_{\chi: X \rightarrow \underline{n}} V\left[X_{1}\right] \otimes \cdots \otimes V\left[X_{n}\right]\right)_{\mathbb{S}_{n}},
\end{aligned}
$$

where $X_{i}:=\chi^{-1}(i)$, and the $\mathbb{S}_{n}$-action is given by

$$
\sigma \cdot\left(\chi, v_{1} \otimes \cdots \otimes v_{n}\right)= \pm\left(\sigma \chi, v_{\sigma^{-1}(1)} \otimes \cdots \otimes v_{\sigma^{-1}(n)}\right)
$$

with the appropriate Koszul sign. For a morphism $f^{\text {op }}: X \rightarrow X^{\prime}$ of 0 -Forest ${ }^{\text {op }}$, we define $V\left[f^{\text {op }}\right]$ to be the universal morphism mapping a component $\chi: X \rightarrow \underline{n}$ to $\chi^{\prime}:=f^{*} \chi: X^{\prime} \rightarrow$ $\underline{n}$ by application of

$$
V\left[f_{1}^{\mathrm{op}}\right] \otimes \cdots \otimes V\left[f_{n}^{\mathrm{op}}\right]
$$

where $f_{i}$ are the appropriate restrictions of $f$.

Using this notation, we associate to any $\operatorname{dg}$ collection $M$ an endofunctor on spaces, called its Schur functor, by

$$
\begin{aligned}
M(V)[1]:= & \operatorname{colim}_{\substack{1 \leftarrow X \\
\in 1-\operatorname{Tree}^{o p}}} M[1 \leftarrow X] \otimes V[X] \\
= & \bigoplus \bigoplus_{1 \leftarrow \underline{n}}(M[1 \leftarrow \underline{n}] \otimes V[\{1\}] \otimes \cdots V[\{n\}])_{\mathbb{S}_{n}} .
\end{aligned}
$$

Similarly, we define an endofunctor $\widehat{M}$ of spaces for any dg collection $M$, called its complete Schur functor, by

$$
\widehat{M}(V)[1]:=\prod_{1 \leftarrow \underline{n}}(M[1 \leftarrow \underline{n}] \otimes V[\{1\}] \otimes \cdots V[\{n\}])_{\mathbb{S}_{n}} .
$$

Note that the complete Schur functor $\widehat{M}$ is not the dual of $M$ since we still use coinvariants (and not invariants). This is absolutely essential for the constructions in later sections to work over a ring.

The definitions given above may seem unnecessarily complicated, however this is because they are written in a general way to capture all three types of collections we consider. In the case of undecorated trees, spaces are just chain complexes and the 
definitions collapse to the much simpler

$$
M(V) \cong \bigoplus_{n \geq 1}\left(M(n) \otimes V^{\otimes n}\right)_{\mathbb{S}_{n}} \quad \text { resp. } \quad \widehat{M}(V) \cong \prod_{n \geq 1}\left(M(n) \otimes V^{\otimes n}\right)_{\mathbb{S}_{n}} .
$$

The construction above associates an endofunctor on spaces to each dg collection, and is, in fact, itself functorial. In particular, we have

$$
(M \circ N)(V) \cong M(N(V)) \quad \text { resp. } \quad \widehat{M \circ N}(V) \cong \widehat{M}(\widehat{N}(V)) .
$$

Note that these isomorphisms involve reordering elements and thus the Koszul sign rule applies (see Figure 1).

Similar definitions can be made for the infinitesimal composite product. Assume $M$ is a dg collection and $V_{1}, V_{2}$ denote spaces. We denote by $M\left(V_{1} ; V_{2}\right)$ the subfunctor of the Schur functor $M\left(V_{1} \oplus V_{2}\right)$ that is linear in $V_{2}$ and by $\widehat{M}\left(V_{1} ; V_{2}\right)$ the subfunctor of the complete Schur functor $\widehat{M}\left(V_{1} \oplus V_{2}\right)$ that is linear in $V_{2}$.

\section{Operads}

The category of dg operads is the category of monoids in dg collections,

$$
\text { dg Opd := Mon(dg Coll) . }
$$

We make this definition explicit below, followed by some basic terminology and an example that will play a key role in the theory of operads. Note that we could just as well (and will in later sections) work with the category $\mathrm{dg} \mathbb{S}$-Mod instead of $\mathrm{dg}$ Coll by the equivalence proven in the Section 1.3

Definition 2.0.1. A $d g$ operad $(\mathcal{P}, \gamma, \eta)$ consists of a dg collection $\mathcal{P}$ with composition map $\gamma: \mathcal{P} \circ \mathcal{P} \rightarrow \mathcal{P}$ and unit $\eta: I \rightarrow \mathcal{P}$ satisfying the associativity and unitality axioms, i.e. the following diagrams must commute:
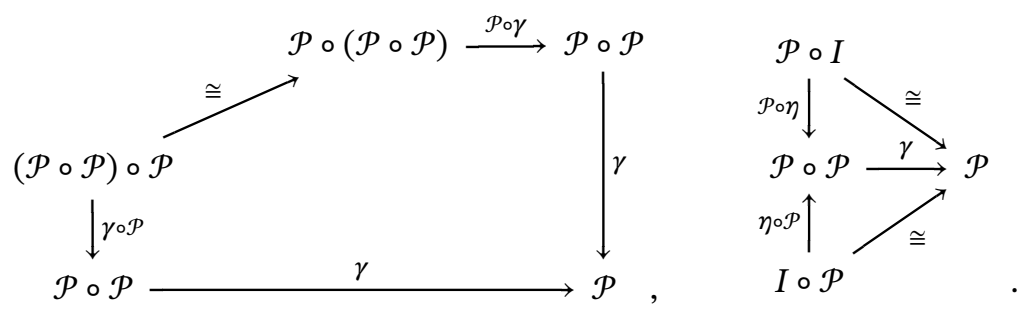

A morphism of $d g$ operads is a morphism of their underlying dg collections $f: \mathcal{P} \rightarrow \mathcal{P}^{\prime}$ commuting with the structure maps in the sense that the following diagrams commute:
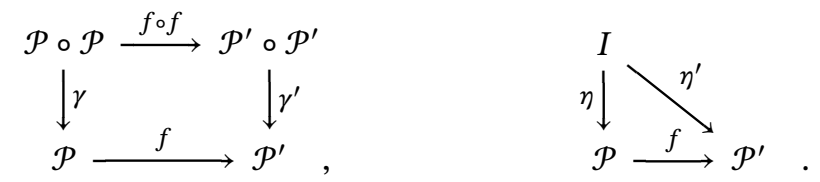

EXAMPLE 2.0.2. The trivial or identity operad $I$ is given by $I[1 \leftarrow 1]=\mathbf{k} \cdot u$ with trivial differential, unit $\eta$ determined by $\eta(1)=u$, and composition $\gamma$ by $\gamma(u \circ(u))=u$. 
An augmentation for $\mathcal{P}$ is a morphism of dg operads $\varepsilon: \mathcal{P} \rightarrow I$ satisfying $\varepsilon \eta=I$. For a morphism $f: \mathcal{P} \rightarrow \mathcal{P}^{\prime}$ of augmented operads, it is assumed that it respects the augmentations, i.e. that $\varepsilon^{\prime} f=\varepsilon$ holds. We denote by $\overline{\mathcal{P}}$ the augmentation ideal $\overline{\mathcal{P}}:=\operatorname{ker} \varepsilon$ and the restriction of $\gamma$ to it by $\bar{\gamma}: \overline{\mathcal{P}} \circ \overline{\mathcal{P}} \rightarrow \overline{\mathcal{P}}$.

The category wdg Opd of weightgraded dg operads or wdg operads is the category of monoids in wdg collections. Explicitly, a wdg operad $(\mathcal{P}, \gamma, \eta)$ is an operad on an underlying weightgraded dg collection $\mathcal{P}$ whose operadic structure maps $\gamma$ and $\eta$ preserve the weight, and morphisms of wdg operads are assumed to preserve the weight as well. A wdg operad is called connected, if $\mathcal{P}^{(0)}=I$. Any connected wdg operad is augmented with augmentation ideal $\overline{\mathcal{P}}=\bigoplus_{w \geq 1} \mathcal{P}^{(w)}$.

Given a dg operad $(\mathcal{P}, \gamma, \eta)$, we denote by $\gamma_{(1)}$ its infinitesimal or partial composition map defined as the composition in the following diagram:

$$
\gamma_{(1)}:=\left(\mathcal{P}_{(1)} \mathcal{P}=\mathcal{P} \circ(I ; \mathcal{P}) \stackrel{\mathcal{P}_{\circ}(\eta ; \mathcal{P})}{\longrightarrow} \mathcal{P} \circ(\mathcal{P} ; \mathcal{P}) \longrightarrow \mathcal{P} \circ \mathcal{P} \stackrel{\gamma}{\longrightarrow} \mathcal{P}\right) .
$$

In fact, this infinitesimal composition determines the full composition map.

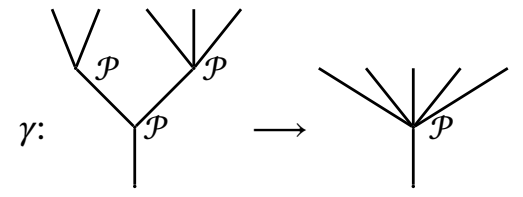

(A) Full composition.

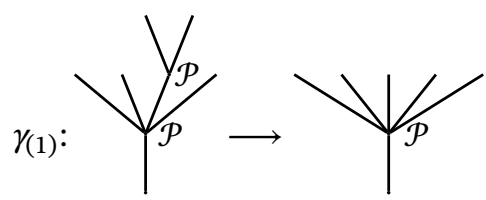

(B) Infinitesimal composition.

FIGURE 2. Comparison of operadic composition maps.

REMARK 2.0.3. We use the notation $\mu \circ_{\chi, i} \nu:=\gamma_{(1)}\left(\mu \otimes_{\chi, i} \nu\right)$ for partial compositions, or $\mu \circ_{i} \nu=\gamma_{(1)}\left(\mu \otimes_{i} \nu\right)$ in case we are working with dg $\mathbb{S}$-modules.

The following example of the operad of concrete multilinear operations on a space plays a fundamental role and is used to model other operads on. Section 2.2 deals with such representations $\mathcal{P} \rightarrow \operatorname{End}_{V}$ of operads, better known as $\mathcal{P}$-algebras.

EXAMPLE 2.0.4. Given a space $V: 0-$ Tree $^{\mathrm{op}} \rightarrow \mathbf{k}$-Ch, we define its endomorphism operad as follows. The underlying collection is given-on objects-by

$$
\operatorname{End}_{V}[1 \leftarrow X]:=\operatorname{hom}(V[X], V[1]),
$$

which, by definition of $V[X]$, expands to

$$
\begin{aligned}
& =\operatorname{hom}\left(\left(\bigoplus_{\chi: X \rightarrow \underline{n}} V\left[X_{1}\right] \otimes \cdots \otimes V\left[X_{n}\right]\right)_{\mathbb{S}_{n}}, V[1]\right) \\
& =\left(\prod_{\chi: X \rightarrow \underline{n}} \operatorname{hom}\left(V\left[X_{1}\right] \otimes \cdots \otimes V\left[X_{n}\right], V[1]\right)\right)^{\mathbb{S}_{n}} .
\end{aligned}
$$


An element $\mu \in \operatorname{End}_{V}[1 \leftarrow X]$ is a collection of maps

$$
\left\{\mu^{(\chi)}: V\left[X_{1}\right] \otimes \cdots \otimes V\left[X_{n}\right] \rightarrow V[1]\right\}_{\chi: X \rightarrow \underline{n}},
$$

satisfying the invariance condition $\mu=\mu \cdot \sigma$ for $\sigma \in \mathbb{S}_{n}$. More explicitly, this means that its components satisfy (see Section 1.7)

$$
\mu^{(\chi)}\left(x_{1}, \ldots, x_{n}\right)=(\mu \cdot \sigma)^{(\chi)}\left(x_{1}, \ldots, x_{n}\right)=\mu^{(\sigma \chi)}\left(x_{\sigma^{-1}(1)}, \ldots, x_{\sigma^{-1}(n)}\right),
$$

and, hence, that $\mu \in \operatorname{End}_{V}[1 \leftarrow X]$ is fully determined by any one its components. On a morphism $\varphi^{\mathrm{op}}: X \rightarrow X^{\prime}$, the collection $\operatorname{End}_{V}\left[\varphi^{\mathrm{op}}\right]$ is given by precomposition with $V\left[\varphi^{-1, \mathrm{op}}\right]$ :

$$
\operatorname{End}_{V}\left[\begin{array}{c}
X \stackrel{\varphi^{\mathrm{op}}}{\longrightarrow} X^{\prime} \\
\searrow
\end{array}\right](V[X] \stackrel{\mu}{\longrightarrow} V[1])=\left(V\left[X^{\prime}\right] \stackrel{V\left[\varphi^{-1, \mathrm{op}}\right]}{\longrightarrow} V[X] \stackrel{\mu}{\longrightarrow} V[1]\right) .
$$

When $\varphi^{\mathrm{op}}: X \rightarrow X$ is an automorphism, we will denote $\operatorname{End}_{V}\left[\varphi^{\mathrm{op}}\right](\mu)$ by $\mu^{\varphi}$. Explicitly, it is given by components

$$
\begin{aligned}
\left(\mu^{\varphi}\right)^{(\chi)}\left(v_{1}, \ldots, v_{n}\right) & =\mu^{\left(\chi \varphi^{-1}\right)}\left(v_{1}, \ldots, v_{n}\right) \\
& =\mu^{\left(\sigma^{-1} \chi\right)}\left(x_{1}, \ldots, x_{n}\right),
\end{aligned}
$$

where $\sigma=\chi \varphi \chi^{-1}$; and, using the $\mathbb{S}_{n}$-invariance, we obtain

$$
=\mu^{(\chi)}\left(x_{\sigma^{-1}(1)}, \ldots, x_{\sigma^{-1}(n)}\right) .
$$

We define the structure of an operad on $\operatorname{End}_{V}$ as follows: the composition map $\gamma:$ End $_{V} \circ$ End $_{V} \rightarrow$ End $_{V}$ is given by components

$$
\operatorname{End}_{V}[1 \leftarrow P] \otimes \operatorname{End}_{V}[P \leftarrow X] \longrightarrow \operatorname{End}_{V}[1 \leftarrow X]
$$

for a partition $P$ of $X$. Given $\mu \in \operatorname{End}_{V}[1 \leftarrow P], \xi: P \rightarrow \underline{m}$, and $\nu_{i} \in \operatorname{End}_{V}\left[1 \leftarrow P_{i}\right]$ with $\chi_{i}: P_{i} \rightarrow \underline{n_{i}}$, the map

$$
\bigotimes_{j=1}^{n} V\left[X_{j}\right] \stackrel{\bigotimes_{i=1}^{m} \nu_{i}^{\left(\chi_{i}\right)}}{\longrightarrow} \bigotimes_{i=1}^{m} V\left[\left\{P_{i}\right\}\right] \stackrel{\mu^{(\xi)}}{\longrightarrow} V[1]
$$

defines the component $\gamma\left(\mu, v_{i}\right)^{(\chi)}$ for

$$
\chi: X \rightarrow \underline{n}, \quad \chi\left(x \in X_{i}\right)=n_{1}+\cdots+n_{i-1}+\chi_{i}(x) .
$$

The unit for the operad $\operatorname{End}_{V}$ is the identity map id: $V[1] \rightarrow V[1]$ in $\operatorname{End}_{V}[1 \leftarrow 1]$.

2.1. Modules over an operad. By definition of operads as monoids in dg Coll, they come with notions of left and right modules as well as bimodules. We give explicit definitions and consider some examples. Throughout this section, let $(\mathcal{P}, \gamma, \eta)$ and $(\mathcal{Q}, \gamma, \eta)$ denote dg operads.

Definition 2.1.1. A left $d g$ P -module $(M, \gamma)$ is a $\operatorname{dg}$ collection $M$ with a left multiplication map $\gamma: \mathcal{P} \circ M \rightarrow M$. This structure map is required to be compatible with the operad 
multiplication and unit maps, i.e. the following diagrams must commute:
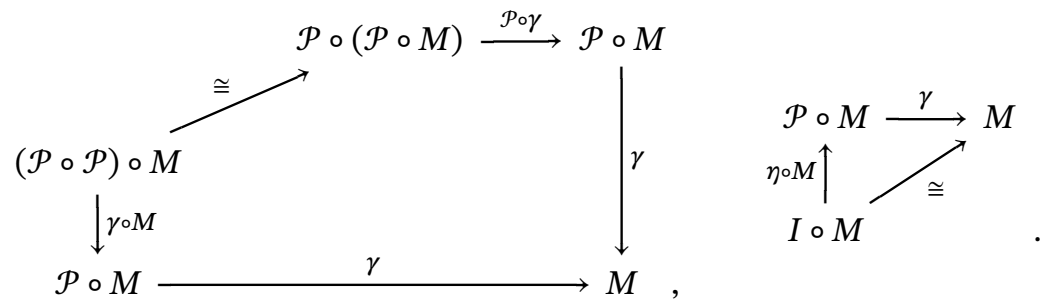

A morphism of left $d g \mathcal{P}$-modules is a morphism of their underlying dg collections $f: M \rightarrow M^{\prime}$ compatible with the $\mathcal{P}$-module multiplication maps in the sense that the following diagram commutes:

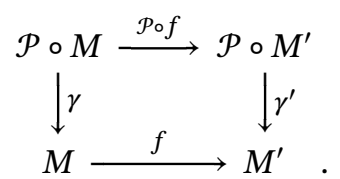

We denote the category of left $d g \mathcal{P}$-modules by $\mathrm{dg} \mathcal{P}$-Mod.

An analogous definition of right modules exists, which we give below. The relevant diagrams are obtained from the diagrams of Equations (29) and (30) above by mirror symmetry.

Definition 2.1.2. A right $d g \mathcal{P}$-module $(M, \gamma)$ is a dg collection $M$ with a right multiplication map $\gamma: M \circ \mathcal{P} \rightarrow M$. This structure map is required to be compatible with the operad multiplication and unit maps. A morphism of right $d g$ P -modules is a morphism of their underlying dg collections $f: M \rightarrow M^{\prime}$ compatible with the module multiplication maps. We denote the category of right $d g \mathcal{P}$-modules by $\mathrm{dg} \operatorname{Mod}-\mathcal{P}$.

Definition 2.1.3. A $d g(\mathcal{P}, Q)$-bimodule (or $d g(\mathcal{P}, \mathcal{Q})$-module) is a triple $\left(M, \gamma^{L}, \gamma^{R}\right)$ consisting of a dg collection $M$, a left multiplication map $\gamma^{L}: \mathcal{P} \circ M \rightarrow M$, and a right multiplication map $\gamma^{R}: M \circ \mathcal{Q} \rightarrow M$, such that $\left(M, \gamma^{L}\right)$ is a left $\operatorname{dg} \mathcal{P}$-module and $\left(M, \gamma^{R}\right)$ is a right $\mathrm{dg} Q$-module. In addition, we require that the left and right multiplication maps are compatible in the sense that the following diagram must commute:

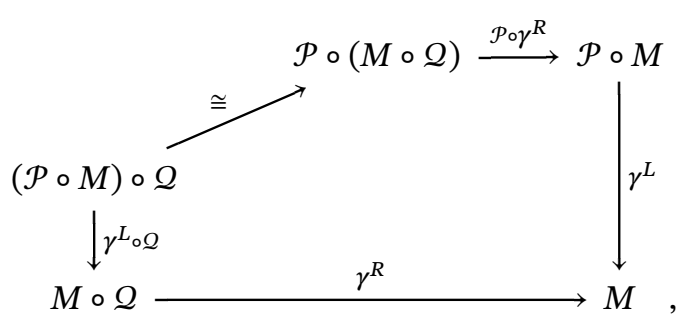

A morphism of $d g(\mathcal{P}, \mathcal{Q})$-bimodules is a morphism of dg collections $f: M \rightarrow M^{\prime}$ which is simultaneously a morphism of left $\operatorname{dg} \mathcal{P}$-modules and of right $\mathrm{dg} \mathcal{Q}$-modules. We denote the category of $\operatorname{dg}(\mathcal{P}, \mathcal{Q})$-bimodules by $\mathrm{dg}(\mathcal{P}, \mathcal{Q})$-Mod.

EXAMPLE 2.1.4. The tuple $(\mathcal{P}, \gamma)$ is both a left and a right $\mathcal{P}$-module. The triple $(\mathcal{P}, \gamma, \gamma)$ is a $(\mathcal{P}, \mathcal{P})$-bimodule and $(\mathcal{P} \circ \mathcal{Q}, \gamma \circ \mathcal{Q}, \mathcal{P} \circ \gamma)$ is a $(\mathcal{P}, \mathcal{Q})$-bimodule. 
EXAMPLE 2.1.5. Given spaces $V, V^{\prime}$, we define $\operatorname{End}_{V^{\prime}}$ to be sub dg collection of maps

$$
\operatorname{End}_{V^{\prime}}^{V}[1 \leftarrow X]:=\operatorname{hom}\left(V[X], V^{\prime}[1]\right) \subset \operatorname{End}_{V \oplus V^{\prime}}[1 \leftarrow X] .
$$

While $\operatorname{End}_{V^{\prime}}^{V}$ is not a sub dg operad, it does have an obvious $\left(\operatorname{End}_{V^{\prime}}\right.$, End $\left._{V}\right)$-bimodule structure given by restriction of the composition map in $\operatorname{End}_{V \oplus V^{\prime}}$.

EXAMPLE 2.1.6. Let $M$ a dg collection. We equip the composite product $\mathcal{P} \circ M$ with a composition map

$$
\gamma:=(\mathcal{P} \circ(\mathcal{P} \circ M) \stackrel{\cong}{\longrightarrow}(\mathcal{P} \circ \mathcal{P}) \circ M \stackrel{\gamma \circ M}{\longrightarrow} \mathcal{P} \circ M)
$$

and call $(\mathcal{P} \circ M, \gamma)$ the free left $d g \mathcal{P}$-module on $M$. Analogously we may define the free right $d g \mathcal{P}$-module on $M$ as $(M \circ \mathcal{P}, \gamma)$ with composition given as

$$
\gamma:=((M \circ \mathcal{P}) \circ \mathcal{P} \stackrel{\cong}{\longrightarrow} M \circ(\mathcal{P} \circ \mathcal{P}) \stackrel{M \circ \gamma}{\longrightarrow} M \circ \mathcal{P})
$$

Combining both structures gives a triple $\left(\mathcal{P} \circ M \circ \mathcal{Q}, \gamma^{L}, \gamma^{R}\right)$ which we call the free $d g$ $(\mathcal{P}, Q)$-bimodule on $M$.

The name free module is justified by the following result.

Lemma 2.1.7. The forgetful functor $U$ mapping a left $d g \mathcal{P}$-module $(M, \gamma)$ to its underlying dg collection $M$ admits a left adjoint functor $F$ as in

$$
F: \mathrm{dg} \text { Coll } \rightleftarrows \mathrm{dg} \mathcal{P} \text {-Mod }: U,
$$

which on objects is given by $F(M)=\mathcal{P} \circ M$ with the structure as defined in Example 2.1.6. The corresponding statements hold for right $d g \mathcal{P}$-modules and $d g(\mathcal{P}, \mathcal{Q})$-bimodules.

Let $f: \mathcal{P} \rightarrow \mathcal{P}^{\prime}$ be a morphism of dg operads and $M^{\prime}$ a $\operatorname{dg} \mathcal{P}^{\prime}$-module. The composition

$$
\mathcal{P} \circ M^{\prime} \stackrel{f \circ M^{\prime}}{\longrightarrow} \mathcal{P}^{\prime} \circ M^{\prime} \stackrel{\gamma}{\longrightarrow} M^{\prime}
$$

defines a $\operatorname{dg} \mathcal{P}$-module structure on $M^{\prime}$ and this construction extends to a functor

$$
f^{*}: \operatorname{dg} \mathcal{P}^{\prime} \text {-Mod } \longrightarrow \text { dg } \mathcal{P} \text {-Mod } .
$$

By symmetry one obtains a similiar functor for right modules, and given another morphism $g: \mathcal{Q} \rightarrow \mathcal{Q}^{\prime}$ of dg operads we may define

$$
(f, g)^{*}: \operatorname{dg}\left(\mathcal{P}^{\prime}, Q^{\prime}\right)-\operatorname{Mod} \longrightarrow \operatorname{dg}(\mathcal{P}, Q)-\operatorname{Mod} .
$$

Definition 2.1.8. We denote by dg Mod the category of all modules defined as follows. Objects in dg Mod are triples $(\mathcal{P}, M, \mathcal{Q})$ consisting of $\operatorname{dg}$ operads $\mathcal{P}$ and $\mathcal{Q}$ and a $(\mathcal{P}, \mathcal{Q})$ module $M$. A morphism $(\mathcal{P}, M, Q) \rightarrow\left(\mathcal{P}^{\prime}, M^{\prime}, Q^{\prime}\right)$ is a triple $(f, h, g)$ consisting of morphisms $f: \mathcal{P} \rightarrow \mathcal{P}^{\prime}$ and $g: \mathcal{Q} \rightarrow \mathcal{Q}^{\prime}$ of dg operads and a morphism $h: M \rightarrow(f, g)^{*} M^{\prime}$ of $(\mathcal{P}, Q)$-modules.

2.2. Algebras over an operad. Let $(\mathcal{P}, \gamma, \eta)$ denote a dg operad. An algebra over $\mathcal{P}$, or dg $\mathcal{P}$-algebra for short, is a representation of $\mathcal{P}$ on a space in a sense made precise 
below. Note that here and in other parts of this thesis, we overload certain notation, e.g. the symbol $\gamma$ is used for the operad, the coalgebra, the comodule, and the coalgebra comodule composition maps. Which map is to be used is determined by its domain and codomain.

Definition 2.2.1. A $d g \mathcal{P}$-algebra $(A, \gamma)$ is a space $A$ equipped with an operad action, i.e. a morphism $\gamma: \mathcal{P}(A) \rightarrow A$ of spaces satisfying
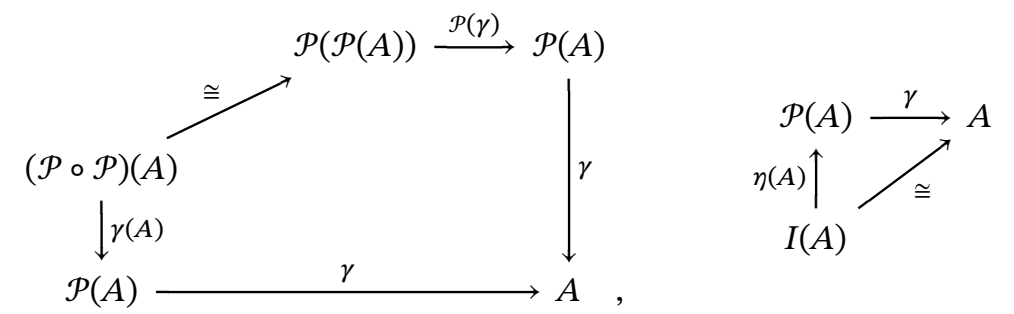

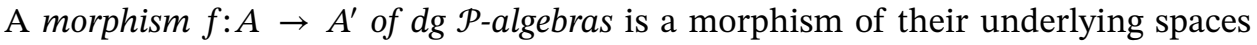
commuting with the structure in the sense that

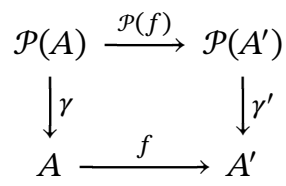

commutes.

By definition, a map $\gamma: \mathcal{P}(A) \rightarrow A$ is determined by its components

$$
\gamma[1 \leftarrow X]: \mathcal{P}[1 \leftarrow X] \otimes A[X] \longrightarrow A[1],
$$

which by currying correspond to

$$
g[1 \leftarrow X]: \mathcal{P}[1 \leftarrow X] \longrightarrow \operatorname{hom}(A[X], A[1])=\operatorname{End}_{A}[1 \leftarrow X] .
$$

In fact, one can show that a $\mathrm{dg} \mathcal{P}$-algebra $(A, \gamma)$ is equivalently determined by a morphism of dg operads

$$
\mathrm{g}: \mathcal{P} \longrightarrow \text { End }_{A} \text {. }
$$

The compatibility of a morphism $f: A \rightarrow A^{\prime}$ of spaces with the $\operatorname{dg} \mathcal{P}$-algebra structures in these terms can be expressed as commutativity of the diagram

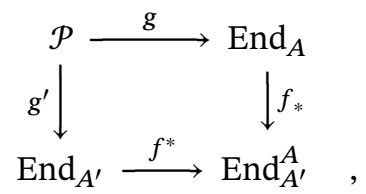

where $f_{*}$ resp. $f^{*}$ denote post- resp. pre-composition with $f$, i.e.

$$
\begin{aligned}
f_{*}: \operatorname{End}_{A} \longrightarrow \operatorname{End}_{A^{\prime}}^{A}, \quad m \longmapsto\left(f \circ m:\left(x_{1}, \ldots\right) \mapsto f\left(m\left(x_{1}, \ldots\right)\right),\right. \\
f^{*}: \operatorname{End}_{A^{\prime}} \longrightarrow \operatorname{End}_{A^{\prime}}^{A}, \quad m^{\prime} \longmapsto\left(m^{\prime} \circ(f, \ldots, f):\left(x_{1}, \ldots\right) \mapsto m^{\prime}\left(f\left(x_{1}\right), \ldots\right)\right) .
\end{aligned}
$$

Consider a morphism $h: \mathcal{P} \rightarrow\left(g^{\prime}, g\right)^{*} \operatorname{End}_{A^{\prime}}^{A}$ of $\operatorname{dg}(\mathcal{P}, \mathcal{P})$-modules. Any such morphism is uniquely determined by its value on the unit $u \in \mathcal{P}$. For $\mu \in \mathcal{P}, u \circ \mu=\mu=\mu \circ(u, \ldots, u)$, 
and thus for $m:=g(\mu), m^{\prime}:=g^{\prime}(\mu)$, and $f:=h(u)$ we obtain

$$
\begin{aligned}
h(\mu) & =h(u) \circ g(\mu)=f \circ m \\
& =g^{\prime}(\mu) \circ(h(u), \ldots, h(u))=m^{\prime} \circ(f, \ldots, f) .
\end{aligned}
$$

Lemma 2.2.2. Let $A$ be a $d g$ space. $A d g \mathcal{P}$-algebra structure $\gamma$ on $A$ is equivalently determined by a morphism of dg operads

$$
g: \mathcal{P} \longrightarrow \operatorname{End}_{A} .
$$

Let $A, A^{\prime}$ be dg spaces with dg $\mathcal{P}$-algebra structures given by $g: \mathcal{P} \rightarrow \operatorname{End}_{A}, g^{\prime}: \mathcal{P} \rightarrow \operatorname{End}_{A^{\prime}}$. A morphism $f: A \rightarrow A^{\prime}$ of dg operads is equivalently determined by a morphism

$$
h: \mathcal{P} \longrightarrow \operatorname{End}_{A^{\prime}}^{A}
$$

of $d g(\mathcal{P}, \mathcal{P})$-modules.

Let us finally look at some examples of operads and the types of algebras they describe.

EXAMPLE 2.2.3. Consider the endomorphism operad $\operatorname{End}_{V}$ for the space $V=\mathbf{k}$ (see Example 2.0.4). Its underlying dg collection is

$$
\begin{aligned}
\operatorname{End}_{\mathbf{k}}[1 \leftarrow X] & =\left(\prod_{\chi: X \rightarrow \underline{n}} \operatorname{hom}\left(\mathbf{k}\left[X_{1}\right] \otimes \cdots \otimes \mathbf{k}\left[X_{n}\right], \mathbf{k}[1]\right)\right)^{\mathbb{S}_{n}} \\
& \cong \mathbf{k} \cdot \mu_{X},
\end{aligned}
$$

where $\mu_{X}$ is the map defined by $\mu_{X}^{(\chi)}(1, \ldots, 1)=1$. Operadic composition of

$$
\mu_{P} \circ_{\xi}\left(\mu_{P_{1}}, \ldots, \mu_{P_{m}}\right) \in \operatorname{End}_{\mathbf{k}}[1 \leftarrow P] \circ \operatorname{End}_{\mathbf{k}}[P \leftarrow X]
$$

for some partition $P=\left\{P_{i}\right\}$ of $X$ is given by

$$
\gamma\left(\mu_{P} \circ \xi\left(\mu_{P_{1}}, \ldots, \mu_{P_{m}}\right)\right)=\mu_{P_{1} \amalg \cdots \amalg P_{m}}=\mu_{X} .
$$

Let now $A$ be another space. An End $_{\mathbf{k}}$-algebra structure on $A$ is a morphism

$$
g: \text { End }_{\mathbf{k}} \longrightarrow \text { End }_{A}
$$

of operads. By naturality of $g$, such a morphism is fully determined by its components on a skeleton for the domain category, here e.g. by its components on $\mathbb{S}^{\text {op }} \subset 1$-Tree ${ }^{\text {op }}$. As a consequence, an $\mathrm{End}_{\mathbf{k}}$-algebra structure on $A$ is defined by a choice of structure maps

$$
\left\{g_{n}:=g[1 \leftarrow \underline{n}]\left(\mu_{\underline{n}}\right) \in \operatorname{hom}(A[\underline{n}], A[1])_{0}\right\}_{n \geq 1} .
$$

These maps are required to satisfy some conditions: $(i) g_{1}=\mathrm{id}_{A}$, since a morphism of operads must preserve the unit; (ii) $g_{n}^{\sigma}=g_{n}$, by naturality of $g$ and the fact that the maps $\mu_{X}$ as defined above satisfy $\mu_{X}^{\varphi}=\mu_{X}$; and $(i i i) \gamma\left(g_{m} \circ\left(g_{n_{1}}, \ldots, g_{n_{m}}\right)\right)=g_{n_{1}+\cdots+n_{m}}$. Note that, in particular,

$$
g_{n+1}=\gamma\left(g_{2} \circ\left(g_{n}, g_{1}\right)\right)=\gamma\left(g_{2} \circ\left(g_{1}, g_{n}\right)\right), \quad \text { for } n \geq 1,
$$


and hence any $g_{n}$ for $n>2$ can be expressed inductively using just $g_{2}$ and the unit $g_{1}$. Thus an End $_{\mathbf{k}}$-algebra structure on a space $A$ is fully defined by its structure map

$$
g_{2}: A \otimes A \longrightarrow A,
$$

and $g_{2}$ is commutative and associative. The operad End $_{\mathbf{k}}$ is known as the operad of commutative algebras and usually denoted as Com.

EXAMPLE 2.2.4. We define an operad Ass as follows. Let Ass be the dg collection defined on objects by

$$
\operatorname{Ass}[1 \leftarrow X]:=\mathbf{k}[\chi: X \rightarrow \underline{n}],
$$

and on morphisms $\varphi^{\mathrm{op}}: X \rightarrow Y$ by

$$
\operatorname{Ass}\left[\varphi^{\mathrm{op}}\right](X \stackrel{\chi}{\longrightarrow} \underline{n}):=\varphi^{*} \chi=(Y \stackrel{\varphi}{\longrightarrow} X \stackrel{\chi}{\longrightarrow} \underline{n}) .
$$

The operadic composition for

$$
\xi \circ_{\xi}\left(\chi_{1}, \ldots, \chi_{m}\right) \in \operatorname{Ass}[1 \leftarrow P] \circ \operatorname{Ass}[P \leftarrow X]
$$

is defined to be the map

$$
\chi: X=\coprod P_{i} \longrightarrow \underline{n}, \quad \chi\left(x \in P_{i}\right)=\sum_{j=1}^{i-1} n_{j}+\chi_{i}(x) .
$$

An Ass-algebra structure on a space $A$ is a map

$$
\mathrm{g}: \text { Ass } \longrightarrow \operatorname{End}_{A}
$$

of operads. As was mentioned in Example 2.2.3, such a morphism is fully determined by its components $g[1 \leftarrow \underline{n}]$ on a skeleton for the domain category, e.g. on $\mathbb{S}^{\text {op }} \subset 1-T_{r e} e^{\text {op }}$. Thus, an Ass-algebra structure on $A$ is defined by a choice of structure maps

$$
\left\{g_{n}^{\sigma}:=g[1 \leftarrow \underline{n}](\sigma) \in \operatorname{hom}(A[\underline{n}], A[1])_{0}\right\}_{n \geq 1, \sigma \in \mathbb{S}_{n}} .
$$

As in the previous example, these structure maps are again required to satisfy some conditions: (i) $g_{1}=\mathrm{id}_{A}$, since $g$ must preserve the unit; $(i i)\left(g_{n}^{\sigma}\right)^{\tau}=g_{n}^{\sigma \tau}$, by definition of the action; and $(i i i) \gamma\left(g_{m}^{\sigma} \circ\left(g_{n_{1}}^{\tau_{1}}, \ldots, g_{n_{m}}^{\tau_{m}}\right)\right)=g_{n}^{\sigma \circ\left(\tau_{1}, \ldots, \tau_{m}\right)}$, where $n=\sum n_{i}$ and $\sigma \circ\left(\tau_{1}, \ldots, \tau_{m}\right)$ denotes composition of permutations as introduced in Section 2.1 of Chapter 1. Note that

$$
g_{n+1}^{\sigma}=\left(g_{n+1}\right)^{\sigma}=\gamma\left(g_{2} \circ\left(g_{n}, g_{1}\right)\right)^{\sigma}=\gamma\left(g_{2} \circ\left(g_{1}, g_{n}\right)\right)^{\sigma}, \quad \text { for } n \geq 1 \text { and } \sigma \in \mathbb{S}_{n},
$$

and hence any $g_{n}^{\sigma}$ for $n>2$ can be expressed inductively using just $g_{2}$ and the unit $g_{1}$. In this way, we see that an Ass-algebra structure on a space $A$ is fully defined by its structure map

$$
g_{2}: A \otimes A \rightarrow A,
$$

and $g_{2}$ is associative. The operad Ass is known as the operad of associative algebras.

REMARK 2.2.5. In Section 2.1 of Chapter 1 we introduced the symmetric groupoid $\mathbb{S}$ and equipped it with a composition map. The symmetric groupoid with this composition 
map is the fundamental example of a set-theoretic operad. The operad Ass defined above is the $\mathbf{k}$-linear version of $\mathbb{S}$.

EXAMPLE 2.2.6. Let $(\mathcal{P}, \gamma, \eta)$ be a dg operad and $V$ a $(\mathrm{dg})$ space. We equip $\mathcal{P}(V)$ with a $\mathcal{P}$-action

$$
\gamma=(\mathcal{P}(\mathcal{P}(V)) \stackrel{\cong}{\rightarrow}(\mathcal{P} \circ \mathcal{P})(V) \stackrel{\gamma(V)}{\longrightarrow} \mathcal{P}(V))
$$

and call $(\mathcal{P}(V), \gamma)$ the free dg $\mathcal{P}$-algebra on $V$. The name is justified by the following result.

Lemma 2.2.7. The forgetful functor $U$ mapping a dg $\mathcal{P}$-algebra $(A, \gamma)$ to its underlying dg space $A$ admits a left adjoint functor $F$ as in

$$
F: \mathrm{dg} \mathrm{Sp} \rightleftharpoons \mathrm{dg} \mathcal{P} \text {-Alg }: U,
$$

which on objects is given by $F(V)=\mathcal{P}(V)$ with the structure as defined in Example 2.2.6.

2.3. Modules over an algebra. A dg $\mathcal{P}$-algebra $(A, \gamma)$ over a dg operad $(\mathcal{P}, \gamma, \eta)$ admits a notion of module which we define here. Note that there is no distinction between left and right modules in the definition below.

Definition 2.3.1. A $d g A$-module $(M, \gamma)$ is a space $M$ with a module multiplication map $\gamma: \mathcal{P}(A ; M) \rightarrow M$. This structure map is required to be compatible with the operad multiplication and unit maps, i.e. the following diagrams must commute:

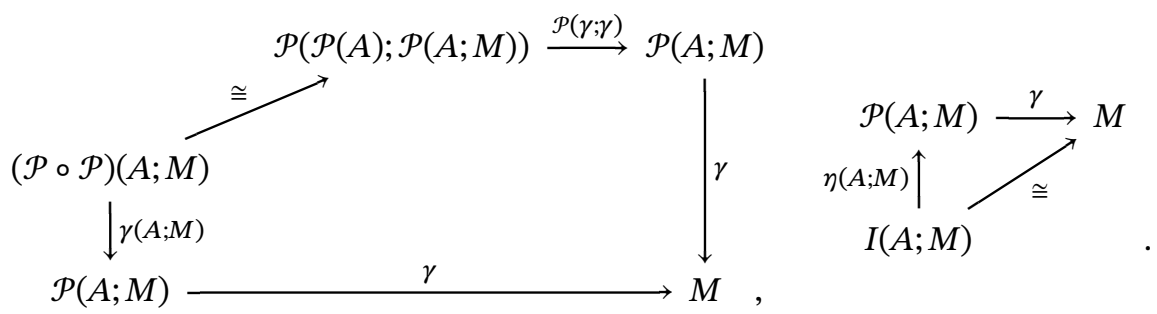

A morphism of $d g$ A-modules is a morphism of the underlying spaces $f: M \rightarrow M^{\prime}$ commuting with the $A$-module multiplication maps in the sense that the following diagram commutes:

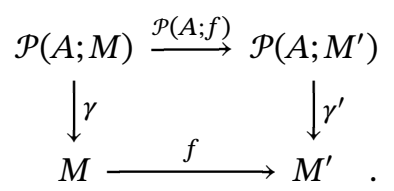

We denote the category of $d g A$-modules by $\mathrm{dg} A$-Mod.

EXAMPLE 2.3.2. Let $A$ be an algebra over the operad Com introduced in Example 2.2.3 An $A$-module in the sense of Definition 2.3.1 is equivalent to a left or right $A$-module in the classical sense.

EXAMPLE 2.3.3. Let $A$ be an algebra over the operad Ass introduced in Example 2.2.4 An $A$-module in the sense of Definition 2.3.1 is precisely an $(A, A)$-bimodule. 
Consider the dg subcollection $\operatorname{End}_{M}^{A ; M} \subset \operatorname{End}_{M \oplus A}$ of maps taking exactly one input in $M$ and with output in $M$. By reasoning similar to that for $\mathrm{dg} \mathcal{P}$-algebras, a map $\gamma: \mathcal{P}(A ; M) \rightarrow M$ corresponds to a morphism of dg collections

$$
h: \mathcal{P} \longrightarrow \operatorname{End}_{M}^{A ; M}
$$

Moreover, the map $\gamma$ defines an $A$-module structure on $M$ iff.

$$
g+h: \mathcal{P} \longrightarrow \operatorname{End}_{A} \oplus \operatorname{End}_{M}^{A ; M} \longrightarrow \operatorname{End}_{M \oplus A}
$$

defines a $\mathcal{P}$-algebra structure on $M \oplus A$. In this case, we call $M \oplus A$ the semidirect product of $A$ with $M$ and denote it by $M \rtimes A$.

2.4. The Hadamard tensor product. Given operads $\mathcal{P}, \mathcal{P}^{\prime}$, we can equip the aritywise tensor product $\mathcal{P} \otimes \mathcal{P}^{\prime}$ of their underlying collections with an operad structure as follows. We define the operadic unit as the map

$$
\eta=\left(I \stackrel{\cong}{\longrightarrow} I \otimes I \stackrel{\eta \otimes \eta^{\prime}}{\longrightarrow} \mathcal{P} \otimes \mathcal{P}^{\prime}\right)
$$

and its composition map $\gamma$ is determined by components

$$
\begin{aligned}
(\mathcal{P} \otimes & \left.\mathcal{P}^{\prime}\right)[1 \leftarrow P] \otimes \bigotimes\left(\mathcal{P} \otimes \mathcal{P}^{\prime}\right)\left[P_{i} \leftarrow X_{i}\right] \\
& =\mathcal{P}[1 \leftarrow P] \otimes \mathcal{P}^{\prime}[1 \leftarrow P] \otimes \bigotimes\left(\mathcal{P}\left[P_{i} \leftarrow X_{i}\right] \otimes \mathcal{P}^{\prime}\left[P_{i} \leftarrow X_{i}\right]\right) \\
& \cong \mathcal{P}[1 \leftarrow P] \otimes \bigotimes \mathcal{P}\left[P_{i} \leftarrow X_{i}\right] \otimes \mathcal{P}^{\prime}[1 \leftarrow P] \otimes \bigotimes \mathcal{P}^{\prime}\left[P_{i} \leftarrow X_{i}\right] \\
& \stackrel{\gamma \otimes \gamma^{\prime}}{\longrightarrow} \mathcal{P}[1 \leftarrow X] \otimes \mathcal{P}^{\prime}[1 \leftarrow X]=\left(\mathcal{P} \otimes \mathcal{P}^{\prime}\right)[1 \leftarrow X]
\end{aligned}
$$

i.e. for $\mu \otimes \mu^{\prime} \in\left(\mathcal{P} \otimes \mathcal{P}^{\prime}\right)[1 \leftarrow P]$ and $\nu_{i} \otimes v_{i}^{\prime} \in\left(\mathcal{P} \otimes \mathcal{P}^{\prime}\right)\left[P_{i} \leftarrow X_{i}\right]$ we have

$$
\gamma\left(\left(\mu \otimes \mu^{\prime}\right){ }_{\chi}\left(\nu_{1} \otimes \nu_{1}^{\prime}, \ldots, \nu_{m} \otimes \nu_{m}^{\prime}\right)\right):=\varepsilon \cdot \gamma\left(\mu \circ_{\chi}\left(\nu_{1}, \ldots, \nu_{m}\right)\right) \otimes \gamma^{\prime}\left(\mu^{\prime} \circ_{\chi}\left(\nu_{1}^{\prime}, \ldots, v_{m}^{\prime}\right)\right) \text {, }
$$

where $\varepsilon$ denotes the appropriate Koszul sign. We call the resulting operad $\mathcal{P} \otimes \mathcal{P}^{\prime}$ the Hadamard product of $\mathcal{P}$ and $\mathcal{P}^{\prime}$. Note that the operad Com provides a unit with respect to the Hadamard tensor product.

Lemma 2.4.1. Let $A$ be a $d g \mathcal{P}$-algebra and $A^{\prime}$ a $d g \mathcal{P}^{\prime}$-algebra. The tensor product of spaces $A \otimes A^{\prime}$ is naturally a $d g\left(\mathcal{P} \otimes \mathcal{P}^{\prime}\right)$-algebra.

Proof. We define a $\left(\mathcal{P} \otimes \mathcal{P}^{\prime}\right)$-algebra structure on $A \otimes A^{\prime}$. It's components are given by

$$
\begin{aligned}
\left(\mathcal{P} \otimes \mathcal{P}^{\prime}\right)[1 \leftarrow X] \otimes\left(A \otimes A^{\prime}\right)[X] & \stackrel{\cong}{\longrightarrow}[1 \leftarrow X] \otimes \mathcal{P}^{\prime}[1 \leftarrow X] \otimes A[X] \otimes A^{\prime}[X] \\
& \stackrel{\cong}{\longrightarrow}[1 \leftarrow X] \otimes A[X] \otimes \mathcal{P}^{\prime}[1 \leftarrow X] \otimes A^{\prime}[X] \\
& \stackrel{\gamma \otimes \gamma^{\prime}}{\longrightarrow} A[1] \otimes A^{\prime}[1]=\left(A \otimes A^{\prime}\right)[1] .
\end{aligned}
$$

Explicitly, this means that for $\mu \otimes \mu^{\prime} \in\left(\mathcal{P} \otimes \mathcal{P}^{\prime}\right)[1 \leftarrow X]$ and $x_{i} \otimes x_{i}^{\prime} \in\left(A \otimes A^{\prime}\right)\left[X_{i}\right]$, we define $\gamma$ by

$$
\gamma\left(\left(\mu \otimes \mu^{\prime}\right)^{(\chi)}\left(x_{1} \otimes x_{1}^{\prime}, \ldots, x_{m} \otimes x_{m}^{\prime}\right)\right):=\varepsilon \cdot \gamma\left(\mu^{(\chi)}\left(x_{1}, \ldots, x_{m}\right)\right) \otimes \gamma^{\prime}\left(\mu^{\prime}(\chi)\left(x_{1}^{\prime}, \ldots, x_{m}^{\prime}\right)\right),
$$


where $\varepsilon$ denotes the appropriate Koszul sign. We omit the verification that this indeed defines the structure of an algebra here (see e.g. [46, §5.3.2]).

EXAMPLE 2.4.2. Denote by $\mathcal{S}:=\operatorname{End}_{s \mathbf{k}}$ resp. $\mathcal{S}^{-1}:=\operatorname{End}_{S^{-1} \mathbf{k}}$ the suspension resp. desuspension operad. Clearly, sk is naturally an $\mathcal{S}$-algebra by

$$
\mathcal{S}=\text { End }_{s \mathbf{k}} \longrightarrow \text { End }_{\mathbf{s k}},
$$

and hence for any $\mathcal{P}$-algebra $A$ we find that by Lemma 2.4.1 its suspension $s A=s \mathbf{k} \otimes A$ is naturally an $\mathcal{S} \otimes \mathcal{P}$-algebra. Similarly, the desuspension $s^{-1} A$ of a $\mathcal{P}$-algebra $A$ is naturally an $\mathcal{S}^{-1} \otimes \mathcal{P}$-algebra.

Definition 2.4.3. We call $\mathcal{S P}:=\mathcal{S} \otimes \mathcal{P}$ resp. $\mathcal{S}^{-1} \mathcal{P}:=\mathcal{S}^{-1} \otimes \mathcal{P}$ the operadic suspension resp. desuspension of $\mathcal{P}$.

2.5. The free operad. In this section, we construct a left adjoint to the forgetful functor $U$ mapping a dg operad to its underlying $\mathrm{dg} \mathbb{S}$-module. By definition of operads as monoids, one might consider using a construction similar to that of the tensor algebra. While this is possible, as shown by Vallette [68], nonlinearity of the composite product on the right makes this more complicated than necessary. In our situation, we can get away with a simpler construction using the linearity of the composite product on the left. For more details on this and other constructions, we refer once again to [46].

Let $M$ be a dg collection. We define a sequence of dg collections

$$
\mathcal{T}_{0} M:=I, \quad \mathcal{T}_{n+1} M:=I \oplus M \circ \mathcal{T}_{n} M,
$$

and inclusion maps

$$
\begin{aligned}
& \iota_{0}: \mathcal{T}_{0} M=I \longleftrightarrow I \oplus M=\mathcal{T}_{1} M, \\
& \iota_{n}: \mathcal{T}_{n} M=I \oplus M \circ \mathcal{T}_{n-1} M \stackrel{I \oplus M \circ \iota_{n-1}}{\longrightarrow} I \oplus M \circ \mathcal{T}_{n} M=\mathcal{T}_{n+1} M .
\end{aligned}
$$

We denote by $\mathcal{T} M$ the colimit

$$
\mathcal{T} M:=\operatorname{colim}_{n} \mathcal{T}_{n} M
$$

and call it the tree module on $M$.

We now equip this tree module with an operad structure. The operadic unit is given by the inclusion

$$
\eta: I \cong \mathcal{T}_{0} M \longrightarrow \mathcal{T} M,
$$

and the composition $\gamma$ is defined inductively as follows:

$$
\begin{aligned}
& \gamma_{0, m}: \mathcal{T}_{0} M \circ \mathcal{T}_{m} M=I \circ \mathcal{T}_{m} M \stackrel{\cong}{\longrightarrow} \mathcal{T}_{0+m} M, \\
& \gamma_{n, m}: \mathcal{T}_{n} M \circ \mathcal{T}_{m} M=\left(I \oplus M \circ \mathcal{T}_{n-1} M\right) \circ \mathcal{T}_{m} M \\
& \begin{aligned}
\stackrel{\cong}{\longrightarrow} \mathcal{T}_{m} M \oplus M \circ\left(\mathcal{T}_{n-1} M \circ \mathcal{T}_{m} M\right) \\
\stackrel{\mathcal{J}_{m} M \oplus M \circ \gamma_{n-1, m}}{\longrightarrow} \mathcal{T}_{m} M \oplus M \circ \mathcal{T}_{n-1+m} M \longrightarrow \mathcal{T}_{n+m} M .
\end{aligned}
\end{aligned}
$$


A straightforward verification shows that $(i)$ the maps $\gamma_{m, n}$ are compatible with the inclusions and therefore they define a composition map $\gamma: \mathcal{T} M \circ \mathcal{T} M \rightarrow \mathcal{T} M$, and (ii) this composition $\gamma$ satisfies the associativity and unitality axioms. This shows that $(\mathcal{T} M, \gamma, \eta)$ constructed above is indeed an operad. The projection

$$
\varepsilon: \mathcal{T} M \longrightarrow \mathcal{T _ { 0 }} M \cong I
$$

defines an augmentation for $\mathcal{T} M$.

Lemma 2.5.1. The operad $(\mathcal{T} M, \gamma, \eta)$ is the free $d g$ operad on the dg collection $M$, i.e. the above construction provides a left adjoint for the forgetful functor U mapping a dg operad to its underlying dg collection

$$
\mathcal{T}: \mathrm{dg} \text { Coll } \rightleftarrows \mathrm{dg} \text { Opd }: U \text {. }
$$

Proof. We construct the unit $\psi$ and counit $\varphi$ for the adjunction as follows. We define the component $\psi(\mathcal{P}): \mathcal{P} \rightarrow \mathcal{T} \mathcal{P}$ to be the inclusion $\mathcal{P} \hookrightarrow I \oplus \mathcal{P}=\mathcal{T}_{1} \mathcal{P}$, and construct $\varphi(\mathcal{P}): \mathcal{T P} \rightarrow \mathcal{P}$ inductively via

$$
\begin{aligned}
& \varphi(\mathcal{P})_{0}: \mathcal{\mathcal { T } _ { 0 }} \mathcal{P}=I \stackrel{\eta}{\longrightarrow} \mathcal{P}, \\
& \varphi(\mathcal{P})_{n}: \mathcal{T}_{n} \mathcal{P}=I \oplus \mathcal{P} \circ \mathcal{T}_{n-1} \mathcal{P} \stackrel{\eta+\gamma\left(\mathcal{P}_{\circ} \varphi(\mathcal{P})_{n-1}\right)}{\longrightarrow} \mathcal{P} .
\end{aligned}
$$

We omit the necessary verifications here; they can be found in [46, §5.5.1].

The tree module $\mathcal{T} M$ on a dg collection $M$ carries a canonical weightgrading in which an element of $M$ is assigned weight 1 . More precisely, one may define a weightgrading on $\mathcal{T}_{n} M$ inductively by

$$
\mathcal{T}_{n} M^{(w)}= \begin{cases}I, & \text { for } w=0 \\ M \circ \mathcal{T}_{n-1} M^{(w-1)}, & \text { for } w>0\end{cases}
$$

The inclusion maps $\iota_{n}$ preserve the weightgrading and thus this defines a weightgrading on the colimit $\mathcal{T} M=\operatorname{colim}_{n} \mathcal{T}_{n} M$. The operadic structure defined on $\mathcal{T} M$ is compatible with the weightgrading and $\mathcal{T} M^{(0)}=I$; in other words, the free operad $\mathcal{T} M$ on a dg collection is a connected wdg operad.

2.6. Derivations. Let $(\mathcal{P}, \gamma, \eta)$ be a graded operad. By a derivation of $\mathcal{P}$, we mean a morphism of graded collections $\mathrm{d}: \mathcal{P} \rightarrow \mathcal{P}$ of degree -1 , satisfying
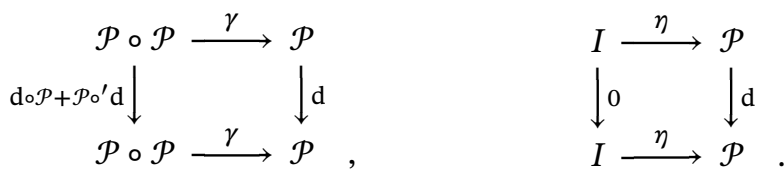

When $\mathcal{P}$ is augmented, we additionally assume commutativity of

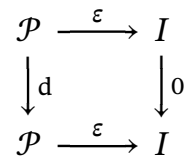


for any derivation. The set of derivations for an operad $\mathcal{P}$ is denoted $\operatorname{Der}(\mathcal{P})$. A squarezero derivation, i.e. a derivation d satisfying $\mathrm{d}^{2}=0$, is called a differential. The set of differentials on $\mathcal{P}$ is denoted $\operatorname{Diff}(\mathcal{P})$.

Consider now the free graded operad $\mathcal{T} M$ on a graded collection $M$. We call $\mathcal{T} M$ equipped with a differential d a quasi-free dg operad on $M$. A derivation for $\mathcal{T} M$ is uniquely determined by its value on generators, i.e. by its restriction to $M \subset \mathcal{T} M$. In fact, we have the following result.

Lemma 2.6.1. There is a one-to-one correspondence between derivations of the free graded operad $\mathcal{T} M$ and morphisms of graded collections $M \rightarrow \overline{\mathcal{T} M}$ of degree -1 :

$$
\operatorname{Der}(\mathcal{T} M) \cong \operatorname{hom}(M, \overline{\mathcal{T} M})_{-1} .
$$

Proof. Given a map $f: M \rightarrow \overline{\mathcal{T} M}$ of degree -1 , we define a map $\mathrm{d}^{f}: \mathcal{T} M \rightarrow \mathcal{T} M$ inductively:

$$
\begin{gathered}
\mathrm{d}_{0}^{f}: \mathcal{T}_{0} M=I \stackrel{0}{\longrightarrow} I=\mathcal{T}_{0} M, \\
\mathrm{~d}_{n}^{f}: \mathcal{T}_{n} M=I \oplus M \circ \mathcal{T}_{n-1} M \stackrel{0 \oplus f \circ \mathcal{F}_{n-1} M+M \circ{ }^{\prime} \mathrm{d}_{n-1}^{f}}{\longrightarrow} \overline{\mathcal{T} M} \circ \mathcal{T}_{n-1} M \oplus M \circ\left(\mathcal{T}_{n-1} M ; \mathcal{T} M\right) \\
\longrightarrow \mathcal{T} M \circ \mathcal{T} M \stackrel{\gamma}{\longrightarrow} \mathcal{T} M .
\end{gathered}
$$

Clearly, when we restrict this map $\mathrm{d}^{f}$ to $M \subset \mathcal{T} M$, we recover $f$. It remains to show that $\mathrm{d}^{f}$ is indeed a derivation.

Let $(\mathcal{P}, \gamma, \eta)$ be a dg operad and $A$ a graded $\mathcal{P}$-algebra. A derivation of $A$ is a degree -1 morphism d: $A \rightarrow A$ of graded collections such that the following diagram commutes,

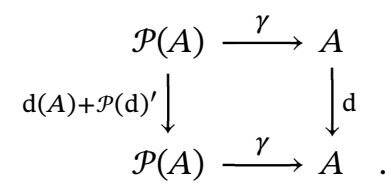

We denote by $\operatorname{Der}(A)$ the set of derivations of $A$ and by $\operatorname{Diff}(A)$ the subset of square-zero derivations or differentials.

Consider now the free graded $\mathcal{P}$-algebra $\mathcal{P}(V)$ on a space $V$. We call $\mathcal{P}(V)$ equipped

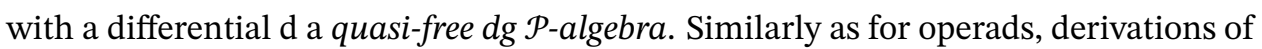
$\mathcal{P}(V)$ are uniquely determined by their value on generators.

Lemma 2.6.2. There is a one-to-one correspondence between derivations of the free graded $\mathcal{P}$-algebra $\mathcal{P}(V)$ and morphisms of graded spaces $\theta: V \rightarrow \mathcal{P}(V)$ of degree -1 :

$$
\operatorname{Der}(\mathcal{P}(V)) \cong \operatorname{hom}(V, \mathcal{P}(V))_{-1} .
$$

Proof. Given a map $\theta: V \rightarrow \mathcal{P}(V)$, we define $\mathrm{d}_{\theta}: \mathcal{P}(V) \rightarrow \mathcal{P}(V)$ as the sum of

$$
\mathcal{P}(V) \stackrel{\mathrm{d}(V)}{\longrightarrow} \mathcal{P}(V)
$$

and

$$
\mathcal{P}(V) \stackrel{\mathcal{P}(\theta)^{\prime}}{\longrightarrow} \mathcal{P}(V ; \mathcal{P}(V)) \stackrel{\cong}{\rightarrow}\left(\mathcal{P} \circ_{(1)} \mathcal{P}\right)(V) \stackrel{\gamma_{(1)}(V)}{\longrightarrow} \mathcal{P}(V)
$$


This is easily seen to define a derivation, i.e. $\mathrm{d}_{\theta} \in \operatorname{Der}(\mathcal{P}(V))$. It remains to prove that (i) $\left.\mathrm{d}_{\theta}\right|_{V}=\theta$, and (ii) $\mathrm{d}_{\left.\mathrm{d}\right|_{V}}=\mathrm{d}$. We omit these verifications since they are essentially dual to those which we will give in the proof of Lemma 3.6.3

2.7. Presentations. Let $(\mathcal{P}, \gamma, \eta)$ be a dg operad. A sub $\mathrm{dg}$ collection $\mathcal{J} \subset \mathcal{P}$ is called a (two-sided) operadic ideal if $\gamma\left(\mu \circ \circ_{\chi}\left(\nu_{1}, \ldots, \nu_{m}\right)\right) \in \mathcal{J}$ whenever any of $\mu, \nu_{1}, \ldots, \nu_{m}$ is. In this case, the quotient $\mathcal{P} / \mathcal{J}$ is naturally a $\mathrm{dg}$ operad. Note that any sub $\mathrm{dg}$ collection $J \subset \mathcal{P}$ generates an operadic ideal $(J)$, and that there is a bijection between morphisms of operads $\mathcal{P} /(J) \rightarrow \mathcal{Q}$ and morphisms $\mathcal{P} \rightarrow \mathcal{Q}$ vanishing on $J$.

Given a dg collection $E$ and a sub dg collection $R \subset \mathcal{T} E$, we use the notation $\mathcal{P}(E, R)$ for the operad $\mathcal{T} E /(R)$. We call the operad $\mathcal{P}(E, R)$ the operad generated by $E$ with relations $R$ and the tuple $(E, R)$ a presentation for $\mathcal{P}(E, R)$.

Let $\mathcal{P}$ be an operad with presentation $(E, R)$, and let $V$ be a space. By definition, a $\mathcal{P}$-algebra structure on $V$ is a morphism $\mathcal{T} E /(R) \rightarrow \operatorname{End}_{V}$ of operads. Equivalently, it is a morphism $\mathcal{T} E \rightarrow$ End $_{V}$ of operads vanishing on the sub dg collection $R$, and such a morphism is uniquely determined by its restriction to generators $E$ by Lemma 2.5.1 Hence, a $\mathcal{P}$-algebra structure on $V$ is given by generating operations indexed by $E$, and subject to the relations of $R$.

EXAMPLE 2.7.1. Let $E$ be the $\mathbb{S}$-module determined by

$$
E=E(2)=\mu_{2} \cdot \mathbf{k}\left[\mathbb{S}_{2}\right]
$$

and denote by $R \subset E{ }_{(1)} E$ the sub $\mathbb{S}_{3}$-module generated by the relation

$$
r=\mu_{2} \circ_{1} \mu_{2}-\mu_{2} \circ_{2} \mu_{2} .
$$

A $\mathcal{P}(E, R)$-algebra structure on $V$ is determined by a morphism $g: E \rightarrow$ End $_{V}$ of $\mathbb{S}$ modules, i.e. by the image $m_{2}:=g\left(\mu_{2}\right): V \otimes V \rightarrow V$. This structure map is required to satisfy the associativity relation

$$
0=g(r)=g\left(\mu_{2} \circ_{1} \mu_{2}-\mu_{2} \circ_{2} \mu_{2}\right)=m_{2} \circ_{1} m_{2}-m_{2} \circ_{2} m_{2} .
$$

We see that the operad $\mathcal{P}(E, R)$ is isomorphic to the operad Ass of associative algebras as defined in Example 2.2.4.

\section{Cooperads}

The category of dg cooperads is the category of comonoids in dg collections,

$$
\text { dg Coopd }=\text { Comon }(\text { dg Coll }) \text {. }
$$

We make their definition explicit below, followed by some basic terminology.

Definition 3.0.1. A $d g$ cooperad $(\mathcal{C}, \Delta, \varepsilon)$ consists of a $d g$ collection $\mathcal{C}$ with decomposition map $\Delta: \mathcal{C} \rightarrow \mathcal{C} \circ \mathcal{C}$ and counit $\varepsilon: \mathcal{C} \rightarrow I$ satisfying the coassociativity and counitality 
axioms, i.e. the following diagrams must commute:

(40)

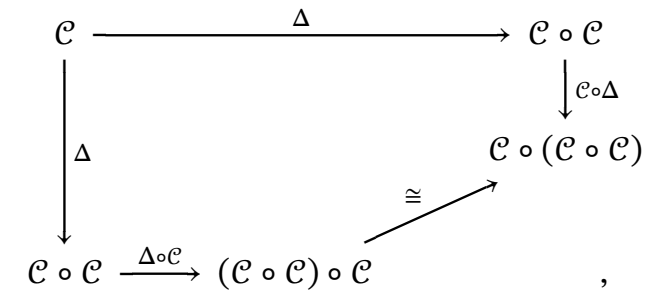

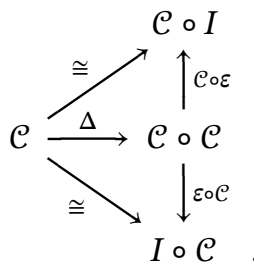

A morphism of dg cooperads is a morphism of the underlying dg collections $f: \mathcal{C} \rightarrow \mathcal{C}^{\prime}$ commuting with the structure maps in the sense that the following diagrams commute:
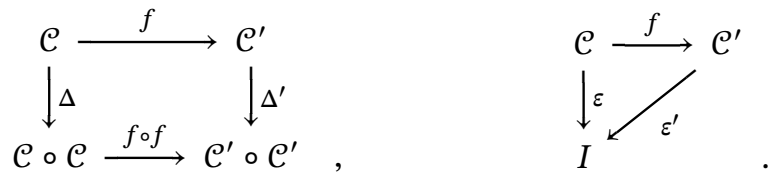

EXAMPLE 3.0.2. The dg collection $I$ underlying the identity operad carries a cooperad structure with counit $\varepsilon$ given by $\varepsilon(u)=1$ and decomposition $\Delta$ by $\Delta(u)=u \circ(u)$. This cooperad is called the trivial or identity cooperad.

A coaugmentation for $\mathcal{C}$ is a morphism of dg cooperads $\eta: I \rightarrow \mathcal{C}$ satisfying $\varepsilon \eta=I$. We denote by $\overline{\mathcal{C}}$ the coaugmentation coideal $\overline{\mathcal{C}}:=$ coker $\eta$ and define a reduced decomposition map $\bar{\Delta}: \overline{\mathcal{C}} \rightarrow \overline{\mathcal{C}} \circ \mathcal{C}$ by $\Delta=\overline{\mathcal{C}} \circ \eta+\bar{\Delta}+\eta \circ \mathcal{C}$, i.e. for $\mu \in \overline{\mathcal{C}}$ we have

$$
\Delta(\mu)=\mu \circ(1, \ldots, 1)+\bar{\Delta}(\mu)+1 \circ(\mu) .
$$

Consider also the map $\widetilde{\Delta}: I \oplus \overline{\mathcal{C}} \rightarrow \mathcal{C} \circ \mathcal{C}$ defined by

$$
\widetilde{\Delta}(1)=1 \circ 1, \quad \widetilde{\Delta}(\mu)=\bar{\Delta}(\mu)+\mu \circ(1, \ldots, 1),
$$

and denote by $\widetilde{\Delta}^{n}$ its right iteration

$$
\widetilde{\Delta}^{0}=\mathcal{C}, \quad \widetilde{\Delta}^{1}=\widetilde{\Delta}, \quad \widetilde{\Delta}^{n}=(\mathcal{C} \circ \widetilde{\Delta}) \widetilde{\Delta}^{n-1} .
$$

We define the coradical filtration $\mathcal{F}$. $\mathcal{C}$ for a cooperad $\mathcal{C}$ via

$$
\mathcal{F}_{0} \mathcal{C}=I, \quad \mathcal{F}_{n} \mathcal{C}=\operatorname{ker}\left(\widetilde{\Delta}^{n}-\left(\mathcal{C}^{\circ n} \circ \eta\right) \widetilde{\Delta}^{n-1}\right),
$$

and call $\mathcal{C}$ conilpotent if its coradical filtration is exhaustive, i.e. if $\mathcal{C}=\operatorname{colim}_{n} \mathcal{F}_{n} \mathcal{C}$. Equivalently, this means that $\widetilde{\Delta}^{n}$ stabilizes for any element of $\mathcal{C}$.

The category wdg Coopd of weightgraded dg cooperads or wdg cooperads is the category of comonoids in wdg collections. Explicitly, a wdg cooperad $(\mathcal{C}, \Delta, \varepsilon)$ is a cooperad on an underlying weightgraded dg collection $\mathcal{C}$ whose cooperadic structure maps $\Delta$ and $\varepsilon$ preserve the weight, and morphisms of wdg cooperads are assumed to preserve the weight as well. A wdg cooperad is called connected, if $\mathcal{C}^{(0)}=I$. Any connected wdg cooperad is coaugmented with coaugmentation coideal $\overline{\mathcal{C}}=\bigoplus_{w \geq 1} \mathcal{C}^{(w)}$ and in fact conilpotent. 
Given a cooperad $(\mathcal{C}, \Delta, \varepsilon)$, we define its infinitesimal or partial decomposition map by

$$
\Delta_{(1)}:=\left(\mathcal{C} \stackrel{\Delta}{\longrightarrow} \mathcal{C} \circ \mathcal{C} \hookrightarrow \mathcal{C} \circ(\mathcal{C} ; \mathcal{C}) \stackrel{\mathcal{C} \circ(\varepsilon ; \mathcal{C})}{\longrightarrow} \mathcal{C} \circ(I ; \mathcal{C})=\mathcal{C} \circ{ }_{(1)} \mathcal{C}\right)
$$

As for operads, this partial decomposition map already determines the full decomposition $\Delta$.

3.1. Comodules over a cooperad. As comonoids in $\mathrm{dg}$ Coll, dg cooperads come with a natural notion of left and right comodules as well as bi-comodules. We make these notions explicit in this section. In the following, we denote by $(\mathcal{C}, \Delta, \varepsilon)$ and $(\mathcal{D}, \Delta, \varepsilon)$ dg cooperads.

Definition 3.1.1. A left $d g \mathcal{C}$-comodule $(M, \Delta)$ is a dg collection $M$ with a left comultiplication map $\Delta: M \rightarrow \mathcal{C} \circ M$. This structure map is required to be compatible with the cooperad comultiplication and counit maps, i.e. the following diagrams must commute:
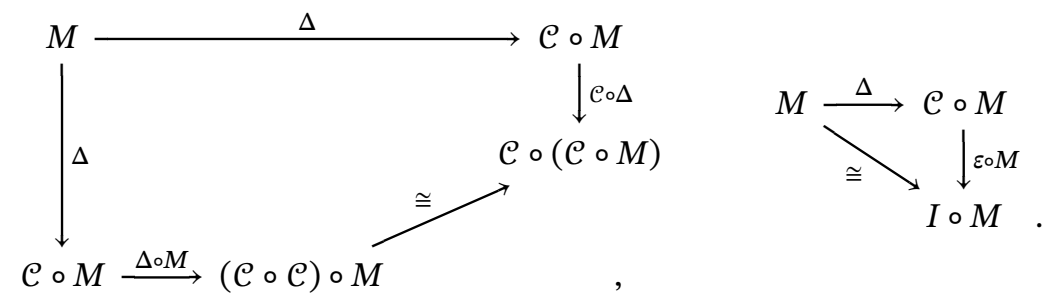

A morphism of left $d g \mathcal{C}$-comodules is a morphism of their underlying dg collections $f: M \rightarrow M^{\prime}$ commuting with the $\mathcal{C}$-comodule comultiplication maps in the sense that the following diagram commutes:

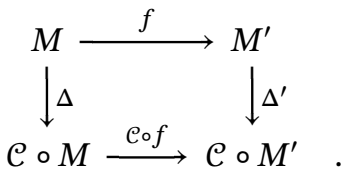

We denote the category of left $d g \mathcal{C}$-comodules by $\mathrm{dg} \mathcal{C}$-Comod.

An analogous definition of right comodules exists, which we give below. The relevant diagrams are obtained from the diagrams of Equations (42) and (43) above by mirror symmetry.

Definition 3.1.2. A right $d g \mathcal{C}$-comodule $(M, \Delta)$ is a dg collection $M$ with a right comultiplication map $\Delta: M \rightarrow M \circ \mathcal{C}$. This structure map is required to be compatible with the cooperad comultiplication and counit maps. A morphism of right $d g \mathcal{C}$-comodules is a morphism of their underlying dg collections $f: M \rightarrow M^{\prime}$ compatible with the comodule comultiplication maps. We denote the category of right $d g \mathcal{C}$-comodules by $\mathrm{dg}$ Comod-C.

Definition 3.1.3. A $d g(\mathcal{C}, \mathcal{D})$-bi-comodule (or simply $d g(\mathcal{C}, \mathcal{D})$-comodule) is a triple $\left(M, \Delta^{L}, \Delta^{R}\right)$ consisting of a dg collection $M$, a left comultiplication map $\Delta^{L}: M \rightarrow \mathcal{C} \circ M$, and a right comultiplication map $\Delta^{R}: M \rightarrow M \circ \mathcal{D}$ such that $\left(M, \Delta^{L}\right)$ is a left dg $\mathcal{C}$-comodule and $\left(M, \Delta^{R}\right)$ is a right $\mathrm{dg} \mathcal{D}$-comodule. In addition, we require that the left and right 
comultiplication maps are compatible in the sense that the following diagram must commute:

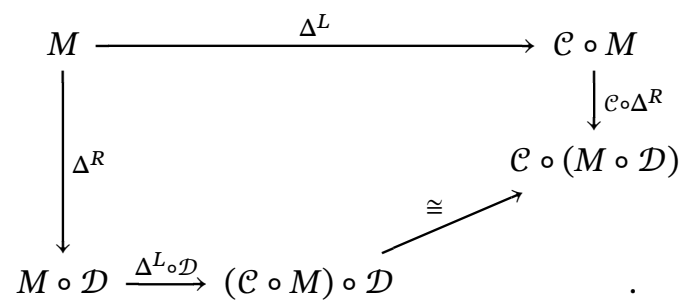

A morphism of $d g(\mathcal{C}, \mathcal{D})$-bi-comodules is a morphism of $\operatorname{dg}$ collections $f: M \rightarrow M^{\prime}$ which is both, a morphism of left $\mathrm{dg} \mathcal{C}$-comodules and of right $\mathrm{dg} \mathcal{D}$-comodules. We denote the category of $d g(\mathcal{C}, \mathcal{D})$-bi-comodules by $\mathrm{dg}(\mathcal{C}, \mathcal{D})$-Comod.

EXAMPLE 3.1.4. The tuple $(\mathcal{C}, \Delta)$ is simultaneously a left and a right $\mathcal{C}$-comodule. The tuple $(\mathcal{C}, \Delta, \Delta)$ is a $(\mathcal{C}, \mathcal{C})$-comodule and $(\mathcal{C} \circ \mathcal{D}, \Delta \circ \mathcal{D}, \mathcal{C} \circ \Delta)$ is a $(\mathcal{C}, \mathcal{D})$-comodule.

EXAMPLE 3.1.5. Let $M$ a dg collection. We equip the composite product $\mathcal{C} \circ M$ with a structure map

$$
\Delta=(\mathcal{C} \circ M \stackrel{\Delta \circ M}{\longrightarrow}(\mathcal{C} \circ \mathcal{C}) \circ M \stackrel{\cong}{\longrightarrow} \mathcal{C} \circ(\mathcal{C} \circ M))
$$

and call $(\mathcal{C} \circ M, \Delta)$ the cofree left dg $\mathcal{C}$-comodule on $M$. Analogously we may define the cofree right dg $\mathcal{C}$-comodule on $M$ as $(M \circ \mathcal{C}, \Delta)$ with decomposition given as

$$
\Delta=(M \circ \mathcal{C} \stackrel{M \circ \Delta}{\longrightarrow} M \circ(\mathcal{C} \circ \mathcal{C}) \stackrel{\cong}{\longrightarrow}(M \circ \mathcal{C}) \circ \mathcal{C}) .
$$

Combining both structures on $\mathcal{C} \circ M \circ \mathcal{D}$ gives a triple $\left(\mathcal{C} \circ M \circ \mathcal{D}, \Delta^{L}, \Delta^{R}\right)$ which we call the cofree $d g(\mathcal{C}, \mathcal{D})$-bi-comodule on $M$.

The name cofree comodule is justified by the following result.

Lemma 3.1.6. The forgetful functor $U$ mapping a left dg $\mathcal{C}$-comodule $(M, \Delta)$ to its underlying dg collection $M$ admits a right adjoint functor $F^{c}$ as in

$$
U: \mathrm{dg} \text { C-Comod } \rightleftharpoons \mathrm{dg} \text { Coll }: F^{c},
$$

which on objects is given by $F^{c}(M)=\mathcal{C} \circ M$ with the structure as defined in Example 3.1.5. The corresponding statements hold for right $d g \mathcal{C}$-comodules and $d g(\mathcal{C}, \mathcal{D})$-bi-comodules.

Let $f: \mathcal{C} \rightarrow \mathcal{C}^{\prime}$ be a morphism of dg cooperads and $M$ a dg $\mathcal{C}$-comodule. The composition

$$
M \stackrel{\Delta}{\longrightarrow} \mathcal{C} \circ M \stackrel{f \circ M}{\longrightarrow} \mathcal{C}^{\prime} \circ M
$$

defines a dg $\mathcal{C}^{\prime}$-comodule structure on $M$ and this construction extends to a functor

$$
f_{*}: \text { dg } \mathcal{C} \text {-Comod } \longrightarrow \text { dg } \mathcal{C}^{\prime} \text {-Comod } .
$$

By symmetry one obtains a similiar functor for right comodules, and given another morphism $g: \mathcal{D} \rightarrow \mathcal{D}^{\prime}$ of dg cooperads we may define

$$
(f, g)_{*}: \operatorname{dg}(\mathcal{P}, Q) \text {-Comod } \longrightarrow \operatorname{dg}\left(\mathcal{P}^{\prime}, Q^{\prime}\right) \text {-Comod } .
$$


Definition 3.1.7. We denote by dg Comod the category of all comodules defined as follows. Objects in $\mathrm{dg}$ Comod are triples $(\mathcal{C}, M, \mathcal{D})$ consisting of $\mathrm{dg}$ cooperads $\mathcal{C}$ and $\mathcal{D}$ and a $(\mathcal{C}, \mathcal{D})$-comodule $M$. A morphism $(\mathcal{C}, M, \mathcal{D}) \rightarrow\left(\mathcal{C}^{\prime}, M^{\prime}, \mathcal{D}^{\prime}\right)$ is a triple $(f, h, g)$ consisting of morphisms $f: \mathcal{C} \rightarrow \mathcal{C}^{\prime}$ and $g: \mathcal{D} \rightarrow \mathcal{D}^{\prime}$ of dg cooperads and a morphism $h:(f, g)_{*} M \rightarrow M^{\prime}$ of $\left(\mathcal{C}^{\prime}, \mathcal{D}^{\prime}\right)$-comodules.

3.2. Coalgebras over a cooperad. Let $(\mathcal{C}, \Delta, \varepsilon)$ denote a dg cooperad. A dg $\mathcal{C}$ coalgebra is a corepresentation of $\mathcal{C}$ on a space in a sense made precise below. As pointed out in Section 2.2. we overload certain notation: the same symbol $\Delta$ as used for the cooperad comultiplication is re-used in the definition below. Which map is to be applied is determined by its domain and codomain.

Definition 3.2.1. A coalgebra $(C, \Delta)$ over $\mathcal{C}$, or $d g \mathcal{C}$-coalgebra for short, is a space $C$ equipped with a cooperad coaction $\Delta: C \rightarrow \widehat{\mathcal{C}}(C)$ satisfying
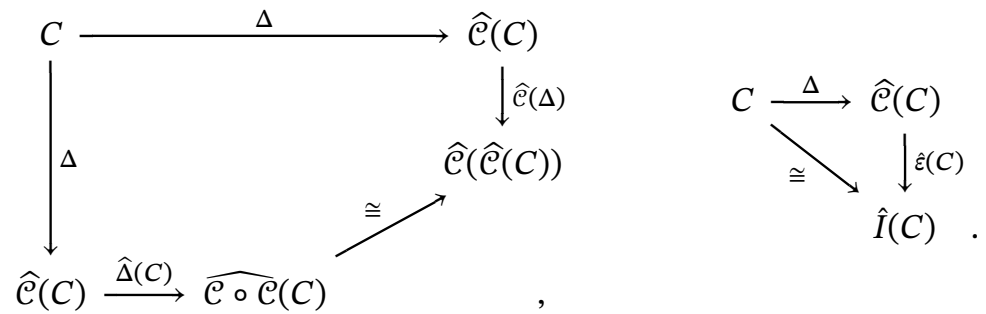

A morphism of $\operatorname{dg} \mathcal{C}$-coalgebras $f: C \rightarrow C^{\prime}$ is a morphism of their underlying spaces compatible with the structure in the sense that

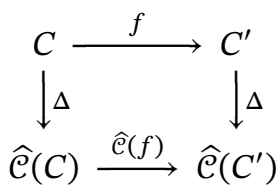

commutes. We denote the category of $\mathrm{dg} \mathcal{C}$-coalgebras by $\mathrm{dg} \mathcal{C}$-Coalg.

A dg $\mathcal{C}$-coalgebra $(C, \Delta)$ is conilpotent if its decomposition map $\Delta: C \rightarrow \widehat{\mathcal{C}}(C)$ factors through $\mathcal{C}(C)$. In this case, we will often implicitly view decomposition as a map $\Delta: C \rightarrow \mathcal{C}(C)$. We denote the full subcategory of $\mathrm{dg} \mathcal{C}$-Coalg consisting of conilpotent dg $\mathcal{C}$-coalgebras by conil dg $\mathcal{C}$-Coalg.

EXAMPLE 3.2.2. Let $(\mathcal{C}, \Delta, \varepsilon)$ be a dg cooperad and $V$ a $(\mathrm{dg})$ space. We equip $\mathcal{C}(V)$ with a $\mathcal{C}$-coaction map

$$
\Delta=(\mathcal{C}(V) \stackrel{\Delta(V)}{\longrightarrow}(\mathcal{C} \circ \mathcal{C})(V) \stackrel{\cong}{\longrightarrow}(\mathcal{C}(V)))
$$

and call $(\mathcal{C}(V), \Delta)$ the cofree conilpotent $d g \mathcal{C}$-coalgebra on $V$. The name is justified by the following lemma.

Lemma 3.2.3. The forgetful functor $U$ mapping a conilpotent dg $\mathcal{C}$-coalgebra to its underlying dg space admits a right adjoint functor $F^{c}$ as in

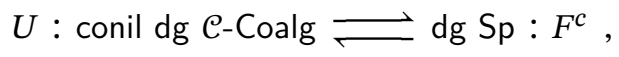


which on objects is given by $F^{c}(V)=\mathcal{C}(V)$ with the structure as defined in Example 3.2.2.

PROof. Given a dg space $V$, we show that the conilpotent dg $\mathcal{C}$-coalgebra $\mathcal{C}(V)$ with its canonical projection map

$$
p:=(\mathcal{C}(V) \stackrel{\varepsilon(V)}{\longrightarrow} I(V) \stackrel{\cong}{\longrightarrow} V)
$$

satisfies the following universal property: given a conilpotent $\operatorname{dg} \mathcal{C}$-coalgebra $C$ with a morphism $q: C \rightarrow V$ of dg spaces, there is a unique morphism $f: C \rightarrow \mathcal{C}(V)$ of dg $\mathcal{C}$-coalgebras such that $p f=q$. We define

$$
f:=(C \stackrel{\Delta}{\longrightarrow} \mathcal{C}(C) \stackrel{\mathcal{C}(q)}{\longrightarrow} \mathcal{C}(V))
$$

and show that (i) $f$ is in fact a morphism of $\operatorname{dg} \mathcal{C}$-coalgebras, (ii) it satisfies $p f=q$, and (iii) $f$ is unique as such.

The first property holds by commutativity of the diagram

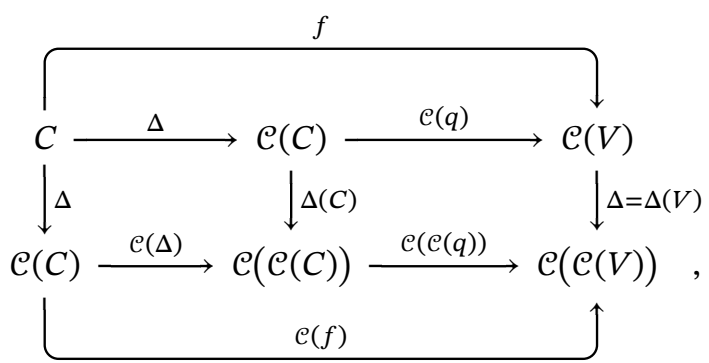

and the second follows immediatly from commutativity of

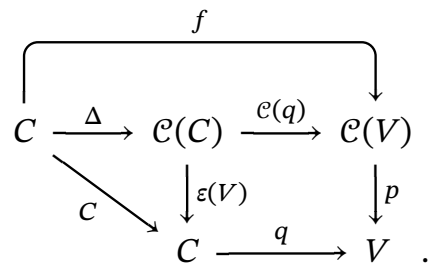

Suppose now that $g: C \rightarrow \mathcal{C}(V)$ is another morphism of $\mathrm{dg} \mathcal{C}$-coalgebras satisfying $p g=q$. The diagram

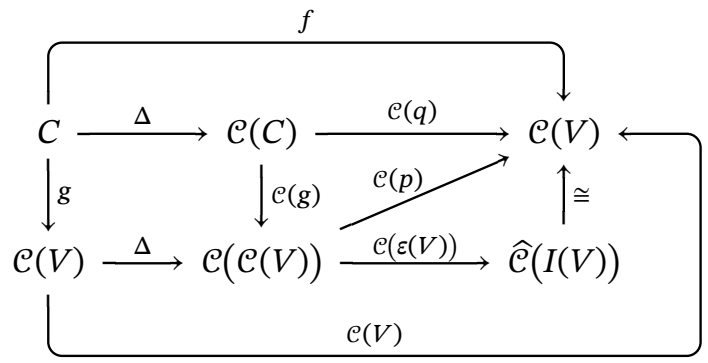

is commutative, proving the third property.

3.3. Comodules over a coalgebra. Let $(\mathcal{C}, \Delta, \varepsilon)$ denote a dg cooperad and $(C, \Delta)$ a dg $\mathcal{C}$-coalgebra. 
Definition 3.3.1. A $d g C$-comodule $(M, \Delta)$ is a space $M$ equipped with a comodule comultiplication map $\Delta: M \rightarrow \widehat{\mathcal{C}}(C ; M)$. This structure map is required to be compatible with the cooperad comultiplication and counit maps, i.e. the following diagrams must commute:

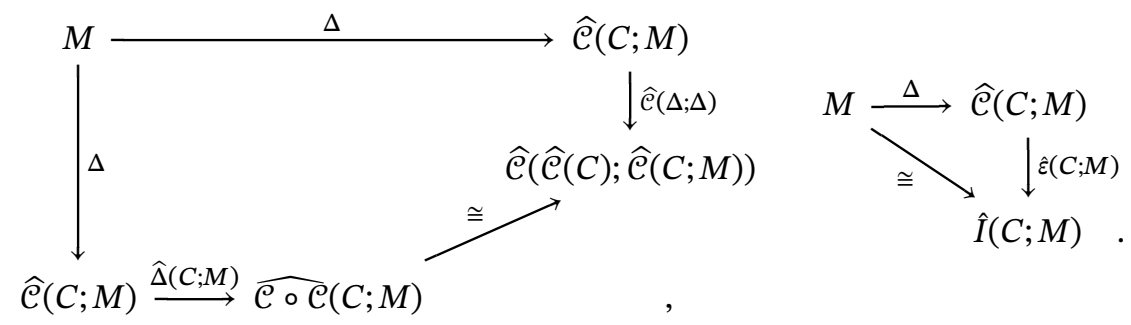

A morphism of $d g C$-comodules $f: M \rightarrow M^{\prime}$ is a morphism of their underlying spaces compatible with the structure in the sense that the following diagram commutes:

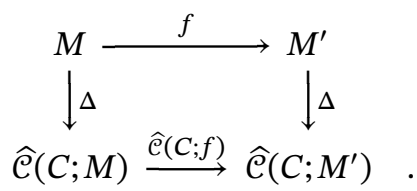

We denote the category of $d g C$-comodules by $\mathrm{dg} C$-Comod.

A dg $C$-comodule $(M, \Delta)$ over a conilpotent dg $\mathcal{C}$-coalgebra $(C, \Delta)$ is conilpotent, if its comultiplication map $\Delta: M \rightarrow \widehat{\mathcal{C}}(C ; M)$ factors through $\mathcal{C}(C ; M)$. In this case we will often implicitly view comultiplication as a map $\Delta: M \rightarrow \mathcal{C}(C ; M)$. We denote the full subcategory of $\mathrm{dg} C$-Comod consisting of conilpotent $\mathrm{dg} C$-comodules over a conilpotent dg $\mathcal{C}$-coalgebra $C$ by conil dg $C$-Comod.

EXAMPLE 3.3.2. Any conilpotent $\mathrm{dg} \mathcal{C}$-coalgebra $C$ can be viewed as a conilpotent $\mathrm{dg}$ $C$-comodule with comultiplication defined by

$$
\Delta:=(C \stackrel{\Delta}{\longrightarrow} \mathcal{C}(C) \longrightarrow \mathcal{C}(C ; C))
$$

EXAMPLE 3.3.3. Let $(\mathcal{C}, \Delta, \varepsilon)$ be a dg cooperad, $V$ a $(\mathrm{dg})$ space, and $\mathcal{C}(V)$ the cofree conilpotent dg $\mathcal{C}$-coalgebra on $V$. Given another space $N$, we equip the space $\mathcal{C}(V ; N)$ with a comultiplication map

$$
\Delta:=(\mathcal{C}(V ; N) \stackrel{\Delta(V ; N)}{\longrightarrow}(\mathcal{C} \circ \mathcal{C})(V ; N) \stackrel{\cong}{\longrightarrow}(\mathcal{C}(V) ; \mathcal{C}(V ; N)))
$$

and call $(\mathcal{C}(V ; N), \Delta)$ the cofree conilpotent $d g \mathcal{C}(V)$-comodule on $N$. The name is justified by the following lemma.

Lemma 3.3.4. The forgetful functor $U$ mapping a conilpotent $d g \mathcal{C}(V)$-comodule to its underlying dg space admits a right adjoint functor $F^{c}$ as in

$$
U: \text { conil dg } \mathcal{C}(V) \text {-Comod } \rightleftharpoons \mathrm{dg} \mathrm{Sp}: F^{c},
$$

which on objects is given by $F^{c}(N)=\mathcal{C}(V ; N)$ with the structure as defined in Example 3.3.3. 
Proof. Given dg spaces $V$ and $N$, we show that the $\operatorname{dg} \mathcal{C}(V)$-comodule $\mathcal{C}(V ; N)$ with its canonical projection map

$$
p:=(\mathcal{C}(V ; N) \stackrel{\varepsilon(V ; N)}{\longrightarrow} I(V ; N) \stackrel{\cong}{\longrightarrow} N)
$$

satisfies the following universal property: given a conilpotent $\operatorname{dg} \mathcal{C}(V)$-comodule $M$ with a morphism $q: M \rightarrow N$ of $\operatorname{dg}$ spaces, there is a unique morphism $f: M \rightarrow \mathcal{C}(V ; N)$ of $\operatorname{dg} \mathcal{C}(V)$-comodules such that $p f=q$. To this end, we define

$$
f:=(M \stackrel{\Delta}{\longrightarrow} \mathcal{C}(\mathcal{C}(V) ; M) \stackrel{\mathcal{C}(p ; q)}{\longrightarrow} \mathcal{C}(V ; N))
$$

and show that $(i) f$ is in fact a morphism of $\operatorname{dg} \mathcal{C}(V)$-comodules, (ii) it satisfies $p f=q$, and (iii) $f$ is unique as such.

The first property holds by commutativity of the diagram

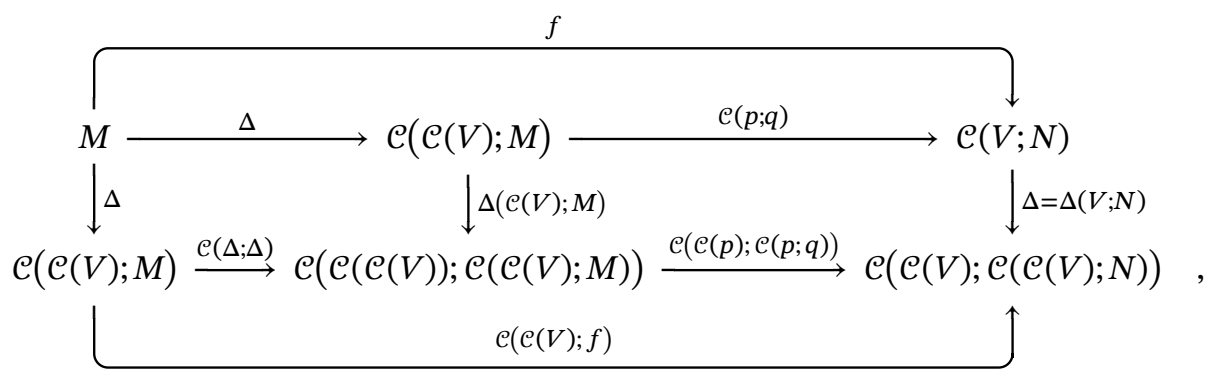

and the second follows immediatly from commutativity of

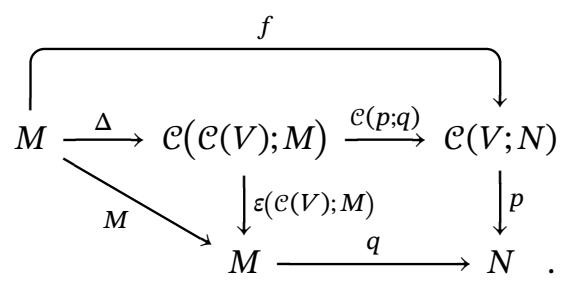

Suppose now that $g: M \rightarrow \mathcal{C}(V ; N)$ is another morphism of $\operatorname{dg} \mathcal{C}(V)$-comodules satisfying $p g=q$. The diagram

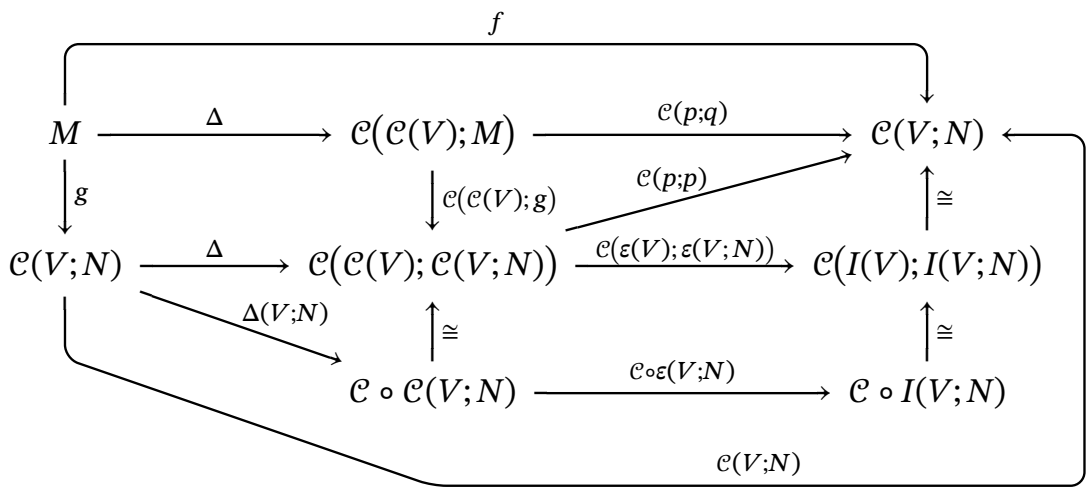

is commutative, proving the third and final property. 
3.4. The Hadamard tensor product. Given cooperads $\mathcal{C}, \mathcal{C}^{\prime}$, we can equip the aritywise tensor product $\mathcal{C} \otimes \mathcal{C}^{\prime}$ of their underlying collections with a cooperad structure as follows. We define the cooperadic counit as the map

$$
\varepsilon=\left(\mathcal{C} \otimes \mathcal{C}^{\prime} \stackrel{\varepsilon \otimes \varepsilon^{\prime}}{\longrightarrow} I \otimes I \stackrel{\cong}{\longrightarrow} I\right)
$$

and its decomposition map $\Delta$ is determined by components

$\left(\mathcal{C} \otimes \mathcal{C}^{\prime}\right)[1 \leftarrow X] \cong \mathcal{C}[1 \leftarrow X] \otimes \mathcal{C}^{\prime}[1 \leftarrow X]$

$$
\begin{aligned}
\stackrel{\Delta[1 \leftarrow X] \otimes \Delta^{\prime}[1 \leftarrow X]}{\longrightarrow} & (\mathcal{C} \circ \mathcal{C})[1 \leftarrow X] \otimes\left(\mathcal{C}^{\prime} \circ \mathcal{C}^{\prime}\right)[1 \leftarrow X] \\
& \cong \bigoplus \begin{array}{l} 
\\
P \in \operatorname{Part}(X)
\end{array}(\mathcal{C}[1 \leftarrow P] \otimes \mathcal{C}[P \leftarrow X]) \otimes \bigoplus_{P^{\prime} \in \operatorname{Part}(X)}\left(\mathcal{C}^{\prime}\left[1 \leftarrow P^{\prime}\right] \otimes \mathcal{C}^{\prime}\left[P^{\prime} \leftarrow X\right]\right) \\
& \cong \bigoplus \mathcal{C}[1 \leftarrow P] \otimes \mathcal{C}[P \leftarrow X] \otimes \mathcal{C}^{\prime}\left[1 \leftarrow P^{\prime}\right] \otimes \mathcal{C}^{\prime}\left[P^{\prime} \leftarrow X\right] \\
& P, P^{\prime} \in \operatorname{Part}(X) \\
\longrightarrow & \bigoplus_{P \in \operatorname{Part}(X)} \mathcal{C}[1 \leftarrow P] \otimes \mathcal{C}[P \leftarrow X] \otimes \mathcal{C}^{\prime}[1 \leftarrow P] \otimes \mathcal{C}^{\prime}[P \leftarrow X] \\
& \cong \bigoplus\left(\mathcal{C} \otimes \mathcal{C}^{\prime}\right)[1 \leftarrow P] \otimes\left(\mathcal{C} \otimes \mathcal{C}^{\prime}\right)[P \leftarrow X] \\
& P \in \operatorname{Part}(X) \\
& =\left(\left(\mathcal{C} \otimes \mathcal{C}^{\prime}\right) \circ\left(\mathcal{C} \otimes \mathcal{C}^{\prime}\right)\right)[1 \leftarrow X] .
\end{aligned}
$$

We call the resulting cooperad $\mathcal{C} \otimes \mathcal{C}^{\prime}$ the Hadamard product of $\mathcal{C}$ and $\mathcal{C}^{\prime}$.

Lemma 3.4.1. Let $C$ be a dg $\mathcal{C}$-coalgebra and $C^{\prime}$ a $d g \mathcal{C}^{\prime}$-coalgebra. The tensor product of spaces $C \otimes C^{\prime}$ is naturally a $d g\left(\mathcal{C} \otimes \mathcal{C}^{\prime}\right)$-coalgebra.

EXAMPLE 3.4.2. We denote by $\mathcal{S}^{c}:=\left(\mathcal{S}^{-1}\right)^{*}=\operatorname{End}_{S^{-1} \mathbf{k}}^{*}$ the linear dual of the desuspension operad and call it the suspension cooperad. Since $\left(s^{-1} \mathbf{k}\right)^{*} \cong s \mathbf{k}, s \mathbf{k}$ is naturally an $\mathcal{S}^{c}$-coalgebra. Hence, for any $\mathcal{C}$-coalgebra $C$, by Lemma 3.4.1 its suspension $s C=s \mathbf{k} \otimes C$ is naturally an $\mathcal{S} \otimes \mathcal{C}$-coalgebra. Similarly, we denote $\mathcal{S}^{-1, c}$ the linear dual of the suspension operad and call it the desuspension cooperad. The desuspension $s^{-1} C$ of a $\mathcal{C}$-coalgebra $C$ is naturally an $\mathcal{S}^{-1, c} \otimes \mathcal{C}$-coalgebra.

Definition 3.4.3. We call $\mathcal{S}^{c} \mathcal{C}:=\mathcal{S}^{c} \otimes \mathcal{C}$ resp. $\mathcal{S}^{-1, c} \mathcal{C}:=\mathcal{S}^{-1, c} \otimes \mathcal{C}$ the cooperadic suspension resp. desuspension of $\mathcal{C}$.

3.5. The cofree cooperad. Let $M$ be a dg collection and $\mathcal{T} M$ its tree module as defined in Section 2.5. We equip $\mathcal{T} M$ with a dg cooperad structure, see [46] for more details. The counit is given by the projection

$$
\varepsilon: \mathcal{T} M \longrightarrow \mathcal{T}_{0} M \cong I
$$

and the decomposition map $\Delta: \mathcal{T} M \rightarrow \mathcal{T} M \circ \mathcal{T} M$ is the limit over

$$
\Delta_{0}: \mathcal{T}_{0} M=I \stackrel{\cong}{\longrightarrow} I \circ I=\mathcal{T}_{0} M \circ \mathcal{T}_{0} M,
$$




$$
\begin{aligned}
\Delta_{n}: \mathcal{T}_{n} M & =I \oplus M \circ \mathcal{T}_{n-1} M \\
& \stackrel{\mathcal{T}_{n} M \oplus M \circ \Delta_{n-1}}{\longrightarrow} I \circ \mathcal{T}_{n} M \oplus M \circ\left(\mathcal{T}_{n-1} M \circ \mathcal{T}_{n-1} M\right) \\
& \stackrel{\cong}{\longrightarrow} \circ \mathcal{T}_{n} M \oplus\left(M \circ \mathcal{T}_{n-1} M\right) \circ \mathcal{T}_{n-1} M \\
& \left(I \oplus M \circ \mathcal{T}_{n-1} M\right) \circ \mathcal{T}_{n} M=\mathcal{T}_{n} M \circ \mathcal{T}_{n} M .
\end{aligned}
$$

A straightforward verification shows that $(i) \Delta$ is welldefined since $\Delta_{n}$ commute with the inclusions: $\Delta_{n+1} \iota_{n}=\left(\iota_{n} \circ l_{n}\right) \Delta_{n}$; and (ii) this decomposition $\Delta$ satisfies the coassociativity and counitality axioms. This shows that $(\mathcal{T} M, \Delta, \varepsilon)$ is indeed a dg cooperad. We will sometimes use the notation $\mathcal{T}^{c} M$ for this cooperad to distinguish it from the free operad on $M$. The inclusion

$$
\eta: I \cong \mathcal{T}_{0}^{c} M \longrightarrow \mathcal{T}^{c} M
$$

defines a coaugmentation for $\mathcal{T}^{c} M$.

Note that the coaugmented $\mathrm{dg}$ cooperad $\mathcal{T}^{c} M=(\mathcal{T} M, \Delta, \varepsilon, \eta)$ is in fact conilpotent. On $\mathcal{T}_{n} M$ the map $\widetilde{\Delta}$ is given by

$$
\begin{aligned}
& \widetilde{\Delta}_{0}: \mathcal{T}_{0} M=I \stackrel{\cong}{\longrightarrow} I \circ I=\mathcal{T}_{0} M \circ \mathcal{T}_{0} M, \\
& \widetilde{\Delta}_{n}: \mathcal{T}_{n} M=I \oplus M \circ \mathcal{T}_{n-1} M \stackrel{\Delta_{I} \oplus M \circ \Delta_{n-1}}{\longrightarrow} I \circ I \oplus M \circ\left(\mathcal{T}_{n-1} M \circ \mathcal{T}_{n-1} M\right) \\
& \cong I \circ I \oplus\left(M \circ \mathcal{T}_{n-1} M\right) \circ \mathcal{T}_{n-1} M \\
& \mathcal{T}_{n} M \circ \mathcal{T}_{n-1} M
\end{aligned}
$$

Thus, the right iteration of $\widetilde{\Delta}$ gives a map

$$
\widetilde{\Delta}^{n}: \mathcal{T}_{n} M \longrightarrow \mathcal{T}_{n} M \circ \mathcal{T}_{n-1} M \circ \cdots \circ \mathcal{T}_{0} M
$$

and hence $\mathcal{T}_{n} M \subset \mathcal{F}_{n}\left(\mathcal{T}^{c} M\right)$. By definition of $\mathcal{T}^{c} M$ as colimit over $\mathcal{T}_{n} M$, this implies that the coradical filtration is exhaustive.

Lemma 3.5.1. The cooperad $\mathcal{T}^{c} M$ is the cofree conilpotent dg cooperad on the $d g$ collection $M$, i.e. the above construction provides a right adjoint for the forgetful functor $\bar{U}$ mapping a dg cooperad to its coaugmentation coideal (viewed as a dg collection)

$$
\bar{U}: \text { conil dg Coopd } \rightleftharpoons \text { dg Coll }: \mathcal{T}^{c} \text {. }
$$

Proof. We construct the counit $\varphi$ and the unit $\psi$ for the adjunction as follows. The components of $\varphi: \bar{U} \mathcal{T}^{c} \Rightarrow 1$ are simply the projections $\varphi(M): \overline{\mathcal{T}^{c} M} \rightarrow \overline{\mathcal{T}^{c} M}=M$, and $\psi(\mathcal{C}): \mathcal{C} \rightarrow \mathcal{T}^{c}(\overline{\mathcal{C}})$ are constructed inductively via

$$
\begin{aligned}
& \psi(\mathcal{C})_{0}: \mathcal{C} \stackrel{\varepsilon}{\rightarrow} I \cong \mathcal{T}_{0}^{c} \overline{\mathcal{C}} \\
& \psi(\mathcal{C})_{n}: \mathcal{C} \stackrel{\varepsilon \oplus \widetilde{\Delta}}{\longrightarrow} I \oplus \overline{\mathcal{C}} \circ \mathcal{C} \stackrel{I \oplus \overline{\mathcal{C}} \circ \psi(\mathcal{C})_{n-1}}{\longrightarrow} I \oplus \overline{\mathcal{C}} \circ \mathcal{T}_{n-1} \overline{\mathcal{C}}=\mathcal{T}_{n} \overline{\mathcal{C}}
\end{aligned}
$$

To show that $(\varphi, \psi)$ form an adjunction, one must show that: $(i) \varphi \bar{U} \circ \bar{U} \psi=1$, and (ii) $\mathcal{T}^{c} \varphi \circ \psi \mathcal{T}^{c}=1$. 
The first statement follows immediatly from commutativity of the following diagram:

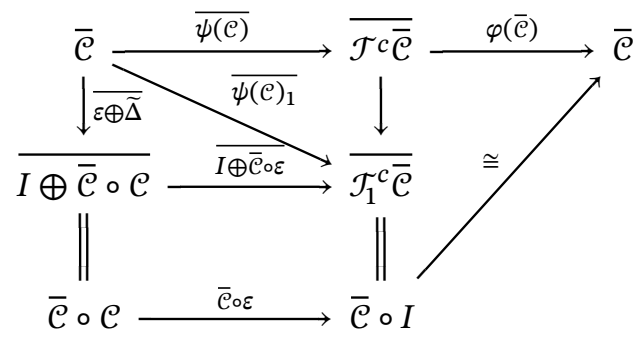

The second holds by inductive commutativity of the diagram

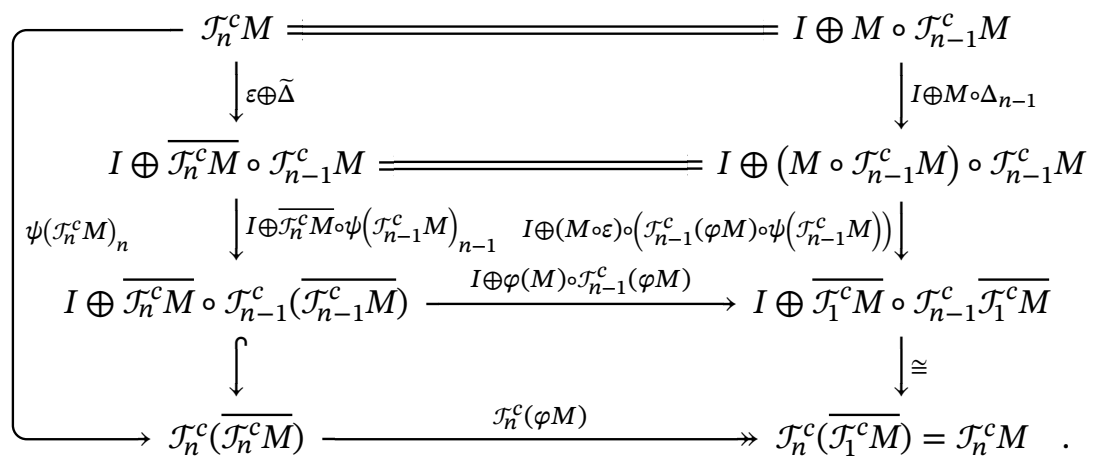

This concludes the proof.

A canonical weightgrading on the tree module $\mathcal{T} M$, in which an element of the generating dg collection $M$ is assigned weight 1 , was defined in Section 2.5. The cooperadic structure of $\mathcal{T}^{c} M$ is compatible with the weightgrading and $\mathcal{T}^{c} M^{(0)}=I$; in other words, the cofree cooperad $\mathcal{T}^{c} M$ on a dg collection is a connected wdg cooperad.

3.6. Coderivations. Let $(\mathcal{C}, \Delta, \varepsilon)$ be a graded cooperad. By a coderivation of $\mathcal{C}$, we mean a morphism of graded collections $\mathrm{d}: \mathcal{C} \rightarrow \mathcal{C}$ of degree -1 , satisfying
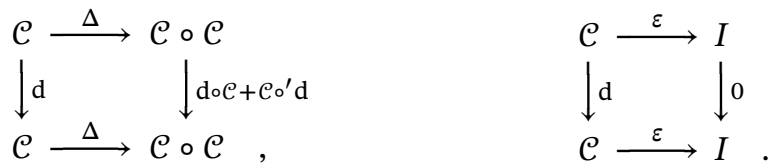

When $\mathcal{C}$ is coaugmented, we additionally assume commutativity of

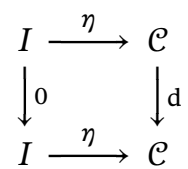

for any coderivation. The set of coderivations for a cooperad $\mathcal{C}$ is denoted $\operatorname{Coder}(\mathcal{C})$. A square-zero coderivation, i.e. a coderivation d satisfying $\mathrm{d}^{2}=0$, is called a codifferential, and the set of codifferentials on $\mathcal{C}$ is denoted $\operatorname{Codiff}(\mathcal{C})$.

Consider the cofree conilpotent graded cooperad $\mathcal{T}^{c} M$ on a graded collection $M$. We call $\mathcal{T}^{c} M$ equipped with a codifferential d a quasi-cofree conilpotent $d g$ cooperad 
on $M$. Note that any coderivation for $\mathcal{T}^{c} M$ is uniquely determined by its projection $\mathcal{T}^{c} M \rightarrow M$ to cogenerators as per the following result.

Lemma 3.6.1. There is a one-to-one correspondence between coderivations of the cofree conilpotent graded cooperad $\mathcal{T}^{c} M$ and morphisms of graded collections $\overline{\mathcal{T}^{c} M} \rightarrow$ of degree-1:

$$
\operatorname{Coder}\left(\mathcal{T}^{c} M\right) \cong \operatorname{hom}\left(\overline{\mathcal{T}^{c} M}, M\right)_{-1}
$$

Proof. Given a map $f: \overline{\mathcal{T}^{c} M} \rightarrow M$ of degree -1 , we define a coderivation $\mathrm{d}^{f}$ on $\mathcal{T}^{c} M$ inductively as follows:

$$
\begin{aligned}
& \mathrm{d}_{0}^{f}: \mathcal{T}_{0} M=I \stackrel{0}{\longrightarrow} I=\mathcal{T}_{0} M, \\
& \mathrm{~d}_{n}^{f}: \mathcal{T}_{n} M=I \oplus M \circ \mathcal{T}_{n-1} M \stackrel{0 \oplus \overline{\mathrm{d}_{n}^{f}}}{\longrightarrow} I \oplus M \circ \mathcal{T}_{n-1} M,
\end{aligned}
$$

where $\overline{\mathrm{d}_{n}^{f}}$ is the sum of

$$
M \circ \mathcal{T}_{n-1} M \stackrel{M \circ \Delta}{\longrightarrow} M \circ \mathcal{T}_{n-1} M \circ \mathcal{T}_{n-1} M \stackrel{f \circ \mathcal{T}_{n-1} M}{\longrightarrow} M \circ \mathcal{T}_{n-1} M
$$

and

$$
M \circ \mathcal{T}_{n-1} M \stackrel{M \circ \mathrm{d}_{n-1}^{f}}{\longrightarrow} M \circ \mathcal{T}_{n-1} M
$$

The first of these two terms amounts to applying $f$ to subtrees containing the root vertex, the second amounts to inductively applying $f$ to all other subtrees.

Let $\mathcal{C}, \mathcal{D}$ be dg cooperads and $M$ a graded $(\mathcal{C}, \mathcal{D})$-comodule. A coderivation of $M$ is a degree -1 morphism d: $M \rightarrow M$ of graded collections such that the following diagrams commute:

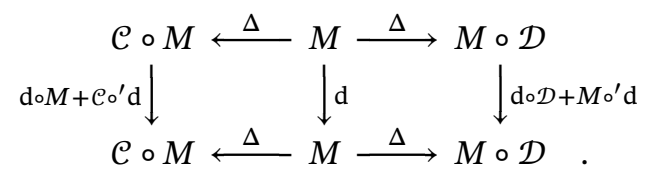

We denote by $\operatorname{Coder}(M)$ the set of coderivations of $M$ and by $\operatorname{Codiff}(M)$ the subset of square-zero coderivations or codifferentials.

Consider the cofree graded $(\mathcal{C}, \mathcal{D})$-comodule $\mathcal{C} \circ N \circ \mathcal{D}$ on a collection $N$. We call $\mathcal{C} \circ N \circ \mathcal{D}$ equipped with a codifferential d a quasi-cofree $d g(\mathcal{C}, \mathcal{D})$-comodule.

Lemma 3.6.2. There is a one-to-one correspondence between coderivations of the cofree graded $(\mathcal{C}, \mathcal{D})$-comodule $\mathcal{C} \circ N \circ \mathcal{D}$ and morphisms of graded collections $p: \mathcal{C} \circ N \circ \mathcal{D} \rightarrow N$ of degree -1 :

$$
\operatorname{Coder}(\mathcal{C} \circ N \circ \mathcal{D}) \cong \operatorname{hom}(\mathcal{C} \circ N \circ \mathcal{D}, N)_{-1} \cdot
$$

PROOF. The coderivation $d$ corresponding to $p$ is the sum of the following three maps:

$$
\begin{aligned}
& \mathcal{C} \circ N \circ \mathcal{D} \stackrel{\mathrm{d} \circ N \circ \mathcal{D}}{\longrightarrow} \mathcal{C} \circ N \circ \mathcal{D}, \\
& \mathcal{C} \circ N \circ \mathcal{D} \stackrel{\mathcal{C} \circ N \circ{ }^{\prime} \mathrm{d}}{\longrightarrow} \mathcal{C} \circ N \circ \mathcal{D},
\end{aligned}
$$


and

$$
\begin{aligned}
\mathcal{C} \circ N \circ \mathcal{D} & \stackrel{\Delta_{(1)} \circ N \circ \mathcal{D}}{\longrightarrow}(\mathcal{C} \circ(1) \\
& \stackrel{\mathcal{C} \circ(N \circ \mathcal{D} ; \mathcal{C} \circ N \circ \Delta)}{\longrightarrow} \mathcal{C} \circ(N \circ \mathcal{D} ; \mathcal{C} \circ N \circ \mathcal{D} \circ \mathcal{D}) \cong \mathcal{C} \circ(N ; \mathcal{C} \circ N \circ \mathcal{D}) \circ \mathcal{D} \\
& \stackrel{\mathcal{C} \circ(N+P) \circ \mathcal{D}}{\longrightarrow} \mathcal{C} \circ N \circ \mathcal{D} .
\end{aligned}
$$

Let $(\mathcal{C}, \Delta, \varepsilon)$ be a dg cooperad and $C$ a conilpotent graded $\mathcal{C}$-coalgebra. A coderivation of $C$ is a degree -1 morphism $\mathrm{d}: C \rightarrow C$ of graded collections such that the following diagram commutes,

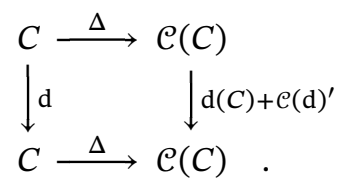

We denote by $\operatorname{Coder}(C)$ the set of coderivations of $C$ and by $\operatorname{Codiff}(C)$ the subset of square-zero coderivations or codifferentials.

Consider now the cofree conilpotent graded $\mathcal{C}$-coalgebra $\mathcal{C}(V)$ on a space $V$. We call $\mathcal{C}(V)$ equipped with a codifferential d a quasi-cofree conilpotent dg $\mathcal{C}$-coalgebra. Similarly as for cooperads, coderivations of $\mathcal{C}(V)$ are uniquely determined by their projection to cogenerators.

Lemma 3.6.3. There is a one-to-one correspondence between coderivations of the cofree conilpotent graded $\mathcal{C}$-coalgebra $\mathcal{C}(V)$ and morphisms of graded spaces $\mu: \mathcal{C}(V) \rightarrow V$ of degree -1 :

$$
\operatorname{Coder}(\mathcal{C}(V)) \cong \operatorname{hom}(\mathcal{C}(V), V)_{-1}
$$

Proof. Given a map $\mu: \mathcal{C}(V) \rightarrow V$, we define $\mathrm{d}_{\mu}: \mathcal{C}(V) \rightarrow \mathcal{C}(V)$ as the sum of

$$
\mathcal{C}(V) \stackrel{\mathrm{d}(V)}{\longrightarrow} \mathcal{C}(V)
$$

and

$$
\mathcal{C}(V) \stackrel{\Delta_{(1)}(V)}{\longrightarrow}\left(\mathcal{C} \circ_{(1)} \mathcal{C}\right)(V) \stackrel{\cong}{\longrightarrow} \mathcal{C}(V ; \mathcal{C}(V)) \stackrel{C(V+\mu)}{\longrightarrow} \mathcal{C}(V)
$$

This is easily seen to define a coderivation, i.e. $\mathrm{d}_{\mu} \in \operatorname{Coder}(\mathcal{C}(V))$. It remains to prove that (i) $\mu=p \mathrm{~d}_{\mu}$, and (ii) $\mathrm{d}=\mathrm{d}_{p \mathrm{~d}}$.

As in the proof of Lemma 3.2.3 the projection $p$ is given by $\varepsilon(V): \mathcal{C}(V) \rightarrow I(V) \cong V$, where $\varepsilon$ denotes the counit of $\mathcal{C}$. By definition of a dg cooperad we have $\varepsilon d=0$, and thus the composition

$$
\mathcal{C}(V) \stackrel{\mathrm{d}(V)}{\longrightarrow} \mathcal{C}(V) \stackrel{\varepsilon(V)}{\longrightarrow} I(V) \cong V
$$


vanishes. The fact that $\mu=p \mathrm{~d}_{\mu}$ then follows from commutativity of the diagram

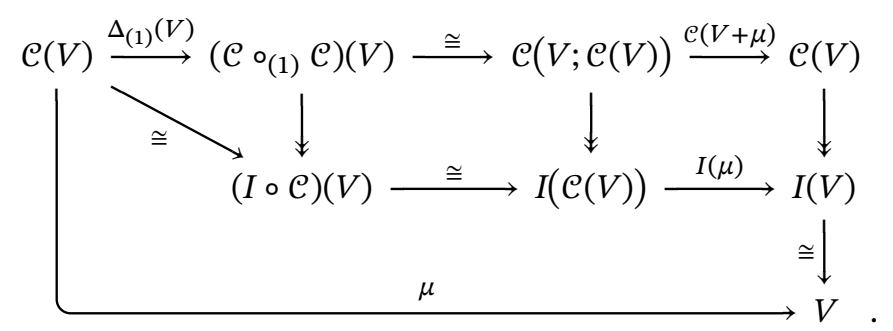

The second statement, $d=d_{p d}$, follows by commutativity of the diagram

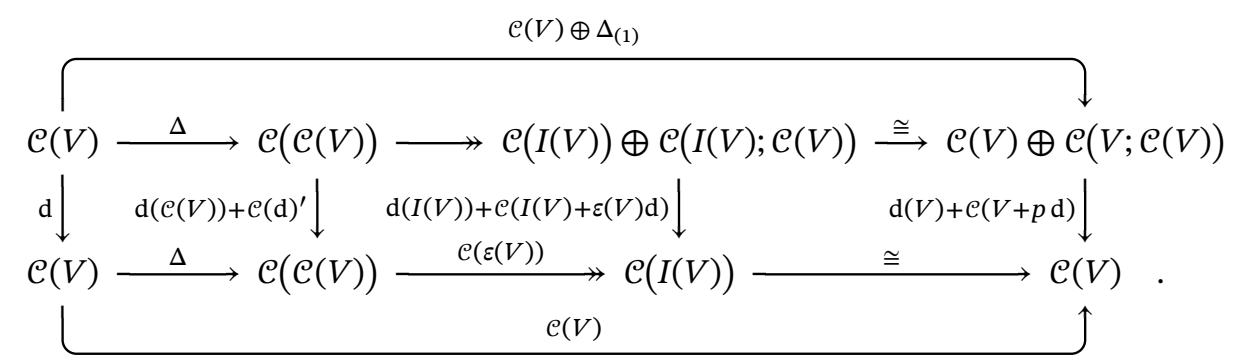

This proves the desired bijection.

Let $\mathcal{C}$ be a dg cooperad, $C$ a conilpotent $\operatorname{dg} \mathcal{C}$-coalgebra and $M$ a conilpotent graded $C$ comodule. A coderivation of $M$ is a degree -1 morphism d: $M \rightarrow M$ of graded collections such that the following diagram commutes,

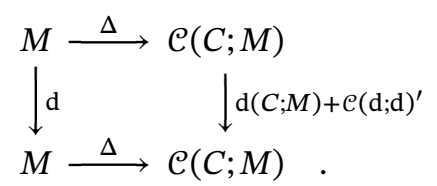

We denote by $\operatorname{Coder}(M)$ the set of coderivations of $M$ and by Codiff( $M)$ the subset of square-zero coderivations or codifferentials.

Consider now the cofree conilpotent graded $\mathcal{C}(V)$-comodule $\mathcal{C}(V ; N)$ on a space $N$ over the cofree conilpotent dg $\mathcal{C}$-coalgebra $\mathcal{C}(V)$ on a space $V$. Coderivations of $\mathcal{C}(V ; N)$ are uniquely determined by their projection to cogenerators.

Lemma 3.6.4. There is a one-to-one correspondence between coderivations of the cofree conilpotent graded $\mathcal{C}(V)$-comodule $\mathcal{C}(V ; N)$ and degree -1 morphisms of graded spaces $\rho: \mathcal{C}(V ; N) \rightarrow N:$

$$
\operatorname{Coder}(\mathcal{C}(V ; N)) \cong \operatorname{hom}(\mathcal{C}(V ; N), N)_{-1}
$$

Proof. Given a map $\rho: \mathcal{C}(V ; N) \rightarrow V$, we define $\mathrm{d}_{\rho}: \mathcal{C}(V ; N) \rightarrow \mathcal{C}(V ; N)$ as the sum of

(54) $\mathcal{C}(V ; N) \stackrel{\mathrm{d}(V ; N)}{\longrightarrow} \mathcal{C}(V ; N)$ 
and the composition

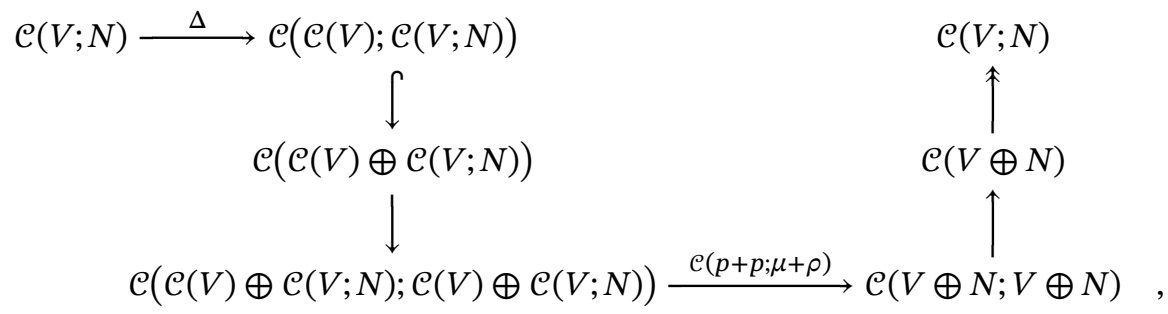

where the second term amounts to applying $\mu$ or $\rho$ to subtrees as indicated in Figure 3 . and $p$ denotes the projections as defined in the proofs of Lemmas 3.2.3 and 3.3.4

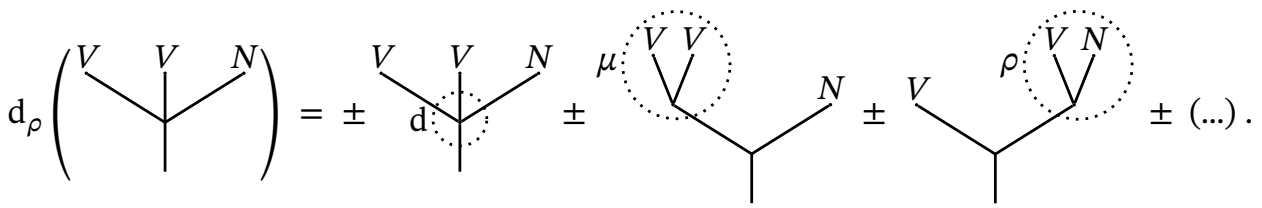

FIGURE 3. Coextension of $\rho$ to a $\left(\mathcal{C}(V), \mathrm{d}_{\mu}\right)$-comodule coderivation.

The map $\mathrm{d}_{\rho}$ is easily seen to define a coderivation on the $\mathcal{C}(V)$-comodule $\mathcal{C}(V ; N)$. It remains to show that $(i) \rho=p \mathrm{~d}_{\rho}$, and $(i i) \mathrm{d}=\mathrm{d}_{p \mathrm{~d}}$. Since the proofs of these statements are analogous to those of Lemma 3.6.3 they are omitted here.

\section{Resolutions of Operads}

Let $\mathcal{C}$ be a conilpotent dg cooperad and $\mathcal{P}$ an augmented dg operad. We define the chain complex

$$
\operatorname{Conv}(\mathcal{C}, \mathcal{P}):=\lim _{\substack{(1 \leftarrow X) \\ \in 1-\text { Tree }}} \operatorname{hom}(\overline{\mathcal{C}}, \overline{\mathcal{P}})[1 \leftarrow X]
$$

and equip it with a binary product as follows: for $\alpha, \beta \in \operatorname{Conv}(\mathcal{C}, \mathcal{P})$, we define

$$
\alpha \star \beta:=\left(\overline{\mathcal{C}} \stackrel{\bar{\Delta}_{(1)}}{\longrightarrow} \overline{\mathcal{C}}_{{ }_{(1)}} \overline{\mathcal{C}} \stackrel{\alpha_{{ }^{o}(1)}^{\beta}}{\longrightarrow} \overline{\mathcal{P}}_{{ }_{(1)}} \overline{\mathcal{P}} \stackrel{\bar{\gamma}_{(1)}}{\longrightarrow} \overline{\mathcal{P}}\right) .
$$

The triple $(\operatorname{Conv}(\mathcal{C}, \mathcal{P}), \star, \partial)$ is a dg pre-Lie algebra, which we call the convolution pre-Lie algebra.

Let now $\mathcal{C}, \mathcal{C}^{\prime}$ be conilpotent dg cooperads, $\mathcal{P}, \mathcal{P}^{\prime}$ augmented dg operads, $M$ a $\left(\mathcal{C}^{\prime}, \mathcal{C}\right)$-comodule, and $N$ a $\left(\mathcal{P}^{\prime}, \mathcal{P}\right)$-module. We define the chain complex

$$
\operatorname{Conv}(M, N):=\lim _{\substack{(1 \leftarrow X) \\ \in 1-\text { Tree }^{o p}}} \operatorname{hom}(M, N)[1 \leftarrow X]
$$

and equip it with the following "actions": for $\varphi \in \operatorname{Conv}(M, N), \alpha \in \operatorname{Conv}(\mathcal{C}, \mathcal{P})$, and $\alpha^{\prime} \in \operatorname{Conv}\left(\mathcal{C}^{\prime}, \mathcal{P}^{\prime}\right)$, let

$$
\varphi * \alpha:=\left(M \stackrel{\bar{\Delta}_{(1)}}{\longrightarrow} M \circ_{(1)} \overline{\mathcal{C}} \stackrel{\varphi_{\circ}^{\circ} \alpha}{\longrightarrow} N \circ_{(1)} \overline{\mathcal{P}} \stackrel{\bar{\gamma}_{(1)}}{\longrightarrow} N\right),
$$


and

$$
\alpha^{\prime} \circledast \varphi:=\left(M \stackrel{\bar{\Delta}}{\longrightarrow} \overline{\mathcal{C}^{\prime}} \circ M \stackrel{\alpha^{\prime} \circ \varphi}{\longrightarrow} \overline{\mathcal{P}^{\prime}} \circ N \stackrel{\bar{\gamma}}{\longrightarrow} N\right) .
$$

The first of these defines a left pre-Lie algebra action by $\operatorname{Conv}(\mathcal{C}, \mathcal{P})$ on $\operatorname{Conv}(M, N)$, the second can be viewed as a right nongraded associative algebra action by $\operatorname{Conv}\left(\mathcal{C}^{\prime}, \mathcal{P}^{\prime}\right)$ on $\operatorname{Conv}(M, N)$ (see $\$ 4610.2 .3])$. We call $\operatorname{Conv}(M, N)$ the convolution module.

4.1. Twisting morphisms. Let $\mathcal{C}, \mathcal{C}^{\prime}$ be conilpotent dg cooperads, $\mathcal{P}, \mathcal{P}^{\prime}$ augmented dg operads, $M$ a $\left(\mathcal{C}^{\prime}, \mathcal{C}\right)$-comodule, and $N$ a $\left(\mathcal{P}^{\prime}, \mathcal{P}\right)$-module. Given an element $\alpha \in \operatorname{Conv}\left(\mathcal{C}, \mathcal{P}^{\prime}\right)_{-1}$, we define a map $D_{\alpha}^{R}: M \circ N \rightarrow M \circ N$ as the composition

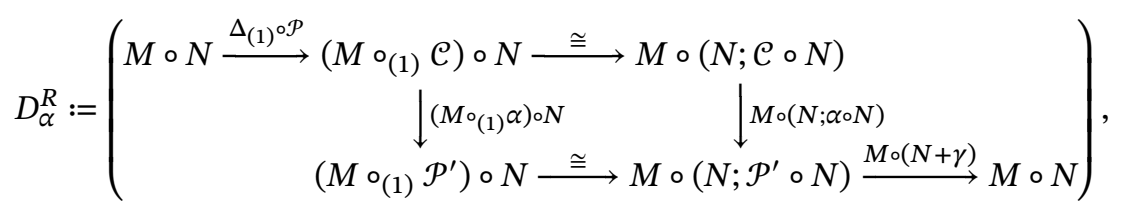

and denote by $\mathrm{d}_{\alpha}^{R}:=\mathrm{d}+D_{\alpha}^{R}$ the sum of the usual differential on $M \circ N$ with $D_{\alpha}^{R}$. Similarly, given an element $\alpha^{\prime} \in \operatorname{Conv}\left(\mathcal{C}^{\prime}, \mathcal{P}\right)_{-1}$, we define $D_{\alpha}^{L}: N \circ M \rightarrow N \circ M$ as

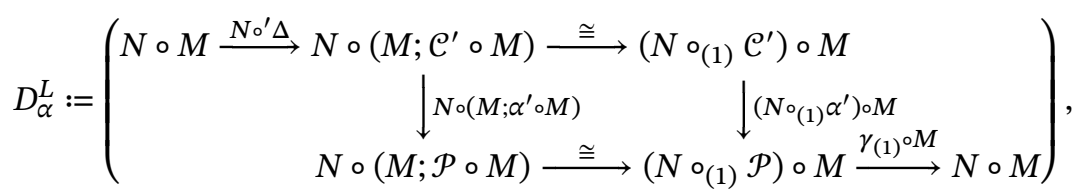

and denote by $\mathrm{d}_{\alpha^{\prime}}^{L}:=\mathrm{d}-D_{\alpha^{\prime}}^{L}$ the difference of the usual differential on $N \circ M$ with $D_{\alpha^{\prime}}^{L}$.

Consider for a moment $M=\mathcal{C}$ as a $(\mathcal{C}, \mathcal{C})$-comodule and $N=\mathcal{P}$ as a $(\mathcal{P}, \mathcal{P})$-module. We call an element $\alpha \in \operatorname{Conv}(\mathcal{C}, \mathcal{P})_{-1}$ a twisting morphism if $\mathrm{d}_{\alpha}^{R}$ on $\mathcal{C} \circ \mathcal{P}$ resp. $\mathrm{d}_{\alpha}^{L}$ on $\mathcal{P} \circ \mathcal{C}$ squares to zero. Note that these conditions are in fact equivalent, even though, by nonlinearity of the composite product, $\mathcal{C} \circ \mathcal{P}$ and $\mathcal{P} \circ \mathcal{C}$ are very different collections. We denote the set of twisting morphisms by $\operatorname{Tw}(\mathcal{C}, \mathcal{P})$.

Lemma 4.1.1. An element $\alpha \in \operatorname{Conv}(\mathcal{C}, \mathcal{P})_{-1}$ is a twisting morphisms iff. it satisfies the (pre-Lie) Maurer-Cartan equation

$$
\partial \alpha+\alpha \star \alpha=0
$$

Returning to the general case where $M$ is a $\left(\mathcal{C}^{\prime}, \mathcal{C}\right)$-comodule and $N$ is a $\left(\mathcal{P}^{\prime}, \mathcal{P}\right)$ module, we find that $\mathrm{d}_{\alpha}^{R}$ on $M \circ N$ (resp. $\mathrm{d}_{\alpha^{\prime}}^{L}$ on $N \circ M$ ) squares to zero for twisting morphisms $\alpha: \mathcal{C} \rightarrow \mathcal{P}^{\prime}$ (resp. $\alpha^{\prime}: \mathcal{C}^{\prime} \rightarrow \mathcal{P}$ ). We call $\mathrm{d}_{\alpha}^{R}$ (resp. $\mathrm{d}_{\alpha^{\prime}}^{L}$ ) the twisted differential on $M \circ N\left(\right.$ resp. $N \circ M$ ) and use the notation $M \circ_{\alpha} N$ (resp. $N \circ_{\alpha^{\prime}} M$ ) for the twisted composite product, i.e. for the composite product with its twisted differential.

We now turn our attention to the convolution module. Given $\varphi \in \operatorname{Conv}(M, N)_{0}$, we define maps $F_{\varphi}^{R}: M \circ \mathcal{P} \rightarrow \mathcal{C}^{\prime} \circ N$ and $F_{\varphi}^{L}: \mathcal{P}^{\prime} \circ M \rightarrow N \circ \mathcal{C}$ as follows:

$$
\begin{aligned}
& F_{\varphi}^{R}:=\left(M \circ \mathcal{P} \stackrel{\Delta \circ \mathcal{P}}{\longrightarrow} \mathcal{C}^{\prime} \circ M \circ \mathcal{P} \stackrel{\mathcal{C}^{\prime} \circ \varphi \circ \mathcal{P}}{\longrightarrow} \mathcal{C}^{\prime} \circ N \circ \mathcal{P} \stackrel{\mathcal{C}^{\prime} \circ \gamma}{\longrightarrow} \mathcal{C}^{\prime} \circ N\right), \\
& F_{\varphi}^{L}:=\left(\mathcal{P}^{\prime} \circ M \stackrel{\mathcal{P}^{\prime} \circ \Delta}{\longrightarrow} \mathcal{P}^{\prime} \circ M \circ \mathcal{C} \stackrel{\mathcal{P}^{\prime} \circ \varphi \circ \mathcal{C}}{\longrightarrow} \mathcal{P}^{\prime} \circ N \circ \mathcal{C} \stackrel{\gamma^{\prime} \circ \mathcal{C}}{\longrightarrow} N \circ \mathcal{C}\right) .
\end{aligned}
$$


We call $\varphi$ a twisting morphism with respect to $\alpha$ and $\alpha^{\prime}$ if $F_{\varphi}^{R}$ (resp. $F_{\varphi}^{L}$ ) defines a morphism of twisted complexes

$$
\left.F_{\varphi}^{R}: M \circ_{\alpha} \mathcal{P} \longrightarrow \mathcal{C}^{\prime} \circ_{\alpha^{\prime}} N \quad \text { (resp. } \quad F_{\varphi}^{L}: \mathcal{P}^{\prime} \circ_{\alpha^{\prime}} M \longrightarrow N \circ_{\alpha} \mathcal{C}\right)
$$

for given twisting morphisms $\alpha \in \operatorname{Tw}(\mathcal{C}, \mathcal{P}), \alpha^{\prime} \in \operatorname{Tw}\left(\mathcal{C}^{\prime}, \mathcal{P}^{\prime}\right)$. Note that, as before, these conditions are equivalent. We denote by $\operatorname{Tw}_{\alpha^{\prime}}^{\alpha}(M, N)$ the set of twisting morphism $\varphi$ for $\alpha, \alpha^{\prime}$, and by $\operatorname{Tw}(M, N)$ the set of triples $\left(\alpha^{\prime}, \varphi, \alpha\right)$ where $\varphi$ is a twisting morphism for $\alpha$, $\alpha^{\prime}$.

Lemma 4.1.2. An element $\varphi \in \operatorname{Conv}(M, N)_{0}$ is a twisting morphism for $\alpha \in \operatorname{Tw}(\mathcal{C}, \mathcal{P})$, $\alpha^{\prime} \in \operatorname{Tw}\left(\mathcal{C}^{\prime}, \mathcal{P}^{\prime}\right)$ iff. it satisfies the Maurer-Cartan equation

$$
\partial \varphi-\varphi * \alpha+\alpha^{\prime} \circledast \varphi=0 .
$$

PROOF. We must show that $\left[\mathrm{d}_{\alpha^{(\prime)}}^{L}, F_{\varphi}^{L}\right]=0$ iff. the Maurer-Cartan equation holds. Note that $F_{\varphi}^{L}$ is a morphism of $\mathcal{P}^{\prime}$-modules and $\mathrm{d}_{\alpha}^{L}, \mathrm{~d}_{\alpha^{\prime}}^{L}$ are derivations of $\mathcal{P}^{\prime}$-modules. This means that the condition $\left[\mathrm{d}_{\alpha^{(\prime)}}^{L}, F_{\varphi}^{L}\right]=0$ can be checked on generators, and we obtain

$$
\begin{aligned}
{\left.\left[\mathrm{d}_{\alpha^{\prime}()^{\prime}}^{L}, F_{\varphi}^{L}\right]\right|_{M} } & =\left.\left(\mathrm{d}-D_{\alpha}^{L}\right) F_{\varphi}^{L}\right|_{M}-\left.F_{\varphi}^{L}\left(\mathrm{~d}-D_{\alpha^{\prime}}^{L}\right)\right|_{M} \\
& =\left.\left(\mathrm{d} F_{\varphi}^{L}-F_{\varphi}^{L} \mathrm{~d}\right)\right|_{M}-\left.D_{\alpha}^{L} F_{\varphi}^{L}\right|_{M}+\left.F_{\varphi}^{L} D_{\alpha^{\prime}}^{L}\right|_{M} .
\end{aligned}
$$

The first of these terms is easily seen to be $\left.F_{\partial \varphi}^{L}\right|_{M}$; the second evaluates to $\left.F_{\varphi * \alpha}^{L}\right|_{M}$ as can be seen from the following diagram:

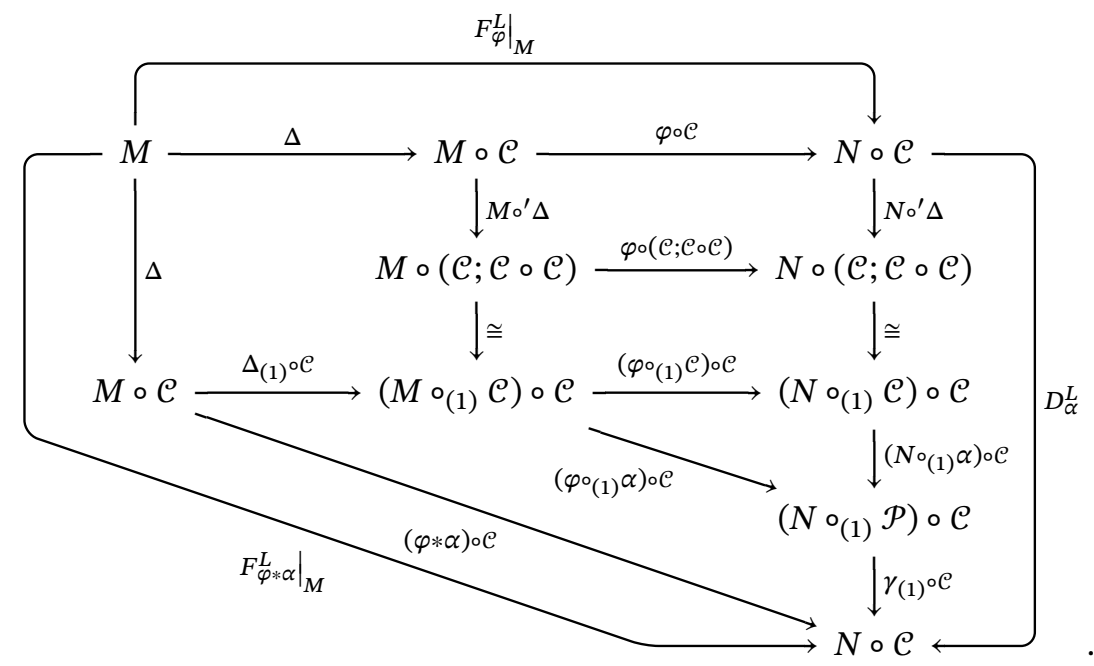


The third and final term gives $\left.F_{\alpha^{\prime} \circledast \varphi}^{L}\right|_{M}$ as shown in this diagram:

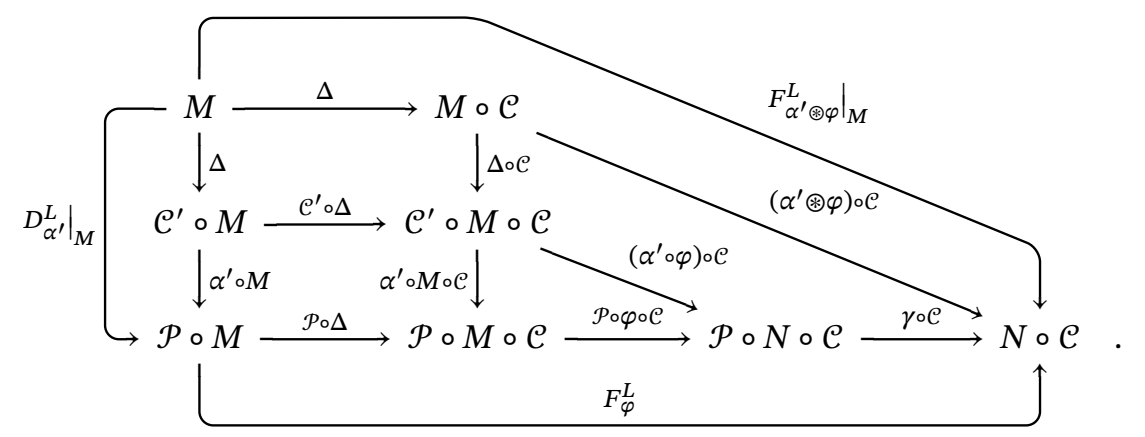

Thus we have shown that

$$
\left.\left[\mathrm{d}_{\alpha^{(\prime)}}^{L}, F_{\varphi}^{L}\right]\right|_{M}=\left.F_{\partial \varphi-\varphi * \alpha+\alpha^{\prime} \circledast \varphi}^{L}\right|_{M},
$$

which concludes the proof.

4.2. The cobar-bar adjunction. Let $\mathcal{C}$ be a coaugmented dg cooperad. Its cobar construction is the quasi-free augmented dg operad $\Omega \mathcal{C}:=\left(\mathcal{T}\left(s^{-1} \overline{\mathcal{C}}\right), \mathrm{d}=\mathrm{d}_{1}-\mathrm{d}_{2}\right)$ with $\mathrm{d}_{1}$ given as the extension of

$$
s^{-1} \overline{\mathcal{C}} \stackrel{\mathrm{d}}{\longrightarrow} s^{-1} \overline{\mathcal{C}} \cong \mathcal{T}\left(s^{-1} \overline{\mathcal{C}}\right)^{(1)} \longleftrightarrow \mathcal{T}\left(s^{-1} \overline{\mathcal{C}}\right)
$$

and $\mathrm{d}_{2}$ as extension of

$$
s^{-1} \overline{\mathcal{C}} \stackrel{s}{\longrightarrow} \overline{\mathcal{C}} \stackrel{\bar{\Delta}_{(1)}}{\longrightarrow} \overline{\mathcal{C}}{ }_{{ }_{(1)}} \overline{\mathcal{C}} \stackrel{s^{-1} o_{(1)} s^{-1}}{\longrightarrow} s^{-1} \overline{\mathcal{C}}{ }_{(1)} s^{-1} \overline{\mathcal{C}} \cong \mathcal{T}\left(s^{-1} \overline{\mathcal{C}}\right)^{(2)} \hookrightarrow \mathcal{T}\left(s^{-1} \overline{\mathcal{C}}\right) \text {. }
$$

A straightforward verification shows that $\mathrm{d}=\mathrm{d}_{1}-\mathrm{d}_{2}$ satisfies $\mathrm{d}^{2}=0$ since $(i) \Delta$ is coassociative, and (ii) $\Delta$ is compatible with the codifferential of $\mathcal{C}$. The cobar construction defines a functor

$$
\Omega: \text { coaug dg Coopd } \longrightarrow \text { aug dg Opd . }
$$

Let $\mathcal{P}$ be an augmented dg operad. Its bar construction is the quasi-cofree conilpotent $\operatorname{dg}$ cooperad $\mathrm{B} \mathcal{P}:=\left(\mathcal{T}^{c}(s \overline{\mathcal{P}}), \mathrm{d}=\mathrm{d}_{1}-\mathrm{d}_{2}\right)$ with $\mathrm{d}_{1}$ given as coextension for

$$
\mathcal{T}^{c}(s \overline{\mathcal{P}}) \longrightarrow \mathcal{T}^{c}(s \overline{\mathcal{P}})^{(1)} \cong s \overline{\mathcal{P}} \stackrel{\mathrm{d}}{\longrightarrow} s \overline{\mathcal{P}}
$$

and $d_{2}$ coextending

$$
\mathcal{T}^{c}(s \overline{\mathcal{P}}) \longrightarrow \mathcal{T}^{c}(s \overline{\mathcal{P}})^{(2)} \cong s \overline{\mathcal{P}}{ }_{{ }_{(}(1)} s \overline{\mathcal{P}} \stackrel{s^{-1} o_{(1)} s^{-1}}{\longrightarrow} \overline{\mathcal{P}}_{{ }^{\circ}} \overline{\mathcal{P}}_{(1)} \stackrel{\bar{\gamma}_{(1)}}{\longrightarrow} \overline{\mathcal{P}} \stackrel{s}{\longrightarrow} s \overline{\mathcal{P}}
$$

A simple verification using that $(i) \gamma$ is associative, and ( $i i) \gamma$ is compatible with the differential of $\mathcal{P}$, shows that the coderivation $d=d_{1}-d_{2}$ satisfies $d^{2}=0$. The bar construction defines a functor

$$
\text { B: aug dg Opd } \longrightarrow \text { conil dg Coopd . }
$$

In plain english, the codifferential of the bar construction amounts to the sum of applying $d$ at each vertex and contracting each internal edge while composing its source 
and target vertex by the partial composition map $\gamma_{(1)}$ (with appropriate signs), as shown in Figure 4

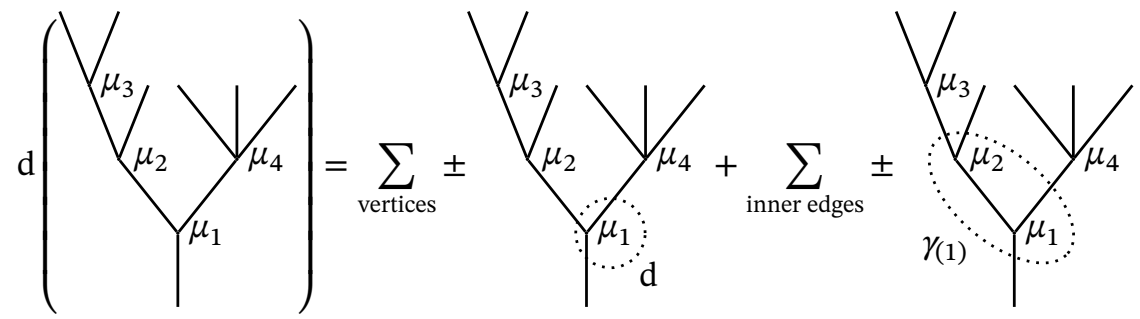

FIGURE 4. Codifferential of the bar construction.

The cobar construction on a cooperad $\mathcal{C}$ is free as a graded operad and therefore a morphism $g: \Omega \mathcal{C} \rightarrow \mathcal{P}$ of graded operads is uniquely determined by its value on generators $s^{-1} \overline{\mathcal{C}}$. A simple computation shows that the shifted restriction

$$
\alpha=\left(\overline{\mathcal{C}} \stackrel{s^{-1}}{\longrightarrow} s^{-1} \overline{\mathcal{C}} \longleftrightarrow \mathcal{T}\left(s^{-1} \overline{\mathcal{C}}\right) \stackrel{g}{\longrightarrow} \mathcal{P}\right)
$$

satisfies the Maurer-Cartan equation if and only if $g$ is compatible with the differentials. The dual statement holds for the bar construction, and we obtain the following result.

Proposition 4.2.1. The cobar and bar functors represent the twisting morphism bifunctor in the sense that

$$
\begin{aligned}
& \operatorname{Hom}_{\text {aug dg Opd }}(\Omega \mathcal{C}, \mathcal{P}) \stackrel{\cong}{\leftrightarrows} \mathrm{Tw}(\mathcal{C}, \mathcal{P}) \stackrel{\cong}{\leftrightarrows} \operatorname{Hom}_{\text {conil dg } \operatorname{Coopd}}(\mathcal{C}, \mathrm{B} \mathcal{P}) \\
& \Psi \quad \Psi \quad U \\
& g_{\alpha} \longleftrightarrow \alpha \longleftrightarrow f_{\alpha} \text {. }
\end{aligned}
$$

Corollary 4.2.2. The cobar and bar constructions form a pair of adjoint functors

$$
\Omega \text { : conil dg Coopd } \rightleftharpoons \text { aug dg Opd : B . }
$$

Under the bijections of Proposition 4.2.1 the identity morphisms on $\Omega \mathcal{C}$ and $\mathrm{B} \mathcal{P}$ correspond to twisting morphisms $l: \mathcal{C} \rightarrow \Omega \mathcal{C}$ and $\pi: \mathrm{B} \mathcal{P} \rightarrow \mathcal{P}$. We call these the canonical twisting morphisms. Given any other twisting morphism $\alpha: \mathcal{C} \rightarrow \mathcal{P}$, we obtain factorizations as indicated in the following diagram:

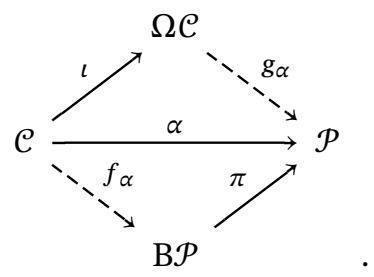

We obtain similar results for modules. Let $\mathcal{C}, \mathcal{C}^{\prime}$ be coaugmented dg cooperads and $M$ a $\left(\mathcal{C}^{\prime}, \mathcal{C}\right)$-comodule. The cobar construction for $M$ is the quasi-free $\left(\Omega \mathcal{C}^{\prime}, \Omega \mathcal{C}\right)$-module $\Omega_{\mathcal{C}^{\prime}}^{\mathcal{C}}, M:=\Omega \mathcal{C}^{\prime}{ }_{\iota^{\prime}} M \circ_{\iota} \Omega \mathcal{C}$. It provides a functor

$$
\Omega_{\mathcal{C}^{\prime}}^{\mathcal{C}}: \operatorname{dg}\left(\mathcal{C}^{\prime}, \mathcal{C}\right) \text {-Comod } \longrightarrow \operatorname{dg}\left(\Omega \mathcal{C}^{\prime}, \Omega \mathcal{C}\right)-\operatorname{Mod} .
$$


Let $\mathcal{P}, \mathcal{P}^{\prime}$ be augmented dg operads and $N$ a $\left(\mathcal{P}^{\prime}, \mathcal{P}\right)$-module. The bar construction for $N$ is the quasi-cofree $\left(\mathrm{B} \mathcal{P}^{\prime}, \mathrm{B} \mathcal{P}\right)$-comodule $\mathrm{B}_{\mathcal{P}^{\prime}}^{\mathcal{P}} N:=\mathrm{B}^{\prime} \circ_{\pi^{\prime}} N \circ_{\pi} \mathrm{B} \mathcal{P}$. It defines a functor

$$
\mathrm{B}_{\mathcal{P}}^{\mathcal{P}}: \mathrm{dg}\left(\mathcal{P}^{\prime}, \mathcal{P}\right) \text {-Mod } \longrightarrow \mathrm{dg}\left(\mathrm{B} \mathcal{P}^{\prime}, \mathrm{B} \mathcal{P}\right) \text {-Comod . }
$$

The cobar and bar constructions for modules defined here readily extend to the full (co)module categories dg Mod resp. dg Comod given in Sections 2.1 and 3.1. We obtain the following analogue of Proposition 4.2.1.

Proposition 4.2.3. The cobar and bar functors represent the twisting morphism bifunctor in the sense that

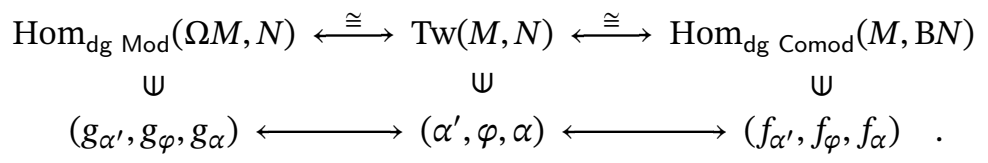

Proof. Given $\left(\alpha^{\prime}, \varphi, \alpha\right) \in \operatorname{Tw}(M, N)$, we define a triple $\left(g_{\alpha^{\prime}}, g_{\varphi}, g_{\alpha}\right)$ as follows: the morphisms $g_{\alpha}: \Omega \mathcal{C} \rightarrow \mathcal{P}$ and $g_{\alpha^{\prime}}: \Omega \mathcal{C}^{\prime} \rightarrow \mathcal{P}^{\prime}$ are given by Proposition 4.2.1 and $g_{\varphi}$ is defined as the composition

$$
g_{\varphi}:=\left(\Omega \mathcal{C}^{\prime} \circ M \circ \Omega \mathcal{C} \stackrel{g_{\alpha^{\prime}} \circ \varphi^{\circ} g_{\alpha}}{\longrightarrow} \mathcal{P}^{\prime} \circ N \circ \mathcal{P} \stackrel{\gamma}{\longrightarrow} N\right) .
$$

To check that $g_{\varphi}$ as defined here is compatible with the differentials, it is sufficient to verify that $\left[\mathrm{d}, g_{\varphi}\right]$ vanishes on generators $M$. Unravelling the definition of $\Omega M$, we find that the differential is given by

$$
\mathrm{d}^{\Omega M}=\mathrm{d}^{\Omega \mathcal{C}^{\prime}} \circ M \circ \Omega \mathcal{C}-D_{l^{\prime}}^{L} \circ \Omega \mathcal{C}+\Omega \mathcal{C}^{\prime} \circ \mathrm{o}^{M} \circ \Omega \mathcal{C}+\Omega \mathcal{C}^{\prime} \circ D_{l}^{R}+\Omega \mathcal{C}^{\prime} \circ M \circ \mathrm{d}^{\Omega \mathcal{C}}
$$

and its restriction to $M$ by

$$
\left.\mathrm{d}^{\Omega M}\right|_{M}=-\left.D_{l^{\prime}}^{L}\right|_{M}+\mathrm{d}^{M}+\left.D_{l}^{R}\right|_{M}
$$

Using the fact that $\left.g_{\varphi}\right|_{M}=\varphi$, we obtain

which evaluates to

$$
\begin{aligned}
{\left.\left[\mathrm{d}, g_{\varphi}\right]\right|_{M} } & =\left.\mathrm{d}^{N} g_{\varphi}\right|_{M}-\left.g_{\varphi} \mathrm{d}^{\Omega M}\right|_{M} \\
& =\mathrm{d}^{N} \varphi-\varphi \mathrm{d}^{M}+\left.g_{\varphi} D_{\iota^{\prime}}^{L}\right|_{M}-\left.g_{\varphi} D_{\iota}^{R}\right|_{M}
\end{aligned}
$$

$$
=\partial \varphi-\varphi * \alpha+\alpha^{\prime} \circledast \varphi,
$$


as can be seen from the following diagram:

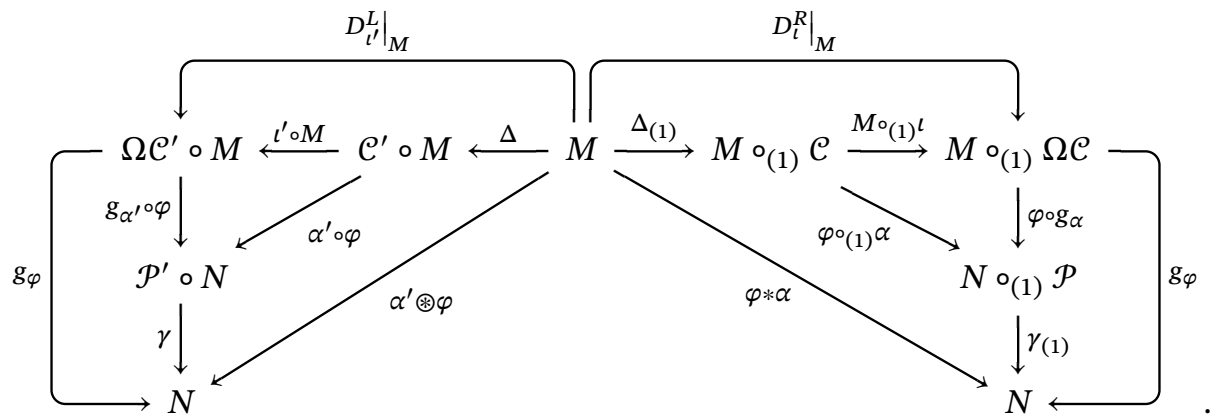

In fact, this proves that $g_{\varphi}$ as defined above is compatible with the differentials iff. $\varphi$ satisfies the Maurer-Cartan equation. Since for any morphism $g: \Omega M \rightarrow N$ in dg Mod $g_{g_{\mid}}=g$, this concludes the proof for the cobar construction. The proof for the bar construction is omitted since it is dual to the above.

Corollary 4.2.4. The cobar and bar constructions form a pair of adjoint functors

$$
\Omega: \mathrm{dg} \operatorname{Mod} \rightleftharpoons \mathrm{dg} \text { Comod }: \mathrm{B} \text {. }
$$

Under the bijections of Proposition 4.2.3 the identity morphisms on $\Omega M$ and $\mathrm{BN}$ correspond to twisting morphisms $t: M \rightarrow \Omega M$ and $\pi: \mathrm{B} N \rightarrow N$. We call these the canonical twisting morphisms for modules. Given any other twisting morphism $\varphi: M \rightarrow N$, we obtain factorizations as indicated in the following diagram:

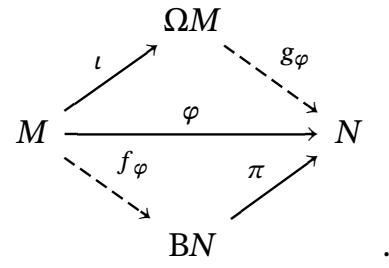

4.3. Koszul twisting morphisms. In this section, we introduce a subset of twisting morphisms $\alpha: \mathcal{C} \rightarrow \mathcal{P}$ which we call Koszul twisting morphisms. Koszul twisting morphisms are of particular significance since, as we will see, they are related to resolutions of operads and their algebras.

Definition 4.3.1. We call an element $\alpha \in \operatorname{Tw}(\mathcal{C}, \mathcal{P})$ a Koszul twisting morphism (or simply Koszul morphism), if the composition

$$
\mathcal{P} \circ_{\alpha} \mathcal{C} \circ \circ_{\alpha} \mathcal{P} \stackrel{\mathcal{P}_{\circ \varepsilon \circ \mathcal{P}}}{\longrightarrow} \mathcal{P} \circ I \circ \mathcal{P} \cong \mathcal{P} \circ \mathcal{P} \stackrel{\gamma}{\longrightarrow} \mathcal{P}
$$

is a quasi-isomorphism of $(\mathcal{P}, \mathcal{P})$-bimodules. The subset of Koszul twisting morphisms $\alpha: \mathcal{C} \rightarrow \mathcal{P}$ is denoted by $\operatorname{Kos}(\mathcal{C}, \mathcal{P}) \subset \operatorname{Tw}(\mathcal{C}, \mathcal{P})$.

The main result of this section provides us with a number of equivalent formulations of the Koszul property for twisting morphisms. 
Theorem 4.3.2 (Fundamental Theorem for Operadic Twisting Morphisms). Let $\mathcal{P}$ resp. $\mathcal{C}$ be a $\mathbf{k}$-projective connected wdg operad resp. cooperad and $\alpha: \mathcal{C} \rightarrow \mathcal{P}$ a twisting morphism. The following are equivalent:

(i) the twisting morphism $\alpha: \mathcal{C} \rightarrow \mathcal{P}$ is Koszul, i.e. $\mathcal{P} \circ_{\alpha} \mathcal{C} \circ_{\alpha} \mathcal{P} \stackrel{\sim}{\rightarrow}$;

(ii) the right twisted composite product $\mathcal{C} \circ_{\alpha} \mathcal{P}$ is acyclic, i.e. $\mathcal{C} \circ_{\alpha} \mathcal{P} \stackrel{\sim}{\rightarrow}$;

(iii) the left twisted composite product $\mathcal{P} \circ_{\alpha} \mathcal{C}$ is acyclic, i.e. $\mathcal{P} \circ_{\alpha} \mathcal{C} \stackrel{\rightarrow}{\rightarrow}$;

(iv) the morphism $g_{\alpha}: \Omega \mathcal{C} \rightarrow \mathcal{P}$ is a quasi-isomorphism; and

(v) the morphism $f_{\alpha}: \mathcal{C} \rightarrow \mathrm{BP}$ is a quasi-isomorphism.

Before we get to the proof of this result, we need to provide its main ingredient: the operadic comparison lemma. We state here its version for right twisted composite products since it is the most important for our purposes.

Lemma 4.3.3 (Operadic Comparison Lemma). Let $f: \mathcal{C} \rightarrow \mathcal{C}^{\prime}$ be a morphism of $\mathbf{k}$ projective connected wdg cooperads, $g: \mathcal{P} \rightarrow \mathcal{P}^{\prime}$ a morphism of $\mathbf{k}$-projective connected $w d g$ operads, and $\alpha: \mathcal{C} \rightarrow \mathcal{P}$ and $\alpha^{\prime}: \mathcal{C}^{\prime} \rightarrow \mathcal{P}^{\prime}$ twisting morphisms compatible with $f$ and $g$, i.e. satisfying $g \alpha=\alpha^{\prime} f$. If two out of the three morphisms $f$, g, and $f \circ g: \mathcal{C} \circ_{\alpha} \mathcal{P} \rightarrow \mathcal{C}^{\prime} \circ_{\alpha^{\prime}} \mathcal{P}^{\prime}$ are quasi-isomorphisms, then so is the third. The analogous statement holds for morphisms of left twisted composite products.

PROOF. The proof can be found in [16 §2.5]. Unfortunately, it is given only for the canonical weightgrading $\mathcal{C}^{(w)}=\mathcal{C}(w+1)$ resp. $\mathcal{P}^{(w)}=\mathcal{P}(w+1)$. A version of the proof allowing for general weightgradings, and hence a weaker connectedness assumption, is given in [46 \$6.7]. However, here a different simplification is used: the commutative ring $\mathbf{k}$ is assumed to be a field of characteristic 0 . The general case of the result stated here can be obtained by considering the filtration

$$
\mathcal{F}_{S}\left(\mathcal{C} \circ_{\alpha} \mathcal{P}\right)^{(w)}:=\bigoplus_{d+u \leq s}\left(\mathcal{C}_{d}^{(u)} \circ \mathcal{P}\right)^{(w)}
$$

as defined in [46] and then proceeding with the proof as given in [16].

The operadic comparison lemma together with the following result on the canonical twisting morphisms $\iota: \mathcal{C} \rightarrow \Omega \mathcal{C}$ and $\pi: \mathrm{B} \mathcal{P} \rightarrow \mathcal{P}$ is enough to prove the fundamental theorem for operadic twisting morphisms.

Theorem 4.3.4. Let $\mathcal{P}$ resp. $\mathcal{C}$ be a $\mathbf{k}$-projective connected $w d g$ operad resp. cooperad. The left twisted composite products $\mathcal{C} \circ_{l} \Omega \mathcal{C}, \mathrm{B} \mathcal{P}{ }_{\pi} \mathcal{P}$ and the right twisted composite products $\Omega \mathcal{C} \circ_{\iota} \mathcal{C}, \mathcal{P} \circ_{\pi} \mathrm{BP}$ are acyclic.

PROOF of Theorem 4.3.2. For the proof of the equivalence of (i) and (iii) we refer the reader to [53, Theorem 2.9]. Only a minor change is required: we have to replace the characteristic 0 assumption by our $\mathbf{k}$-projective requirement.

Consider now for some twisting morphism $\alpha: \mathcal{C} \rightarrow \mathcal{P}$ with associated $g_{\alpha}: \Omega \mathcal{C} \rightarrow \mathcal{P}$ the morphism $\mathcal{C} \circ g_{\alpha}: \mathcal{C} \circ \Omega \mathcal{C} \rightarrow \mathcal{C} \circ_{\alpha} \mathcal{P}$. Since $\mathcal{C} \circ_{\iota} \Omega \mathcal{C}$ is acyclic by Theorem 4.3.4 the same is true for $\mathcal{C} \circ_{\alpha} \mathcal{P}$ if and only if $\mathcal{C} \circ g_{\alpha}$ is a quasi-isomorphism. By the operadic 
comparison lemma, this is the case if and only if $g_{\alpha}$ is a quasi-isomorphism. This proves the equivalence of (ii) and (iv) The remaining equivalences follow along the same lines.

Applying Theorem 4.3.2 to the canonical Koszul twisting morphism $\pi: \mathrm{BP} \rightarrow \mathcal{P}$ leads us to the following result.

Corollary 4.3.5. The counit of the cobar-bar adjunction provides functorial quasi-free resolutions

$$
g_{\pi}: \Omega B \mathcal{P} \stackrel{\sim}{\longrightarrow}
$$

of operads.

4.4. Semiaugmented operads. So far we have dealt only with resolutions of augmented dg operads, despite the fact that the main operad $\mathcal{O}$ of interest to us in Chapter 3 does not fall in this category. We may, however, equip it with a semiaugmentation. An extension of the cobar-bar resolution to this setting was developed by Hirsh-Millès in [31]. In this section, we give a short overview. Another approach was recently introduced by Le Grignou [42].

Definition 4.4.1. A semiaugmented $d g$ operad or $\operatorname{sdg}$ operad $(\mathcal{P}, \gamma, \eta, \varepsilon)$ is a dg operad $(\mathcal{P}, \gamma, \eta)$ equipped with a morphism $\varepsilon: \mathcal{P} \rightarrow I$ of collections such that $\varepsilon \eta=I$. The morphism $\varepsilon$ is called the semiaugmentation for $\mathcal{P}$. A morphism $f: \mathcal{P} \rightarrow \mathcal{P}^{\prime}$ of semiaugmented $\mathrm{dg}$ operads is a morphism of $\mathrm{dg}$ operads preserving the semiaugmentation in the sense that $\varepsilon^{\prime} f=\varepsilon$. We denote the category of semiaugmented dg operads by sdg Opd.

Note the difference between the definition of a semiaugmented operad and an augmented operad: in the case of augmented operads, the map $\varepsilon$ is required to be a morphism of operads, while for semiaugmented operads, it is merely a morphism of collections.

As for augmented operads, we define $\overline{\mathcal{P}}:=\operatorname{ker}(\varepsilon)$ and obtain an isomorphism $\mathcal{P} \cong I \oplus \overline{\mathcal{P}}$. We denote by $\overline{\mathrm{d}}$ the composition

$$
\overline{\mathrm{d}}=(\overline{\mathcal{P}} \longleftrightarrow \mathcal{P} \stackrel{\mathrm{d}}{\longrightarrow} \mathcal{P} \longrightarrow \overline{\mathcal{P}})
$$

and by

$$
\bar{\gamma}=(\overline{\mathcal{P}} \circ \overline{\mathcal{P}} \longrightarrow \mathcal{P} \circ \mathcal{P} \stackrel{\gamma}{\longrightarrow} \mathcal{P} \longrightarrow \overline{\mathcal{P}}) .
$$

Note that in general $\bar{\gamma}$ is not associative and, as a result, if we apply the bar construction as defined in Section 4.2 to $\mathcal{P}$ naively, its derivation will not square to zero. To control this defect, we introduce a (co)curvature term as follows.

Definition 4.4.2. A curved cooperad $(\mathcal{C}, \mathrm{d}, \theta)$ is a graded cooperad $\mathcal{C}$ equipped with a coderivation $\mathrm{d}: \mathcal{C} \rightarrow \mathcal{C}$ of degree -1 and a morphism $\theta: \mathcal{C} \rightarrow I$ of degree -2 called the cocurvature. These maps are required to satisfy the conditions

(i) $\mathrm{d}^{2}=-\left(\theta_{{ }_{(1)}} \mathcal{C}-\mathcal{C} \circ_{(1)} \theta\right) \Delta_{(1)}$, and

(ii) $\theta d=0$. 
A morphism of curved cooperads $f: \mathcal{C} \rightarrow \mathcal{C}^{\prime}$ is simply a morphism of graded operads commuting with the coderivations and preserving the cocurvature in the sense that $\theta^{\prime} f=\theta$. We denote the category of curved cooperads by curved Coopd.

Given a curved cooperad $\mathcal{C}$ and an sdg operad $\mathcal{P}$, we define their convolution algebra as before,

$$
\operatorname{Conv}(\mathcal{C}, \mathcal{P}):=\lim _{\substack{(1 \leftarrow X) \\ \in 1-\operatorname{Tree}^{\mathrm{op}}}} \operatorname{hom}(\overline{\mathcal{C}}, \overline{\mathcal{P}})[1 \leftarrow X],
$$

and equip it with the same binary product

$$
\alpha \star \beta:=\left(\overline{\mathcal{C}} \stackrel{\bar{\Delta}_{(1)}}{\longrightarrow} \overline{\mathcal{C}}_{{ }_{(1)}} \overline{\mathcal{C}} \stackrel{\alpha_{(1)}^{\beta}}{\longrightarrow} \overline{\mathcal{P}}_{{ }_{(1)}} \overline{\mathcal{P}} \stackrel{\bar{\gamma}_{(1)}}{\longrightarrow} \overline{\mathcal{P}}\right) .
$$

In this setting, however, the convolution algebra is not a dg pre-Lie algebra. Instead, it satisfies

(i) $\partial^{2}=\left(\Theta \star{ }_{\lrcorner}\right)-(\star \star \star \Theta)$, and

(ii) $\partial \Theta=0$,

where $\Theta$ is the degree -2 element given by the composition

$$
\Theta:=(\mathcal{C} \stackrel{\theta}{\longrightarrow} I \stackrel{\eta}{\longrightarrow} \mathcal{P}) .
$$

Such a structure is known as a curved pre-Lie algebra and we call $(\operatorname{Conv}(\mathcal{C}, \mathcal{P}), \partial, \star, \Theta)$ the curved pre-Lie convolution algebra. The set of twisting morphisms in the curved convolution algebra is defined as

$$
\operatorname{Tw}(\mathcal{C}, \mathcal{P})=\left\{\alpha \in \operatorname{Conv}(\mathcal{C}, \mathcal{P})_{-1} \mid \partial \alpha+\alpha \star \alpha=\Theta\right\} .
$$

Let $\mathcal{C}$ be a conilpotent curved cooperad. Its cobar construction is the quasi-free semiaugmented dg operad

$$
\Omega \mathcal{C}:=\left(\mathcal{T}\left(s^{-1} \overline{\mathcal{C}}\right), \mathrm{d}=-\mathrm{d}_{0}+\mathrm{d}_{1}-\mathrm{d}_{2}, \varepsilon\right),
$$

where $d_{0}$ is the extension of

$$
s^{-1} \overline{\mathcal{C}} \stackrel{s}{\longrightarrow} \overline{\mathcal{C}} \stackrel{\theta}{\longrightarrow} I \longleftrightarrow \mathcal{T}\left(s^{-1} \overline{\mathcal{C}}\right),
$$

$\mathrm{d}_{1}$ is the extension of

$$
s^{-1} \overline{\mathcal{C}} \stackrel{s^{-1} \mathrm{~d}}{\longrightarrow} s^{-1} \overline{\mathcal{C}} \longleftrightarrow \mathcal{T}\left(s^{-1} \overline{\mathcal{C}}\right),
$$

and $\mathrm{d}_{2}$ extends

$$
s^{-1} \overline{\mathcal{C}} \stackrel{s}{\longrightarrow} \overline{\mathcal{C}} \stackrel{\bar{\Delta}_{(1)}}{\longrightarrow} \overline{\mathcal{C}}_{{ }_{(1)}} \overline{\mathcal{C}} \stackrel{s^{-1 o_{(1)} s^{-1}} \longrightarrow}{\longrightarrow} s^{-1} \overline{\mathcal{C}}_{{ }_{(1)}} s^{-1} \overline{\mathcal{C}} \longleftrightarrow \mathcal{T}\left(s^{-1} \overline{\mathcal{C}}\right)
$$

The semiaugmentation for $\Omega \mathcal{C}$ is given by the canonical projection $\varepsilon: \mathcal{T}\left(s^{-1} \overline{\mathcal{C}}\right) \rightarrow I$. The cobar construction defines a functor

$$
\Omega: \text { coaug curved Coopd } \longrightarrow \text { sdg Opd . }
$$


Let $\mathcal{P}$ be a semiaugmented dg operad. Its bar construction is the quasi-cofree conilpotent curved cooperad

$$
\mathrm{B} \mathcal{P}:=\left(\mathcal{T}^{c}(s \overline{\mathcal{P}}), \mathrm{d}=\mathrm{d}_{1}+\mathrm{d}_{2}, \theta\right),
$$

where $d_{1}$ is the coextension of

$$
\mathcal{T}^{c}(s \overline{\mathcal{P}}) \longrightarrow s \overline{\mathcal{P}} \stackrel{s \mathrm{~d}}{\longrightarrow} s \overline{\mathcal{P}}
$$

$\mathrm{d}_{2}$ coextends

$$
\mathcal{T}^{c}(s \overline{\mathcal{P}}) \longrightarrow s \overline{\mathcal{P}}{ }_{{ }_{(1)}} s \overline{\mathcal{P}} \stackrel{s^{-1}{ }_{(1)} s^{-1}}{\longrightarrow} \mathcal{P}{ }^{\circ}{ }_{(1)} \mathcal{P} \stackrel{\bar{\gamma}_{(1)}}{\longrightarrow} \overline{\mathcal{P}} \stackrel{s}{\longrightarrow} s \overline{\mathcal{P}}
$$

and the cocurvature is given by the composition

$\theta: \mathcal{T}^{c}(s \overline{\mathcal{P}}) \longrightarrow s \overline{\mathcal{P}} \oplus s \overline{\mathcal{P}}_{{ }_{(1)}} s \overline{\mathcal{P}} \stackrel{s^{-1} \oplus s^{-1}{ }_{(1)} s^{-1}}{\longrightarrow} \overline{\mathcal{P}} \oplus \overline{\mathcal{P}}_{{ }_{(1)}} \overline{\mathcal{P}} \stackrel{\mathrm{d}+\gamma_{(1)}}{\longrightarrow} \mathcal{P} \stackrel{\varepsilon}{\longrightarrow} I$

The bar construction defines a functor

$$
\text { B:sdg Opd } \longrightarrow \text { conil curved Coopd . }
$$

With the same construction as used to show Proposition 4.2.1 we obtain its analogous result for semiaugmented dg operads and conilpotent curved cooperads.

Proposition 4.4.3. The cobar and bar functors represent the twisting morphism bifunctor in the sense that

$$
\begin{aligned}
& \operatorname{Hom}_{\text {sdg Opd }}(\Omega \mathcal{C}, \mathcal{P}) \stackrel{\cong}{\longrightarrow} \mathrm{Tw}(\mathcal{C}, \mathcal{P}) \stackrel{\cong}{\longleftrightarrow} \operatorname{Hom}_{\text {conil curved Coopd }}(\mathcal{C}, \mathrm{B} \mathcal{P}) \\
& \Psi \quad \Psi \\
& g_{\alpha} \longleftrightarrow \alpha \longleftrightarrow f_{\alpha} \text {. }
\end{aligned}
$$

Corollary 4.4.4. The cobar and bar constructions form a pair of adjoint functors

$$
\Omega: \text { conil curved Coopd } \rightleftharpoons \text { sdg Opd : B . }
$$

We saw that the counit of the cobar-bar adjunction for dg operads provides functorial quasi-free resolutions of $\mathrm{dg}$ operads (Corollary 4.3.5). An analogous result was shown for semiaugmented dg operads [31, Theorem 3.4.4], however the contracting homotopy used in the proof requires $\mathbf{k}$ to be a field of characteristic 0 . Since our operads are by definition reduced (i.e. have no arity 0 operations), we may use the simpler contracting homotopy as provided in [67. Lemma 4.14] instead. This leads to the following result.

Theorem 4.4.5. Let $\mathcal{P}$ be a k-projective semiaugmented connected wdg operad. The counit of the cobar-bar adjunction provides functorial quasi-free resolutions

$$
g_{\pi}: \Omega B \mathcal{P} \stackrel{\sim}{\longrightarrow}
$$

of semiaugmented wdg operads. 


\section{Resolutions of Algebras}

Let $\mathcal{C}$ be a conilpotent dg cooperad and $\mathcal{P}$ an augmented dg operad. For a conilpotent $\operatorname{dg} \mathcal{C}$-coalgebra $C$ and a $\operatorname{dg} \mathcal{P}$-algebra $A$, we define the complex

$$
\operatorname{Conv}(C, A):=\lim _{0-T_{\text {ree }}^{\text {op }}} \operatorname{hom}(C, A)[1],
$$

which simplifies to $\operatorname{Conv}(C, A)=\operatorname{hom}(C, A)$ in the noncolored case. Given a twisting morphism $\alpha: \mathcal{C} \rightarrow \mathcal{P}$, we may equip $\operatorname{Conv}(C, A)$ with an operator $\star_{\alpha}$ defined by

$$
\star_{\alpha}(\beta):=(C \stackrel{\Delta}{\longrightarrow} \mathcal{C}(C) \stackrel{\alpha(\beta)}{\longrightarrow} \mathcal{P}(A) \stackrel{\gamma}{\longrightarrow} A) .
$$

We call a degree 0 element $\beta \in \operatorname{Conv}(C, A)_{0}$ a twisting morphism if it satisfies the Maurer-Cartan equation

$$
\partial \beta+\star_{\alpha}(\beta)=0
$$

and denote the set of such twisting morphisms by $\operatorname{Tw}_{\alpha}(C, A)$. As is the case for (co)operads, the twisting morphisms for (co)algebras form a bifunctor and we may define a pair of functors-the cobar and bar construction-representing it on either side.

REMARK 5.0.1. In recent work by Robert-Nicoud and Wierstra 61, 70, it was shown that-given a twisting morphism $\alpha: \mathcal{C} \rightarrow \mathcal{P}$ as above-one can construct an $\mathrm{L}_{\infty}$-algebra structure on $\operatorname{Conv}(C, A)$. Maurer-Cartan elements of this $\mathrm{L}_{\infty}$-algebra $\operatorname{Conv}^{\alpha}(C, A)$ correspond to twisting morphisms $\operatorname{Tw}_{\alpha}(C, A)$ as defined above. This provides an interesting new perspective on these twisting morphisms, at least over a field of characteristic 0 .

5.1. The cobar-bar adjunction. The cobar construction for a conilpotent $\mathrm{dg} \mathcal{C}$ coalgebra $C$ with respect to a twisting morphism $\alpha: \mathcal{C} \rightarrow \mathcal{P}$ is the quasi-free $\operatorname{dg} \mathcal{P}$-algebra $\Omega_{\alpha} C:=\left(\mathcal{P}(C), \mathrm{d}_{1}-\mathrm{d}_{2}\right)$, where

$$
\mathrm{d}_{1}:=\left(\mathcal{P}(C) \stackrel{\mathrm{d}(C)+\mathcal{P}(\mathrm{d})^{\prime}}{\longrightarrow} \mathcal{P}(C)\right)
$$

and

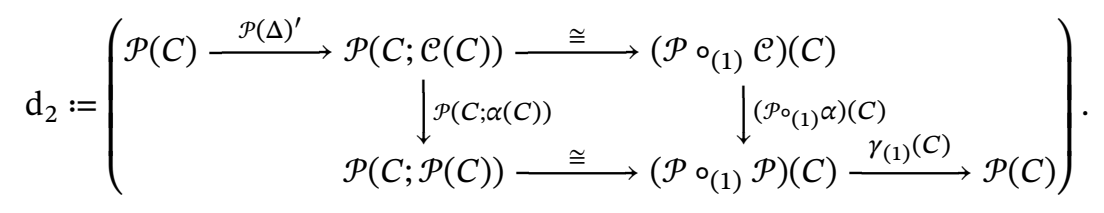

The cobar construction defines a functor

$$
\Omega_{\alpha} \text { : conil dg } \mathcal{C} \text {-Coalg } \longrightarrow \text { dg } \mathcal{P} \text {-Alg . }
$$

The bar construction for a $\operatorname{dg} \mathcal{P}$-algebra $A$ with respect to a twisting morphism $\alpha: \mathcal{C} \rightarrow \mathcal{P}$ is the quasi-cofree conilpotent dg $\mathcal{C}$-coalgebra $\mathrm{B}_{\alpha} C:=\left(\mathcal{C}(A), \mathrm{d}_{1}+\mathrm{d}_{2}\right)$, where

$$
\mathrm{d}_{1}:=\left(\mathcal{C}(A) \stackrel{\mathrm{d}(A)+\mathcal{C}(\mathrm{d})^{\prime}}{\longrightarrow} \mathcal{C}(A)\right)
$$


and

$$
\mathrm{d}_{2}:=\left(\begin{array}{cc}
\mathcal{C}(A) \stackrel{\Delta_{(1)}}{\longrightarrow}\left(\mathcal{C}_{{ }_{(1)}} \mathcal{C}\right)(A) \stackrel{\cong}{\longrightarrow} \mathcal{C}(A ; \mathcal{C}(A)) \\
\left.\mid \mathcal{C}_{(1)}^{\alpha}\right)(A) & \mid \mathcal{C}(A ; \alpha(A)) \\
\left(\mathcal{C}_{{ }^{\circ}(1)} \mathcal{P}\right)(A) \stackrel{\cong}{\longrightarrow} \mathcal{C}(A ; \mathcal{P}(A)) \stackrel{\mathcal{C}(A+\gamma)}{\longrightarrow} \mathcal{C}(A)
\end{array}\right)
$$

The bar construction defines a functor

$$
\mathrm{B}_{\alpha}: \mathrm{dg} \mathcal{P} \text {-Alg } \longrightarrow \text { conil dg } \mathcal{C} \text {-Coalg . }
$$

Proposition 5.1.1. The cobar and bar functors represent the twisting morphism bifunctor in the sense that

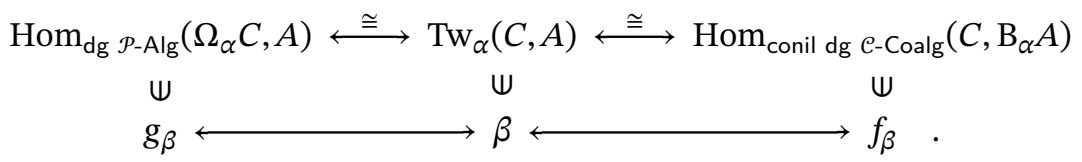

Corollary 5.1.2. The cobar and bar constructions form a pair of adjoint functors

$$
\Omega_{\alpha}: \text { conil dg } \mathcal{C} \text {-Coalg } \rightleftharpoons d g ~ \mathcal{P} \text {-Alg }: B_{\alpha} \text {. }
$$

Under the bijections of Proposition 5.1.1 the identity morphisms on $\Omega_{\alpha} C$ and $\mathrm{B}_{\alpha} A$ correspond to canonical twisting morphisms $t: C \rightarrow \Omega_{\alpha} C$ and $\pi: \mathrm{B}_{\alpha} A \rightarrow A$, and we obtain factorizations for any $\beta \in \mathrm{Tw}_{\alpha}(C, A)$ as indicated in the following diagram:

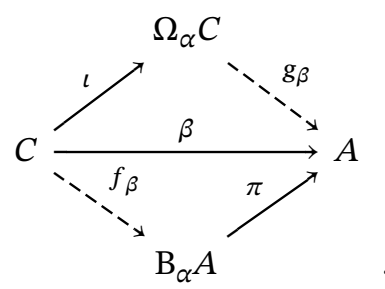

Proposition 5.1.3. Let $\mathcal{C}$ be a $\mathbf{k}$-projective connected wdg cooperad, $\mathcal{P}$ a $\mathbf{k}$-projective connected wdg operad, and $\alpha: \mathcal{C} \rightarrow \mathcal{P}$ a weightgraded Koszul twisting morphism. For any $\mathbf{k}$-projective dg $\mathcal{P}$-algebra $A$, the counit of the cobar-bar adjunction

$$
\Omega_{\alpha} \mathrm{B}_{\alpha} A \stackrel{\sim}{\longrightarrow} A
$$

is a quasi-isomorphism.

5.2. Algebras over sdg operads. We present an extension of the results to the case of algebras over sdg operads developed by Hirsh-Millès in [31]. Let $(\mathcal{P}, \mathrm{d}, \varepsilon)$ be an sdg operad, $(\mathcal{C}, \mathrm{d}, \theta)$ a conilpotent curved cooperad, and $\alpha: \mathcal{C} \rightarrow \mathcal{P}$ a curved twisting morphism. A $d g \mathcal{P}$-algebra over an sdg operad $\mathcal{P}$ is simply an algebra over $\mathcal{P}$ as a dg operad. On the coalgebra side the definition is changed to take the cocurvature into account.

Definition 5.2.1. A conilpotent curved $\mathcal{C}$ - coalgebra over a conilpotent curved cooperad $(\mathcal{C}, \mathrm{d}, \theta)$ is a conilpotent graded algebra $(C, \Delta)$ over the underlying graded cooperad, 
with a coderivation d: $C \rightarrow C$ satisfying

$$
\mathrm{d}^{2}=(C \stackrel{\Delta}{\longrightarrow} \mathcal{C}(C) \stackrel{\theta(C)}{\longrightarrow} I(C) \cong C) .
$$

For a conilpotent curved $\mathcal{C}$-coalgebra $C$ and a $\operatorname{dg} \mathcal{P}$-algebra $A$, we define their convolution algebra $\left(\operatorname{Conv}(C, A), \star_{\alpha}\right)$ as before by Equations (67) and (68) We define twisting morphisms with respect to $\alpha$ again by

$$
\operatorname{Tw}_{\alpha}(C, A):=\left\{\beta \in \operatorname{Conv}(C, A)_{0} \mid \partial \beta+\star_{\alpha}(\beta)=0\right\} .
$$

The cobar construction $\Omega_{\alpha} C$ for a conilpotent curved $\mathcal{C}$-coalgebra $C$ is defined exactly as in Section 5.1, and, in particular, $\left(\mathrm{d}_{1}-\mathrm{d}_{2}\right)^{2}=0$ still holds. The cobar construction defines a functor

$$
\Omega_{\alpha} \text { : conil curved } \mathcal{C} \text {-Coalg } \longrightarrow \text { dg } \mathcal{P} \text {-Alg . }
$$

The bar construction $\mathrm{B}_{\alpha} A$ for a dg $\mathcal{P}$-algebra $A$ is also defined as in Section 5.1 however, the coderivation in this case does not square to zero. A simple calculation shows that

$$
\left(\mathrm{d}_{1}+\mathrm{d}_{2}\right)^{2}=(\theta(C)) \Delta,
$$

and thus $\mathrm{B}_{\alpha} A$ is a curved $\mathcal{C}$-coalgebra. In fact, the bar construction defines a functor

$$
\mathrm{B}_{\alpha}: \mathrm{dg} \mathcal{P} \text {-Alg } \longrightarrow \text { conil curved } \mathcal{C} \text {-Coalg . }
$$

The obvious analogues of Proposition 5.1.1 and Corollary 5.1.2 hold, and we obtain the same factorizations of twisting morphisms through the canonical twisting morphisms $\iota: C \rightarrow \Omega_{\alpha} C$ and $\pi: \mathrm{B}_{\alpha} A \rightarrow A$. Finally, Proposition 5.1.3 extends to the setting presented here.

Proposition 5.2.2. Let $\mathcal{C}$ be a $\mathbf{k}$-projective connected weightgraded curved cooperad, $\mathcal{P}$ a k-projective connected weightgraded semiaugmented dg operad, and $\alpha: \mathcal{C} \rightarrow \mathcal{P}$ a weightgraded curved Koszul twisting morphism. For any $\mathbf{k}$-projective $d g \mathcal{P}$-algebra $A$, the counit of the cobar-bar adjunction

$$
\Omega_{\alpha} \mathrm{B}_{\alpha} A \stackrel{\sim}{\longrightarrow} A
$$

is a quasi-isomorphism.

\section{Homotopy Algebras}

Let $\mathcal{P}$ be an augmented dg operad with a quasi-free resolution given by the cobar construction on some conilpotent $\mathrm{dg}$ cooperad $\mathcal{C}$ and a morphism

$$
g_{\alpha}: \Omega \mathcal{C} \stackrel{\sim}{\longrightarrow}
$$

corresponding to a twisting morphism $\alpha: \mathcal{C} \rightarrow \mathcal{P}$. This could be the cobar-bar resolution $\Omega \mathrm{B} \mathcal{P} \sim \mathcal{P}$ as introduced in the previous section, or any other choice of resolution. In this section, we consider the algebras over such resolutions, their homotopy properties, as well as their modules and some constructions. For further detail on the material in 
this section, we refer the reader to [46, §10.1-3]. While the statements in loc. cit. are made only for $\mathcal{C}=\mathcal{P}^{\mathrm{i}}$, they hold in the more general case presented here.

Recall that an $\Omega \mathcal{C}$-algebra structure on a space $V$ can be given as a morphism $g_{\alpha}: \Omega \mathcal{C} \rightarrow \operatorname{End}_{V}$ or, equivalently, as a twisting morphism $\alpha: \mathcal{C} \rightarrow \operatorname{End}_{V}$. An element $\alpha \in \operatorname{Conv}\left(\mathcal{C}, \operatorname{End}_{V}\right)_{-1}$ corresponds to a degree -1 morphism $p_{\alpha}: \mathcal{C}(V) \rightarrow V$ and this morphism $p_{\alpha}$ admits a unique coextension as a coderivation $\delta_{\alpha}$ of the cofree conilpotent graded $\mathcal{C}$-coalgebra $\mathcal{C}(V)$ by Lemma 3.6.3 A simple calculation shows that the Maurer-Cartan equation for $\alpha$ is equivalent to the condition $\left(\delta_{\alpha}\right)^{2}=0$. This proves the following result.

Theorem 6.0.1 (Rosetta Stone, [46 Theorem 10.1.13]). The set of $\Omega \mathcal{C}$-algebra structures on a space $V$ is equivalently given by

$$
\begin{aligned}
\operatorname{Hom}_{\mathrm{dg} \text { aug Opd }}\left(\Omega \mathcal{C}, \operatorname{End}_{V}\right) \cong \operatorname{Tw}\left(\mathcal{C}, \operatorname{End}_{V}\right) & \cong \operatorname{Hom}_{\mathrm{dg} \text { conil } \operatorname{Coopd}}\left(\mathcal{C}, \mathrm{B} \mathrm{End}_{V}\right) \\
& \cong \operatorname{Codiff}(\mathcal{C}(V)) .
\end{aligned}
$$

Now consider two dg $\Omega \mathcal{C}$-algebras $V, V^{\prime}$. By Lemma 2.2.2 a morphism $f: V \rightarrow V^{\prime}$ is equivalently determined by a morphism

$$
\Omega \mathcal{C} \longrightarrow \operatorname{End}_{V^{\prime}}^{V}
$$

of $\mathrm{dg}(\Omega \mathcal{C}, \Omega \mathcal{C})$-modules. We call this type of morphism a strict morphism of $\Omega \mathcal{C}$ algebras. By contrast, we introduce the following notion using the cobar construction for dg modules introduced in Section 4.2 .

Definition 6.0.2. A homotopy morphism $V \rightsquigarrow V^{\prime}$ is a morphism

$$
g_{\varphi}: \Omega_{\mathcal{C}}^{\mathcal{C}} \longrightarrow \operatorname{End}_{V^{\prime}}^{V}
$$

of $\operatorname{dg}(\Omega \mathcal{C}, \Omega \mathcal{C})$-modules.

By Proposition 4.2.3, a homotopy morphism of $\Omega \mathcal{C}$-algebras corresponds to a twisting morphism $\varphi: \mathcal{C} \rightarrow \operatorname{End}_{V^{\prime}}^{V}$. An element $\varphi \in \operatorname{Conv}\left(\mathcal{C}, \operatorname{End}_{V^{\prime}}^{V}\right)_{0}$ corresponds to a degree 0 morphism $\mathcal{C}(V) \rightarrow V^{\prime}$, and such a map admits a unique coextension to a morphism $F_{\varphi}: \mathcal{C}(V) \rightarrow \mathcal{C}\left(V^{\prime}\right)$ of cofree conilpotent graded $\mathcal{C}$-coalgebras. A simple calculation shows that $F_{\varphi}$ is compatible with the differentials $\delta_{\alpha}, \delta_{\alpha^{\prime}}$, i.e. is a morphism of quasi-cofree conilpotent $\mathrm{dg} \mathcal{C}$-coalgebras, if and only if $\varphi$ satisfies the Maurer-Cartan equation

$$
\partial \varphi-\varphi * \alpha+\alpha^{\prime} \circledast \varphi=0 .
$$

Theorem 6.0.3 (Rosetta Stone for Morphisms). The set of homotopy $\Omega \mathcal{C}$-algebra morphisms from $V$ to $V^{\prime}$ is equivalently given by

$$
\begin{aligned}
& \operatorname{Hom}_{\mathrm{dg}(\Omega \mathcal{C}, \Omega \mathcal{C})-\operatorname{Mod}}\left(\Omega_{\mathcal{C}}^{\mathcal{C}} \mathcal{C}, \operatorname{End}_{V^{\prime}}^{V}\right) \cong \operatorname{Tw}_{\alpha^{\prime}}^{\alpha}\left(\mathcal{C}, \operatorname{End}_{V^{\prime}}^{V}\right) \\
& \cong \operatorname{Hom}_{\mathrm{dg}}\left(\operatorname{End}_{V^{\prime}}, \operatorname{End}_{V}\right)-\operatorname{Comod}\left(\mathcal{C}, \mathrm{B}_{\mathrm{End}_{V^{\prime}}}^{\operatorname{End}_{V}} \operatorname{End}_{V^{\prime}}^{V}\right) \\
& \cong \operatorname{Hom}_{\mathrm{dg} \mathcal{C} \text {-Coalg }}\left(\left(\mathcal{C}(V), \delta_{\alpha}\right),\left(\mathcal{C}\left(V^{\prime}\right), \delta_{\alpha^{\prime}}\right)\right) \text {. }
\end{aligned}
$$


Given homotopy morphisms $V \rightsquigarrow V^{\prime}$ and $V^{\prime} \rightsquigarrow V^{\prime \prime}$ in terms of twisting morphisms

$$
\varphi: \mathcal{C} \longrightarrow \operatorname{End}_{V^{\prime}}^{V} \quad \text { and } \varphi^{\prime}: \mathcal{C} \longrightarrow \operatorname{End}_{V^{\prime \prime}}^{V^{\prime}}
$$

we define their composition to be the morphism $V \rightsquigarrow V^{\prime \prime}$ represented by the twisting morphism

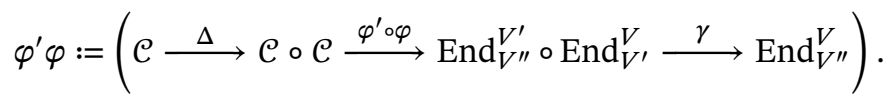

This composition corresponds to composition for morphisms of quasi-cofree $\mathrm{dg} \mathcal{C}$ coalgebras: $F_{\varphi^{\prime}} F_{\varphi}=F_{\varphi^{\prime} \varphi}$.

Definition 6.0.4. Let $\mathcal{P}$ be a dg operad with a quasi-free resolution $\Omega \mathcal{C} \stackrel{\sim}{\rightarrow} \mathcal{P}$. A homotopy $\mathcal{P}$-algebra is a dg $\Omega \mathcal{C}$-algebra, and we denote by hty $\mathcal{P}$-Alg the category of homotopy $\mathcal{P}$-algebras with homotopy morphisms.

6.1. Homotopy transfer. Let $W$ be a deformation retract of $V$, i.e. assume we are given the following data:

$$
h \subset V \underset{i}{\stackrel{p}{\rightleftarrows}} W, \quad \text { such that }\left\{\begin{array}{l}
V-i p=[\mathrm{d}, h], \\
W-p i=0 .
\end{array}\right.
$$

It is a result of Van der Laan [39 that, using these data, one can construct a nontrivial morphism

$$
\Psi: \mathrm{B} \mathrm{End}_{V} \longrightarrow \mathrm{B} \mathrm{End}_{W}
$$

of $\mathrm{dg}$ cooperads. As one of the ways to define an $\Omega \mathcal{C}$-algebra structure on a space $V$ is via a morphism $f_{\alpha}: \mathcal{C} \rightarrow \mathrm{B} \mathrm{End}_{V}$ of dg cooperads, any such structure determines a transferred $\Omega \mathcal{C}$-algebra structure on $W$ by composition with $\Psi$ :

$$
\Psi f_{\alpha}: \mathcal{C} \longrightarrow \mathrm{BEnd}_{V} \longrightarrow \mathrm{BEnd}_{W} .
$$

More precisely, this provides a way to prove the following result.

Theorem 6.1.1 (Homotopy Transfer Theorem). Let $\mathcal{C}$ be a conilpotent dg cooperad and $W$ a deformation retract of a k-projective dg space $V$. Any $\Omega \mathcal{C}$-algebra structure on $V$ can be transferred to an $\Omega \mathcal{C}$-algebra structure on $W$ such that $i$ extends to a homotopy quasi-isomorphism.

Proof. We specify the morphism $\Psi$ by defining its corresponding twisting morphism $\psi: \mathrm{BEnd}_{V} \longrightarrow$ End $_{W}$ via
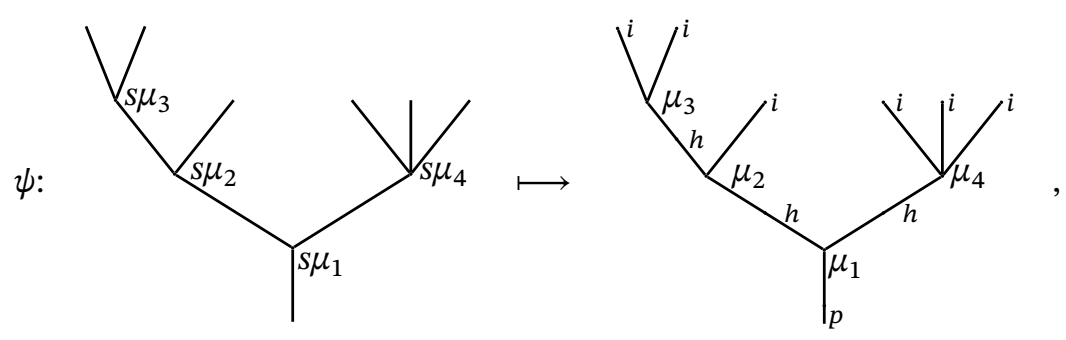
where the tree on the right-hand side is to be understood as composition of (multi-)linear maps. More formally, we define a map of collections $\widetilde{\psi}: \operatorname{BEnd}_{V} \rightarrow \operatorname{End}_{V}^{W}$ inductively by

$$
\begin{aligned}
& \widetilde{\psi}_{(0)}: I \longrightarrow \operatorname{End}_{V}^{W}, \quad u \longmapsto(i: W \rightarrow V), \\
& \widetilde{\psi}_{(n)}:\left\{\begin{array}{l}
I \oplus s \operatorname{End}_{V} \circ \mathcal{T}_{(n-1)}\left(s \operatorname{End}_{V}\right) \longrightarrow \operatorname{End}_{V}^{W}, \\
s \mu \circ\left(t_{1}, \ldots, t_{m}\right) \longmapsto h \mu\left(\widetilde{\psi}_{(n-1)}\left(t_{1}\right), \ldots, \widetilde{\psi}_{(n-1)}\left(t_{1}\right)\right),
\end{array}\right.
\end{aligned}
$$

and—using it—define $\psi:$ B End $_{V} \rightarrow \operatorname{End}_{W}$ via

$$
\psi_{(n)}:\left\{\begin{array}{l}
I \oplus s \operatorname{End}_{V} \circ \mathcal{T}_{(n-1)}\left(s \operatorname{End}_{V}\right) \longrightarrow \operatorname{End}_{W}, \\
s \mu \circ\left(t_{1}, \ldots, t_{m}\right) \longmapsto p \mu\left(\widetilde{\psi}_{(n-1)}\left(t_{1}\right), \ldots, \widetilde{\psi}_{(n-1)}\left(t_{1}\right)\right) .
\end{array}\right.
$$

A straightforward though tedious computation shows that (i) $\partial \psi+\psi \star \psi=0$, and (ii) $\partial \widetilde{\psi}-\widetilde{\psi} * \psi+\varphi \circledast \widetilde{\psi}=0$. In other words, $\psi f_{\alpha}$ defines an $\Omega \mathcal{C}$-algebra structure on $W$ and $\widetilde{\psi} f_{\alpha}$ a homotopy morphism $W \rightsquigarrow V$ extending $i$.

6.2. Homotopy modules. Let $\mathcal{P}$ be a dg operad, $\Omega \mathcal{C} \sim \mathcal{P}$ a quasi-free resolution, and let $V$ be a homotopy $\mathcal{P}$-algebra with structure given by $g_{\alpha}: \Omega \mathcal{C} \rightarrow$ End $_{V}$. A homotopy module or representation up to homotopy for $V$ is a dg space $N$ with a morphism of $\mathrm{dg}$ collections

$$
g_{\rho}: \Omega \mathcal{C} \longrightarrow \operatorname{End}_{N}^{V ; N}
$$

such that

$$
g_{\alpha}+g_{\rho}: \Omega \mathcal{C} \longrightarrow \operatorname{End}_{V} \oplus \operatorname{End}_{N}^{V ; N} \longrightarrow \operatorname{End}_{N \oplus V}
$$

defines a homotopy $\mathcal{P}$-algebra structure on $N \oplus V$. Note that this is simply the definition of a module over an operad algebra as given in Section 2.3

In terms of twisting morphisms, the map $g_{\rho}$ corresponds to

$$
\rho: \mathcal{C} \longrightarrow \operatorname{End}_{N}^{V: N}
$$

and defines a homotopy module structure on $N$ if and only if $\alpha+\rho: \mathcal{C} \rightarrow \operatorname{End}_{N \oplus V}$ satisfies the Maurer-Cartan equation:

$$
\begin{aligned}
0 & =\partial(\alpha+\rho)+(\alpha+\rho) \star(\alpha+\rho) \\
& =(\partial \alpha+\alpha \star \alpha)+(\partial \rho+\rho \star(\alpha+\rho)) .
\end{aligned}
$$

Since $\alpha$ is assumed to be a twisting morphism, this gives the following result.

Lemma 6.2.1. Let $\left(V, g_{\alpha}\right)$ be a dg $\Omega \mathcal{C}$-algebra. A morphism $\rho: \mathcal{C} \rightarrow \operatorname{End}_{N}^{V ; N}$ defines a homotopy $V$-module structure on $N$ iff. $\rho$ satisfies the Maurer-Cartan equation

$$
\partial \rho+\rho \star(\alpha+\rho)=0 .
$$

The map $\rho: \mathcal{C} \rightarrow \operatorname{End}_{N}^{V ; N}$ corresponds to $p_{\rho}: \mathcal{C}(V ; N) \rightarrow N$, and this morphism admits a unique coextension as $\mathcal{C}(V)$-comodule coderivation $\delta_{\rho} \in \operatorname{Coder}(\mathcal{C}(V ; N))$. A simple calculation shows that $\left(\delta_{\rho}\right)^{2}=0$ iff. $\rho$ satisfies the Maurer-Cartan Equation (71) 
Lemma 6.2.2. Let $\left(V, g_{\alpha}\right)$ be a dg $\Omega \mathcal{C}$-algebra. A morphism $\rho: \mathcal{C} \rightarrow \operatorname{End}_{N}^{V ; N}$ defines a homotopy $V$-module structure on $N$ iff. $\left(\mathcal{C}(V ; N), \delta_{\rho}\right)$ is a dg $\left(\mathcal{C}(V), \delta_{\alpha}\right)$-comodule.

6.3. Cohomology. Let $\mathcal{P}$ be a dg operad with quasi-free resolution $\Omega \mathcal{C} \sim \mathcal{P}$, let $(V, \alpha)$ be a homotopy $\mathcal{P}$-algebra, and let $(N, \rho)$ be a homotopy $V$-module. Consider the convolution algebra $\operatorname{Conv}\left(\mathcal{C}, \operatorname{End}_{N \oplus V}\right)$ and its subcomplex of maps

$$
\zeta: \mathcal{C} \longrightarrow \operatorname{End}_{N}^{V} \subset \operatorname{End}_{N \oplus V},
$$

which we denote by $C C(V ; N)$.

Lemma 6.3.1. The map $\delta$ defined on $C C(V ; N)$ by

$$
\delta(\zeta):=\partial \zeta+\rho \star \zeta-(-1)^{\zeta} \zeta \star \alpha
$$

satisfies $\delta^{2}=0$.

Proof. From the fact that $\operatorname{Conv}\left(\mathcal{C}, \operatorname{End}_{N \oplus V}\right)$ is a pre-Lie algebra, one can easily deduce the following identities:

(i) $\rho \star(\rho \star \zeta)=(\rho \star \rho) \star \zeta$

(ii) $(\rho \star \zeta) \star \alpha-\rho \star(\zeta \star \alpha)=(-1)^{\zeta}(\rho \star \alpha) \star \zeta$, and

(iii) $(\zeta \star \alpha) \star \alpha=\zeta \star(\alpha \star \alpha)$.

For any $\zeta \in C C(V ; N)$ we can calculate

$$
\begin{aligned}
\delta(\delta \zeta)= & \partial\left(\delta \zeta+\rho \star \zeta-(-1)^{\zeta \zeta} \alpha \alpha\right) \\
& +\rho \star\left(\partial \zeta+\rho \star \zeta-(-1)^{\zeta} \zeta \star \alpha\right) \\
& -(-1)^{\zeta}\left(\partial \zeta+\rho \star \zeta-(-1)^{\zeta \zeta} \star \alpha\right) \star \alpha \\
= & \partial^{2} \zeta+\partial \rho \star \zeta-\rho \star \partial \zeta-(-1)^{\zeta} \partial \zeta \star \alpha-\zeta \star \partial \alpha \\
& +\rho \star \partial \zeta+\rho \star(\rho \star \zeta)-(-1)^{\zeta} \rho \star(\zeta \star \alpha) \\
& -(-1)^{\zeta-1} \partial \zeta \star \alpha-(-1)^{\zeta-1}(\rho \star \zeta) \star \alpha-(\zeta \star \alpha) \star \alpha
\end{aligned}
$$

and using the above identities this reduces to

$$
=(\partial \rho+\rho \star(\rho+\alpha)) \star \zeta-\zeta \star(\partial \alpha+\alpha \star \alpha)=0 .
$$

Definition 6.3.2. We call $C C^{\cdot}(V ; N):=\left(C C(V ; N)_{-.}, \delta\right)$ the cochain complex for the homotopy $\mathcal{P}$-algebra $(V, \alpha)$ with coefficients in its homotopy module $(N, \rho)$.

Lemma 6.3.3. Let $\zeta$ be a degree 1 cocycle, i.e. $\zeta \in C C(V ; N)_{-1}$ such that $\delta(\zeta)=0$. Then

$$
\alpha+\rho+\zeta \in \operatorname{Conv}\left(\mathcal{C}, \operatorname{End}_{N \oplus V}\right)_{-1}
$$

satisfies the Maurer-Cartan equation and thus defines a homotopy $\mathcal{P}$-algebra structure on $N \oplus V$.

PROOF. This is shown by the following simple calculation:

$$
\begin{aligned}
& \partial(\alpha+\rho+\zeta)+(\alpha+\rho+\zeta) \star(\alpha+\rho+\zeta) \\
& \quad=\partial(\alpha+\rho+\zeta)+\alpha \star(\alpha+\rho+\zeta)+\rho \star(\alpha+\rho+\zeta)+\zeta \star(\alpha+\rho+\zeta)
\end{aligned}
$$


of which $\alpha \star \rho, \alpha \star \zeta, \zeta \star \rho$, and $\zeta \star \zeta$ vanish since their input and output are mismatched, leaving

$$
=(\partial \alpha+\alpha \star \alpha)+(\partial \rho+\rho \star(\alpha+\rho))+(\partial \zeta+\rho \star \zeta+\zeta \star \alpha)
$$

which vanishes by the Maurer-Cartan equations for $\alpha$ and $\rho$ and since $\delta(\zeta)=0$.

A cochain $\zeta: \mathcal{C} \rightarrow \operatorname{End}_{N}^{V}$ corresponds to a map $\mathcal{C}(V) \rightarrow N$, which coextends to a morphism $F_{\zeta}: \mathcal{C}(V) \rightarrow \mathcal{C}(V ; N)$ of graded $\mathcal{C}(V)$-comodules. Under this correspondence, $\delta(\zeta)$ maps to $\partial F_{\zeta}=\delta_{\rho} F_{\zeta}-(-1)^{\zeta} F_{\zeta} \delta_{\alpha}$ and we obtain the following result.

Lemma 6.3.4. There is an isomorphism of cochain complexes

$$
C C^{\cdot}(V ; N) \cong \operatorname{hom}_{\mathrm{dg}} \mathcal{C}(V)-C_{\text {Comod }}(\mathcal{C}(V), \mathcal{C}(V ; N))_{-} .
$$

\section{Koszul Duality}

In Section 4 we saw that the counit of the cobar-bar adjunction provides us with functorial quasi-free resolutions $\Omega B \mathcal{P} \sim \mathcal{\rightarrow} \mathcal{P}$ for $\mathbf{k}$-projective connected wdg operads. In this section, we consider operads given by presentation and show that this additional information can sometimes be used to produce much smaller resolutions. Given an operad $\mathcal{P}$ by presentation, we associate to it a cooperad $\mathcal{P}$. Under certain conditions, this provides us with a resolution $\Omega \mathcal{P} i \stackrel{\sim}{\rightarrow} \mathcal{P}$ of operads, allowing us to apply the machinery developed in the Section 6. In this case, we often use the notation $\mathcal{P}_{\infty}:=\Omega \mathcal{P} i$ and call homotopy morphisms of $\mathcal{P}_{\infty}$-algebras $\infty$-morphisms. We denote by $\mathcal{P}_{\infty}$-Alg the category of $\mathcal{P}_{\infty}$-algebras with strict morphisms, and by $\infty$ - $\mathcal{P}_{\infty}$-Alg the category of $\mathcal{P}_{\infty}$-algebras with $\infty$-morphisms.

7.1. Quadratic presentations. Let $\mathcal{P}=\mathcal{P}(E, R)$ be a dg operad with presentation $(E, R)$ satisfying $R \subset \mathcal{T} E^{(2)}$. Such a presentation is known as a quadratic presentation or quadratic data and $\mathcal{P}(E, R)$ is called a quadratic operad. In Section 2.7 we defined $\mathcal{P}(E, R)$ to be the quotient of the free operad on $E$ by the ideal generated by $R$, i.e. $\mathcal{P}(E, R)=\mathcal{T} E /(R)$. Here we formulate the definition slightly differently: $\mathcal{P}(E, R)$ is the universal dg operad for which the composition $R \hookrightarrow \mathcal{T} E \rightarrow \mathcal{P}(E, R)$ vanishes. Given another dg operad $Q$ with a morphism $\mathcal{T} E \rightarrow Q$ for which the composition $R \hookrightarrow \mathcal{T} E \rightarrow \mathcal{Q}$ vanishes, there is a unique morphism $\mathcal{P}(E, R) \rightarrow \mathcal{Q}$ such that the following diagram commutes:

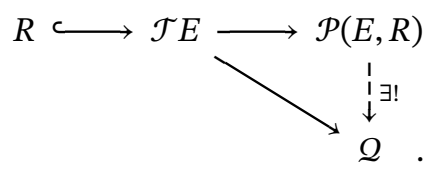

This definition is easily dualizable, which allows us to associate a dg cooperad $\mathcal{C}(E, R)$ to quadratic data $(E, R)$. The quadratic cooperad $\mathcal{C}(E, R)$ is the couniversal $\mathrm{dg}$ cooperad for which the composition $\mathcal{C}(E, R) \rightarrow \mathcal{T}^{c} E \rightarrow \mathcal{T}^{c} E^{(2)} / R$ vanishes. Given another $\mathrm{dg}$ cooperad $\mathcal{D}$ with a morphism $\mathcal{D} \rightarrow \mathcal{T}^{c} E$ for which the composition $\mathcal{D} \rightarrow \mathcal{T}^{c} E \rightarrow$ $\mathcal{T}^{c} E^{(2)} / R$ vanishes, there is a unique morphism $\mathcal{D} \rightarrow \mathcal{C}(E, R)$ such that the following 
diagram commutes:

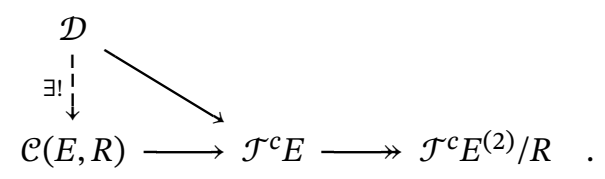

Since the subcollection $R$ of relations is weightgraded, both the dg operad $\mathcal{P}(E, R)$ and the dg cooperad $\mathcal{C}(E, R)$ defined from quadratic data are naturally weightgraded.

Definition 7.1.1. A finitely generated projective quadratic presentation is a quadratic presentation $(E, R)$ for which $E$ is a (componentwise) finitely generated projective collection, such that the short exact sequence

$$
0 \longrightarrow R \hookrightarrow \mathcal{T}^{(2)}(E) \longrightarrow \mathcal{T}^{(2)}(E) / R \longrightarrow 0
$$

splits.

Using the notation $S:=\mathcal{T}^{(2)}(E) / R$, the splitting condition means that $\mathcal{T}^{(2)}(E) \cong$ $R \oplus S$. Since $E$ and therefore $\mathcal{T}^{(2)}(E)$ is finitely generated projective, the same is true for the direct summands $R$ and $S$. In weight 3, we have a decomposition for the tree module

$$
\mathcal{T}^{(3)}(E)=\searrow_{E}^{E} \oplus /_{E}^{E}
$$

Its summands admit decompositions

$$
\begin{aligned}
& \searrow_{E}^{E}=R \oplus S J^{E}=I_{R} V^{E} \oplus\left(\backslash S V^{E} \cap V^{E} \oplus^{\prime} S\right)
\end{aligned}
$$

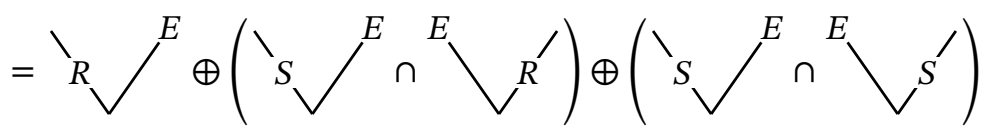

$$
\begin{aligned}
& =\left(\backslash R J^{E}+V^{R}\right) \oplus\left(S^{\prime} V^{E} \cap V^{\prime}\right)
\end{aligned}
$$

and, similarly,

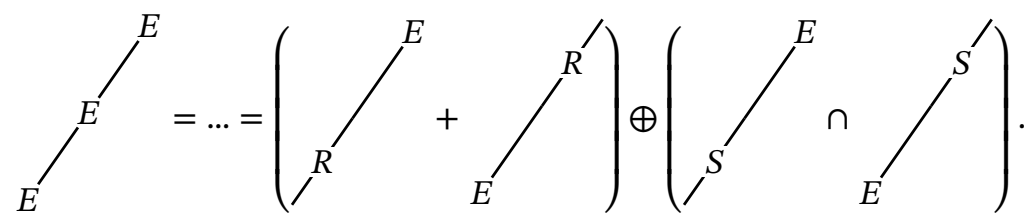

Note that

$$
\mathcal{P}(E, R)^{(3)}=\mathcal{T}^{(3)}(E) /\left(R \circ_{(1)} E+E \circ_{(1)} R\right)
$$



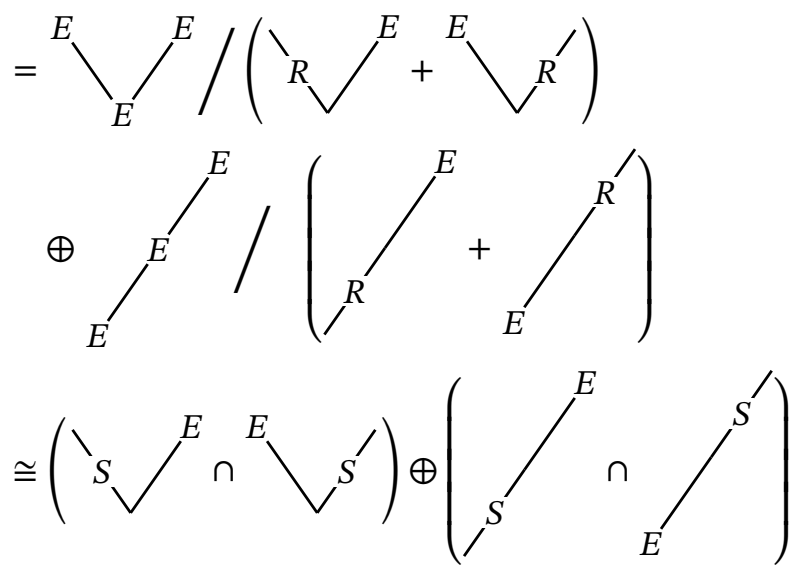

is a direct summand in $\mathcal{T}^{(3)}(E)$ and hence is finitely generated projective. Similar decompositions can be constructed in higher weights, leading to the following result.

Lemma 7.1.2. Let $(E, R)$ be a finitely generated projective quadratic presentation. The operad $\mathcal{P}(E, R)$ is finitely generated $\mathbf{k}$-projective.

With the notation $S=\mathcal{T}^{(2)}(E) / R$, we have $\mathcal{C}(E, S) \cong \mathcal{P}(E, R)$ as collections and, as a consequence, we obtain the following analogous result for cooperads.

Lemma 7.1.3. Let $(E, R)$ be a finitely generated projective quadratic presentation. The cooperad $\mathcal{C}(E, R)$ is finitely generated $\mathbf{k}$-projective.

7.2. Quadratic Koszul duality. We follow the presentation in [25 46]. The theory there is presented for operads in (graded) vector spaces, however the arguments hold more generally for finitely generated $\mathbf{k}$-projective modules over a commutative ring.

Let $(E, R)$ denote quadratic data defining a quadratic operad $\mathcal{P}=\mathcal{P}(E, R)$. Consider the suspension $s E$ of the collection of generators and denote by $s^{2} R$ the image of $R$ under

$$
\mathcal{T}^{(2)}(E)=E{ }_{(1)} E \stackrel{{ }^{{ }^{\circ}\left({ }_{1}\right)^{s}} \longrightarrow}{\longrightarrow} s E{ }^{\circ}{ }_{(1)} s E=\mathcal{T}^{(2)}(s E) .
$$

Definition 7.2.1. Let $\mathcal{P}=\mathcal{P}(E, R)$ be a quadratic operad. The quadratic cooperad $\mathcal{P} i:=\mathcal{C}\left(s E, s^{2} R\right)$ is called the Koszul dual cooperad for $\mathcal{P}(E, R)$.

The Koszul dual cooperad $\mathcal{P}^{i}$ is the key to the construction of a small quasi-free resolution of its corresponding quadratic operad $\mathcal{P}=\mathcal{P}(E, R)$. One may consider the composition

$$
\kappa=\left(\mathcal{P}^{\mathrm{i}}=\mathcal{C}\left(s E, s^{2} R\right) \longleftrightarrow \mathcal{T}^{c}(s E) \longrightarrow s E \stackrel{s^{-1}}{\longrightarrow} E \longleftrightarrow \mathcal{P}(E, R)=\mathcal{P}\right),
$$

which is readily seen to be a twisting morphism.

Definition 7.2.2. A quadratic operad $\mathcal{P}(E, R)$ is called Koszul if the canonical twisting morphism $\kappa: \mathcal{P} i \rightarrow \mathcal{P}$ is Koszul. 
At this point, all of the general theory introduced in Sections $4-6$ may be applied to the twisting morphism $\kappa$ of a $\operatorname{Koszul}$ operad $\mathcal{P}(E, R)$.

To make any of this useful, however, we first need to be able to actually compute the Koszul dual cooperad. One way to compute $\mathcal{P} i$ explicitly is via its (shifted) linear dual.

Definition 7.2.3. Let $\mathcal{P}=\mathcal{P}(E, R)$ be a quadratic operad. The Koszul dual operad for $\mathcal{P}$ is the operad $\mathcal{P}^{!}:=\left(\mathcal{S}^{\mathcal{C}} \otimes \mathcal{P}^{\mathrm{i}}\right)^{*}$.

By reflexivity of finitely generated projective modules, the following result is an immediate consequence of this definition.

Lemma 7.2.4. Let $\mathcal{P}=\mathcal{P}(E, R)$ be a finitely generated $\mathbf{k}$-projective quadratic operad. The Koszul dual cooperad and operad are related by the equation $\mathcal{P}^{i} \cong\left(\mathcal{S} \otimes \mathcal{P}^{!}\right)^{*}$.

To make Lemma 7.2.4 useful in computing the Koszul dual cooperad $\mathcal{P}$, we must provide an independent characterizaton for the Koszul dual operad $\mathcal{P}$ ! To this end, we consider a cooperad $\mathcal{C}=\mathcal{C}(E, R)$ with finitely generated $\mathbf{k}$-projective binary quadratic presentation $(E, R)$, i.e. $E=E(2)$ is concentrated in arity 2 and hence $\mathcal{T}^{(2)}(E)=\mathcal{T} E(3)$. Its linear dual is the operad $\mathcal{C}(E, R)^{*}=\mathcal{P}\left(E^{*}, R^{\perp}\right)$, and its presentation $\left(E^{*}, R^{\perp}\right)$ is again finitely generated k-projective binary quadratic. The generating collection $E^{*}$ is the componentwise linear dual of $E$, and the subcollection $R^{\perp} \subset \mathcal{T} E^{*}(3)$ is constructed below. We define a pairing $\left\langle_{\left.{ }_{⿻},{ }_{-}\right\rangle}\right\rangle: \mathcal{T} E^{*}(3) \otimes \mathcal{T} E(3) \rightarrow \mathbf{k}$ by setting

$$
\left\langle\mu^{*} \circ_{\sigma} \nu^{*}, \alpha \circ_{\tau} \beta\right\rangle= \begin{cases}(-1)^{\left|\nu^{*}\right||\alpha|} \cdot \mu^{*}(\alpha) \nu^{*}(\beta), & \text { if } \sigma=\tau, \\ 0, & \text { otherwise } .\end{cases}
$$

This pairing provides us with an isomorphism

$$
\mathcal{T} E^{*}(3) \longrightarrow \mathcal{T} E(3)^{*}, \quad \mu^{*} o_{\sigma} \nu^{*} \longmapsto\left\langle\mu^{*} \circ_{\sigma} \nu^{*},{ }_{\lrcorner}\right\rangle,
$$

and the preimage of $S^{*}=(\mathcal{T} E(3) / R)^{*}$ under this isomorphism is denoted by $R^{\perp}$. Equivalently, we may define $R^{\perp}$ as the subcollection

$$
R^{\perp}:=\left\{x \in \mathcal{T} E^{*}(3) \mid\langle x, R\rangle=0\right\}
$$

annihilating $R$ under the pairing.

Now let us return our attention to the Koszul dual operad $\mathcal{P}$ ! of an operad $\mathcal{P}=$ $\mathcal{P}(E, R)$ defined by a finitely generated $\mathbf{k}$-projective binary quadratic presentation. In Definition 7.2.3 $\mathcal{P}^{!}$is defined as the linear dual of $\mathcal{S}^{c} \otimes \mathcal{P}$, and this cooperad admits a binary quadratic presentation $\mathcal{S}^{c} \otimes \mathcal{P} i=\mathcal{C}(\widetilde{E}, \widetilde{R})$ constructed as follows. Consider

$$
\left(\mathcal{S}^{c} \otimes \mathcal{P} \mathbf{i}\right)(2)=\operatorname{End}_{s \mathbf{k}}(2) \otimes \mathcal{P}^{\mathrm{i}}(2)=\mathbf{k} \cdot \nu_{2} \otimes s E,
$$

and note the isomorphism of $\mathbb{S}_{2}$-modules

$$
\left(\mathcal{S}^{c} \otimes \mathcal{P}^{\mathrm{i}}\right)(2) \longrightarrow E \otimes \operatorname{sgn}_{2}, \quad \nu_{2} \otimes s \mu \longmapsto \mu .
$$


Accordingly, we define $\widetilde{E}=E \otimes \operatorname{sgn}_{2}$. In arity 3, we obtain

$$
\left(\mathcal{S}^{c} \otimes \mathcal{P} \mathrm{i}\right)(3)=\operatorname{End}_{s \mathbf{k}}(3) \otimes \mathcal{P} \mathrm{i}(3)=\mathbf{k} \cdot v_{3} \otimes s^{2} R \cong R \otimes \mathrm{sgn}_{3},
$$

where the isomorphism is the inverse of the one defined in Equation (73) and we define $\widetilde{R}$ to be the image of $R \otimes \operatorname{sgn}_{3}$ under the isomorphism of $\mathbb{S}_{3}$-modules

$$
\mathcal{T} E(3) \otimes \operatorname{sgn}_{3} \longrightarrow \mathcal{T}\left(E \otimes \operatorname{sgn}_{2}\right)(3), \quad\left\{\begin{array}{l}
\mu \circ_{1} \nu \longmapsto \mu \circ_{1} \nu, \\
\mu \circ_{2} \nu \longmapsto-\mu o_{2} \nu, \\
\left(\mu \circ_{2} \nu\right)^{(12)} \longmapsto\left(\mu \circ_{2} \nu\right)^{(12)} .
\end{array}\right.
$$

Note that simply requiring $\mu \circ_{1} \nu \mapsto \mu \circ_{1} \nu$ fully determines this isomorphism.

This concludes the construction underlying the following result; for a detailed proof we refer the reader to [25, 46]. Be aware that our conventions used here differ slightly from those in loc. cit. In particular, the pairing of Equation (74) contains no signs-instead they appear naturally under the isomorphism of Equation (76)

Proposition 7.2.5. Let $\mathcal{P}=\mathcal{P}(E, R)$ be a binary quadratic operad with finitely generated k-projective presentation. The Koszul dual operad is the binary quadratic operad

$$
\mathcal{P}^{!}=\mathcal{P}\left(\widetilde{E}^{*}, \widetilde{R}^{\perp}\right),
$$

where $\widetilde{E}^{*} \cong E^{*} \otimes \mathbf{k} \cdot \operatorname{sgn}_{2}$ and $\widetilde{R}^{\perp} \subset \mathcal{T}\left(\widetilde{E}^{*}\right)(3)$ denotes the orthogonal space of relations as constructed above.

A more general result for (nonbinary) quadratic operads is given in [46]. Its proof follows roughly along the same lines but is a bit more involved.

Proposition 7.2.6. The Koszul dual operad for a finitely generated $\mathbf{k}$-projective quadratic operad $\mathcal{P}=\mathcal{P}(E, R)$ admits a quadratic presentation

$$
\mathcal{P}^{!}=\mathcal{P}\left(s^{-1} \mathcal{S}^{-1} \otimes E^{*}, R^{\perp}\right) .
$$

And finally, the following property justifies the name Koszul duality. Using the fact that finitely generate projective modules are reflexive, it is an immediate consequence of Proposition 7.2.6 (resp. Proposition 7.2.5).

Corollary 7.2.7. Let $\mathcal{P}=\mathcal{P}(E, R)$ be a binary quadratic operad with finitely generated k-projective presentation. The Koszul dual operad satisfies the relation

$$
\left(\mathcal{P}^{!}\right)^{!} \cong \mathcal{P} \text {. }
$$

7.3. Example: Koszul duality for the operad of commutative algebras. The operad Com of commutative algebras can be defined as the binary quadratic operad Com $=\mathcal{P}(E, R)$ where the generating $\mathbb{S}_{2}$-module is the free $\mathbf{k}$-module on a single generator with trivial $\mathbb{S}_{2}$-action,

$$
E=E(2)=\mathbf{k} \cdot \mu, \quad \mu^{\sigma}=\mu,
$$


and $R \subset \mathcal{T} E(3)$ is the $\mathbb{S}_{3}$-module generated by

$$
r=\mu \circ_{1} \mu-\mu \circ_{2} \mu
$$

Note that

$$
r^{(12)}=\mu \circ_{1} \mu-\left(\mu \circ_{2} \mu\right)^{(12)}
$$

and

$$
\begin{aligned}
r^{(123)} & =\left(\mu \circ_{1} \mu\right)^{(123)}-\left(\mu \circ_{2} \mu\right)^{(123)} \\
& =\left(\mu \circ_{2} \mu\right)^{(12)}-\left(\mu \circ_{1} \mu\right)=-r^{(12)},
\end{aligned}
$$

hence $R$ is k-linearly spanned by $r$ and $r^{(12)}$. Letting $S \subset \mathcal{T} E(3)$ be the sub k-module generated by

$$
r^{\prime}=-\mu \circ_{1} \mu+\mu \circ_{2} \mu+\left(\mu \circ_{2} \mu\right)^{(12)},
$$

it is easy to see that $\mathcal{T} E(3)=R \oplus S$ and the presentation $(E, R)$ is finitely generated projective binary quadratic.

By Definition 7.2.1 the Koszul dual cooperad for Com is $\operatorname{Com}^{i}=\mathcal{C}\left(s E, s^{2} R\right)$, with

$$
s E=s \mathbf{k} \cdot \mu, \quad s \mu^{\sigma}=s \mu,
$$

and $s^{2} R$ the image of $R$ under the isomorphism of Equation (73). Since $\mu$ is of degree $|\mu|=0$, the signs remain the same and $s^{2} R$ is simply $\mathbf{k}$-linearly spanned by

$$
s^{2} r=s \mu \circ_{1} s \mu-s \mu \circ_{2} s \mu, \quad s^{2} r^{(12)}=s \mu \circ_{1} s \mu-\left(s \mu \circ_{2} s \mu\right)^{(12)} .
$$

To give a more explicit description of the Koszul dual cooperad Com', we take the detour via the Koszul dual operad Com!. By Proposition 7.2.5, it has a presentation $\mathrm{Com}^{!}=\mathcal{P}\left(\widetilde{E}^{*}, \widetilde{R}^{\perp}\right)$ constructed as follows. First, define $\widetilde{E}=E \otimes \operatorname{sgn}_{2}=\mathbf{k} \cdot \mu \otimes \mathrm{sgn}_{2}$ and denote by $\widetilde{R}$ the image of $R$ under the isomorphism of Equation (76) Constructed in this way, $\widetilde{R}$ is $\mathbf{k}$-linearly spanned by

$$
\tilde{r}=\mu \circ_{1} \mu+\mu \circ_{2} \mu, \quad \tilde{r}^{(12)}=-\mu \circ_{1} \mu+\left(\mu \circ_{2} \mu\right)^{(12)} .
$$

This provides a presentation $(\widetilde{E}, \widetilde{R})$ for $\mathcal{S}^{c} \otimes$ Com $^{i}$. Next, we consider the linear dual $\operatorname{Com}^{!}=\mathcal{C}(\widetilde{E}, \widetilde{R})^{*}=\mathcal{P}\left(\widetilde{E}^{*}, \widetilde{R}^{\perp}\right)$, where

$$
\widetilde{E}^{*}=E^{*} \otimes \operatorname{sgn}_{2}, \quad \widetilde{R}^{\perp}=\left\{x \in \mathcal{T}\left(\widetilde{E}^{*}\right)(3) \mid\langle x, \widetilde{R}\rangle=0\right\} .
$$

A simple computation verifies that the orthogonal space of relations $\widetilde{R}^{\perp}$ is $\mathbf{k}$-linearly spanned by

$$
\tilde{r}^{\perp}:=\mu^{*} \circ_{1} \mu^{*}-\mu^{*} \circ_{2} \mu^{*}+\left(\mu^{*} \circ_{2} \mu^{*}\right)^{(12)} .
$$

Using the notation $\lambda:=\mu^{*}$, we may characterize the Koszul dual operad Com! as follows: it is the operad defined by a single skewsymmetric binary generator $\lambda$ of degree 0 satisfying the relation

$$
\lambda \circ_{1} \lambda=\lambda \circ_{2} \lambda-\left(\lambda \circ_{2} \lambda\right)^{(12)} .
$$

This leads to the following result. 
Lemma 7.3.1. The Koszul dual operad of the commutative operad is the Lie operad and vice versa,

$$
\mathrm{Com}^{!} \cong \mathrm{Lie}, \quad \text { and } \quad \mathrm{Lie}^{!} \cong \mathrm{Com}
$$

7.4. Curved Koszul duality. Quadratic Koszul duality as described in Section 7.2 is the basic case underlying a number of extensions, one of which is curved Koszul duality. Curved Koszul duality was first introduced by Positselski for algebras [58], see also [57]. The extension to operads was developed by Hirsh-Millès in [31]. In this section, we give a very brief overview.

Let $(E, R)$ be a quadratic-linear-constant (or qlc) presentation, i.e. let $E$ be a graded collection of generators and let $R$ be a subcollection $R \subset \mathcal{T}^{(\leq 2)}(E)=I \oplus E \oplus \mathcal{T}^{(2)}(E)$ of (inhomogeneous) relations of weight at most 2 . We denote by $\mathcal{P}=\mathcal{P}(E, R)=\mathcal{T} E /(R)$ the associated quadratic-linear-constant (or qlc) operad.

Given a qlc operad $\mathcal{P}(E, R)$, assume that the following conditions are satisfied:

(i) $R \cap\{I \oplus E\}=0$,

(ii) $(R) \cap\left\{I \oplus E \oplus \mathcal{T}^{(2)}(E)\right\}=R$.

These conditions are known as minimality of generators (Condition (i) and maximality of relations (Condition (ii)).

Denote by $q: \mathcal{T} E \rightarrow \mathcal{T}^{(2)}(E)$ the projection onto weight 2 , and by $q R \subset \mathcal{T}^{(2)}(E)$ the image of the relations $R$ under this projection. We call the operad $q \mathcal{P}:=\mathcal{P}(E, q R)$ the quadratic analogue for $\mathcal{P}=\mathcal{P}(E, R)$ and denote by

$$
q \mathcal{P} i:=\mathcal{C}\left(s E, s^{2} q R\right) \subset \mathcal{T}^{c}(s E)
$$

its Koszul dual cooperad, which we also refer to as the quadratic analogue for $\mathcal{P} i$.

By Condition (i) there are maps $\varphi_{1}: q R \rightarrow E$ and $\varphi_{0}: q R \rightarrow I$ such that the subcollection of relations can be written in the following way:

$$
R=\left\{x+\varphi_{1}(x)+\varphi_{0}(x) \mid x \in q R\right\} .
$$

Using these maps, we may define a coderivation $\mathrm{d}_{\mathcal{P}_{i}}$ as the coextension of

$$
q \mathcal{P} i \longrightarrow s^{2} q R \stackrel{s^{-1} \otimes \varphi_{1}}{\longrightarrow} s E,
$$

and a map

$$
\theta=\left(q \mathcal{P}^{\mathrm{i}} \longrightarrow s^{2} q R \stackrel{s^{-2} \otimes \varphi_{0}}{\longrightarrow} I\right) .
$$

A straightforward computation shows that the triple $\left(q \mathcal{P}{ }^{i}, \mathrm{~d}_{\mathcal{P}_{i}}, \theta\right)$ defines a curved cooperad.

Definition 7.4.1. The Koszul dual curved cooperad for a qlc operad $\mathcal{P}=\mathcal{P}(E, R)$ satisfying Conditions $(i)$ and $(i i)$ is the curved cooperad $\mathcal{P}^{\mathrm{i}}:=\left(q \mathcal{P}^{\mathrm{i}}, \mathrm{d}_{\mathcal{P}_{\mathrm{i}}}, \theta\right)$.

With this definition, we may define the canonical twisting morphism $\kappa: \mathcal{P} i \rightarrow \mathcal{P}$ in the same way as in the quadratic case:

$$
\kappa=\left(\mathcal{P}^{\mathrm{i}}=\mathcal{C}\left(s E, s^{2} q R\right) \longleftrightarrow \mathcal{T}^{c}(s E) \longrightarrow s E \stackrel{s^{-1}}{\longrightarrow} E \hookrightarrow \mathcal{P}(E, R)=\mathcal{P}\right) .
$$


A simple verification shows that $\kappa$ is indeed a curved twisting morphism in the sense of the definition given in Section 4.4

Definition 7.4.2. A quadratic-linear-constant operad $\mathcal{P}=\mathcal{P}(E, R)$ is Koszul if its quadratic analogue $q \mathcal{P}=\mathcal{P}(E, q R)$ is Koszul and Conditions (i) and (ii) hold.

An issue with this definition is that Condition (ii) is hard to check in general. It can be shown, however, that it is enough to check it in low weights only:

Proposition 7.4.3. A qlc operad $\mathcal{P}=\mathcal{P}(E, R)$ is Koszul if and only if its quadratic analogue $q \mathcal{P}=\mathcal{P}(E, q R)$ is Koszul and

(i) $R \cap\{I \oplus E\}=0$,

(ii) $\left\{R \circ_{(1)} E+E \circ_{(1)} R\right\} \cap\left\{E \oplus \mathcal{T}^{(2)} E\right\} \subset R$.

As in the quadratic case, the cobar construction provides functorial quasi-free resolutions for Koszul operads.

Theorem 7.4.4. Let $\mathcal{P}=\mathcal{P}(E, R)$ be a qlc operad. If $\mathcal{P}$ is Koszul, the (curved) cobar construction on $\mathcal{P}$ i provides us with a quasi-free resolution

$$
g_{\kappa}: \Omega \mathcal{P} i \stackrel{\sim}{\longrightarrow} \text {. }
$$





\section{Symmetric Homotopy Theory for Operads}

This chapter consists essentially of our joint work with Bruno Vallette [12]. Minor changes have been made to the presentation, and references to other parts of this thesis were added.

\section{Introduction}

There are two philosophically different ways of viewing the notion of an operad. The first and classical way is to start from an $\mathbb{S}$-module and consider composition maps acting on it. In this case, the symmetries of the operations are given as part of the underlying $\mathbb{S}$ module and only their compositions are modeled. The second way of viewing an operad is as an $\mathbb{N}$-module, i.e., as a module graded by the arity number, with composition maps and symmetric group actions as the defining structure. This second way is the point of view taken in this chapter. We work over the ground category of differential graded $\mathbb{N}$-modules, which has better homological properties than the category of differential graded $\mathbb{S}$-modules. For instance, the projectivity property is easier to satisfy there. We will then develop a theory similar to that of Ginzburg-Kapranov and Getzler-Jones but in this second context.

To produce such a theory, we do not simply work by hand but instead we use the following conceptual approach: we consider the colored operad $\mathcal{O}$ whose category of algebras over $\mathbb{N}$-modules is the category of nonunital (or, equivalently, augmented) operads. It turns out that this colored operad admits a natural inhomogenous presentation. Therefore, it fits into the framework of curved Koszul duality theory for (colored) operads recently developed by Hirsh-Millès [31]. The first result of this chapter says that $\mathcal{O}$ is actually a Koszul colored operad.

Theorem 1.6.5. The colored operad $\mathcal{O}$ is an inhomogeneous curved Koszul operad.

This theorem is the key point in the following theory: the Koszul property ensures that all of the subsequent constructions are well behaved with respect to homological properties. We describe the general constructions associated to Koszul colored operads in the case of the operad $\mathcal{O}$, see Chapter 2 (in particular its Section 5) and [46. Chapter 11] for more details. This will produce the following notions. First, coalgebras over the Koszul dual colored cooperad $\mathcal{O}^{i}$ give rise to a new category of higher cooperads. Then, there is a new adjunction, called the higher cobar-bar adjunction,

$$
\widetilde{\Omega}: \text { conil higher Coopd } \rightleftarrows \text { nu Opd : } \widetilde{\mathrm{B}} \text {, }
$$


between (nonunital) operads and (conilpotent) higher cooperads. As expected, the counit of this adjunction induces a cofibrant resolution functor.

Theorem 2.5.3. The augmentation of the higher cobar-bar counit

$$
I \oplus \widetilde{\Omega} \widetilde{\mathrm{B}} \overline{\mathcal{P}} \stackrel{\sim}{\longrightarrow} \mathcal{P}
$$

is a functorial resolution of dg augmented operads, which is cofibrant when the underlying chain complex of $\mathcal{P}$ is nonnegatively graded and consists of projective $\mathbf{k}$-modules.

Let us compare this higher cobar-bar construction with the classical cobar-bar adjunction. First, the intermediate category of the higher cobar-bar resolution is made up of higher cooperads, which is more involved than the category of cooperads. The conceptual reason is quite natural: the higher bar construction $\widetilde{\mathrm{B}} \mathcal{P}$ needs to resolve the symmetric group actions of the operad $\mathcal{P}$ up to homotopy in a functorial way. As a result, while the classical cobar-bar construction was cofibrant in characteristic 0 or when the underlying $\mathbb{S}$-module of $\mathcal{P}$ is projective, the higher cobar-bar construction is always cofibrant over any ring providing that the underlying $\mathbb{N}$-module is projective, which is a much weaker assumption.

Let us recall the existence of a particularly well behaved $\mathrm{E}_{\infty}$-operad whose components are defined by the bar resolutions of the symmetric groups: the Barratt-Eccles operad $\mathcal{E}$, see [4]. This operad allows us to relate the cobar-bar construction and the higher cobar-bar construction in the following precise way: there exists a natural isomorphism

$$
I \oplus \widetilde{\Omega} \widetilde{\mathrm{P}} \mathcal{P} \cong \Omega \mathrm{B}(\mathcal{P} \otimes \mathcal{E}),
$$

where $\mathcal{P} \otimes \mathcal{E}$ stands for the aritywise tensor product (see Section 2.4 of Chapter 2 ) and where $I$ is the identity operad (Proposition 2.5.4). It was already known that the latter construction gives a cofibrant replacement functor [6]. On the bright side, one can interpret this result as a new universal property satisfied by the Barratt-Eccles operad.

Applied to the operad encoding commutative algebras, the higher cobar-bar resolution automatically produces a cofibrant $\mathrm{E}_{\infty}$-operad over any ring, which is also a Hopf operad resolution. Under the above isomorphism, this result is not that new-it gives an operad isomorphic to the cobar-bar construction of the Barratt-Eccles operad. On a basic level, having a Hopf operad resolution provides the associated category of algebras with a monoidal structure given by the underlying tensor product. On a more advanced level, the existence of a cofibrant Hopf $\mathrm{E}_{\infty}$-operad plays a crucial role in Fresse's works on the iterated bar construction of $E_{\infty}$-algebras $[19,20]$ and on the rational homotopy theory of operads with application to the Grothendieck-Teichmüller group [22]. However, the actual description of the associated category of algebras does not seem to be present in the literature, for instance in loc. cit. Since we believe that it could be useful to have an explicit description of an $\mathrm{E}_{\infty}$-algebra by means of generating operations and relations, e.g., in order to perform computations of homology groups in algebraic topology along the lines of [20], we include it in this chapter. The advantage 
of our model is that it relies on simple combinatorics of planar trees and permutations. This provides us with a "simple" explicit notion of an $\mathrm{E}_{\infty}$-algebra (Proposition 2.6.3).

The Koszul property of Theorem 1.6.5 gives a cofibrant resolution of the colored operad $\mathcal{O}$. As a result, algebras over this resolution provide us with a new notion of higher homotopy operads, for which the relations for both, the partial composition products and the symmetric group actions, are relaxed coherently up to homotopy. This very general notion includes many of the existing homotopy algebraic notions as special cases: $\mathrm{A}_{\infty}$-algebras, $\mathrm{A}_{\infty}$-modules, and homotopy nonsymmetric operads, to name some examples. It comes naturally equipped with a more general notion of $\infty$-morphism, which is proven to be an efficient tool to describe the homotopy properties of differential graded operads. For instance, the following homotopy transfer theorem shows how to transfer operadic structures through homotopy equivalences of $\mathrm{dg} \mathbb{N}$-modules (which need not be homotopy equivalences of $\mathrm{dg} \mathbb{S}$-modules).

Theorem 3.3.4 (Homotopy Transfer Theorem). Let $\left\{\mathcal{H}(n), \mathrm{d}_{\mathcal{H}(n)}\right\}_{n \in \mathbb{N}}$ be a homotopy retract of $\left\{\mathcal{P}(n), \mathrm{d}_{\mathcal{P}(n)}\right\}_{n \in \mathbb{N}}$ in the category of $d g \mathbb{N}$-modules:

$$
{ }_{h} \bigcirc\left(\mathcal{P}(n), \mathrm{d}_{\mathcal{P}}\right) \underset{i}{\stackrel{p}{\rightleftarrows}}\left(\mathcal{H}(n), \mathrm{d}_{\mathcal{H}}\right), \quad \text { such that }\left\{\begin{array}{l}
\operatorname{id}_{\mathcal{P}}-i p=\left[\mathrm{d}_{\mathcal{P}}, h\right], \\
\operatorname{id}_{\mathcal{H}}-p i=0,
\end{array}\right.
$$

and $i$ is a quasi-isomorphism. Any (higher homotopy) operad structure on $\mathcal{P}$ can be transferred into a higher homotopy operad structure on $\mathcal{H}$ such that the quasi-isomorphism $i$ extends to an $\infty$-quasi-isomorphism.

The methods developed and used in this chapter to solve the above-mentioned operadic issues also allow us to define a suitable notion of group representation up to homotopy. This is treated separately in an appendix.

A few remarks are in order to conclude this introduction. We tried to provide the reader with as many figures of trees as possible to render the exposition more readable. Particular care has been applied to the treatment of the signs; unlike in some articles in this domain (including some written by the second author), all the signs are made explicit. We hope that, in this way, the reader will be able to perform actual computations with the various notions introduced here. Furthermore, one can apply the same methods used here to the categories of cyclic operads, modular operads and unital operads. In this last case, this would help develop the homotopy properties of the partial compositions, symmetric group actions and the unit at the same time. The colored operad which encodes unital operads still admits a natural inhomogeneous presentation, though it is more involved than that of $\mathcal{O}$. It is very likely to be curved Koszul too. The present chapter should represent about two-thirds of the relevant computations to treat this case. Since we were only interested in applying our theory to augmented operads (or, equivalently, to nonunital operads) we focused on this particular case.

We chose to develop the theory presented in this article because we had the example of the operad encoding Lie algebras in mind. Our ultimate goal is to be able to 
produce a suitable version of weak $\mathrm{L}_{\infty}$-algebras where both the Jacobi identity and the skewsymmetry of the Lie bracket are relaxed up to higher coherent homotopies. There already exists a notion of weak Lie 2-algebras due to Roytenberg [56, 63], which is made up of just a first stratum of homotopies. If not the most general relaxed notion, it already admits applications in symplectic geometry and in mathematical physics. Producing a definition including an infinite sequence of coherent higher homotopies by hands is out of reach (at least for us), but the present conceptual theory is the right tool to solve this issue. First computations already show that we can recover Roytenberg's notion of weak Lie 2-algebras and produce, as a next step, a definition of weak Lie 3-algebras (see Chapter 4).

Finally, the present chapter, with these new higher bar and cobar constructions, is the first step toward a new higher Koszul duality theory for operads taking the symmetric group actions faithfully into account. The general pattern for the Koszul duality theory of algebras over a colored operad as detailed by Millès in [53] can be applied to algebras over the operad $\mathcal{O}$, that is to operads. In this way, one should be able to simplify the cofibrant resolutions produced by the higher cobar-bar resolution of Theorem 2.5.3 The case of the operad encoding commutative algebras is of particular interest since it should give an operad related to the cofibrant $\mathrm{E}_{\infty}$-operad given in [21] and to the chain complex of the Fox-Neuwirth cells of the Fulton-MacPherson operad of [24, 50].

This chapter is organised as follows. In Section 1 we introduce the colored operad which encodes nonunital operads, and we prove that its natural inhomogeneous quadratic presentation is curved Koszul. In Section 2 we introduce a new category of higher cooperads and we define a higher cobar-bar adjunction with the category of nonunital differential graded operads. The resulting higher cobar-bar adjunction is shown to give rise to a cofibrant replacement functor, which, applied to the operad encoding commutative algebras, gives a cofibrant $\mathrm{E}_{\infty^{-}}$-operad. In Section 3 . we introduce a new notion of higher homotopy operad where the action of the symmetric groups is also relaxed up to homotopy. This gives rise to new tools to study the homotopy properties of operads over any ring. Finally, the appendix deals with the notion of group representation up to homotopy.

\section{The Colored Operad which Encodes Operads}

In this section, we begin by recalling the definitions of the various types of operads used throughout the text. For further details, we refer the reader to Chapter 2 or the book [46]. We introduce the colored operad which encodes the category of operads with their symmetric groups actions viewed as structure maps. We show that it admits an inhomogeneous quadratic presentation, which is Koszul in the sense of the curved Koszul duality theory of Hirsh-Millès [31].

1.1. Nonunital operads and colored operads. The definition of an operad we give here is sometimes called the "partial definition." It is equivalent to the definition as a monoid given in Chapter 2 . 
Definition 1.1.1. An operad $\mathcal{P}$ is an $\mathbb{N}$-module $\{\mathcal{P}(n)\}_{n \in \mathbb{N}}$ equipped with structure maps

$$
\begin{array}{ll}
()^{\sigma}: \mathcal{P}(n) \longrightarrow \mathcal{P}(n), & \text { for } \sigma \in \mathbb{S}_{n}, \\
\left({ }^{\circ} o_{i}\right): \mathcal{P}(n) \otimes \mathcal{P}(k) \longrightarrow \mathcal{P}(n+k-1), & \text { for } 1 \leq i \leq n, \\
\eta(\lrcorner) \quad: \mathbf{k} \longrightarrow \mathcal{P}(1) . &
\end{array}
$$

These are required to satisfy the axioms of a right group action

$\left(\right.$ Opd-1) $\left(\mu^{\sigma}\right)^{\tau}=\mu^{\sigma \tau}$, and

$\left(\right.$ Opd-2) $\mu^{\text {id }_{n}}=\mu$ for all $\mu \in \mathcal{P}(n)$ and $\sigma, \tau \in \mathbb{S}_{n}$,

the parallel composition and the sequential composition axioms

$(O p d-3)\left(\lambda \circ_{j} \mu\right) \circ_{i} \nu=\left(\lambda \circ_{i} \nu\right) o_{j+k-1} \mu$, when $i<j$, and

$\left(\right.$ Opd-4) $\lambda \circ_{i}\left(\mu \circ_{j} \nu\right)=\left(\lambda \circ_{i} \mu\right) \circ_{j+i-1} \nu$, for all $\lambda \in \mathcal{P}(m), \mu \in \mathcal{P}(n)$, and $\nu \in \mathcal{P}(k)$,

the compatibility axioms

$\left(\right.$ Opd-5) $\mu \circ_{i} \nu^{\sigma}=\left(\mu \circ_{i} \nu\right)^{\mathrm{id}_{n}{ }^{\circ} \sigma}$, for all $\mu \in \mathcal{P}(n), \nu \in \mathcal{P}(k)$, and $\sigma \in \mathbb{S}_{k}$,

$\left(\right.$ Opd-6) $\mu^{\sigma} o_{i} \nu=\left(\mu \circ_{\sigma(i)} \nu\right)^{\sigma \sigma_{i} \mathrm{id}_{k}}$, for all $\mu \in \mathcal{P}(n), \nu \in \mathcal{P}(k)$, and $\sigma \in \mathbb{S}_{n}$,

and, finally, the unit axioms for $u:=\eta(1)$,

(Opd-7) $u \circ_{1} \mu=\mu$ for $\mu \in \mathcal{O}(n)$, and

(Opd-8) $\mu \circ_{i} u=\mu$ for $\mu \in \mathcal{O}(n)$ and $1 \leq i \leq n$.

An $n u$ operad or nonunital operad $\mathcal{P}$ is an $\mathbb{N}$-module equipped with structure maps (\lrcorner$)^{\sigma}$ and $\left(\sim_{\llcorner} \circ_{{ }_{\lrcorner}}\right)$as above, subject to Axioms (Opd-1) $($Opd-6)

An $n$ s operad or nonsymmetric operad $\mathcal{P}$ is an $\mathbb{N}$-module equipped with structure

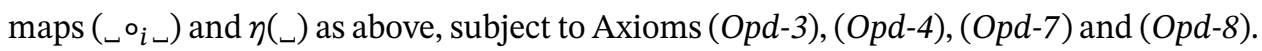

An $n s$ nu operad or nonsymmetric nonunital operad $\mathcal{P}$ is an $\mathbb{N}$-module equipped with partial composition structure maps $\left({ }_{{ }^{\circ}}{ }_{i}{ }_{\lrcorner}\right)$as above, subject to Axioms (Opd-3) and $(O p d-4)$

Since this definition is set-theoretical, it applies to any symmetric (closed) monoidal category, for instance to topological spaces or differential graded vector spaces. In this chapter, we will only work with differential graded nonunital operads. A morphism of dg nonunital operads is a morphism of $d g \mathbb{N}$-modules which respects the structures maps; the associated category is denoted by nu Opd.

Let us consider the identity operad $I$ made up of only the identity operation: $I(1)=$ $\mathbf{k} \cdot$ id and $I(n)=0$, for $n \neq 1$. The usual notion of an operad is defined with a unit $\eta: I \rightarrow \mathcal{P}$ for the partial composition products: $u \circ_{1} \mu=\mu$ and $\mu \circ_{i} u=\mu$, where $u:=\eta(1) \in \mathcal{P}(1)$. However, many operads come equipped with an augmentation, i.e., a morphism of operads $\varepsilon: \mathcal{P} \rightarrow I$. Such an augmented operad splits into $\mathcal{P}=I \oplus \overline{\mathcal{P}}$, where the augmentation ideal $\overline{\mathcal{P}}:=\operatorname{ker} \varepsilon$ is a nonunital operad. In the other way round, one can freely add a unit to a nonunital operad $\mathcal{P}: \mathcal{P}^{+}:=I \oplus \mathcal{P}$. These two constructions extend to functors in the obvious way, and provide us with an equivalence of categories between nonunital operads and augmented operads:

$$
\mathrm{nu} \mathrm{Opd} \cong \text { aug Opd . }
$$


EXAMPLE 1.1.2. Given a dg k-module $V$, we consider the collection of all multilinear maps from $V$ to $V$ :

$$
\operatorname{End}_{V}(n):=\operatorname{hom}\left(V^{\otimes n}, V\right) .
$$

The permutation of the inputs, respecting the Koszul sign rule in the graded case, and the classical composition of functions endow it with a nonunital operad structure (see Section 2.2 of Chapter 2 for details).

Definition 1.1.3. A $\mathcal{P}$-algebra structure on a dg $\mathbf{k}$-module $V$ is a morphism of operads $\mathcal{P} \rightarrow \operatorname{End}_{V}$.

An operad can act on a single $\mathbf{k}$-module. To model operations acting on various $\mathbf{k}$-modules at the same time, we consider the following colored version of operads.

Definition 1.1.4. Let $X$ be a set; its elements are referred to as colors. An $X$-colored operad $\mathcal{O}$, or colored operad for short, consists of an underlying collection of $\mathbf{k}$-modules

$$
\left\{\mathcal{O}\left(x_{0} ; x_{1}, \ldots, x_{n}\right) \mid x_{0}, \ldots, x_{n} \in X\right\},
$$

equipped with symmetric group actions, partial composition maps and a unit map

$$
\begin{gathered}
()^{\sigma}: \mathcal{O}\left(x_{0} ; x_{1}, \ldots, x_{n}\right) \longrightarrow \mathcal{O}\left(x_{0} ; x_{\sigma(1)}, \ldots, x_{\sigma(n)}\right), \quad \text { for } \sigma \in \mathbb{S}_{n}, \\
\left(\left\llcorner_{i \sim}\right): \mathcal{O}\left(x_{0} ; x_{1}, \ldots, x_{n}\right) \otimes \mathcal{O}\left(x_{i} ; y_{1}, \ldots, y_{k}\right) \longrightarrow \mathcal{O}\left(x_{0} ; x_{1}, \ldots, x_{i-1}, y_{1}, \ldots, y_{k}, x_{i+1}, \ldots, x_{n}\right),\right. \\
\quad \text { for } 1 \leq i \leq n \text { and for } x_{0}, x_{1}, \ldots, x_{n}, y_{1}, \ldots, y_{k} \in X,
\end{gathered}
$$

$$
\eta_{x}(\leftarrow): \mathbf{k} \longrightarrow \mathcal{O}(x ; x), \quad \text { for any } x \in X .
$$

They should satisfy the aforementioned Axioms (Opd-1) $($ Opd-6) colorwise. The data of the unit maps is equivalent to unit elements $u_{x} \in \mathcal{O}(x ; x)$. These are required to satisfy the unit axioms

(Opd-7') $u_{x_{0}{ }^{\circ}} \mu=\mu$ for $\mu \in \mathcal{O}\left(x_{0} ; x_{1}, \ldots, x_{n}\right)$, and

$\left(\right.$ Opd-8') $\mu \circ_{i} u_{x_{i}}=\mu$ for $\mu \in \mathcal{O}\left(x_{0} ; x_{1}, \ldots, x_{n}\right)$ and $1 \leq i \leq n$.

EXAMPLE 1.1.5. Given a collection $\left\{V_{x}\right\}$ of $\mathbf{k}$-modules $V_{x}$ indexed by colors $x \in X$, we consider the collection of all multilinear maps from the $V_{x}$ to themselves:

$$
\operatorname{End}_{V}\left(x_{0} ; x_{1}, \ldots, x_{n}\right):=\operatorname{hom}\left(V_{x_{1}} \otimes \cdots \otimes V_{x_{n}}, V_{x_{0}}\right) .
$$

The permutation of the inputs, the classical composition of functions and the identity functions endow it with a colored operad structure (see again Section 2.2 of Chapter 2 for details).

Definition 1.1.6. An $\mathcal{O}$-algebra structure on a family of $\mathbf{k}$-modules $\left\{V_{x}\right\}_{x \in X}$ is a morphism of colored operads $\mathcal{O} \rightarrow$ End $_{V}$.

One can extend the aforementioned definitions to the differential graded setting as usual by replacing $\mathbf{k}$-modules by $\mathbf{d g} \mathbf{k}$-modules and requiring all morphisms to be compatible with the differential. 
1.2. Nonunital operads as algebras over a colored operad. In this section, we introduce a particular colored operad $\mathcal{O}$ whose algebras are nonunital operads.

Definition 1.2.1. We define the $\mathbb{N}$-colored operad $\mathcal{O}=\mathcal{T} E /(R)$ by generators

$$
E(n ; n):=\left\langle\begin{array}{l}
n \\
\sigma \\
n
\end{array} \mid \sigma \in \mathbb{S}_{n} \backslash\left\{\operatorname{id}_{n}\right\}\right\rangle, \quad \text { for } n \geq 1,
$$

and

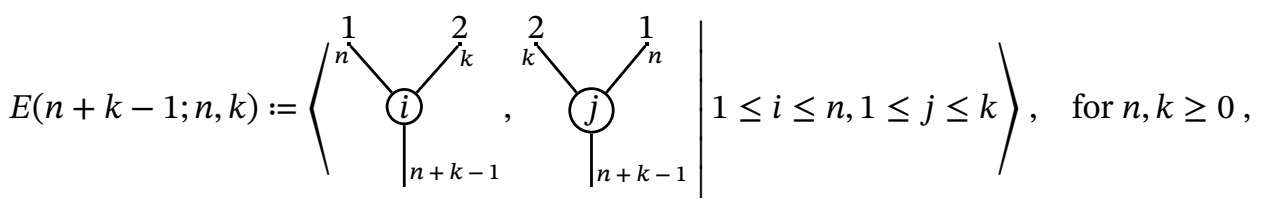

with regular $\mathbb{S}_{2}$-action, that is the action of the transposition (12) maps

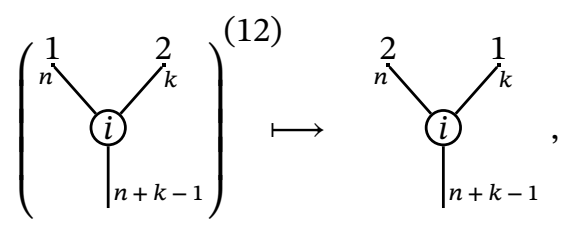

and relations

$\left.(\mathcal{O}-1) \oint_{\tau}^{n} \stackrel{\left(\tau \neq \sigma^{-1}\right)}{=}\right|_{\sigma \tau,} ^{n}$,

$\left\{\begin{array}{l}n \\ \sigma \\ (\mathcal{O}-2) \\ \sigma^{-1}=\mid \mathrm{id}_{n}\end{array}\right.$,

$(\mathcal{O}-3)$

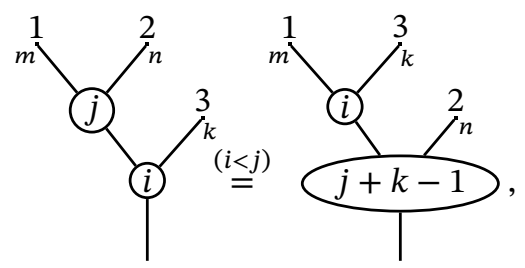

$(\mathcal{O}-4)$

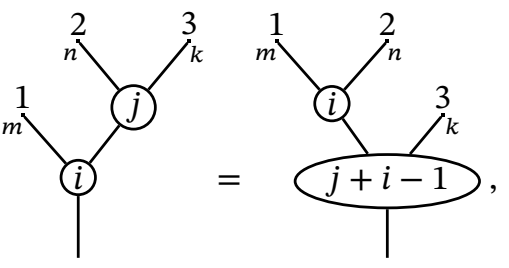

$(\mathcal{O}-5)$

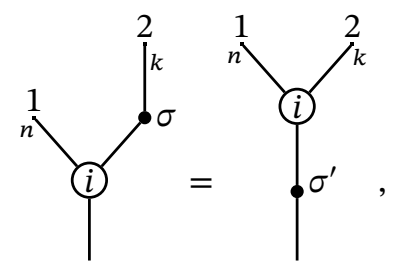

$(\mathcal{O}-6)$

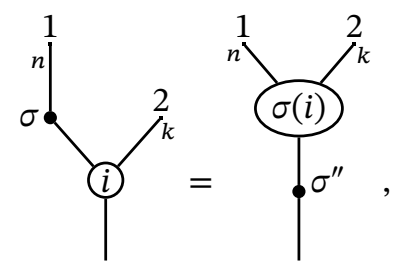

where $\sigma^{\prime}=\mathrm{id}_{n} \circ_{i} \sigma$ and $\sigma^{\prime \prime}=\sigma \circ_{i} \mathrm{id}_{k}$.

Lemma 1.2.2. The algebras over the colored operad $\mathcal{O}$ are the nonunital operads.

PROOF. This is straightforward from Definition 1.1.1 The unary generators correspond to the application of symmetric group elements; the binary generators correspond to the $o_{i}$-composition of their right-hand branch with the $i$-th input of their left-hand branch. 
We consider the following two suboperads of $\mathcal{O}$. The operad $\mathcal{O}_{n s}$ is generated by the binary generators $E(n+k-1 ; n, k)$ with Relations (O-3) and $(\mathcal{O}-4)$ Algebras over the operad $\mathcal{O}_{n s}$ are nonunital nonsymmetric operads. It was shown by Van der Laan in [39, Proposition 4.2] that one basis for $\mathcal{O}_{n s}$ is made up of planar rooted trees with vertices indexed bijectively by $1, \ldots, k$. The partial compositions then correspond to the substitution of trees.

The colored operad $\mathbf{k}[\mathbb{S}]$ is generated by the unary generators $E(n ; n)$ with Relations (O-1) and $(\mathcal{O}-2)$ This is the trivial presentation of the group algebras $\mathbf{k}\left[\mathbb{S}_{n}\right]$. So the colored operad $\mathbf{k}[\mathbb{S}]$ is a colored operad concentrated in arity 1 equal to the colored group algebra of symmetric groups $\left\{\mathbf{k}\left[\mathbb{S}_{n}\right]\right\}_{n \in \mathbb{N}}$.

The full colored operad $\mathcal{O}$ can be constructed from its two suboperads $\mathcal{O}_{n s}$ and $\mathbf{k}[\mathbb{S}$ ] by means of a distributive law (see [46, §8.6]) as follows. Recall that the notation ${ }_{{ }^{\circ}}{ }_{-}$ stands for the composite product of $(\mathbb{N}$-colored) $\mathbb{S}$-modules.

Proposition 1.2.3. The rewriting rule defined by Relations (O-5) and (O-6), read from left to right, induces a distributive law $\mathcal{O}_{n s} \circ \mathbf{k}[\mathbb{S}] \rightarrow \mathbf{k}[\mathbb{S}] \circ \mathcal{O}_{n s}$. Therefore, the underlying colored $\mathbb{S}$-module of $\mathcal{O}$ is isomorphic to $\mathbf{k}[\mathbb{S}] \circ \mathcal{O}_{n s}$.

PROOF. One has to check that the surjection $\mathbf{k}[\mathbb{S}] \circ \mathcal{O}_{n s} \rightarrow \mathcal{O}$ is injective. It is well known that the free nonunital operad on an $\mathbb{S}$-module $M$ is given by the module of nontrivial trees with vertices labeled by the elements of $M$ and with the leaves labeled by a permutation of $\mathbb{S}_{n}[46 \S 5.6]$. Together with [39, Proposition 4.2], this proves that the colored operad $\mathcal{O}$ is isomorphic to $\mathbf{k}[\mathbb{S}] \circ \mathcal{O}_{n s}$.

\section{REMARKS.}

- This proposition proves that the colored operad $\mathcal{O}$ defined here by generators and relations is the reduced version, i.e., without anything in arity 0 , of the colored operad $S$ of Berger-Moerdijk [7, §1.5.6] defined by labeled trees and encoding operads.

- The colored operad $\mathcal{O}$ is made up of regular representations of the symmetric groups, however it is not a regular operad. This means that the colored operad $\mathcal{O}$ is not obtained from a nonsymmetric operad by tensoring it with the regular representation of the symmetric goups since Relation $(\mathcal{O}-3)$ contains a nontrivial permutation of the inputs.

- Proposition 1.2.3 is actually equivalent to the commutativity of the following diagram of forgetful functors

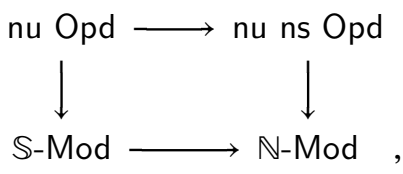

where ns Opd stands for the category of nonsymmetric operads. The induced commutative diagram of left adjoint functors shows that the free $\mathcal{O}$-algebra 
on an $\mathbb{N}$-module $\mathcal{P}$ is isomorphic to

$$
\mathcal{O}(\mathcal{P}) \cong \overline{\mathcal{T}}(\mathcal{P} \otimes \mathbf{k}[\mathbb{S}]),
$$

where $\overline{\mathcal{T}}$ stands for the nonunital free operad functor of an $\mathbb{S}$-module, see 46 . $\S 5.5]$.

1.3. Trees, bases, orders and signs. The presentation of the colored operad $\mathcal{O}$ given in Definition 1.2.1 suggests we consider the following type of trees. We call composite trees the planar trees with only unary and binary vertices labeled respectively by elements of the symmetric groups and by positive integers. Composite trees form a basis for the free colored operad $\mathcal{T} E$. Composite trees which are left combs with nondecreasing binary vertices from top to bottom, are called standard composite trees. They provide us with a basis for the colored operad $\mathcal{O}$ as follows.

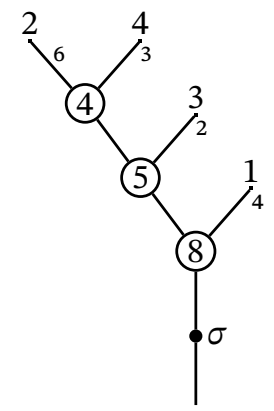

Proposition 1.3.1. A k-linear basis for the colored operad $\mathcal{O}$ is given by the left combs with nondecreasing binary vertices, seen top to bottom, at most one unary vertex labeled by a permutation at the bottom and a labeling of the leaves with a permutation on the number of them.

This is a corollary of Proposition 1.2.3 the permutations labeling the root of a composite tree form a basis of $\mathbf{k}[\mathbb{S}]$ and we claim that the left combs with nondecreasing binary vertices from top to bottom form a basis of $\mathcal{O}_{n s}$. In order to prove this last point, we consider planar trees, without any restriction on the valence of the vertices, which can be equal to 0 ; we call them operadic trees.

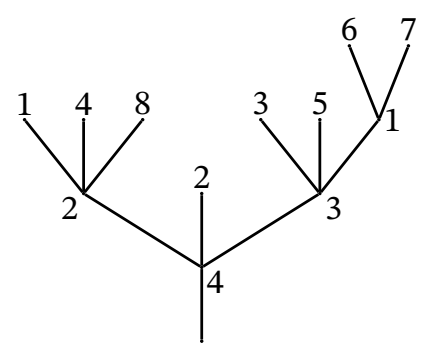

Proposition 1.3.2. The set of planar trees labeled with one permutation on the number of leaves and one permutation on the number of vertices forms a $\mathbf{k}$-linear basis of the colored operad $\mathcal{O}$. 
PROOF. This is a direct corollary of Proposition 1.2.3. since planar trees form a basis of the colored operad $\mathcal{O}_{n s}$. The permutation labeling the leaves comes from the suboperad $\mathbf{k}[\mathbb{S}]$.

In the rest of this chapter, we will consider the following total order on the set of vertices of operadic trees.

left-recursive/depth-first: Starting at the root, we recursively order child vertices from left to right.

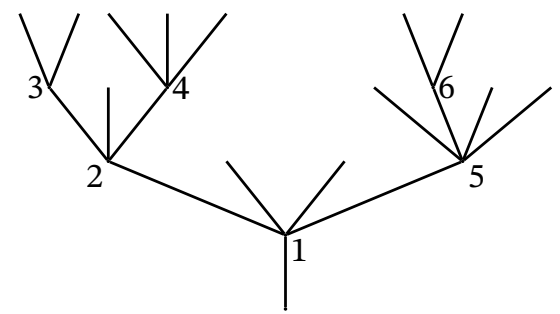

Proof of Proposition 1.3.1 The key point lies in the bijection induced by the correspondence between the binary generators of the colored operad $\mathcal{O}$ and trees with two vertices:

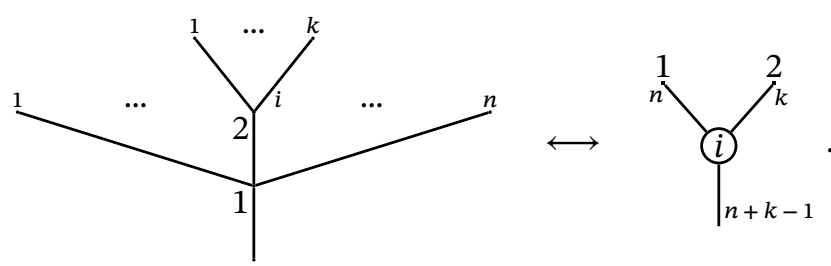

The labels of the vertices of any operadic tree given by the left-recursive order coincide with the labels of the leaves of the associated standard composition tree: if we traverse the operadic tree in a left-recursive manner, the inner edges found in this order are encoded as binary vertices in the composition tree. In the other way round, any standard composition tree corresponds to the canonical way of creating an operadic tree by grafting the corollas in the left-recursive way, i.e., from bottom to top and from left to right. In this way, we get a bijection between standard composite trees and operadic trees.
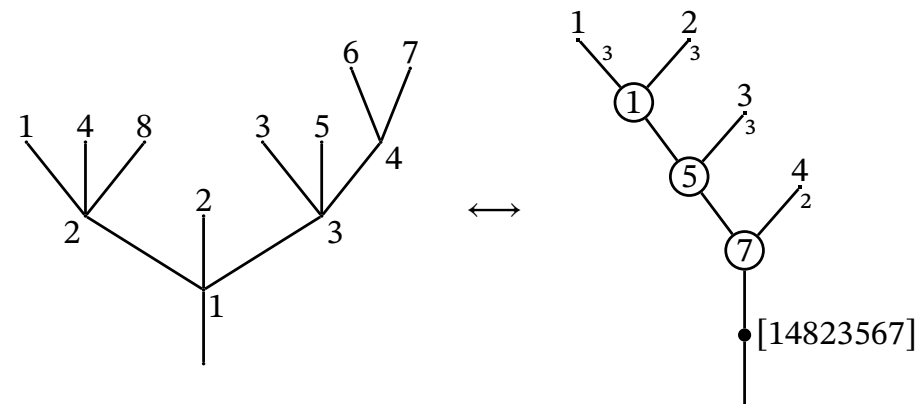

In order to treat the signs, we consider the following total order on the set of vertices of composite trees. 
left-levelwise/breadth-first: Starting at the root, order child vertices left to right before proceeding to the next level/generation.

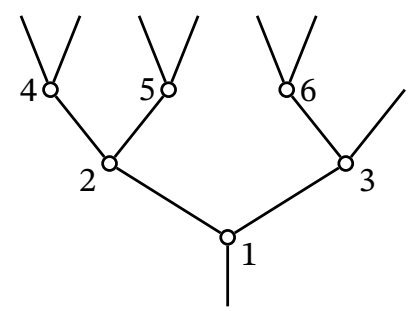

We follow the Koszul sign rule for trees, see [46, Chapter 6]. This means that we equip composite trees with the left-levelwise ordering on the vertices, i.e., we have vertices $\nu_{1}, \ldots, \nu_{n}$. We then identify the space of labelings of a given tree by elements of graded k-modules $V_{i}$ at $\nu_{i}$ with the tensor product $V_{1} \otimes \cdots \otimes V_{n}$. A relabeling of the vertices corresponds to a permutation of the factors $V_{i}$ and the isomorphism honors the classical Koszul sign rule: $x \otimes y \mapsto(-1)^{|x||y|} \cdot y \otimes x$ (see Remark 1.2.1 of Chapter 1).

1.4. Quadratic analogue. Recall the presentation $(E, R)$ for $\mathcal{O}$ of Definition 1.2.1 We consider the projection $q: \mathcal{T} E \rightarrow \mathcal{T} E^{(2)}$ onto the weight 2 part of the free operad. The quadratic analogue of the colored operad $\mathcal{O}$ is the quadratic colored operad

$$
q \mathcal{O}:=\mathcal{T} E /(q R) .
$$

In the case of the colored operad $\mathcal{O}$, the space of relations $q R$ differs from $R$ only in its unary part: the right-hand sides of the $\operatorname{Relations~(\mathcal {O}-1)}$ and $(\mathcal{O}-2)$ project to 0, i.e.,

$$
\int_{\tau}^{n} \sigma=0 .
$$

REMARK 1.4.1. The quadratic analogue $q \mathcal{O}$ is just a technical step in the curved Koszul duality theory for the operad $\mathcal{O}$. Though we will never work with them, we give the definition of a $q \mathcal{O}$-algebra for the interested reader: roughly, this is an "operad" where the action of two consecutive nontrivial elements of the symmetric group vanishes.

The first step in the curved Koszul duality theory is to show that the homogenous quadratic colored operad $q \mathcal{O}$ is Koszul (see Section 7.4 of Chapter 2). To this extent, we consider the nilpotent quadratic unital colored algebra $\mathbf{k}\{\mathbb{S}\}$ generated by all the elements of $\mathbb{S}$ except for the identities and with the maximal set of relations, i.e., any product is equal to zero. Its underlying module is the same as the colored algebra $\mathbf{k}[\mathbb{S}]$.

\section{Proposition 1.4.2.}

(i) The rewriting rule defined by the Relations (O-5) and (O-6), read from left to right, induces a distributive law $\mathcal{O}_{n s} \circ \mathbf{k}\{\mathbb{S}\} \rightarrow \mathbf{k}\{\mathbb{S}\} \circ \mathcal{O}_{n s}$. Therefore, the underlying colored $\mathbb{S}$-module of $q \mathcal{O}$ is isomorphic to $\mathbf{k}\{\mathbb{S}\} \circ \mathcal{O}_{n s}$.

(ii) The homogenous quadratic colored operad qO $\mathcal{O}$ is Koszul. 
PROOF. These two points are actually proven at once by the Diamond Lemma for distributive laws [46, Theorem 8.6.5]. Both quadratic colored operads $\mathbf{k}\{\mathbb{S}\}$ and $\mathcal{O}_{n s}$ are Koszul; the first one is nilpotent with maximal set of relations, and the second one was shown to be Koszul in [39. Theorem 4.3]. Strictly speaking, Van der Laan's Koszul duality theory for colored operads was proven over a field of characteristic zero. One can extend it over a commutative ring using the methods of Fresse [16], see Chapter 2 This applies to the colored operad $q \mathcal{O}$ since it is made up of free finitely generated $\mathbf{k}$-modules and free $\mathbf{k}\left[\mathbb{S}_{n}\right]$-modules.

It remains to prove that the natural map $\left(\mathbf{k}\{\mathbb{S}\} \circ \mathcal{O}_{n s}\right)^{(3)} \rightarrow q \mathcal{O}$ is injective in weight 3. Since the left-hand term is concentrated in weight 1 (except for its unit), it is enough to prove that $\overline{\mathbf{k}\{\mathbb{S}\}} \circ \mathcal{O}_{n s}^{(2)}$ imbeds into $q \mathcal{O}$. We use the relations of $q \mathcal{O}$, read from left to right, as rewriting rules. In this way, one can always pull down the symmetric group elements. So if a tree presents at least two such elements, it vanishes in the colored operad $q \mathcal{O}$. Let us now restrict to the summand generated by trees with one element coming from the symmetric group; it is a set-theoretical summand, that is linearly spanned by trees subject to the Relations (O-3) $(\mathcal{O}-6)$ For our purpose, it is now enough to prove that in each equivalence class of trees with two binary vertices and one unary vertex, there exists a unique standard composition tree with the unary vertex at the bottom. This is shown by checking that the ambiguities are all confluent; these ambiguities are given by the left-hand terms of Relations (O)-3) and $(\mathcal{O}-4)$ with a symmetric group element labeling one leaf. Let us give one such verification below; the other ones follow the same pattern.

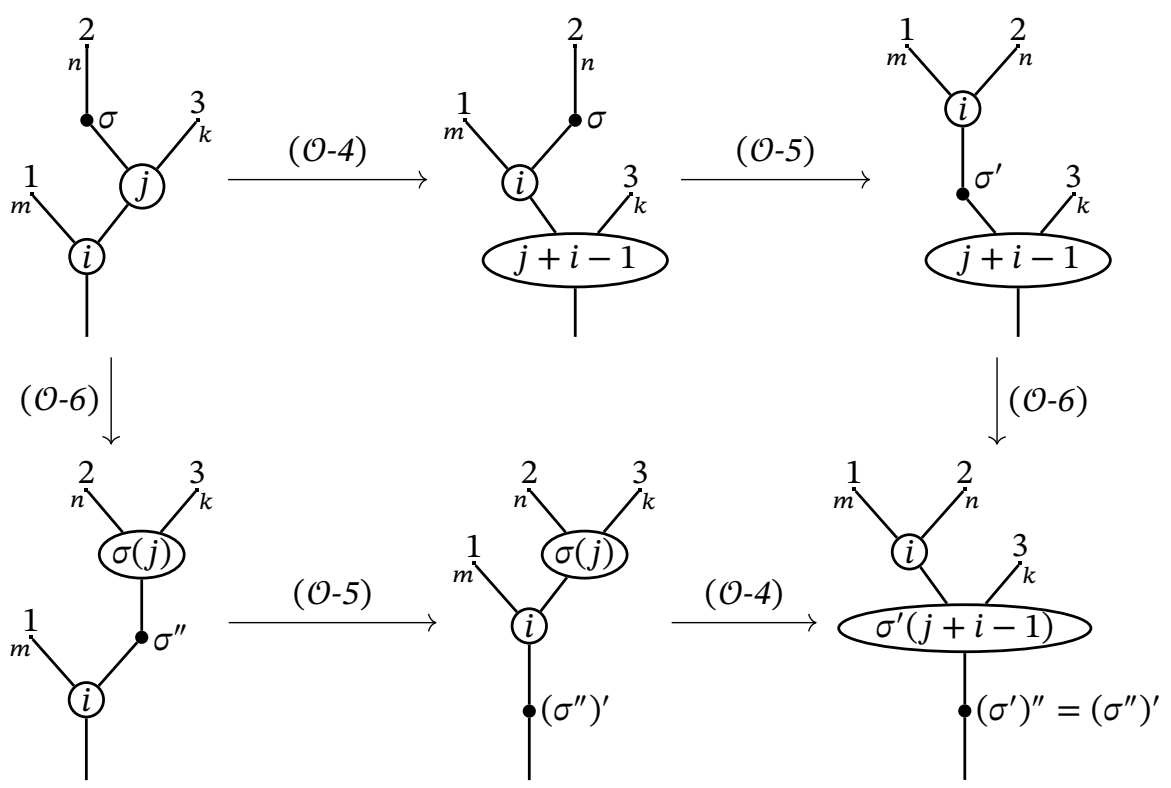

The second step in the curved Koszul duality theory is to calculate the Koszul dual operad and cooperad of the quadratic analogue $q \mathcal{O}$. 
Proposition 1.4.3. The Koszul dual operad $q \mathcal{O}^{!}$of the quadratic analogue $q \mathcal{O}$ is generated by

$$
\begin{aligned}
& s^{-1} E(n ; n)^{*}=\left\langle\left.\right|_{l_{n}^{n}} ^{n} s^{-1} \sigma^{*} \mid \sigma \in \mathbb{S}_{n} \backslash\left\{\mathrm{id}_{n}\right\}\right\rangle,
\end{aligned}
$$

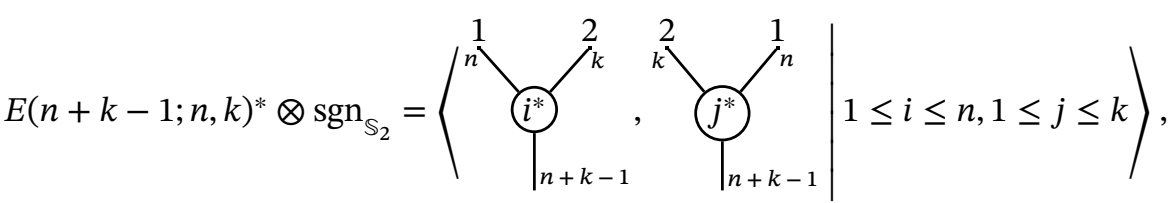

with the following quadratic relations:

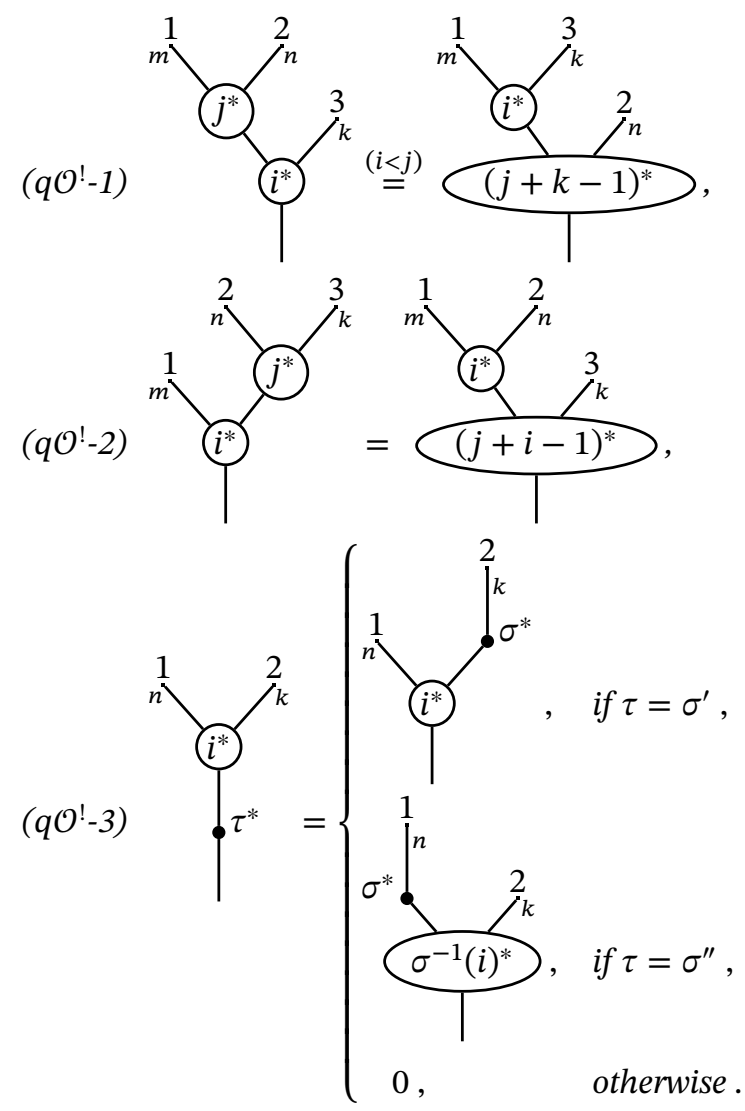

PROOF. The method of computing the Koszul dual of an operad recalled in Section 7 of Chapter 2 (based on [46 Chapter 7]) still holds mutatis mutandis for colored operads, see also [39]. The only new point here is to manage the nonbinary parts. Notice that the operad $q \mathcal{O}$ is colorwise finitely generated $\mathbf{k}$-projective.

The Koszul dual cooperad $q \mathcal{O}^{i}$ is the quadratic (colored) cooperad with suspended presentation

$$
q \mathcal{O}^{i}:=\mathcal{C}\left(s E, s^{2} q R\right)
$$


The piecewise and colorwise linear dual $\left(q \mathcal{O}^{i}\right)^{*}$ is a quadratic colored operad. By the same arguments as for [46. Proposition 7.2.1], its operadic suspension

$$
q \mathcal{O}^{!}:=\left(q \mathcal{O}^{i}\right)^{*} \otimes \mathcal{S}^{-1}
$$

is still a quadratic operad, where $\mathcal{S}^{-1}$, in the colored setting, is defined by the endomorphism operad of the k-module $\bigoplus_{n \in \mathbb{N}} \mathbf{k} s_{n}^{-1}$, where the index $n$ stands for the color. Its quadratic presentation is given by

$$
q \mathcal{O}^{!}=\mathcal{T}\left(s^{-1} E(1)^{*} \oplus E(2)^{*} \otimes \operatorname{sgn}_{\mathbb{S}_{2}}\right) /\left(q R^{\perp}\right),
$$

where the orthogonal space is obtained by the scalar product $\left\langle_{-},{ }_{-}\right\rangle$defined on the arity 3 part in [46, Section 7.6.3]. Using this, one proves the two first relations. By the same arguments as for [46, Theorem 7.6.2], the extension of the scalar product $\left\langle_{\triangle},{ }_{-}\right\rangle$to $\mathcal{T}\left(s^{-1} E(1)^{*} \oplus E(2)^{*} \otimes \operatorname{sgn}_{\mathbb{S}_{2}}\right)^{(2)}(2) \otimes \mathcal{T} E^{(2)}(2)$ given by

$$
\left\{\begin{array}{l}
\left\langle\alpha^{*} \circ_{1} \sigma^{*}, \mu \circ_{1} \tau\right\rangle:=+\alpha^{*}(\mu) \sigma^{*}(\tau), \\
\left\langle\alpha^{*} \circ_{2} \sigma^{*}, \mu \circ_{2} \tau\right\rangle:=+\alpha^{*}(\mu) \sigma^{*}(\tau), \\
\left\langle\sigma^{*} \circ_{1} \alpha^{*}, \tau \circ_{1} \mu\right\rangle:=-\sigma^{*}(\tau) \alpha^{*}(\mu),
\end{array}\right.
$$

for $\alpha^{*} \in E(2)^{*}, \mu \in E(2), \sigma^{*} \in E(1)^{*}$, and $\tau \in E(1)$, allows one to compute the Koszul dual operad. This gives the last 3 relations and concludes the proof.

Lemma 1.4.4. The underlying colored $\mathbb{S}$-module of the operad $q \mathcal{O}^{!}$is isomorphic to

$$
q \mathcal{O}^{!} \cong \mathcal{O}_{n s} \circ T\left(s^{-1} \overline{\mathbb{S}}^{*}\right)
$$

where $T\left(s^{-1} \overline{\mathbb{S}}^{*}\right)$ is the free colored algebra generated by $\left\{s^{-1} \sigma^{*} \mid \sigma \in \mathbb{S}_{n} \backslash\left\{\mathrm{id}_{n}\right\}, n \in \mathbb{N}\right\}$.

PROOF. This is a straightforward consequence of the Diamond Lemma for distributive laws [46. Theorem 8.6.5] and Proposition 1.4.2 since the colored operad $\mathcal{O}_{n s}$ is Koszul auto-dual [39. Theorem 4.3], i.e., $\mathcal{O}_{n s}^{!} \cong \mathcal{O}_{n s}$, and since the Koszul dual of the quadratic nilpotent algebra $\mathbf{k}\{\mathbb{S}\}$ is the free algebra $T\left(s^{-1} \overline{\mathbb{S}}^{*}\right)$.

Proposition 1.4.5. A $\mathbf{k}[\mathbb{S}]$-linear basis for the colored operad $q \mathcal{O}^{!}$is given by the left combs with nondecreasing binary vertices, seen top to bottom, and strings of symmetric group elements labeling the leaves as in

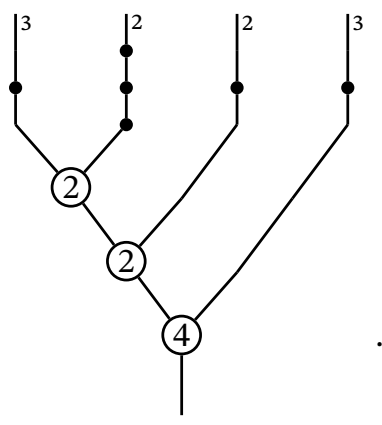


Since the context is obvious, we do not use the linear dual star notation for the basis of $q \mathcal{O}^{!}$anymore. In addition, we will often omit inessential labels such as those for the symmetric group elements above.

PROOF. This is a direct corollary of Lemma 1.4.4 since standard composition trees form a basis of the colored operad $\mathcal{O}_{n s}$.

On this basis, the partial composition of the colored operad $q \mathcal{O}^{!}$is given by the grafting of trees as usual. Then one has to rewrite the result in terms of standard composition trees keeping track of the order on generators and sticking to the Koszul sign rule as in the examples below. From now on, we order the vertices of composition trees with leaves labeled by strings of permutations by, first, ordering all the binary vertices left-levelwise and then ordering the strings of permutations left-levelwise.

EXAMPLE 1.4.6 (Partial composition in $q \mathcal{O}^{!}$).

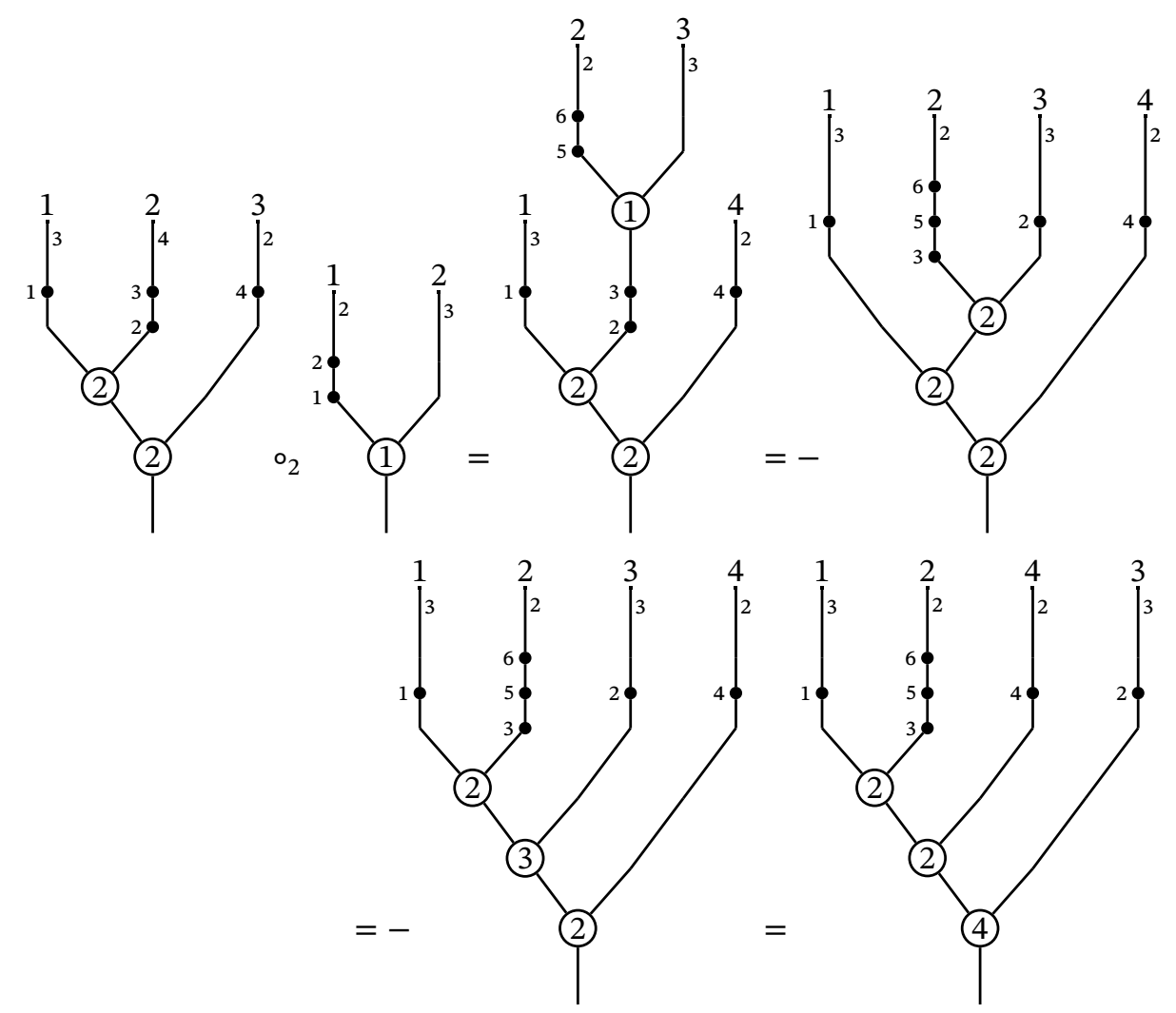

(Assuming the permutation at 2 is [1342] and at 3 it is [4123].) In this example, we reorder the labeling permutations bottom to top in leaf order. Following the Koszul sign rule, the sign is that of the permutation [135642], i.e., +1 .

Proposition 1.4.7. The set of planar trees with vertices labeled by (possibly empty) strings of permutations on the number of leaves of the vertex forms another $\mathbf{k}[\mathbb{S}$ ]-linear basis of 
the colored operad $q \mathcal{O}$ !

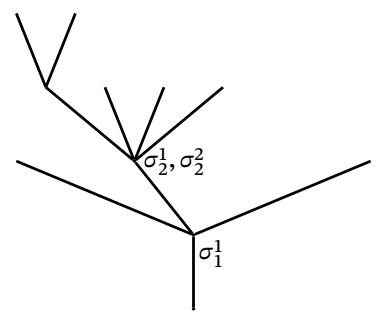

PROOF. The proof is a consequence of Proposition 1.3.2 for the "tree" part and Proposition 1.4.5 for the "permutations" part: strings of symmetric group elements on top of composition trees correspond to strings of symmetric group elements labeling the vertices of operadic trees as follows:

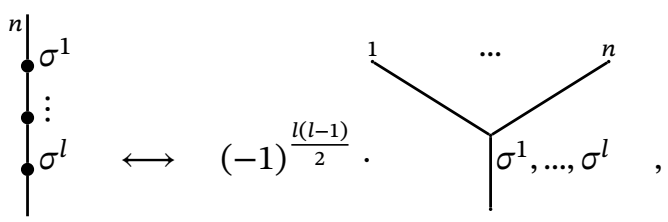

where the sign comes from the reordering of $l$ degree -1 terms.

The vertices of the operadic trees are ordered by the left-recursive order. Hence we denote such a labeled tree by $t\left(\bar{\sigma}_{1}, \ldots, \bar{\sigma}_{n}\right)$, where $t$ stands for the underlying planar tree and where each $\bar{\sigma}_{j}=\left(\sigma_{j}^{1}, \ldots, \sigma_{j}^{i_{j}}\right)$ is a (possibly empty) string of permutations. The above example is $t\left(\left(\sigma_{1}^{1}\right),\left(\sigma_{2}^{1}, \sigma_{2}^{2}\right), \varnothing\right)$.

The bijection mentioned in Section 1.3 between standard composition trees and operadic trees induces a bijection between the two above bases of the colored operad $q \mathcal{O}$ !: we add the strings of symmetric group elements found at the corresponding vertices of the operadic tree to the leaves of the standard composition tree. Note that the change of order for the strings permutations induces a sign, e.g.,
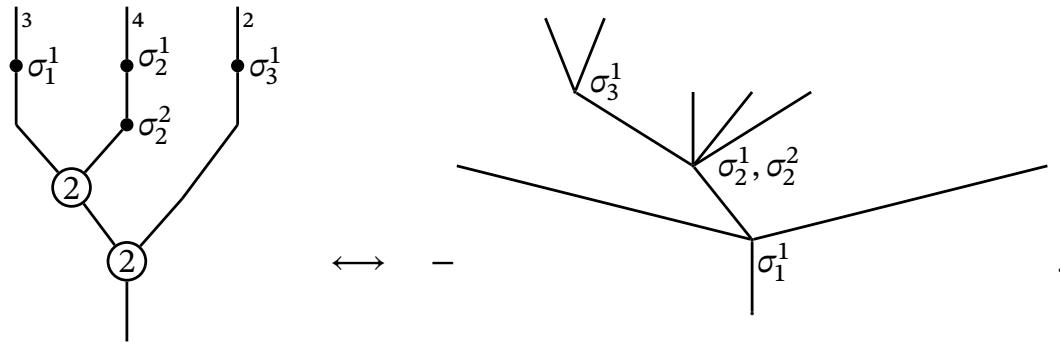

Under this bijection, the partial composition of the colored operad $q \mathcal{O}^{!}$can also be described on the operadic trees basis. Since the leaves of composition trees correspond to the vertices of operadic trees, and since the binary generators correspond to the internal edges, the partial composition amounts to the substitution of operadic trees. 
EXAMPLE 1.4.8.

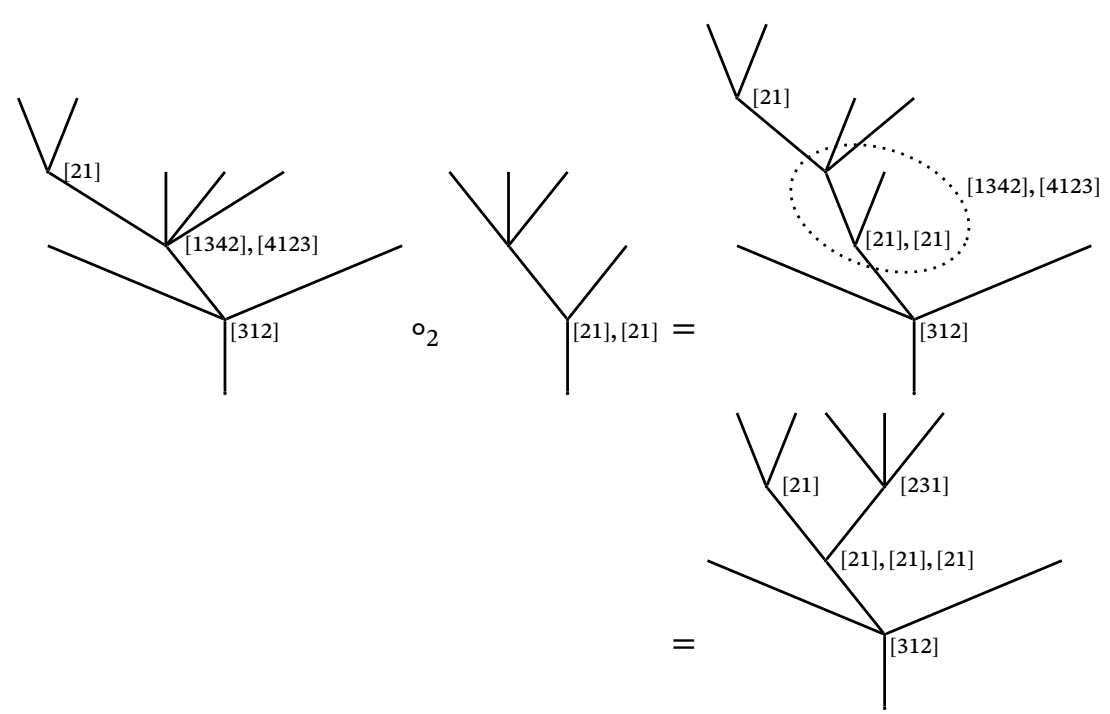

This example depicts the same partial composition as in Example 1.4.6 but this time in terms of operadic trees.

The main advantage here of operadic trees over composition trees lies in the fact that in the colored operad $q \mathcal{O}^{!}$the former have no relations, unlike the latter: they are related by Relations $\left(q \mathcal{O}^{!}-1\right)$ and $\left(q \mathcal{O}^{!}-2\right)$.

1.5. Koszul dual cooperad of the quadratic analogue. The Koszul dual cooperad $q \mathcal{O}^{i}$ is the linear dual of the operad $q \mathcal{O}^{!} \otimes \mathcal{S}$, as explained above in the proof of Proposition 1.4.3. This latter operad admits the same presentation as the operad $q \mathcal{O}^{\text {! }}$ except that all the generators are now in degree -1 and that all the relations hold up to a minus sign. So the degree of the underlying planar tree $t$ of an operadic tree depicting a basis element of the cooperad $q \mathcal{O}^{i}$ is equal to the number of its internal edges; we denote it by $|t|$.

Dualizing the aforementioned partial composition of the operad $q \mathcal{O}^{\text {! }}$, we get the infinitesimal decomposition map $\Delta_{(1)}$ of the cooperad $q \mathcal{O}^{i}$ as follows. Let $t\left(\bar{\sigma}_{1}, \ldots, \bar{\sigma}_{n}\right) \in$ $q \mathcal{O}^{i}$ be an operadic tree basis element. Let $s \subset t$ be any subtree, which can be just a corolla. It is completely characterized by the associated subset $\left\{s_{1}, \ldots, s_{k}\right\} \subset\{1, \ldots, n\}$ of vertices of $t$. The infinitesimal coproduct of $q \mathcal{O}^{i}$ extracts any such subtree $s$ of $t$ and produces the tree $t / s$ obtained by contracting $s$ inside $t$, i.e., replacing the subset $s$ by a corolla of same arity.

The strings of permutations $\left\{\bar{\sigma}_{s_{1}}, \ldots, \bar{\sigma}_{s_{k}}\right\}$ labeling the vertices of $s$ splits into two parts, in all possible ways, even empty. For this, we use the notation

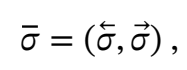

where the left-hand parts $\tilde{\sigma}$ remain in s. (It can be empty only when the subtree $s$ is not a corolla). The right-hand parts $\vec{\sigma}$ leave $s$ one by one in all possible orders, change its shape according to Relation $\left(q \mathcal{O}^{!}-3\right)$ and label the new corolla in the contracted tree $t / s$. We denote the new string of permutations by $\amalg\left(\vec{\sigma}_{s_{1}}, \ldots, \vec{\sigma}_{s_{k}}\right)$ because all the shuffles 
of the $k$ strings appear in the formula. (We should use here notation $\sigma^{\prime}$ and $\sigma^{\prime \prime}$ for these induced permutations.) We denote by $\tilde{s}$, the new subtree $s$. The other vertices of $t$ remain labeled by the same strings of permutations. The overall sign is denoted by $\varepsilon_{s, t, \omega}$

Proposition 1.5.1. The infinitesimal decomposition map of the cooperad $q \mathcal{O}^{i}$ is equal to

$$
\Delta_{(1)}\left(t\left(\bar{\sigma}_{1}, \ldots, \bar{\sigma}_{n}\right)\right)=\sum_{s \subseteq t, \omega} \varepsilon_{s, t, \omega} \cdot t / s\left(\bar{\sigma}_{1}, \ldots, ш\left(\vec{\sigma}_{s_{1}}, \ldots, \vec{\sigma}_{s_{k}}\right), \ldots, \bar{\sigma}_{n}\right) \otimes \tilde{s}\left(\overleftarrow{\sigma}_{s_{1}}, \ldots, \overleftarrow{\sigma}_{s_{k}}\right) .
$$

PROOF. This formula is the linear dual of the partial composition map of the operad $q \mathcal{O}^{!}$

Let us explain this procedure on an example and give the computation of the associated signs at the same time. Recall that the signs all come from the left-recursive order on composition trees.

EXAMPLE 1.5.2 (Infinitesimal decomposition map of $q \mathcal{O}^{i}$ ). We consider the operadic trees

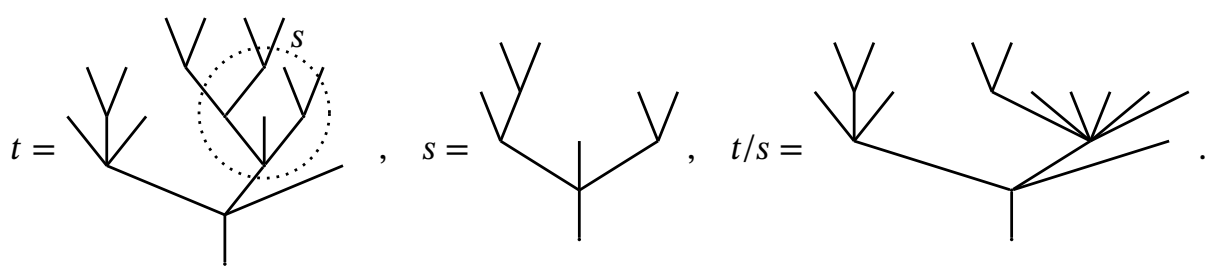

We ommited the symmetric group elements in the above pictures. See the composition tree pictures below.

Step 1. We associate to the operadic tree $t\left(\bar{\sigma}_{1}, \ldots, \bar{\sigma}_{n}\right)$ its standard composition tree. The reversal of order in the strings of permutations induces the sign

$$
(-1)^{\frac{\left|\bar{\sigma}_{1}\right|\left(\left|\bar{\sigma}_{1}\right|-1\right)}{2}+\cdots+\frac{\left|\bar{\sigma}_{n}\right|\left(\left|\bar{\sigma}_{n}\right|-1\right)}{2}} \text {. }
$$

Step 2. We isolate the subtree $s$ inside $t$ as follows. The label $s_{1}$ is the smallest index of a vertex of $s$ viewed inside $t$. We relabel the vertices of $t$ by, first, keeping the labels $1, \ldots, s_{1}-1$, then, labeling only the vertices of $s$ by $s_{1}, s_{1}+1, \ldots, s_{1}+$ $k-1$, and, finally, by labeling the rest of the vertices of $t$. This implies a permutation of vertices, denoted by $\nu$, and an induced permutation of strings of symmetric group elements, which all have degree 1 . The induced sign is the signature of $\nu$ times the sign produces by the Koszul sign rule. We denote this sign by $\operatorname{sgn}\left(\nu\left(\left|\bar{\sigma}_{1}\right|, \ldots,\left|\bar{\sigma}_{n}\right|\right)\right)$.

On the level of the equivalent standard composition tree, we rewrite it as a left-comb but isolating the subtree $s$. The signature of the permutation $\nu$ comes from the use of the (signed) Relation $\left(q \mathcal{O}^{!}-1\right)$.

Step 3. We extract the subtree $s$ out of the tree $t$. On the level of the left-comb composition tree, this amounts to using Relations $\left(q \mathcal{O}^{!}-1\right)$ and $\left(q \mathcal{O}^{!}-2\right)$ to write the tree $t$ with a first left-comb giving $t / s$ and an attached parallel 
left-comb giving the subtree $s$. Then, we pull the left-comb representing $s$ above the one representing $t / s$ with its strings of permutations. The sign for the underlying trees is $(-1)^{|s|\left(s_{1}-1\right)}$; it should be multiplied with the sign coming from the change of order of the strings of permutations.

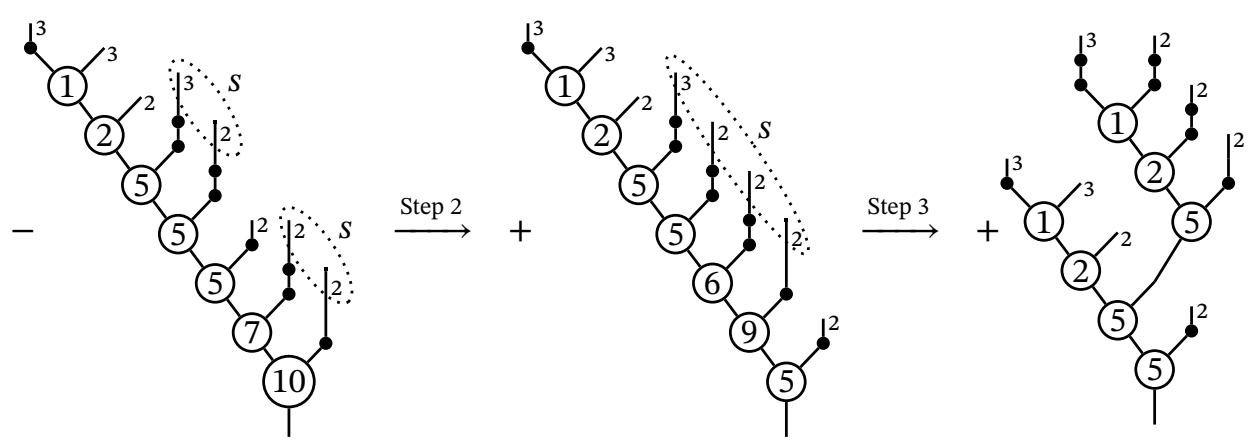

Step 4. For each choice of sub-strings labeling the vertices of $s$ and for each shuffle of them, we pull them down and we change them into $\sigma^{\prime}$ or $\sigma^{\prime \prime}$ at each step. And we push down these elements inside the bottom tree representing $t / s$. The sign is two-fold: each use of (signed) Relation $\left(q \mathcal{O}^{!}-3\right)$ creates a sign and the permutations of the order of the symmetric group elements produce a sign.

Step 5. By pulling down the symmetric group elements using Relation $\left(q \mathcal{O}^{!}-3\right)$ the labels of the binary vertices of the standard left-comb giving $s$ might be changed (second case of Relation $\left(q \mathcal{O}^{!}-3\right)$. So we might have to rewrite this left-comb into a standard form, i.e., with nondecreasing binary vertices from top to bottom, using Relation $\left(q \mathcal{O}^{!}-1\right)$, this gives a sign similar to the sign in Step 1.

In our example, assume that $\rho_{1}=$ [321], $\rho_{2}=\rho_{3}=$ [21]. In the pictures we then use the $(1,0,2,0)$-shuffle [213] to pull down the symmetric group elements. This induces permutations (in this order) $\tilde{\rho}_{2}=[132456]$, $\tilde{\rho}_{1}=[456312]$ and $\tilde{\rho}_{3}=[123465]$.
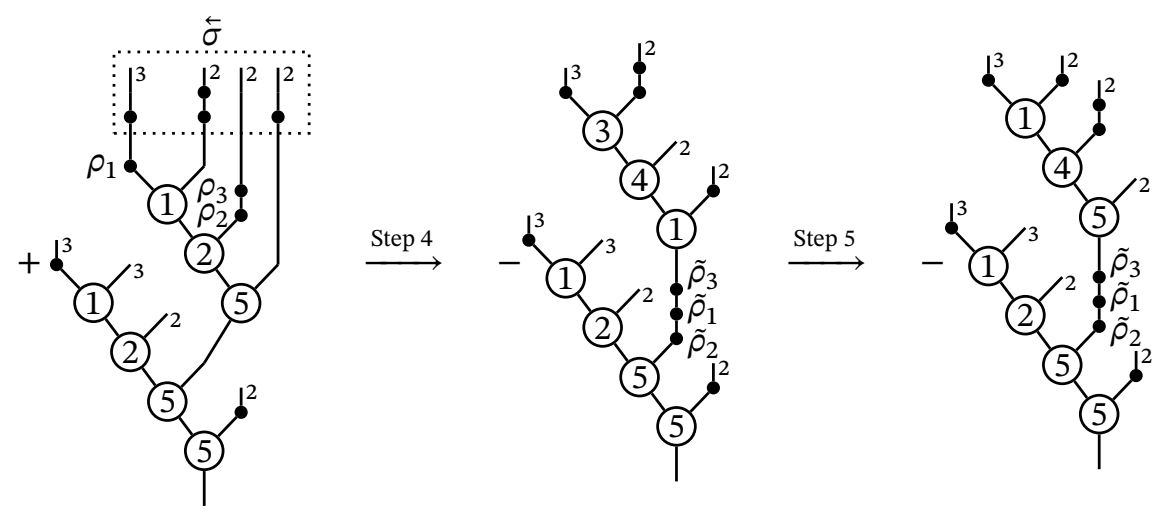
Step 6. We write the operadic tree $\tilde{s}\left(\overleftarrow{\sigma}_{S_{1}}, \ldots, \overleftarrow{\sigma}_{s_{k}}\right)$ corresponding to the top standard composition tree on the right of $t / s\left(\bar{\sigma}_{1}, \ldots, \omega\left(\vec{\sigma}_{s_{1}}, \ldots, \vec{\sigma}_{s_{k}}\right), \ldots, \bar{\sigma}_{n}\right)-$ the operadic tree corresponding to the bottom standard composition tree. There is a sign coming from the bijection between operadic trees and standard composition trees with strings of permutations.

$$
\stackrel{\text { Step } 6}{\longrightarrow} \quad-t / s\left((\bullet), \varnothing, \varnothing,\left(\tilde{\rho}_{3}, \tilde{\rho}_{1}, \tilde{\rho}_{2}\right),(\bullet)\right) \otimes \tilde{s}((\bullet),(\bullet),(\bullet, \bullet), \varnothing) .
$$

1.6. Curved Koszul dual cooperad. To apply the curved Koszul duality theory to the colored operad $\mathcal{O}$, one has to check first the following conditions on the presentation of this operad, according to [31. Section 4.1], see also our summary in Section 7 of Chapter 2 .

Lemma 1.6.1. The following conditions hold:

(I) Minimality of generators: $R \cap\{I \oplus E\}=0$,

(II) Maximality of relations: $(R) \cap\left\{I \oplus E \oplus \mathcal{T} E^{(2)}\right\}=R$.

PROOF. The first condition is obviously satisfied. Regarding the second condition, since the operad $\mathcal{O}$ is the free $\mathbf{k}$-linear version of a set-theoretical operad, any element of $(R) \cap\left\{I \oplus E \oplus \mathcal{T} E^{(2)}\right\}$ is a linear combination of elements of the form $x_{1}-x_{2}$, where $x_{1}, x_{2}$ are set-theoretical elements of $I \oplus E \oplus \mathcal{T} E^{(2)}$ which give the same element in $\mathcal{O}$. If they are of arity 1, then one is made up of two elements $\sigma$ and $\tau$ of a symmetric group $\mathbb{S}_{n}$. So, the other one is either the identity if $\tau=\sigma^{-1}$ or made up by the element $\sigma \tau$. In both cases, $x_{1}-x_{2}$ lives in $R$ by Relations (O)-1) and (O-2) If they are of arity 2, they are both made up of one binary generator of $E$ and one element of the symmetric

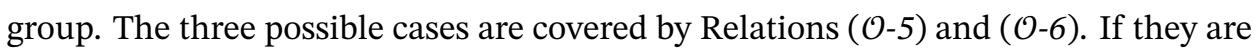
of arity 3, they are both made up of two binary generators of $E$ and Relations (O-3) and (O-4) express all the possible ways to write such elements. Thus, we have shown that $(R) \cap\left\{I \oplus E \oplus \mathcal{T} E^{(2)}\right\} \subset R$, which concludes the proof.

Under Conditions (I) and (II) one can endow the quadratic Koszul dual cooperad $q \mathcal{O}^{i}$ with a curved cooperad structure

$$
\mathcal{O}^{i}:=\left(q \mathcal{O}^{i}, \mathrm{~d}_{\mathcal{O}^{i}}, \theta\right),
$$

defining the Koszul dual curved cooperad. Such a structure amounts to a cocurvature $\theta: q \mathcal{O}^{i} \rightarrow I$, which is a degree -2 map satisfying $\theta \circ \mathrm{d}_{\mathcal{O}^{i}}=0$ and controlling the defect for the degree -1 coderivation $d_{\mathcal{O}}$ to square to zero:

$$
\left(\mathrm{d}_{\mathcal{O}_{\mathrm{i}}}\right)^{2}=(\mathrm{id} \otimes \theta-\theta \otimes \mathrm{id}) \circ \Delta_{(1)} .
$$

Condition (I) ensures that there exists a map $\varphi: q R \rightarrow E \oplus I$ such that the space of relations $R$ is the graph of $\varphi$, i.e., $R=\{X-\varphi(X) \mid X \in q R\}$. Let us denote the two summands of this map by $\varphi=\varphi_{1}+\varphi_{0}$, where $\varphi_{1}: q R \rightarrow E$ and $\varphi_{0}: q R \rightarrow I$ are defined 
by

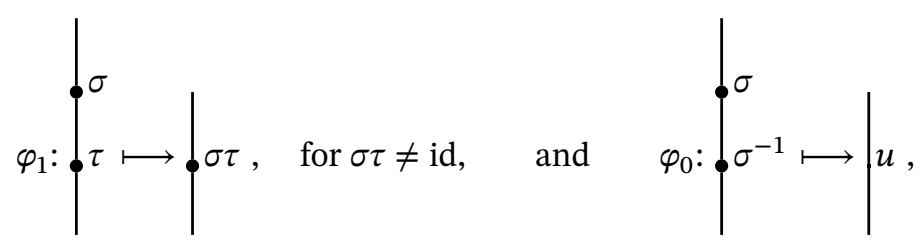

and 0 otherwise.

There exists a unique coderivation $q \mathcal{O}^{i} \rightarrow \mathcal{T}^{c}(s E)$ which extends the map

$$
q \mathcal{O}^{i} \longrightarrow s^{2} q R \stackrel{s^{-1} \varphi_{1}}{\longrightarrow} s E
$$

Condition (II) implies that its image lies in the subcooperad $q \mathcal{O}^{i} \subset \mathcal{T}^{c}(s E)$, see 31 , Lemma 4.1.1]. This defines the coderivation $\mathrm{d}_{\mathcal{O}_{i}}: q \mathcal{O}^{i} \rightarrow q \mathcal{O}^{i}$.

Proposition 1.6.2. The coderivation $\mathrm{d}_{\mathcal{O}^{i}}: q \mathcal{O}^{i} \rightarrow q \mathcal{O}^{i}$ is equal to

$$
\mathrm{d}_{\mathcal{O}}\left(t\left(\bar{\sigma}_{1}, \ldots, \bar{\sigma}_{k}\right)\right)=\sum_{j=1}^{k} \sum_{l=1}^{i_{j}-1} \pm t\left(\bar{\sigma}_{1}, \ldots, \bar{\sigma}_{j-1},(\sigma_{j}^{1}, \ldots, \underbrace{\sigma_{j}^{l} \sigma_{j}^{l+1}}_{\neq \text {id }}, \ldots, \sigma_{j}^{i_{j}}), \bar{\sigma}_{j+1}, \ldots, \bar{\sigma}_{k}\right),
$$

where the sign is equal to

$$
(-1)^{|t|+\left|\bar{\sigma}_{1}\right|+\cdots+\left|\bar{\sigma}_{j-1}\right|+l} \text {. }
$$

ProOF. This a direct consequence of [46. Proposition 6.3.7], i.e., one applies the map $\varphi_{1}$ everywhere one can. The sign is given by the Koszul sign rule with the leftrecursive order on composition trees.

EXAMPLE 1.6.3. Assume $\rho \sigma \neq$ id and $\sigma \tau \neq$ id. We compute

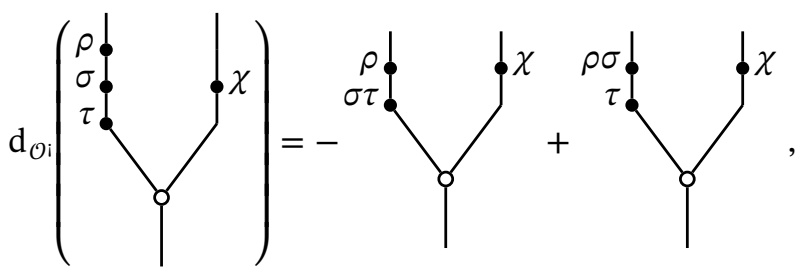

or, equivalently,

$$
\mathrm{d}_{\mathcal{O}}(t((\rho, \sigma, \tau), \chi))=t((\rho, \sigma \tau), \chi)-t((\rho \sigma, \tau), \chi)
$$

The cocurvature $\theta$ is the map defined by the composition

$$
\theta=\left(q \mathcal{O} i \longrightarrow s^{2} q R \stackrel{s^{-2} \otimes \varphi_{0}}{\longrightarrow} I\right) \text {. }
$$

Proposition 1.6.4. The cocurvature $\theta: q \mathcal{O}^{i} \rightarrow I$ is equal to zero, except for

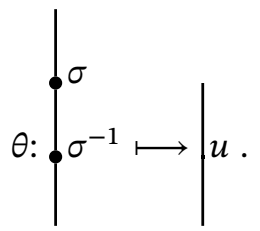

PROOF. Straightforward from the definition. 
Theorem 1.6.5. The colored operad $\mathcal{O}$, with its presentation given in Definition 1.2.1, is an inhomogeneous Koszul operad. The cobar construction of the curved Koszul dual cooperad $\mathcal{O}^{i}$ is a cofibrant resolution of the operad $\mathcal{O}$ :

$$
\Omega \mathcal{O}^{\mathrm{i}} \stackrel{\sim}{\longrightarrow} \mathcal{O} \text {. }
$$

Proof. Conditions (I) and (II)] of [31] are satisfied by Lemma 1.6.1 The homogeneous quadratic colored operad $q \mathcal{O}$ is Koszul by the distributive law method, see Proposition 1.4.2 Hence the colored operad $\mathcal{O}$ is Koszul. The second statement is a consequence of the first one by [31, Theorem 4.3.1]. Again, the proofs of [31] are only given over a field of characteristic 0 . One can extend them over a commutative ring along the lines of [16], provided that the components of the colored operad $\mathcal{O}$ are finitely generated projective $\mathbf{k}$-modules and projective $\mathbf{k}\left[\mathbb{S}_{n}\right]$-modules. This is the case here since the components of the colored operad $\mathcal{O}$ are free finitely generated $\mathbf{k}$-modules and free $\mathbf{k}\left[\mathbb{S}_{n}\right]$-modules.

\section{Homotopy Theory of Symmetric Operads}

We now apply this new Koszul model to the study of the homotopy properties of dg operads with the required properties for the symmetric group action over any ring. This gives rise to a new category of cooperads, dual to the category of nu operads, to a new bar construction, and to a new cobar construction, which properly take into account the symmetric group actions. These notions turn out to be enriched generalisations of the classical notions. Again, this is produced automatically and conceptually by the Koszul duality theory applied to the colored operad $\mathcal{O}$.

In the end, the iteration of these new bar and cobar constructions provides us with a functorial cofibrant resolution for augmented dg operads over any ring. We relate it to the classical cobar-bar construction of the Barratt-Eccles operad. These results, applied to the operad encoding commutative algebras, give rise to a cofibrant $\mathrm{E}_{\infty}$-operad and to an explicit notion of $\mathrm{E}_{\infty}$-algebra by means of generators and relations.

2.1. Higher cooperads. In this section, we give some basic definitions related to resolutions of algebras over the colored operad $\mathcal{O}$. For a recollection of the general approach, see Section 5 of Chapter 2.

Definition 2.1.1. A higher cooperad $\mathcal{C}$ is an $\mathcal{O}^{i}$-coalgebra over the curved colored cooperad $\mathcal{O}^{i}$.

Let us now make this definition explicit.

Proposition 2.1.2. A higher cooperad is a graded $\mathbb{N}$-module $\{\mathcal{C}(n)\}_{n \in \mathbb{N}}$ equipped with symmetric group "coactions" and partial decomposition maps:

$$
\begin{array}{ll}
\delta_{\sigma}: \mathcal{C}(n) \longrightarrow \mathcal{C}(n), & \text { for } \sigma \in \mathbb{S}_{n} \backslash\left\{\mathrm{id}_{n}\right\}, \\
\Delta_{i}: \mathcal{C}(n+k-1) \longrightarrow \mathcal{C}(n) \otimes \mathcal{C}(k), & \text { for } 1 \leq i \leq n,
\end{array}
$$

both of degree $\left|\delta_{\sigma}\right|=\left|\Delta_{i}\right|=-1$. These are required to satisfy the signed parallel and the signed sequential decomposition axioms 
(i) $\left(\Delta_{j} \otimes \mathrm{id}\right) \Delta_{i}=-(23)\left(\Delta_{i} \otimes \mathrm{id}\right) \Delta_{j+k-1}$ when $i<j$,

(ii) $\left(\mathrm{id} \otimes \Delta_{j}\right) \Delta_{i}=-\left(\Delta_{i} \otimes \mathrm{id}\right) \Delta_{j+i-1}$,

and the compatibility axiom

(iii) $\Delta_{i} \delta_{\tau}= \begin{cases}-\left(\mathrm{id} \otimes \delta_{\sigma}\right) \Delta_{i}, & \text { if } \tau=\sigma^{\prime}, \\ -\left(\delta_{\sigma} \otimes \mathrm{id}\right) \Delta_{\sigma^{-1}(i)}, & \text { if } \tau=\sigma^{\prime \prime}, \\ 0, & \text { otherwise. }\end{cases}$

A higher cooperad is also endowed with a degree -1 map of $\mathbb{N}$-modules $\mathrm{d}_{\mathcal{C}}: \mathcal{C} \rightarrow \mathcal{C}$ satisfying the coderivation property, i.e.,

$$
\Delta_{i} \mathrm{~d}_{\mathcal{C}}=-\left(\mathrm{d}_{\mathcal{C}} \otimes \mathrm{id}+\mathrm{id} \otimes \mathrm{d}_{\mathcal{C}}\right) \Delta_{i},
$$

and

$$
\delta_{\sigma} \mathrm{d}_{\mathcal{C}}=-\mathrm{d}_{\mathcal{C}} \delta_{\sigma}+\sum_{\substack{\tau, \omega \in \mathbb{S}_{n}\{\{\mathrm{id}\} \\ \tau \omega=\sigma}} \delta_{\tau} \delta_{\omega}
$$

and the curved differential property

$$
\left(\mathrm{d}_{\mathcal{C}}\right)^{2}=-\sum_{\sigma \in \mathbb{S}_{n} \backslash\left\{\mathrm{id}_{n}\right\}} \delta_{\sigma} \delta_{\sigma^{-1}}, \quad \text { on } \mathcal{C}(n) .
$$

Proof. By definition, an $\mathcal{O}^{\mathrm{i}}$-coalgebra over the curved colored cooperad $\mathcal{O}^{\mathrm{i}}=$ $\left(q \mathcal{O}^{\mathrm{i}}, \mathrm{d}_{\mathcal{O}^{i}}, \theta\right)$ is a triple $\left(\mathcal{C}, \Delta_{\mathcal{C}}, \mathrm{d}_{\mathcal{C}}\right)$ consisting of a $q \mathcal{O}^{\mathrm{i}}$-coalgebra and a degree -1 coderivation satisfying

$$
\left(\mathrm{d}_{\mathcal{C}}\right)^{2}=\left(-\theta \circ \mathrm{id}_{\mathcal{C}}\right) \Delta_{\mathcal{C}}
$$

From the results of Section 1.4 we see that a $q \mathcal{O}^{\mathrm{i}}$-coalgebra is a graded $\mathbb{N}$-module $\{\mathcal{C}(n)\}_{n \in \mathbb{N}}$ equipped with a structure map

$$
\Delta_{\mathcal{C}}: \mathcal{C} \longrightarrow \widehat{q \mathcal{O}^{\mathrm{i}}}(\mathcal{C})=\prod_{n}\left(q \mathcal{O}^{\mathrm{i}}(n) \otimes \mathcal{C}^{\otimes n}\right)^{\mathbb{S}_{n}}
$$

compatible with the decomposition map $\Delta_{q \mathcal{O}}$ of the cooperad $q \mathcal{O}^{i}$. Therefore the data of this structure map $\Delta_{\mathcal{C}}$ is completely determined by its image on the space of cogenerators $s E$ of $q \mathcal{O}^{i}$. The part made up of the unary generators $s \sigma$ in $s E(\mathcal{C})$ produces the maps $\delta_{\sigma}$ and the part consisting of the binary generators corresponding to the $o_{i}$-products in $s E(\mathcal{C})$ produces the maps $\Delta_{i}$. The corelations $s^{2} q R$ of $q \mathcal{O}^{i}$ give the Relations $\left(q \mathcal{O}^{\text {! }}\right.$ 1) $\left(q \mathcal{O}^{!}-3\right)$. Note that there are no relations among the coactions of the symmetric groups $\mathbb{S}_{n}$.

The coderivation property of the map $d_{\mathcal{C}}$ amounts to the commutativity of the following diagram:

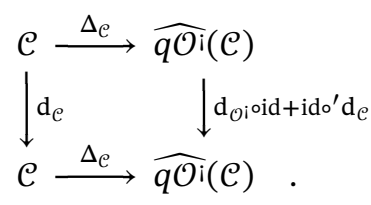

Since the structure map $\Delta_{\mathcal{C}}$ of a higher cooperad is compatible with the decomposition map $\Delta_{q \mathcal{O}}$ of the cooperad $q \mathcal{O}^{i}$, it is enough to check the commutativity of the above diagram on the part of the image which lands on the space of cogenerators $s E$ of $q \mathcal{O}^{i}$. 
The part which gives the binary generator corresponding to the $\circ_{i}$-product in $s E(\mathcal{C})$ produces the coderivation property with respect to $\Delta_{i}$ (Equation (77)), and the part which gives the unary generators $s \sigma$ in $s E(\mathcal{C})$ produces the coderivation property with respect to $\delta_{\sigma}($ Equation (78) $)$. Note that in the first case, the coderivation $\mathrm{d}_{\mathcal{O}}$ plays no role and that, in the second case, it is responsible for the term

$$
\sum_{\tau, \omega \in \mathbb{S}_{n} \backslash\{\mathrm{id}\}, \tau \omega=\sigma} \delta_{\tau} \delta_{\omega} .
$$

Finally, the cocurvature relation $\left(\mathrm{d}_{\mathcal{C}}\right)^{2}=\left(-\theta \circ \mathrm{id}_{\mathcal{C}}\right) \Delta_{\mathcal{C}}$ gives the (co)curved differential property (Equation (79)).

REMARK 2.1.3. As usual, the underlying suspension $s \mathcal{C}$ of a $q \mathcal{O}^{i}$-coalgebra $\mathcal{C}$ gives a notion with fewer signs, see [46, §11.1.3]. For instance, the category made up of suspensions of higher cooperads $s \mathcal{C}$ with trivial symmetric group coactions is isomorphic to the category made up of suspensions of dg ns cooperads without counits. In general, the suspension of a higher cooperad amounts to an ns cooperad without counit equipped with compatible free coactions of the symmetric groups, i.e., Axioms (i) (iii) without sign, equipped with a curved "differential". Note that a cooperad is not an example of a higher cooperad, due to the discrepancy between symmetric group actions in the former and symmetric group coactions is the latter.

By definition, a coalgebra over a cooperad is a coalgebra over the associated comonad, which is

$$
\mathcal{C} \longrightarrow \widehat{q \mathcal{O}}(\mathcal{C})=\prod_{n}\left(q \mathcal{O}^{i}(n) \otimes \mathcal{C}^{\otimes n}\right)^{\mathbb{S}_{n}}
$$

here. Since the cooperad $q \mathcal{O}^{i}$ is weight graded, so is the induced comonad, that is $\mathcal{C} \rightarrow \prod_{r} q \mathcal{O}^{i(r)}(\mathcal{C})$. One can introduce the following coradical filtration to detect which part of a coalgebra is decomposed in each weight component. We consider

$$
\mathcal{F}_{1}:=\left\{c \in \mathcal{C} \mid \Delta_{i}(c)=0 \text { and } \delta_{\sigma}(c)=0 \text {, for any } i \text { and any } \sigma\right\} .
$$

More generally, the space $\mathcal{F}_{r}$ is made up of elements $c \in \mathcal{C}$ which vanish under the composition of $r$ maps among $\Delta_{i}$ or $\delta_{\sigma}$.

Definition 2.1.4. A conilpotent higher cooperad is a higher cooperad whose coradical filtration is exhaustive.

Equivalently, this means that its structure map factors through the direct sum of the weight components, that is $\mathcal{C} \rightarrow \bigoplus_{r} q \mathcal{O}^{i}{ }^{(r)}(\mathcal{C})$. From now on, the notation $q \mathcal{O}^{i}\left(\_\right)$ will always mean $\bigoplus_{r} q \mathcal{O}^{i(r)}\left({ }^{(}\right)$.

EXAMPLE 2.1.5. Let us give the most important example of a $q \mathcal{O}^{i}$-coalgebra used in this chapter, which is cofree among conilpotent $q \mathcal{O}^{i}$-coalgebras. For any graded $\mathbb{N}$-module 
$\mathcal{P}$, we consider the graded $\mathbb{N}$-module

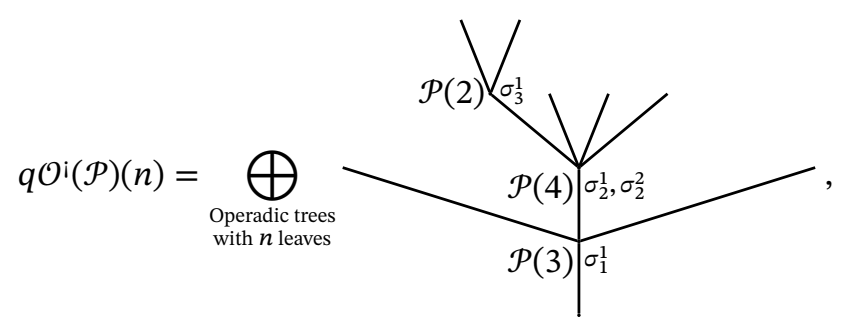

where the underlying operadic trees with vertices labeled by strings $\sigma_{j}^{1}, \sigma_{j}^{2}, \ldots, \sigma_{j}^{i_{j}}$ of permutations correspond to the basis given in Proposition 1.4.7. If an operadic tree can be obtained by grafting a subtree with $k$ leaves on a subtree with $n$ leaves at the $i$-th leaf, then its image under the map

$$
\Delta_{i}: q \mathcal{O}^{\mathrm{i}}(\mathcal{P})(n+k-1) \longrightarrow q \mathcal{O}^{\mathrm{i}}(\mathcal{P})(n) \otimes q^{\mathrm{O}}(\mathcal{P})(k)
$$

is the pair of these two subtrees; otherwise, it is 0 . The image of an operadic tree under $\delta_{\tau}$ is the sum of several contributions. For each vertex $j$, one considers the last permutation $\sigma=\sigma_{j}^{i_{j}}$ of the string and pulls it out of the operadic tree step by step changing it into $\sigma^{\prime}$ or $\sigma^{\prime \prime}$. (This process is similar to Step 4 of Section 1.5). If the final permutation is equal to $\tau$, the remaining operadic tree contributes to $\delta_{\tau}$.

The subcategory of conilpotent higher cooperads provides us with the correct target (resp. source) category for the forthcoming new bar (resp. cobar) construction.

2.2. Higher bar construction. Recall from the general theory described in Section 5 of Chapter 2 (or see [46. Chapter 11] and [31, §5.2] for details), that any curved twisting morphism $\kappa: \mathcal{O}^{i} \rightarrow \mathcal{O}$ gives rise to a pair of adjoint functors

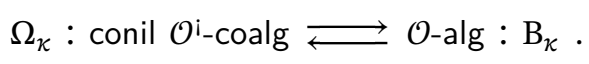

We consider here the canonical Koszul morphism $\kappa: \mathcal{O}^{i} \rightarrow \mathcal{O}$ which desuspends the generators

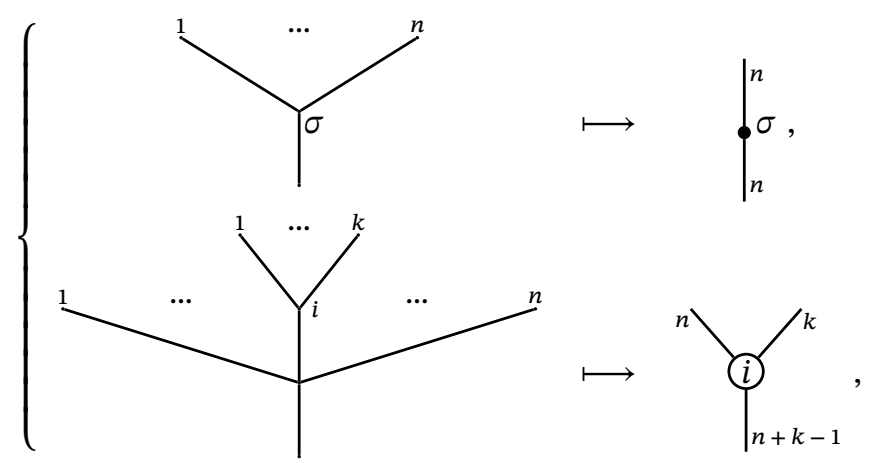

and which is equal to zero otherwise. This gives rise to the following higher cobar-bar pair of adjoint functors:

$$
\widetilde{\Omega}: \text { conil higher Coopd } \rightleftarrows \text { nu Opd : } \widetilde{\mathrm{B}} \text {. }
$$


Proposition 2.2.1. The higher bar construction

$$
\widetilde{\mathrm{B}} \mathcal{P}:=\mathrm{B}_{\kappa} \mathcal{P}=\left(q \mathcal{O}(\mathcal{P}), \mathrm{d}_{1}+\mathrm{d}_{2}\right)
$$

of a nonunital operad $\mathcal{P}$ is the $\mathcal{O}^{\mathrm{i}}$-coalgebra defined on the cofree $q \mathcal{O}^{\mathrm{i}}$-coalgebra $q \mathcal{O}^{\mathrm{i}}(\mathcal{P})$ by the curved codifferential $\mathrm{d}_{1}+\mathrm{d}_{2}$, where $\mathrm{d}_{1}$ is the coderivation

$$
\mathrm{d}_{1}: q \mathcal{O}^{\mathrm{i}}(\mathcal{P}) \stackrel{\mathrm{d}_{\mathcal{O} \mathrm{i}}(\mathcal{P})+q \mathcal{O}^{\mathrm{i}}\left(\mathrm{d}_{\mathcal{P}}\right)^{\prime}}{\longrightarrow} q \mathcal{O}^{\mathrm{i}}(\mathcal{P}) \text {, }
$$

and where $\mathrm{d}_{2}$ is the unique coderivation extending the action of $\kappa$

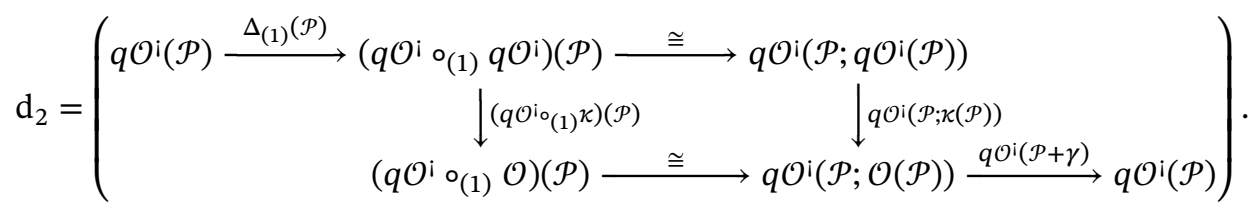

REMARK 2.2.2. Since the category of nonunital operads is equivalent to the category of augmented operads, one can define the higher bar construction of an augmented operad by $\widetilde{\mathrm{B}} \overline{\mathcal{P}}:=\left(q \mathcal{O}^{\mathrm{i}}(\overline{\mathcal{P}}), \mathrm{d}_{1}+\mathrm{d}_{2}\right)$.

The higher bar construction $\widetilde{\mathrm{B}} \mathcal{P}$ of a dg nu operad $\mathcal{P}$ is given by the sum of operadic trees with vertices labeled by the elements of $\mathcal{P}$ and by strings of permutations that we denote by

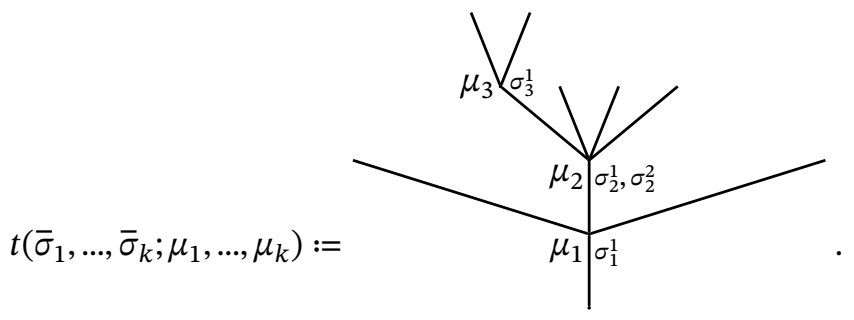

The coderivation $d_{1}$ is equal to the sum

$$
\begin{aligned}
& \sum_{j=1}^{k} \sum_{l=1}^{i_{j}-1}(-1)^{|t|+\left|\bar{\sigma}_{1}\right|+\cdots+\left|\bar{\sigma}_{j-1}\right|+l} t\left(\bar{\sigma}_{1}, \ldots, \bar{\sigma}_{j-1},(\sigma_{j}^{1}, \ldots, \underbrace{\sigma_{j}^{l} \sigma_{j}^{l+1}}_{\neq \text {id }}, \ldots, \sigma_{j}^{i_{j}}), \bar{\sigma}_{j+1}, \ldots, \bar{\sigma}_{k} ; \mu_{1}, \ldots, \mu_{k}\right) \\
& +\sum_{j=1}^{k}(-1)^{|t(\sigma)|+\left|\mu_{1}\right|+\cdots+\left|\mu_{j-1}\right|} t\left(\bar{\sigma}_{1}, \ldots, \bar{\sigma}_{k} ; \mu_{1}, \ldots, \mathrm{d}_{\mathcal{P}}\left(\mu_{j}\right), \ldots, \mu_{k}\right) .
\end{aligned}
$$

The coderivation $d_{2}$ is equal to

$$
\begin{aligned}
& \sum_{j=1}^{k}(-1)^{|t|+\left|\bar{\sigma}_{1}\right|+\cdots+\left|\bar{\sigma}_{j-1}\right|} t\left(\bar{\sigma}_{1}, \ldots,\left(\sigma_{j}^{2}, \ldots, \sigma_{j}^{i j}\right), \ldots, \bar{\sigma}_{k} ; \mu_{1}, \ldots, \mu_{k}^{\sigma_{j}^{1}}, \ldots, \mu_{k}\right) \\
& \quad+\sum_{\substack{\text { internal edges } \\
j-e-l}} \pm t / e\left(\bar{\sigma}_{1}, \ldots, w\left(\bar{\sigma}_{j}, \bar{\sigma}_{l}\right), \ldots, \bar{\sigma}_{l-1}, \bar{\sigma}_{l+1}, \ldots, \bar{\sigma}_{k} ; \mu_{1}, \ldots, \mu_{j} \circ_{m} \mu_{l}, \ldots, \mu_{l-1}, \mu_{l+1}, \ldots, \mu_{k}\right),
\end{aligned}
$$

where the internal edge $e$ joins the vertex $j$ to the vertex $l$ at the $i^{\text {th }}$-place, where $m=$ $\sigma_{j}^{1} \cdots \sigma_{j}^{i_{j}}(i)$ and where the sign \pm is given by the same procedure as in Section 1.5

2.3. Higher cobar construction. Below, we make the left adjoint $\widetilde{\Omega}$ of the higher bar construction $\widetilde{\mathrm{B}}$ explicit. 
Proposition 2.3.1. The higher cobar construction

$$
\widetilde{\Omega} \mathcal{C}:=\Omega_{\mathcal{K}} \mathcal{C}=\left(\mathcal{O}(\mathcal{C}), \mathrm{d}_{1}-\mathrm{d}_{2}\right)
$$

of a higher cooperad $\left(\mathcal{C}, \Delta_{\mathcal{C}}, \mathrm{d}_{\mathcal{C}}\right)$ is the dg nu operad defined on $\mathcal{O}(\mathcal{C})$ by the differential $\mathrm{d}_{1}-\mathrm{d}_{2}$, where $\mathrm{d}_{1}$ is the underlying derivation

$$
\mathrm{d}_{1}: \mathcal{O}(\mathcal{C}) \stackrel{\mathcal{O}\left(\mathrm{d}_{\mathcal{C}}\right)^{\prime}}{\longrightarrow} \mathcal{O}(\mathcal{C})
$$

and where $\mathrm{d}_{2}$ is the unique derivation extending the action of $\kappa$

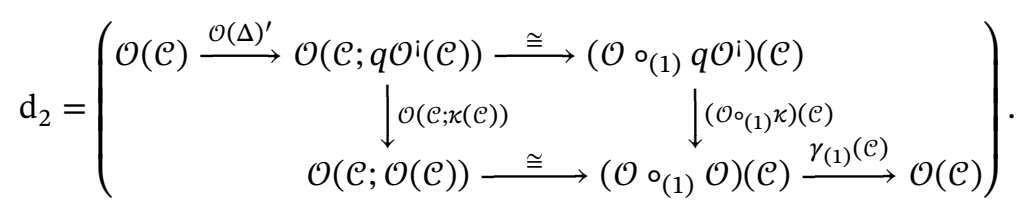

The cobar construction $\widetilde{\Omega} \mathcal{C}$ of a higher cooperad $\mathcal{C}$ is given by the sum of operadic trees with the vertices labeled by the elements of $\mathcal{C}$ and with the leaves globally labeled by a permutation, that we denote by

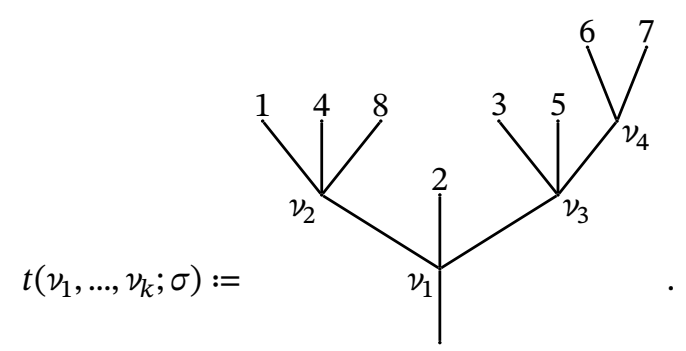

The derivation $d_{1}$ is equal to

$$
\sum_{j=1}^{k}(-1)^{\left|\nu_{1}\right|+\cdots+\left|\nu_{j-1}\right|} t\left(\nu_{1}, \ldots, \mathrm{d}_{\mathcal{C}}\left(\nu_{j}\right), \ldots, \nu_{k} ; \sigma\right) .
$$

The derivation $\mathrm{d}_{2}$ is equal to

$$
\begin{aligned}
& \sum_{j=1}^{k} \sum_{i=1}^{n_{j}}(-1)^{\left|\nu_{1}\right|+\cdots+\left|\nu_{j-1}\right|} t \sqcup e_{j}^{i}\left(\nu_{1}, \ldots, \Delta_{i}^{\prime}\left(\nu_{j}\right), \Delta_{i}^{\prime \prime}\left(\nu_{j}\right), \ldots, v_{k} ; \sigma\right) \\
& +\sum_{j=1}^{k} \sum_{\tau \in \mathbb{S}_{n_{j}} \backslash\left\{\mathrm{id}_{n_{j}}\right\}}(-1)^{\left|\nu_{1}\right|+\cdots+\left|\nu_{j-1}\right|} \tilde{t}\left(\nu_{1}, \ldots, \delta_{\tau}\left(\nu_{j}\right), \ldots, \nu_{k} ; \tilde{\tau} \sigma\right),
\end{aligned}
$$

where $n_{j}$ is the arity of vertex $j$, where $t \sqcup e_{j}^{i}$ represents the tree $t$ with an extra edge at vertex $j$ and where $\Delta_{i}\left(\nu_{j}\right)=\Delta_{i}^{\prime}\left(\nu_{j}\right) \otimes \Delta_{i}^{\prime \prime}\left(\nu_{j}\right)$.

2.4. Higher cobar-bar adjunction. Let $\mathcal{C}$ be a higher cooperad and let $\mathcal{P}$ be a $\mathrm{dg}$ nu operad. We consider the mapping space

$$
\operatorname{Hom}(\mathcal{C}, \mathcal{P}):=\prod_{n \in \mathbb{N}} \operatorname{Hom}(\mathcal{C}(n), \mathcal{P}(n))
$$


endowed with the derivation $\partial(f):=\mathrm{d}_{\mathcal{P}} \circ f-(-1)^{|f|} \cdot f \circ \mathrm{d}_{\mathcal{C}}$ and the following two degree -1 operations:

$$
f * g:=\sum_{i}\left(\mathcal{C} \stackrel{\Delta_{i}}{\longrightarrow} \mathcal{C} \otimes \mathcal{C} \stackrel{f \otimes g}{\longrightarrow} \mathcal{P} \otimes \mathcal{P} \stackrel{\mathcal{L}^{\circ i}}{\longrightarrow} \mathcal{P}\right)
$$

and

$$
\Delta(f):=\sum_{\sigma \in \mathbb{S}_{n} \backslash\left\{\mathrm{id}_{n}\right\}}\left(\mathcal{C}(n) \stackrel{\delta_{\sigma}}{\longrightarrow} \mathcal{C}(n) \stackrel{f(n)}{\longrightarrow} \mathcal{P}(n) \stackrel{(\lrcorner)^{\sigma}}{\longrightarrow} \mathcal{P}(n)\right) .
$$

Proposition 2.4.1. These operations satisfy the following relations:

(80) $(-1)^{|\gamma|}(\alpha * \beta) * \gamma+\alpha *(\beta * \gamma)+(-1)^{|\beta||\gamma|}\left((-1)^{|\beta|}(\alpha * \gamma) * \beta+\alpha *(\gamma * \beta)\right)=0$,

$$
\Delta(\alpha * \beta)+(-1)^{|\beta|}(\Delta \alpha) * \beta+\alpha *(\Delta \beta)=0 .
$$

PROOF. These relations are checked in a straightforward way using the axioms defining the notions of an nu operad and a higher cooperad. The "shifted" pre-Lie relation Equation (80) is proven using Axioms (Opd-3) and (Opd-4) of the definition of an nu operad and Axioms (i) and (ii) in the definition of a higher cooperad. The "shifted" derivation relation (Equation (81) $)$ is proven using Axioms (Opd-5) and (Opd-6) in the definition of an nu operad and Axiom (iii) in the definition of a higher cooperad.

REMARK 2.4.2. In the classical case, the convolution algebra made up of maps from a cooperad to an operad forms a pre-Lie algebra, see [46, \$6.4.2]. Because of the slightly different sign convention chosen here, the $*$-product satisfies the relation of a "shifted" pre-Lie algebra. Since $\mathcal{C}$ is a higher cooperad, this enrichment gives rise to the operator $\Delta$ which is a "shifted" derivation with respect to the $*$-product.

We call a degree 0 morphism of $\mathbb{N}$-modules $\alpha: \mathcal{C} \rightarrow \mathcal{P}$ satisfying the Maurer-Cartan equation

$$
\partial \alpha+\alpha * \alpha+\Delta \alpha=0
$$

a twisting morphism with respect to $\kappa$, or simply a twisting morphism when the context is obvious. The associated set of solutions is denoted by $\operatorname{Tw}(\mathcal{C}, \mathcal{P})$.

Proposition 2.4.3. The higher cobar and bar constructions $\widetilde{\Omega}$ and $\widetilde{\mathrm{B}}$ form a pair of adjoint functors

$$
\widetilde{\Omega}: \text { conil higher Coopd } \rightleftarrows \text { nu Opd : } \widetilde{\mathrm{B}}
$$

and are represented by the set of twisting morphisms:

$$
\operatorname{Hom}_{\mathrm{dg} \text { nu Opd }}(\widetilde{\Omega} \mathcal{C}, \mathcal{P}) \cong \operatorname{Tw}(\mathcal{C}, \mathcal{P}) \cong \operatorname{Hom}_{\text {conil higher Coop }}(\mathcal{C}, \widetilde{\mathrm{B}} \mathcal{P}) .
$$

PROOF. The arguments and computations are similar to the ones of [46, §11.1].

2.5. Higher cobar-bar resolution. In this section, we show that the counit of the higher cobar-bar adjunction provides us with a resolution for augmented operads with nice homotopy properties. 
Proposition 2.5.1. Let $\mathcal{C}$ be a nonnegatively graded conilpotent higher cooperad $\mathcal{C}$ made up of projective $\mathbf{k}$-modules. The augmentation $I \oplus \widetilde{\Omega} \mathcal{C}$ of its cobar construction is a cofibrant dg operad.

PROOF. Let us first mention that we are working with the semimodel category structure defined on dg operads in [64], where weak equivalences are aritywise quasiisomorphisms and where fibrations are aritywise epimorphisms. Let $f$ and $b$ be two morphisms of dg operads

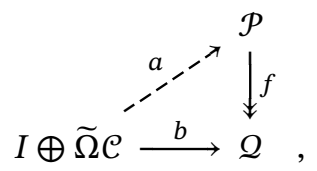

where $f$ is an acyclic fibration. And let us look for a morphism $a$ of $\mathrm{dg}$ operads which factors $b$ through $f$. The augmentation $I \oplus \widetilde{\Omega} \mathcal{C}$ of a dg nu operad gives an augmented operad. And the data of a morphism of dg operads $I \oplus \widetilde{\Omega} \mathcal{C} \rightarrow \mathcal{Q}$ is equivalent to the data of a morphism $\widetilde{\Omega} \mathcal{C} \rightarrow \mathcal{Q}$ of dg nu operads. So, according to Proposition 2.4.3, the data of a morphism of dg operads $b: I \oplus \widetilde{\Omega} \mathcal{C} \rightarrow \mathcal{Q}$ is equivalent to the data of a twisting morphism with respect to $\kappa$, i.e., a degree $0 \operatorname{map} \beta: \mathcal{C} \rightarrow \mathcal{Q}$ satisfying the Maurer-Cartan Equation (82)

We first set $a(\mathrm{id}):=\mathrm{id}_{\mathcal{P}}$ and the rest of the morphism $a$ is completely determined by its restriction $\alpha:=\left.a\right|_{\mathcal{C}}$ on $\mathcal{C}$, which has to satisfy the Maurer-Cartan Equation (82) We construct $\alpha$ by induction on the homological degree; we use the notation $\alpha_{d}$ for the restriction of $\alpha$ to $\mathcal{C}_{d}$. The Maurer-Cartan Equation (82) for an element $c \in \mathcal{C}_{d}$ of degree $d$ reads

$$
\mathrm{d}_{\mathcal{P}}(\alpha(c))=\alpha\left(\mathrm{d}_{\mathcal{C}}(c)\right)-(\alpha * \alpha)(c)-\Delta(\alpha)(c),
$$

where $\left|\mathrm{d}_{\mathcal{C}}(c)\right|=d-1,\left|\Delta_{i}(c)\right|=d-1$ and $\left|\delta_{\sigma}(c)\right|=d-1$. Therefore, the right-hand term amounts to applying the map $\alpha$ to elements of degree at most $d-1$. Since $\mathcal{C}$ is nonnegatively graded, we define $\alpha_{d}$ to be trivial on $\mathcal{C}_{d}$, for $d<0$. We suppose now that $\alpha$ is known up to degree $d-1$ and that is satisfies

$$
\mathrm{d}_{\mathcal{P}} \alpha_{e}=\alpha_{e-1} \mathrm{~d}_{\mathcal{C}}-\left(\sum_{\substack{k+l=e-1 \\ k, l \leq e-1}} \alpha_{k} * \alpha_{l}\right)-\Delta\left(\alpha_{e-1}\right)
$$

for $e \leq d-1$. Let us now construct $\alpha_{d}$; we consider the following diagram

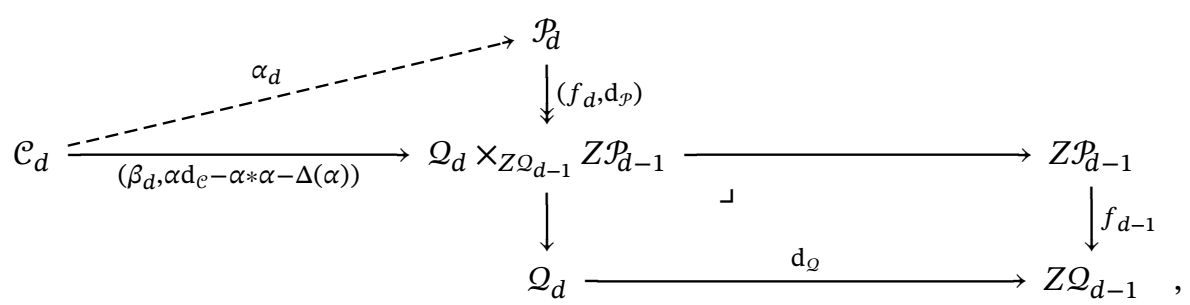

where $Z \mathcal{P}_{d-1}$ and $Z \mathcal{Q}_{d-1}$ stand for the set of cycles in $\mathcal{P}_{d-1}$ and $\mathcal{Q}_{d-1}$. As explained above, the map $\alpha \mathrm{d}_{\mathcal{C}}-\alpha * \alpha-\Delta(\alpha)$ has already been defined on $\mathcal{C}_{d}$. We still have to prove that the image of $\left(\beta_{d}, \alpha \mathrm{d}_{\mathcal{C}}-\alpha * \alpha-\Delta(\alpha)\right)$ lands in $Q_{d} \times_{Z Q_{d-1}} Z \mathcal{P}_{d-1}$. We first compute the 
composition with $d_{\mathcal{P}}$ of the right-hand term. By the induction hypothesis, since $d_{\mathcal{P}}$ is a derivation of the operad $\mathcal{P}$ and by the definition of $\mathcal{C}$ being a higher cooperad, we have

$$
\begin{aligned}
& \mathrm{d}_{\mathcal{P}}\left(\alpha_{d-1} \mathrm{~d}_{\mathcal{C}}-\left(\sum_{\substack{k+l=d-1 \\
k, l \leq d-1}} \alpha_{k} * \alpha_{l}\right)-\Delta\left(\alpha_{d-1}\right)\right) \\
& =\mathrm{d}_{\mathcal{P}} \alpha_{d-1} \mathrm{~d}_{\mathcal{C}}-\sum_{i} \sum_{\substack{k+l=d-1 \\
k, l \leq d-1}} \mathrm{~d}_{\mathcal{P}} \circ_{i}\left(\alpha_{k} \otimes \alpha_{l}\right) \Delta_{i}-\sum_{\sigma \in \overline{\mathbb{S}}_{n}} \mathrm{~d}_{\mathcal{P}}\left({ }^{\sigma}\right)^{\sigma} \alpha_{d-1} \delta_{\sigma} \\
& =\mathrm{d}_{\mathcal{P}} \alpha_{d-1} \mathrm{~d}_{\mathcal{C}}-\sum_{i} \sum_{\substack{k+l=d-1 \\
k, l \leq d-1}} \circ_{i}\left(\mathrm{~d}_{\mathcal{P}} \alpha_{k} \otimes \alpha_{l}\right) \Delta_{i}-\sum_{i} \sum_{\substack{k+l=d-1 \\
k, l \leq d-1}} \circ_{i}\left(\alpha_{k} \otimes \mathrm{d}_{\mathcal{P}} \alpha_{l}\right) \Delta_{i} \\
& -\sum_{\sigma \in \overline{\mathbb{S}}_{n}}\left({ }_{-}\right)^{\sigma} \mathrm{d}_{\mathcal{P}} \alpha_{d-1} \delta_{\sigma} \\
& =\mathrm{d}_{\mathcal{P}} \alpha_{d-1} \mathrm{~d}_{\mathcal{C}}-\sum_{i} \sum_{\substack{k+l=d-1 \\
k, l \leq d-1}} \circ_{i}\left(\left(\alpha_{k-1} \mathrm{~d}_{\mathcal{C}}-\sum_{\substack{j+m=k-1 \\
j, m \leq k-1}} \alpha_{j} * \alpha_{m}-\Delta\left(\alpha_{k-1}\right)\right) \otimes \alpha_{l}\right) \Delta_{i} \\
& -\sum_{i} \sum_{\substack{k+l=d-1 \\
k, l \leq d-1}} \circ_{i}\left(\alpha_{k} \otimes\left(\alpha_{l-1} \mathrm{~d}_{\mathcal{C}}-\sum_{\substack{j+m=l-1 \\
j, m \leq l-1}} \alpha_{j} * \alpha_{m}-\Delta\left(\alpha_{l-1}\right)\right) \Delta_{i}\right.
\end{aligned}
$$

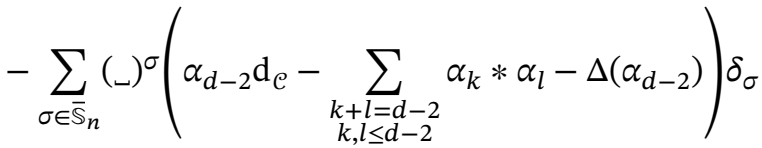

$$
\begin{aligned}
& =\mathrm{d}_{\mathcal{P}} \alpha_{d-1} \mathrm{~d}_{\mathcal{C}}+\sum_{\substack{k+l=d-2 \\
k, l \leq d-1}}\left(\alpha_{k} * \alpha_{l}\right) \mathrm{d}_{\mathcal{C}}+\Delta\left(\alpha_{d-2}\right) \mathrm{d}_{\mathcal{C}} \\
& +\sum_{\substack{j+k+l=d-2 \\
j, k, l \leq d-2}}\left(\left(\alpha_{j} * \alpha_{k}\right) * \alpha_{l}+\alpha_{j} *\left(\alpha_{k} * \alpha_{l}\right)\right) \\
& +\sum_{i} \sum_{\substack{k+l=d-2 \\
k, l \leq d-2}}\left(\Delta\left(\alpha_{k}\right) * \alpha_{l}+\alpha_{k} * \Delta\left(\alpha_{l}\right)+\Delta\left(\alpha_{k} * \alpha_{l}\right)\right)+\Delta^{2}\left(\alpha_{d-2}\right) \\
& -\sum_{\sigma \in \overline{\mathbb{S}}_{n}} \sum_{\substack{\tau, \omega \in \overline{\mathbb{S}}_{n} \\
\tau \omega=\sigma}}()^{\omega}\left({ }^{\tau}\right)^{\tau} \alpha_{d-2} \delta_{\tau} \delta_{\omega} .
\end{aligned}
$$

Since $\alpha$ is of degree 0 , the shifted pre-Lie relation (Equation (80) implies

$$
(\alpha * \alpha) * \alpha+\alpha *(\alpha * \alpha)=0
$$

and the shifted derivation relation (Equation (81) of Proposition 2.4.1 implies

$$
\Delta(\alpha * \alpha)+(\Delta \alpha) * \alpha+\alpha *(\Delta \alpha)=0
$$

The only remaining terms are the first three ones and the last two ones, whose sum is equal to

$$
\left(\mathrm{d}_{\mathcal{P}} \alpha_{d-1}+\left(\sum_{\substack{k+l=d-2 \\ k, l \leq d-1}}\left(\alpha_{k} * \alpha_{l}\right)\right)+\Delta\left(\alpha_{d-2}\right)\right) \mathrm{d}_{\mathcal{C}}+\Delta^{2}\left(\alpha_{d-2}\right)-\sum_{\substack{\sigma, \tau, \omega \in \overline{\mathbb{S}}_{n} \\ \tau \omega=\sigma}}()^{\omega}\left({ }^{\tau}\right)^{\tau} \alpha_{d-2} \delta_{\tau} \delta_{\omega}
$$




$$
\begin{aligned}
& =\alpha_{d-2} \mathrm{~d}_{\mathcal{C}} \mathrm{d}_{\mathcal{C}}+\Delta^{2}\left(\alpha_{d-2}\right)-\sum_{\substack{\sigma, \tau, \omega \in \overline{\mathbb{S}}_{n} \\
\tau \omega=\sigma}}()^{\omega}\left({ }^{\tau}\right)^{\tau} \alpha_{d-2} \delta_{\tau} \delta_{\omega} \\
& \left.\left.\left.=-\sum_{\substack{\tau, \omega \in \overline{\mathbb{S}}_{n} \\
\tau \omega=\mathrm{id}_{n}}} \alpha_{d-2} \delta_{\tau} \delta_{\omega}+\sum_{\tau, \omega \in \overline{\mathbb{S}}_{n}}(\lrcorner\right)^{\omega}(\lrcorner\right)^{\tau} \alpha_{d-2} \delta_{\tau} \delta_{\omega}-\sum_{\substack{\sigma, \tau, \omega \in \overline{\mathbb{S}}_{n} \\
\tau \omega=\sigma}}(\lrcorner\right)^{\omega}\left({ }^{\tau}\right)^{\tau} \alpha_{d-2} \delta_{\tau} \delta_{\omega} \\
& =0,
\end{aligned}
$$

by the induction hypothesis and the defining relation Equation (79) of a higher cooperad. Thus we conclude that

$$
\mathrm{d}_{\mathcal{P}}\left(\alpha_{d-1} \mathrm{~d}_{\mathcal{C}}-\left(\sum_{\substack{k+l=d-1 \\ k, l \leq d-1}} \alpha_{k} * \alpha_{l}\right)-\Delta\left(\alpha_{d-1}\right)\right)=0 .
$$

We now prove that

$$
\mathrm{d}_{\mathcal{Q}} \beta_{d}=f_{d-1}\left(\alpha_{d-1} \mathrm{~d}_{\mathcal{C}}-\left(\sum_{\substack{k+l=d-1 \\ k, l \leq d-1}} \alpha_{k} * \alpha_{l}\right)-\Delta\left(\alpha_{d-1}\right)\right) .
$$

Since the map $\beta$ satisfies the Maurer-Cartan Equation (82), the left-hand term is equal to

$$
\mathrm{d}_{\mathcal{Q}} \beta_{d}=\beta_{d-1} \mathrm{~d}_{\mathcal{C}}-\left(\sum_{\substack{k+l=d-1 \\ k, l \leq d-1}} \beta_{k} * \beta_{l}\right)-\Delta\left(\beta_{d-1}\right) .
$$

Since $f$ is a morphism of operads and by the induction hypothesis, the right-hand term is equal to

$$
\begin{aligned}
& f_{d-1}\left(\alpha_{d-1} \mathrm{~d}_{\mathcal{C}}-\left(\sum_{\substack{k+l=d-1 \\
k, l \leq d-1}} \alpha_{k} * \alpha_{l}\right)-\Delta\left(\alpha_{d-1}\right)\right) \\
& =\beta_{d-1} \mathrm{~d}_{\mathcal{C}}-\left(\sum_{\substack{k+l=d-1 \\
k, l \leq d-1}}\left(f_{k} \alpha_{k}\right) *\left(f_{l} \alpha_{l}\right)\right)-\Delta\left(f_{d-1} \alpha_{d-1}\right) \\
& =\beta_{d-1} \mathrm{~d}_{\mathcal{C}}-\left(\sum_{\substack{k+l=d-1 \\
k, l \leq d-1}} \beta_{k} * \beta_{l}\right)-\Delta\left(\beta_{d-1}\right) .
\end{aligned}
$$

which proves the above claim.

To conclude, we use a lemma which asserts that a morphism $f: \mathcal{P} \rightarrow Q$ is a surjective quasi-isomorphism if and only if every map $\left(f_{d}, \mathrm{~d}_{\mathcal{P}}\right): \mathcal{P}_{d} \rightarrow \mathcal{Q}_{d} \times_{Z Q_{d-1}} Z \mathcal{P}_{d-1}$ is surjective. In fact, we need just the "only if" direction of this lemma, which is proven by the following diagram chasing. Suppose that $f$ is a surjective quasi-isomorphism and let $(q, p) \in Q_{d} \times_{Z Q_{d-1}} Z \mathcal{P}_{d-1}$, that is $\mathrm{d}_{Q}(q)=f_{d-1}(p)$. Since $f_{d}$ is surjective, there exists $x \in \mathcal{P}_{d}$ such that $f_{d}(x)=q$. The element $p-\mathrm{d}_{\mathcal{P}}(x) \in Z \mathcal{P}_{d-1}$ is a cycle, so it represents a homology class in $\mathcal{P}$, which is sent to the trivial homology class in $\mathcal{Q}$ under $f$ since

$$
f_{d-1}\left(p-\mathrm{d}_{\mathcal{P}}(x)\right)=f_{d-1}(p)-\mathrm{d}_{\mathcal{Q}}\left(f_{d}(x)\right)=f_{d-1}(p)-\mathrm{d}_{\mathcal{Q}}(q)=0 .
$$


Since $f$ is a quasi-isomorphism, there exists an element $y \in \mathcal{P}_{d}$ such that $\mathrm{d}_{\mathcal{P}}(y)=$ $p-\mathrm{d}_{\mathcal{P}}(x)$. The element $f_{d}(y) \in Z Q_{d}$ is a cycle of $\mathcal{Q}$ since $\mathrm{d}_{\mathcal{Q}}\left(f_{d}(y)\right)=f_{d-1}\left(\mathrm{~d}_{\mathcal{P}}(y)\right)=0$. The map $f$ being a quasi-isomorphism, there exists an element $z \in Z \mathcal{P}_{d}$ and an element $\xi \in Q_{d+1}$ such that $f(z)=f(y)+\mathrm{d}_{\mathcal{Q}}(\xi)$. Since the map $f_{d+1}$ is surjective, there exists $t \in \mathcal{P}_{d+1}$ such that $f_{d+1}(t)=\xi$. In the end, we have

$$
\left(f_{d}, \mathrm{~d}_{\mathcal{P}}\right)\left(x+y-z-\mathrm{d}_{\mathcal{P}}(t)\right)=(q, p) .
$$

Finally, the $\mathbf{k}$-modules $\mathcal{C}_{d}(n)$ are projective for any $n \in \mathbb{N}$, so there exists a map of $\mathbb{N}$-modules $\alpha_{d}: \mathcal{C}_{d} \rightarrow \mathcal{P}_{d}$, which fills the above diagram. By construction, this map satisfies both $\beta_{d}=f_{d} \alpha_{d}$ and $\mathrm{d}_{\mathcal{P}} \alpha_{d}=\alpha \mathrm{d}_{\mathcal{C}}-\alpha * \alpha-\Delta(\alpha)$, which concludes the proof.

REMARK 2.5.2. The higher cobar construction $\widetilde{\Omega} \mathcal{C}$ of a higher cooperad is a quasi-free $\mathcal{O}$-algebra. So, forgetting the differential, it is isomorphic to the free operad on the free $\mathbb{S}$-module generated by the $\mathbb{N}$-module $\mathcal{C}$. However since this latter one is not a free k-module, but only a projective one, we cannot apply (easily) the usual results, like [18, Proposition 12.2.3], which give the form of cofibrant dg operads: retracts of quasi-free dg operads generated by free $\mathbb{S}$-modules endowed with a filtration lowered by the differential. Instead, we prove directly the lifting property with respect to acyclic fibrations using the Maurer-Cartan equation.

Theorem 2.5.3. The augmentation of the higher cobar-bar counit

$$
I \oplus \widetilde{\Omega} \widetilde{\mathrm{B}} \overline{\mathcal{P}} \stackrel{\sim}{\longrightarrow} \mathcal{P}
$$

is a functorial resolution of dg augmented operads, which is cofibrant when the underlying chain complex of $\mathcal{P}$ is nonnegatively graded and made up of projective $\mathbf{k}$-modules.

Proof. As a graded $\mathbb{N}$-module, the higher cobar-bar construction is isomorphic to $\widetilde{\Omega} \widetilde{\mathrm{B}} \overline{\mathcal{P}} \cong \mathcal{O} \circ q \mathcal{O}^{i} \circ \overline{\mathcal{P}}$. So its underyling space is made up partitioned planar rooted trees with vertices labeled by the elements of $\overline{\mathcal{P}}$ and by strings of (nontrivial) permutations and with the leaves globally labeled by a permutation.

The differential d on $\widetilde{\Omega} \widetilde{\mathrm{B}} \overline{\mathcal{P}}$ is a sum of several parts. Summing up all the components of the differential maps of the higher bar construction and the higher cobar construction from Sections 2.2 and 2.3 we obtain 6 components for the differential d. First, there are four terms $d_{1}=d_{1}^{\mathcal{P}}+d_{1}^{\text {sh }}+d_{1}^{l}+d_{1}^{\text {int }}$, coming from the higher bar construction, which apply only inside each partition. The term $\mathrm{d}_{1}^{\mathcal{P}}$ comes from the differential of the operad $\mathcal{P}$. The term $\mathrm{d}_{1}^{\text {sh }}$ contracts inner edges, composes the two elements of the operad $\mathcal{P}$ labeling the associated two vertices, and shuffles the two strings of permutations labeling the two associated vertices. The term $\mathrm{d}_{1}^{1}$ removes the first element of each string of permutations and makes it act on the element of $\mathcal{P}$ labeling the vertex. The term $d_{1}^{\text {int }}$ is equal to the sum of the products of two consecutive elements of a string labeling a vertex, provided that it is not the identity, in which case it gives 0 . Then, there are two terms $d_{2}=d_{2}^{\text {split }}+d_{2}^{r}$, coming from the higher cobar construction, which apply to each partition. The term $\mathrm{d}_{2}^{\text {split }}$ splits each of the partitions into two along an inner edge. The term $\mathrm{d}_{2}^{\mathrm{r}}$ extracts the right most external term of each string labeling the 
vertex of the tree inside the partition, pulls it out of the partition and makes it act on the permutation labeling the leaves of the global tree. The total differential is then the sum

$$
\mathrm{d}=\mathrm{d}_{1}-\mathrm{d}_{2}=\left(\mathrm{d}_{1}^{\mathcal{P}}+\mathrm{d}_{1}^{\mathrm{sh}}+\mathrm{d}_{1}^{\mathrm{l}}+\mathrm{d}_{1}^{\mathrm{int}}\right)-\left(\mathrm{d}_{2}^{\mathrm{split}}+\mathrm{d}_{2}^{\mathrm{r}}\right) .
$$

We consider the following bounded below and exhaustive filtration on the higher cobar-bar construction: the component $\mathcal{F}_{r} \widetilde{\Omega} \widetilde{\mathrm{B}} \overline{\mathcal{P}}$ consists of elements such that the sum of their degree and the number of their partitions is at most $r+1$. All the above terms of the differential send $\mathcal{F}_{r}$ to $\mathcal{F}_{r-1}$, except for $\mathrm{d}_{2}^{\text {split }}: \mathcal{F}_{r} \rightarrow \mathcal{F}_{r}$. On the right-hand side, we consider the filtration on $\mathcal{P}$ defined by the homological degree. The morphism of dg nu operads $\widetilde{\Omega} \widetilde{\mathrm{B}} \overline{\mathcal{P}} \rightarrow \overline{\mathcal{P}}$ is equal to the composition

$$
\widetilde{\Omega} \widetilde{\mathrm{B}} \overline{\mathcal{P}} \cong \mathcal{O} \circ q \mathcal{O}^{i} \circ \overline{\mathcal{P}} \longrightarrow \mathcal{O} \circ \overline{\mathcal{P}} \longrightarrow \overline{\mathcal{P}},
$$

where the first map comes from the augmentation projection $q \mathcal{O}^{i} \rightarrow I$ and where the second map is given by the composition map in the operad $\mathcal{P}$. Thus, this morphism preserves the respective filtrations, and therefore it induces a morphism between the two associated spectral sequences.

The underlying space of the first page of the spectral sequence associated to $\mathcal{F}_{r} \widetilde{\Omega} \widetilde{\mathrm{B}} \overline{\mathcal{P}}$ is isomorphic to the chain complex consisting of the same partitioned labeled planar trees with differential equal only to $\mathrm{d}_{2}^{\text {split }}$. This chain complex decomposes as a direct sum of the sub-chain complexes consisting of partitioned labeled planar trees with the same underlying planar tree. When the number of inner edges is greater than 1 , this chain complex is acyclic, since it is isomorphic to the $k^{\text {th }}$-tensor power of the acyclic chain complex

$$
0 \longrightarrow \mathbf{k} \longrightarrow \mathbf{k} \longrightarrow 0
$$

where $k$ is the number of internal edges. Hence, the second page of this spectral sequence is isomorphic to $\overline{\mathcal{P}}(n) \otimes \mathrm{T}\left(s \overline{\mathbb{S}}_{n}\right)$, with differential equal to the one of $\mathcal{P}$ and the differential of the normalized bar construction of the symmetric groups, see next paragraph and next proof for more details. Since $\mathcal{P}$ consists of projective $\mathbf{k}$-modules and since the normalized bar complex of the symmetric groups is a resolution of $\mathbf{k}$, the third page of this spectral sequence is isomorphic to the underlying homology of $\overline{\mathcal{P}}$, that is $H .\left(\overline{\mathcal{P}}, \mathrm{d}_{\mathcal{P}}\right)$. This is also the case for the other spectral sequence, the one associated to the operad $\overline{\mathcal{P}}$. In the end, the morphism $\widetilde{\Omega} \widetilde{\mathrm{B}} \overline{\mathcal{P}} \rightarrow \overline{\mathcal{P}}$ induces an isomorphism between the third pages of the two spectral sequences. So the convergence theorem for spectral sequences implies that $\widetilde{\Omega} \widetilde{\mathrm{B}} \overline{\mathcal{P}} \rightarrow \overline{\mathcal{P}}$ is a quasi-isomorphism.

Recall that the underlying graded colored $\mathbb{S}$-module of the cooperad $q \mathcal{O}^{i}$ is nonnegatively graded. So, when the underlying graded $\mathbb{N}$-module of the operad $\mathcal{P}$ is nonnegatively graded, this is also the case for the underlying graded $\mathbb{N}$-module of its higher bar construction since $\widetilde{\mathrm{B}} \overline{\mathcal{P}}=q \mathcal{O}^{\mathrm{i}}(\overline{\mathcal{P}})$. By definition, one can see that the $\mathbf{k}$-module components $q \mathcal{O}(\overline{\mathcal{P}})_{d}(n)$ of the higher bar construction are isomorphic to tensor products of $\mathbf{k}$-modules of the form $\overline{\mathcal{P}}_{e}(m)$. Since these latter ones are all projective, the $\mathbf{k}$-module components of the higher bar construction are projective. Finally, Proposition 2.5.1 applies and proves the cofibrancy property. 
It turns out that this functorial resolution for augmented operads is actually isomorphic to the classical cobar-bar resolution of the Hadamard tensor product of $\mathcal{P}$ with the Barratt-Eccles operad $\varepsilon$ :

$$
(\mathcal{P} \otimes \mathcal{E})(n):=\mathcal{P}(n) \otimes \mathcal{E}(n)
$$

Recall that the Barratt-Eccles operad $\mathcal{E}$ is defined as follows, see [4] for more details. The underlying graded $\mathbb{S}$-module is defined by

$$
\mathcal{E}(n)_{d}:=\left\langle\left(\sigma_{1}, \ldots, \sigma_{d+1}\right) \mid \sigma_{i} \in \mathbb{S}_{n}, \sigma_{i} \neq \sigma_{i+1}\right\rangle,
$$

in arity $n$ and degree $d$. The symmetric group acts diagonally on the right:

$$
\left(\sigma_{1}, \ldots, \sigma_{d+1}\right)^{\sigma}:=\left(\sigma_{1} \sigma, \ldots, \sigma_{d+1} \sigma\right) \text {. }
$$

(Note that this convention differs from [4].)

The partial composition maps in the Barratt-Eccles operad are given as follows. First recall that the set-theoretical operad $\operatorname{Ass}(n)=\mathbb{S}_{n}$, made up of the symmetric groups, carries partial composition maps. For $\sigma \in \mathbb{S}_{n}$ and $\tau \in \mathbb{S}_{k}$, the partial composition $\sigma \circ_{i} \tau$ is equal to

$\sigma \circ_{i} \tau:=[f(\sigma(1)) \cdots f(\sigma(i-1))(\tau(1)+\sigma(i)-1) \cdots(\tau(k)+\sigma(i)-1) f(\sigma(i+1)) \cdots f(\sigma(n))]$, where $f$ denotes the function defined by $f(x)=x$, for $x \leq \sigma(i)$, and by $f(x)=x+k-1$, for $x>\sigma(i)$. Put more plainly, this amounts to inserting the permutation $\tau$ at the $i^{\text {th }}$ place of the permutation $\sigma$. Note that we have

$$
\sigma \circ_{i} \tau=\left(\sigma \circ_{i} \operatorname{id}_{k}\right)\left(\operatorname{id}_{n} \circ_{i} \tau\right)=\sigma^{\prime \prime} \tau^{\prime}=\left(\operatorname{id}_{n} \circ \sigma(i) \tau\right)\left(\sigma \circ_{i} \mathrm{id}_{k}\right)=\tau^{\prime} \sigma^{\prime \prime},
$$

with the notations introduced in Definition 1.1.1 Now the partial composition map in the Barratt-Eccles operad is equal to

$$
\left(\sigma_{1}, \ldots, \sigma_{d+1}\right) \circ_{i}\left(\tau_{1}, \ldots, \tau_{e+1}\right):=\sum_{\left(x_{.}, y_{.}\right)} \pm\left(\sigma_{x_{0}+1} \circ_{i} \tau_{y_{0}+1}, \cdots, \sigma_{x_{d+e}+1} \circ_{i} \tau_{y_{d+e}+1}\right),
$$

where the sum is taken over the nondecreasing paths $\left(x_{\bullet}, y_{\bullet}\right)=\left\{\left(x_{i}, y_{i}\right)\right\}_{0 \leq i \leq d+e}$ from $(0,0)$ to $(d, e)$ on the grid $\mathbb{N} \times \mathbb{N}$.

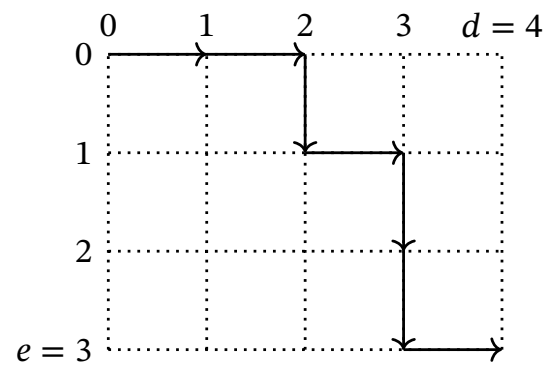

Such a path is made up of $d$ horizontal segments and $e$ vertical segments. Let us number the segments from 0 to $d+e$, beginning with $\left(x_{0}, y_{0}\right) \rightarrow\left(x_{1}, y_{1}\right)$ and following the path. We consider the shuffle permutation reordering the segments, such that the $d$ horizontal segments appear first and the $e$ vertical segments appear after. In the above example, 
we get [1247356]. The sign in the formula defining the above partial composition map is the signature of this shuffle permutation.

Since the components of the Barratt-Eccles operad are defined by the normalized bar complex of the symmetric groups $\mathbb{S}_{n}$, the differential map is equal to

$$
\mathrm{d}_{\mathcal{E}}\left(\sigma_{1}, \ldots, \sigma_{d+1}\right):=\sum_{i=1}^{d+1}(-1)^{i-1} \cdot\left(\sigma_{1}, \ldots, \hat{\sigma}_{i}, \ldots, \sigma_{d+1}\right),
$$

where a summand of the right-hand side is zero when $\sigma_{i-1}=\sigma_{i+1}$.

Proposition 2.5.4. There exists a natural isomorphism of augmented dg operads

$$
I \oplus \widetilde{\Omega} \widetilde{\mathrm{B}} \overline{\mathcal{P}} \cong \Omega \mathrm{B}(\mathcal{P} \otimes \mathcal{E})
$$

between the augmentation of the higher cobar-bar construction of $\overline{\mathcal{P}}$ and the cobar-bar construction of the Hadamard tensor product of the operad $\mathcal{P}$ with the Barratt-Eccles operad.

Proof. We consider first the case when $\mathcal{P}=$ Com and we note that Com $\otimes \mathcal{E}=\mathcal{E}$. We define an isomorphism of dg operads $\Psi: I \oplus \widetilde{\Omega} \widetilde{\mathrm{B}} \overline{\mathrm{Com}} \rightarrow \Omega \mathrm{B} \mathcal{E}$. Such a map is uniquely determined by its restriction to the space of generators $\widetilde{\mathrm{B}} \overline{\mathrm{Com}}$. As a graded $\mathbb{N}$-module, $\widetilde{\mathrm{B}} \overline{\mathrm{Com}}=q \mathcal{O}^{i} \circ \overline{\mathrm{Com}} \cong \mathcal{O}_{n s}^{i} \circ \mathrm{T}^{c}(s \overline{\mathrm{S}}) \circ \overline{\mathrm{Com}}$, as shown in Lemma 1.4.4 We first define a morphism of graded $\mathbb{N}$-modules $\psi: \mathrm{T}^{c}(s \overline{\mathrm{S}}) \circ \overline{\mathrm{Com}} \rightarrow \mathcal{E}$ by

$$
\psi\left(s \sigma_{1}, \ldots, s \sigma_{d}\right):=\left(\sigma_{1} \cdots \sigma_{d}, \sigma_{2} \cdots \sigma_{d}, \ldots, \sigma_{d}, \mathrm{id}_{n}\right),
$$

in arity $n$ and degree $d$. Note that the image of $\psi$ is indeed nondegenerate, since $\sigma_{i} \neq$ id for all $i$. This map $\psi$ extends to

$$
\left.\Psi\right|_{\widetilde{\mathrm{B}} \overline{\mathrm{Com}}}: \widetilde{\mathrm{B}} \overline{\mathrm{Com}} \longrightarrow s^{-1} \mathrm{~B} \mathcal{E} \longleftrightarrow \Omega \mathrm{B} \mathcal{E}
$$

as follows. Since $\mathcal{O}_{n s}^{i}$ has a basis made up of planar rooted trees, there exists a basis of $\widetilde{\mathrm{B}} \overline{\mathrm{Com}} \cong \mathcal{O}_{n s}^{i} \circ \mathrm{T}^{c}(s \overline{\mathrm{S}}) \circ \overline{\mathrm{Com}}$ made up of planar rooted trees such that each vertex has arity $n \geq 2$ and is labeled by a finite sequence of permutations of $\mathbb{S}_{n}$. The image of such an element under $\left.\Psi\right|_{\tilde{\mathrm{B}} \overline{\mathrm{Com}}}$ is the same underling tree, forgetting its planar structure, where we apply $\psi$ at each vertex. Each vertex of the resulting tree is suspended and the global tree is desuspended, resulting in an element of $s^{-1} \mathrm{~B} \mathcal{E}=s^{-1} \mathcal{T}(\mathrm{T}(s \overline{\mathcal{E}}))$. Since the degree of a planar tree representing an element of $\mathcal{O}_{n s}^{i}$ is given by the number of internal edges, the map $\left.\Psi\right|_{\widetilde{\mathrm{B}} \overline{\mathrm{Com}}}$ has degree 0 .

The map $\Psi: I \oplus \widetilde{\Omega} \widetilde{\mathrm{B}} \overline{\mathrm{Com}} \rightarrow \Omega \mathrm{B} \mathcal{E}$ is by definition a morphism of operads. The underlying space of the bar construction of an operad is made up of rooted trees (in space, i.e., nonplanar) with leaves labeled by $\{1, \ldots, n\}$ and with vertices labeled by the elements of the operad together with an identification of the inputs with the action of the symmetric group, see [46, \$5.6.1]. Therefore, the underlying space of the bar construction $\mathrm{B} \mathcal{E}$ of the Barratt-Eccles operad $\mathcal{E}$ admits a basis made up of planar rooted trees where each vertex has arity $n \geq 2$ and is labeled by a sequence of permutations of the form $\left(\sigma_{1}, \ldots, \sigma_{d}, \mathrm{id}_{n}\right)$. (One can use the last element of the strings to provide the 
rooted tree with a planar structure.) Since the map $\psi$ is an isomorphism, this shows that the map $\Psi$ is an isomorphisms of graded operads.

It remains to show, that the morphism of graded operads $\Psi$ respects the differentials. This again can be checked on the space of generators $\widetilde{\mathrm{B}} \overline{\mathrm{Com}}$. Recall from the previous proof that the higher cobar-bar construction is made up of partitioned planar rooted trees with vertices of arity $n \geq 2$ labeled by strings of nontrivial permutations of $\mathbb{S}_{n}$. The differential d on $\widetilde{\Omega} \widetilde{\mathrm{B}} \overline{\mathrm{Com}}$ is a sum of several parts. First, there are three terms $\mathrm{d}_{1}=\mathrm{d}_{1}^{\text {sh }}+\mathrm{d}_{1}^{1}+\mathrm{d}_{1}^{\text {int }}$, coming from the higher bar construction, which apply only inside each partition. The term $d_{1}^{\text {sh }}$ contracts inner edges and shuffles the two strings of permutations labeling the two associated vertices. The term $\mathrm{d}_{1}^{1}$ drops the first element of each string of permutations. (This corresponds to the trivial action of this element on the corolla viewed as an element of Com). The term $d_{1}^{\text {int }}$ is equal to the sum of the products of two consecutive elements of a string labeling a vertex, provided that it is not the identity (in which case it gives 0 ). Then, there are two terms $d_{2}=d_{2}^{\text {split }}+d_{2}^{r}$, coming from the higher cobar construction, which apply to each partition. The term $\mathrm{d}_{2}^{\text {split }}$ splits each part of the partition into two along an inner edge. The term $\mathrm{d}_{2}^{\mathrm{r}}$ extracts the right most external term of each string labeling the vertex of the tree inside the partition, pulls it out of the partition and make it act on the permutation labeling the leaves of the global tree. The total differential is then the sum

$$
\mathrm{d}=\mathrm{d}_{1}-\mathrm{d}_{2}=\left(\mathrm{d}_{1}^{\mathrm{sh}}+\mathrm{d}_{1}^{\mathrm{l}}+\mathrm{d}_{1}^{\mathrm{int}}\right)-\left(\mathrm{d}_{2}^{\text {split }}+\mathrm{d}_{2}^{\mathrm{r}}\right) .
$$

On the cobar-bar construction of the Barratt-Eccles operad $\Omega B \mathcal{E}$, the differential is equal to the sum of the following three parts. First, there is the internal differential $d_{\text {int }}$ induced by the differential $\mathrm{d}_{\mathcal{E}}$ on $\mathcal{E}$. Second, there is the differential $\mathrm{d}_{\text {bar }}$ coming from the differential on the bar construction $\mathrm{B} \mathcal{E}$ which is produced by the partial composition maps in $\mathcal{E}$. And finally, there is the differential $\mathrm{d}_{\text {cobar }}$ coming from the differential of the cobar construction, which splits each partition into two by cutting along any internal edge inside each part of the partition.

The sum $d_{1}^{1}+d_{1}^{\text {int }}-d_{2}^{r}$ of the three parts of the differential on $\widetilde{\Omega} \widetilde{\mathrm{B}} \overline{\mathrm{Com}}$ involving the strings of symmetric group elements is sent to the internal differential $d_{\text {int }}$ of the Barratt-Eccles operad under the morphism $\Psi$ :

$$
\begin{aligned}
\psi\left(\left(\mathrm{d}_{1}^{1}\right.\right. & \left.\left.+\mathrm{d}_{1}^{\mathrm{int}}-\mathrm{d}_{2}^{\mathrm{r}}\right)\left(\sigma_{1}, \ldots, \sigma_{d}\right)\right) \\
& =\psi\left(\left(\sigma_{2}, \ldots, \sigma_{d}\right)+\left(\sum_{i=2}^{d}(-1)^{i-1}\left(\sigma_{1}, \ldots, \sigma_{i-1} \sigma_{i}, \ldots, \sigma_{d}\right)\right)-(-1)^{d-1}\left(\sigma_{1}, \ldots, \sigma_{d-1} \mid \sigma_{d}\right)\right) \\
& =\left(\sum_{i=1}^{d}(-1)^{i-1}\left(\sigma_{1} \cdots \sigma_{d}, \ldots, \widehat{\sigma_{i} \cdots \sigma_{d}}, \ldots, \sigma_{d}, \mathrm{id}_{n}\right)\right)+(-1)^{d}\left(\sigma_{1} \cdots \sigma_{d-1}, \ldots, \sigma_{d-1}, \mathrm{id}_{n}\right)^{\sigma_{d}}
\end{aligned}
$$

and

$$
\begin{aligned}
\mathrm{d}_{\text {int }}( & \left.\psi\left(\sigma_{1}, \ldots, \sigma_{d}\right)\right) \\
& =\mathrm{d}_{\text {int }}\left(\sigma_{1} \cdots \sigma_{d}, \ldots, \sigma_{d}, \mathrm{id}_{n}\right)
\end{aligned}
$$




$$
=\left(\sum_{i=1}^{d}(-1)^{i-1}\left(\sigma_{1} \cdots \sigma_{d}, \ldots, \widehat{\sigma_{i} \cdots \sigma_{d}}, \ldots, \sigma_{d}, \operatorname{id}_{n}\right)\right)+(-1)^{d}\left(\sigma_{1} \cdots \sigma_{d}, \ldots, \sigma_{d}\right) .
$$

Note that in the first case, the last term amounts to splitting apart $\sigma_{d}$ and making it act on the underlying tree. In the tree module underlying $\Omega \mathrm{B} \mathcal{E}$, this action on rooted trees is identified with the symmetric group action on the strings labeling the vertices.

The next term $-\mathrm{d}_{2}^{\text {split }}$ of the differential on $\widetilde{\Omega} \widetilde{\mathrm{B}} \overline{\mathrm{Com}}$ amounts to cutting each partition into two along any inner edge. It is sent to $-\mathrm{d}_{\text {cobar }}$ in $\Omega \mathrm{B} \mathcal{E}$, which does the same things on partitioned rooted trees and with the same minus sign, see [46, \$6.5.2] where the sign is creating by the formula $\Delta_{s}\left(s^{-1}\right)=-s^{-1} \otimes s^{-1}$.

The last part $\mathrm{d}_{1}^{\text {sh }}$ contracts inner edges inside partions and shuffles the two strings of permutations labeling the two associated vertices. Let $t\left(\left(\sigma_{1}, \ldots, \sigma_{d}\right),\left(\sigma_{d+1}, \ldots, \sigma_{d+e}\right)\right)$ be a planar rooted subtree with two vertices of arity $n$ and $k$ and labeled respectively by two strings $\left(\sigma_{1}, \ldots, \sigma_{d}\right) \in \mathbb{S}_{n}^{d}$ and $\left(\sigma_{d+1}, \ldots, \sigma_{d+e}\right) \in \mathbb{S}_{k}^{e}$. Since each element of the symmetric group in the higher bar construction comes in degree 1 , for each shuffle permutation there is a sign, which is equal to the signature of the permutation.

The image under the composition $\psi \circ \mathrm{d}_{2}^{\text {sh }}$ of the above labeled tree is equal to

$$
\begin{aligned}
\psi\left(\mathrm{d}_{2}^{\mathrm{sh}}\right. & \left.\left.t\left(\left(\sigma_{1}, \ldots, \sigma_{d}\right),\left(\sigma_{d+1}, \ldots, \sigma_{d+e}\right)\right)\right)\right) \\
& =\psi\left(\sum_{\alpha \in \operatorname{Sh}(d, e)} \operatorname{sgn}(\alpha)\left(\tilde{\sigma}_{\alpha^{-1}(1)}, \ldots, \tilde{\sigma}_{\alpha^{-1}(d+e)}\right)\right) \\
& =\sum_{\alpha \in \operatorname{Sh}(d, e)} \operatorname{sgn}(\alpha)\left(\tilde{\sigma}_{\alpha^{-1}(1)} \cdots \tilde{\sigma}_{\alpha^{-1}(d+e)}, \ldots, \tilde{\sigma}_{\alpha^{-1}(d+e)}, \operatorname{id}_{n+m-1}\right),
\end{aligned}
$$

where we use the overall notation $\tilde{\sigma}_{j}=\sigma_{j}^{\prime \prime}$ for $j \leq d$ and $\tilde{\sigma}_{j}=\sigma_{j}^{\prime}$ for $j>d$. Note that the $(d, e)$-shuffles are in one-to-one correspondence with nondecreasing paths $\left(x_{.}, y_{.}\right)=\left\{\left(x_{i}, y_{i}\right)\right\}_{0 \leq i \leq d+e}$ from $(0,0)$ to $(d, e)$ on the grid $\mathbb{N} \times \mathbb{N}$. On the other hand, we have

$$
\begin{aligned}
& \mathrm{d}_{\mathrm{bar}}(\Psi\left.\left(t\left(\left(\sigma_{1}, \ldots, \sigma_{d}\right),\left(\sigma_{d+1}, \ldots, \sigma_{d+e}\right)\right)\right)\right) \\
&= \mathrm{d}_{\mathrm{bar}}\left(t\left(\psi\left(\sigma_{1}, \ldots, \sigma_{d}\right), \psi\left(\sigma_{d+1}, \ldots, \sigma_{d+e}\right)\right)\right) \\
&= \psi\left(\sigma_{1}, \ldots, \sigma_{d}\right) \circ_{i} \psi\left(\sigma_{d+1}, \ldots, \sigma_{d+e}\right) \\
&=\left(\sigma_{1} \cdots \sigma_{d}, \ldots, \sigma_{d}, \mathrm{id}_{n}\right) \circ_{i}\left(\sigma_{d+1} \cdots \sigma_{d+e}, \ldots, \sigma_{d+e}, \mathrm{id}_{k}\right) \\
&= \sum_{(x, y .)} \pm\left(\left(\sigma_{x_{0}+1} \cdots \sigma_{d}\right) \circ_{i}\left(\sigma_{y_{0}+d+1} \cdots \sigma_{d+e}\right),\right. \\
&\left.\quad \ldots,\left(\sigma_{x_{d+e}+1} \cdots \sigma_{d}\right) \circ_{i}\left(\sigma_{y_{d+e}+d+1} \cdots \sigma_{d+e}\right), \mathrm{id}_{n} \circ_{i} \mathrm{id}_{k}\right),
\end{aligned}
$$

where $i$ is the leaf of the root corolla of the tree $t$ where the second corolla is attached to. It remains to show that

$$
\left(\sigma_{x_{j}+1} \cdots \sigma_{d}\right) \circ_{i}\left(\sigma_{y_{j}+d+1} \cdots \sigma_{d+e}\right)=\tilde{\sigma}_{\alpha^{-1}(j+1)} \cdots \tilde{\sigma}_{\alpha^{-1}(d+e)} .
$$

First, it is straightforward to prove $\alpha^{-1}([j, d+e])=\left[x_{j}+1, d\right] \cup\left[y_{j}+d+1, d+e\right]$, for instance by induction on $j$. Then, using Equation (83) with $(\sigma \tau)^{\prime}=\sigma^{\prime} \tau^{\prime}$ and 
$(\sigma \tau)^{\prime \prime}=\sigma^{\prime \prime} \tau^{\prime \prime}$, we get

$$
\begin{aligned}
\tilde{\sigma}_{\alpha^{-1}(j+1)} \cdots \tilde{\sigma}_{\alpha^{-1}(d+e)} & =\sigma_{x_{j}+1}^{\prime \prime} \cdots \sigma_{d}^{\prime \prime} \sigma_{y_{j}+d+1}^{\prime} \cdots \sigma_{d+e}^{\prime} \\
& =\left(\sigma_{x_{j}+1} \cdots \sigma_{d}\right)^{\prime \prime}\left(\sigma_{y_{j}+d+1} \cdots \sigma_{d+e}\right)^{\prime} \\
& =\left(\sigma_{x_{j}+1} \cdots \sigma_{d}\right) \circ_{i}\left(\sigma_{y_{j}+d+1} \cdots \sigma_{d+e}\right) .
\end{aligned}
$$

This completes the proof for $\mathcal{P}=$ Com.

The general case of any augmented dg operad $\mathcal{P}$ is treated in the same way. We consider now the following morphism of graded $\mathbb{N}$-modules $\psi: \mathrm{T}^{c}(s \bar{\S}) \circ \overline{\mathcal{P}} \rightarrow \mathcal{E}$ by

$$
\psi\left(\mu ; s \sigma_{1}, \ldots, s \sigma_{d}\right):=\left(\mu^{\sigma_{1} \cdots \sigma_{d}} ; \sigma_{1} \cdots \sigma_{d}, \sigma_{2} \cdots \sigma_{d}, \ldots, \sigma_{d}, \mathrm{id}_{n}\right) .
$$

and the induced morphism of operads $\Psi: I \oplus \widetilde{\Omega} \widetilde{\mathrm{B}} \overline{\mathcal{P}} \rightarrow \Omega \mathrm{B}(\mathcal{P} \otimes \mathcal{E})$.

The vertices of the partitioned planar rooted trees of $\widetilde{\Omega} \widetilde{\mathrm{B}} \overline{\mathcal{P}}$ are now labeled by elements of $\mathcal{P}$ and strings of permutation and the vertices of the partitioned rooted trees of $\Omega \mathrm{B}(\mathcal{P} \otimes \mathcal{E})$ are labelled by elements of $\overline{\mathcal{P}} \otimes \overline{\mathcal{E}}$. Therefore, the same arguments apply to prove that $\Psi$ is an isomorphism.

Then, one has to deal with the internal differential $\mathrm{d}_{\mathcal{P}}$ of the $\mathrm{dg}$ operad $\mathcal{P}$. It is easy to see that the extra term induced by $\mathrm{d}_{\mathcal{P}}$ on the differentials of $\widetilde{\Omega} \widetilde{\mathrm{B}} \overline{\mathcal{P}}$ and $\Omega \mathrm{B}(\mathcal{P} \otimes \mathcal{E})$ respectively are sent to one another under the map $\Psi$. The term $\mathrm{d}_{1}^{1}$ now incorporates the action of the symmetric group on $\mathcal{P}$ :

$$
\mathrm{d}_{1}^{\mathrm{l}}\left(\mu ; \sigma_{1}, \ldots, \sigma_{d}\right)=\left(\mu^{\sigma_{1}} ; \sigma_{2}, \ldots, \sigma_{d}\right) .
$$

And the terms $\mathrm{d}_{1}^{\mathrm{l}}+\mathrm{d}_{1}^{\mathrm{int}}-\mathrm{d}_{2}^{\mathrm{r}}$ are still sent to $\mathrm{d}_{\text {int }}$ under the map $\Psi$ since

$$
\begin{aligned}
\psi\left(\left(\mathrm{d}_{1}^{\mathrm{l}}+\right.\right. & \left.\left.\mathrm{d}_{1}^{\mathrm{int}}-\mathrm{d}_{2}^{\mathrm{r}}\right)\left(\mu ; \sigma_{1}, \ldots, \sigma_{d}\right)\right) \\
= & \mathrm{d}_{\mathrm{int}}\left(\psi\left(\mu ; \sigma_{1}, \ldots, \sigma_{d}\right)\right) \\
= & \sum_{i=1}^{d}(-1)^{i-1}\left(\mu^{\sigma_{1} \cdots \sigma_{d}} ; \sigma_{1} \cdots \sigma_{d}, \ldots, \widehat{\sigma_{i} \cdots \sigma_{d}}, \ldots, \sigma_{d}, \mathrm{id}_{n}\right) \\
& +(-1)^{d}\left(\mu^{\sigma_{1} \cdots \sigma_{d}} ; \sigma_{1} \cdots \sigma_{d}, \ldots, \sigma_{d}\right) .
\end{aligned}
$$

Since they do not involve the operad $\mathcal{P}$, the two terms $-\mathrm{d}_{2}^{\text {split }}$ and $-\mathrm{d}_{\text {bar }}$ still correspond to each other under $\Psi$.

The component $d_{1}^{\text {sh }}$ now also includes the partial composition ${ }_{i}$ of the two elements of $\overline{\mathcal{P}}$ labeling the two vertices located on both sides of internal edges. This is also the case for the component $d_{\text {bar }}$. They actually satisfy

$$
\begin{aligned}
& \psi\left(\mathrm{d}_{2}^{\mathrm{sh}}\left(t\left(\left(\mu ; \sigma_{1}, \ldots, \sigma_{d}\right),\left(\nu ; \sigma_{d+1}, \ldots, \sigma_{d+e}\right)\right)\right)\right) \\
& =\sum_{\alpha \in \operatorname{Sh}(d, e)}(-1)^{\alpha} \cdot\left(\left(\mu \circ_{i} \nu\right)^{\sigma_{\alpha^{-1}(1)} \cdots \widetilde{\sigma}_{\alpha^{-1}(d+e)}} ; \tilde{\sigma}_{\alpha^{-1}(1)} \cdots \tilde{\sigma}_{\alpha^{-1}(d+e)}, \ldots, \tilde{\sigma}_{\alpha^{-1}(d+e)}, \mathrm{id}_{n+m-1}\right)
\end{aligned}
$$

and

$$
\begin{aligned}
& \mathrm{d}_{\mathrm{bar}}\left(\Psi\left(t\left(\left(\mu ; \sigma_{1}, \ldots, \sigma_{d}\right),\left(\nu ; \sigma_{d+1}, \ldots, \sigma_{d+e}\right)\right)\right)\right) \\
& =\sum_{(x, y .)} \pm\left(\mu^{\sigma_{1} \cdots \sigma_{d} o_{i}} \nu^{\sigma_{d+1} \cdots \sigma_{d+e}} ;\left(\sigma_{x_{0}+1} \cdots \sigma_{d}\right) \circ_{i}\left(\sigma_{y_{0}+d+1} \cdots \sigma_{d+e}\right)\right. \text {, } \\
& \left.\ldots,\left(\sigma_{x_{d+e}+1} \cdots \sigma_{d}\right) \circ_{i}\left(\sigma_{y_{d+e}+d+1} \cdots \sigma_{d+e}\right), \operatorname{id}_{n} \circ_{i} \operatorname{id}_{k}\right) \text {. }
\end{aligned}
$$


It remains to notice that

$$
\left(\mu \circ_{i} \nu\right)^{\widetilde{\sigma}_{\alpha^{-1}(1)} \cdots \widetilde{\sigma}_{\alpha^{-1}(d+e)}}=\left(\mu \circ_{i} \nu\right)^{\left(\sigma_{1} \cdots \sigma_{d}\right)^{\prime \prime}\left(\sigma_{d+1} \cdots \sigma_{d+e}\right)^{\prime}}=\mu^{\sigma_{1} \cdots \sigma_{d} o_{i}} \nu^{\sigma_{d+1} \cdots \sigma_{d+e}}
$$

to conclude the proof.

2.6. $\mathrm{E}_{\infty}$-operad. Recall that an $\mathrm{E}_{\infty}$-operad is an operad quasi-isomorphic to the operad Com and whose components are free (or projective) $\mathbf{k}\left[\mathbb{S}_{n}\right]$-modules. By $[5$, Proposition 4.3], any cofibrant resolution of the operad Com is an $\mathrm{E}_{\infty}$-operad. In applications, however, for instance in algebraic topology (e.g., [19,20]), it is sometimes necessary to work with a cofibrant $\mathrm{E}_{\infty}$-operad and not only an $\mathrm{E}_{\infty}$-operad.

We made the higher cobar-bar construction of the operad $\mathcal{P}=$ Com of commutative algebras explicit in the core of the proof of Proposition 2.5.4 This construction provides us with a cofibrant $\mathrm{E}_{\infty}$-operad over any ring.

Theorem 2.6.1. The augmentation of the higher cobar-bar counit

$$
\mathcal{E}_{\infty}:=I \oplus \widetilde{\Omega} \widetilde{\mathrm{B}} \overline{\mathrm{Com}} \stackrel{\sim}{\rightarrow} \mathrm{Com}
$$

is a cofibrant $\mathrm{E}_{\infty}$-operad over any ring, which is moreover a Hopf operad resolution.

PROOF. The operad Com is nonnegatively graded and the components of its underlying $\mathbb{S}$-module are the ground $\operatorname{ring} \operatorname{Com}(n)=\mathbf{k}$ for any $n \geq 1$, so they are projective k-modules. Therefore Theorem 2.5.3 applies and gives the result.

Proposition 2.5.4 shows that the operad $\mathcal{E}_{\infty} \cong \Omega B \mathcal{E}$ is isomorphic to the cobar-bar construction of the Barratt-Eccles operad. Since the Barratt-Eccles operad is a Hopf operad, this is also the case for its cobar-bar construction by [17. Theorem $\S 2 . A(c)]$. Finally, the composition $\mathcal{E}_{\infty} \cong \Omega \mathrm{B} \mathcal{E} \tilde{\rightarrow} \mathcal{E} \tilde{\rightarrow}$ Com is a quasi-isomorphism of Hopf operads.

REMARK 2.6.2. Let us emphasize that, by Proposition 2.5.4 this theorem was already known in the following form: the present cofibrant $\mathrm{E}_{\infty}$-operad is isomorphic to the classical cobar-bar construction of the Barratt-Eccles operad, which is a cofibrant $\mathrm{E}_{\infty}$-operad over any ring by [7, Theorem 5.1].

The operad $\varepsilon_{\infty}$ is given by partitioned planar rooted trees with vertices of valence at least 2 , labeled by strings of nontrivial permutations and with the leaves globally labeled by a permutation

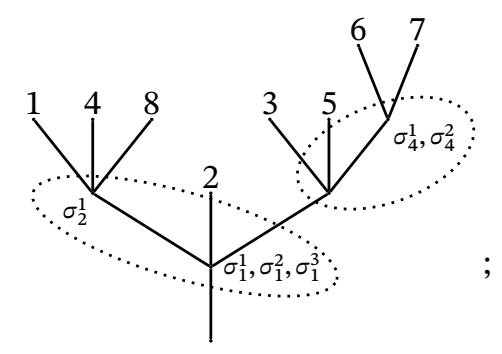

it is equipped with a differential given by the sum of three types of terms: 
(i) at each vertex, one deletes the left-most permutation $\sigma_{j}^{1}$, one composes pairs of permutations when they are not inverse to each other, and one removes the last permutation $\sigma_{j}^{i_{j}}$ of the string, pulls it out of the underlying tree (action which may modify this tree) and rearranges the labels of the leaves accordingly;

(ii) inside each subtree, one contracts every edge and shuffles the two strings of permutations in all possible ways;

(iii) for each subtree, one splits it into two subtrees in all possible ways, the number of which is equal to the number of internal edges.

By definition, an $\mathrm{E}_{\infty}$-algebra is an algebra over an $\mathrm{E}_{\infty}$-operad. This definition offers an interesting degree of freedom. But it is sometimes useful to have at hand an explicit definition by means of generators and relations. The form of the present $\mathrm{E}_{\infty}$-operad $\mathcal{E}_{\infty}$ allows us to give such a definition. Moreover, this definition carries canonical properties since the resolution $\varepsilon_{\infty}$ that we use is a cofibrant and Hopf operad.

Proposition 2.6.3. Let $\left(A, \mathrm{~d}_{A}\right)$ be a chain complex equipped with operations

$$
\left\{\mu_{t(\sigma)}: A^{\otimes n} \rightarrow A\right\}
$$

indexed by planar rooted trees $t$ with $n$ leaves and vertices of valence $m_{j} \geq 2$ labeled by strings $\bar{\sigma}_{j}$ of nontrivial permutations of $\mathbb{S}_{m_{j}}$. The operations are supposed to be of degree

$$
\left|\mu_{t(\sigma)}\right|=\text { number of internal edges }+ \text { number of permutations }
$$

and to satisfy the relations

$$
\begin{aligned}
& \partial_{A} \mu_{t(\sigma)}-\sum_{\begin{array}{c}
\text { internal edges } \\
j-e-l
\end{array}} \varepsilon_{1} \cdot \mu_{t / e\left(\bar{\sigma}_{1}, \ldots, \omega\left(\bar{\sigma}_{j}, \bar{\sigma}_{l}\right), \ldots, \bar{\sigma}_{k}\right)}+\sum_{\begin{array}{c}
\text { internal edges } \\
t=t_{1} 0_{i} t_{2}
\end{array}} \varepsilon_{2} \cdot \mu_{t_{1}\left(\bar{\sigma}_{1}, \ldots\right)} o_{i} \mu_{t_{2}\left(\bar{\sigma}_{l}, \ldots\right)} \\
& -\sum_{j=1}^{k} \varepsilon_{3} \cdot \mu_{t\left(\bar{\sigma}_{1}, \ldots,\left(\sigma_{j}^{2}, \ldots, \sigma_{j}^{i}\right), \ldots, \bar{\sigma}_{k}\right)}-\sum_{j=1}^{k} \sum_{l=1}^{i_{j}-1} \varepsilon_{4} \cdot \mu_{t\left(\bar{\sigma}_{1}, \ldots,(\sigma_{j}^{1}, \ldots, \underbrace{\sigma_{j}^{l} \sigma_{j}^{l+1}, \ldots, \sigma_{j}^{i}}_{\neq i \mathrm{~d}}), \ldots, \bar{\sigma}_{k}\right)} \\
& +\sum_{j=1}^{k} \varepsilon_{5} \cdot\left(\mu_{t\left(\bar{\sigma}_{1}, \ldots,\left(\sigma_{j}^{1}, \ldots, \sigma_{j}^{j^{-1}}\right), \ldots, \bar{\sigma}_{k}\right)}\right)^{\widetilde{\sigma}_{j}^{i}}=0,
\end{aligned}
$$

where the sign $\varepsilon_{1}$ comes from the permutations of the $\sigma$ 's, as described in Section 1.5, the sign $\varepsilon_{2}$ comes from the reordering of the tree into the two parts, the sign $\varepsilon_{3}$ is equal to $(-1)^{|t|+\left|\bar{\sigma}_{1}\right|+\cdots+\left|\bar{\sigma}_{j-1}\right|}$, the sign $\varepsilon_{4}$ is equal to $(-1)^{|t|+\left|\bar{\sigma}_{1}\right|+\cdots+\left|\bar{\sigma}_{j-1}\right|+l}$, and the sign $\varepsilon_{5}$ is equal to $(-1)^{|t|+\left|\bar{\sigma}_{1}\right|+\cdots+\left|\bar{\sigma}_{j-1}\right|+\left|\sigma_{j}^{1}\right|+\cdots+\mid \sigma_{j}^{i_{j}-1}}$. Such a data $\left(A, \mathrm{~d}_{A},\left\{\mu_{t(\sigma)}\right\}\right)$ is an $\mathrm{E}_{\infty}$-algebra.

Proof. An $\mathcal{E}_{\infty}$-algebra structure on the chain complex $\left(A, \mathrm{~d}_{A}\right)$ is a morphism of $\mathrm{dg}$ operads $\varepsilon_{\infty} \rightarrow \mathrm{End}_{A}$; data which is equivalent to a morphism of $\mathbb{N}$-modules $\mu: \widetilde{\mathrm{BCom}} \rightarrow$ End $_{A}$ satisfying the Maurer-Cartan Equation (82) $\partial \mu+\mu * \mu+\Delta \mu=0$, by Proposition 2.4.3. Since $\mu$ is concentrated in degree 0 , all the signs are directly given by the ones of the higher bar construction $\widetilde{\mathrm{B}} \overline{\mathrm{Com}}$ described in Section 2.2

REMARK 2.6.4. As mentioned before, an equivalent explicit definition can be obtained using the cobar-bar construction of the Barratt-Eccles operad. The existence of a 
cofibrant Hopf $\mathrm{E}_{\infty}$-operad plays a crucial role, for instance in B. Fresse's works [19 20]. But the actual description of the category of algebras over it does not seem to be present in the existing literature. We believe that it could be useful to have such an explicit description at hand. Moreover, the operad which emerges from the higher cobar-bar construction seems to provide us with a "simple" model since it is made up of planar trees and easy combinatorics of permutations.

In characteristic 0 , one can use the Koszul model of the operad Com to get a suitable notion of an $\mathrm{E}_{\infty}$-algebra. This only amounts to resolving the associative relation of commutative algebras. Over any ring, the first operadic syzygies can be found by hand, see [49. Example 4.8]. In the above definition, there are strings of permutations whose role is to resolve, in a coherent way, the trivial symmetric group actions on the components of the operad Com over any ring. Let us unravel the above definition a little bit.

An $E_{\infty}$-algebra, in the above sense, is equipped with a degree 0 operation

$$
\mu_{Y}: A^{\otimes 2} \rightarrow A .
$$

The above relation applied to the tree $Y$ is equivalent to the fact that $d_{A}$ is a derivation with respect to $\mu_{Y}$. It is also equipped with a degree 1 operation

$$
\mu_{\Upsilon_{(12)}}: A^{\otimes 2} \rightarrow A
$$

which satisfies

$$
\partial_{A} \mu_{Y(12)}=\mu_{Y}-\left(\mu_{Y}\right)^{(12)} .
$$

So $\mu_{Y_{(12)}}$ is a homotopy for the commutativity relation of $\mu_{Y}$. It also comes equipped with a degree 0 operation

$$
\mu \mho: A^{\otimes 3} \rightarrow A .
$$

and two degree 1 operations

$$
\mu_{Y}: A^{\otimes 3} \rightarrow A \quad \text { and } \quad \mu_{Y}: A^{\otimes 3} \rightarrow A,
$$

satisfying respectively

$$
\partial_{A} \mu_{Y}=\mu_{Y}-\mu_{Y} \circ_{1} \mu_{Y} \quad \text { and } \quad \partial_{A} \mu_{Y}=\mu_{Y}-\mu_{Y} o_{2} \mu_{Y} .
$$

So $-\mu_{Y}+\mu_{Y}$ is a homotopy for the associativity relation of $\mu_{Y}$. The higher operations coming from more elaborate labeled planar trees provide us with coherent higher homotopies for these relations.

2.7. Higher Koszul duality theory. The higher cobar-bar construction of Theorem 2.5.3 shares nice properties: functorial, cofibrant over any ring. However, the underlying $\mathbb{N}$-module is quite huge. Given a particular operad $\mathcal{P}$ with some description, like a presentation by means of generators and relations, one might want to describe a smaller cofibrant resolution of $\mathcal{P}$. This is precisely what the Koszul duality theory for operads performs, see [46, Chapter 7]. However, the original Koszul duality theory for operads of [24 25] works well over a field of characterstic 0 . 
REMARK (Further study). The general pattern of the Koszul duality theory of algebras over an (colored) operad $\mathcal{O}$ was given by Millès in [53]. One should be able to apply it to the present case and thus obtain minimal models for operads generated by free $\mathbb{S}$-modules. In this way, the present chapter already settles the first step of this tentative higher Koszul duality theory with the definitions of the higher bar and the higher cobar constructions. It remains to define the Koszul dual higher cooperad of an operad. This issue is more subtle in the present case since the underlying space of the higher bar construction is not a differential graded module but a curved one. So one cannot define the Koszul dual directly by the homology groups of the bar construction.

The case of the operad Com is particularly interesting since this would simplify the above $\mathrm{E}_{\infty}$-operad. This should give an operad related to the cofibrant $\mathrm{E}_{\infty}$-operad given in [21] or to the chain complex of the Fox-Neuwirth cells of the Fulton-MacPherson operad and solve the issues raised by the presence of "bad cells" in [24].

\section{Higher Homotopy Operads}

The main result of Section 1 allows us to define a new notion of an operad up to homotopy. Since we take into account the action of the symmetric groups on the same footing as the partial composition maps, the upshot is a higher notion of a homotopy operad where all the defining relations of an nu operad are relaxed. Such a higher notion includes all the previously known cases like $\mathrm{A}_{\infty}$-algebras, $\mathrm{A}_{\infty}$-modules, and homotopy nonsymmetric operads, for instance.

Since this new notion is conceptually produced by the curved Koszul duality theory, we can endow it with a suitable notion of $\infty$-morphisms, which are shown to satisfy the required interesting homotopy properties. For instance, this allows us to describe the homotopy theory of dg augmented operads, like the homotopy transfer theorem, over any ring.

3.1. Definition. The cofibrant resolution $\mathcal{O}_{\infty}:=\Omega \mathcal{O}^{i}$ of the colored operad $\mathcal{O}$ allows us to define a notion of operads up to homotopy with the required homotopy properties over any ring.

Definition 3.1.1. A higher homotopy operad, also called a higher operad ${ }_{\infty}$, is an $\mathcal{O}_{\infty}$ algebra.

Using our computation of the Koszul dual curved cooperad $\mathcal{O}^{i}=\left(q \mathcal{O}^{i}, \mathrm{~d}_{\mathcal{O}^{i}}, \theta\right)$, we can make the definition of higher homotopy operads explicit. Let $\left\{\left(\mathcal{P}(n), \mathrm{d}_{\mathcal{P}(n)}\right)\right\}_{n \in \mathbb{N}}$ be a differential graded $\mathbb{N}$-module. We consider the space of maps from the Koszul dual curved cooperad $\mathcal{O}^{i}$ to the endomorphism operad End $_{\mathcal{P}}$ :

$$
\begin{aligned}
& \operatorname{Hom}_{\mathbb{S}}\left(\mathcal{O}^{\mathrm{i}}, \operatorname{End}_{\mathcal{P}}\right):= \\
& \prod_{k \in \mathbb{N}}\left(\prod_{n_{1}, \ldots, n_{k} \in \mathbb{N}} \operatorname{Hom}\left(\overline{\mathcal{O}}^{\mathrm{i}}\left(n ; n_{1}, \ldots, n_{k}\right), \operatorname{Hom}\left(\mathcal{P}\left(n_{1}\right) \otimes \cdots \otimes \mathcal{P}\left(n_{k}\right), \mathcal{P}(n)\right)\right)\right)^{\mathbb{S}_{k}},
\end{aligned}
$$


where $n=n_{1}+\cdots+n_{k}-k+1$. This mapping space is endowed with a convolution pre-Lie product $f \star g$ defined by the following composition

$$
f \star g: \mathcal{O}^{i} \stackrel{\Delta_{(1)}}{\longrightarrow} \mathcal{O}^{i} \otimes \mathcal{O}^{i} \stackrel{f \otimes g}{\longrightarrow} \operatorname{End}_{\mathcal{P}} \otimes \operatorname{End}_{\mathcal{P}} \stackrel{\gamma_{\text {End }}}{\longrightarrow} \operatorname{End}_{\mathcal{P}} .
$$

As usual, the antisymmetrized bracket gives rise to a convolution Lie algebra structure

$$
[f, g]:=f \star g-(-1)^{|f||g|} g \star f .
$$

Let us denote by $\partial_{\mathcal{P}}$ the differential of the endomorphism operad $\operatorname{End}_{\mathcal{P}}$. We consider the classical derivation

$$
\partial(f):=\partial_{\mathcal{P}} \circ f-(-1)^{|f|} \cdot f \circ \mathrm{d}_{\mathcal{O} i}
$$

on the mapping space $\operatorname{Hom}_{\mathbb{S}}\left(\mathcal{O}^{i}, \operatorname{End}_{\mathcal{P}}\right)$. Finally, the cocurvature $\theta$ of the Koszul dual curved cooperad $\mathcal{O}^{i}$ induces the following curvature

$$
\Theta: \mathcal{O}^{i} \stackrel{-\theta}{\longrightarrow} I \hookrightarrow \operatorname{End}_{\mathcal{P}} .
$$

Lemma 3.1.2 ([31] §3.2.3]). The quadruple $\left(\operatorname{Hom}_{\mathbb{S}}\left(\mathcal{O}^{i}, \operatorname{End}_{\mathcal{P}}\right), \star, \partial, \Theta\right)$ forms a curved pre-Lie algebra, i.e.,

$$
\partial(\Theta)=0 \quad \text { and } \quad \partial^{2}\left({ }_{\lrcorner}\right)=\left({ }_{\triangleleft} \star \Theta\right)-\left(\Theta \star{ }_{\lrcorner}\right) .
$$

The quadruple $\left(\operatorname{Hom}_{\mathbb{S}}\left(\mathcal{O}^{i}, \operatorname{End}_{\mathcal{P}}\right),\left[\_, \_\right], \partial, \Theta\right)$ forms a curved Lie algebra, i.e.,

$$
\left.\partial(\Theta)=0 \quad \text { and } \quad \partial^{2}(\lrcorner\right)=\left[\_, \Theta\right] .
$$

Proposition 3.1.3. A higher homotopy operad structure on a $d g \mathbb{N}$-module $\mathcal{P}$ is equivalent to a degree -1 solution to the curved Maurer-Cartan equation

$$
\partial \gamma+\gamma \star \gamma=\Theta
$$

in the curved convolution algebra $\operatorname{Hom}_{\mathbb{S}}\left(\mathcal{O}^{i}\right.$, End $\left._{\mathcal{P}}\right)$ which vanishes on $I \subset \mathcal{O}^{i}$.

PROOF. This is a direct application of [31, Theorem 3.4.1].

Theorem 3.1.4. A higher homotopy operad structure on a dg $\mathbb{N}$-module $\left\{\mathcal{P}(n), \mathrm{d}_{\mathcal{P}(n)}\right\}_{n \in \mathbb{N}}$ amounts to a collection of maps

$$
\left\{\gamma_{t(\sigma)}: \mathcal{P}\left(n_{1}\right) \otimes \cdots \otimes \mathcal{P}\left(n_{k}\right) \rightarrow \mathcal{P}(n) \mid\right.
$$

labeled by the set of planar rooted trees $t(\sigma)=t\left(\bar{\sigma}_{1}, \ldots, \bar{\sigma}_{k}\right)$ with each vertex of arity $n_{j} \geq 0$ labeled by a possibly empty string $\bar{\sigma}_{j}=\left(\sigma_{j}^{1}, \ldots, \sigma_{j}^{i_{j}}\right)$ of nontrivial permutations $\sigma_{j}^{l} \in \mathbb{S}_{n_{j}} \backslash\left\{\mathrm{id}_{n_{j}}\right\}$, and with at least one internal edge or one permutation. These structure maps have degree equal to

$$
\left|\gamma_{t(\sigma)}\right|=|t|+\left|\bar{\sigma}_{1}\right|+\cdots+\left|\bar{\sigma}_{k}\right|-1,
$$


and they satisfy the relations

$$
\begin{aligned}
& \partial_{\mathcal{P}}\left(\gamma_{t(\sigma)}\right)+\sum_{j=1}^{k} \sum_{l=1}^{i_{j}-1}(-1)^{|t|+\left|\bar{\sigma}_{1}\right|+\cdots+\left|\bar{\sigma}_{j-1}\right|+l} \cdot \gamma_{t\left(\bar{\sigma}_{1}, \ldots, \bar{\sigma}_{j-1},(\sigma_{j}^{1}, \ldots, \underbrace{\sigma_{j}^{l} \sigma_{j}^{l+1}, \ldots, \sigma_{j}}_{\neq i d} i_{j} \bar{\sigma}_{j+1}, \ldots, \bar{\sigma}_{k})\right.} \\
& +\sum_{s \subset t, \omega}(-1)^{|t / s(\vec{\sigma})|} \varepsilon_{S, t, \omega} \cdot \gamma_{t / s(\vec{\sigma})}{ }^{\circ} s_{1} \gamma_{\tilde{s}(\hat{\sigma})}
\end{aligned}
$$

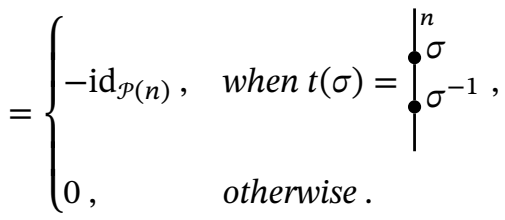

ProOF. The map $\gamma$ is completely characterized by its image on the operadic tree basis: $\gamma_{t(\sigma)}:=\gamma(t(\sigma))$. These structure maps have to satisfy the curved Maurer-Cartan Equation (84), which, when evaluated on the operadic tree $t(\sigma)$, gives Equation (85). Its second term, equal to $(\gamma \star \gamma)(t(\sigma))$ in End $_{\mathcal{P}}$, is given by the infinitesimal decomposition map of the Koszul dual cooperad described in Proposition 1.5.1 as follows. One first considers the image of $t(\sigma)$ under $\Delta_{(1)}$, which produces pairs $(t / s(\vec{\sigma}), \tilde{s}(\stackrel{\leftarrow}{\sigma}))$ of operadic trees. Then, we compose the associated structure map $\gamma_{t / s(\vec{\sigma})}$ at the entry $s_{1}$ of the structure $\operatorname{map} \gamma_{\tilde{S}(\overleftarrow{\sigma})}$.

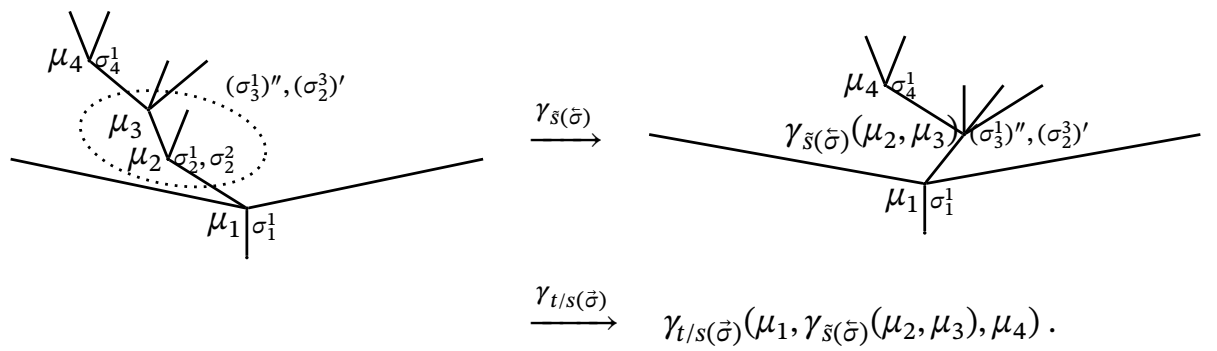

The second term of Equation (85), equal to $\gamma \circ \mathrm{d}_{\mathcal{O}_{i}}(t(\sigma))$, is given by the formula for the coderivation of the Koszul dual cooperad described in Proposition 1.6.2. Finally, the right-hand side of Equation (85) is the cocurvature described in Proposition 1.6.4

A morphism of higher homotopy operads is a morphism of $\mathrm{dg} \mathbb{N}$-modules which respects the structure maps. The associated category is denoted by $\mathrm{Opd}_{\infty}$.

Since the Koszul dual colored cooperad $q \mathcal{O}^{i}$ is homogenous quadratic, it admits a weight grading given by the numbers of generators. So the structure maps $\gamma_{t(\sigma)}$ are stratified by the weight, greater or equal than $l$, of the operadic trees $t(\sigma)$, which is equal to the number of internal edges plus the total number of permutations.

Proposition 3.1.5. There is a canonical embedding of categories

$$
\mathrm{nu} \mathrm{Opd} \longrightarrow \mathrm{Opd}_{\infty} \text {, }
$$

given by the higher homotopy operads with trivial structure maps of weight greater than or equal to 2. 
PROOF. In the case at hand, the weight one structure maps $\gamma_{t}$ labeled by operadic trees with one internal edge and no permutation correspond to the partial compositions ${ }_{-}{ }_{i \dashv}$, and the weight one operations $\gamma_{c(\sigma)}$ labeled by corollas with only one permutation correspond to the symmetric group actions. Since $\gamma$ vanishes on operadic trees with 2 internal edges and no permutation, Equation (85) for these types of trees corresponds to the defining Axioms (Opd-3) and (Opd-4) In the same way, Equation (85) for corollas with 2 permutations corresponds to the defining Axiom (Opd-1), and for trees with 1 internal edge and 1 permutation it corresponds to the defining Axioms (Opd-5) and $(\mathrm{Opd}-$ 6)

The structure maps $\gamma_{t(\sigma)}$ of weight 2 are the first homotopies for these relations. And then, each higher stratum corresponds to a higher level of coherent homotopies for the defining relations of the notion of an nu operad.

REMARK (Further study). In the very same way as explained above, since the colored operad $\mathcal{O}$ which encodes nu operads is set-theoretical, one can look for a topological colored operad whose chain complex would give the resolution $\mathcal{O}_{\infty}$. In this case, one would be able to define a suitable notion of a higher homotopy topological operad.

3.2. Comparison with other similar notions. The Koszul dual colored cooperad $\mathcal{O}^{i}$ actually admits several gradings, for instance by arity, number of internal edges, or total number of permutations. Using them, one can get many full subcategories of the category of higher homotopy operads by requiring that the Maurer-Cartan elements vanish outside some degree. This shows that the above definition of homotopy operads generalizes the following well-known notions, see Figure 1. One can go from one category to another by forgetting some structure. In the other way round, one can consider some trivial structure, or, in the case of symmetric groups action, the regular representations.

Proposition 3.2.1. The following categories are canonical full subcategories of the category $\mathrm{Opd}_{\infty}$ of higher homotopy operads.

(i) When $\gamma$ vanishes on operadic trees of arity not equal to 1, we get the notions of an $\mathrm{A}_{\infty}$-algebra of [65] and, respectively, an associative algebra, when moreover, it vanishes on operadic trees with more than 2 vertices.

(ii) When $\gamma$ vanishes on operadic trees of arity not equal to 0 or 1 , we get the notion of a homotopy associative algebra together with a homotopy left module over it. When, moreover, it vanishes on operadic trees with more than 2 vertices, we get the strict notion of an associative algebra together with a left module over it.

(iii) When $\gamma$ vanishes outside corollas of arity $n$, we get a new notion of a homotopy $\mathbb{S}_{n}$-module, see Section Afor more details.

(iv) When $\gamma$ vanishes everywhere except on the 2-vertex operadic trees with no permutation, we recover the notion of a nonsymmetric operad. When $\gamma$ vanishes outside operadic trees labeled with no permutation, we recover the notion of a homotopy nonsymmetric operad of Van der Laan [39. \$4.4]. 


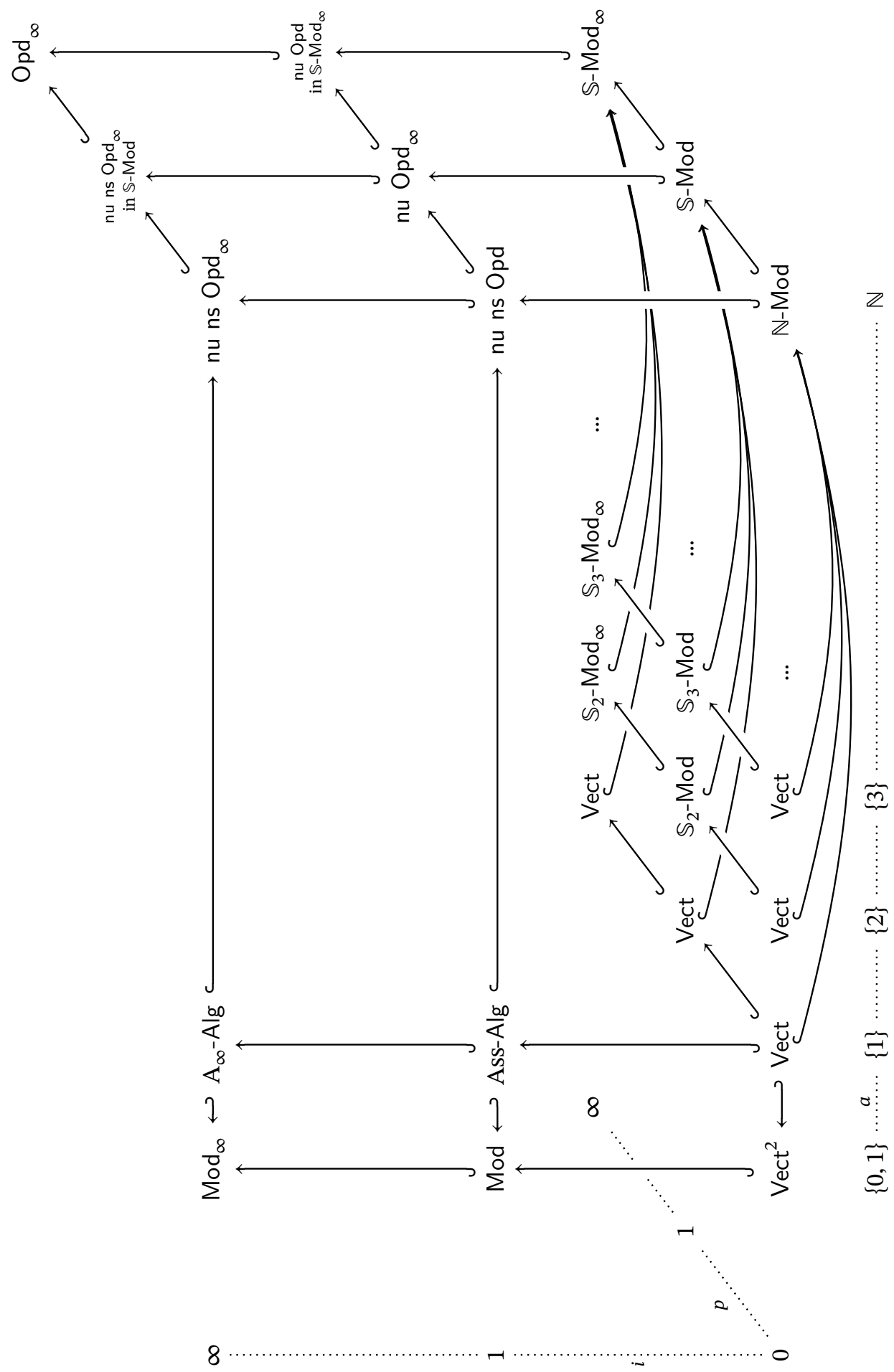

$a$ : arity, $i$ : number of internal edges, $p$ : total number of permutations

FIGURE 1. Various full subcategories of the category $\mathrm{Opd}_{\infty}$. 
(v) When $\gamma$ vanishes on operadic trees labeled by at least 2 permutations, we recover the notion of a symmetric homotopy operad of Van der Laan [39. \$4.5], where the partial operadic composition is relaxed up to homotopy, but not the action of the symmetric groups.

(vi) When $\gamma$ vanishes on operadic trees with at least 2 internal edges, we get a new notion of an operad in the category of homotopy $\mathbb{S}$-modules.

PROOF. This is directly checked.

Brinkmeier defined in [9] a notion called lax operad by applying the topological W-construction of Boardman-Vogt [8] to the set-theoretical colored operad which encodes operads (with unit). In this way, he obtains a notion of homotopy operad where everything, the partial composition, the symmetric group actions, and the unit, are relaxed up to homotopy. Let us consider the same method applied to the set-theoretical colored operad $O$ encoding nonunital operads and satisfying $\mathbf{k} O=\mathcal{O}$. Applying the chain complex functor to $\mathrm{W} O$, one gets the cobar-bar construction $\Omega \mathrm{B} \mathcal{O}$ of the associated linear colored operad $\mathcal{O}$, according to [6]. So such "lax operads" are made up of more structure maps, which are labeled by trees with vertices labeled by operadic trees with one permutation (the ones of Proposition 1.3.2). The natural colored operad map $\Omega \mathcal{O}^{i} \rightarrow \Omega \mathrm{BO}$ induces a functor from lax operads to our notion of homotopy operads, which amounts to defining maps $\gamma_{t(\sigma)}$ by sums of the above structure maps over some set of trees of trees. So, finally, the notion of lax operad of [9] is more complex than our homotopy operads in two directions: the unit is encoded and relaxed up to homotopy and there are many more homotopies for the partial compositions and the symmetric group actions.

There are also two (homotopically equivalent) notions of $\infty$-operads introduced respectively by Moerdijk-Weiss and Lurie [47. 55], which are colored set-theoretical operads in $\infty$-categories. These notions are different from the differential graded notion of higher homotopy operad developed here. However, one can relate them precisely as follows. Recently, Le Grignou built a homotopy coherent nerve functor from the category of homotopy operads à la Van der Laan, which relaxes only the partial composition products up to homotopy, to the category of $\infty$-operads, see [41]. (Le Grignou's construction is actually an operadic generalization of the homotopy coherent nerve functor of Lurie and Faonte [15, 47] between dg categories or $\mathrm{A}_{\infty}$-categories to $\infty$-categories.) By considering the slightly extended version with strict units of the present notion of higher homotopy operads and the suitable notion of $\infty$-morphism introduced in the next section, one can define a homotopy coherent nerve functor by the same kind of formula as Le Grignou or Faonte. Such a functor is expected to share the same nice homotopy properties as the one of Le Grignou.

3.3. Infinity-morphisms. As usual, the strict notion of morphisms of higher homotopy operads does not share the suitable homotopy properties. Instead, we consider a more general version called $\infty$-morphism and defined as follows. 
The curved Koszul duality theory provides us with a third equivalent definition of a higher homotopy operad structure on a $\operatorname{dg} \mathbb{N}$-module $\mathcal{P}$, see $[46, \S 10.1 .8]$. It amounts to the data of a curved codifferential on the cofree $q \mathcal{O}^{i}$-coalgebra $q \mathcal{O}^{i}(\mathcal{P})$ :

$$
\left(\mathcal{P}, \mathrm{d}_{\mathcal{P}}, \gamma\right) \stackrel{1-1}{\longrightarrow}\left(q \mathcal{O}^{\mathrm{i}}(\mathcal{P}), \mathrm{d}_{\gamma}:=\mathrm{d}_{\mathcal{O}(\mathcal{P})}+\mathrm{d}_{\gamma}^{r}\right)
$$

Recall that a morphism of $\mathcal{O}^{i}$-coalgebras is a morphism of $q \mathcal{O}^{i}$-coalgebras which preserves the curved codifferentials.

Definition 3.3.1. An $\infty$-morphism $\mathcal{P} \leadsto \mathcal{Q}$ between two higher homotopy operads $\left(\mathcal{P}, \mathrm{d}_{\mathcal{P}}, \gamma\right)$ and $\left(\mathcal{Q}, \mathrm{d}_{\mathcal{Q}}, \lambda\right)$ is a morphism of quasi-cofree $\mathcal{O}^{i}$-coalgebras from $\left(q \mathcal{O}^{\mathrm{i}}(\mathcal{P}), \mathrm{d}_{\gamma}\right)$ to $\left(q \mathcal{O}^{\mathrm{i}}(\mathcal{Q}), \mathrm{d}_{\lambda}\right)$.

By definition, $\infty$-morphisms are composable; they form a new category denoted $\infty-\mathrm{Opd}_{\infty}$ which includes the category $\mathrm{Opd}_{\infty}$, but which is better behaved with respect to homotopy properties of higher homotopy operads. Note that the subcategories of $\mathrm{Opd}_{\infty}$ given in Proposition 3.2.1 correspond to full subcategories of $\infty$-Opd ${ }_{\infty}$ consisting of the same object, but considered with their respective notions of $\infty$-morphisms.

Definition 3.3.2. An $\infty$-morphism $\mathcal{P} \stackrel{\sim}{\rightarrow} \mathcal{Q}$ is called an $\infty$-isomorphism (resp. an $\infty$ quasi-isomorphism) when its first component $\mathcal{P} \rightarrow \mathcal{Q}$ is an isomorphism (resp. a quasiisomorphism).

Proposition 3.3.3. The class of $\infty$-isomorphisms is the class of the isomorphisms of the category $\infty-\mathrm{Opd}_{\infty}$.

PROOF. The arguments of [46, \$10.4] apply mutatis mutandis.

Theorem 3.3.4 (Homotopy Transfer Theorem). Let $\left\{\mathcal{H}(n), \mathrm{d}_{\mathcal{H}(n)}\right\}_{n \in \mathbb{N}}$ be a homotopy retract of $\left\{\mathcal{P}(n), \mathrm{d}_{\mathcal{P}(n)}\right\}_{n \in \mathbb{N}}$ in the category of $d g \mathbb{N}$-modules:

$$
h \subset\left(\mathcal{P}(n), \mathrm{d}_{\mathcal{P}}\right) \stackrel{p}{\rightleftarrows}\left(\mathcal{H}(n), \mathrm{d}_{\mathcal{H}}\right), \text { such that }\left\{\begin{array}{l}
\mathrm{id}_{\mathcal{P}}-i p=\left[\mathrm{d}_{\mathcal{P}}, h\right], \\
\operatorname{id}_{\mathcal{H}}-p i=0,
\end{array}\right.
$$

and $i$ is a quasi-isomorphism. Any (higher homotopy) operad structure on $\mathcal{P}$ can be transferred into a higher homotopy operad structure on $\mathcal{H}$ such that the quasi-isomorphism $i$ extends to an $\infty$-quasi-isomorphism.

PROOF. The arguments of [46 §10.3] apply mutatis mutandis.

Corollary 3.3.5. For any $\infty$-quasi-isomorphism $\mathcal{P} \stackrel{\sim}{\rightarrow} \mathcal{Q}$ of higher homotopy operads, there exists $\infty$-quasi-isomorphism $\mathcal{Q} \sim \mathcal{P}$ which is the inverse of $\mathrm{H}(\mathcal{P}) \cong \mathrm{H}(\mathcal{Q})$ on the level of homology.

PROOF. The arguments of [46 §10.4] apply mutatis mutandis.

3.4. Symmetric homotopy theory of operads. The general theory of [31 §5.2] and [46, \$11.4] allows us to use the Koszul duality for the colored operad $\mathcal{O}$ to provide new tools to study the homotopy properties of operads over any ring. 
We first consider the cobar-bar adjunction

$$
\Omega_{\iota}: \text { conil higher Coopd } \rightleftharpoons \mathrm{Opd}_{\infty}: \mathrm{B}_{\iota}
$$

associated to the universal twisting morphism $\iota: \mathcal{O}^{i} \rightarrow \Omega \mathcal{O}^{i}$. The image $\mathrm{B}_{l} \mathcal{P}$ of a higher homotopy operad is the equivalent definition mentioned at the end of Section 2.1 in terms of quasi-free $\mathcal{O}^{i}$-coalgebras. By definition of $\infty$-morphisms, the functor $\mathrm{B}_{\iota}$ extends to an isomorphism of categories

$$
\widetilde{\mathrm{B}}_{l}: \infty-\mathrm{Opd}_{\infty} \stackrel{\cong}{\longrightarrow} \text { quasi-free higher Coopd . }
$$

All these constructions can be summed up in the following diagram:

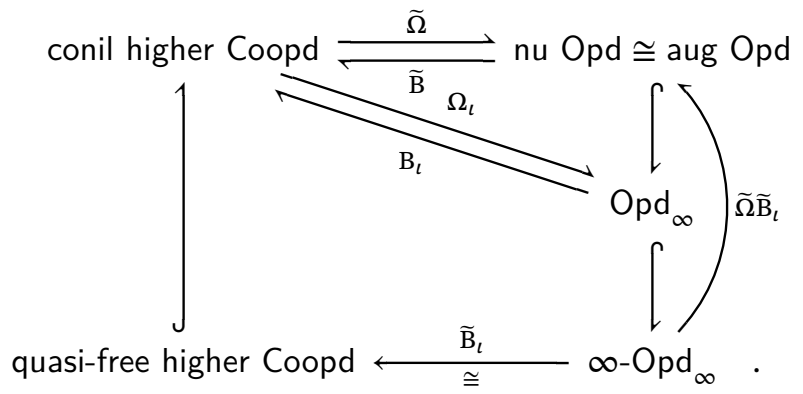

The right column is made up of a pair of adjoint functors

$$
\widetilde{\Omega} \widetilde{\mathrm{B}}_{l}: \infty-\mathrm{Opd}_{\infty} \rightleftharpoons \mathrm{nu} \text { Opd }: i
$$

which satisfy the following property.

Theorem 3.4.1 (Rectification). Any higher homotopy operad $\left(\mathcal{P}, \mathrm{d}_{\mathcal{P}}, \gamma\right)$ is naturally $\infty$ quasi-isomorphic to the dg nu operad $\widetilde{\Omega} \mathrm{B}_{l} \mathcal{P}$,

$$
\mathcal{P} \sim \tilde{\sim} \sim \widetilde{\Omega} \mathrm{B}_{l} \mathcal{P} \text {. }
$$

PROOF. The arguments of [31, 46] apply mutatis mutandis.

Rectification is unique up to unique $\infty$-isomorphism, see [46, Proposition 11.4.6].

Proposition 3.4.2 (Homotopy properties).

- For any pair of dg augmented operads $\mathcal{P}$ and $\mathcal{Q}$, there exists a zigzag of quasiisomorphisms

$$
\mathcal{P} \stackrel{\sim}{\longleftarrow} \stackrel{\sim}{\longrightarrow} \cdots \stackrel{\sim}{\longleftarrow} \stackrel{\sim}{\longrightarrow} Q
$$

if and only if there exists an $\infty$-quasi-isomorphism

$$
\mathcal{P} \leadsto \mathcal{\sim} \rightarrow
$$

- The homotopy category of dg augmented operads and the homotopy category of homotopy operads are equivalent:

$$
\mathrm{Ho}(\mathrm{dg} \text { aug } \mathrm{Opd}) \cong \mathrm{Ho}\left(\infty-\mathrm{Opd}_{\infty}\right) \text {. }
$$

PROOF. The arguments of $[31,46]$ apply mutatis mutandis. 
REMARK (Further study). At this stage, one would like to go one step further, that is to write these two localized categories as an "honest" category. Following [43, 69], one should be able to prove an equivalence like

$$
\mathrm{Ho}(\mathrm{dg} \text { aug } \mathrm{Opd}) \cong \infty-\mathrm{Opd}_{\infty} / \sim_{h}
$$

with a good notion of homotopy equivalence of $\infty$-morphisms. There are two main conceptual difficulties here since one would have to endow the category of conilpotent $\mathcal{O}^{i}$-coalgebras with a suitable model category structure: the underlying objects are not chain complexes, the "differential" map does not square to 0 , and one will have to deal with the general homotopy properties of modules over a ring, not a field. The first point is manageable, for instance by using the methods of Le Grignou [40].

\section{A. Homotopy Group Representation}

The methods used in this chapter allow us to treat an interesting question: how can one define a notion of a module over a group relaxed up to homotopy with good properties. Let $(\mathbb{G}, \cdot, e)$ be a group; a representation of $\mathbb{G}$ is a $\mathbf{k}[\mathbb{G}]$-module. So one can use the Koszul duality theory to find resolutions of the group algebra $\mathbf{k}[\mathbb{G}]$. Any presentation of the group $\mathbb{G}$ gives a presentation of the group algebra $\mathbf{k}[\mathbb{G}]$ that one can use to get "small" resolutions. Without any particular presentation, one can only consider the trivial presentation where the set of generators is the full group itself. At that point, there are two ways to apply the Koszul duality theory; we show that they are equivalent.

First, one can consider the presentation where the set of generators $\overline{\mathbb{G}}$ is the group without the unit, that is

$$
\mathbf{k}[\mathbb{G}] \cong \mathrm{T}(\overline{\mathbb{G}}) /\left(g \otimes g^{-1}-1, g \otimes h-g h \mid g h \neq e\right) .
$$

This presentation is semiaugmented according to the terminology of [31, §3.3.1], this means that the augmentation map is not required to be an algebra morphism. Then, the curved Koszul duality theory of loc. cit. applies; that is what we did in the case of the symmetric groups $\mathbb{G}=\mathbb{S}_{n}$ in the arity 1 part of the colored operad $\mathcal{O}$.

Lemma A.0.1. The group algebra $\mathbf{k}[\mathbb{G}]$ with this quadratic-linear-constant presentation is curved Koszul.

PROOF. Since the above quadratic-linear-constant presentation forms a Gröbner basis of the group algebra, Conditions (I) and (II) (already mentioned in Lemma 1.6.1) hold and the algebra is Koszul.

In this case, the Koszul dual curved coalgebra is given by the cofree coalgebra $\left(\mathrm{T}^{c}(s \overline{\mathbb{G}}), \mathrm{d}, \theta\right)$, where

$$
\mathrm{d}\left(g_{1} \otimes \cdots \otimes g_{n}\right)=\sum_{i=1}^{n-1}(-1)^{i-1} \cdot g_{1} \otimes \cdots \otimes \underbrace{g_{i} g_{i+1}}_{\neq e} \otimes \cdots \otimes g_{n}
$$

and $\theta\left(g \otimes g^{-1}\right)=1$. 
Proposition A.0.2. The cobar construction of the Koszul dual curved coalgebra forms a cofibrant resolution of the group algebra

$$
\Omega_{1}:=\left(\mathrm{T}\left(s^{-1} \overline{\mathrm{T}^{c}}(s \overline{\mathbb{G}})\right), \mathrm{d}_{2}+\mathrm{d}_{1}-\mathrm{d}_{0}\right) \stackrel{\sim}{\longrightarrow} \mathbf{k}[\mathbb{G}] .
$$

On the other hand, one can consider the augmentation

$$
\varepsilon: \mathbf{k}[\mathbb{G}] \longrightarrow \mathbf{k}, \quad g \longmapsto 1
$$

which induces the splitting $\mathbf{k}[\mathbb{G}] \cong \mathbf{k} \oplus \operatorname{ker} \varepsilon$. The augmentation ideal admits $\{\tilde{g}:=1-g \mid$ $g \in \overline{\mathbb{G}}\}$ as basis; we can use it to provide the group algebra $\mathbf{k}[\mathbb{G}]$ with a quadratic-linear presentation:

$$
\mathbf{k}[\mathbb{G}] \cong \mathrm{T}(\overline{\mathbb{G}}) /\left(\tilde{g} \otimes \widetilde{g^{-1}}-\tilde{g}-\widetilde{g^{-1}}, \tilde{g} \otimes \tilde{h}-\tilde{g}-\tilde{h}+\widetilde{g h} \mid g h \neq e\right) .
$$

Such formulas using $\tilde{g}$ instead of group elements $g$ can be interpreted as some kind of "linearization" of the group $\mathbb{G}$. The first presentation of the group algebra is settheoretical, this second one is not.

Lemma A.0.3. The group algebra $\mathbf{k}[\mathbb{G}]$ with this quadratic-linear presentation is inhomogeneous Koszul.

PROOF. Again this presentation provides the group algebra with a Gröbner basis. Hence it proves Conditions (I) and (II) (already mentioned in Lemma 1.6.1) and that this presentation is inhomogeneous Koszul.

In this case, the Koszul dual is the dg coalgebra $\left(\mathrm{T}^{c}(s \overline{\mathbb{G}}), \mathrm{d}_{\varphi}\right)$, where

$$
\mathrm{d}_{\varphi}\left(\tilde{g}_{1} \otimes \cdots \otimes \tilde{g}_{n}\right)=\sum_{i=1}^{n-1}(-1)^{i-1} \cdot \tilde{g}_{1} \otimes \cdots \otimes\left(\tilde{g}_{i}+\tilde{g}_{i+1}-\widetilde{g_{i} g_{i+1}}\right) \otimes \cdots \otimes \tilde{g}_{n} .
$$

Proposition A.0.4. The cobar construction of the Koszul dual dg coalgebra forms a cofibrant resolution of the group algebra

$$
\Omega_{2}:=\left(\mathrm{T}\left(s^{-1} \overline{\mathrm{Tc}^{c}}(s \overline{\mathbb{G}})\right), \mathrm{d}_{2}^{\prime}+\mathrm{d}_{1}^{\prime}\right) \stackrel{\sim}{\longrightarrow} \mathbf{k}[\mathbb{G}] .
$$

So both $\Omega_{1}$-algebras and $\Omega_{2}$-algebras define a notion of homotopy $\mathbb{G}$-module with the required homotopy properties: $\infty$-morphisms, Homotopy Transfer Theorem, description of the homotopy category of $\mathbb{G}$-modules, for instance. By abstract nonsense, the two cofibrant resolutions $\Omega_{1}$ and $\Omega_{2}$ are quasi-isomorphic so these two notions of homotopy $\mathbb{G}$-modules are homotopy equivalent. But, in this case, one can prove the following stronger result.

Theorem A.0.5. The two resolutions $\Omega_{1}$ and $\Omega_{2}$ of the group algebra $\mathbf{k}[\mathbb{G}]$ are isomorphic, which makes the two associated notions of homotopy $\mathbb{G}$-modules isomorphic.

PROOF. One can consider the isomorphism $\Omega_{2} \rightarrow \Omega_{1}$ defined on the generators by

$$
\left\{\begin{array}{lll}
\tilde{g} & \longmapsto 1-g, \\
s^{-1}\left(s \tilde{g}_{1} \otimes \cdots \otimes s \tilde{g}_{n}\right) & \longmapsto(-1)^{n} \cdot s^{-1}\left(s g_{1} \otimes \cdots \otimes s g_{n}\right) \quad \text { for } n \geq 2,
\end{array}\right.
$$


with the reverse isomorphism $\Omega_{1} \rightarrow \Omega_{2}$ given by

$$
\left\{\begin{array}{lll}
g & \longmapsto 1-\tilde{g}, \\
s^{-1}\left(s g_{1} \otimes \cdots \otimes s g_{n}\right) & \longmapsto(-1)^{n} \cdot s^{-1}\left(s \tilde{g}_{1} \otimes \cdots \otimes s \tilde{g}_{n}\right) \quad \text { for } n \geq 2 .
\end{array}\right.
$$

We choose the first resolution to define the homotopy notion of $\mathbb{G}$-module

Definition A.0.6. A homotopy $\mathbb{G}$-module, or $\mathbb{G}_{\infty}$-module, is a chain complex $(V$,d) equipped with degree $n-1$ maps

$$
\gamma\left(g_{1}, \ldots, g_{n}\right): V \rightarrow V
$$

for any $n$-tuples $\left(g_{1}, \ldots, g_{n}\right) \in \overline{\mathbb{G}}^{n}$ satisfying

$$
\begin{gathered}
\sum_{i=1}^{n-1}(-1)^{i-1} \cdot \gamma\left(g_{1}, \ldots, g_{i}\right) \circ \gamma\left(g_{i+1}, \ldots, g_{n}\right)-\sum_{i=1}^{n-1}(-1)^{i-1} \cdot \gamma(g_{1}, \ldots, \underbrace{g_{i} g_{i+1}}_{\neq e}, g_{n}) \\
-\partial\left(\gamma\left(g_{1}, \ldots, g_{n}\right)\right)= \begin{cases}\operatorname{id}_{V}, & \text { when }\left(g_{1}, \ldots, g_{n}\right)=\left(g, g^{-1}\right), \\
0, & \text { otherwise } .\end{cases}
\end{gathered}
$$

A notion of "representation up to homotopy" was introduced in [1] as follows. Instead of considering algebra morphisms $\mathbf{k}[\mathbb{G}] \rightarrow \operatorname{Hom}(V, V)$, one can consider the wider class of $\mathrm{A}_{\infty}$-morphisms. Such a data is actually equivalent to an algebra morphism from the bar-cobar construction of $\mathbf{k}[\mathbb{G}$ ], as explained in [46, §10.5.5]. Notice that the Koszul resolution obtained from the trivial presentation with all the elements for generators is the bar-cobar construction. Hence the formulas in loc. cit. are similar to the one given here, except that the specific role of the group unit is not taken into account there. One obtains the definition of [1] by applying the bar-cobar construction to the group algebra after forgetting its unit. The interpretation in terms of Maurer-Cartan elements of [1, §3] then follows directly from the general theory [46. Chapter 2].

REMARK A.0.7. In this chapter, we could have chosen to work with the linearized presentation of the symmetric group algebras. Only Relations (O-1) and (O-2) of Definition 1.2.1 would change, not $\operatorname{Relations}(\mathcal{O}-5)$ and $(\mathcal{O}-6)$ which would remain exactly the same with $\tilde{\sigma}$ instead of $\sigma$. So all the results would still hold with nearly the same formulas and we would get a cofibrant resolution isomorphic to the one given in Theorem 1.6.5. With that approach, we would get a Koszul dual dg colored cooperad instead of a curved colored cooperad but the underlying colored cooperad would be the same, the Maurer-Cartan equation encoding homotopy operads would have no curvature terms and only the third term on the left-hand side of Equation (85) would be modified using the above formula for $\mathrm{d}_{\varphi}$. However, the (homotopy) action of the symmetric groups would be less transparent and not set-theoretical: it is the linearized action that would be relaxed up to homotopy. 


\section{On Weak Lie 3-Algebras}

This chapter consists of our article [11]. Minor modifications were made to the structure and references were added to earlier parts of this thesis.

\section{Introduction}

Our interest in this article lies in finding a small cofibrant replacement $\mathrm{EL}_{\infty} \stackrel{\sim}{\rightarrow}$ Lie for the Lie operad over any commutative unital ring $\mathbf{k}$. Since the operad Lie is not $\mathbb{S}$-cofibrant, we cannot use the classical Koszul duality methods. Within the context of the new cobar-bar adjunction $\widetilde{\Omega} \dashv \widetilde{\mathrm{B}}$ we introduced in Chapter 3 . however, a Koszul duality approach is not yet available. We will therefore resort to a more ad hoc approach to build our resolution: we start from the usual Koszul dual cooperad Lie ${ }^{i}$ and build an $\mathbb{S}$-free resolution step by step. Assume for a moment that we had completed this process, i.e., we have an $\mathbb{S}$-free resolution $\psi: \mathrm{Lie}^{\diamond} \tilde{\rightarrow} \mathrm{Lie}^{i}$ of $\mathrm{dg}$ cooperads. Since $\mathrm{Lie}^{\diamond}$ is $\mathbb{S}$-free, $\Omega \mathrm{Lie}^{\diamond}$ is then a cofibrant operad. To show that the composition

$$
g_{\kappa} \circ \Omega \psi: \Omega \mathrm{Lie}^{\diamond} \longrightarrow \Omega \mathrm{Lie}^{\mathrm{i}} \stackrel{\sim}{\longrightarrow} \mathrm{Lie}
$$

forms a cofibrant resolution, we need to verify that $\Omega \psi$ is a quasi-isomorphism or, equivalently, that the twisted composite product $\mathrm{Lie}^{\diamond}{ }_{\kappa \circ \psi}$ Lie is acyclic. In this case, we can apply the standard machinery of algebraic operads to obtain a category of $\mathrm{EL}_{\infty}{ }^{-}$ algebras with homotopy morphisms, satisfying a version of the homotopy transfer theorem. We recall the relevant background material in Chapter 2

While we do not have a complete $\mathbb{S}$-free resolution of $\mathrm{dg}$ cooperads as described above, in Section 1 we do construct such a resolution in low degrees and show that at least truncated versions of the relevant statements hold. As a first step, we introduce an explicit $\mathbb{S}$-free resolution $\psi: \mathrm{Lie}_{3}^{\diamond} \rightarrow \mathrm{Lie}^{i}$ of $\mathrm{dg} \mathbb{S}$-modules in low degrees, i.e., such that $\mathrm{H}_{r}(\psi)$ are isomorphisms for $r \leq 3$. In the second step, we equip Lie ${ }^{\diamond}$ with a decomposition map turning it into a dg cooperad. Since Leibniz algebras are essentially nonsymmetric Lie algebras, it makes sense to use their $\mathbb{S}$-free Koszul dual cooperad Leib $^{i}$ as a starting point for both steps. The higher degrees of $\mathrm{Lie}^{\diamond}$ can be viewed as a coherent system of higher homotopies for the (missing) skewsymmetry. We extend the decomposition map of Leib ${ }^{i}$ to the higher degrees in a way that is compatible with the differential by solving systems of linear diophantine equations, and verify that it is actually coassociative. Finally we prove the following result, which-while of no immediate consequences - is a necessary condition if we intend to extend our low degree resolution to a full cofibrant resolution. 
Proposition 1.4.1. The twisted composite product $\mathrm{Lie}_{3}^{\diamond}{ }^{\circ} \circ \psi$ Lie satisfies

$$
\mathrm{H}_{r}\left(\left(\operatorname{Lie}_{3}^{\diamond} \circ_{\kappa \circ \psi} \operatorname{Lie}\right)(n)\right)=0
$$

for all $r \leq 3$ in all arities $n$.

In Section 2, we use the resolution $\mathrm{Lie}_{3}^{\diamond}$ to define weak Lie 3-algebras as $\mathrm{SLie}_{3}^{\diamond}$ algebras on a 3-term complex, and we introduce the corresponding notion of weak morphisms. Since we used Leib ${ }^{i}$ as a starting point for our resolution, weak Lie 3-algebras and their weak morphisms consist of extra structure on top of Leibniz 3-algebras and homotopy morphisms of such. We make the definitions explicit in terms of structure maps and equations. We proceed to show explicitly that, given a deformation retract of 3-term chain complexes, i.e., chain maps $p$ and $i$ and a chain homotopy $h$ as in

$$
h \subset(L, \mathrm{~d}) \stackrel{p}{\rightleftarrows}\left(L^{\prime}, \mathrm{d}^{\prime}\right), \quad \text { such that }\left\{\begin{array}{l}
\mathrm{id}_{L}-i \circ p=[\mathrm{d}, h], \\
\operatorname{id}_{L^{\prime}}-p \circ i=0,
\end{array}\right.
$$

the following homotopy transfer property holds.

Proposition 2.4.1. Let $(L, \mathrm{~d}, \lambda)$ be a weak Lie 3-algebra and let $\left(L^{\prime}, \mathrm{d}^{\prime}\right)$ be a deformation retract of $(L, \mathrm{~d})$. Then $\left(L^{\prime}, \mathrm{d}^{\prime}\right)$ can be equipped with a transferred weak Lie 3-algebra structure in such a way, that the map i admits an extension to a weak morphism of weak Lie 3-algebras.

In [2] the notion of a Lie 2-algebra is introduced using a very different approach known as categorification. It is then shown that the definition is equivalent to that of a 2-term $\mathrm{L}_{\infty}$-algebra, i.e., a 2-term chain complex with a binary graded skewsymmetric bracket satisfying the Jacobi identity up to homotopy. In [63], the definition of a weak Lie 2-algebra is introduced, again as a categorification of Lie algebras, this time with the skewsymmetry of the Lie bracket relaxed up to homotopy in addition to the Jacobi identity. Truncating the complexes underlying our weak Lie 3-algebras to 2-term complexes, we recover Roytenberg's definitions of weak Lie 2-algebras and their weak morphisms. Similarly, we recover his homotopy transfer theorem [63. Theorem 4.1] for weak Lie 2-algebras as a truncation of Proposition 2.4.1.

By construction of $\mathrm{Lie}_{3}^{\diamond}$, we have morphisms of dg cooperads

$$
\operatorname{Leib}_{3}^{i} \hookrightarrow \operatorname{Lie}_{3}^{\diamond} \longrightarrow \operatorname{Lie}_{3}^{i}
$$

and therefore functors

$$
\text { Leibniz 3-algebras } \longleftarrow \text { Weak Lie 3-algebras } \longleftarrow \text { Lie 3-algebras . }
$$

While (homotopy) Lie algebras are precisely (homotopy) Leibniz algebras with skewsymmetric structure maps, in general skewsymmetrizing the bracket(s) of a (homotopy) Leibniz algebra does not give a (homotopy) Lie algebra. Operadically speaking, this means that there is no simple nontrivial morphism of cooperads Lie $^{i} \rightarrow$ Leib $^{i}$. Using the higher degree terms in $\mathrm{Lie}_{3}^{\diamond}$ we can, however, construct a morphism $\mathrm{Lie}_{3}^{\diamond} \leadsto \mathrm{Leib}_{3}^{\mathrm{i}}$ of dg cooperads up to homotopy. Precisely, we will prove the following result in Section 3 . 
Lemma 3.1.1. The morphism $\Omega \psi$ admits a right inverse, i.e., a morphism $\Phi$ of $d g$ operads

$$
\Omega \psi: \Omega \operatorname{Lie}_{3}^{\diamond} \rightleftharpoons \Omega \operatorname{Lie}_{3}^{i}: \Phi, \text { such that } \Omega \psi \circ \Phi=\mathrm{id} .
$$

This leads to a skewsymmetrization construction producing for each weak Lie 3algebra $(L, \mathrm{~d}, \lambda)$ a (semistrict) Lie 3-algebra $(L, \mathrm{~d}, \bar{\lambda})$. We then introduce an ad hoc definition of skewsymmetrization for weak morphisms of weak Lie 3-algebras and proceed to show that this construction-while not strictly functorial-is in some sense functorial up to homotopy.

We end this article with a discussion of applications of our results in higher differential geometry. In [3, 62, the concept of $n$-plectic manifolds is introduced as a higher analogue for symplectic manifolds. To any $n$-plectic manifold $(M, \omega)$, two algebraic structures on an $n$-term truncation of the de Rham complex are associated: an $\mathrm{L}_{\infty}$-algebra $\mathrm{L}_{\infty}(M, \omega)$ and a dg Leibniz algebra $\operatorname{Leib}(M, \omega)$ with a certain hidden skewsymmetry. In the case of 3-plectic manifolds, both are examples of weak Lie 3-algebras. We show that $\mathrm{L}_{\infty}(M, \omega)$ and $\operatorname{Leib}(M, \omega)$ are isomorphic as such. The analogous result for 2-plectic manifolds is due to Rogers [62, Appendix A].

In recent work of Liu-Sheng [44], the notion of a CLWX 2-algebroid is introduced as a higher Courant algebroid, and it is shown that a Lie 3-algebra can be assigned to any CLWX 2-algebroid. We refine the construction to give a weak Lie 3-algebra and show that the Lie 3-algebra constructed in op. cit. is in fact the skewsymmetrization of our weak Lie 3-algebra, thereby giving a new proof for [44, Theorem 3.10].

\section{An $\mathbb{S}$-Free Resolution of the Koszul Dual Cooperad of the Lie Operad}

In this section, we attempt to construct an $\mathbb{S}$-free resolution $\psi: \mathrm{Lie}^{\diamond} \tilde{\rightarrow} \mathrm{Lie}^{i}$ of dg cooperads over $\mathbb{Z}$ for the Koszul dual cooperad of the Lie operad. Assume for a moment that we have such a resolution. If we can show that $\psi$ is in fact a weak equivalence, i.e., $\Omega \psi$ is a quasi-isomorphism, then this gives us a small cofibrant resolution

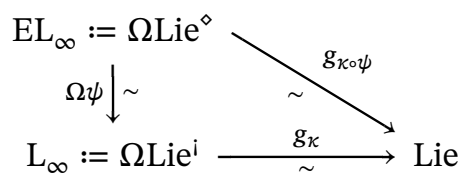

of the Lie operad over $\mathbb{Z}$, and therefore over any unital commutative ring $\mathbf{k}$. Unfortunately, we do not yet have a general method to obtain such an $\mathbb{S}$-free resolution. Instead, we proceed degreewise to construct a dg cooperad $\mathrm{Lie}_{3}^{\diamond}$ that satisfies the desired conditions in low degrees (see the following sections for the precise meaning of "in low degrees.”)

REMARK. While our notation below may suggest that we are working with truncations of a dg cooperad Lie ${ }^{\diamond}$, this is not a proven result. We do not have a complete resolution $\mathrm{Lie}^{\diamond} \stackrel{\sim}{\rightarrow} \mathrm{Lie}^{i}$, nor do we have a proof that our $\mathrm{Lie}_{3}^{\diamond}$ extends to such a resolution.

The remainder of this section is organized as follows. In Section 1.1 we recall the Koszul dual cooperads Lie ${ }^{i}$ and Leib ${ }^{i}$ of the Lie and Leibniz operads, respectively. In 
Section 1.2, we construct an explicit $\mathbb{S}$-free resolution $\psi: \mathrm{Lie}_{3}^{\diamond} \rightarrow \mathrm{Lie}^{\mathrm{i}}$ as a dg $\mathbb{S}$-module in low degrees. In Section 1.3 we equip Lie ${ }_{3}^{\diamond}$ with a decomposition map and show that our definition turns it into a dg cooperad. Finally, in Section 1.4 we prove that the homology of the twisted composite product $\mathrm{Lie}_{3}^{\diamond}{ }^{\circ}{ }^{\circ} \psi$ Lie vanishes in low degrees.

1.1. The Koszul dual cooperads of the Lie and Leibniz operads. In Section 7 of Chapter 2 we saw that the Koszul dual cooperad for Lie is

$$
\operatorname{Lie}^{i}=\left(\mathcal{S} \otimes \operatorname{Lie}^{!}\right)^{*}=\mathcal{S}^{*} .
$$

Explicitly, this means the $\mathbb{S}$-module underlying Lie is given by

$$
\operatorname{Lie}^{\mathrm{i}}(0)=0 \quad \text { and } \quad \operatorname{Lie}^{\mathrm{i}}(n)=\left(\bar{l}_{n} \cdot \mathbf{k} \cdot \operatorname{sgn}_{n}\right)[n-1] \quad \text { for } n \geq 1,
$$

and its decomposition map is given by

$$
\Delta\left(\bar{l}_{n}\right)=\sum_{\substack{1 \leq j \leq n \\ i_{1}+\cdots+i_{j}=n}}(-1)^{(j-1)(n-j)} \cdot(-1)^{\sum_{p=1}^{j}(p-1)\left(i_{p}-1\right)} \sum_{\sigma \in \overline{S h}^{-1}\left(i_{1}, \ldots, i_{j}\right)}\left(-1|\sigma| \cdot \bar{l}_{j} \circ\left(\bar{l}_{i_{1}}, \ldots, \bar{l}_{i_{j}}\right)^{\sigma} .\right.
$$

From this we obtain, by projection to $\mathrm{Lie}^{\mathrm{i}}{ }_{(1)} \mathrm{Lie}^{\mathrm{i}}$, the partial decomposition map

$$
\Delta_{(1)}\left(\bar{l}_{n}\right)=\sum_{i+j=n+1}(-1)^{(j-1)(i-1)} \sum_{p=1}^{j}(-1)^{(p-1)(i-1)} \sum_{\sigma \in \overline{S h}^{-1}(p-1, i)}(-1)^{|\sigma|} \cdot\left(\bar{l}_{j} \otimes_{p} \bar{l}_{i}\right)^{\sigma} .
$$

Using the skewsymmetry of the $\bar{l}_{n}$, it can be rewritten as

$$
\Delta_{(1)}\left(\bar{l}_{n}\right)=\sum_{i+j=n+1}(-1)^{(j-1)(i-1)} \sum_{\sigma \in \mathrm{Sh}^{-1}(i, j-1)}(-1)^{|\sigma|} \cdot\left(\bar{l}_{j} \otimes_{1} \bar{l}_{i}\right)^{\sigma} .
$$

The Koszul dual operad Leib ${ }^{\prime}=$ Zinb for Leib was first introduced by Loday in [45]. Algebras over Leib! were originally referred to as dual Leibniz algebras, but are now more commonly known as Zinbiel algebras. As before, the Koszul dual cooperad Leib ${ }^{i}$ can be computed as Leib ${ }^{i}=(\mathcal{S} \otimes \text { Zinb })^{*}$. Explicitly, it is given by

$$
\operatorname{Leib}^{\mathrm{i}}(0)=0 \quad \text { and } \quad \operatorname{Leib}^{\mathrm{i}}(n)=\left(l_{n} \cdot \mathbf{k}\left[\mathbb{S}_{n}\right]\right)[n-1] \quad \text { for } n \geq 1,
$$

and with (partial) decomposition map given as in Equations (86) and (87)] substituting $l_{n}$ for $\bar{l}_{n}$. Note however, that Equations (87) and (88) are not equivalent in this case, since the $l_{n}$ are not skewsymmetric.

There is an obvious morphism of dg cooperads,

$$
\psi: \text { Leib }^{\mathrm{i}} \rightarrow \mathrm{Lie}^{\mathrm{i}}, \quad \psi\left(l_{n}^{\sigma}\right)=(-1)^{\sigma} \cdot \bar{l}_{n} .
$$

Since Leib is $\mathbb{S}$-free and this morphism is surjective, this provides us with a good starting point for our resolution of Liei.

1.2. The resolution as an $\mathbb{S}$-free $\mathbf{d g} \mathbb{S}$-module. In this section, we fix an $\mathbb{S}$-free resolution of $\mathrm{Lie}^{i}$ as an $\mathbb{S}$-module in low degrees. We describe, in general, a way to

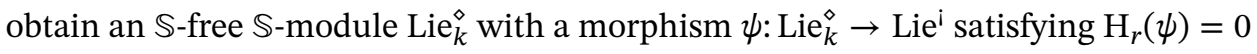
for $r \leq k$. We make such an $\mathbb{S}$-module explicit for $k=3$. 
We construct, for each arity $n \geq 1$, an exact augmented complex

$$
0 \leftarrow \operatorname{Lie}^{i}(n)_{n-1} \leftarrow \operatorname{Lie}_{k}^{\diamond}(n)
$$

in $\mathbf{k}\left[\mathbb{S}_{n}\right]$-modules up to degree $k+1$. As indicated in Section 1.1, we may choose $\operatorname{Lie}_{k}^{\diamond}(n)_{n-1}:=\operatorname{Leib}^{\mathrm{i}}(n)_{n-1}$ and the augmentation map to be $\psi$ as defined by Equation (89) i.e., our complexes are of the following shape:

$$
\begin{array}{r}
0 \longleftarrow \operatorname{Lie}^{\mathrm{i}}(n)_{n-1} \stackrel{\psi=\mathrm{d}_{n-1}}{\longleftarrow} \operatorname{Leib}^{\mathrm{i}}(n)_{n-1} \stackrel{\mathrm{d}_{n}}{\longleftarrow} \operatorname{Lie}_{k}^{\diamond}(n)_{n} \stackrel{\mathrm{d}_{n+1}}{\longleftarrow} \ldots \\
\ldots \stackrel{\mathrm{d}_{k+1}}{\longleftarrow} \operatorname{Lie}_{k}^{\diamond}(n)_{k+1} .
\end{array}
$$

Our general approach to determining the higher degrees of $\operatorname{Lie}_{k}^{\diamond}(n)$ is to construct a resolution of syzygies as follows. For $r=n-1, \ldots, k$, successively, we extend the complex using the following steps:

(i) compute $\operatorname{ker}\left(\mathrm{d}_{r}\right)$,

(ii) choose generators $\left\{x_{i}\right\}_{i \in I}$ for $\operatorname{ker}\left(\mathrm{d}_{r}\right)$ as a $\mathbf{k}\left[\mathbb{S}_{n}\right]$-module, and

(iii) define $\operatorname{Lie}_{k}^{\diamond}(n)_{r+1}:=\left\langle\hat{x}_{i}\right\rangle_{i \in I}$ to be the free $\mathbf{k}\left[\mathbb{S}_{n}\right]$-module generated by symbols $\left\{\hat{x}_{i}\right\}_{i \in I}$ and the differential by $\mathrm{d}_{r+1}\left(\hat{x}_{i}\right):=x_{i}$.

Obviously, any complex $\operatorname{Lie}_{k}^{\diamond}(n)$ constructed in this way will be exact. We describe the beginning of these computations explicitly for general $n$.

Degree $\mathbf{r}=\mathbf{n}-\mathbf{1}:$ Since

$$
\mathrm{d}_{n-1}\left(l_{n}-(-1)^{|\sigma|} \cdot l_{n}^{\sigma}\right)=\psi\left(l_{n}-(-1)^{|\sigma|} \cdot l_{n}^{\sigma}\right)=\bar{l}_{n}-(-1)^{|\sigma|} \cdot(-1)^{|\sigma|} \cdot \bar{l}_{n}^{\sigma}=0
$$

and $\operatorname{dim}_{\mathbf{k}}\left(\mathrm{im}\left(\mathrm{d}_{n-1}\right)\right)=1$, we obtain

$$
\operatorname{ker}\left(\mathrm{d}_{n-1}\right)=\operatorname{span}_{\mathbf{k}}\left\{l_{n}-(-1)^{|\sigma|} \cdot l_{n}^{\sigma} \mid \sigma \in \mathbb{S}_{n} \backslash \mathrm{id}\right\} .
$$

As a $\mathbf{k}\left[\mathbb{S}_{n}\right]$-module, the kernel is generated by the set $\left\{l_{n}+l_{n}^{\sigma_{i}}\right\}_{i=1}^{n-1}$ for the adjacent transpositions $\sigma_{i}=(i i+1)$. We define

$$
\operatorname{Lie}_{k}^{\diamond}(n)_{n}:=\left\langle l_{n ; i} \mid 1 \leq i<n\right\rangle, \quad \text { with } \quad \mathrm{d}_{n}\left(l_{n ; i}\right):=-l_{n}-l_{n}^{\sigma_{i}} .
$$

Degree $\mathbf{r}=\mathbf{n}$ : Clearly, the following hold

$$
\begin{aligned}
& \mathrm{d}_{n}\left(l_{n ; i}-l_{n ; i}^{\sigma_{i}}\right)=\left(-l_{n}-l_{n}^{\sigma_{i}}\right)-\left(-l_{n}-l_{n}^{\sigma_{i}}\right)^{\sigma_{i}}=0, \\
& \mathrm{~d}_{n}\left(l_{n ; i}-l_{n ; i+1}^{\sigma_{i}}+l_{n ; i}^{\sigma_{i+1} \sigma_{i}}-l_{n ; i+1}+l_{n ; i}^{\sigma_{i+1}}+l_{n ; i+1}^{\sigma_{i} \sigma_{i+1}}\right) \\
& \quad=\left(-l_{n}-l_{n}^{\sigma_{i}}\right)-\left(-l_{n}-l_{n}^{\sigma_{i+1}}\right)^{\sigma_{i}}+\left(-l_{n}-l_{n}^{\sigma_{i}}\right)^{\sigma_{i+1} \sigma_{i}} \\
& \quad-\left(-l_{n}-l_{n}^{\sigma_{i+1}}\right)+\left(-l_{n}-l_{n}^{\sigma_{i}}\right)^{\sigma_{i+1}}-\left(-l_{n}-l_{n}^{\sigma_{i+1}}\right)^{\sigma_{i} \sigma_{i+1}}=0,
\end{aligned}
$$

and, for $|i-j|>1$,

$\mathrm{d}_{n}\left(l_{n ; i}-l_{n ; j}^{\sigma_{i}}-l_{n ; j}+l_{n ; i}^{\sigma_{j}}\right)=\left(-l_{n}-l_{n}^{\sigma_{i}}\right)-\left(-l_{n}-l_{n}^{\sigma_{j}}\right)^{\sigma_{i}}-\left(-l_{n}-l_{n}^{\sigma_{j}}\right)+\left(-l_{n}-l_{n}^{\sigma_{i}}\right)^{\sigma_{j}}=0$.

In fact, these elements generate the kernel of $\mathrm{d}_{n}$ under the $\mathbf{k}\left[\mathbb{S}_{n}\right]$-action. We omit the general proof here, since we will only need this result in arity 3 where it is a trivial computation of the rank of a $12 \times 18$ matrix over $\mathbf{k}$. 
We define

$$
\operatorname{Lie}_{k}^{\diamond}(n)_{n+1}:=\left\langle l_{n ; i, j} \mid 1 \leq i \leq j<n\right\rangle,
$$

and

$$
\mathrm{d}_{n+1}\left(l_{n ; i, j}\right):= \begin{cases}-l_{n ; i}+l_{n ; i}^{\sigma_{i}}, & \text { for } j=i, \\ l_{n ; i}-l_{n ; i+1}^{\sigma_{i}}+l_{n ; i}^{\sigma_{i+1} \sigma_{i}}-l_{n ; i+1}+l_{n ; i}^{\sigma_{i+1}}+l_{n ; i+1}^{\sigma_{i} \sigma_{i+1},}, & \text { for } j=i+1, \\ l_{n ; i}-l_{n ; j}^{\sigma_{i}}-l_{n ; j}+l_{n ; i}^{\sigma_{j}}, & \text { for } j>i+1 .\end{cases}
$$

The above computations are already enough for our purpose. In summary, we obtain the explicit dg $\mathbb{S}$-module $\mathrm{Lie}_{3}^{\diamond}$ as presented in Figure 1. Note that we included the results of computations for one additional degree. So far, we left out arity 1 . We will need to define $\operatorname{Lie}_{3}^{\diamond}(1):=\operatorname{Lie}_{3}^{i}(1)=\mathbf{k}$ for the counit of the cooperad structure introduced in the following section.

1.3. The resolution as a dg cooperad. In this section, we describe how to equip a dg $\mathbb{S}$-module $\mathrm{Lie}_{k}^{\diamond}$, as obtained in the previous section, with a decomposition map $\Delta$ in such a way that

(i) $\left(\mathrm{Lie}_{k}^{\diamond}, \mathrm{d}, \Delta\right)$ becomes a dg cooperad, and

(ii) $\psi:$ Lie $_{k}^{\diamond} \rightarrow$ Lie $^{i}$ becomes a morphism of dg cooperads.

As before, we begin by explaining the general approach and then proceed to make such a structure explicit for the case $k=3$, i.e., on the $\mathrm{dg} \mathbb{S}$-module $\mathrm{Lie}_{3}^{\diamond}$ of the previous section.

Since we started our resolution of dg $\mathbb{S}$-modules by $\psi: \operatorname{Lie}_{k}^{\diamond}(n)_{n-1}=\operatorname{Leib}^{\mathrm{i}}(n) \rightarrow$ $\operatorname{Lie}^{\mathrm{i}}(n)$, and this is in fact a morphism of dg cooperads, we define the decomposition map $\Delta$ on $\operatorname{Lie}_{k}^{\diamond}(n)_{n-1}$ in the same way as on Leibi $(n)$, i.e., by Equation (86). It remains to extend the definition of the decomposition map to the higher degree terms of $\operatorname{Lie}_{k}^{\diamond}(n)$. Clearly, any such extension will satisfy Condition (ii)

Consider now Condition $(i)$ Note that it implies, in particular, that the decomposition map be a map of dg $\mathbb{S}$-modules, i.e., $\Delta$ has to commute with the differential as in the diagram

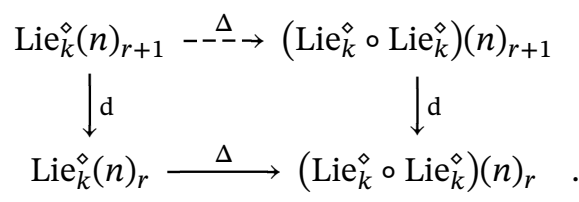

We use this condition to define $\Delta$ as follows: In each arity $n$, proceed degreewise for $r=n-1, \ldots, k+1$. Given a $\mathbf{k}\left[\mathbb{S}_{n}\right]$-basis $\left\{x_{i}\right\}_{i \in I}$ for $\operatorname{Lie}_{k}^{\diamond}(n)_{r+1}$, solve the equations $\mathrm{d}\left(y_{i}\right)=\Delta\left(\mathrm{d} x_{i}\right)$ for $y_{i}$ and define $\Delta$ by $\Delta\left(x_{i}\right):=y_{i}$ and $\mathbf{k}\left[\mathbb{S}_{n}\right]$-linearity. Finally, it remains to check that our decomposition map $\Delta$ satisfies the coassociativity condition.

The remainder of this section consists of the explicit computations for the case of $\mathrm{Lie}_{3}^{\diamond}$. As mentioned in Chapter 1 we work over the integers $\mathbb{Z}$. Finding a decomposition map as described above amounts to solving systems of linear diophantine equations. For $\mathrm{Lie}_{3}^{\diamond}$ these are still manageable by hand; for $\mathrm{Lie}_{k}^{\diamond}$ for $k \geq 4$, a computer can be used 


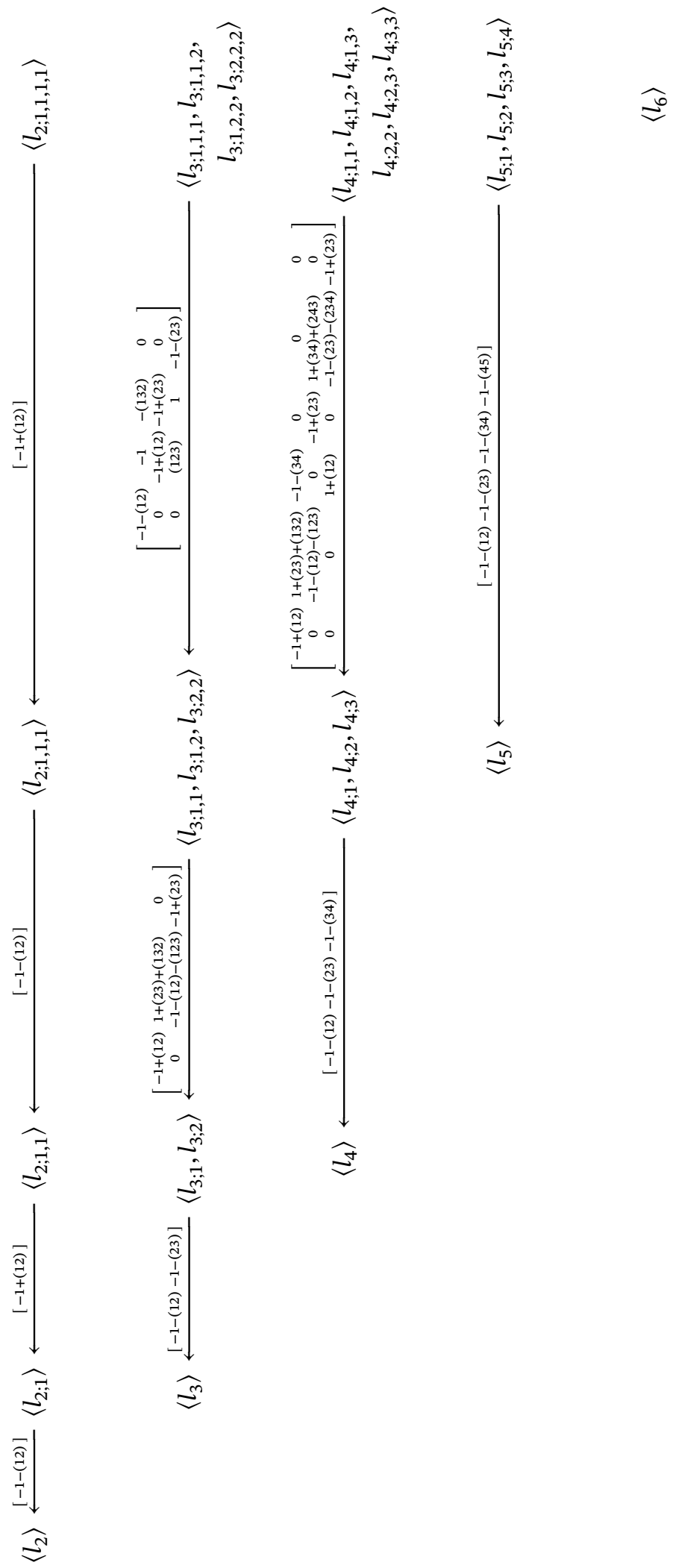

FIGURE 1. The $\mathbb{S}$-free dg $\mathbb{S}$-module Lie ${ }^{\diamond}$ 
to solve them. To save a bit of space, we work with the reduced decomposition map $\bar{\Delta}$ instead of the full decomposition $\Delta$ below.

Arity $\mathbf{n}=2$ : The (reduced) decomposition $\bar{\Delta}$ vanishes for degree reasons, i.e., we have

$$
\bar{\Delta}\left(l_{2}\right):=\bar{\Delta}\left(l_{2 ; 1}\right):=\bar{\Delta}\left(l_{2 ; 1,1}\right):=\bar{\Delta}\left(l_{2 ; 1,1,1}\right):=0 .
$$

Arity $\mathbf{n}=3$ : For $l_{3}$, we have defined $\bar{\Delta}$ by Equation (86) i.e.,

$$
\bar{\Delta}\left(l_{3}\right)=-l_{2} \circ\left(l_{2}, 1\right)+l_{2} \circ\left(1, l_{2}\right)-l_{2} \circ\left(1, l_{2}\right)^{(12)},
$$

as in Leib ${ }^{i}(3)$. To extend the decomposition map $\bar{\Delta}$ to the next degree, we compute for $l_{3 ; 1}$,

$$
\begin{aligned}
\bar{\Delta}\left(\mathrm{d} l_{3 ; 1}\right)= & \bar{\Delta}\left(-l_{3}-l_{3}^{(12)}\right)=-\bar{\Delta}\left(l_{3}\right)-\bar{\Delta}\left(l_{3}\right)^{(12)} \\
= & -\left(-l_{2} \circ\left(l_{2}, 1\right)+l_{2} \circ\left(1, l_{2}\right)-l_{2} \circ\left(1, l_{2}\right)^{(12)}\right) \\
& -\left(-l_{2} \circ\left(l_{2}, 1\right)+l_{2} \circ\left(1, l_{2}\right)-l_{2} \circ\left(1, l_{2}\right)^{(12)}\right)^{(12)} \\
= & l_{2} \circ\left(l_{2}, 1\right)+l_{2} \circ\left(l_{2}^{(12)}, 1\right),
\end{aligned}
$$

then solve for a preimage under $\mathrm{d}$,

$$
=-l_{2} \circ\left(\mathrm{d} l_{2 ; 1}, 1\right)=\mathrm{d}\left(l_{2} \circ\left(l_{2 ; 1}, 1\right)\right) .
$$

We proceed for $l_{3 ; 2}$ in the same way,

$$
\begin{aligned}
\bar{\Delta}\left(\mathrm{d} l_{3 ; 2}\right)= & \bar{\Delta}\left(-l_{3}-l_{3}^{(23)}\right)=-\bar{\Delta}\left(l_{3}\right)-\bar{\Delta}\left(l_{3}\right)^{(23)} \\
= & -\left(-l_{2} \circ\left(l_{2}, 1\right)+l_{2} \circ\left(1, l_{2}\right)-l_{2} \circ\left(1, l_{2}\right)^{(12)}\right) \\
& -\left(-l_{2} \circ\left(l_{2}, 1\right)+l_{2} \circ\left(1, l_{2}\right)-l_{2} \circ\left(1, l_{2}\right)^{(12)}\right)^{(23)} \\
= & l_{2} \circ\left(l_{2}, 1\right)-l_{2} \circ\left(1, l_{2}\right)+l_{2} \circ\left(1, l_{2}\right)^{(12)} \\
& +l_{2}^{(12)} \circ\left(1, l_{2}\right)^{(12)}-l_{2} \circ\left(1, l_{2}^{(12)}\right)+l_{2}^{(12)} \circ\left(l_{2}, 1\right) \\
= & \mathrm{d}\left(-l_{2 ; 1} \circ\left(l_{2}, 1\right)-l_{2} \circ\left(1, l_{2 ; 1}\right)-l_{2 ; 1} \circ\left(1, l_{2}\right)^{(12)}\right) .
\end{aligned}
$$

This gives us candidates for the definition of $\bar{\Delta}$ for which the diagram 91 commutes. We define

$$
\begin{aligned}
& \bar{\Delta}\left(l_{3 ; 1}\right):=l_{2} \circ\left(l_{2 ; 1}, 1\right), \\
& \bar{\Delta}\left(l_{3 ; 2}\right):=-l_{2 ; 1} \circ\left(l_{2}, 1\right)-l_{2} \circ\left(1, l_{2 ; 1}\right)-l_{2 ; 1} \circ\left(1, l_{2}\right)^{(12)} .
\end{aligned}
$$

Using these definitions, we continue in the next degree and find

$$
\begin{aligned}
\bar{\Delta}\left(\mathrm{d} l_{3 ; 1,1}\right) & =\bar{\Delta}\left(-l_{3 ; 1}+l_{3 ; 1}^{(12)}\right)=-\bar{\Delta}\left(l_{3 ; 1}\right)+\bar{\Delta}\left(l_{3 ; 1}\right)^{(12)} \\
& =-l_{2} \circ\left(l_{2 ; 1}, 1\right)+l_{2} \circ\left(l_{2 ; 1}, 1\right)^{(12)}=-l_{2} \circ\left(l_{2 ; 1}, 1\right)+l_{2} \circ\left(l_{2 ; 1}^{(12)}, 1\right) \\
& =l_{2} \circ\left(\mathrm{d} l_{2 ; 1,1}, 1\right)=\mathrm{d}\left(-l_{2} \circ\left(l_{2 ; 1,1}, 1\right)\right), \\
\bar{\Delta}\left(\mathrm{d} l_{3 ; 1,2}\right) & =\bar{\Delta}\left(l_{3 ; 1}+l_{3 ; 1}^{(23)}+l_{3 ; 1}^{(132)}-l_{3 ; 2}-l_{3 ; 2}^{(12)}-l_{3 ; 2}^{(123)}\right)
\end{aligned}
$$




$$
\begin{aligned}
= & l_{2} \circ\left(l_{2 ; 1}, 1\right)+l_{2} \circ\left(l_{2 ; 1}, 1\right)^{(23)}+l_{2} \circ\left(l_{2 ; 1}, 1\right)^{(132)}-\left(-l_{2 ; 1} \circ\left(l_{2}, 1\right)-l_{2} \circ\left(1, l_{2 ; 1}\right)\right. \\
& \left.-l_{2 ; 1} \circ\left(1, l_{2}\right)^{(12)}\right)-\left(-l_{2 ; 1} \circ\left(l_{2}, 1\right)-l_{2} \circ\left(1, l_{2 ; 1}\right)-l_{2 ; 1} \circ\left(1, l_{2}\right)^{(12)}\right)^{(12)} \\
& -\left(-l_{2 ; 1} \circ\left(l_{2}, 1\right)-l_{2} \circ\left(1, l_{2 ; 1}\right)-l_{2 ; 1} \circ\left(1, l_{2}\right)^{(12)}\right)^{(123)} \\
= & l_{2} \circ\left(l_{2 ; 1}, 1\right)+l_{2}^{(12)} \circ\left(1, l_{2 ; 1}\right)^{(12)}+l_{2}^{(12)} \circ\left(1, l_{2 ; 1}\right)+l_{2 ; 1} \circ\left(l_{2}, 1\right)+l_{2} \circ\left(1, l_{2 ; 1}\right) \\
& +l_{2 ; 1} \circ\left(1, l_{2}\right)^{(12)}+l_{2 ; 1} \circ\left(l_{2}^{(12)}, 1\right)+l_{2} \circ\left(1, l_{2 ; 1}\right)^{(12)}+l_{2 ; 1} \circ\left(1, l_{2}\right) \\
& +l_{2 ; 1}^{(12)} \circ\left(1, l_{2}^{(12)}\right)^{(12)}+l_{2}^{(12)} \circ\left(l_{2 ; 1}, 1\right)+l_{2 ; 1} \circ\left(1, l_{2}^{(12)}\right) \\
= & -\left(\mathrm{d} l_{2 ; 1} \circ \circ\left(l_{2 ; 1}, 1\right)-\left(\mathrm{d} l_{2 ; 1} \circ\left(1, l_{2 ; 1}\right)^{(12)}-\left(\mathrm{d} l_{2 ; 1}\right) \circ\left(1, l_{2 ; 1}\right)-l_{2 ; 1} \circ\left(\mathrm{d} l_{2 ; 1}, 1\right)\right.\right. \\
& -l_{2 ; 1} \circ\left(1, \mathrm{~d} l_{2 ; 1}\right)^{(12)}-l_{2 ; 1} \circ\left(1, \mathrm{~d} l_{2 ; 1}\right)+\left(\mathrm{d} l_{2 ; 1,1}\right) \circ\left(1, l_{2}\right)^{(132)} \\
= & \mathrm{d}\left(-l_{2 ; 1} \circ\left(l_{2 ; 1}, 1\right)-l_{2 ; 1} \circ\left(1, l_{2 ; 1}\right)-l_{2 ; 1} \circ\left(1, l_{2 ; 1}\right)^{(12)}+l_{2 ; 1,1} \circ\left(1, l_{2}\right)^{(132)}\right), \\
\bar{\Delta}\left(\mathrm{d} l_{3 ; 2,2}\right)= & \bar{\Delta}\left(-l_{3 ; 2}+l_{3 ; 2}^{(23)}\right)=-\bar{\Delta}\left(l_{3 ; 2}\right)+\bar{\Delta}\left(l_{3 ; 2}\right)^{(23)} \\
= & -\left(-l_{2 ; 1} \circ\left(l_{2}, 1\right)-l_{2} \circ\left(1, l_{2 ; 1}\right)-l_{2 ; 1} \circ\left(1, l_{2}\right)^{(12)}\right) \\
& +\left(-l_{2 ; 1} \circ\left(l_{2}, 1\right)-l_{2} \circ\left(1, l_{2 ; 1}\right)-l_{2 ; 1} \circ\left(1, l_{2}\right)^{(12)}\right)^{(23)} \\
= & l_{2 ; 1} \circ\left(l_{2}, 1\right)+l_{2} \circ\left(1, l_{2 ; 1}\right)+l_{2 ; 1} \circ\left(1, l_{2}\right)^{(12)}-l_{2 ; 1}^{(12)} \circ\left(1, l_{2}\right)^{(12)}-l_{2} \circ\left(1, l_{2 ; 1}^{(12)}\right) \\
& -l_{2 ; 1}^{(12)} \circ\left(l_{2}, 1\right) \\
= & -\left(\mathrm{d} l_{2 ; 1,1} \circ \circ\left(l_{2}, 1\right)-l_{2} \circ\left(1, \mathrm{~d} l_{2 ; 1,1}\right)-\left(\mathrm{d} l_{2 ; 1,1}\right) \circ\left(1, l_{2}\right)^{(12)}\right. \\
= & \mathrm{d}\left(-l_{2 ; 1,1} \circ\left(l_{2}, 1\right)+l_{2} \circ\left(1, l_{2 ; 1,1}\right)-l_{2 ; 1,1} \circ\left(1, l_{2}\right)^{(12)}\right) .
\end{aligned}
$$

This again gives us candidates for the definition of $\bar{\Delta}$ and we define

(96) $\bar{\Delta}\left(l_{3 ; 1,1}\right):=-l_{2} \circ\left(l_{2 ; 1,1}, 1\right)$,

(97) $\bar{\Delta}\left(l_{3 ; 1,2}\right):=-l_{2 ; 1} \circ\left(l_{2 ; 1}, 1\right)-l_{2 ; 1} \circ\left(1, l_{2 ; 1}\right)-l_{2 ; 1} \circ\left(1, l_{2 ; 1}\right)^{(12)}+l_{2 ; 1,1} \circ\left(1, l_{2}\right)^{(132)}$,

(98) $\bar{\Delta}\left(l_{3 ; 2,2}\right):=-l_{2 ; 1,1} \circ\left(l_{2}, 1\right)+l_{2} \circ\left(1, l_{2 ; 1,1}\right)-l_{2 ; 1,1} \circ\left(1, l_{2}\right)^{(12)}$.

Arity $\mathbf{n}=4$ : For $l_{4}$ we have

$$
\begin{aligned}
\bar{\Delta}\left(l_{4}\right)= & l_{2} \circ\left(l_{3}, 1\right)+l_{2} \circ\left(1, l_{3}\right)-l_{2} \circ\left(1, l_{3}\right)^{(12)}+l_{2} \circ\left(1, l_{3}\right)^{(123)}-l_{2} \circ\left(l_{2}, l_{2}\right) \\
& +l_{2} \circ\left(l_{2}, l_{2}\right)^{(23)}-l_{2} \circ\left(l_{2}, l_{2}\right)^{(132)}+l_{3} \circ\left(l_{2}, 1,1\right)-l_{3} \circ\left(1, l_{2}, 1\right) \\
& +l_{3} \circ\left(1, l_{2}, 1\right)^{(12)}+l_{3} \circ\left(1,1, l_{2}\right)-l_{3} \circ\left(1,1, l_{2}\right)^{(23)}+l_{3} \circ\left(1,1, l_{2}\right)^{(132),}
\end{aligned}
$$

and analogous to the arity 3 computations, we obtain (indicating by the ellipses $\cdots$ terms that cancel on the nose)

$$
\begin{aligned}
\bar{\Delta}\left(\mathrm{d} l_{4 ; 1}\right)= & \bar{\Delta}\left(-l_{4}-l_{4}^{(12)}\right)=-\bar{\Delta}\left(l_{4}\right)-\bar{\Delta}\left(l_{4}\right)^{(12)} \\
= & -\left(l_{2} \circ\left(l_{3}, 1\right)+l_{2} \circ\left(1, l_{3}\right)^{(123)}-l_{2} \circ\left(l_{2}, l_{2}\right)+l_{3} \circ\left(l_{2}, 1,1\right)+l_{3} \circ\left(1,1, l_{2}\right)\right. \\
& +\cdots)-\left(l_{2} \circ\left(l_{3}^{(12)}, 1\right)+l_{2} \circ\left(1, l_{3}^{(12)}\right)^{(123)}-l_{2} \circ\left(l_{2}^{(12)}, l_{2}\right)+l_{3} \circ\left(l_{2}^{(12)}, 1,1\right)\right. \\
& \left.+l_{3}^{(12)} \circ\left(1,1, l_{2}\right)+\cdots\right) \\
= & l_{2} \circ\left(\mathrm{d} l_{3 ; 1}, 1\right)+l_{2} \circ\left(1, \mathrm{~d} l_{3 ; 1}\right)^{(123)}-l_{2} \circ\left(\mathrm{d} l_{2 ; 1}, l_{2}\right)+l_{3} \circ\left(\mathrm{d} l_{2 ; 1}, 1,1\right) \\
& +\left(\mathrm{d} l_{3 ; 1}\right) \circ\left(1,1, l_{2}\right)
\end{aligned}
$$




$$
\begin{aligned}
& =\mathrm{d}\left(-l_{2} \circ\left(l_{3 ; 1}, 1\right)-l_{2} \circ\left(1, l_{3 ; 1}\right)^{(123)}+l_{2} \circ\left(l_{2 ; 1}, l_{2}\right)+l_{3} \circ\left(l_{2 ; 1}, 1,1\right)\right. \\
& \left.+l_{3 ; 1} \circ\left(1,1, l_{2}\right)\right) \text {, } \\
& \bar{\Delta}\left(\mathrm{d} l_{4 ; 2}\right)=\bar{\Delta}\left(-l_{4}-l_{4}^{(23)}\right)=-\bar{\Delta}\left(l_{4}\right)-\bar{\Delta}\left(l_{4}\right)^{(23)} \\
& =-\left(l_{2} \circ\left(l_{3}, 1\right)+l_{2} \circ\left(1, l_{3}\right)-l_{2} \circ\left(l_{2}, l_{2}\right)^{(132)}+l_{3} \circ\left(l_{2}, 1,1\right)-l_{3} \circ\left(1, l_{2}, 1\right)\right. \\
& \left.+l_{3} \circ\left(1, l_{2}, 1\right)^{(12)}+l_{3} \circ\left(1,1, l_{2}\right)^{(132)}+\cdots\right)-\left(l_{2} \circ\left(l_{3}^{(23)}, 1\right)+l_{2} \circ\left(1, l_{3}^{(12)}\right)\right. \\
& -l_{2} \circ\left(l_{2}^{(12)}, l_{2}\right)^{(132)}+l_{3}^{(12)} \circ\left(1, l_{2}, 1\right)^{(12)}-l_{3} \circ\left(1, l_{2}^{(12)}, 1\right)+l_{3}^{(12)} \circ\left(l_{2}, 1,1\right) \\
& \left.+l_{3}^{(12)} \circ\left(1,1, l_{2}\right)^{(132)}+\cdots\right) \\
& =l_{2} \circ\left(\mathrm{d} l_{3 ; 2}, 1\right)+l_{2} \circ\left(1, \mathrm{dl}_{3 ; 1}\right)-l_{2} \circ\left(\mathrm{dl}_{2 ; 1}, l_{2}\right)^{(132)}+\left(\mathrm{d} l_{3 ; 1}\right) \circ\left(l_{2}, 1,1\right) \\
& +\left(\mathrm{d} l_{3 ; 1}\right) \circ\left(1, l_{2}, 1\right)^{(12)}-l_{3} \circ\left(1, \mathrm{~d} l_{2 ; 1}, 1\right)+\left(\mathrm{d} l_{3 ; 1}\right) \circ\left(1,1, l_{2}\right)^{(132)} \\
& =\mathrm{d}\left(-l_{2} \circ\left(l_{3 ; 2}, 1\right)-l_{2} \circ\left(1, l_{3 ; 1}\right)+l_{2} \circ\left(l_{2 ; 1}, l_{2}\right)^{(132)}+l_{3 ; 1} \circ\left(l_{2}, 1,1\right)\right. \\
& \left.+l_{3 ; 1} \circ\left(1, l_{2}, 1\right)^{(12)}-l_{3} \circ\left(1, l_{2 ; 1}, 1\right)+l_{3 ; 1} \circ\left(1,1, l_{2}\right)^{(132)}\right) \text {, } \\
& \bar{\Delta}\left(\mathrm{d} l_{4 ; 3}\right)=\bar{\Delta}\left(-l_{4}-l_{4}^{(34)}\right)=-\bar{\Delta}\left(l_{4}\right)-\bar{\Delta}\left(l_{4}\right)^{(34)} \\
& =-\left(l_{2} \circ\left(l_{3}, 1\right)+l_{2} \circ\left(1, l_{3}\right)-l_{2} \circ\left(1, l_{3}\right)^{(12)}+l_{2} \circ\left(1, l_{3}\right)^{(123)}-l_{2} \circ\left(l_{2}, l_{2}\right)\right. \\
& +l_{2} \circ\left(l_{2}, l_{2}\right)^{(23)}-l_{2} \circ\left(l_{2}, l_{2}\right)^{(132)}+l_{3} \circ\left(l_{2}, 1,1\right)-l_{3} \circ\left(1, l_{2}, 1\right) \\
& \left.+l_{3} \circ\left(1, l_{2}, 1\right)^{(12)}+l_{3} \circ\left(1,1, l_{2}\right)-l_{3} \circ\left(1,1, l_{2}\right)^{(23)}+l_{3} \circ\left(1,1, l_{2}\right)^{(132)}+\cdots\right) \\
& -\left(l_{2}^{(12)} \circ\left(1, l_{3}\right)^{(123)}+l_{2} \circ\left(1, l_{3}^{(23)}\right)-l_{2} \circ\left(1, l_{3}^{(23)}\right)^{(12)}+l_{2}^{(12)} \circ\left(l_{3}, 1\right)\right. \\
& -l_{2} \circ\left(l_{2}, l_{2}^{(12)}\right)-l_{2}^{(12)} \circ\left(l_{2}, l_{2}\right)^{(132)}+l_{2}^{(12)} \circ\left(l_{2}, l_{2}\right)^{(23)}+l_{3}^{(23)} \circ\left(l_{2}, 1,1\right) \\
& -l_{3}^{(23)} \circ\left(1,1, l_{2}\right)^{(23)}+l_{3}^{(23)} \circ\left(1,1, l_{2}\right)^{(132)}+l_{3} \circ\left(1,1, l_{2}^{(12)}\right)-l_{3}^{(23)} \circ\left(1, l_{2}, 1\right) \\
& \left.+l_{3}^{(23)} \circ\left(1, l_{2}, 1\right)^{(12)}+\cdots\right) \\
& =\left(\mathrm{d} l_{2 ; 1}\right) \circ\left(1, l_{3}\right)^{(123)}+l_{2} \circ\left(1, \mathrm{~d} l_{3 ; 2}\right)-l_{2} \circ\left(1, \mathrm{~d} l_{3 ; 2}\right)^{(12)}+\left(\mathrm{d} l_{2 ; 1}\right) \circ\left(l_{3}, 1\right) \\
& -l_{2} \circ\left(l_{2}, \mathrm{~d} l_{2 ; 1}\right)-\left(\mathrm{d} l_{2 ; 1}\right) \circ\left(l_{2}, l_{2}\right)^{(132)}+\left(\mathrm{d} l_{2 ; 1}\right) \circ\left(l_{2}, l_{2}\right)^{(23)}+\left(\mathrm{d} l_{3 ; 2}\right) \circ\left(l_{2}, 1,1\right) \\
& -\left(\mathrm{d} l_{3 ; 2}\right) \circ\left(1,1, l_{2}\right)^{(23)}+\left(\mathrm{dl}_{3 ; 2}\right) \circ\left(1,1, l_{2}\right)^{(132)}+l_{3} \circ\left(1,1, \mathrm{~d} l_{2 ; 1}\right) \\
& -\left(\mathrm{d} l_{3 ; 2}\right) \circ\left(1, l_{2}, 1\right)+\left(\mathrm{d} l_{3 ; 2}\right) \circ\left(1, l_{2}, 1\right)^{(12)} \\
& =\mathrm{d}\left(l_{2 ; 1} \circ\left(1, l_{3}\right)^{(123)}-l_{2} \circ\left(1, l_{3 ; 2}\right)+l_{2} \circ\left(1, l_{3 ; 2}\right)^{(12)}+l_{2 ; 1} \circ\left(l_{3}, 1\right)-l_{2} \circ\left(l_{2}, l_{2 ; 1}\right)\right. \\
& -l_{2 ; 1} \circ\left(l_{2}, l_{2}\right)^{(132)}+l_{2 ; 1} \circ\left(l_{2}, l_{2}\right)^{(23)}+l_{3 ; 2} \circ\left(l_{2}, 1,1\right)-l_{3 ; 2} \circ\left(1,1, l_{2}\right)^{(23)} \\
& \left.+l_{3 ; 2} \circ\left(1,1, l_{2}\right)^{(132)}+l_{3} \circ\left(1,1, l_{2 ; 1}\right)-l_{3 ; 2} \circ\left(1, l_{2}, 1\right)+l_{3 ; 2} \circ\left(1, l_{2}, 1\right)^{(12)}\right) \text {. }
\end{aligned}
$$

As before, this gives us candidates for the definition of $\bar{\Delta}$. We define

(100)

$$
\bar{\Delta}\left(l_{4 ; 1}\right):=-l_{2} \circ\left(l_{3 ; 1}, 1\right)-l_{2} \circ\left(1, l_{3 ; 1}\right)^{(123)}+l_{2} \circ\left(l_{2 ; 1}, l_{2}\right)+l_{3} \circ\left(l_{2 ; 1}, 1,1\right)
$$

$$
+l_{3 ; 1} \circ\left(1,1, l_{2}\right) \text {, }
$$

(101)

$$
\begin{aligned}
\bar{\Delta}\left(l_{4 ; 2}\right):= & -l_{2} \circ\left(l_{3 ; 2}, 1\right)-l_{2} \circ\left(1, l_{3 ; 1}\right)+l_{2} \circ\left(l_{2 ; 1}, l_{2}\right)^{(132)}+l_{3 ; 1} \circ\left(l_{2}, 1,1\right) \\
& +l_{3 ; 1} \circ\left(1, l_{2}, 1\right)^{(12)}-l_{3} \circ\left(1, l_{2 ; 1}, 1\right)+l_{3 ; 1} \circ\left(1,1, l_{2}\right)^{(132)},
\end{aligned}
$$




$$
\begin{aligned}
\bar{\Delta}\left(l_{4 ; 3}\right):= & l_{2 ; 1} \circ\left(1, l_{3}\right)^{(123)}-l_{2} \circ\left(1, l_{3 ; 2}\right)+l_{2} \circ\left(1, l_{3 ; 2}\right)^{(12)}+l_{2 ; 1} \circ\left(l_{3}, 1\right) \\
& -l_{2} \circ\left(l_{2}, l_{2 ; 1}\right)-l_{2 ; 1} \circ\left(l_{2}, l_{2}\right)^{(132)}+l_{2 ; 1} \circ\left(l_{2}, l_{2}\right)^{(23)} \\
& +l_{3 ; 2} \circ\left(l_{2}, 1,1\right)-l_{3 ; 2} \circ\left(1,1, l_{2}\right)^{(23)}+l_{3 ; 2} \circ\left(1,1, l_{2}\right)^{(132)} \\
& +l_{3} \circ\left(1,1, l_{2 ; 1}\right)-l_{3 ; 2} \circ\left(1, l_{2}, 1\right)+l_{3 ; 2} \circ\left(1, l_{2}, 1\right)^{(12)} .
\end{aligned}
$$

Arity $\mathbf{n}=\mathbf{5}$ : We have already defined the decomposition map for $l_{5}$. Explicitly, it is given by

$$
\begin{aligned}
\bar{\Delta}\left(l_{5}\right)= & -l_{2} \circ\left(l_{4}, 1\right)+l_{2} \circ\left(l_{3}, l_{2}\right)-l_{2} \circ\left(l_{3}, l_{2}\right)^{(34)}+l_{2} \circ\left(l_{3}, l_{2}\right)^{(243)}-l_{2} \circ\left(l_{3}, l_{2}\right)^{(1432)} \\
& -l_{2} \circ\left(l_{2}, l_{3}\right)+l_{2} \circ\left(l_{2}, l_{3}\right)^{(23)}-l_{2} \circ\left(l_{2}, l_{3}\right)^{(234)}-l_{2} \circ\left(l_{2}, l_{3}\right)^{(132)} \\
& +l_{2} \circ\left(l_{2}, l_{3}\right)^{(1342)}-l_{2} \circ\left(l_{2}, l_{3}\right)^{(13)(24)}+l_{2} \circ\left(1, l_{4}\right)-l_{2} \circ\left(1, l_{4}\right)^{(12)} \\
& +l_{2} \circ\left(1, l_{4}\right)^{(123)}-l_{2} \circ\left(1, l_{4}\right)^{(1234)}+l_{3} \circ\left(l_{3}, 1,1\right)-l_{3} \circ\left(l_{2}, l_{2}, 1\right) \\
& +l_{3} \circ\left(l_{2}, l_{2}, 1\right)^{(23)}-l_{3} \circ\left(l_{2}, l_{2}, 1\right)^{(132)}+l_{3} \circ\left(l_{2}, 1, l_{2}\right)+l_{3} \circ\left(l_{2}, 1, l_{2}\right)^{(243)} \\
& -l_{3} \circ\left(l_{2}, 1, l_{2}\right)^{(34)}+l_{3} \circ\left(1, l_{3}, 1\right)-l_{3} \circ\left(1, l_{3}, 1\right)^{(12)}+l_{3} \circ\left(1, l_{3}, 1\right)^{(123)} \\
& -l_{3} \circ\left(1, l_{2}, l_{2}\right)+l_{3} \circ\left(1, l_{2}, l_{2}\right)^{(34)}-l_{3} \circ\left(1, l_{2}, l_{2}\right)^{(243)}+l_{3} \circ\left(1, l_{2}, l_{2}\right)^{(12)} \\
& -l_{3} \circ\left(1, l_{2}, l_{2}\right)^{(12)(34)}+l_{3} \circ\left(1, l_{2}, l_{2}\right)^{(1243)}-l_{3} \circ\left(1, l_{2}, l_{2}\right)^{(143)} \\
& +l_{3} \circ\left(1, l_{2}, l_{2}\right)^{(1432)}+l_{3} \circ\left(1,1, l_{3}\right)-l_{3} \circ\left(1,1, l_{3}\right)^{(23)}+l_{3} \circ\left(1,1, l_{3}\right)^{(234)} \\
& +l_{3} \circ\left(1,1, l_{3}\right)^{(132)}-l_{3} \circ\left(1,1, l_{3}\right)^{(1342)}+l_{3} \circ\left(1,1, l_{3}\right)^{(13)(24)}-l_{4} \circ\left(l_{2}, 1,1,1\right) \\
& +l_{4} \circ\left(1, l_{2}, 1,1\right)-l_{4} \circ\left(1, l_{2}, 1,1\right)^{(12)}-l_{4} \circ\left(1,1, l_{2}, 1\right)+l_{4} \circ\left(1,1, l_{2}, 1\right)^{(23)} \\
& -l_{4} \circ\left(1,1, l_{2}, 1\right)^{(132)}+l_{4} \circ\left(1,1,1, l_{2}\right)-l_{4} \circ\left(1,1,1, l_{2}\right)^{(34)} \\
& +l_{4} \circ\left(1,1,1, l_{2}\right)^{(243)}-l_{4} \circ\left(1,1,1, l_{2}\right)^{(1432)} \cdot
\end{aligned}
$$

Since there are no higher degree terms in $\operatorname{Lie}_{3}^{\diamond}(5)$, we are done here.

Lemma 1.3.1. The triple ( $\left.\mathrm{Lie}_{3}^{\diamond}, \mathrm{d}, \Delta\right)$ consisting of the $d g \mathbb{S}$-module ( $\mathrm{Lie}_{3}^{\diamond}, \mathrm{d}$ ) defined in Figure 1 and the decomposition map as defined by Equations (92)(103) is a dg cooperad.

The decomposition structure map we defined is compatible with the differential by its construction. What is left to do, in order to show that it defines a dg cooperad structure on the dg $\mathbb{S}$-module $\mathrm{Lie}_{3}^{\diamond}$ defined in the previous section, is to check that it satisfies the coassociativity condition $(\Delta \circ \mathrm{id}) \Delta=($ id $\circ \Delta) \Delta$. Note that coassociativity is automatic in arities $n \leq 3$ and we already know that it holds for $l_{n} \in \operatorname{Lie}_{3}^{\diamond}(n)_{n-1}=\operatorname{Leib}^{i}(n)$. Thus, it is sufficient to check it for the elements $l_{4 ; 1}, l_{4 ; 2}$, and $l_{4 ; 3}$. See Section A.1 for this long and tedious computation.

1.4. The twisted composite product. As explained in the introduction to this section, if we could show that for an $\mathbb{S}$-free resolution $\psi: \mathrm{Lie}^{\diamond} \rightarrow \mathrm{Lie}^{\mathrm{i}}$ of $\mathrm{dg}$ cooperads the map $\Omega \psi$ is a quasi-isomorphism, this would give us a cofibrant resolution of the Lie operad over $\mathbb{Z}$. One way to do this would be to show, that the twisted composite product Lie $^{\diamond} \circ_{\widetilde{\kappa}}$ Lie is acyclic for $\tilde{\kappa}=\kappa \circ \psi$. Since we do not have the full dg cooperad $\mathrm{Lie}^{\diamond}$, we will show the following truncated statement. This is of course a neccessary 
condition for our dg cooperad $\mathrm{Lie}_{3}^{\diamond}$ to be a truncation of an $\mathbb{S}$-free resolution $\mathrm{Lie}^{\diamond}$ with the desired properties.

Proposition 1.4.1. The twisted composite product $\mathrm{Lie}_{3}^{\diamond} \circ_{\widetilde{\kappa}}$ Lie satisfies

$$
\mathrm{H}_{r}\left(\left(\operatorname{Lie}_{3}^{\diamond} \circ_{\widetilde{\mathcal{K}}} \operatorname{Lie}\right)(n)\right)=0,
$$

for all $r \leq 3$ in all arities $n$.

PROOF. Since the result actually holds for any $\mathrm{Lie}_{k}^{\diamond}$ constructed as in the previous sections, we phrase the proof for arbitrary $k$ instead of just $k=3$.

Consider the bigrading on the composite product $\operatorname{Lie}_{k}^{\diamond} \circ$ Lie given by

$$
\left(\operatorname{Lie}_{k}^{\diamond} \circ \operatorname{Lie}\right)(n)_{p, q}=\left(\operatorname{Lie}_{k}^{\diamond}(p+1) \otimes_{S_{p+1}}\left(\operatorname{Lie}^{\otimes p+1}\right)(n)\right)_{p+q} .
$$

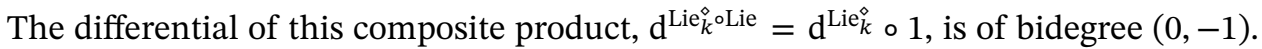
On the twisted composite product $\operatorname{Li}_{k}^{\diamond} \circ_{\widetilde{\kappa}}$ Lie, the differential has another term, $\mathrm{d}_{\widetilde{\kappa}}$, defined as in Equation (61) Note that $\mathrm{d}_{\tilde{\mathcal{\kappa}}}$ is of bidegree $(-1,0)$, since $\tilde{\mathcal{\kappa}}:=\kappa \circ \psi$ vanishes everywhere except on $\operatorname{Lie}_{k}^{\diamond}(2)_{1}$. Thus, with the above bigrading, the twisted composite product becomes a first quadrant bicomplex. Below, we consider its spectral sequence $E(n)$ for each arity $n$.

Since Lie $(0)=0$, the action of $\mathbb{S}_{p+1}$ on Lie ${ }^{\otimes p+1}(n)$ is free and hence $E(n)_{p, q}^{0}$ admits the following expansion (see Section 1.3 of Chapter 2):

$$
\begin{aligned}
E(n)_{p, q}^{0} & =\left(\operatorname{Lie}_{k}^{\diamond} \circ \operatorname{Lie}\right)(n)_{p, q} \\
& =\left(\operatorname{Lie}_{k}^{\diamond}(p+1) \otimes_{\mathbb{S}_{p+1}}\left(\operatorname{Lie}^{\otimes p+1}\right)(n)\right)_{p+q} \\
& =\left(\bigoplus_{n=k_{1}+\cdots+k_{p+1}} \operatorname{Lie}_{k}^{\diamond}(p+1) \otimes_{\mathbb{S}_{p+1}}\left(\operatorname{Ind}_{\mathbb{S}_{k_{1}} \times \cdots \times \mathbb{S}_{k_{p+1}}}^{\mathbb{S}_{n}} \operatorname{Lie}\left(k_{1}\right) \otimes \cdots \otimes \operatorname{Lie}\left(k_{p+1}\right)\right)\right)_{p+q} \\
& =\left(\bigoplus_{n=k_{1}+\cdots+k_{m}} \operatorname{Lie}_{k}^{\diamond}(p+1) \otimes \operatorname{Lie}\left(k_{1}\right) \otimes \cdots \otimes \operatorname{Lie}\left(k_{p+1}\right) \otimes \mathbf{k}\left[\operatorname{Sh}\left(k_{1}, \ldots, k_{p+1}\right)\right]\right)_{p+q} .
\end{aligned}
$$

Since Lie is k-projective, i.e., Lie $(n)$ are projective $\mathbf{k}$-modules for all arities $n$, this implies that the first page $E(n)_{p, q}^{1}$ is given by

$$
\begin{aligned}
E(n)_{p, q}^{1} & =\mathrm{H}_{p+q}\left(\operatorname{Lie}_{k}^{\diamond}(p+1) \otimes_{\mathbb{S}_{p+1}}\left(\operatorname{Lie}^{\otimes p+1}\right)(n), \mathrm{d}^{\left.\mathrm{Lie}_{k}^{\circ} \circ 1\right)}\right. \\
& =\mathrm{H}_{p+q}\left(\operatorname{Lie}_{k}^{\diamond}(p+1), \mathrm{d}^{\mathrm{Lie}_{k}^{\circ}}\right) \otimes_{\mathbb{S}_{p+1}}\left(\operatorname{Lie}^{\otimes p+1}\right)(n) .
\end{aligned}
$$

By the construction of Section 1.2 we have for $n \leq k+1$ :

$$
\mathrm{H}_{r}\left(\operatorname{Lie}_{k}^{\diamond}(n)\right)= \begin{cases}\operatorname{Lie}^{\mathrm{i}}(n)_{r}, & \text { if } r \leq k, \\ \operatorname{kerd}_{r}, & \text { if } r=k+1, \\ 0, & \text { otherwise } .\end{cases}
$$


Hence, for $p+q \leq k$ we obtain

(105) $E(n)_{p, q}^{1}=\left(\operatorname{Lie}^{\mathrm{i}}(p+1) \otimes_{S_{p+1}}\left(\operatorname{Lie}^{\otimes p+1}\right)(n)\right)_{p+q}= \begin{cases}\left(\operatorname{Lie}^{i} \circ \operatorname{Lie}\right)(n)_{p}, & \text { if } q=0, \\ 0, & \text { otherwise . }\end{cases}$

Since $\psi$ is a morphism of dg cooperads and $\tilde{\kappa}=\kappa \circ \psi$, it follows from the definition of the twisted differential in Equation (61), that the following diagram commutes:

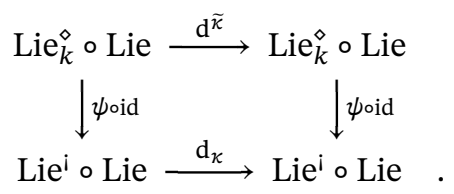

Since $\psi$ is a quasi-isomorphism, this shows that $\mathrm{d}_{p, 0}^{1}=\left(\mathrm{d}_{\kappa}\right)_{p}$ for $p \leq k+1$. Thus we obtain $E_{p, q}^{2}=0$ for $p+q \leq k$, since the operad Lie is Koszul.

The proof for $n>k+1$ is essentially the same, except for the computation of $E(n)_{k, 0}^{2}$. We have

$$
\mathrm{H}_{r}\left(\operatorname{Lie}_{k}^{\diamond}(k+2)\right)=\operatorname{Lie}_{k}^{\diamond}(k+2)_{r}= \begin{cases}\operatorname{Lie}_{k}^{\diamond}(k+2)_{k+1}, & \text { if } r=k+1, \\ 0, & \text { otherwise },\end{cases}
$$

and hence $\mathrm{d}_{k+1,0}^{1} \neq\left(\mathrm{d}_{\kappa}\right)_{k+1}$. However, we do find

$$
\begin{aligned}
\operatorname{im}\left(E(n)_{k+1,0}^{1} \stackrel{\mathrm{d}_{k+1,0}^{1}}{\longrightarrow} E(n)_{k, 0}^{1}\right) & =\operatorname{im}\left(\left(\operatorname{Lie}_{k}^{\diamond} \circ \mathrm{Lie}\right)(n)_{k+1} \stackrel{(\psi \circ \mathrm{oid}) \mathrm{d}_{\tilde{\mathrm{K}}}}{\longrightarrow}\left(\operatorname{Lie}^{\mathrm{i}} \circ \mathrm{Lie}\right)(n)_{k}\right) \\
& =\operatorname{im}\left(\left(\operatorname{Lie}^{\mathrm{i}} \circ \mathrm{Lie}\right)(n)_{k+1} \stackrel{\mathrm{d}_{\kappa}}{\longrightarrow}\left(\operatorname{Lie}^{\mathrm{i}} \circ \mathrm{Lie}\right)(n)_{k}\right),
\end{aligned}
$$

since $\psi$ is surjective. Thus we obtain $E_{p, q}^{2}=0$ for $p+q \leq k$ again.

\section{The Category of Weak Lie 3-Algebras}

Assume again that we had an $\mathbb{\$}$-free resolution $\psi: \mathrm{Lie}^{\diamond} \rightarrow \mathrm{Lie}^{i}$ of dg cooperads, and in addition that $\mathrm{Lie}^{\diamond} o_{\kappa \circ \psi}$ Lie is acyclic and therefore $\mathrm{EL}_{\infty}=\Omega \mathrm{Lie}^{\diamond} \tilde{\rightarrow}$ Lie is a cofibrant resolution. Now consider a 3-term complex

$$
(L, \mathrm{~d})=\left(L_{0} \stackrel{\mathrm{d}}{\longleftarrow} L_{1} \stackrel{\mathrm{d}}{\longleftarrow} L_{2}\right),
$$

and note that its endomorphism operad $\operatorname{End}_{L}$ vanishes in degrees $r>2$. Since the Maurer-Cartan equation for a twisting morphism $\lambda: \mathrm{Lie}^{\diamond} \rightarrow \mathrm{End}_{L}$ is of degree -2 , only degrees $\leq 4$ of Lie $e^{\diamond}$ play a role in the definition of 3-term $\mathrm{EL}_{\infty}$-algebras. A homotopy transfer theorem for such 3-term $\mathrm{EL}_{\infty}$-algebras holds as a special case of the general HTT for algebras over a cofibrant operad. For our resolution $\mathrm{Lie}_{3}^{\diamond}$, however, we do not know that it is the truncation of such a dg cooperad Lie ${ }^{\diamond}$. Nonetheless, we show constructively that our weak Lie 3-algebras satisfy a homotopy transfer theorem (Proposition 2.4.1.

This section is organized as follows. In Section 2.1, we recall the definition of a homotopy Leibniz algebra and of its homotopy morphisms. We spell out the details for the case of a homotopy Leibniz algebra on a 3-term complex or Leibniz 3-algebra, and for homotopy morphisms between such. In Section 2.2, we introduce the notion 
of a weak Lie 3-algebra as extra structure on a Leibniz 3-algebra. In Section 2.3 the accompanying notion of weak morphisms is made explicit. Finally, in Section 2.4 we prove a version of the homotopy transfer theorem for weak Lie 3-algebras.

2.1. Homotopy Leibniz algebras. From the description of the Koszul dual cooperad Leib in Section 1.1. we obtain the following explicit definitions of algebras and homotopy morphisms over Leib $_{\infty}=\Omega$ Leib $^{\mathrm{i}}$ via the Maurer-Cartan Equations (63) and (64). For a more thorough exposition, we refer the reader to [36].

Definition 2.1.1. A homotopy Leibniz algebra or Leib ${ }_{\infty}$-algebra $(L, \mathrm{~d}, \lambda)$ consists of a chain complex $(L, \mathrm{~d})$ with structure maps

$$
\lambda_{n}: L^{\otimes n} \rightarrow L[n-2], \quad \text { for } n \geq 2,
$$

satisfying the following generalized Jacobi identities:

$$
\partial\left(\lambda_{n}\right)=-\sum_{i+j=n+1}(-1)^{(j-1) i} \sum_{p=1}^{j}(-1)^{(p-1)(i-1)} \sum_{\sigma \in \overline{\mathrm{Sh}}^{-1}(p-1, i)}(-1)^{|\sigma|} \cdot\left(\lambda_{j} \otimes_{p} \lambda_{i}\right)^{\sigma}, \quad \text { for } n \geq 3 .
$$

Let $(L, \mathrm{~d}, \lambda),\left(L^{\prime}, \mathrm{d}^{\prime}, \lambda^{\prime}\right)$ be homotopy Leibniz algebras. A homotopy morphism or Leib $_{\infty}$-morphism $f: L \rightsquigarrow L^{\prime}$ consists of maps

$$
f_{n}: L^{\otimes n} \rightarrow L^{\prime}[n-1], \quad \text { for } n \geq 1,
$$

satisfying the following equations (for $n \geq 1$ ):

$$
\begin{aligned}
& \partial\left(f_{n}\right)=\sum_{i+j=n+1}(-1)^{(j-1) i} \sum_{p=1}^{j}(-1)^{(p-1)(i-1)} \sum_{\sigma \in \overline{\mathrm{Sh}}^{-1}(p-1, i)}(-1)^{|\sigma|} \cdot\left(f_{j} \otimes_{p} \lambda_{i}\right)^{\sigma} \\
& \underset{\substack{1 \leq j \leq n \\
i_{1}+\cdots+i_{j}=n}}{-\sum^{1}(-1)^{(j-1)(n-j)}} \cdot(-1)^{\sum_{p=1}^{j}(p-1)\left(i_{p}-1\right)} \sum_{\sigma \in \overline{\mathrm{Sh}}^{-1}\left(i_{1}, \ldots, i_{j}\right)}(-1)^{|\sigma|} \cdot \lambda_{j}^{\prime} \circ\left(f_{i_{1}}, \ldots, f_{i_{j}}\right)^{\sigma} .
\end{aligned}
$$

A Leibniz 3-algebra is just a homotopy Leibniz algebra on a 3-term complex. Since this is the basis for our definition of a weak Lie 3-algebra, we spell out the definition here.

Definition 2.1.2. A Leibniz, 3-algebra $(L, \mathrm{~d}, \lambda)$ consists of a 3-term chain complex

$$
(L, \mathrm{~d})=\left(L_{0} \stackrel{\mathrm{d}}{\longleftarrow} L_{1} \stackrel{\mathrm{d}}{\longleftarrow} L_{2}\right),
$$

equipped with structure maps

$$
\lambda_{2}: L^{\otimes 2} \rightarrow L, \quad \lambda_{3}: L^{\otimes 3} \rightarrow L[1], \quad \lambda_{4}: L^{\otimes 4} \rightarrow L[2],
$$

satisfying the following generalized Jacobi identities:

(110) $\partial\left(\lambda_{2}\right)=0$,

(111) $\partial\left(\lambda_{3}\right)=\lambda_{2} \circ_{2} \lambda_{2}-\lambda_{2} \circ_{1} \lambda_{2}-\left(\lambda_{2} \circ_{2} \lambda_{2}\right)^{(12)}$,

$$
\begin{aligned}
\partial\left(\lambda_{4}\right)= & \lambda_{2} \circ_{1} \lambda_{3}+\lambda_{2} \circ_{2} \lambda_{3}-\left(\lambda_{2} \circ_{2} \lambda_{3}\right)^{(12)}+\left(\lambda_{2} \circ_{2} \lambda_{3}\right)^{(123)}-\lambda_{3} \circ_{1} \lambda_{2} \\
& +\lambda_{3} \circ_{2} \lambda_{2}-\left(\lambda_{3} \circ_{2} \lambda_{2}\right)^{(12)}-\lambda_{3} \circ_{3} \lambda_{2}+\left(\lambda_{3} \circ_{3} \lambda_{2}\right)^{(23)}-\left(\lambda_{3} \circ_{3} \lambda_{2}\right)^{(132)},
\end{aligned}
$$


and

$$
\begin{aligned}
0= & \lambda_{2} \circ_{1} \lambda_{4}-\lambda_{2} \circ_{2} \lambda_{4}+\left(\lambda_{2} \circ_{2} \lambda_{4}\right)^{(12)}-\left(\lambda_{2} \circ_{2} \lambda_{4}\right)^{(123)}+\left(\lambda_{2} \circ_{2} \lambda_{4}\right)^{(1234)} \\
& +\lambda_{3} \circ_{1} \lambda_{3}+\lambda_{3} \circ_{2} \lambda_{3}-\left(\lambda_{3} \circ_{2} \lambda_{3}\right)^{(12)}+\left(\lambda_{3} \circ_{2} \lambda_{3}\right)^{(123)}+\lambda_{3} \circ_{3} \lambda_{3} \\
& -\left(\lambda_{3} \circ_{3} \lambda_{3}\right)^{(23)}+\left(\lambda_{3} \circ_{3} \lambda_{3}\right)^{(132)}+\left(\lambda_{3} \circ_{3} \lambda_{3}\right)^{(234)}-\left(\lambda_{3} \circ_{3} \lambda_{3}\right)^{(1342)} \\
& +\left(\lambda_{3} \circ_{3} \lambda_{3}\right)^{(13)(24)}+\lambda_{4} \circ_{1} \lambda_{2}-\lambda_{4} \circ_{2} \lambda_{2}+\left(\lambda_{4} \circ_{2} \lambda_{2}\right)^{(12)}+\lambda_{4} \circ_{3} \lambda_{2} \\
& -\left(\lambda_{4} \circ_{3} \lambda_{2}\right)^{(23)}+\left(\lambda_{4} \circ_{3} \lambda_{2}\right)^{(132)}-\lambda_{4} \circ_{4} \lambda_{2}+\left(\lambda_{4} \circ_{4} \lambda_{2}\right)^{(34)}-\left(\lambda_{4} \circ_{4} \lambda_{2}\right)^{(243)} \\
& +\left(\lambda_{4} \circ_{4} \lambda_{2}\right)^{(1432)}
\end{aligned}
$$

A morphism of Leibniz 3-algebras is just a homotopy morphism between 3-term homotopy Leibniz algebras.

Definition 2.1.3. Let $(L, \mathrm{~d}, \lambda)$ and $\left(L^{\prime}, \mathrm{d}^{\prime}, \lambda^{\prime}\right)$ be Leibniz 3-algebras. A homotopy morphism $f: L \rightsquigarrow L^{\prime}$ consists of maps

$$
f_{1}: L \rightarrow L^{\prime}, \quad f_{2}: L^{\otimes 2} \rightarrow L^{\prime}[1], \quad f_{3}: L^{\otimes 3} \rightarrow L^{\prime}[2],
$$

satisfying the following equations,

$$
\begin{aligned}
\partial\left(f_{1}\right)= & 0 \\
\partial\left(f_{2}\right)= & f_{1} \circ \lambda_{2}-\lambda_{2}^{\prime} \circ\left(f_{1}, f_{1}\right), \\
\partial\left(f_{3}\right)= & f_{1} \circ \lambda_{3}-f_{2} \circ{ }_{2} \lambda_{2}+f_{2} \circ \lambda_{2}+\left(f_{2} \circ \lambda_{2} \lambda_{2}\right)^{(12)} \\
& -\lambda_{3}^{\prime} \circ\left(f_{1}, f_{1}, f_{1}\right)-\lambda_{2}^{\prime} \circ\left(f_{1}, f_{2}\right)+\lambda_{2}^{\prime} \circ\left(f_{2}, f_{1}\right)+\lambda_{2}^{\prime} \circ\left(f_{1}, f_{2}\right)^{(12)},
\end{aligned}
$$

and

$$
\begin{aligned}
f_{1} \circ \lambda_{4} & -\lambda_{4}^{\prime} \circ\left(f_{1}, f_{1}, f_{1}, f_{1}\right) \\
= & f_{2} \circ \lambda_{3}+f_{2} \circ \circ_{2} \lambda_{3}-\left(f_{2} \circ \lambda_{3}\right)^{(12)}+\left(f_{2} \circ \lambda_{3}\right)^{(123)}-f_{3} \circ \lambda_{2}+f_{3} \circ \lambda_{2} \lambda_{2} \\
& -\left(f_{3} \circ \lambda_{2}\right)^{(12)}-f_{3} \circ \lambda_{2}+\left(f_{3} \circ \lambda_{2}\right)^{(23)}-\left(f_{3} \circ \lambda_{2}\right)^{(132)}+\lambda_{2}^{\prime} \circ\left(f_{3}, f_{1}\right) \\
& +\lambda_{2}^{\prime} \circ\left(f_{1}, f_{3}\right)-\lambda_{2}^{\prime} \circ\left(f_{1}, f_{3}\right)^{(12)}+\lambda_{2}^{\prime} \circ\left(f_{1}, f_{3}\right)^{(123)}-\lambda_{2}^{\prime} \circ\left(f_{2}, f_{2}\right) \\
& +\lambda_{2}^{\prime} \circ\left(f_{2}, f_{2}\right)^{(23)}-\lambda_{2}^{\prime} \circ\left(f_{2}, f_{2}\right)^{(132)}+\lambda_{3}^{\prime} \circ\left(f_{2}, f_{1}, f_{1}\right)-\lambda_{3}^{\prime} \circ\left(f_{1}, f_{2}, f_{1}\right) \\
& +\lambda_{3}^{\prime} \circ\left(f_{1}, f_{2}, f_{1}\right)^{(12)}+\lambda_{3}^{\prime} \circ\left(f_{1}, f_{1}, f_{2}\right)-\lambda_{3}^{\prime} \circ\left(f_{1}, f_{1}, f_{2}\right)^{(23)} \\
& +\lambda_{3}^{\prime} \circ\left(f_{1}, f_{1}, f_{2}\right)^{(132)} .
\end{aligned}
$$

Let $f: L \rightsquigarrow L^{\prime}$ and $f^{\prime}: L^{\prime} \rightsquigarrow L^{\prime \prime}$ be homotopy morphisms of Leibniz 3-algebras. Their composition is defined by the following components:

(119) $\left(f^{\prime} \circ f\right)_{1}:=f_{1}^{\prime} \circ f_{1}$,

(120) $\left(f^{\prime} \circ f\right)_{2}:=f_{2}^{\prime} \circ\left(f_{1}, f_{1}\right)+f_{1}^{\prime} \circ f_{2}$,

(121) $\left(f^{\prime} \circ f\right)_{3}:=f_{3}^{\prime} \circ\left(f_{1}, f_{1}, f_{1}\right)-f_{2}^{\prime} \circ\left(f_{2}, f_{1}\right)+f_{2}^{\prime} \circ\left(f_{1}, f_{2}\right)-f_{2}^{\prime} \circ\left(f_{1}, f_{2}\right)^{(12)}+f_{1}^{\prime} \circ f_{3}$.

2.2. Weak Lie 3-algebras. In this section, we spell out the definition of weak Lie 3-algebras as solutions to the Maurer-Cartan Equation (63) on a 3-term complex. To do this, we evaluate the Maurer-Cartan equation for a twisting morphism $\lambda: \operatorname{Lie}_{3}^{\diamond} \rightarrow \operatorname{End}_{L}$ on the $\mathbf{k}\left[\mathbb{S}_{n}\right]$-generators introduced in Section 1.2 and use the shorthand notation $\lambda_{*}$ 
for $\lambda\left(l_{*}\right)$. E.g., for $l_{3 ; 1}$ we find

$$
\begin{aligned}
0=(\partial \lambda+\lambda \star \lambda)\left(l_{3 ; 1}\right) & =\partial\left(\lambda\left(l_{3 ; 1}\right)\right)+\lambda\left(\mathrm{d} l_{3 ; 1}\right)+\left(\gamma_{(1)} \circ\left(\lambda \circ{ }_{(1)} \lambda\right) \circ \Delta_{(1)}\right)\left(l_{3 ; 1}\right) \\
& =\partial\left(\lambda_{3 ; 1}\right)+\lambda\left(-l_{3}-l_{3}^{(12)}\right)+\left(\gamma_{(1)} \circ\left(\lambda \circ \circ_{(1)} \lambda\right)\right)\left(l_{2} \circ_{1} l_{2 ; 1}\right) \\
& =\partial\left(\lambda_{3 ; 1}\right)-\lambda_{3}-\lambda_{3}^{(12)}-\lambda_{2} \circ{ }_{1} \lambda_{2 ; 1},
\end{aligned}
$$

which is equivalent to Equation (124) Doing this for all generators of $\mathrm{Lie}_{3}^{\circ}$ and restricting to 3-term complexes leads to the following Definition.

Definition 2.2.1. A weak Lie 3-algebra $(L, \mathrm{~d}, \lambda)$ is a 3-term chain complex

$$
(L, \mathrm{~d})=\left(L_{0} \stackrel{\mathrm{d}}{\longleftarrow} L_{1} \stackrel{\mathrm{d}}{\longleftarrow} L_{2}\right),
$$

equipped with structure maps

$$
\begin{array}{ll}
\lambda_{2}: L^{\otimes 2} \rightarrow L, & \lambda_{2 ; 1}: L^{\otimes 2} \rightarrow L[1], \\
\lambda_{3}: L^{\otimes 3} \rightarrow L[1], & \lambda_{2 ; 1,1}: L^{\otimes 2} \rightarrow L[2], \\
& \lambda_{3 ; 1}: L^{\otimes 3} \rightarrow L[2], \\
& \lambda_{3 ; 2}: L^{\otimes 3} \rightarrow L[2], \\
& \lambda_{4}: L^{\otimes 4} \rightarrow L[2] .
\end{array}
$$

We require these to satisfy the Equations (110) $(113)$ i.e., $(L, \mathrm{~d}, \lambda)$ to be a Leibniz 3algebra, and in addition we require the following equations to hold,

(126) $\lambda_{2 ; 1,1}+\lambda_{2 ; 1,1}^{(12)}=0$,

$$
\begin{aligned}
\partial\left(\lambda_{2 ; 1}\right) & =\lambda_{2}+\lambda_{2}^{(12)}, \\
\partial\left(\lambda_{2 ; 1,1}\right) & =\lambda_{2 ; 1}-\lambda_{2 ; 1}^{(12)}, \\
\partial\left(\lambda_{3 ; 1}\right) & =\lambda_{3}+\lambda_{3}^{(12)}+\lambda_{2} \circ_{1} \lambda_{2 ; 1}, \\
\partial\left(\lambda_{3 ; 2}\right) & =\lambda_{3}+\lambda_{3}^{(23)}-\lambda_{2} \circ_{2} \lambda_{2 ; 1}+\lambda_{2 ; 1} \circ_{1} \lambda_{2}+\left(\lambda_{2 ; 1} \circ_{2} \lambda_{2}\right)^{(12)},
\end{aligned}
$$$$
\lambda_{2 ; 1,1}+\lambda_{2 ; 1,1}^{(12)}=0
$$$$
\lambda_{3 ; 1}-\lambda_{3 ; 1}^{(12)}=\lambda_{2} \circ_{1} \lambda_{2 ; 1,1},
$$$$
\lambda_{3 ; 1}-\lambda_{3 ; 2}^{(12)}+\lambda_{3 ; 1}^{(132)}-\lambda_{3 ; 2}+\lambda_{3 ; 1}^{(23)}-\lambda_{3 ; 2}^{(123)}
$$$$
=\lambda_{2 ; 1} \circ_{2} \lambda_{2 ; 1}+\lambda_{2 ; 1} \circ_{1} \lambda_{2 ; 1}+\left(\lambda_{2 ; 1} \circ_{2} \lambda_{2 ; 1}\right)^{(12)}+\left(\lambda_{2 ; 1,1} \circ_{2} \lambda_{2}\right)^{(132)},
$$$$
\lambda_{3 ; 2}-\lambda_{3 ; 2}^{(23)}=-\lambda_{2} \circ_{2} \lambda_{2 ; 1,1}+\lambda_{2 ; 1,1} \circ_{1} \lambda_{2}+\left(\lambda_{2 ; 1,1} \circ_{2} \lambda_{2}\right)^{(12)} \text {, }
$$$$
\lambda_{4}+\lambda_{4}^{(12)}=\lambda_{2} \circ_{1} \lambda_{3 ; 1}-\lambda_{3 ; 1} \circ_{3} \lambda_{2}+\left(\lambda_{2} \circ_{2} \lambda_{3 ; 1}\right)^{(123)}+\lambda_{3} \circ_{1} \lambda_{2 ; 1},
$$$$
\lambda_{4}+\lambda_{4}^{(23)}=\lambda_{2} \circ_{1} \lambda_{3 ; 2}-\lambda_{3} \circ_{2} \lambda_{2 ; 1}+\lambda_{2} \circ_{2} \lambda_{3 ; 1}-\lambda_{3 ; 1} \circ_{1} \lambda_{2}-\left(\lambda_{3 ; 1} \circ_{2} \lambda_{2}\right)^{(12)}
$$$$
-\left(\lambda_{3 ; 1} \circ_{3} \lambda_{2}\right)^{(132)}
$$$$
\lambda_{4}+\lambda_{4}^{(34)}=\lambda_{2 ; 1} \circ_{1} \lambda_{3}-\lambda_{3 ; 2} \circ_{1} \lambda_{2}+\lambda_{3 ; 2} \circ_{2} \lambda_{2}-\left(\lambda_{3 ; 2} \circ_{2} \lambda_{2}\right)^{(12)}+\lambda_{3} \circ_{3} \lambda_{2 ; 1}
$$$$
+\left(\lambda_{3 ; 2} \circ_{3} \lambda_{2}\right)^{(23)}-\left(\lambda_{3 ; 2} \circ_{3} \lambda_{2}\right)^{(132)}+\lambda_{2} \circ_{2} \lambda_{3 ; 2}-\left(\lambda_{2} \circ_{2} \lambda_{3 ; 2}\right)^{(12)}
$$$$
+\left(\lambda_{2 ; 1} \circ_{2} \lambda_{3}\right)^{(123)} \text {. }
$$

When in the above definition we assume $L_{2}=0$, this forces the structure maps $\lambda_{2 ; 1,1}, \lambda_{3 ; 1}, \lambda_{3 ; 2}$, and $\lambda_{4}$ to vanish for degree reasons. The Equations (113) and (126) (132) 
hold for degree reasons in this case, and thus the left-hand sides of Equations (112) and (123) (125) become 0. In this way, we recover Roytenberg's definition of a 2-term $\mathrm{EL}_{\infty}$-algebra 63, Definition 2.16] (which we will call weak Lie 2-algebras).

2.3. Morphisms of weak Lie 3-algebras. Since weak Lie 3-algebras are algebras over the operad $\Omega \mathrm{Lie}_{3}^{\diamond}$, they come with a general notion of morphism of operadic algebras. Such a morphism of weak Lie 3-algebras $L \rightarrow L^{\prime}$ is a morphisms of the underlying chain complexes $(L, \mathrm{~d}) \rightarrow\left(L^{\prime}, \mathrm{d}^{\prime}\right)$ commuting with all structure maps. This notion of strict morphisms is of limited use, however (see the explanations in Section 6 of Chapter 2, and we will introduce another type of morphisms below.

Consider again weak Lie 3-algebras $L$ and $L^{\prime}$, i.e., chain complexes $(L, \mathrm{~d}),\left(L^{\prime}, \mathrm{d}^{\prime}\right)$ equipped with structure maps given by twisting morphisms

$$
\lambda \in \operatorname{Tw}\left(\operatorname{Lie}_{3}^{\diamond}, \operatorname{End}_{L}\right), \quad \quad \lambda^{\prime} \in \operatorname{Tw}\left(\operatorname{Lie}_{3}^{\diamond}, \operatorname{End}_{L^{\prime}}\right) .
$$

Following the general theory described in Section 6 of Chapter 2 a weak morphism of weak Lie 3 -algebras is a degree 0 solution to the following Maurer-Cartan equation:

$$
f: \operatorname{Lie}_{3}^{\diamond} \rightarrow \operatorname{End}_{L^{\prime}}^{L}, \quad \text { such that } \partial(f)-f * \lambda+\lambda^{\prime} \circledast f=0 .
$$

We again evaluate this Maurer-Cartan equation for the $\mathbf{k}\left[\mathbb{S}_{n}\right]$-generators of $\mathrm{Lie}_{3}^{\diamond}$, using the shorthand notation $f_{*}$ for $f\left(l_{*}\right)$. E.g., for $l_{2 ; 1}$ this amounts to

$$
\begin{aligned}
0 & =\left(\partial(f)-f * \lambda+\lambda^{\prime} \circledast f\right)\left(l_{2 ; 1}\right) \\
& =\partial\left(f\left(l_{2 ; 1}\right)\right)-f\left(\mathrm{~d} l_{2 ; 1}\right)-(f * \lambda)\left(l_{2 ; 1}\right)+\left(\lambda^{\prime} \circledast f\right)\left(l_{2 ; 1}\right) \\
& =\partial\left(f_{2 ; 1}\right)+f\left(l_{2}\right)+f\left(l_{2}\right)^{(12)}-f(\mathrm{id}) \circ 1 \\
& \left.=\partial\left(f_{2 ; 1}\right)+f_{2}\right)+f_{2}+f_{2 ; 1}^{(12)}-f_{1} \circ \lambda_{2 ; 1}+\lambda_{2 ; 1}^{\prime} \circ\left(f\left(f_{1}, f_{1}\right),\right.
\end{aligned}
$$

which is equivalent to Equation (134). In summary, we obtain the following definition.

Definition 2.3.1. A weak morphism of weak Lie 3-algebras $f:(L, \mathrm{~d}, \lambda) \rightsquigarrow\left(L^{\prime}, \mathrm{d}^{\prime}, \lambda^{\prime}\right)$ consists of a collection of $\mathbf{k}$-linear maps,

$$
\begin{aligned}
f_{1}: L \rightarrow L^{\prime}, \quad f_{2}: L^{\otimes 2} \rightarrow L^{\prime}[1], \quad f_{2 ; 1}: L^{\otimes 2} & \rightarrow L^{\prime}[2], \\
f_{3}: L^{\otimes 3} & \rightarrow L^{\prime}[2] .
\end{aligned}
$$

We assume that the Equations (115) (118) hold, i.e., $f$ is a homotopy morphism of Leibniz 3-algebras. In addition the maps are required to satisfy the following equations:

$$
\begin{aligned}
\partial\left(f_{2 ; 1}\right)= & -f_{2}-f_{2}^{(12)}+f_{1} \circ \lambda_{2 ; 1}-\lambda_{2 ; 1}^{\prime} \circ\left(f_{1}, f_{1}\right), \\
f_{1} \circ \lambda_{2 ; 1,1}-\lambda_{2 ; 1,1}^{\prime} \circ\left(f_{1}, f_{1}\right)= & f_{2 ; 1}-f_{2 ; 1}^{(12)}, \\
f_{1} \circ \lambda_{3 ; 1}-\lambda_{3 ; 1}^{\prime} \circ\left(f_{1}, f_{1}, f_{1}\right)= & f_{3}+f_{3}^{(12)}+f_{2} \circ \lambda_{2 ; 1}+\lambda_{2}^{\prime} \circ\left(f_{2 ; 1}, f_{1}\right), \\
f_{1} \circ \lambda_{3 ; 2}-\lambda_{3 ; 2}^{\prime} \circ\left(f_{1}, f_{1}, f_{1}\right)= & f_{3}+f_{3}^{(23)}-f_{2} \circ \lambda_{2 ; 1}+f_{2 ; 1} \circ \lambda_{2}+\left(f_{2 ; 1} \circ \circ_{2} \lambda_{2}\right)^{(12)} \\
& -\lambda_{2}^{\prime} \circ\left(f_{1}, f_{2 ; 1}\right)-\lambda_{2 ; 1}^{\prime} \circ\left(f_{2}, f_{1}\right)-\lambda_{2 ; 1}^{\prime} \circ\left(f_{1}, f_{2}\right)^{(12)}
\end{aligned}
$$


Let $f: L \rightsquigarrow L^{\prime}$ and $f^{\prime}: L^{\prime} \rightsquigarrow L^{\prime \prime}$ be weak morphisms of weak Lie 3-algebras. Their composition is defined to be the composition of the underlying homotopy morphisms of Leibniz 3-algebras, i.e., by Equations (119) (121), with the additional component

$$
\left(f^{\prime} \circ f\right)_{2 ; 1}:=f_{2 ; 1}^{\prime} \circ\left(f_{1}, f_{1}\right)+f_{1}^{\prime} \circ f_{2 ; 1} \text {. }
$$

If we assume that $L$ and $L^{\prime}$ in the above definition are in fact weak Lie 2-algebras, then for degree reasons $f_{2 ; 1}$ and $f_{3}$ must vanish. Equations (118) and (135) (137) hold again for degree reasons, and the left-hand sides of Equations (117) and (134) are zero. In this way we recover Roytenberg's notion of a morphism of 2-term $\mathrm{EL}_{\infty}$-algebras 63 . Definition 2.18].

2.4. The homotopy transfer theorem. While we explicitly prove a homotopy transfer result for weak Lie 3-algebras below, let us remark again that for a cofibrant resolution of any operad such a result is automatic.

Let $(L, d, \lambda)$ be a weak Lie 3-algebra. Assume that we are given a deformation retract of the underlying chain complex, i.e., chain maps $p$ and $i$, and a chain homotopy $h$ as follows:

$$
{ }_{h} \subset(L, \mathrm{~d}) \stackrel{p}{\rightleftarrows}\left(L^{\prime}, \mathrm{d}^{\prime}\right), \quad \text { such that }\left\{\begin{array}{l}
\operatorname{id}_{L}-i \circ p=[\mathrm{d}, h], \\
\operatorname{id}_{L^{\prime}}-p \circ i=0 .
\end{array}\right.
$$

In this setting, the following homotopy transfer theorem for weak Lie 3-algebras holds.

Proposition 2.4.1. Let $(L, \mathrm{~d}, \lambda)$ be a weak Lie 3-algebra and let $\left(L^{\prime}, \mathrm{d}^{\prime}\right)$ be a deformation retract of $(L, \mathrm{~d})$ as in Equation (139), Then $\left(L^{\prime}, \mathrm{d}^{\prime}\right)$ can be equipped with a transferred weak Lie 3-algebra structure in such a way, that the map $i$ admits an extension to a weak morphism of weak Lie 3-algebras.

PROOF. This follows directly from Lemma 2.4.2 and Lemma 2.4.3 below.

By the homotopy transfer theorem for Leib ${ }_{\infty}$-algebras we know that one can define a Leibniz 3-algebra structure on $\left(L^{\prime}, \mathrm{d}^{\prime}\right)$ by the structure maps

$$
\begin{aligned}
& \lambda_{2}^{\prime}:=p \circ \lambda_{2} \circ(i, i), \\
& \lambda_{3}^{\prime}:=p \circ \lambda_{3} \circ(i, i, i)+p \circ\left(\lambda_{2} \circ\left(h \circ \lambda_{2}\right)-\left(\lambda_{2} \circ{ }_{2}\left(h \circ \lambda_{2}\right)\right)^{1-(12)}\right) \circ(i, i, i),
\end{aligned}
$$

and

$$
\begin{aligned}
\lambda_{4}^{\prime}:= & p \circ \lambda_{4} \circ(i, i, i, i)-p \circ\left(\lambda_{2} \circ\left(h \circ \lambda_{3}\right)+\left(\lambda_{2} \circ \circ_{2}\left(h \circ \lambda_{3}\right)\right)^{1-(12)+(123)}\right. \\
& \left.\left.+\lambda_{3} \circ\left(h \circ \lambda_{2}\right)-\left(\lambda_{3} \circ\left(h \circ \lambda_{2}\right)\right)^{1-(12)}+\left(\lambda_{3} \circ 3 \circ \lambda_{2}\right)\right)^{1-(23)+(132)}\right) \circ(i, i, i, i) \\
& -p \circ\left(\lambda_{2} \circ\left(h \circ \lambda_{2}\right) \circ\left(h \circ \lambda_{2}\right)-\left(\lambda_{2} \circ\left(h \circ \lambda_{2}\right) \circ\left(h \circ \lambda_{2}\right)\right)^{1-(12)}\right. \\
& +\lambda_{2} \circ\left(h \circ \lambda_{2}, h \circ \lambda_{2}\right)^{1-(23)+(132)}+\left(\lambda_{2} \circ\left(h \circ \lambda_{2}\right) \circ\left(h \circ \lambda_{2}\right)\right)^{1-(12)+(123)} \\
& \left.-\left(\lambda_{2} \circ \circ_{2}\left(h \circ \lambda_{2}\right) \circ_{3}\left(h \circ \lambda_{2}\right)\right)^{1-(12)+(123)-(23)+(132)-(13)}\right) \circ(i, i, i, i),
\end{aligned}
$$


and that the following components define an extension of $i$ to a homotopy morphism of Leibniz 3-algebras $i:\left(L^{\prime}, \mathrm{d}^{\prime}, \lambda^{\prime}\right) \rightsquigarrow(L, \mathrm{~d}, \lambda)$ :

$$
\begin{aligned}
& \text { (143) } i_{1}=i, \\
& \text { (144) } i_{2}=-h \circ \lambda_{2} \circ(i, i), \\
& \text { (145) } i_{3}=-h \circ \lambda_{3} \circ(i, i, i)-h \circ\left(\lambda_{2} \circ\left(h \circ \lambda_{2}\right)-\left(\lambda_{2} \circ{ }_{2}\left(h \circ \lambda_{2}\right)\right)^{1-(12)}\right) \circ(i, i, i) .
\end{aligned}
$$

We extend these constructions to weak Lie 3-algebras following the same pattern.

Lemma 2.4.2. The Leibniz 3-algebra $\left(L^{\prime}, \mathrm{d}^{\prime}\right)$ with structure maps $\lambda_{*}^{\prime}$ as defined by Equations (140) (142) admits an extension to a weak Lie 3-algebra. Explicitly, such an extension is given by the structure maps

$$
\begin{aligned}
\lambda_{2 ; 1}^{\prime}:= & p \circ \lambda_{2 ; 1} \circ(i, i), \\
\lambda_{2 ; 1,1}^{\prime}:= & p \circ \lambda_{2 ; 1,1} \circ(i, i), \\
\lambda_{3,1}^{\prime}:= & p \circ \lambda_{3 ; 1} \circ(i, i, i)-p \circ\left(\lambda_{2} \circ\left(h \circ \lambda_{2 ; 1}\right)\right) \circ(i, i, i), \\
\lambda_{3,2}^{\prime}:= & p \circ \lambda_{3 ; 2} \circ(i, i, i)+p \circ\left(\lambda_{2 ; 1} \circ\left(h \circ \lambda_{2}\right)+\lambda_{2} \circ\left(h \circ \lambda_{2 ; 1}\right)\right. \\
& \left.+\left(\lambda_{2 ; 1} \circ \circ_{2}\left(h \circ \lambda_{2}\right)\right)^{(12)}\right) \circ(i, i, i) .
\end{aligned}
$$

The proof is a straightforward verification of Equations (122) (132), which we postpone to Section A.2

Lemma 2.4.3. The homotopy morphism $i:\left(L^{\prime}, \mathrm{d}^{\prime}\right) \leadsto(L, \mathrm{~d})$ of Leibniz 3-algebras with components defined by Equations (143) (145) extends to a morphism of weak Lie 3algebras with the additional component

$$
i_{2 ; 1}=-h \circ \lambda_{2 ; 1} \circ(i, i) .
$$

PROOF. We verify that with this definition of the additional component $i_{2 ; 1}$ Equations (134) (137) hold:

$$
\begin{aligned}
\partial\left(i_{2 ; 1}\right) & =-\partial\left(h \circ \lambda_{2 ; 1} \circ(i, i)\right) \\
& =-(\mathrm{id}-i p) \circ \lambda_{2 ; 1} \circ(i, i)+h \circ\left(\lambda_{2}+\lambda_{2}^{(12)}\right) \circ(i, i) \\
& =-i_{2}-i_{2}^{(12)}+i_{1} \circ \lambda_{2 ; 1}^{\prime}-\lambda_{2 ; 1} \circ\left(i_{1}, i_{1}\right), \\
i_{1} \circ \lambda_{2 ; 1,1}^{\prime}-\lambda_{2 ; 1,1} \circ\left(i_{1}, i_{1}\right) & \\
& =i \circ\left(p \circ \lambda_{2 ; 1,1} \circ(i, i)\right)-\lambda_{2 ; 1,1} \circ(i, i) \\
& =-\partial h \circ \lambda_{2 ; 1,1} \circ(i, i) \\
& =-h \circ\left(\lambda_{2 ; 1}-\lambda_{2 ; 1}^{(12)}\right) \circ(i, i) \\
& =i_{2 ; 1}-i_{2 ; 1}^{(12)}, \\
i_{1} \circ \lambda_{3 ; 1}^{\prime} & -\lambda_{3 ; 1} \circ\left(i_{1}, i_{1}, i_{1}\right) \\
& =i \circ\left(p \circ\left(\lambda_{3 ; 1}-\lambda_{2} \circ\left(h \circ \lambda_{2 ; 1}\right)\right) \circ(i, i, i)\right)-\lambda_{3 ; 1} \circ(i, i, i) \\
& =-\partial h \circ \lambda_{3 ; 1} \circ(i, i, i)-(\mathrm{id}-\partial h) \circ\left(\lambda_{2} \circ\left(h \circ \lambda_{2 ; 1}\right)\right) \circ(i, i, i)
\end{aligned}
$$




$$
\begin{aligned}
& =-h \circ\left(\lambda_{3}+\lambda_{3}^{(12)}+\lambda_{2} \circ{ }_{1} \lambda_{2 ; 1}\right) \circ(i, i, i)+\lambda_{2} \circ\left(i_{2 ; 1}, i_{1}\right) \\
& +h \circ\left(\lambda_{2} \circ{ }_{1}\left(\partial h \circ \lambda_{2 ; 1}\right)\right) \circ(i, i, i)-h \circ\left(\lambda_{2} \circ\left(h \circ\left(\lambda_{2}+\lambda_{2}^{(12)}\right)\right)\right) \circ(i, i, i) \\
& =-h \circ\left(\lambda_{3}+\lambda_{2} \circ\left(h \circ \lambda_{2 ; 1}\right)\right) \circ(i, i, i)-h \circ\left(\lambda_{3}+\lambda_{2} \circ{ }_{1}\left(h \circ \lambda_{2 ; 1}\right)\right)^{(12)} \circ(i, i, i) \\
& +\lambda_{2} \circ\left(i_{2 ; 1}, i_{1}\right)-h \circ\left(\lambda_{2} \circ\left(i p \circ \lambda_{2 ; 1}\right)\right) \circ(i, i, i) \\
& =i_{3}+i_{3}^{(12)}+\lambda_{2} \circ\left(i_{2 ; 1}, i_{1}\right)+i_{2} \circ_{1} \lambda_{2 ; 1}^{\prime} \text {, } \\
& i_{1} \circ \lambda_{3 ; 2}^{\prime}-\lambda_{3 ; 2} \circ\left(i_{1}, i_{1}, i_{1}\right) \\
& =i \circ p \circ\left(\lambda_{3 ; 2}+\lambda_{2 ; 1} \circ \circ_{1}\left(h \circ \lambda_{2}\right)+\lambda_{2} \circ_{2}\left(h \circ \lambda_{2 ; 1}\right)+\left(\lambda_{2 ; 1} \circ\left(h \circ \lambda_{2}\right)\right)^{(12)}\right) \circ(i, i, i) \\
& -\lambda_{3 ; 2} \circ(i, i, i) \\
& =-\partial h \circ \lambda_{3 ; 2} \circ(i, i, i)+(\mathrm{id}-\partial h) \circ\left(\lambda_{2 ; 1} \circ \circ_{1}\left(h \circ \lambda_{2}\right)+\lambda_{2} \circ_{2}\left(h \circ \lambda_{2 ; 1}\right)\right. \\
& \left.+\left(\lambda_{2 ; 1} \circ_{2}\left(h \circ \lambda_{2}\right)\right)^{(12)}\right) \circ(i, i, i) \\
& =-h \circ\left(\lambda_{3}+\lambda_{3}^{(23)}+\lambda_{2 ; 1} \circ_{1} \lambda_{2}-\lambda_{2} \circ_{2} \lambda_{2 ; 1}+\left(\lambda_{2 ; 1} \circ_{2} \lambda_{2}\right)^{(12)}\right) \circ(i, i, i) \\
& +\left(\lambda_{2 ; 1} \circ \circ_{1}\left(h \circ \lambda_{2}\right)+\lambda_{2} \circ_{2}\left(h \circ \lambda_{2 ; 1}\right)+\left(\lambda_{2 ; 1} \circ_{2}\left(h \circ \lambda_{2}\right)\right)^{(12)}\right) \circ(i, i, i) \\
& -h \circ\left(\left(\lambda_{2}+\lambda_{2}^{(12)}\right) \circ_{1}\left(h \circ \lambda_{2}\right)\right) \circ(i, i, i)+h \circ\left(\lambda_{2 ; 1} \circ{ }_{1}\left((\mathrm{id}-i p) \circ \lambda_{2}\right) \circ(i, i, i)\right. \\
& -h \circ\left(\lambda_{2} \circ 2\left((\mathrm{id}-i p) \circ \lambda_{2 ; 1}\right) \circ(i, i, i)+h \circ\left(\lambda_{2} \circ 2\left(h \circ\left(\lambda_{2}+\lambda_{2}^{(12)}\right)\right)\right) \circ(i, i, i)\right. \\
& -h \circ\left(\left(\lambda_{2}+\lambda_{2}^{(12)}\right) \circ_{2}\left(h \circ \lambda_{2}\right)\right)^{(12)} \circ(i, i, i) \\
& +h \circ\left(\lambda_{2 ; 1} \circ 2\left((\mathrm{id}-i p) \circ \lambda_{2}\right)^{(12)} \circ(i, i, i)\right. \\
& =-h \circ\left(\lambda_{3}+\lambda_{2} \circ\left(h \circ \lambda_{2}\right)-\left(\lambda_{2} \circ 2\left(h \circ \lambda_{2}\right)\right)^{1-(12)}\right) \circ(i, i, i) \\
& -h \circ\left(\lambda_{3}+\lambda_{2} \circ\left(h \circ \lambda_{2}\right)-\left(\lambda_{2} \circ \circ_{2}\left(h \circ \lambda_{2}\right)\right)^{1-(12)}\right)^{(23)} \circ(i, i, i) \\
& -\lambda_{2 ; 1} \circ\left(i_{2}, i_{1}\right)-\lambda_{2} \circ\left(i_{1}, i_{2 ; 1}\right)-\lambda_{2 ; 1} \circ\left(i_{1}, i_{2}\right)^{(12)} \\
& +\left(-h \circ \lambda_{2 ; 1} \circ(i, i)\right) \circ_{1}\left(p \circ \lambda_{2} \circ(i, i)\right)-\left(-h \circ \lambda_{2} \circ(i, i)\right) \circ_{2}\left(p \circ \lambda_{2 ; 1} \circ(i, i)\right) \\
& +\left(\left(-h \circ \lambda_{2 ; 1} \circ(i, i)\right) \circ_{2}\left(p \circ \lambda_{2} \circ(i, i)\right)\right)^{(12)} \\
& =i_{3}+i_{3}^{(23)}-\lambda_{2 ; 1} \circ\left(i_{2}, i_{1}\right)-\lambda_{2} \circ\left(i_{1}, i_{2 ; 1}\right)-\lambda_{2 ; 1} \circ\left(i_{1}, i_{2}\right)^{(12)} \\
& +i_{2 ; 1} \circ_{1} \lambda_{2}^{\prime}-i_{2} \circ_{2} \lambda_{2 ; 1}^{\prime}+\left(i_{2 ; 1} \circ_{2} \lambda_{2}^{\prime}\right)^{(12)} \text {. }
\end{aligned}
$$

This concludes the proof.

\section{Skewsymmetrization}

Since an $\mathrm{L}_{\infty}$-algebra is just a Leib ${ }_{\infty}$-algebra with skewsymmetric structure maps, it seems natural to try and construct an $\mathrm{L}_{\infty}$-algebra by skewsymmetrizing the structure maps of a Leib ${ }_{\infty}$-algebra. One way to put this more formally is in terms of the Koszul dual cooperads: assume for a moment that we can construct a right inverse to the morphism $\psi$ defined by Equation (89), i.e., a morphism $\phi$ of dg cooperads

$$
\psi: \text { Leib }^{\mathrm{i}} \rightleftarrows \text { Lie }^{\mathrm{i}}: \phi, \text { such that } \psi \circ \phi=\mathrm{id} \text {. }
$$

In this case, we obtain for any Leib $_{\infty}$-algebra $(L, \mathrm{~d}, \lambda)$ given by a twisting morphism $\lambda:$ Leib $^{i} \rightarrow$ End $_{L}$, an $\mathrm{L}_{\infty}$-algebra $(L, \mathrm{~d}, \bar{\lambda})$ via precomposition of the twisting morphism 
with $\phi$, i.e., $\bar{\lambda}:=\lambda \circ \phi$. We shall make a naive attempt at defining such a morphism $\phi$ below to see how it fails.

Define $\phi$ on $\mathbf{k}\left[\mathbb{S}_{n}\right]$-generators by

$$
\phi\left(\bar{l}_{n}\right):=\frac{1}{n !} \sum_{\sigma \in \mathbb{S}_{n}}(-1)^{|\sigma|} \cdot l_{n}^{\sigma} .
$$

Clearly, this is a welldefined morphism of dg $\mathbb{S}$-modules and satisfies $\psi \circ \phi=\mathrm{id}$, provided the $1 / n$ ! exist. The calculation below, however, shows that the map $\phi$ does not define a morphism of dg cooperads since $\phi$ does not commute with the decomposition map $\Delta$ already in arity 3 . We find

$$
\begin{aligned}
\Delta\left(\phi\left(\bar{l}_{3}\right)\right) & =\frac{1}{6} \sum_{\sigma \in \mathbb{S}_{3}}(-1)^{|\sigma|} \cdot\left(-l_{2} \circ\left(l_{2}, 1\right)+l_{2} \circ\left(1, l_{2}\right)-l_{2} \circ\left(1, l_{2}\right)^{(12)}\right)^{\sigma} \\
& =-\frac{1}{6} \sum_{\sigma \in \mathbb{S}_{3}}(-1)^{|\sigma|} \cdot l_{2} \circ\left(l_{2}, 1\right)^{\sigma}+\frac{1}{3} \sum_{\sigma \in \mathbb{S}_{3}}(-1)^{|\sigma|} \cdot l_{2} \circ\left(1, l_{2}\right)^{\sigma},
\end{aligned}
$$

while

$$
\phi\left(\Delta\left(\bar{l}_{3}\right)\right)=-\frac{1}{4} \sum_{\sigma \in \mathbb{S}_{3}}(-1)^{|\sigma|} \cdot\left(l_{2} \circ\left(l_{2}, 1\right)-l_{2} \circ\left(1, l_{2}\right)\right)^{\sigma} .
$$

Note that the difference

$$
(\Delta \circ \phi-\phi \circ \Delta)\left(\bar{l}_{3}\right)=\frac{1}{12} \sum_{\sigma \in \mathbb{S}_{3}}(-1)^{|\sigma|} \cdot\left(l_{2} \circ\left(l_{2}, 1\right)+l_{2} \circ\left(1, l_{2}\right)\right)^{\sigma}
$$

is actually a coboundary when we view $\operatorname{Leib}_{3}^{i} \subset \mathrm{Lie}_{3}^{\diamond}$ as a dg subcooperad,

$$
=-\frac{1}{12} \sum_{\sigma \in \mathbb{S}_{3}}(-1)^{|\sigma|} \cdot\left(\mathrm{d} l_{2 ; 1} \circ\left(l_{2}, 1\right)\right)^{\sigma} .
$$

This suggests that $(i)$ while Leib $b_{\infty}$-algebras do not admit a skewsymmetrization in general, for weak Lie 3-algebras (and more generally $\mathrm{EL}_{\infty}$-algebras) such a construction may exist, and (ii) we should try to extend $\phi$ to a homotopy morphism of dg cooperads.

The remainder of this section is organized as follows. In Section 3.1 we construct a right inverse $\Phi$ for $\Omega \psi: \Omega \mathrm{Lie}_{3}^{\diamond} \rightarrow \Omega \mathrm{Lie}_{3}^{\mathrm{i}}$. In Section 3.2 we use $\Phi$ to define a skewsymmetrization construction for weak Lie 3-algebras. In Section 3.3, we define an ad hoc skewsymmetrization for homotopy morphisms of weak Lie 3-algebras and show that it is functorial up to homotopy.

In this entire section, we assume that $2,3 \in \mathbf{k}^{\times}$are units.

3.1. A right inverse homotopy morphism for the cooperad resolution. Below we construct a right inverse for $\Omega \psi: \Omega \mathrm{Lie}_{3}^{\diamond} \rightarrow \Omega \mathrm{Lie}_{3}^{i}$. We think of such a map as a homotopy morphism of dg cooperads.

Lemma 3.1.1. The morphism $\Omega \psi$ admits a right inverse, i.e., a morphism $\Phi$ of $d g$ operads

$$
\Omega \psi: \Omega \mathrm{Lie}_{3}^{\diamond} \rightleftarrows \Omega \mathrm{Lie}_{3}^{\mathrm{i}}: \Phi, \text { such that } \Omega \psi \circ \Phi=\mathrm{id} .
$$

One such morphism $\Phi$ is defined by

$$
\Phi\left(s^{-1} \bar{l}_{2}\right)=\frac{1}{2} \sum_{\sigma \in \mathbb{S}_{2}}(-1)^{|\sigma|} \cdot s^{-1} l_{2}^{\sigma},
$$




$$
\begin{aligned}
\Phi\left(s^{-1} \bar{l}_{3}\right)= & \frac{1}{6} \sum_{\sigma \in \mathbb{S}_{3}}(-1)^{|\sigma|} \cdot s^{-1} l_{3}^{\sigma}-\frac{1}{24} \sum_{\sigma \in \mathbb{S}_{3}}(-1)^{|\sigma|} \cdot\left(s^{-1} l_{2 ; 1} \circ_{1} s^{-1} l_{2}+s^{-1} l_{2 ; 1} \circ_{2} s^{-1} l_{2}\right)^{\sigma}, \\
\Phi\left(s^{-1} \bar{l}_{4}\right)= & \frac{1}{24} \sum_{\sigma \in \mathbb{S}_{4}}(-1)^{|\sigma|} \cdot s^{-1} l_{4}^{\sigma} \\
& +\frac{1}{48} \sum_{\sigma \in \mathbb{S}_{4}}(-1)^{|\sigma|} \cdot\left(s^{-1} l_{2 ; 1} \circ_{1} s^{-1} l_{3}-s^{-1} l_{3 ; 1} \circ_{1} s^{-1} l_{2}+s^{-1} l_{3 ; 2} \circ_{2} s^{-1} l_{2}\right. \\
& \left.\quad-s^{-1} l_{2 ; 1} \circ_{2} s^{-1} l_{3}-s^{-1} l_{3 ; 1} \circ_{2} s^{-1} l_{2}+s^{-1} l_{3 ; 2} \circ_{3} s^{-1} l_{2}\right)^{\sigma} .
\end{aligned}
$$

PROOF. Any morphism of free dg operads is completely determined by its value on generators, i.e., it is sufficient to define $\left.\Phi\right|_{S^{-1} \operatorname{Lie}_{3}^{i}}$ and its extension $\Phi$ is then automatically a morphism of operads. It remains to verify that $\Phi$ commutes with the differential, which we do below:

$$
\begin{aligned}
& \mathrm{d} \Phi\left(s^{-1} \bar{l}_{2}\right)=\frac{1}{2} \sum_{\sigma \in \mathbb{S}_{2}}(-1)^{|\sigma|} \cdot \mathrm{d} s^{-1} l_{2}^{\sigma}=0=\Phi(0)=\Phi\left(\mathrm{d} s^{-1} \bar{l}_{2}\right), \\
& \mathrm{d} \Phi\left(s^{-1} \bar{l}_{3}\right)=-\frac{1}{6} \sum_{\sigma \in \mathbb{S}_{3}}(-1)^{|\sigma|} \cdot \mathrm{d}_{2} s^{-1} l_{3}^{\sigma} \\
& -\frac{1}{24} \sum_{\sigma \in \mathbb{S}_{3}}(-1)^{|\sigma|} \cdot\left(\mathrm{d}_{1} s^{-1} l_{2 ; 1} \circ_{1} s^{-1} l_{2}+\mathrm{d}_{1} s^{-1} l_{2 ; 1} \circ_{2} s^{-1} l_{2}\right)^{\sigma} \\
& =-\frac{1}{6} \sum_{\sigma \in \mathbb{S}_{3}}(-1)^{|\sigma|} \cdot\left(-s^{-1} l_{2} \circ_{1} s^{-1} l_{2}+s^{-1} l_{2} \circ_{2} s^{-1} l_{2}-\left(s^{-1} l_{2} \circ_{2} s^{-1} l_{2}\right)^{(12)}\right)^{\sigma} \\
& -\frac{1}{12} \sum_{\sigma \in \mathbb{S}_{3}}(-1)^{|\sigma|} \cdot\left(\left(s^{-1} l_{2}+s^{-1} l_{2}^{(12)}\right) \circ_{1} s^{-1} l_{2}\right)^{\sigma} \\
& =-\frac{1}{4} \sum_{\sigma \in \mathbb{S}_{3}}(-1)^{|\sigma|} \cdot\left(s^{-1} l_{2} \circ_{1} s^{-1} l_{2}-s^{-1} l_{2} \circ_{2} s^{-1} l_{2}\right)^{\sigma} \\
& =-\Phi\left(s^{-1} \bar{l}_{2}\right) \circ_{1} \Phi\left(s^{-1} \bar{l}_{2}\right)+\Phi\left(s^{-1} \bar{l}_{2}\right) \circ_{2} \Phi\left(s^{-1} \bar{l}_{2}\right)-\left(\Phi\left(s^{-1} \bar{l}_{2}\right) \circ_{2} \Phi\left(s^{-1} \bar{l}_{2}\right)\right)^{(12)} \\
& =\Phi\left(-s^{-1} \bar{l}_{2} \circ_{1} s^{-1} \bar{l}_{2}+s^{-1} \bar{l}_{2} \circ_{2} s^{-1} \bar{l}_{2}-\left(s^{-1} \bar{l}_{2} \circ_{2} s^{-1} \bar{l}_{2}\right)^{(12)}\right) \\
& =\Phi\left(-\mathrm{d}_{2} s^{-1} \bar{l}_{3}\right)=\Phi\left(\mathrm{d} s^{-1} \bar{l}_{3}\right) \text {, } \\
& \mathrm{d} \Phi\left(s^{-1} \bar{l}_{4}\right)=-\frac{1}{24} \sum_{\sigma \in \mathbb{S}_{4}}(-1)^{|\sigma|} \cdot \mathrm{d}_{2} s^{-1} l_{4}^{\sigma} \\
& +\frac{1}{48} \sum_{\sigma \in \mathbb{S}_{4}}(-1)^{|\sigma|} \cdot\left(\left(\mathrm{d}_{1} s^{-1} l_{2 ; 1}\right) \circ_{1} s^{-1} l_{3}+s^{-1} l_{2 ; 1} \circ_{1}\left(\mathrm{~d}_{2} s^{-1} l_{3}\right)\right. \\
& \left.-\left(\mathrm{d}_{1} s^{-1} l_{2 ; 1}\right) \circ_{2} s^{-1} l_{3}-s^{-1} l_{2 ; 1} \circ_{2}\left(\mathrm{~d}_{2} s^{-1} l_{3}\right)\right)^{\sigma} \\
& -\frac{1}{48} \sum_{\sigma \in \mathbb{S}_{4}}(-1)^{|\sigma|} \cdot\left(\left(\mathrm{d}_{1} s^{-1} l_{3 ; 1}\right) \circ_{1} s^{-1} l_{2}-\left(\mathrm{d}_{2} s^{-1} l_{3 ; 1}\right) \circ_{1} s^{-1} l_{2}\right. \\
& \left.+\left(\mathrm{d}_{1} s^{-1} l_{3 ; 1}\right) \circ_{2} s^{-1} l_{2}-\left(\mathrm{d}_{2} s^{-1} l_{3 ; 1}\right) \circ_{2} s^{-1} l_{2}\right)^{\sigma} \\
& +\frac{1}{48} \sum_{\sigma \in \mathbb{S}_{4}}(-1)^{|\sigma|} \cdot\left(\left(\mathrm{d}_{1} s^{-1} l_{3 ; 2}\right) \circ_{2} s^{-1} l_{2}-\left(\mathrm{d}_{2} s^{-1} l_{3 ; 2}\right) \circ_{2} s^{-1} l_{2}\right. \\
& \left.+\left(\mathrm{d}_{1} s^{-1} l_{3 ; 2}\right) \circ_{2} s^{-1} l_{2}-\left(\mathrm{d}_{2} s^{-1} l_{3 ; 2}\right) \circ_{2} s^{-1} l_{2}\right)^{\sigma}
\end{aligned}
$$




$$
\begin{aligned}
& =-\frac{1}{24} \sum_{\sigma \in \mathbb{S}_{4}}(-1)^{|\sigma|} \cdot\left(-s^{-1} l_{2} \circ_{1} s^{-1} l_{3}-3 s^{-1} l_{2} \circ_{2} s^{-1} l_{3}\right. \\
& \left.+s^{-1} l_{3} \circ_{1} s^{-1} l_{2}-2 s^{-1} l_{3} o_{2} s^{-1} l_{2}+3 s^{-1} l_{3} o_{3} s^{-1} l_{2}\right)^{\sigma} \\
& +\frac{1}{48} \sum_{\sigma \in \mathbb{S}_{4}}(-1)^{|\sigma|} \cdot\left(s^{-1} l_{2} \circ_{1} s^{-1} l_{3}+\left(s^{-1} l_{2} \circ_{2} s^{-1} l_{3}\right)^{(1234)}\right. \\
& -s^{-1} l_{2} \circ_{2} s^{-1} l_{3}-\left(s^{-1} l_{2} \circ_{1} s^{-1} l_{3}\right)^{(1432)} \\
& +s^{-1} l_{2 ; 1} \circ_{1}\left(s^{-1} \bar{l}_{2} \circ_{1} s^{-1} \bar{l}_{2}-s^{-1} \bar{l}_{2} \circ_{2} s^{-1} \bar{l}_{2}\right. \\
& \left.+\left(s^{-1} \bar{l}_{2} \circ_{2} s^{-1} \bar{l}_{2}\right)^{(12)}\right) \\
& -s^{-1} l_{2 ; 1} \circ_{2}\left(s^{-1} \bar{l}_{2} \circ_{1} s^{-1} \bar{l}_{2}-s^{-1} \bar{l}_{2} \circ_{2} s^{-1} \bar{l}_{2}\right. \\
& \left.\left.+\left(s^{-1} \bar{l}_{2} \circ_{2} s^{-1} \bar{l}_{2}\right)^{(12)}\right)\right)^{\sigma} \\
& -\frac{1}{48} \sum_{\sigma \in \mathbb{S}_{4}}(-1)^{|\sigma|} \cdot\left(s^{-1} l_{3} \circ_{1} s^{-1} l_{2}+\left(s^{-1} l_{3} \circ_{2} s^{-1} l_{2}\right)^{(123)}\right. \\
& +\left(s^{-1} l_{2} \circ_{1} s^{-1} l_{2 ; 1}\right) \circ_{1} s^{-1} l_{2} s^{-1} l_{3} \circ_{2} s^{-1} l_{2} \\
& \left.+\left(s^{-1} l_{3} \circ_{1} s^{-1} l_{2}\right)^{(132)}+\left(s^{-1} l_{2} \circ_{1} s^{-1} l_{2 ; 1}\right) \circ_{2} s^{-1} l_{2}\right)^{\sigma} \\
& +\frac{1}{48} \sum_{\sigma \in \mathbb{S}_{4}}(-1)^{|\sigma|} \cdot\left(s^{-1} l_{3} o_{2} s^{-1} l_{2}+\left(s^{-1} l_{3} o_{3} s^{-1} l_{2}\right)^{(234)}\right. \\
& +\left(s^{-1} l_{2 ; 1} \circ_{1} s^{-1} l_{2}-s^{-1} l_{2} \circ_{2} s^{-1} l_{2 ; 1}\right. \\
& \left.+\left(s^{-1} l_{2 ; 1} \circ_{2} s^{-1} l_{2}\right)^{(12)}\right) \circ_{2} s^{-1} l_{2} \\
& +s^{-1} l_{3} \circ_{3} s^{-1} l_{2}+\left(s^{-1} l_{3} \circ_{2} s^{-1} l_{2}\right)^{(243)} \\
& +\left(s^{-1} l_{2 ; 1} \circ_{1} s^{-1} l_{2}-s^{-1} l_{2} \circ_{2} s^{-1} l_{2 ; 1}\right. \\
& \left.\left.+\left(s^{-1} l_{2 ; 1} \circ_{2} s^{-1} l_{2}\right)^{(12)}\right) \circ_{3} s^{-1} l_{2}\right)^{\sigma} \\
& =\frac{1}{12} \sum_{\sigma \in \mathbb{S}_{4}}(-1)^{|\sigma|} \cdot\left(s^{-1} l_{2} \circ_{1} s^{-1} l_{3}+s^{-1} l_{2} \circ_{2} s^{-1} l_{3}-s^{-1} l_{3} \circ_{1} s^{-1} l_{2}\right. \\
& \left.+s^{-1} l_{3} \circ_{2} s^{-1} l_{2}-s^{-1} l_{3} \circ_{3} s^{-1} l_{2}\right)^{\sigma} \\
& -\frac{1}{48} \sum_{\sigma \in \mathbb{S}_{4}}(-1)^{|\sigma|} \cdot\left(s^{-1} l_{2} \circ_{1} s^{-1} l_{2 ; 1} \circ_{1} s^{-1} l_{2}+s^{-1} l_{2} \circ_{1} s^{-1} l_{2 ; 1} \circ_{2} s^{-1} l_{2}\right. \\
& +s^{-1} l_{2} \circ_{2} s^{-1} l_{2 ; 1} \circ_{2} s^{-1} l_{2}+s^{-1} l_{2} \circ_{2} s^{-1} l_{2 ; 1} \circ_{3} s^{-1} l_{2} \\
& -s^{-1} l_{2 ; 1} \circ_{1} s^{-1} l_{2} \circ_{1} s^{-1} l_{2}-s^{-1} l_{2 ; 1} \circ_{2} s^{-1} l_{2} \circ_{1} s^{-1} l_{2} \\
& +s^{-1} l_{2 ; 1} \circ_{1} s^{-1} l_{2} \circ_{2} s^{-1} l_{2}+s^{-1} l_{2 ; 1} \circ_{2} s^{-1} l_{2} \circ_{2} s^{-1} l_{2} \\
& \left.-s^{-1} l_{2 ; 1} \circ_{1} s^{-1} l_{2} \circ_{3} s^{-1} l_{2}-s^{-1} l_{2 ; 1} \circ_{2} s^{-1} l_{2} \circ_{3} s^{-1} l_{2}\right)^{\sigma} \\
& =\Phi\left(s^{-1} \bar{l}_{2} \circ_{1} s^{-1} \bar{l}_{3}+s^{-1} \bar{l}_{2} \circ_{2} s^{-1} \bar{l}_{3}-\left(s^{-1} \bar{l}_{2} \circ_{2} s^{-1} \bar{l}_{3}\right)^{(12)}\right. \\
& +\left(s^{-1} \bar{l}_{2} \circ_{2} s^{-1} \bar{l}_{3}\right)^{(123)}-s^{-1} \bar{l}_{3} \circ_{1} s^{-1} \bar{l}_{2} \\
& +s^{-1} \bar{l}_{3} \circ_{2} s^{-1} \bar{l}_{2}-\left(s^{-1} \bar{l}_{3} \circ_{2} s^{-1} \bar{l}_{2}\right)^{(12)} \\
& \left.-s^{-1} \bar{l}_{3} o_{3} s^{-1} \bar{l}_{2}+\left(s^{-1} \bar{l}_{3} o_{3} s^{-1} \bar{l}_{2}\right)^{(23)}-\left(s^{-1} \bar{l}_{3} o_{3} s^{-1} \bar{l}_{2}\right)^{(132)}\right) \\
& =\Phi\left(\mathrm{d} s^{-1} \bar{l}_{4}\right) \text {. }
\end{aligned}
$$

Finally, note that indeed $\Omega \psi \circ \Phi=$ id. 
3.2. Skewsymmetrization for weak Lie 3-algebras. In Section 2.2 we defined a weak Lie 3-algebra $(L, \mathrm{~d}, \lambda)$ as a 3-term complex $(L, \mathrm{~d})$ with a twisting morphism $\lambda: \mathrm{Lie}_{3}^{\diamond} \rightarrow \mathrm{End}_{L}$. Such a twisting morphism corresponds to a morphism of $\mathrm{dg}$ operads $g_{\lambda}: \Omega \operatorname{Lie}_{3}^{\diamond} \rightarrow \operatorname{End}_{L}$ via $\left.g_{\lambda}\right|_{s^{-1} \operatorname{Lie}_{3}^{\diamond}}\left(s^{-1} l_{*}\right)=\lambda_{*}$, see Proposition 4.2.1 of Chapter 2 By precomposition with $\Phi$ we obtain a morphism

$$
\Phi^{*} g_{\lambda}: \Omega \mathrm{Lie}_{3}^{i} \rightarrow \Omega \mathrm{Lie}_{3}^{\diamond} \rightarrow \operatorname{End}_{L}
$$

of $\mathrm{dg}$ operads, which in turn corresponds to a 3-term $\mathrm{L}_{\infty}$-algebra or (semistrict) Lie 3-algebra. We make the result of this construction explicit below.

Definition 3.2.1. Let $(L, \mathrm{~d}, \lambda)$ be a weak Lie 3-algebra. Its skewsymmetrization is the (semistrict) Lie 3-algebra $(L, \mathrm{~d}, \bar{\lambda})$ given by the following structure maps:

$$
\begin{aligned}
\bar{\lambda}_{2}:= & \frac{1}{2} \sum_{\sigma \in \mathbb{S}_{2}}(-1)^{|\sigma|} \cdot \lambda_{2}^{\sigma}, \\
\bar{\lambda}_{3}:= & \frac{1}{6} \sum_{\sigma \in \mathbb{S}_{3}}(-1)^{|\sigma|} \cdot \lambda_{3}^{\sigma}-\frac{1}{24} \sum_{\sigma \in \mathbb{S}_{3}}(-1)^{|\sigma|} \cdot\left(\lambda_{2 ; 1} \circ_{1} \lambda_{2}+\lambda_{2 ; 1} \circ_{2} \lambda_{2}\right)^{\sigma}, \\
\bar{\lambda}_{4}:= & \frac{1}{24} \sum_{\sigma \in \mathbb{S}_{4}}(-1)^{|\sigma|} \cdot \lambda_{4}^{\sigma} \\
& +\frac{1}{48} \sum_{\sigma \in \mathbb{S}_{4}}(-1)^{|\sigma|} \cdot\left(\lambda_{2 ; 1} \circ_{1} \lambda_{3}-\lambda_{3 ; 1} \circ_{1} \lambda_{2}+\lambda_{3 ; 2} \circ_{2} \lambda_{2}\right. \\
& \left.\quad-\lambda_{2 ; 1} \circ_{2} \lambda_{3}-\lambda_{3 ; 1} \circ_{2} \lambda_{2}+\lambda_{3 ; 2} \circ_{3} \lambda_{2}\right)^{\sigma} .
\end{aligned}
$$

Note that for weak Lie 2-algebras, $\lambda_{2 ; 1}$ is symmetric and we recover Roytenberg's skewsymmetrization construction in this case.

3.3. Skewsymmetrization for morphisms of weak Lie 3-algebras. A weak morphism $f:(L, \mathrm{~d}, \lambda) \rightsquigarrow\left(L^{\prime}, \mathrm{d}^{\prime}, \lambda^{\prime}\right)$ of weak Lie 3-algebras was defined in Section 2.3 as a morphism $f: \mathrm{Lie}_{3}^{\diamond} \rightarrow \operatorname{End}_{L^{\prime}}^{L}$ satisfying a certain Maurer-Cartan type Equation (133) Such a morphism in general does not correspond to a morphism $\Omega \mathrm{Lie}_{3}^{\diamond} \rightarrow$ End $_{L^{\prime}}^{L}$ and there is no obvious way to precompose $f$ with $\Phi$. Below we give an ad hoc construction for a skewsymmetrization of morphisms instead.

Lemma 3.3.1. Let $f:(L, \mathrm{~d}, \lambda) \rightsquigarrow\left(L^{\prime}, \mathrm{d}^{\prime}, \lambda^{\prime}\right)$ be a weak morphism of weak Lie 3-algebras. The following components define a homotopy morphism $\bar{f}:(L, \mathrm{~d}, \bar{\lambda}) \rightsquigarrow\left(L^{\prime}, \mathrm{d}^{\prime}, \bar{\lambda}^{\prime}\right)$ of (semistrict) Lie 3-algebras:

(154) $\bar{f}_{1}:=f_{1}$,

(155) $\bar{f}_{2}:=\frac{1}{2} \sum_{\sigma \in \mathbb{S}_{2}}(-1)^{|\sigma|} \cdot f_{2}^{\sigma}$,

$$
\begin{aligned}
\bar{f}_{3}:= & \frac{1}{6} \sum_{\sigma \in \mathbb{S}_{3}}(-1)^{|\sigma|} \cdot f_{3}^{\sigma} \\
& -\frac{1}{24} \sum_{\sigma \in \mathbb{S}_{3}}(-1)^{|\sigma|} \cdot\left(f_{2 ; 1} \circ{ }_{1} \lambda_{2}+f_{2 ; 1} \circ_{2} \lambda_{2}-\lambda_{2 ; 1}^{\prime} \circ\left(f_{2}, f_{1}\right)-\lambda_{2 ; 1}^{\prime} \circ\left(f_{1}, f_{2}\right)\right)^{\sigma} .
\end{aligned}
$$

We call $\bar{f}$ the skewsymmetrization of $f$. 
The proof is a direct verification of Equations (115) (118) Since it is quite tedious and long, we postpone it to Section A.3

In low degrees, skewsymmetrization of weak morphisms commutes with their composition,

$$
\begin{aligned}
\left(\overline{f^{\prime} \circ f}\right)_{1} & =\left(f^{\prime} \circ f\right)_{1}=f_{1}^{\prime} \circ f_{1}={\overline{f^{\prime}}}_{1} \circ \bar{f}_{1}=\left(\overline{f^{\prime}} \circ \bar{f}\right)_{1}, \\
\left(\overline{f^{\prime} \circ f}\right)_{2} & =\frac{1}{2}\left(\left(f^{\prime} \circ f\right)_{2}-\left(f^{\prime} \circ f\right)_{2}^{(12)}\right) \\
& =\frac{1}{2}\left(f_{2}^{\prime} \circ\left(f_{1}, f_{1}\right)+f_{1}^{\prime} \circ f_{2}-f_{2}^{\prime} \circ\left(f_{1}, f_{1}\right)^{(12)}-f_{1}^{\prime} \circ f_{2}^{(12)}\right) \\
& ={\overline{f^{\prime}}}_{2} \circ\left(\bar{f}_{1}, \bar{f}_{1}\right)+{\overline{f^{\prime}}}_{1} \circ \bar{f}_{2} \\
& =\left(\overline{f^{\prime}} \circ \bar{f}\right)_{2} .
\end{aligned}
$$

In particular, this implies that skewsymmetrization of weak Lie 2-algebras forms a functor 63. Theorem 3.2]. However, for weak Lie 3-algebras this is no longer the case as the following computation shows:

$$
\begin{aligned}
& \left(\overline{f^{\prime} \circ f}-\overline{f^{\prime}} \circ \bar{f}\right)_{3} \\
& =\frac{1}{6} \sum_{\sigma \in \mathbb{S}_{3}}(-1)^{|\sigma|} \cdot\left(f^{\prime} \circ f\right)_{3}^{\sigma} \\
& \begin{array}{r}
-\frac{1}{24} \sum_{\sigma \in \mathbb{S}_{3}}(-1)^{|\sigma|} \cdot\left(\left(f^{\prime} \circ f\right)_{2 ; 1} \circ{ }_{1} \lambda_{2}+\left(f^{\prime} \circ f\right)_{2 ; 1} \circ_{2} \lambda_{2}\right. \\
-\lambda_{2 ; 1}^{\prime \prime} \circ\left(\left(f^{\prime} \circ f\right)_{2},\left(f^{\prime} \circ f\right)_{1}\right)
\end{array} \\
& \left.-\lambda_{2 ; 1}^{\prime \prime} \circ\left(\left(f^{\prime} \circ f\right)_{1},\left(f^{\prime} \circ f\right)_{2}\right)\right)^{\sigma} \\
& -\left(\bar{f}_{3} \circ\left(\bar{f}_{1}, \bar{f}_{1}, \bar{f}_{1}\right)-{\overline{f^{\prime}}}_{2} \circ\left(\bar{f}_{2}, \bar{f}_{1}\right)+{\overline{f^{\prime}}}_{2} \circ\left(\bar{f}_{1}, \bar{f}_{2}\right)-{\overline{f^{\prime}}}_{2} \circ\left(\bar{f}_{1}, \bar{f}_{2}\right)^{(12)}\right. \\
& \left.+\bar{f}_{1} \circ \bar{f}_{3}\right)
\end{aligned}
$$




$$
\begin{gathered}
=\frac{1}{6} \sum_{\sigma \in \mathbb{S}_{3}}(-1)^{|\sigma|} \cdot\left(f_{3}^{\prime} \circ\left(f_{1}, f_{1}, f_{1}\right)-f_{2}^{\prime} \circ\left(f_{2}, f_{1}\right)+f_{2}^{\prime} \circ\left(f_{1}, f_{2}\right)-f_{2}^{\prime} \circ\left(f_{1}, f_{2}\right)^{(12)}\right. \\
+\frac{1}{24} \sum_{\sigma \in \mathbb{S}_{3}}(-1)^{|\sigma|} \cdot\left(\left(f_{2 ; 1}^{\prime} \circ\left(f_{1}, f_{1}\right)+f_{1}^{\prime} \circ f_{2 ; 1}\right) \circ \lambda_{2}\right. \\
+\left(f_{2 ; 1}^{\prime} \circ\left(f_{1}, f_{1}\right)+f_{1}^{\prime} \circ f_{2 ; 1}\right) \circ \lambda_{2} \\
-\lambda_{2 ; 1}^{\prime \prime} \circ\left(f_{2}^{\prime} \circ\left(f_{1}, f_{1}\right)+f_{1}^{\prime} \circ f_{2}, f_{1}^{\prime} \circ f_{1}\right) \\
\left.-\lambda_{2 ; 1}^{\prime \prime} \circ\left(f_{1}^{\prime} \circ f_{1}, f_{2}^{\prime} \circ\left(f_{1}, f_{1}\right)+f_{1}^{\prime} \circ f_{2}\right)\right)^{\sigma}
\end{gathered}
$$

Note that the defect of functoriality is a coboundary. We say that skewsymmetrization is functorial up to homotopy.

\section{Applications}

In this section, we give two examples of applications of the theory developed in the earlier sections. The first is an extension of a result of Rogers [62] on algebraic structures on $n$-plectic manifolds. The second is a construction of a weak Lie 3-algebra associated to a CLWX 2-algebroid, whose skewsymmetrization is precisely the Lie 3algebra introduced by Liu-Sheng [44. Theorem 3.10]. We thereby give an alternative proof for the theorem cited. 
4.1. Higher symplectic geometry. In 3,62 the concept of $n$-plectic manifolds is introduced. We recall some of the basic definitions here. We then consider the case of a 3-plectic manifold and compare two associated algebraic structures, an $\mathrm{L}_{\infty}$-algebra and a dg Leibniz algebra with a certain hidden skewsymmetry. Both structures are examples of weak Lie 3-algebras and turn out to be isomorphic as such. The analogous result for 2-plectic manifolds was shown by Rogers in [62 Appendix A].

Definition 4.1.1. An $n$-plectic manifold $(M, \omega)$ is a smooth manifold $M$ with a closed, nondegenerate $(n+1)$-form $\omega \in \Omega^{n+1}(M)$, i.e., $\mathrm{d} \omega=0$ and $\iota(v) \omega=0$ implies $v=0$. An $(n-1)$-form $\alpha \in \Omega^{n-1}(M)$ is called Hamiltonian, if there exists a vector field $v_{\alpha} \in \mathfrak{X}(M)$ such that $\mathrm{d} \alpha=-l\left(v_{\alpha}\right) \omega$. In order to simplify notation, we let $v_{\alpha}=0$ whenever $\alpha$ is not Hamiltonian, in particular when $\alpha$ is not an $(n-1)$-form.

Let $(M, \omega)$ be an $n$-plectic manifold. Two algebraic structures are introduced on the chain complex

$$
L_{i}:= \begin{cases}\Omega_{\mathrm{Ham}}^{n-1}(M), & \text { for } i=0, \\ \Omega^{n-1-i}(M), & \text { for } 0<i \leq n-1,\end{cases}
$$

with differential the usual de Rham differential. Note that this is welldefined since closed forms are always Hamiltonian. The first structure we introduce is that of an $n$-term $\mathrm{L}_{\infty}$-algebra or (semistrict) Lie $n$-algebra $\mathrm{L}_{\infty}(M, \omega)$ given by the brackets

$$
\lambda_{k}\left(\alpha_{1}, \ldots, \alpha_{k}\right):= \pm l\left(v_{\alpha_{1}}, \ldots, v_{\alpha_{k}}\right) \omega
$$

We will provide the sign in low degrees in the proof of Proposition 4.1.2 For more details, including the general definition of the sign, we refer the reader to loc. cit. The second structure on the complex of Equation (157) is a dg Leibniz algebra given by

$$
\lambda_{2}^{\prime}(\alpha, \beta):=\mathcal{L}\left(v_{\alpha}\right) \beta
$$

and denoted by $\operatorname{Leib}(M, \omega)$. This dg Leibniz algebra actually satisfies a certain symmetry up to homotopy, and in the case $n=2$ is shown to be a weak Lie 2 -algebra with alternator bracket

$$
\lambda_{2 ; 1}^{\prime}(\alpha, \beta):=\iota\left(v_{\alpha}\right) \beta+\iota\left(v_{\beta}\right) \alpha .
$$

This result can be extended to the case $n=3$, i.e., the same binary bracket and alternator form a weak Lie 3-algebra on the underlying 3-term chain complex in that case.

Proposition 4.1.2. Let $(M, \omega)$ be a 3-plectic manifold. Then $\mathrm{L}_{\infty}(M, \omega)$ and $\operatorname{Leib}(M, \omega)$ are isomorphic as weak Lie 3-algebras.

Proof. For a 3-plectic manifold $(M, \omega)$, the chain complex underlying $\mathrm{L}_{\infty}(M, \omega)$ and $\operatorname{Leib}(M, \omega)$ is

$$
\left(L, \mathrm{~d}^{L}\right):=\left(\Omega_{\mathrm{Ham}}^{2}(M) \stackrel{\mathrm{d}^{L}}{\longleftarrow} \Omega^{1}(M) \stackrel{\mathrm{d}^{L}}{\longleftarrow} \Omega^{0}(M)=C^{\infty}(M)\right) .
$$


Note that for a Hamiltonian form $\alpha, \mathrm{d} \alpha$ need not vanish, while $\mathrm{d}^{L} \alpha=0$ for degree reasons. The structure maps for $\mathrm{L}_{\infty}(M, \omega)$ are explicitly given by

$$
\begin{aligned}
& \lambda_{2}(\alpha, \beta) \quad:=\iota\left(v_{\alpha}, v_{\beta}\right) \omega, \\
& \lambda_{3}(\alpha, \beta, \gamma):=-l\left(v_{\alpha}, v_{\beta}, v_{\gamma}\right) \omega, \\
& \lambda_{4}(\alpha, \beta, \gamma, \eta):=-l\left(v_{\alpha}, v_{\beta}, v_{\gamma}, v_{\eta}\right) \omega .
\end{aligned}
$$

We define a weak morphism $f: \mathrm{L}_{\infty}(M, \omega) \rightsquigarrow \operatorname{Leib}(M, \omega)$ of weak Lie 3-algebras by

$$
\begin{array}{ll}
f_{1}(\alpha)=\alpha, \quad f_{2}(\alpha, \beta)=-l\left(v_{\alpha}\right) \beta, & f_{2 ; 1}(\alpha, \beta)=0, \\
f_{3}(\alpha, \beta, \gamma) & =-l\left(v_{\alpha}, v_{\beta}\right) \gamma .
\end{array}
$$

To show that these maps define a weak morphism of weak Lie 3-algebras, we begin by verifying that they indeed satisfy Equations (115) (118), i.e., define a homotopy morphism of Leibniz 3-algebras.

Clearly $\partial f_{1}=0$ and so Equation (115) holds. When $|\alpha|>0$, Equation (116) is trivially satisfied. In case $|\alpha|=|\beta|=0$ we obtain

$$
\begin{aligned}
\left(\partial f_{2}-f_{1} \circ \lambda_{2}+\lambda_{2}^{\prime} \circ\left(f_{1}, f_{1}\right)\right)(\alpha, \beta) & =\mathrm{d}^{L} f_{2}(\alpha, \beta)-\lambda_{2}(\alpha, \beta)+\lambda_{2}^{\prime}(\alpha, \beta) \\
& =\mathrm{d}^{L}\left(-\iota\left(v_{\alpha}\right) \beta\right)-\iota\left(v_{\alpha}, v_{\beta}\right) \omega+\mathcal{L}\left(v_{\alpha}\right) \beta,
\end{aligned}
$$

which, using Cartan's formula and the fact that $\beta$ is Hamiltonian, can be written as

$$
=-\mathrm{d} \iota\left(v_{\alpha}\right) \beta-\iota\left(v_{\alpha}\right) \mathrm{d} \beta+\mathrm{d} \iota\left(v_{\alpha}\right) \beta+\iota\left(v_{\alpha}\right) \mathrm{d} \beta=0,
$$

while for $|\alpha|=0,|\beta|>0$ we have

$$
\begin{aligned}
\left(\partial f_{2}-f_{1} \circ \lambda_{2}+\lambda_{2}^{\prime} \circ\left(f_{1}, f_{1}\right)\right)(\alpha, \beta) & =\mathrm{d}^{L} f_{2}(\alpha, \beta)+f_{2}\left(\alpha, \mathrm{d}^{L} \beta\right)-\lambda_{2}(\alpha, \beta)+\lambda_{2}^{\prime}(\alpha, \beta) \\
& =\mathrm{d}^{L}\left(-\iota\left(v_{\alpha}\right) \beta\right)-\iota\left(v_{\alpha}\right) \mathrm{d}^{L} \beta-\iota\left(v_{\alpha}, v_{\beta}\right) \omega+\mathcal{L}\left(v_{\alpha}\right) \beta,
\end{aligned}
$$

which, using that $\beta$ is not Hamiltonian, reduces to

$$
=-\mathrm{d} \iota\left(v_{\alpha}\right) \beta-\iota\left(v_{\alpha}\right) \mathrm{d} \beta+\mathcal{L}\left(v_{\alpha}\right) \beta=0 .
$$

Evaluating Equation (117) for $|\alpha|=|\beta|=0$, we obtain—omitting $f_{1}=$ id for brevity from now on

$$
\begin{aligned}
\left(\partial\left(f_{3}\right)-\right. & \lambda_{3}+f_{2} \circ_{2} \lambda_{2}-f_{2} \circ_{1} \lambda_{2}-\left(f_{2} \circ_{2} \lambda_{2}\right)^{(12)}+\lambda_{2}^{\prime} \circ_{2} f_{2}-\lambda_{2}^{\prime} \circ_{1} f_{2} \\
- & \left.-\lambda_{2}^{\prime} \circ_{2} f_{2}^{(12)}\right)(\alpha, \beta, \gamma) \\
= & \mathrm{d}^{L} f_{3}(\alpha, \beta, \gamma)-f_{3}\left(\alpha, \beta, \mathrm{d}^{L} \gamma\right)-\lambda_{3}(\alpha, \beta, \gamma)+f_{2}\left(\alpha, \lambda_{2}(\beta, \gamma)\right)-f_{2}\left(\lambda_{2}(\alpha, \beta), \gamma\right) \\
& -f_{2}\left(\beta, \lambda_{2}(\alpha, \gamma)\right)+\lambda_{2}^{\prime}\left(\alpha, f_{2}(\beta, \gamma)\right)-\lambda_{2}^{\prime}\left(f_{2}(\alpha, \beta), \gamma\right)-\lambda_{2}^{\prime}\left(\beta, f_{2}(\alpha, \gamma)\right) \\
= & \mathrm{d}^{L}\left(-\iota\left(v_{\alpha}, v_{\beta}\right) \gamma\right)+\iota\left(v_{\alpha}, v_{\beta}\right) \mathrm{d}^{L} \gamma+\iota\left(v_{\alpha}, v_{\beta}, v_{\gamma}\right) \omega-\iota\left(v_{\alpha}\right) \iota\left(v_{\beta}, v_{\gamma}\right) \omega+\iota\left(v_{\lambda_{2}(\alpha, \beta)}\right) \gamma \\
& +\iota\left(v_{\beta}\right) \iota\left(v_{\alpha}, v_{\gamma}\right) \omega-\mathcal{L}\left(v_{\alpha}\right) \iota\left(v_{\beta}\right) \gamma+\mathcal{L}\left(v_{\beta}\right) \iota\left(v_{\alpha}\right) \gamma,
\end{aligned}
$$

which, using the identities $v_{\lambda_{2}(\alpha, \beta)}=\left[v_{\alpha}, v_{\beta}\right]$ and $\iota\left(\left[v_{\alpha}, v_{\beta}\right]\right) \omega=\left[\mathcal{L}\left(v_{\alpha}\right), \iota\left(v_{\beta}\right)\right] \omega$, becomes

$$
=-\mathrm{d} \iota\left(v_{\alpha}, v_{\beta}\right) \gamma+\iota\left(v_{\alpha}, v_{\beta}\right) \mathrm{d}^{L} \gamma-\iota\left(v_{\alpha}, v_{\beta}, v_{\gamma}\right) \omega-\iota\left(v_{\beta}\right) \mathcal{L}\left(v_{\alpha}\right) \gamma+\mathcal{L}\left(v_{\beta}\right) \iota\left(v_{\alpha}\right) \gamma .
$$


Now consider two cases: when $|\gamma|=0, \mathrm{~d}^{L} \gamma=0$ we have

$$
=-\mathrm{d} \iota\left(v_{\alpha}, v_{\beta}\right) \gamma-\iota\left(v_{\alpha}, v_{\beta}, v_{\gamma}\right) \omega-\iota\left(v_{\alpha}, v_{\beta}\right) \mathrm{d} \gamma+\mathrm{d} \iota\left(v_{\alpha}, v_{\beta}\right) \gamma=0,
$$

while for $|\gamma|>0, \iota\left(v_{\alpha}, v_{\beta}\right) \gamma=0$ and $v_{\gamma}=0$, we obtain

$$
=\iota\left(v_{\alpha}, v_{\beta}\right) \mathrm{d} \gamma-\iota\left(v_{\alpha}, v_{\beta}\right) \mathrm{d} \gamma=0 .
$$

To verify Equation (118), it is sufficient to consider the case $|\alpha|=|\beta|=|\gamma|=|\eta|=0$. Leaving out the terms vanishing for degree reasons, we obtain

$$
\begin{aligned}
\left(\lambda_{4}-f_{2} \circ_{2} \lambda_{3}+\left(f_{2} \circ_{2} \lambda_{3}\right)^{(12)}-\left(f_{2} \circ_{2} \lambda_{3}\right)^{(123)}+f_{3} \circ_{1} \lambda_{2}-f_{3} \circ_{2} \lambda_{2}\right. \\
+\left(f_{3} \circ_{2} \lambda_{2}\right)^{(12)}+f_{3} \circ_{3} \lambda_{2}-\left(f_{3} \circ_{3} \lambda_{2}\right)^{(23)}+\left(f_{3} \circ_{3} \lambda_{2}\right)^{(132)} \\
\left.\quad-\lambda_{2}^{\prime} \circ_{2} f_{3}+\left(\lambda_{2}^{\prime} \circ_{2} f_{3}\right)^{(12)}-\left(\lambda_{2}^{\prime} \circ_{2} f_{3}\right)^{(123)}\right)(\alpha, \beta, \gamma, \eta) \\
=\lambda_{4}(\alpha, \beta, \gamma, \eta)-f_{2}\left(\alpha, \lambda_{3}(\beta, \gamma, \eta)\right)+f_{2}\left(\beta, \lambda_{3}(\alpha, \gamma, \eta)\right)-f_{2}\left(\gamma, \lambda_{3}(\alpha, \beta, \eta)\right) \\
+f_{3}\left(\lambda_{2}(\alpha, \beta), \gamma, \eta\right)-f_{3}\left(\alpha, \lambda_{2}(\beta, \gamma), \eta\right)+f_{3}\left(\beta, \lambda_{2}(\alpha, \gamma), \eta\right)+f_{3}\left(\alpha, \beta, \lambda_{2}(\gamma, \eta)\right) \\
-f_{3}\left(\alpha, \gamma, \lambda_{2}(\beta, \eta)\right)+f_{3}\left(\beta, \gamma, \lambda_{2}(\alpha, \eta)\right)-\lambda_{2}^{\prime}\left(\alpha, f_{3}(\beta, \gamma, \eta)\right)+\lambda_{2}^{\prime}\left(\beta, f_{3}(\alpha, \gamma, \eta)\right) \\
-\lambda_{2}^{\prime}\left(\gamma, f_{3}(\alpha, \beta, \eta)\right) .
\end{aligned}
$$

Note that the terms $f_{2} \circ_{2} \lambda_{3}$ cancel with $f_{3} \circ_{3} \lambda_{2}$, leaving us with

$$
\begin{aligned}
= & -\iota\left(v_{\alpha}, v_{\beta}, v_{\gamma}, v_{\eta}\right) \omega-\iota\left(v_{\lambda_{2}(\alpha, \beta)}, v_{\gamma}\right) \eta+\iota\left(v_{\alpha}, v_{\lambda_{2}(\beta, \gamma)}\right) \eta-\iota\left(v_{\beta}, v_{\lambda_{2}(\alpha, \gamma)}\right) \eta \\
& +\mathcal{L}\left(v_{\alpha}\right) \iota\left(v_{\beta}, v_{\gamma}\right) \eta-\mathcal{L}\left(v_{\beta}\right) \iota\left(v_{\alpha}, v_{\gamma}\right) \eta+\mathcal{L}\left(v_{\gamma}\right) \iota\left(v_{\alpha}, v_{\beta}\right) \eta, \\
= & -\iota\left(v_{\alpha}, v_{\beta}, v_{\gamma}, v_{\eta}\right) \omega+\iota\left(v_{\gamma}\right) \iota\left(v_{\beta}\right) \mathcal{L}\left(v_{\alpha}\right) \eta-\iota\left(v_{\gamma}\right) \mathcal{L}\left(v_{\beta}\right) \iota\left(v_{\alpha}\right) \eta+\mathcal{L}\left(v_{\gamma}\right) \iota\left(v_{\alpha}, v_{\beta}\right) \eta \\
= & -\iota\left(v_{\alpha}, v_{\beta}, v_{\gamma}, v_{\eta}\right) \omega+\iota\left(v_{\gamma}\right) \iota\left(v_{\beta}\right) \iota\left(v_{\alpha}\right) \mathrm{d} \eta+\mathrm{d} \iota\left(v_{\gamma}\right) \iota\left(v_{\alpha}, v_{\beta}\right) \eta=0 .
\end{aligned}
$$

We proceed to show that $f$ additionally satisfies Equations (134)-(137) and hence defines a weak morphism of weak Lie 3-algebras. For Equation (134), we find

$$
\left(f_{2}+f_{2}^{(12)}+\lambda_{2 ; 1}^{\prime}\right)(\alpha, \beta)=-\imath\left(v_{\alpha}\right) \beta-\iota\left(v_{\beta}\right) \alpha+\iota\left(v_{\alpha}\right) \beta+\iota\left(v_{\beta}\right) \alpha=0 .
$$

Since all involved maps vanish, Equation (135) is trivially satisfied. Finally we consider Equation (136)

$$
\left(f_{3}+f_{3}^{(12)}\right)(\alpha, \beta, \gamma)=-\imath\left(v_{\alpha}, v_{\beta}\right) \gamma-\iota\left(v_{\beta}, v_{\alpha}\right) \gamma=0,
$$

and Equation (137).

$$
\begin{aligned}
\left(f_{3}+\right. & \left.f_{3}^{(23)}-\lambda_{2 ; 1}^{\prime} \circ_{1} f_{2}-\left(\lambda_{2 ; 1}^{\prime} \circ_{2} f_{2}\right)^{(12)}\right)(\alpha, \beta, \gamma) \\
& =-\iota\left(v_{\alpha}, v_{\beta}\right) \gamma-\iota\left(v_{\alpha}, v_{\gamma}\right) \beta+\iota\left(v_{\gamma}\right) \iota\left(v_{\alpha}\right) \beta+\iota\left(v_{\beta}\right) \iota\left(v_{\alpha}\right) \gamma=0 .
\end{aligned}
$$

This concludes the proof.

4.2. Higher Courant algebroids. In [44], Liu and Sheng introduce the notion of a CLWX 2-algebroid as a higher analogue of a Courant algebroid. A Lie 3-algebra is associated to any CLWX 2-algebroid in [44. Theorem 3.10]. In this section, we give a construction of a weak Lie 3-algebra for any CLWX 2-algebroid. We then show that the 
Lie 3-algebra constructed in loc. cit. is in fact the skewsymmetrization of our weak Lie 3-algebra.

Definition 4.2.1. Let $E=\left(E_{0} \stackrel{\partial}{\leftarrow} E_{1}\right)$ be a 2-term dg vector bundle over $M$ equipped with a morphism $\rho: E \rightarrow T M$ of dg vector bundles, a (graded) bilinear map $\circ: \Gamma E \otimes \Gamma E \rightarrow$ $\Gamma E$ which is skewsymmetric on $\Gamma E_{0} \otimes \Gamma E_{0}$, a (graded) 3-form $\Omega: \Gamma E \otimes \Gamma E \otimes \Gamma E \rightarrow \Gamma E[1]$, and a nondegenerate symmetric bilinear form $S: \Gamma E \otimes \Gamma E \rightarrow C^{\infty}(M)$. Using these data, we define a map $\mathcal{D}: C^{\infty}(M) \rightarrow \Gamma E_{1}$ by

$$
S(e, \mathcal{D} f)=\rho(e)(f), \quad \text { for all } e \in \Gamma E .
$$

We call $\mathcal{E}=\left(E_{0} \stackrel{\partial}{\leftarrow} E_{1}, \rho, \circ, \Omega, S\right)$ a $C L W X 2$-algebroid, if the following conditions are satisfied:

(i) $E_{0}, E_{1}$ are isotropic, i.e., $S\left(E_{i}, E_{i}\right)=0$ for $i=0,1$,

(ii) $\left(\Gamma E_{0} \stackrel{\partial}{\leftarrow} \Gamma E_{1}, \circ, \Omega\right)$ is a Leibniz 2-algebra,

(iii) $e \circ e=\frac{1}{2} \mathcal{D} S(e, e)$ for all $e \in \Gamma E$,

(iv) $S\left(\partial e_{1}, e_{2}\right)=S\left(e_{1}, \partial e_{2}\right)$ for all $e_{i} \in \Gamma E$,

(v) $\rho\left(e_{1}\right) S\left(e_{2}, e_{3}\right)=S\left(e_{1} \circ e_{2}, e_{3}\right)+S\left(e_{2}, e_{1} \circ e_{3}\right)$ for all $e_{i} \in \Gamma E$, and

(vi) $S\left(\Omega\left(e_{1}, e_{2}, e_{3}\right), e_{4}\right)=-S\left(e_{3}, \Omega\left(e_{1}, e_{2}, e_{4}\right)\right)$ for all $e_{i} \in \Gamma E$.

Proposition 4.2.2. For any CLWX 2-algebroid, there is an associated complex

$$
(L, \mathrm{~d}):=\left(\Gamma E_{0} \stackrel{\partial}{\longleftarrow} \Gamma E_{1} \stackrel{\mathcal{D}}{\longleftarrow} C^{\infty}(M)\right),
$$

which, equipped with structure maps

$$
\begin{array}{rlrl}
\lambda_{2}=(-\circ-)+S \circ_{2} \mathcal{D}, & \lambda_{2 ; 1}=S, & \lambda_{2 ; 1,1}=0, \\
\lambda_{3}=\Omega, & \lambda_{3 ; 1}=0, \\
& \lambda_{3 ; 2}=0, \\
& \lambda_{4}=0,
\end{array}
$$

defines a weak Lie 3-algebra.

Proof. The fact that $(L, \mathrm{~d})$ is indeed a complex is equivalent to $\partial \mathcal{D}=0$, which is shown in [44. Lemma 3.6].

We begin by showing that $(L, \mathrm{~d}, \lambda)$ is a Leibniz 3-algebra, i.e., by checking Equations (110)-(113). Since, by Condition (ii), we already know that the 2-term truncation of $L$ forms a Leibniz 2-algebra, it is sufficient to verify that these equations hold on tuples containing at least one degree 2 element. To keep notation as simple as possible, we denote arbitrary sections of $E$ by $e \in \Gamma E$ and indicate degree using superscript, i.e., $e^{i} \in \Gamma E_{i}$, and use $f$ for functions $f \in L_{2}=C^{\infty}(M)$. We use subscripts to distinguish multiple elements of the same degree, e.g., $e_{1}^{0}$ and $e_{2}^{0}$.

Evaluating Equation (110), we obtain

$$
\partial\left(\lambda_{2}\right)\left(e^{0}, f\right)=\mathrm{d}\left(\lambda_{2}\left(e^{0}, f\right)\right)-\lambda_{2}\left(e^{0}, \mathcal{D} f\right)=\mathcal{D} S\left(e^{0}, \mathcal{D} f\right)-e^{0} \circ \mathcal{D} f=0,
$$


and

$$
\partial\left(\lambda_{2}\right)\left(f, e^{0}\right)=-\lambda_{2}\left(\mathcal{D} f, e^{0}\right)=-\mathcal{D} f \circ e^{0}=0,
$$

which are both shown to hold in [44. Lemma 3.6]. In addition we have

$$
\partial\left(\lambda_{2}\right)\left(e^{1}, f\right)=-\lambda_{2}\left(\partial e^{1}, f\right)=-S\left(\partial e^{0}, \mathcal{D} f\right)=-S\left(e^{0}, \partial \mathcal{D} f\right)=0
$$

which holds since $\partial \mathcal{D}=0$, as we have seen above. In the remaining cases, i.e., for $\left(f, e^{1}\right)$ and $\left(f_{1}, f_{2}\right)$, the equation holds trivially since all structure maps vanish. For Equation (111) we obtain

$$
\begin{aligned}
& \left(\partial \lambda_{3}-\lambda_{2} \circ_{2} \lambda_{2}+\lambda_{2} \circ_{1} \lambda_{2}+\left(\lambda_{2} \circ_{2} \lambda_{2}\right)^{(12)}\right)\left(e_{1}^{0}, e_{2}^{0}, f\right) \\
& \quad=-\lambda_{2}\left(e_{1}^{0}, \lambda_{2}\left(e_{2}^{0}, f\right)\right)+\lambda_{2}\left(\lambda_{2}\left(e_{1}^{0}, e_{2}^{0}\right), f\right)+\lambda_{2}\left(e_{2}^{0}, \lambda_{2}\left(e_{1}^{0}, f\right)\right) \\
& \quad=-S\left(e_{1}^{0}, \mathcal{D} S\left(e_{2}^{0}, \mathcal{D} f\right)\right)+S\left(e_{1}^{0} \circ e_{2}^{0}, \mathcal{D} f\right)+S\left(e_{2}^{0}, \mathcal{D} S\left(e_{1}^{0}, \mathcal{D} f\right)\right),
\end{aligned}
$$

which, by Equation (162). can be written as

$$
=-S\left(e_{1}^{0}, \mathcal{D} S\left(e_{2}^{0}, \mathcal{D} f\right)\right)+S\left(e_{1}^{0} \circ e_{2}^{0}, \mathcal{D} f\right)+S\left(e_{2}^{0}, e_{1}^{0} \circ \mathcal{D} f\right),
$$

and using Condition (v) becomes

$$
=-S\left(e_{1}^{0}, \mathcal{D} S\left(e_{2}^{0}, \mathcal{D} f\right)\right)+\rho\left(e_{1}^{0}\right) S\left(e_{2}^{0}, \mathcal{D} f\right)=0,
$$

where the last equality holds by definition of $\mathcal{D}$. Since Equation (112) is of degree 1, it holds for degree reasons when evaluated on any tuple containing a degree 2 element. Similarly, Equation (113) is always satisfied for degree reasons. This completes the proof that $L$ is a Leibniz 3-algebra.

We proceed to show that $L$ is in fact a weak Lie 3-algebra. Consider Equation (122) By isotropy of $E_{0}$ and skewsymmetry of ${ }_{\iota^{\circ}}{ }_{-}$on $L_{0} \otimes L_{0}$ we see that

$$
\left(\partial\left(\lambda_{2 ; 1}\right)-\lambda_{2}-\lambda_{2}^{(12)}\right)\left(e_{1}^{0}, e_{2}^{0}\right)=\partial S\left(e_{1}^{0}, e_{2}^{0}\right)-e_{1}^{0} \circ e_{2}^{0}-e_{2}^{0} \circ e_{1}^{0}=0 .
$$

In addition we find

$$
\begin{aligned}
\left(\partial\left(\lambda_{2 ; 1}\right)-\lambda_{2}-\lambda_{2}^{(12)}\right)\left(e^{0}, e^{1}\right) & =\mathcal{D} S\left(e^{0}, e^{1}\right)-e^{0} \circ e^{1}-e^{1} \circ e^{0} \\
& =\frac{1}{2} \mathcal{D} S\left(e^{0}+e^{1}, e^{0}+e^{1}\right)-\left(e^{0}+e^{1}\right) \circ\left(e^{0}+e^{1}\right)=0,
\end{aligned}
$$

which holds using Condition (iii) and

$$
\left(\partial\left(\lambda_{2 ; 1}\right)-\lambda_{2}-\lambda_{2}^{(12)}\right)\left(e^{1}, e^{1}\right)=S\left(\partial e_{1}^{1}, e_{2}^{1}\right)-S\left(e_{1}^{1}, \partial e_{2}^{1}\right)=0,
$$

which holds by Condition (iv) In the remaining cases the equation is trivially satisfied, e.g.,

$$
\left(\partial\left(\lambda_{2 ; 1}\right)-\lambda_{2}-\lambda_{2}^{(12)}\right)\left(e^{0}, f\right)=S\left(e^{0}, \mathcal{D} f\right)-S\left(e^{0}, \mathcal{D} f\right)=0 .
$$

Next, we consider Equation (123). Since $\lambda_{2 ; 1,1}=0$, the only nontrivial case is

$$
\left(\partial\left(\lambda_{2 ; 1,1}\right)+\lambda_{2 ; 1}-\lambda_{2 ; 1}^{(12)}\right)\left(e^{0}, e^{1}\right)=S\left(e^{0}, e^{1}\right)-S\left(e^{1}, e^{0}\right)=0,
$$


which holds by symmetry of $S$. For Equations (124) and (125) we obtain

$$
\left(\partial\left(\lambda_{3 ; 1}\right)-\lambda_{3}-\lambda_{3}^{(12)}-\lambda_{2} \circ_{1} \lambda_{2 ; 1}\right)\left(e_{1}^{0}, e_{2}^{0}, e_{3}^{0}\right)=-\Omega\left(e_{1}^{0}, e_{2}^{0}, e_{3}^{0}\right)-\Omega\left(e_{2}^{0}, e_{1}^{0}, e_{3}^{0}\right)=0,
$$

which holds by skewsymmetry of $\Omega$, and

$$
\begin{aligned}
& \left(\partial\left(\lambda_{3 ; 2}\right)-\lambda_{3}-\lambda_{3}^{(23)}+\lambda_{2} \circ_{2} \lambda_{2 ; 1}-\lambda_{2 ; 1} \circ_{1} \lambda_{2}-\left(\lambda_{2 ; 1} \circ_{2} \lambda_{2}\right)^{(12)}\right)\left(e_{1}^{0}, e_{2}^{0}, e_{3}^{0}\right) \\
& \quad=-\Omega\left(e_{1}^{0}, e_{2}^{0}, e_{3}^{0}\right)-\Omega\left(e_{1}^{0}, e_{3}^{0}, e_{2}^{0}\right)+S\left(e_{1}^{0}, \mathcal{D} S\left(e_{2}^{0}, e_{3}^{0}\right)\right)-S\left(e_{1}^{0} \circ e_{2}^{0}, e_{3}^{0}\right)-S\left(e_{2}^{0}, e_{1}^{0} \circ e_{3}^{0}\right) \\
& \quad=0
\end{aligned}
$$

again from skewsymmetry of $\Omega$ and using Condition (v) Equations (126) (131) hold trivially since each term vanishes for degree reasons or because one of the relevant structure maps is zero. Finally, Equation (132) reduces to

$\left(\lambda_{2 ; 1} \circ_{1} \lambda_{3}+\left(\lambda_{2 ; 1} \circ_{2} \lambda_{3}\right)^{(123)}\right)\left(e_{1}^{0}, e_{2}^{0}, e_{3}^{0}, e_{4}^{0}\right)=S\left(\Omega\left(e_{1}^{0}, e_{2}^{0}, e_{3}^{0}\right), e_{4}^{0}\right)+S\left(e_{3}^{0}, \Omega\left(e_{1}^{0}, e_{2}^{0}, e_{4}^{0}\right)\right)=0$, which is precisely Condition (vi)

Corollary 4.2.3. The skewsymmetrization $\bar{L}$ of $L$ is a Lie 3-algebra. Its structure maps are explicitly given by

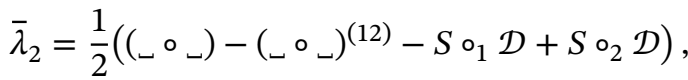

$$
\begin{aligned}
& \bar{\lambda}_{3}=\Omega-\frac{1}{12} \sum_{\sigma \in \mathbb{S}_{3}}(-1)^{|\sigma|} \cdot\left(S \circ_{1}\left(\sim^{\circ} \circ_{-}\right)\right)^{\sigma} \text {, } \\
& \bar{\lambda}_{4}=S \circ_{1} \Omega \text {. }
\end{aligned}
$$

PROOF. Taking into account the skewsymmetry of $\Omega$, the symmetry of $S$, and Condition (vi) it is clear that the given terms are precisely the skewsymmetrized structure maps of Definition 3.2.1.

The structure maps of Corollary 4.2.3 are precisely as given in [44. Theorem 3.10]. Hence, our construction in Proposition 4.2.2 gives an alternative proof of the cited theorem, based on the methods developed in Section 3

\section{A. Computations}

This appendix contains some long and tedious computations that were removed from the main text as to not disturb its flow.

A.1. Proof of Lemma 1.3.1. We verify that $(\Delta \circ$ id $) \Delta=($ id $\circ \Delta) \Delta$ when evaluated on $l_{4 ; 1}, l_{4 ; 2}$, or $l_{4 ; 3}$ by computing both sides of the equation individually:

$$
\begin{aligned}
(\Delta \circ \text { id }) \Delta\left(l_{4 ; 1}\right)= & 1 \circ(1) \circ\left(l_{4 ; 1}\right)-\left(1 \circ\left(l_{2}\right)+l_{2} \circ(1,1)\right) \circ\left(l_{3 ; 1}, 1\right)-\left(1 \circ\left(l_{2}\right)\right. \\
& \left.+l_{2} \circ(1,1)\right) \circ\left(1, l_{3 ; 1}\right)^{(123)}+\left(1 \circ\left(l_{2}\right)+l_{2} \circ(1,1)\right) \circ\left(l_{2 ; 1}, l_{2}\right)+\left(1 \circ\left(l_{3}\right)\right. \\
& \left.-l_{2} \circ\left(l_{2}, 1\right)+l_{2} \circ\left(1, l_{2}\right)-l_{2} \circ\left(1, l_{2}\right)^{(12)}+l_{3} \circ(1,1,1)\right) \circ\left(l_{2 ; 1}, 1,1\right) \\
& +\left(1 \circ\left(l_{3 ; 1}\right)+l_{2} \circ\left(l_{2 ; 1}, 1\right)+l_{3 ; 1} \circ(1,1,1)\right) \circ\left(1,1, l_{2}\right)+\left(1 \circ\left(l_{4 ; 1}\right)\right. \\
& -l_{2} \circ\left(l_{3 ; 1}, 1\right)-l_{2} \circ\left(1, l_{3 ; 1}\right)^{(123)}+l_{2} \circ\left(l_{2 ; 1}, l_{2}\right)+l_{3} \circ\left(l_{2 ; 1}, 1,1\right)
\end{aligned}
$$




$$
\begin{aligned}
& \left.+l_{3 ; 1} \circ\left(1,1, l_{2}\right)+l_{4 ; 1} \circ(1,1,1,1)\right) \circ(1,1,1,1), \\
(\text { id } \circ) \Delta\left(l_{4 ; 1}\right)= & 1 \circ\left(1 \circ\left(l_{4 ; 1}\right)-l_{2} \circ\left(l_{3 ; 1}, 1\right)-l_{2} \circ\left(1, l_{3 ; 1}\right)^{(123)}+l_{2} \circ\left(l_{2 ; 1}, l_{2}\right)\right. \\
& \left.+l_{3} \circ\left(l_{2 ; 1}, 1,1\right)+l_{3 ; 1} \circ\left(1,1, l_{2}\right)+l_{4 ; 1} \circ(1,1,1,1)\right)-l_{2} \circ\left(1 \circ\left(l_{3 ; 1}\right)\right. \\
& \left.+l_{2} \circ\left(l_{2 ; 1}, 1\right)+l_{3 ; 1} \circ(1,1,1), 1 \circ(1)\right)-l_{2} \circ\left(1 \circ(1), 1 \circ\left(l_{3 ; 1}\right)\right. \\
& \left.+l_{2} \circ\left(l_{2 ; 1}, 1\right)+l_{3 ; 1} \circ(1,1,1)\right)^{(123)}+l_{2} \circ\left(1 \circ\left(l_{2 ; 1}\right)+l_{2 ; 1} \circ(1,1), 1 \circ\left(l_{2}\right)\right. \\
& \left.+l_{2} \circ(1,1)\right)+l_{3} \circ\left(1 \circ\left(l_{2 ; 1}\right)+l_{2 ; 1} \circ(1,1), 1 \circ(1), 1 \circ(1)\right) \\
& +l_{3 ; 1} \circ\left(1 \circ(1), 1 \circ(1), 1 \circ\left(l_{2}\right)+l_{2} \circ(1,1)\right)+l_{4 ; 1} \circ(1 \circ(1), 1 \circ(1), \\
& 1 \circ(1), 1 \circ(1)),
\end{aligned}
$$

and see (using the associativity of the composite product $\_{ }_{-}$) that they agree. In the same way we compute

$$
\begin{aligned}
& (\Delta \circ \mathrm{id}) \Delta\left(l_{4 ; 2}\right)=1 \circ(1) \circ\left(l_{4 ; 2}\right)-\left(1 \circ\left(l_{2}\right)+l_{2} \circ(1,1)\right) \circ\left(l_{3 ; 2}, 1\right)-\left(1 \circ\left(l_{2}\right)\right. \\
& \left.+l_{2} \circ(1,1)\right) \circ\left(1, l_{3 ; 1}\right)+\left(1 \circ\left(l_{2}\right)+l_{2} \circ(1,1)\right) \circ\left(l_{2 ; 1}, l_{2}\right)^{(132)}+\left(1 \circ\left(l_{3 ; 1}\right)\right. \\
& \left.+l_{2} \circ\left(l_{2 ; 1}, 1\right)+l_{3 ; 1} \circ(1,1,1)\right) \circ\left(l_{2}, 1,1\right)+\left(1 \circ\left(l_{3 ; 1}\right)+l_{2} \circ\left(l_{2 ; 1}, 1\right)\right. \\
& \left.+l_{3 ; 1} \circ(1,1,1)\right) \circ\left(1, l_{2}, 1\right)^{(12)}-\left(1 \circ\left(l_{3}\right)-l_{2} \circ\left(l_{2}, 1\right)+l_{2} \circ\left(1, l_{2}\right)\right. \\
& \left.-l_{2} \circ\left(1, l_{2}\right)^{(12)}+l_{3} \circ(1,1,1)\right) \circ\left(1, l_{2 ; 1}, 1\right)+\left(1 \circ\left(l_{3 ; 1}\right)+l_{2} \circ\left(l_{2 ; 1}, 1\right)\right. \\
& \left.+l_{3 ; 1} \circ(1,1,1)\right) \circ\left(1,1, l_{2}\right)^{(132)}+\left(1 \circ\left(l_{4 ; 2}\right)-l_{2} \circ\left(l_{3 ; 2}, 1\right)-l_{2} \circ\left(1, l_{3 ; 1}\right)\right. \\
& +l_{2} \circ\left(l_{2 ; 1}, l_{2}\right)^{(132)}+l_{3 ; 1} \circ\left(l_{2}, 1,1\right)+l_{3 ; 1} \circ\left(1, l_{2}, 1\right)^{(12)}-l_{3} \circ\left(1, l_{2 ; 1}, 1\right) \\
& \left.+l_{3 ; 1} \circ\left(1,1, l_{2}\right)^{(132)}+l_{4 ; 2} \circ(1,1,1,1)\right) \circ(1,1,1,1) \text {, } \\
& (\text { id } \circ \Delta) \Delta\left(l_{4 ; 2}\right)=1 \circ\left(1 \circ\left(l_{4 ; 2}\right)-l_{2} \circ\left(l_{3 ; 2}, 1\right)-l_{2} \circ\left(1, l_{3 ; 1}\right)+l_{2} \circ\left(l_{2 ; 1}, l_{2}\right)^{(132)}\right. \\
& +l_{3 ; 1} \circ\left(l_{2}, 1,1\right)+l_{3 ; 1} \circ\left(1, l_{2}, 1\right)^{(12)}-l_{3} \circ\left(1, l_{2 ; 1}, 1\right)+l_{3 ; 1} \circ\left(1,1, l_{2}\right)^{(132)} \\
& \left.+l_{4 ; 2} \circ(1,1,1,1)\right)-l_{2} \circ\left(1 \circ\left(l_{3 ; 2}\right)-l_{2 ; 1} \circ\left(l_{2}, 1\right)-l_{2} \circ\left(1, l_{2 ; 1}\right)\right. \\
& \left.-l_{2 ; 1} \circ\left(1, l_{2}\right)^{(12)}+l_{3 ; 2} \circ(1,1,1), 1 \circ(1)\right)-l_{2} \circ\left(1 \circ(1), 1 \circ\left(l_{3 ; 1}\right)\right. \\
& \left.+l_{2} \circ\left(l_{2 ; 1}, 1\right)+l_{3 ; 1} \circ(1,1,1)\right)+l_{2} \circ\left(1 \circ\left(l_{2 ; 1}\right)+l_{2 ; 1} \circ(1,1), 1 \circ\left(l_{2}\right)\right. \\
& \left.+l_{2} \circ(1,1)\right)^{(132)}+l_{3 ; 1} \circ\left(1 \circ\left(l_{2}\right)+l_{2} \circ(1,1), 1 \circ(1), 1 \circ(1)\right) \\
& +l_{3 ; 1} \circ\left(1 \circ(1), 1 \circ\left(l_{2}\right)+l_{2} \circ(1,1), 1 \circ(1)\right)^{(12)}-l_{3} \circ\left(1 \circ(1), 1 \circ\left(l_{2 ; 1}\right)\right. \\
& \left.+l_{2 ; 1} \circ(1,1), 1 \circ(1)\right)+l_{3 ; 1} \circ\left(1 \circ(1), 1 \circ(1), 1 \circ\left(l_{2}\right)+l_{2} \circ(1,1)\right)^{(132)} \\
& +l_{4 ; 2} \circ(1 \circ(1), 1 \circ(1), 1 \circ(1), 1 \circ(1)) \text {, }
\end{aligned}
$$

and

$$
\begin{aligned}
(\Delta \circ \mathrm{id}) \Delta\left(l_{4 ; 3}\right)= & 1 \circ(1) \circ\left(l_{4 ; 3}\right)+\left(1 \circ\left(l_{2 ; 1}\right)+l_{2 ; 1} \circ(1,1)\right) \circ\left(1, l_{3}\right)^{(123)}-\left(1 \circ\left(l_{2}\right)\right. \\
& \left.+l_{2} \circ(1,1)\right) \circ\left(1, l_{3 ; 2}\right)+\left(1 \circ\left(l_{2}\right)+l_{2} \circ(1,1)\right) \circ\left(1, l_{3 ; 2}\right)^{(12)}+\left(1 \circ\left(l_{2 ; 1}\right)\right. \\
& \left.+l_{2 ; 1} \circ(1,1)\right) \circ\left(l_{3}, 1\right)-\left(1 \circ\left(l_{2}\right)+l_{2} \circ(1,1)\right) \circ\left(l_{2}, l_{2 ; 1}\right)-\left(1 \circ\left(l_{2 ; 1}\right)\right. \\
& \left.+l_{2 ; 1} \circ(1,1)\right) \circ\left(l_{2}, l_{2}\right)^{(132)}+\left(1 \circ\left(l_{2 ; 1}\right)+l_{2 ; 1} \circ(1,1)\right) \circ\left(l_{2}, l_{2}\right)^{(23)} \\
& +\left(1 \circ\left(l_{3 ; 2}\right)-l_{2 ; 1} \circ\left(l_{2}, 1\right)-l_{2} \circ\left(1, l_{2 ; 1}\right)-l_{2 ; 1} \circ\left(1, l_{2}\right)^{(12)}\right.
\end{aligned}
$$




$$
\begin{aligned}
& \left.+l_{3 ; 2} \circ(1,1,1)\right) \circ\left(l_{2}, 1,1\right)-\left(1 \circ\left(l_{3 ; 2}\right)-l_{2 ; 1} \circ\left(l_{2}, 1\right)-l_{2} \circ\left(1, l_{2 ; 1}\right)\right. \\
& \left.-l_{2 ; 1} \circ\left(1, l_{2}\right)^{(12)}+l_{3 ; 2} \circ(1,1,1)\right) \circ\left(1,1, l_{2}\right)^{(23)}+\left(1 \circ\left(l_{3 ; 2}\right)\right. \\
& -l_{2 ; 1} \circ\left(l_{2}, 1\right)-l_{2} \circ\left(1, l_{2 ; 1}\right)-l_{2 ; 1} \circ\left(1, l_{2}\right)^{(12)} \\
& \left.+l_{3 ; 2} \circ(1,1,1)\right) \circ\left(1,1, l_{2}\right)^{(132)}+\left(1 \circ\left(l_{3}\right)-l_{2} \circ\left(l_{2}, 1\right)+l_{2} \circ\left(1, l_{2}\right)\right. \\
& \left.-l_{2} \circ\left(1, l_{2}\right)^{(12)}+l_{3} \circ(1,1,1)\right) \circ\left(1,1, l_{2 ; 1}\right)-\left(1 \circ\left(l_{3 ; 2}\right)-l_{2 ; 1} \circ\left(l_{2}, 1\right)\right. \\
& \left.-l_{2} \circ\left(1, l_{2 ; 1}\right)-l_{2 ; 1} \circ\left(1, l_{2}\right)^{(12)}+l_{3 ; 2} \circ(1,1,1)\right) \circ\left(1, l_{2}, 1\right)+\left(1 \circ\left(l_{3 ; 2}\right)\right. \\
& -l_{2 ; 1} \circ\left(l_{2}, 1\right)-l_{2} \circ\left(1, l_{2 ; 1}\right)-l_{2 ; 1} \circ\left(1, l_{2}\right)^{(12)} \\
& \left.+l_{3 ; 2} \circ(1,1,1)\right) \circ\left(1, l_{2}, 1\right)^{(12)}+\left(1 \circ\left(l_{4 ; 3}\right)+l_{2 ; 1} \circ\left(1, l_{3}\right)^{(123)}\right. \\
& -l_{2} \circ\left(1, l_{3 ; 2}\right)+l_{2} \circ\left(1, l_{3 ; 2}\right)^{(12)}+l_{2 ; 1} \circ\left(l_{3}, 1\right)-l_{2} \circ\left(l_{2}, l_{2 ; 1}\right) \\
& -l_{2 ; 1} \circ\left(l_{2}, l_{2}\right)^{(132)}+l_{2 ; 1} \circ\left(l_{2}, l_{2}\right)^{(23)}+l_{3 ; 2} \circ\left(l_{2}, 1,1\right)-l_{3 ; 2} \circ\left(1,1, l_{2}\right)^{(23)} \\
& +l_{3 ; 2} \circ\left(1,1, l_{2}\right)^{(132)}+l_{3} \circ\left(1,1, l_{2 ; 1}\right)-l_{3 ; 2} \circ\left(1, l_{2}, 1\right)+l_{3 ; 2} \circ\left(1, l_{2}, 1\right)^{(12)} \\
& \left.+l_{4 ; 3} \circ(1,1,1,1)\right) \circ(1,1,1,1) \text {, } \\
& (\mathrm{id} \circ \Delta) \Delta\left(l_{4 ; 3}\right)=1 \circ\left(1 \circ\left(l_{4 ; 3}\right)+l_{2 ; 1} \circ\left(1, l_{3}\right)^{(123)}-l_{2} \circ\left(1, l_{3 ; 2}\right)+l_{2} \circ\left(1, l_{3 ; 2}\right)^{(12)}\right. \\
& +l_{2 ; 1} \circ\left(l_{3}, 1\right)-l_{2} \circ\left(l_{2}, l_{2 ; 1}\right)-l_{2 ; 1} \circ\left(l_{2}, l_{2}\right)^{(132)}+l_{2 ; 1} \circ\left(l_{2}, l_{2}\right)^{(23)} \\
& +l_{3 ; 2} \circ\left(l_{2}, 1,1\right)-l_{3 ; 2} \circ\left(1,1, l_{2}\right)^{(23)}+l_{3 ; 2} \circ\left(1,1, l_{2}\right)^{(132)}+l_{3} \circ\left(1,1, l_{2 ; 1}\right) \\
& \left.-l_{3 ; 2} \circ\left(1, l_{2}, 1\right)+l_{3 ; 2} \circ\left(1, l_{2}, 1\right)^{(12)}+l_{4 ; 3} \circ(1,1,1,1)\right)+l_{2 ; 1} \circ(1 \circ(1) \text {, } \\
& \left.1 \circ\left(l_{3}\right)-l_{2} \circ\left(l_{2}, 1\right)+l_{2} \circ\left(1, l_{2}\right)-l_{2} \circ\left(1, l_{2}\right)^{(12)}+l_{3} \circ(1,1,1)\right)^{(123)} \\
& -l_{2} \circ\left(1 \circ(1), 1 \circ\left(l_{3 ; 2}\right)-l_{2 ; 1} \circ\left(l_{2}, 1\right)-l_{2} \circ\left(1, l_{2 ; 1}\right)-l_{2 ; 1} \circ\left(1, l_{2}\right)^{(12)}\right. \\
& \left.+l_{3 ; 2} \circ(1,1,1)\right)+l_{2} \circ\left(1 \circ(1), 1 \circ\left(l_{3 ; 2}\right)-l_{2 ; 1} \circ\left(l_{2}, 1\right)-l_{2} \circ\left(1, l_{2 ; 1}\right)\right. \\
& \left.-l_{2 ; 1} \circ\left(1, l_{2}\right)^{(12)}+l_{3 ; 2} \circ(1,1,1)\right)^{(12)}+l_{2 ; 1} \circ\left(1 \circ\left(l_{3}\right)-l_{2} \circ\left(l_{2}, 1\right)\right. \\
& \left.+l_{2} \circ\left(1, l_{2}\right)-l_{2} \circ\left(1, l_{2}\right)^{(12)}+l_{3} \circ(1,1,1), 1 \circ(1)\right)-l_{2} \circ\left(1 \circ\left(l_{2}\right)\right. \\
& \left.+l_{2} \circ(1,1), 1 \circ\left(l_{2 ; 1}\right)+l_{2 ; 1} \circ(1,1)\right)-l_{2 ; 1} \circ\left(1 \circ\left(l_{2}\right)+l_{2} \circ(1,1), 1 \circ\left(l_{2}\right)\right. \\
& \left.+l_{2} \circ(1,1)\right)^{(132)}+l_{2 ; 1} \circ\left(1 \circ\left(l_{2}\right)+l_{2} \circ(1,1), 1 \circ\left(l_{2}\right)+l_{2} \circ(1,1)\right)^{(23)} \\
& +l_{3 ; 2} \circ\left(1 \circ\left(l_{2}\right)+l_{2} \circ(1,1), 1 \circ(1), 1 \circ(1)\right)-l_{3 ; 2} \circ(1 \circ(1), 1 \circ(1) \text {, } \\
& \left.1 \circ\left(l_{2}\right)+l_{2} \circ(1,1)\right)^{(23)}+l_{3 ; 2} \circ\left(1 \circ(1), 1 \circ(1), 1 \circ\left(l_{2}\right)+l_{2} \circ(1,1)\right)^{(132)} \\
& +l_{3} \circ\left(1 \circ(1), 1 \circ(1), 1 \circ\left(l_{2 ; 1}\right)+l_{2 ; 1} \circ(1,1)\right)-l_{3 ; 2} \circ\left(1 \circ(1), 1 \circ\left(l_{2}\right)\right. \\
& \left.+l_{2} \circ(1,1), 1 \circ(1)\right)+l_{3 ; 2} \circ\left(1 \circ(1), 1 \circ\left(l_{2}\right)+l_{2} \circ(1,1), 1 \circ(1)\right)^{(12)} \\
& +l_{4 ; 3} \circ(1 \circ(1), 1 \circ(1), 1 \circ(1), 1 \circ(1)) \text {, }
\end{aligned}
$$

and again we see that both sides agree. This concludes the proof of Lemma 1.3.1

A.2. Proof of Lemma 2.4.2. We verify that the structure maps defined in Equations (146) (149) satisfy Equations (122) (132) and hence form a weak Lie 3-algebra:

$$
\partial\left(\lambda_{2 ; 1}^{\prime}\right)=p \circ\left(\partial \lambda_{2 ; 1}\right) \circ(i, i)=p \circ\left(\lambda_{2}+\lambda_{2}^{(12)}\right) \circ(i, i)=\lambda_{2}^{\prime}+\lambda_{2}^{(12)},
$$




$$
\begin{aligned}
& \partial\left(\lambda_{2 ; 1,1}^{\prime}\right)=p \circ\left(\partial \lambda_{2 ; 1,1}\right) \circ(i, i)=p \circ\left(\lambda_{2 ; 1}-\lambda_{2 ; 1}^{(12)}\right) \circ(i, i)=\lambda_{2 ; 1}^{\prime}-\lambda_{2 ; 1}^{\prime(12)} \text {, } \\
& \partial\left(\lambda_{3 ; 1}^{\prime}\right)=p \circ\left(\partial \lambda_{3 ; 1}\right) \circ(i, i, i)-p \circ \partial\left(\lambda_{2} \circ 1\left(h \circ \lambda_{2 ; 1}\right)\right) \circ(i, i, i) \\
& =p \circ\left(\lambda_{3}+\lambda_{3}^{(12)}+\lambda_{2} \circ \lambda_{1} \lambda_{2 ; 1}\right) \circ(i, i, i) \\
& -p \circ\left(\lambda_{2} \circ \circ_{1}\left(\partial h \circ \lambda_{2 ; 1}\right)-\lambda_{2} \circ_{1}\left(h \circ \partial \lambda_{2 ; 1}\right)\right) \circ(i, i, i) \\
& =p \circ\left(\lambda_{3}+\lambda_{2} \circ\left(h \circ \lambda_{2}\right)\right) \circ(i, i, i) \\
& +p \circ\left(\lambda_{3}^{(12)}+\left(\lambda_{2} \circ\left(h \circ \lambda_{2}\right)\right)^{(12)}\right) \circ(i, i, i) \\
& +p \circ\left(\lambda_{2} \circ \circ_{1}\left((\mathrm{id}-\partial h) \lambda_{2 ; 1}\right)\right) \circ(i, i, i) \\
& =\lambda_{3}^{\prime}+\lambda_{3}^{\prime(12)}+\lambda_{2}^{\prime} \circ_{1} \lambda_{2 ; 1}^{\prime}, \\
& \partial\left(\lambda_{3 ; 2}^{\prime}\right)=p \circ\left(\partial \lambda_{3 ; 2}\right) \circ(i, i, i) \\
& +p \circ \partial\left(\lambda_{2 ; 1} \circ_{1}\left(h \circ \lambda_{2}\right)+\lambda_{2} \circ_{2}\left(h \circ \lambda_{2 ; 1}\right)+\left(\lambda_{2 ; 1} \circ_{2}\left(h \circ \lambda_{2}\right)\right)^{(12)}\right) \circ(i, i, i) \\
& =p \circ\left(\lambda_{3}+\lambda_{3}^{(23)}+\lambda_{2 ; 1} \circ_{1}\left(h \circ \lambda_{2}\right)-\lambda_{2} \circ_{2}\left(h \circ \lambda_{2 ; 1}\right)\right. \\
& \left.+\left(\lambda_{2 ; 1} \circ 2\left(h \circ \lambda_{2}\right)\right)^{(12)}\right) \circ(i, i, i) \\
& +p \circ\left(\partial \lambda_{2 ; 1} \circ \circ_{1}\left(h \circ \lambda_{2}\right)+\lambda_{2} \circ_{2}\left(\partial h \circ \lambda_{2 ; 1}\right)\right. \\
& +\left(\partial \lambda_{2 ; 1} \circ_{2}\left(h \circ \lambda_{2}\right)\right)^{(12)}-\lambda_{2 ; 1} \circ_{1}\left(\partial h \circ \lambda_{2}\right) \\
& \left.-\lambda_{2} \circ_{2}\left(h \circ \partial \lambda_{2 ; 1}\right)-\left(\lambda_{2 ; 1} \circ_{2}\left(\partial h \circ \lambda_{2}\right)\right)^{(12)}\right) \circ(i, i, i) \\
& =p \circ\left(\lambda_{3}+\lambda_{2} \circ\left(h \circ \lambda_{2}\right)-\lambda_{2} \circ_{2}\left(h \circ \lambda_{2}\right)+\left(\lambda_{2} \circ o_{2}\left(h \circ \lambda_{2}\right)\right)^{(12)}\right) \circ(i, i, i) \\
& +p \circ\left(\lambda_{3}^{(23)}+\lambda_{2}^{(12)} \circ_{1}\left(h \circ \lambda_{2}\right)-\lambda_{2} \circ \circ_{2}\left(h \circ \lambda_{2}^{(12)}\right)\right. \\
& \left.+\left(\lambda_{2}^{(12)} \circ_{2}\left(h \circ \lambda_{2}\right)\right)^{(12)}\right) \circ(i, i, i) \\
& +p \circ\left(\lambda_{2 ; 1} \circ{ }_{1}\left((\mathrm{id}-\partial h) \circ \lambda_{2}\right)-\lambda_{2} \circ_{2}\left((\mathrm{id}-\partial h) \circ \lambda_{2 ; 1}\right)\right. \\
& \left.+\left(\lambda_{2 ; 1} \circ_{2}\left((\mathrm{id}-\partial h) \circ \lambda_{2}\right)\right)^{(12)}\right) \circ(i, i, i) \\
& =\lambda_{3}^{\prime}+\lambda_{3}^{\prime(23)}+\lambda_{2 ; 1}^{\prime} \circ_{1} \lambda_{2}^{\prime}-\lambda_{2}^{\prime} \circ_{2} \lambda_{2 ; 1}^{\prime}+\left(\lambda_{2 ; 1}^{\prime} \circ_{2} \lambda_{2}^{\prime}\right)^{(12)} \text {, } \\
& \lambda_{2 ; 1,1}^{\prime}+\lambda_{2 ; 1,1}^{(12)}=p \circ\left(\lambda_{2 ; 1,1}+\lambda_{2 ; 1,1}^{(12)}\right) \circ(i, i)=0, \\
& \lambda_{3 ; 1}^{\prime}-\lambda_{3 ; 1}^{\prime(12)}=p \circ\left(\lambda_{3 ; 1}-\lambda_{3 ; 1}^{(12)}\right) \circ(i, i, i) \\
& -p \circ\left(\lambda_{2} \circ_{1}\left(h \circ \lambda_{2 ; 1}\right)-\left(\lambda_{2} \circ{ }_{1}\left(h \circ \lambda_{2 ; 1}\right)\right)^{(12)}\right) \circ(i, i, i) \\
& =p \circ\left(\lambda_{2} \circ_{1} \lambda_{2 ; 1,1}\right) \circ(i, i, i)-p \circ\left(\lambda_{2} \circ_{1}\left(h \circ \partial \lambda_{2 ; 1,1}\right)\right) \circ(i, i, i) \\
& =p \circ\left(\lambda_{2} \circ{ }_{1}\left((\mathrm{id}-\partial h) \lambda_{2 ; 1,1}\right)\right) \circ(i, i, i) \\
& =\lambda_{2}^{\prime} \circ_{1} \lambda_{2 ; 1,1}^{\prime} \text {, } \\
& \lambda_{3 ; 1}^{\prime}-\lambda_{3 ; 2}^{\prime(12)}+\lambda_{3 ; 1}^{\prime(132)}-\lambda_{3 ; 2}^{\prime}+\lambda_{3 ; 1}^{\prime(23)}-\lambda_{3 ; 2}^{(123)} \\
& =p \circ\left(\lambda_{3 ; 1}-\lambda_{3 ; 2}^{(12)}+\lambda_{3 ; 1}^{(132)}-\lambda_{3 ; 2}+\lambda_{3 ; 1}^{(23)}-\lambda_{3 ; 2}^{(123)}\right) \circ(i, i, i) \\
& -p \circ\left(\lambda_{2} \circ\left(h \circ \lambda_{2 ; 1}\right)\right)^{1+(23)+(132)} \circ(i, i, i)
\end{aligned}
$$




$$
\begin{aligned}
& -p \circ\left(\lambda_{2 ; 1} \circ_{1}\left(h \circ \lambda_{2}\right)+\lambda_{2} \circ_{2}\left(h \circ \lambda_{2 ; 1}\right)\right. \\
& \left.+\left(\lambda_{2 ; 1} \circ \circ_{2}\left(h \circ \lambda_{2}\right)\right)^{(12)}\right)^{1+(12)+(123)} \circ(i, i, i) \\
& =p \circ\left(\lambda_{2 ; 1} \circ_{1} \lambda_{2 ; 1}+\left(\lambda_{2 ; 1} \circ_{2} \lambda_{2 ; 1}\right)^{1+(12)}+\left(\lambda_{2 ; 1,1} \circ_{2} \lambda_{2}\right)^{(132)}\right) \circ(i, i, i) \\
& -p \circ\left(\partial \lambda_{2 ; 1} \circ 1\left(h \circ \lambda_{2 ; 1}\right)+\lambda_{2 ; 1} \circ_{1}\left(h \circ \partial \lambda_{2 ; 1}\right)+\partial \lambda_{2 ; 1} \circ 2\left(h \circ \lambda_{2 ; 1}\right)\right. \\
& +\lambda_{2 ; 1} \circ 2\left(h \circ \partial \lambda_{2 ; 1}\right)+\left(\partial \lambda_{2 ; 1} \circ 2\left(h \circ \lambda_{2 ; 1}\right)\right)^{(12)} \\
& \left.+\left(\lambda_{2 ; 1} \circ 2\left(h \circ \partial \lambda_{2 ; 1}\right)\right)^{(12)}+\left(\partial \lambda_{2 ; 1,1} \circ_{2}\left(h \circ \lambda_{2}\right)\right)^{(132)}\right) \\
& =\lambda_{2 ; 1}^{\prime} \circ_{1} \lambda_{2 ; 1}^{\prime}+\left(\lambda_{2 ; 1}^{\prime} \circ_{2} \lambda_{2 ; 1}^{\prime}\right)^{1+(12)}+\left(\lambda_{2 ; 1,1}^{\prime} \circ_{2} \lambda_{2}^{\prime}\right)^{(132)} \text {, } \\
& \lambda_{3 ; 2}^{\prime}-\lambda_{3 ; 2}^{\prime(23)}=p \circ\left(\lambda_{3 ; 2}-\lambda_{3 ; 2}^{(23)}\right) \circ(i, i, i) \\
& +p \circ\left(\lambda_{2 ; 1} \circ \circ_{1}\left(h \circ \lambda_{2}\right)+\lambda_{2} \circ_{2}\left(h \circ \lambda_{2 ; 1}\right)\right. \\
& +\left(\lambda_{2 ; 1} \circ 2\left(h \circ \lambda_{2}\right)\right)^{(12)}-\left(\lambda_{2 ; 1} \circ 1\left(h \circ \lambda_{2}\right)\right)^{(23)} \\
& \left.-\left(\lambda_{2} \circ 2\left(h \circ \lambda_{2 ; 1}\right)\right)^{(23)}-\left(\lambda_{2 ; 1} \circ_{2}\left(h \circ \lambda_{2}\right)\right)^{(123)}\right) \circ(i, i, i) \\
& =p \circ\left(\lambda_{2 ; 1,1} \circ_{1} \lambda_{2}-\lambda_{2} \circ_{2} \lambda_{2 ; 1,1}+\left(\lambda_{2} \circ_{2} \lambda_{2 ; 1,1}\right)^{(12)}\right) \circ(i, i, i) \\
& -p \circ\left(\partial \lambda_{2 ; 1,1} \circ 1\left(h \circ \lambda_{2}\right)+\lambda_{2} \circ_{2}\left(h \circ \partial \lambda_{2 ; 1,1}\right)\right. \\
& \left.+\left(\partial \lambda_{2 ; 1,1} \circ_{2}\left(h \circ \lambda_{2}\right)\right)^{(12)}\right) \circ(i, i, i) \\
& =p \circ\left(\lambda_{2 ; 1,1} \circ_{1} \lambda_{2}-\lambda_{2} \circ_{2} \lambda_{2 ; 1,1}+\left(\lambda_{2 ; 1,1} \circ_{2} \lambda_{2}\right)^{(12)}\right) \circ(i, i, i) \\
& =p \circ\left(\lambda_{2 ; 1,1} \circ_{1}\left((\mathrm{id}-\partial h) \lambda_{2}\right)-\lambda_{2} \circ_{2}\left((\mathrm{id}-\partial h) \lambda_{2 ; 1,1}\right)\right. \\
& \left.+\left(\lambda_{2 ; 1,1} \circ_{2}(\mathrm{id}-\partial h) \lambda_{2}\right)^{(12)}\right) \circ(i, i, i) \\
& =\lambda_{2 ; 1,1}^{\prime} \circ_{1} \lambda_{2}^{\prime}-\lambda_{2}^{\prime} \circ_{2} \lambda_{2 ; 1,1}^{\prime}+\left(\lambda_{2 ; 1,1}^{\prime} \circ_{2} \lambda_{2}^{\prime}\right)^{(12)} \text {, } \\
& \lambda_{4}^{\prime}+\lambda_{4}^{(12)}=p \circ\left(\lambda_{4}+\lambda_{4}^{(12)}\right) \circ(i, i, i, i) \\
& -p \circ\left(\lambda_{2} \circ\left(h \circ \lambda_{3}\right)+\left(\lambda_{2} \circ 2\left(h \circ \lambda_{3}\right)\right)^{(123)}\right. \\
& \left.+\lambda_{3} \circ_{1}\left(h \circ \lambda_{2}\right)+\lambda_{3} \circ_{3}\left(h \circ \lambda_{2}\right)\right)^{1+(12)} \circ(i, i, i, i) \\
& -p \circ\left(\lambda_{2} \circ \circ_{1}\left(h \circ \lambda_{2}\right) \circ\left(h \circ \lambda_{2}\right)+\lambda_{2} \circ\left(h \circ \lambda_{2}, h \circ \lambda_{2}\right)\right. \\
& \left.+\left(\lambda_{2} \circ_{1}\left(h \circ \lambda_{2}\right) \circ_{1}\left(h \circ \lambda_{2}\right)\right)^{(123)}\right)^{1+(12)} \circ(i, i, i, i)
\end{aligned}
$$




$$
\begin{aligned}
& =p \circ\left(\lambda_{2} \circ_{1} \lambda_{3 ; 1}+\left(\lambda_{2} \circ_{2} \lambda_{3 ; 1}\right)^{(123)}+\lambda_{3} \circ_{1} \lambda_{2 ; 1}-\lambda_{3 ; 1} \circ_{3} \lambda_{2}\right) \circ(i, i, i, i) \\
& -p \circ\left(\lambda_{2} \circ_{1}\left(h \circ\left(\lambda_{3}+\lambda_{3}^{(12)}+\lambda_{2} \circ_{1} \lambda_{2 ; 1}\right)\right)\right. \\
& +\left(\lambda_{2} \circ_{2}\left(h \circ\left(\lambda_{3}+\lambda_{3}^{(12)}+\lambda_{2} \circ_{1} \lambda_{2 ; 1}\right)\right)\right)^{(123)} \\
& -\left(\lambda_{2} \circ_{1} \lambda_{2}-\left(\lambda_{2} \circ_{2} \lambda_{2}\right)^{1-(12)}\right) \circ_{1}\left(h \circ \lambda_{2 ; 1}\right) \\
& +\lambda_{3} \circ_{1}\left(h \circ\left(\lambda_{2}+\lambda_{2}^{(12)}\right)\right) \\
& \left.+\left(\lambda_{3}+\lambda_{3}^{(12)}+\lambda_{2} \circ_{1} \lambda_{2 ; 1}\right) \circ_{3}\left(h \circ \lambda_{2}\right)\right) \circ(i, i, i, i) \\
& -p \circ\left(\lambda_{2} \circ \lambda_{2} \circ_{1}\left(h \circ \lambda_{2 ; 1}\right)-\lambda_{2} \circ_{1}\left(\partial h \circ \lambda_{2}\right) \circ_{1}\left(h \circ \lambda_{2 ; 1}\right)\right. \\
& +\left(\lambda_{2} \circ \circ_{2} \lambda_{2} \circ 2\left(h \circ \lambda_{2 ; 1}\right)\right)^{(123)}-\left(\lambda_{2} \circ \circ_{2}\left(\partial h \circ \lambda_{2}\right) \circ_{2}\left(h \circ \lambda_{2 ; 1}\right)\right)^{(123)} \\
& -\lambda_{2} \circ_{1}\left(h \circ \lambda_{2}\right) \circ_{1} \lambda_{2 ; 1}+\lambda_{2} \circ_{1}\left(h \circ \lambda_{2}\right) \circ_{1}\left(\partial h \circ \lambda_{2 ; 1}\right) \\
& +\left(\lambda_{2} \circ \circ_{2}\left(h \circ \lambda_{2}\right)\right)^{1-(12)} \circ_{1} \lambda_{2 ; 1} \\
& -\left(\lambda_{2} \circ 2\left(h \circ \lambda_{2}\right)\right)^{1-(12)} \circ_{1}\left(\partial h \circ \lambda_{2 ; 1}\right)-\lambda_{2} \circ\left(h \circ \lambda_{2 ; 1}\right) \circ_{3} \lambda_{2} \\
& \left.+\lambda_{2} \circ\left(h \circ \lambda_{2 ; 1}\right) \circ_{3}\left(\partial h \circ \lambda_{2}\right)\right) \circ(i, i, i, i) \\
& =p \circ\left(\lambda_{2} \circ\left((\mathrm{id}-\partial h) \circ \lambda_{3 ; 1}\right)-\lambda_{2} \circ_{1}\left((\mathrm{id}-\partial h) \circ \lambda_{2}\right) \circ\left(h \circ \lambda_{2 ; 1}\right)\right. \\
& +\left(\lambda_{2} \circ 2\left((\mathrm{id}-\partial h) \circ \lambda_{3 ; 1}\right)\right)^{(123)} \\
& -\left(\lambda_{2} \circ{ }_{2}\left((\mathrm{id}-\partial h) \circ \lambda_{2}\right) \circ_{2}\left(h \circ \lambda_{2 ; 1}\right)\right)^{(123)} \\
& +\lambda_{3} \circ_{1}\left((\mathrm{id}-\partial h) \circ \lambda_{2 ; 1}\right)+\lambda_{2} \circ_{1}\left(h \circ \lambda_{2}\right) \circ_{1}\left((\mathrm{id}-\partial h) \circ \lambda_{2 ; 1}\right) \\
& -\left(\lambda_{2} \circ\left(h \circ \lambda_{2}\right)\right)^{1-(12)} \circ_{1}\left((\mathrm{id}-\partial h) \circ \lambda_{2 ; 1}\right) \\
& -\lambda_{3 ; 1} \circ_{3}\left((\mathrm{id}-\partial h) \circ \lambda_{2}\right) \\
& \left.+\lambda_{2} \circ_{1}\left(h \circ \lambda_{2 ; 1}\right) \circ_{3}\left((\mathrm{id}-\partial h) \circ \lambda_{2}\right)\right) \circ(i, i, i, i) \\
& =p \circ\left(\lambda_{2} \circ\left(i \circ p \circ\left(\lambda_{3 ; 1}-\lambda_{2} \circ\left(h \circ \lambda_{2 ; 1}\right)\right)\right)\right. \\
& +\left(\lambda_{2} \circ \circ_{2}\left(i \circ p \circ\left(\lambda_{3 ; 1}-\lambda_{2} \circ{ }_{1}\left(h \circ \lambda_{2 ; 1}\right)\right)\right)\right)^{(123)} \\
& +\left(\lambda_{3}+\lambda_{2} \circ\left(h \circ \lambda_{2}\right)-\left(\lambda_{2} \circ_{2}\left(h \circ \lambda_{2}\right)\right)^{1-(12)}\right) \circ_{1}\left(i \circ p \circ \lambda_{2 ; 1}\right) \\
& \left.-\left(\lambda_{3 ; 1}-\lambda_{2} \circ_{1}\left(h \circ \lambda_{2 ; 1}\right)\right) \circ_{3}\left(i \circ p \circ \lambda_{2}\right)\right) \circ(i, i, i, i) \\
& =\lambda_{2}^{\prime} \circ_{1} \lambda_{3 ; 1}^{\prime}+\left(\lambda_{2}^{\prime} \circ_{2} \lambda_{3 ; 1}^{\prime}\right)^{(123)}+\lambda_{3}^{\prime} \circ_{1} \lambda_{2 ; 1}^{\prime}-\lambda_{3 ; 1}^{\prime} \circ_{3} \lambda_{2}^{\prime} \text {, } \\
& \lambda_{4}^{\prime}+\lambda_{4}^{(23)}=p \circ\left(\lambda_{4}+\lambda_{4}^{(23)}\right) \circ(i, i, i, i) \\
& -p \circ\left(\lambda_{2} \circ\left(h \circ \lambda_{3}\right)+\lambda_{2} \circ\left(h \circ \lambda_{3}\right)+\lambda_{3} \circ\left(h \circ \lambda_{2}\right)\right. \\
& \left.-\left(\lambda_{3} \circ \circ_{2}\left(h \circ \lambda_{2}\right)\right)^{1-(12)}+\left(\lambda_{3} \circ_{3}\left(h \circ \lambda_{2}\right)\right)^{(132)}\right)^{1+(23)} \circ(i, i, i, i) \\
& -p \circ\left(\lambda_{2} \circ\left(h \circ \lambda_{2}\right) \circ\left(h \circ \lambda_{2}\right)-\left(\lambda_{2} \circ\left(h \circ \lambda_{2}\right) \circ_{2}\left(h \circ \lambda_{2}\right)\right)^{1-(12)}\right. \\
& +\lambda_{2} \circ\left(h \circ \lambda_{2}, h \circ \lambda_{2}\right)^{(132)} \\
& \left.+\lambda_{2} \circ_{2}\left(h \circ \lambda_{2}\right) \circ_{2}\left(h \circ \lambda_{2}\right)\right)^{1+(23)} \circ(i, i, i, i)
\end{aligned}
$$




$$
\begin{aligned}
& =p \circ\left(\lambda_{2} \circ_{1} \lambda_{3 ; 2}+\lambda_{2} \circ_{2} \lambda_{3 ; 1}-\lambda_{3 ; 1} \circ_{1} \lambda_{2}-\lambda_{3} \circ_{2} \lambda_{2 ; 1}-\left(\lambda_{3 ; 1} \circ_{2} \lambda_{2}\right)^{(12)}\right. \\
& \left.-\left(\lambda_{3 ; 1} \circ_{3} \lambda_{2}\right)^{(132)}\right) \circ(i, i, i, i) \\
& -p \circ\left(\lambda_{2} \circ_{1}\left(h \circ\left(\lambda_{3}+\lambda_{3}^{(23)}+\lambda_{2 ; 1} \circ_{1} \lambda_{2}-\lambda_{2} \circ_{2} \lambda_{2 ; 1}+\left(\lambda_{2 ; 1} \circ_{2} \lambda_{2}\right)^{(12)}\right)\right)\right. \\
& +\lambda_{2} \circ_{2}\left(h \circ\left(\lambda_{3}+\lambda_{3}^{(12)}+\lambda_{2} \circ_{1} \lambda_{2 ; 1}\right)\right) \\
& +\left(\lambda_{3}+\lambda_{3}^{(12)}+\lambda_{2} \circ_{1} \lambda_{2 ; 1}\right) \circ_{1}\left(h \circ \lambda_{2}\right) \\
& +\left(\lambda_{2} \circ_{1} \lambda_{2}-\left(\lambda_{2} \circ_{2} \lambda_{2}\right)^{1-(12)}\right) \circ_{2}\left(h \circ \lambda_{2 ; 1}\right) \\
& -\lambda_{3} \circ_{2}\left(h \circ\left(\lambda_{2}+\lambda_{2}^{(12)}\right)\right) \\
& +\left(\left(\lambda_{3}+\lambda_{3}^{(12)}+\lambda_{2} \circ_{1} \lambda_{2 ; 1}\right) \circ_{2}\left(h \circ \lambda_{2}\right)\right)^{(12)} \\
& \left.+\left(\left(\lambda_{3}+\lambda_{3}^{(12)}+\lambda_{2} \circ_{1} \lambda_{2 ; 1}\right) \circ_{3}\left(h \circ \lambda_{2}\right)\right)^{(132)}\right) \circ(i, i, i, i) \\
& +p \circ\left(\lambda_{2} \circ_{1}\left(\lambda_{2 ; 1} \circ_{1}\left(h \circ \lambda_{2}\right)+\lambda_{2} \circ_{2}\left(h \circ \lambda_{2 ; 1}\right)+\left(\lambda_{2 ; 1} \circ_{2}\left(h \circ \lambda_{2}\right)\right)^{(12)}\right)\right. \\
& -\lambda_{2} \circ \circ_{1}\left(\partial h \circ \left(\lambda_{2 ; 1} \circ\left(h \circ \lambda_{2}\right)+\lambda_{2} \circ_{2}\left(h \circ \lambda_{2 ; 1}\right)\right.\right. \\
& \left.\left.+\left(\lambda_{2 ; 1} \circ_{2}\left(h \circ \lambda_{2}\right)\right)^{(12)}\right)\right) \\
& -\lambda_{2} \circ_{2}\left(\lambda_{2} \circ \circ_{1}\left(h \circ \lambda_{2 ; 1}\right)\right)+\lambda_{2} \circ_{2}\left(\partial h \circ\left(\lambda_{2} \circ\left(h \circ \lambda_{2 ; 1}\right)\right)\right) \\
& +\left(\lambda_{2} \circ{ }_{1}\left(h \circ \lambda_{2 ; 1}\right)\right) \circ_{1} \lambda_{2}-\left(\lambda_{2} \circ \circ_{1}\left(h \circ \lambda_{2 ; 1}\right)\right) \circ_{1}\left(\partial h \circ \lambda_{2}\right) \\
& -\left(\lambda_{2} \circ\left(h \circ \lambda_{2}\right)-\left(\lambda_{2} \circ\left(h \circ \lambda_{2}\right)\right)^{1-(12)}\right) \circ_{2} \lambda_{2 ; 1} \\
& +\left(\lambda_{2} \circ \circ_{1}\left(h \circ \lambda_{2}\right)-\left(\lambda_{2} \circ 2\left(h \circ \lambda_{2}\right)\right)^{1-(12)}\right) \circ_{2}\left(\partial h \circ \lambda_{2 ; 1}\right) \\
& +\left(\left(\lambda_{2} \circ_{1}\left(h \circ \lambda_{2 ; 1}\right)\right) \circ_{2} \lambda_{2}\right)^{(12)}-\left(\left(\lambda_{2} \circ_{1}\left(h \circ \lambda_{2 ; 1}\right)\right) \circ_{2}\left(\partial h \circ \lambda_{2}\right)\right)^{(12)} \\
& +\left(\left(\lambda_{2} \circ \circ_{1}\left(h \circ \lambda_{2 ; 1}\right)\right) \circ_{3} \lambda_{2}\right)^{(132)} \\
& \left.-\left(\left(\lambda_{2} \circ \circ_{1}\left(h \circ \lambda_{2 ; 1}\right)\right) \circ_{3}\left(\partial h \circ \lambda_{2}\right)\right)^{(132)}\right) \circ(i, i, i, i) \\
& =p \circ\left(\lambda_{2} \circ\left((\mathrm{id}-\partial h) \circ \lambda_{3 ; 2}\right)\right. \\
& +\lambda_{2} \circ_{1}\left(( \mathrm { id } - \partial h ) \circ \left(\lambda_{2 ; 1} \circ_{1}\left(h \circ \lambda_{2}\right)+\lambda_{2} \circ_{2}\left(h \circ \lambda_{2 ; 1}\right)\right.\right. \\
& \left.\left.+\left(\lambda_{2 ; 1} \circ_{2}\left(h \circ \lambda_{2}\right)\right)^{(12)}\right)\right) \\
& +\lambda_{2} \circ_{2}\left((\mathrm{id}-\partial h) \circ \lambda_{3 ; 1}\right)-\lambda_{2} \circ_{2}\left((\mathrm{id}-\partial h) \circ\left(\lambda_{2} \circ_{1}\left(h \circ \lambda_{2 ; 1}\right)\right)\right) \\
& -\lambda_{3 ; 1} \circ_{1}\left((\mathrm{id}-\partial h) \circ \lambda_{2}\right)+\left(\lambda_{2} \circ_{1}\left(h \circ \lambda_{2 ; 1}\right)\right) \circ_{1}\left((\mathrm{id}-\partial h) \circ \lambda_{2}\right) \\
& -\lambda_{3} \circ_{2}\left((\mathrm{id}-\partial h) \circ \lambda_{2 ; 1}\right) \\
& -\left(\lambda_{2} \circ \circ_{1}\left(h \circ \lambda_{2}\right)-\left(\lambda_{2} \circ_{2}\left(h \circ \lambda_{2}\right)\right)^{1-(12)}\right) \circ_{2}\left((\mathrm{id}-\partial h) \circ \lambda_{2 ; 1}\right) \\
& -\left(\lambda_{3 ; 1} \circ 2\left((\mathrm{id}-\partial h) \circ \lambda_{2}\right)\right)^{(12)} \\
& +\left(\left(\lambda_{2} \circ{ }_{1}\left(h \circ \lambda_{2 ; 1}\right)\right) \circ_{2}\left((\mathrm{id}-\partial h) \circ \lambda_{2}\right)\right)^{(12)} \\
& -\left(\lambda_{3 ; 1} \circ_{3}\left((\mathrm{id}-\partial h) \circ \lambda_{2}\right)\right)^{(132)} \\
& \left.+\left(\left(\lambda_{2} \circ_{1}\left(h \circ \lambda_{2 ; 1}\right)\right) \circ_{3}\left((\mathrm{id}-\partial h) \circ \lambda_{2}\right)\right)^{(132)}\right) \circ(i, i, i, i) \\
& =\lambda_{2}^{\prime} \circ_{1} \lambda_{3 ; 2}^{\prime}+\lambda_{2}^{\prime} \circ_{2} \lambda_{3 ; 1}^{\prime}-\lambda_{3 ; 1}^{\prime} \circ_{1} \lambda_{2}^{\prime}-\lambda_{3}^{\prime} \circ_{2} \lambda_{2 ; 1}^{\prime}
\end{aligned}
$$




$$
\begin{aligned}
& -\left(\lambda_{3 ; 1}^{\prime} \circ_{2} \lambda_{2}^{\prime}\right)^{(12)}-\left(\lambda_{3 ; 1}^{\prime} \circ_{3} \lambda_{2}^{\prime}\right)^{(132)}, \\
& \lambda_{4}^{\prime}+\lambda_{4}^{(34)}=p \circ\left(\lambda_{4}+\lambda_{4}^{(34)}\right) \circ(i, i, i, i) \\
& -p \circ\left(\lambda_{2} \circ{ }_{1}\left(h \circ \lambda_{3}\right)+\left(\lambda_{2} \circ_{2}\left(h \circ \lambda_{3}\right)\right)^{1-(12)+(123)}+\lambda_{3} \circ\left(h \circ \lambda_{2}\right)\right. \\
& -\left(\lambda_{3} \circ{ }_{2}\left(h \circ \lambda_{2}\right)\right)^{1-(12)} \\
& +\left(\lambda_{3} \circ o_{3}\left(h \circ \lambda_{2}\right)\right)^{1-(23)+(132))^{1+(34)}} \circ(i, i, i, i) \\
& -p \circ\left(\lambda_{2} \circ 1\left(h \circ \lambda_{2}\right) \circ\left(h \circ \lambda_{2}\right)-\left(\lambda_{2} \circ{ }_{1}\left(h \circ \lambda_{2}\right) \circ\left(h \circ \lambda_{2}\right)\right)^{1-(12)}\right. \\
& +\lambda_{2} \circ\left(h \circ \lambda_{2}, h \circ \lambda_{2}\right)^{1-(23)+(132)} \\
& +\left(\lambda_{2} \circ \circ_{2}\left(h \circ \lambda_{2}\right) \circ_{2}\left(h \circ \lambda_{2}\right)\right)^{1-(12)+(123)} \\
& \left.-\left(\lambda_{2} \circ \circ_{2}\left(h \circ \lambda_{2}\right) \circ_{3}\left(h \circ \lambda_{2}\right)\right)^{1-(12)+(123)-(23)+(132)-(13)}\right)^{1+(34)} \\
& \text {... }(i, i, i, i) \\
& =p \circ\left(\lambda_{2 ; 1} \circ_{1} \lambda_{3}+\left(\lambda_{2} \circ_{2} \lambda_{3 ; 2}\right)^{1-(12)}+\left(\lambda_{2 ; 1} \circ_{2} \lambda_{3}\right)^{(123)}-\lambda_{3 ; 2} \circ_{1} \lambda_{2}\right. \\
& \left.+\left(\lambda_{3 ; 2} \circ_{2} \lambda_{2}\right)^{1-(12)}+\lambda_{3} \circ_{3} \lambda_{2 ; 1}+\left(\lambda_{3 ; 2} \circ_{3} \lambda_{2}\right)^{(23)-(132)}\right) \circ(i, i, i, i) \\
& -p \circ\left(\left(\lambda_{2}+\lambda_{2}^{(12)}\right) \circ_{1}\left(h \circ \lambda_{3}\right)\right. \\
& +\lambda_{2 ; 1} \circ_{1}\left(h \circ\left(-\lambda_{2} \circ \lambda_{2}+\left(\lambda_{2} \circ_{2} \lambda_{2}\right)^{1-(12)}\right)\right) \\
& +\left(\lambda _ { 2 } \circ { } _ { 2 } \left(h \circ \left(\lambda_{3}+\lambda_{3}^{(23)}+\lambda_{2 ; 1} \circ_{1} \lambda_{2}-\lambda_{2} \circ_{2} \lambda_{2 ; 1}\right.\right.\right. \\
& \left.\left.\left.+\left(\lambda_{2 ; 1} \circ_{2} \lambda_{2}\right)^{(12)}\right)\right)\right)^{1-(12)} \\
& +\left(\left(\lambda_{2}+\lambda_{2}^{(12)}\right) \circ_{2}\left(h \circ \lambda_{3}\right)\right)^{(123)} \\
& +\left(\lambda_{2 ; 1} \circ_{2}\left(h \circ\left(-\lambda_{2} \circ_{1} \lambda_{2}+\left(\lambda_{2} \circ_{2} \lambda_{2}\right)^{1-(12)}\right)\right)\right)^{(123)} \\
& +\left(\lambda_{3}+\lambda_{3}^{(23)}+\lambda_{2 ; 1} \circ_{1} \lambda_{2}-\lambda_{2} \circ_{2} \lambda_{2 ; 1}+\left(\lambda_{2 ; 1} \circ_{2} \lambda_{2}\right)^{(12)}\right) \\
& \cdots \circ \circ_{1}\left(h \circ \lambda_{2}\right) \\
& -\left(\left(\lambda_{3}+\lambda_{3}^{(23)}+\lambda_{2 ; 1} \circ_{1} \lambda_{2}-\lambda_{2} \circ_{2} \lambda_{2 ; 1}+\left(\lambda_{2 ; 1} \circ_{2} \lambda_{2}\right)^{(12)}\right)\right. \\
& \left.\cdots \circ_{2}\left(h \circ \lambda_{2}\right)\right)^{1-(12)} \\
& +\left(-\lambda_{2} \circ \lambda_{2}+\left(\lambda_{2} \circ_{2} \lambda_{2}\right)^{1-(12)}\right) \circ_{3}\left(h \circ \lambda_{2 ; 1}\right) \\
& +\lambda_{3} \circ \circ_{3}\left(h \circ\left(\lambda_{2}+\lambda_{2}^{(12)}\right)\right) \\
& -\left(\left(\lambda_{3}+\lambda_{3}^{(23)}+\lambda_{2 ; 1} \circ_{1} \lambda_{2}-\lambda_{2} \circ_{2} \lambda_{2 ; 1}+\left(\lambda_{2 ; 1} \circ_{2} \lambda_{2}\right)^{(12)}\right)\right. \\
& \left.\left.\cdots \circ_{3}\left(h \circ \lambda_{2}\right)\right)^{(23)-(132)}\right) \circ(i, i, i, i)
\end{aligned}
$$




$$
\begin{aligned}
& +p \circ\left(-\lambda_{2 ; 1} \circ_{1}\left(-\lambda_{2} \circ\left(h \circ \lambda_{2}\right)+\left(\lambda_{2} \circ_{2}\left(h \circ \lambda_{2}\right)\right)^{1-(12)}\right)\right. \\
& +\lambda_{2 ; 1} \circ{ }_{1}\left(\partial h \circ\left(-\lambda_{2} \circ{ }_{1}\left(h \circ \lambda_{2}\right)+\left(\lambda_{2} \circ\left(h \circ \lambda_{2}\right)\right)^{1-(12)}\right)\right) \\
& +\left(\lambda _ { 2 } \circ \circ _ { 2 } \left(\lambda_{2 ; 1} \circ \circ_{1}\left(h \circ \lambda_{2}\right)+\lambda_{2} \circ \circ_{2}\left(h \circ \lambda_{2 ; 1}\right)\right.\right. \\
& \left.\left.+\left(\lambda_{2 ; 1} \circ 2\left(h \circ \lambda_{2}\right)\right)^{(12)}\right)\right)^{1-(12)} \\
& -\left(\lambda _ { 2 } \circ 2 \left(\partial h \circ \left(\lambda_{2 ; 1} \circ \circ_{1}\left(h \circ \lambda_{2}\right)+\lambda_{2} \circ_{2}\left(h \circ \lambda_{2 ; 1}\right)\right.\right.\right. \\
& \left.\left.\left.+\left(\lambda_{2 ; 1} \circ \circ_{2}\left(h \circ \lambda_{2}\right)\right)^{(12)}\right)\right)\right)^{1-(12)} \\
& -\left(\lambda_{2 ; 1} \circ \circ_{2}\left(-\lambda_{2} \circ \circ_{1}\left(h \circ \lambda_{2}\right)+\left(\lambda_{2} \circ_{2}\left(h \circ \lambda_{2}\right)\right)^{1-(12)}\right)\right)^{(123)} \\
& +\left(\lambda_{2 ; 1} \circ{ }_{2}\left(\partial h \circ\left(-\lambda_{2} \circ \circ_{1}\left(h \circ \lambda_{2}\right)+\left(\lambda_{2} \circ \circ_{2}\left(h \circ \lambda_{2}\right)\right)^{1-(12)}\right)\right)\right)^{(123)} \\
& -\left(\lambda_{2 ; 1} \circ \circ_{1}\left(h \circ \lambda_{2}\right)+\lambda_{2} \circ_{2}\left(h \circ \lambda_{2 ; 1}\right)+\left(\lambda_{2 ; 1} \circ_{2}\left(h \circ \lambda_{2}\right)\right)^{(12)}\right) \circ_{1} \lambda_{2} \\
& +\left(\lambda_{2 ; 1} \circ \circ_{1}\left(h \circ \lambda_{2}\right)+\lambda_{2} \circ_{2}\left(h \circ \lambda_{2 ; 1}\right)+\left(\lambda_{2 ; 1} \circ\left(h \circ \lambda_{2}\right)\right)^{(12)}\right) \\
& \cdots \circ \circ_{1}\left(\partial h \circ \lambda_{2}\right) \\
& +\left(\left(\lambda_{2 ; 1} \circ \circ_{1}\left(h \circ \lambda_{2}\right)+\lambda_{2} \circ_{2}\left(h \circ \lambda_{2 ; 1}\right)+\left(\lambda_{2 ; 1} \circ\left(h \circ \lambda_{2}\right)\right)^{(12)}\right)\right. \\
& \left.\cdots \circ_{2} \lambda_{2}\right)^{1-(12)} \\
& -\left(\left(\lambda_{2 ; 1} \circ{ }_{1}\left(h \circ \lambda_{2}\right)+\lambda_{2} \circ\left(h \circ \lambda_{2 ; 1}\right)\right.\right. \\
& \left.\left.+\left(\lambda_{2 ; 1} \circ \circ_{2}\left(h \circ \lambda_{2}\right)\right)^{(12)}\right) \circ_{2}\left(\partial h \circ \lambda_{2}\right)\right)^{1-(12)} \\
& -\left(-\lambda_{2} \circ \circ_{1}\left(h \circ \lambda_{2}\right)+\left(\lambda_{2} \circ\left(h \circ \lambda_{2}\right)\right)^{1-(12)}\right) \circ_{3} \lambda_{2 ; 1} \\
& +\left(-\lambda_{2} \circ \circ_{1}\left(h \circ \lambda_{2}\right)+\left(\lambda_{2} \circ \circ_{2}\left(h \circ \lambda_{2}\right)\right)^{1-(12)}\right) \circ_{3}\left(\partial h \circ \lambda_{2 ; 1}\right) \\
& +\left(\left(\lambda_{2 ; 1} \circ \circ_{1}\left(h \circ \lambda_{2}\right)+\lambda_{2} \circ 2\left(h \circ \lambda_{2 ; 1}\right)+\left(\lambda_{2 ; 1} \circ\left(h \circ \lambda_{2}\right)\right)^{(12)}\right)\right. \\
& \left.\cdots \circ_{3} \lambda_{2}\right)^{(23)-(132)} \\
& -\left(\left(\lambda_{2 ; 1} \circ{ }_{1}\left(h \circ \lambda_{2}\right)+\lambda_{2} \circ{ }_{2}\left(h \circ \lambda_{2 ; 1}\right)\right.\right. \\
& \left.\left.\left.+\left(\lambda_{2 ; 1} \circ \circ_{2}\left(h \circ \lambda_{2}\right)\right)^{(12)}\right) \circ_{3}\left(\partial h \circ \lambda_{2}\right)\right)^{(23)-(132)}\right) \circ(i, i, i, i)
\end{aligned}
$$




$$
\begin{aligned}
& =p \circ\left(\lambda_{2 ; 1} \circ \circ_{1}\left((\mathrm{id}-\partial h) \circ \lambda_{3}\right)\right. \\
& -\lambda_{2 ; 1} \circ_{1}\left((\mathrm{id}-\partial h) \circ\left(-\lambda_{2} \circ_{1}\left(h \circ \lambda_{2}\right)+\left(\lambda_{2} \circ_{2}\left(h \circ \lambda_{2}\right)\right)^{1-(12)}\right)\right) \\
& +\left(\lambda_{2} \circ_{2}\left((\mathrm{id}-\partial h) \circ \lambda_{3 ; 2}\right)\right)^{1-(12)} \\
& +\left(\lambda _ { 2 } \circ _ { 2 } \left(( \mathrm { id } - \partial h ) \circ \left(\lambda_{2 ; 1} \circ_{1}\left(h \circ \lambda_{2}\right)+\lambda_{2} \circ_{2}\left(h \circ \lambda_{2 ; 1}\right)\right.\right.\right. \\
& \left.\left.\left.+\left(\lambda_{2 ; 1} \circ 2\left(h \circ \lambda_{2}\right)\right)^{(12)}\right)\right)\right)^{1-(12)} \\
& +\left(\lambda_{2 ; 1} \circ_{2}\left((\mathrm{id}-\partial h) \circ \lambda_{3}\right)\right)^{(123)} \\
& -\left(\lambda_{2 ; 1} \circ_{2}((\mathrm{id}-\partial h)\right. \\
& \left.\left.\cdots \circ\left(-\lambda_{2} \circ{ }_{1}\left(h \circ \lambda_{2}\right)+\left(\lambda_{2} \circ 2\left(h \circ \lambda_{2}\right)\right)^{1-(12)}\right)\right)\right)^{(123)} \\
& -\lambda_{3 ; 2} \circ_{1}\left((\mathrm{id}-\partial h) \circ \lambda_{2}\right) \\
& -\left(\lambda_{2 ; 1} \circ \circ_{1}\left(h \circ \lambda_{2}\right)+\lambda_{2} \circ_{2}\left(h \circ \lambda_{2 ; 1}\right)\right. \\
& \left.+\left(\lambda_{2 ; 1} \circ_{2}\left(h \circ \lambda_{2}\right)\right)^{(12)}\right) \circ_{1}\left((\mathrm{id}-\partial h) \circ \lambda_{2}\right) \\
& +\left(\lambda_{3 ; 2} \circ_{2}\left((\mathrm{id}-\partial h) \circ \lambda_{2}\right)\right)^{1-(12)} \\
& +\left(\left(\lambda_{2 ; 1} \circ \circ_{1}\left(h \circ \lambda_{2}\right)+\lambda_{2} \circ_{2}\left(h \circ \lambda_{2 ; 1}\right)\right.\right. \\
& \left.\left.+\left(\lambda_{2 ; 1} \circ \circ_{2}\left(h \circ \lambda_{2}\right)\right)^{(12)}\right) \circ_{2}\left((\mathrm{id}-\partial h) \circ \lambda_{2}\right)\right)^{1-(12)} \\
& +\lambda_{3} \circ_{3}\left((\mathrm{id}-\partial h) \circ \lambda_{2 ; 1}\right) \\
& -\left(-\lambda_{2} \circ{ }_{1}\left(h \circ \lambda_{2}\right)+\left(\lambda_{2} \circ_{2}\left(h \circ \lambda_{2}\right)\right)^{1-(12)}\right) \circ_{3}\left((\mathrm{id}-\partial h) \circ \lambda_{2 ; 1}\right) \\
& +\left(\lambda_{3 ; 2} \circ_{3}\left((\mathrm{id}-\partial h) \circ \lambda_{2}\right)\right)^{(23)-(132)} \\
& +\left(\left(\lambda_{2 ; 1} \circ \circ_{1}\left(h \circ \lambda_{2}\right)+\lambda_{2} \circ_{2}\left(h \circ \lambda_{2 ; 1}\right)\right.\right. \\
& \left.\left.\left.+\left(\lambda_{2 ; 1} \circ_{2}\left(h \circ \lambda_{2}\right)\right)^{(12)}\right) \circ_{3}\left((\mathrm{id}-\partial h) \circ \lambda_{2}\right)\right)^{(23)-(132)}\right) \circ(i, i, i, i) \\
& =\lambda_{2 ; 1}^{\prime} \circ_{1} \lambda_{3}^{\prime}+\left(\lambda_{2}^{\prime} \circ_{2} \lambda_{3 ; 2}^{\prime}\right)^{1-(12)}+\left(\lambda_{2 ; 1}^{\prime} \circ_{2} \lambda_{3}^{\prime}\right)^{(123)}-\lambda_{3 ; 2}^{\prime} \circ_{1} \lambda_{2}^{\prime} \\
& +\left(\lambda_{3 ; 2}^{\prime} \circ_{2} \lambda_{2}^{\prime}\right)^{1-(12)}+\lambda_{3}^{\prime} \circ_{3} \lambda_{2 ; 1}^{\prime}+\left(\lambda_{3 ; 2}^{\prime} \circ_{3} \lambda_{2}^{\prime}\right)^{(23)-(132)} \text {. }
\end{aligned}
$$

This proves Lemma 2.4 .2

A.3. Proof of Lemma 3.3.1. The components of $\bar{f}$ are clearly skewsymmetric, hence the proof boils down to checking Equations (115) (118). The first three are easily verified:

$$
\begin{aligned}
\partial\left(\bar{f}_{1}\right) & =\partial f_{1}=0, \\
\partial\left(\bar{f}_{2}\right) & =\frac{1}{2} \partial\left(f_{2}-f_{2}^{(12)}\right) \\
& =\frac{1}{2}\left(f_{1} \circ \lambda_{2}-\lambda_{2}^{\prime} \circ\left(f_{1}, f_{1}\right)-f_{1} \circ \lambda_{2}^{(12)}+\lambda_{2}^{\prime} \circ\left(f_{1}, f_{1}\right)^{(12)}\right) \\
& =\bar{f}_{1} \circ \bar{\lambda}_{2}-\bar{\lambda}_{2}^{\prime} \circ\left(\bar{f}_{1}, \bar{f}_{1}\right),
\end{aligned}
$$




$$
\begin{aligned}
& \partial\left(\bar{f}_{3}\right)=\frac{1}{6} \sum_{\sigma \in \mathbb{S}_{3}}(-1)^{|\sigma|} \cdot \partial\left(f_{3}\right)^{\sigma} \\
& -\frac{1}{24} \sum_{\sigma \in \mathbb{S}_{3}}(-1)^{|\sigma|} \cdot\left(\partial\left(f_{2 ; 1}\right) \circ \circ_{1} \lambda_{2}-\partial\left(\lambda_{2 ; 1}^{\prime}\right) \circ\left(f_{2}, f_{1}\right)+\lambda_{2 ; 1}^{\prime} \circ\left(\partial\left(f_{2}\right), f_{1}\right)\right. \\
& \left.+\partial\left(f_{2 ; 1}\right) \circ_{2} \lambda_{2}-\partial\left(\lambda_{2 ; 1}^{\prime}\right) \circ\left(f_{1}, f_{2}\right)+\lambda_{2 ; 1}^{\prime} \circ\left(f_{1}, \partial\left(f_{2}\right)\right)\right)^{\sigma} \\
& =\frac{1}{6} \sum_{\sigma \in \mathbb{S}_{3}}(-1)^{|\sigma|} \cdot\left(f_{1} \circ \lambda_{3}+f_{2} \circ \circ_{1} \lambda_{2}-f_{2} \circ \circ_{2} \lambda_{2}+\left(f_{2} \circ \circ_{2} \lambda_{2}\right)^{(12)}+\lambda_{2}^{\prime} \circ\left(f_{2}, f_{1}\right)\right. \\
& \left.-\lambda_{2}^{\prime} \circ\left(f_{1}, f_{2}\right)+\lambda_{2}^{\prime} \circ\left(f_{1}, f_{2}\right)^{(12)}-\lambda_{3}^{\prime} \circ\left(f_{1}, f_{1}, f_{1}\right)\right)^{\sigma} \\
& -\frac{1}{24} \sum_{\sigma \in \mathbb{S}_{3}}(-1)^{|\sigma|} \cdot\left(\left(-f_{2}-f_{2}^{(12)}+f_{1} \circ \lambda_{2 ; 1}-\lambda_{2 ; 1}^{\prime} \circ\left(f_{1}, f_{1}\right)\right) \circ{ }_{1} \lambda_{2}\right. \\
& +\left(-f_{2}-f_{2}^{(12)}+f_{1} \circ \lambda_{2 ; 1}-\lambda_{2 ; 1}^{\prime} \circ\left(f_{1}, f_{1}\right)\right) \circ_{2} \lambda_{2} \\
& -\left(\lambda_{2}^{\prime}+\lambda_{2}^{\prime(12)}\right) \circ\left(f_{2}, f_{1}\right)+\lambda_{2 ; 1}^{\prime} \circ\left(f_{1} \circ \lambda_{2}-\lambda_{2}^{\prime} \circ\left(f_{1}, f_{1}\right), f_{1}\right) \\
& \left.-\left(\lambda_{2}^{\prime}+\lambda_{2}^{\prime(12)}\right) \circ\left(f_{1}, f_{2}\right)+\lambda_{2 ; 1}^{\prime} \circ\left(f_{1}, f_{1} \circ \lambda_{2}-\lambda_{2}^{\prime} \circ\left(f_{1}, f_{1}\right)\right)\right)^{\sigma} \\
& =\bar{f}_{1} \circ \bar{\lambda}_{3}-\bar{\lambda}_{3}^{\prime} \circ\left(\bar{f}_{1}, \bar{f}_{1}, \bar{f}_{1}\right) \\
& +\frac{1}{12} \sum_{\sigma \in \mathbb{S}_{3}}(-1)^{|\sigma|} \cdot\left(2 f_{2} \circ \circ_{1} \lambda_{2}-4 f_{2} \circ \circ_{2} \lambda_{2}\right)^{\sigma} \\
& +\frac{1}{24} \sum_{\sigma \in \mathbb{S}_{3}}(-1)^{|\sigma|} \cdot\left(\left(f_{2}+f_{2}^{(12)}\right) \circ_{1} \lambda_{2}+\left(f_{2}+f_{2}^{(12)}\right) \circ_{2} \lambda_{2}\right)^{\sigma} \\
& +\frac{1}{12} \sum_{\sigma \in \mathbb{S}_{3}}(-1)^{|\sigma|} \cdot\left(2 \lambda_{2}^{\prime} \circ\left(f_{2}, f_{1}\right)-4 \lambda_{2}^{\prime} \circ\left(f_{1}, f_{2}\right)\right)^{\sigma} \\
& +\frac{1}{24} \sum_{\sigma \in \mathbb{S}_{3}}(-1)^{|\sigma|} \cdot\left(\left(\lambda_{2}^{\prime}+\lambda_{2}^{(12)}\right) \circ\left(f_{2}, f_{1}\right)+\left(\lambda_{2}^{\prime}+\lambda_{2}^{(12)}\right) \circ\left(f_{1}, f_{2}\right)\right)^{\sigma} \\
& =\bar{f}_{1} \circ \bar{\lambda}_{3}+\bar{f}_{2} \circ{ }_{1} \bar{\lambda}_{2}-\bar{f}_{2} \circ_{2} \bar{\lambda}_{2}+\left(\bar{f}_{2} \circ_{2} \bar{\lambda}_{2}\right)^{(12)} \\
& +\bar{\lambda}_{2}^{\prime} \circ\left(\bar{f}_{2}, \bar{f}_{1}\right)-\bar{\lambda}_{2}^{\prime} \circ\left(\bar{f}_{1}, \bar{f}_{2}\right)+\bar{\lambda}_{2}^{\prime} \circ\left(\bar{f}_{1}, \bar{f}_{2}\right)^{(12)}-\bar{\lambda}_{3}^{\prime} \circ\left(\bar{f}_{1}, \bar{f}_{1}, \bar{f}_{1}\right) \text {. }
\end{aligned}
$$

A trick to showing Equation (118) is to define

$$
\begin{aligned}
\bar{f}_{4}:=\frac{1}{24} \sum_{\sigma \in \mathbb{S}_{4}}(-1)^{|\sigma|} \cdot f_{4}^{\sigma} & \\
+\frac{1}{48} \sum_{\sigma \in \mathbb{S}_{4}}(-1)^{|\sigma|} \cdot & \left(f_{2 ; 1} \circ \circ_{1} \lambda_{3}-f_{2 ; 1} \circ_{2} \lambda_{3}-\lambda_{2 ; 1}^{\prime} \circ\left(f_{3}, f_{1}\right)+\lambda_{2 ; 1}^{\prime} \circ\left(f_{1}, f_{3}\right)\right. \\
& \quad-f_{3 ; 1} \circ_{1} \lambda_{2}-f_{3 ; 1} \circ_{2} \lambda_{2}-\lambda_{3 ; 1}^{\prime} \circ\left(f_{2}, f_{1}, f_{1}\right)-\lambda_{3 ; 1}^{\prime} \circ\left(f_{1}, f_{2}, f_{1}\right) \\
& \left.+f_{3 ; 2} \circ_{2} \lambda_{2}+f_{3 ; 2} \circ_{3} \lambda_{2}+\lambda_{3 ; 2}^{\prime} \circ\left(f_{1}, f_{2}, f_{1}\right)+\lambda_{3 ; 2}^{\prime} \circ\left(f_{1}, f_{1}, f_{2}\right)\right)^{\sigma},
\end{aligned}
$$

which for degree reasons must vanish, and verify $0=\partial\left(\bar{f}_{4}\right)$ 


$$
\begin{aligned}
& =\frac{1}{24} \sum_{\sigma \in \mathbb{S}_{4}}(-1)^{|\sigma|} \cdot \partial\left(f_{4}\right)^{\sigma} \\
& +\frac{1}{48} \sum_{\sigma \in \mathbb{S}_{4}}(-1)^{|\sigma|} \cdot\left(\partial\left(f_{2 ; 1}\right) \circ \circ_{1} \lambda_{3}+f_{2 ; 1} \circ \circ_{1} \partial\left(\lambda_{3}\right)-\partial\left(\lambda_{2 ; 1}^{\prime}\right) \circ\left(f_{3}, f_{1}\right)+\lambda_{2 ; 1}^{\prime} \circ\left(\partial\left(f_{3}\right), f_{1}\right)\right. \\
& -\partial\left(f_{2 ; 1}\right) \circ_{2} \lambda_{3}-f_{2 ; 1} \circ_{2} \partial\left(\lambda_{3}\right)+\partial\left(\lambda_{2 ; 1}^{\prime}\right) \circ\left(f_{1}, f_{3}\right) \\
& -\lambda_{2 ; 1}^{\prime} \circ\left(f_{1}, \partial\left(f_{3}\right)\right)-\partial\left(f_{3 ; 1}\right) \circ{ }_{1} \lambda_{2}-\partial\left(\lambda_{3 ; 1}^{\prime}\right) \circ\left(f_{2}, f_{1}, f_{1}\right) \\
& -\lambda_{3 ; 1}^{\prime} \circ\left(\partial\left(f_{2}\right), f_{1}, f_{1}\right)-\partial\left(f_{3 ; 1}\right) \circ_{2} \lambda_{2}-\partial\left(\lambda_{3 ; 1}^{\prime}\right) \circ\left(f_{1}, f_{2}, f_{1}\right) \\
& -\lambda_{3 ; 1}^{\prime} \circ\left(f_{1}, \partial\left(f_{2}\right), f_{1}\right)+\partial\left(f_{3 ; 2}\right) \circ_{2} \lambda_{2}+\partial\left(\lambda_{3 ; 2}^{\prime}\right) \circ\left(f_{1}, f_{2}, f_{1}\right) \\
& +\lambda_{3 ; 2}^{\prime} \circ\left(f_{1}, \partial\left(f_{2}\right), f_{1}\right)+\partial\left(f_{3 ; 2}\right) \circ_{3} \lambda_{2}+\partial\left(\lambda_{3 ; 2}^{\prime}\right) \circ\left(f_{1}, f_{1}, f_{2}\right) \\
& \left.+\lambda_{3 ; 2}^{\prime} \circ\left(f_{1}, f_{1}, \partial\left(f_{2}\right)\right)\right)^{\sigma} \\
& =\frac{1}{24} \sum_{\sigma \in \mathbb{S}_{4}}(-1)^{|\sigma|} \cdot\left(f_{1} \circ \lambda_{4}-\lambda_{4}^{\prime} \circ\left(f_{1}, f_{1}, f_{1}, f_{1}\right)\right)^{\sigma} \\
& -\frac{1}{24} \sum_{\sigma \in \mathbb{S}_{4}}(-1)^{|\sigma|} \cdot\left(f_{2} \circ_{1} \lambda_{3}+3 f_{2} \circ_{2} \lambda_{3}\right)^{\sigma} \\
& +\frac{1}{48} \sum_{\sigma \in \mathbb{S}_{4}}(-1)^{|\sigma|} \cdot\left(\left(-f_{2}-f_{2}^{(12)}+f_{1} \circ \lambda_{2 ; 1}-\lambda_{2 ; 1}^{\prime} \circ\left(f_{1}, f_{1}\right)\right) \circ{ }_{1} \lambda_{3}\right. \\
& \left.-\left(-f_{2}-f_{2}^{(12)}+f_{1} \circ \lambda_{2 ; 1}-\lambda_{2 ; 1}^{\prime} \circ\left(f_{1}, f_{1}\right)\right) \circ_{2} \lambda_{3}\right)^{\sigma} \\
& +\frac{1}{24} \sum_{\sigma \in \mathbb{S}_{4}}(-1)^{|\sigma|} \cdot\left(f_{3} \circ_{1} \lambda_{2}-2 f_{3} \circ_{2} \lambda_{2}+3 f_{3} \circ_{3} \lambda_{2}\right)^{\sigma} \\
& -\frac{1}{48} \sum_{\sigma \in \mathbb{S}_{4}}(-1)^{|\sigma|} \cdot\left(\quad \left(-f_{3}-f_{3}^{(12)}+f_{1} \circ \lambda_{3 ; 1}-\lambda_{3 ; 1}^{\prime} \circ\left(f_{1}, f_{1}, f_{1}\right)\right.\right. \\
& \left.-f_{2} \circ \lambda_{2 ; 1}-\lambda_{2}^{\prime} \circ\left(f_{2 ; 1}, f_{1}\right)\right) \circ_{1} \lambda_{2} \\
& -\left(-f_{3}-f_{3}^{(12)}+f_{1} \circ \lambda_{3 ; 1}-\lambda_{3 ; 1}^{\prime} \circ\left(f_{1}, f_{1}, f_{1}\right)\right. \\
& \left.\left.-f_{2} \circ \lambda_{1} \lambda_{2 ; 1}-\lambda_{2}^{\prime} \circ\left(f_{2 ; 1}, f_{1}\right)\right) \circ_{2} \lambda_{2}\right)^{\sigma} \\
& +\frac{1}{48} \sum_{\sigma \in \mathbb{S}_{4}}(-1)^{|\sigma|} \cdot\left(\quad \left(-f_{3}-f_{3}^{(23)}+f_{1} \circ \lambda_{3 ; 2}-\lambda_{3 ; 2}^{\prime} \circ\left(f_{1}, f_{1}, f_{1}\right)\right.\right. \\
& -f_{2 ; 1} \circ_{1} \lambda_{2}+f_{2} \circ_{2} \lambda_{2 ; 1}-\left(f_{2 ; 1} \circ_{2} \lambda_{2}\right)^{(12)} \\
& \left.+\lambda_{2 ; 1}^{\prime} \circ\left(f_{2}, f_{1}\right)+\lambda_{2}^{\prime} \circ\left(f_{1}, f_{2 ; 1}\right)+\lambda_{2 ; 1}^{\prime} \circ\left(f_{1}, f_{2}\right)^{(12)}\right) \circ_{2} \lambda_{2} \\
& -\left(-f_{3}-f_{3}^{(23)}+f_{1} \circ \lambda_{3 ; 2}-\lambda_{3 ; 2}^{\prime} \circ\left(f_{1}, f_{1}, f_{1}\right)\right. \\
& -f_{2 ; 1} \circ_{1} \lambda_{2}+f_{2} \circ_{2} \lambda_{2 ; 1}-\left(f_{2 ; 1} \circ_{2} \lambda_{2}\right)^{(12)} \\
& \left.\left.+\lambda_{2 ; 1}^{\prime} \circ\left(f_{2}, f_{1}\right)+\lambda_{2}^{\prime} \circ\left(f_{1}, f_{2 ; 1}\right)+\lambda_{2 ; 1}^{\prime} \circ\left(f_{1}, f_{2}\right)^{(12)}\right) \circ_{3} \lambda_{2}\right)^{\sigma} \\
& -\frac{1}{24} \sum_{\sigma \in \mathbb{S}_{4}}(-1)^{|\sigma|} \cdot\left(\lambda_{2}^{\prime} \circ\left(f_{3}, f_{1}\right)+\lambda_{2}^{\prime} \circ\left(f_{1}, f_{3}\right)\right)^{\sigma} \\
& -\frac{1}{48} \sum_{\sigma \in \mathbb{S}_{4}}(-1)^{|\sigma|} \cdot\left(\left(\lambda_{2}^{\prime}+\lambda_{2}^{(12)}\right) \circ\left(f_{3}, f_{1}\right)-\left(\lambda_{2}^{\prime}+\lambda_{2}^{(12)}\right) \circ\left(f_{1}, f_{3}\right)\right)^{\sigma} \\
& -\frac{1}{24} \sum_{\sigma \in \mathbb{S}_{4}}(-1)^{|\sigma|} \cdot\left(\lambda_{3}^{\prime} \circ\left(f_{2}, f_{1}, f_{1}\right)-2 \lambda_{3}^{\prime} \circ\left(f_{1}, f_{2}, f_{1}\right)+3 \lambda_{3}^{\prime} \circ\left(f_{1}, f_{1}, f_{2}\right)\right)^{\sigma}
\end{aligned}
$$




$$
\begin{aligned}
& -\frac{1}{48} \sum_{\sigma \in \mathbb{S}_{4}}(-1)^{|\sigma|} \cdot\left(\left(\lambda_{3}^{\prime}+\lambda_{3}^{(12)}+\lambda_{2}^{\prime} \circ_{1} \lambda_{2 ; 1}^{\prime}\right) \circ\left(f_{2}, f_{1}, f_{1}\right)\right. \\
& \left.+\left(\lambda_{3}^{\prime}+\lambda_{3}^{\prime(12)}+\lambda_{2}^{\prime} \circ_{1} \lambda_{2 ; 1}^{\prime}\right) \circ\left(f_{1}, f_{2}, f_{1}\right)\right)^{\sigma} \\
& +\frac{1}{48} \sum_{\sigma \in \mathbb{S}_{4}}(-1)^{|\sigma|} \cdot\left(\left(\lambda_{3}^{\prime}+\lambda_{3}^{\prime(23)}+\lambda_{2 ; 1}^{\prime} \circ_{1} \lambda_{2}^{\prime}-\lambda_{2}^{\prime} \circ_{2} \lambda_{2 ; 1}^{\prime}+\left(\lambda_{2 ; 1}^{\prime} \circ_{2} \lambda_{2}^{\prime}\right)^{(12)}\right) \circ\left(f_{1}, f_{2}, f_{1}\right)\right. \\
& +\left(\lambda_{3}^{\prime}+\lambda_{3}^{\prime(23)}+\lambda_{2 ; 1}^{\prime} \circ_{1} \lambda_{2}^{\prime}-\lambda_{2}^{\prime} \circ_{2} \lambda_{2 ; 1}^{\prime}+\left(\lambda_{2 ; 1}^{\prime} \circ_{2} \lambda_{2}^{\prime}\right)^{(12)}\right) \\
& \left.\cdots \circ\left(f_{1}, f_{1}, f_{2}\right)\right)^{\sigma} \\
& +\frac{1}{24} \sum_{\sigma \in \mathbb{S}_{4}}(-1)^{|\sigma|} \cdot\left(3 \lambda_{2}^{\prime} \circ\left(f_{2}, f_{2}\right)\right)^{\sigma} \\
& +\frac{1}{48} \sum_{\sigma \in \mathbb{S}_{4}}(-1)^{|\sigma|} \cdot\left(f_{2 ; 1} \circ_{1}\left(-\lambda_{2}^{\prime} \circ_{1} \lambda_{2}^{\prime}+2 \lambda_{2}^{\prime} \circ_{2} \lambda_{2}^{\prime}\right)\right. \\
& \left.-f_{2 ; 1} \circ_{2}\left(-\lambda_{2}^{\prime} \circ_{1} \lambda_{2}^{\prime}+2 \lambda_{2}^{\prime} \circ_{2} \lambda_{2}^{\prime}\right)\right)^{\sigma} \\
& +\frac{1}{48} \sum_{\sigma \in \mathbb{S}_{4}}(-1)^{|\sigma|} \cdot\left(\lambda _ { 2 ; 1 } ^ { \prime } \circ \left(\left(f_{1} \circ \lambda_{3}+f_{2} \circ \lambda_{1} \lambda_{2}-2 f_{2} \circ{ }_{2} \lambda_{2}+\lambda_{2}^{\prime} \circ\left(f_{2}, f_{1}\right)\right.\right.\right. \\
& \left.\left.-2 \lambda_{2}^{\prime} \circ\left(f_{1}, f_{2}\right)-\lambda_{3}^{\prime} \circ\left(f_{1}, f_{1}, f_{1}\right)\right), f_{1}\right) \\
& -\lambda_{2 ; 1}^{\prime} \circ\left(f_{1},\left(f_{1} \circ \lambda_{3}+f_{2} \circ{ }_{1} \lambda_{2}-2 f_{2} \circ \circ_{2} \lambda_{2}+\lambda_{2}^{\prime} \circ\left(f_{2}, f_{1}\right)\right.\right. \\
& \left.\left.\left.-2 \lambda_{2}^{\prime} \circ\left(f_{1}, f_{2}\right)-\lambda_{3}^{\prime} \circ\left(f_{1}, f_{1}, f_{1}\right)\right)\right)\right)^{\sigma} \\
& -\frac{1}{48} \sum_{\sigma \in \mathbb{S}_{4}}(-1)^{|\sigma|} \cdot\left(\lambda_{3 ; 1}^{\prime} \circ\left(f_{1} \circ \lambda_{2}-\lambda_{2}^{\prime} \circ\left(f_{1}, f_{1}\right), f_{1}, f_{1}\right)\right. \\
& \left.+\lambda_{3 ; 1}^{\prime} \circ\left(f_{1}, f_{1} \circ \lambda_{2}-\lambda_{2}^{\prime} \circ\left(f_{1}, f_{1}\right), f_{1}\right)\right)^{\sigma} \\
& +\frac{1}{48} \sum_{\sigma \in \mathbb{S}_{4}}(-1)^{|\sigma|} \cdot\left(\lambda_{3 ; 2}^{\prime} \circ\left(f_{1}, f_{1} \circ \lambda_{2}-\lambda_{2}^{\prime} \circ\left(f_{1}, f_{1}\right), f_{1}\right)\right. \\
& \left.+\lambda_{3 ; 2}^{\prime} \circ\left(f_{1}, f_{1}, f_{1} \circ \lambda_{2}-\lambda_{2}^{\prime} \circ\left(f_{1}, f_{1}\right)\right)\right)^{\sigma} \\
& =\bar{f}_{1} \circ \bar{\lambda}_{4}-\bar{f}_{2} \circ \bar{\lambda}_{3}-\bar{f}_{2} \circ_{2} \bar{\lambda}_{3}+\left(\bar{f}_{2} \circ_{2} \bar{\lambda}_{3}\right)^{(12)}-\left(\bar{f}_{2} \circ_{2} \bar{\lambda}_{3}\right)^{(123)}+\bar{f}_{3} \circ_{1} \bar{\lambda}_{2}-\bar{f}_{3} \circ_{2} \bar{\lambda}_{2} \\
& +\left(\bar{f}_{3} \circ_{2} \bar{\lambda}_{2}\right)^{(12)}+\bar{f}_{3} \circ_{3} \bar{\lambda}_{2}-\left(\bar{f}_{3} \circ_{3} \bar{\lambda}_{2}\right)^{(23)}+\left(\bar{f}_{3} \circ_{3} \bar{\lambda}_{2}\right)^{(132)}-\bar{\lambda}_{2}^{\prime} \circ\left(\bar{f}_{3}, \bar{f}_{1}\right) \\
& -\bar{\lambda}_{2}^{\prime} \circ\left(\bar{f}_{1}, \bar{f}_{3}\right)+\bar{\lambda}_{2}^{\prime} \circ\left(\bar{f}_{1}, \bar{f}_{3}\right)^{(12)}-\bar{\lambda}_{2}^{\prime} \circ\left(\bar{f}_{1}, \bar{f}_{3}\right)^{(123)}+\bar{\lambda}_{2}^{\prime} \circ\left(\bar{f}_{2}, \bar{f}_{2}\right) \\
& -\bar{\lambda}_{2}^{\prime} \circ\left(\bar{f}_{2}, \bar{f}_{2}\right)^{(23)}+\bar{\lambda}_{2}^{\prime} \circ\left(\bar{f}_{2}, \bar{f}_{2}\right)^{(132)}-\bar{\lambda}_{3}^{\prime} \circ\left(\bar{f}_{2}, \bar{f}_{1}, \bar{f}_{1}\right)+\bar{\lambda}_{3}^{\prime} \circ\left(\bar{f}_{1}, \bar{f}_{2}, \bar{f}_{1}\right) \\
& -\bar{\lambda}_{3}^{\prime} \circ\left(\bar{f}_{1}, \bar{f}_{2}, \bar{f}_{1}\right)^{(12)}-\bar{\lambda}_{3}^{\prime} \circ\left(\bar{f}_{1}, \bar{f}_{1}, \bar{f}_{2}\right)+\bar{\lambda}_{3}^{\prime} \circ\left(\bar{f}_{1}, \bar{f}_{1}, \bar{f}_{2}\right)^{(23)}-\bar{\lambda}_{3}^{\prime} \circ\left(\bar{f}_{1}, \bar{f}_{1}, \bar{f}_{2}\right)^{(132)} \\
& -\bar{\lambda}_{4}^{\prime} \circ\left(\bar{f}_{1}, \bar{f}_{1}, \bar{f}_{1}, \bar{f}_{1}\right) \text {. }
\end{aligned}
$$

This concludes the proof of Lemma 3.3.1 


\section{Bibliography}

[1] Camilo Arias Abad, Marius Crainic, and Benoit Dherin. "Tensor products of representations up to homotopy”. In: Journal of Homotopy and Related Structures 6.2 (2011), pp. 239-288.

[2] John Baez and Alissa Crans. "Higher-dimensional algebra VI: Lie 2-algebras". In: Theory and Applications of Categories 12.15 (2004), pp. 492-538.

[3] John Baez, Alexander Hoffnung, and Christopher Rogers. "Categorified symplectic geometry and the classical string”. In: Communications in Mathematical Physics 293.3 (2010), pp. 701-725.

[4] Clemens Berger and Benoit Fresse. "Combinatorial operad actions on cochains". In: Mathematical Proceedings of the Cambridge Philosophical Society 137.1 (2004), pp. 135-174.

[5] Clemens Berger and Ieke Moerdijk. "Axiomatic homotopy theory for operads". In: Commentarii Mathematici Helvetici 78.4 (2003), pp. 805-831.

[6] Clemens Berger and Ieke Moerdijk. "The Boardman-Vogt resolution of operads in monoidal model categories". In: Topology 45.5 (2006), pp. 807-849.

[7] Clemens Berger and Ieke Moerdijk. "Resolution of coloured operads and rectification of homotopy algebras". In: Categories in algebra, geometry and mathematical physics. Vol. 431. Contemporary Mathematics. American Mathematical Society, 2007, pp. 31-58.

[8] J. Michael Boardman and Rainer M. Vogt. Homotopy invariant algebraic structures on topological spaces. Lecture Notes in Mathematics 347. Springer-Verlag, 1973.

[9] Michael Brinkmeier. "On Operads". PhD thesis. 2000.

[10] Claude Chevalley and Samuel Eilenberg. "Cohomology theory of Lie groups and Lie algebras". In: Transactions of the American Mathematical Society 63 (1948), pp. 85-124.

[11] Malte Dehling. “On weak Lie 3-algebras”. In: Preprint (2017). arXiv: 1710.11104

[12] Malte Dehling and Bruno Vallette. "Symmetric homotopy theory for operads". In: Preprint (2015). arXiv: 1503.02701 To appear in: "Algebraic \& Geometric Topology".

[13] Vladimir Drinfeld. Letter to Schechtman. 1988.

[14] Samuel Eilenberg and Saunders Mac Lane. "On the groups of $H(\Pi, n)$. I". In: Annals of Mathematics. 2nd ser. 58 (1953), pp. 55-106.

[15] Giovanni Faonte. "Simplicial nerve of an A-infinity category". In: Theory and Applications of Categories 32.2 (2017), pp. 31-52. 
[16] Benoit Fresse. "Koszul duality of operads and homology of partition posets". In: Homotopy theory and its applications. Vol. 346. Contemporary Mathematics. American Mathematical Society, 2004, pp. 115-215.

[17] Benoit Fresse. "The universal Hopf operads of the bar construction". In: Preprint (2007). arXiv: 0701245

[18] Benoit Fresse. Modules over operads and functors. Lecture Notes in Mathematics 1967. Springer-Verlag, 2009.

[19] Benoit Fresse. "The bar complex of an E-infinity algebra”. In: Advances in Mathematics 223.6 (2010), pp. 2049-2096.

[20] Benoit Fresse. "Iterated bar complexes of $E$-infinity algebras and homology theories”. In: Algebraic \& Geometric Topology 11.2 (2011), pp. 747-838.

[21] Benoit Fresse. "Koszul duality of $E_{n}$-operads". In: Selecta Mathematica. New ser. 17.2 (2011), pp. 363-434.

[22] Benoit Fresse. Homotopy of operads and Grothendieck-Teichmüller groups. Part 2. Mathematical Surveys and Monographs 217. American Mathematical Society, 2017.

[23] Ezra Getzler. "Lie theory for nilpotent $L_{\infty}$-algebras". In: Annals of Mathematics. 2nd ser. 170.1 (2009), pp. 271-301.

[24] Ezra Getzler and John D.S. Jones. "Operads, homotopy algebra and iterated integrals for double loop spaces”. In: Preprint (1994). arXiv: hep-th/9403055.

[25] Victor Ginzburg and Mikhail Kapranov. "Koszul duality for operads”. In: Duke Mathematical Journal 76.1 (1994), pp. 203-272.

[26] Javier J. Gutiérrez et al. "Motivic slices and coloured operads". In: Journal of Topology 5.3 (2012), pp. 727-755.

[27] David K. Harrison. "Commutative algebras and cohomology". In: Transactions of the American Mathematical Society 104 (1962), pp. 191-204.

[28] Vladimir Hinich. "Homological algebra of homotopy algebras". In: Communications in Algebra 25.10 (1997), pp. 3291-3323.

[29] Vladimir Hinich. "Erratum to 'Homological algebra of homotopy algebras"' In: Preprint (2003). arXiv: math/0309453

[30] Vladimir Hinich and Vadim Schechtman. "Homotopy Lie algebras”. In: I. M. Gelfand Seminar. Vol. 16. Advances in Soviet Mathematics. American Mathematical Society, 1993, pp. 1-28.

[31] Joseph Hirsh and Joan Millès. "Curved Koszul duality theory”. In: Mathematische Annalen 354.4 (2012), pp. 1465-1520.

[32] Mark Hovey. Model categories. Mathematical Surveys and Monographs. American Mathematical Society, 1999.

[33] Tornike Kadeishvili. "The structure of the $A(\infty)$-algebra, and the Hochschild and Harrison cohomologies". In: Trudy Tbiliss. Mat. Inst. Razmadze Akad. Nauk Gruzin. SSR 91 (1988), pp. 19-27.

[34] Mikhail Kapranov. "Operads and algebraic geometry". In: Extra Vol., ICM Berlin 1998. Vol. II. Documenta Mathematica. 1998, pp. 277-286. 
[35] Max Kelly. Basic concepts of enriched category theory. Lecture Notes in Mathematics 64. Cambridge University Press, 1982.

[36] David Khudaverdyan, Norbert Poncin, and Jian Qiu. "On the infinity category of homotopy Leibniz algebras”. In: Theory and Applications of Categories 29.12 (2014), pp. 332-370.

[37] Joachim Kock. “Polynomial functors and trees”. In: International Mathematics Research Notices 2011.3 (2010), pp. 609-673.

[38] Maxim Kontsevich. "Deformation quantization of Poisson manifolds". In: Letters in Mathematical Physics 66.3 (2003), pp. 157-216.

[39] Pepijn van der Laan. "Coloured Koszul duality and strongly homotopy operads". In: Preprint (2003). arXiv: math.QA/0312147.

[40] Brice Le Grignou. “Théories homotopiques des algèbres unitaires et des opérades”. PhD thesis. 2016.

[41] Brice Le Grignou. "From homotopy operads to infinity-operads". In: Journal of Noncommutative Geometry 11.1 (2017), pp. 309-366.

[42] Brice Le Grignou. "Homotopy theory of unital algebras". In: Algebraic \& Geometric Topology 19.3 (2019), pp. 1541-1618.

[43] Kenji Lefevre-Hasegawa. "Sur les A-infini catégories". In: Preprint (2003). arXiv: math/0310337

[44] Jiefeng Liu and Yunhe Sheng. "QP-structures of degree 3 and CLWX2-algebroids”. In: Journal of Symplectic Geometry 17.6 (2019).

[45] Jean-Louis Loday. "Cup-product for Leibniz cohomology and dual Leibniz algebras”. In: Mathematica Scandinavica 77.2 (1995), pp. 189-196.

[46] Jean-Louis Loday and Bruno Vallette. Algebraic operads. Grundlehren der Mathematischen Wissenschaften 346. Springer-Verlag, 2012.

[47] Jacob Lurie. Higher algebra. Preprint, 2014.

[48] Michael A. Mandell. "Cochains and homotopy type”. In: Publications mathématiques de l'IHÉS 103 (2006), pp. 213-246.

[49] Martin Markl. "Models for operads”. In: Communications in Algebra 24.4 (1996), pp. 1471-1500.

[50] Martin Markl. "An E-infty-extension of the associahedra and the Tamarkin cell mystery”. In: Journal of Geometry and Physics 98 (2015), pp. 446-471.

[51] Martin Markl, Steve Shnider, and Jim Stasheff. Operads in algebra, topology and physics. Mathematical Surveys and Monographs 96. American Mathematical Society, 2002.

[52] J. Peter May. The geometry of iterated loop spaces. Springer-Verlag, 1972.

[53] Joan Millès. “The Koszul complex is the cotangent complex”. In: International Mathematical Research Notices 2012.3 (2012), pp. 607-650.

[54] Ieke Moerdijk and Bertrand Toën. Simplicial methods for operads and algebraic geometry. Advanced Courses in Mathematics - CRM Barcelona. Springer-Verlag, 2010. 
[55] Ieke Moerdijk and Ittay Weiss. "On inner Kan complexes in the category of dendroidal sets”. In: Advances in Mathematics 221.2 (2009), pp. 343-389.

[56] Behrang Noohi. "Integrating morphisms of Lie 2-algebras". In: Compositio Mathematica 149.2 (2013), pp. 264-294.

[57] Alexander Polishchuk and Leonid Positselski. Quadratic algebras. University Lecture Series 37. American Mathematical Society, 2005.

[58] Leonid Positselski. "Nonhomogeneous quadratic duality and curvature". In: Functional Analysis and its Applications 27.3 (1993), pp. 197-204.

[59] Daniel Quillen. Homotopical algebra. Lecture Notes in Mathematics 43. SpringerVerlag, 1967.

[60] Daniel Quillen. "Rational homotopy theory”. In: Annals of Mathematics. 2nd ser. 90 (1969), pp. 205-295.

[61] Daniel Robert-Nicoud and Felix Wierstra. "Homotopy morphisms between convolution homotopy Lie algebras”. In: Journal of Noncommutative Geometry 13.4 (2020), pp. 1463-1520.

[62] Christopher Rogers. "Higher symplectic geometry”. PhD thesis. 2011.

[63] Dmitry Roytenberg. "On weak Lie 2-algebras". In: Geometrical Methods in Physics. Vol. 956. American Institute of Physics Conference Series. 2007, pp. 180-198.

[64] Markus Spitzweck. "Operads, Algebras and Modules in General Model Categories”. PhD thesis. 2001.

[65] James D. Stasheff. "Homotopy associativity of H-spaces I, II”. In: Transactions of the American Mathematical Society 108 (1963), pp. 293-312.

[66] Dennis Sullivan. "Infinitesimal computations in topology". In: Publications mathématiques de l'IHÉS 47 (1977), pp. 269-331.

[67] Bruno Vallette. "A Koszul duality for props". In: Transactions of the American Mathematical Society 359 (2007), pp. 4865-4993.

[68] Bruno Vallette. "Free monoid in monoidal abelian categories". In: Appl. Categ. Structures 17.1 (2009), pp. 43-61.

[69] Bruno Vallette. "Homotopy theory of homotopy algebras". In: Annales de l'Institute Fourier 70.2 (2020), pp. 683-738.

[70] Felix Wierstra. "Algebraic Hopf invariants and rational models for mapping spaces”. In: Journal of Homotopy and Related Structures 14 (2019), pp. 719-747. 
APPENDIX A

Curriculum Vitae 



\section{Malte Dehling}

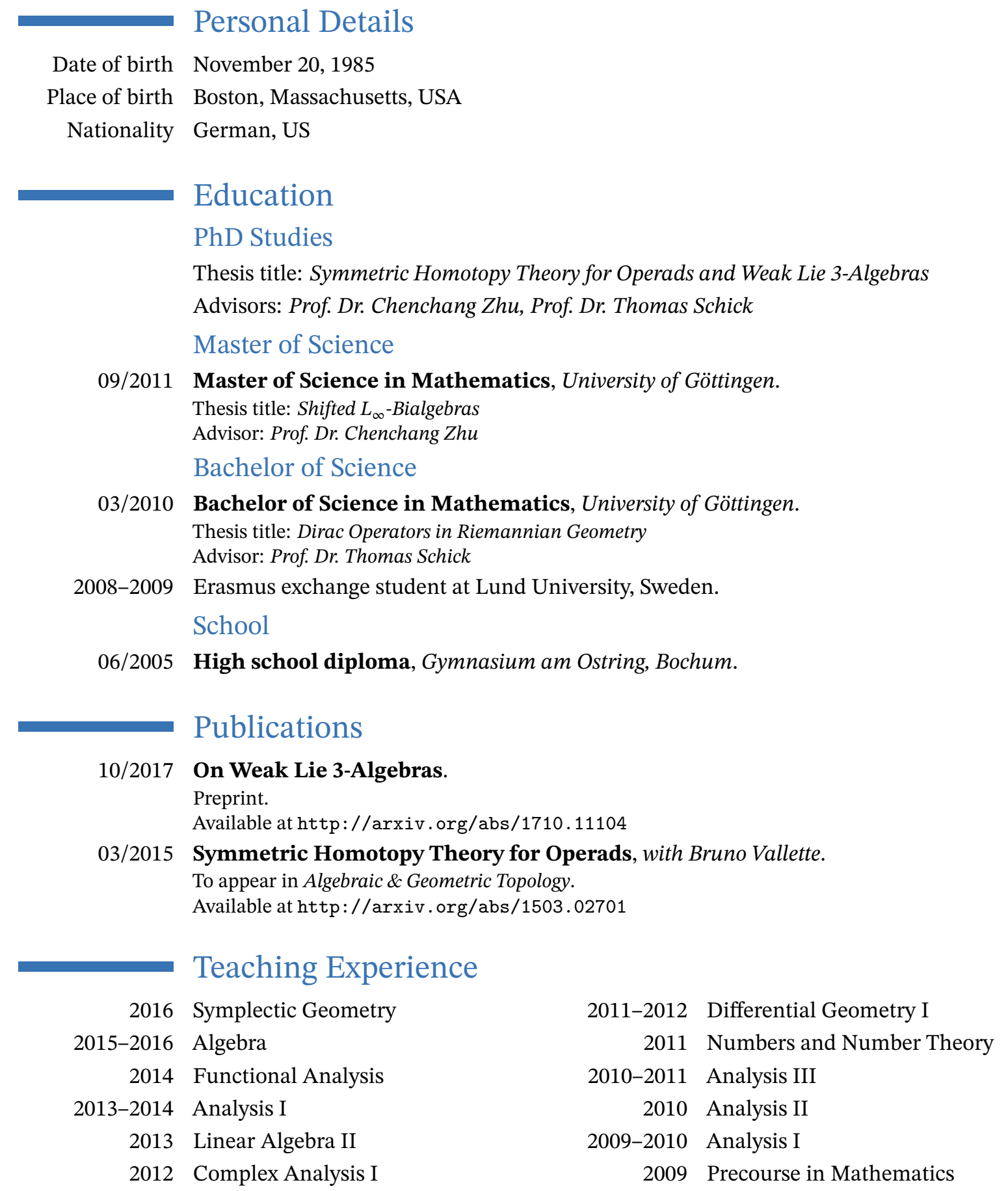

